Cochrane Database of Systematic Reviews

\title{
Certolizumab pegol (CDP870) for rheumatoid arthritis in adults
} (Review)

Ruiz Garcia V, Burls A, Cabello JB, Vela Casasempere P, Bort-Marti S, Bernal JA

Ruiz Garcia V, Burls A, Cabello JB, Vela Casasempere P, Bort-Marti S, Bernal JA. Certolizumab pegol (CDP870) for rheumatoid arthritis in adults.

Cochrane Database of Systematic Reviews 2017, Issue 9. Art. No.: CD007649. DOI: 10.1002/14651858.CD007649.pub4.

www.cochranelibrary.com 
TABLE OF CONTENTS

HEADER

ABSTRACT

PLAIN LANGUAGE SUMMARY

SUMMARY OF FINDINGS

2

BACKGROUND

OBJECTIVES

METHODS

RESULTS

Figure 1.

Figure 2.

Figure 3.

Figure 4.

DISCUSSION

AUTHORS' CONCLUSIONS

ACKNOWLEDGEMENTS

REFERENCES

CHARACTERISTICS OF STUDIES

DATA AND ANALYSES

Analysis 1.1. Comparison 1 Efficacy at 12 weeks, any dose, Outcome 1 ACR20.

Analysis 1.2. Comparison 1 Efficacy at 12 weeks, any dose, Outcome 2 ACR50.

Analysis 1.3. Comparison 1 Efficacy at 12 weeks, any dose, Outcome 3 ACR70.

Analysis 2.1. Comparison 2 ACR50 24 weeks, 200 mg certolizumab pegol, Outcome 1 ACR 50.

Analysis 3.1. Comparison 3 ACR50 at 24 weeks, $400 \mathrm{mg}$ certolizumab, Outcome 1 ACR 50.

Analysis 4.1. Comparison 4 ACR50 at 52 weeks, $200 \mathrm{mg}$ certolizumab, Outcome 1 ACR 50.

Analysis 5.1. Comparison 5 ACR50 at 52 weeks, $400 \mathrm{mg}$ certolizumab, Outcome 1 ACR 50.

Analysis 6.1. Comparison 6 Mean HAQ-DI from baseline at week 12, Outcome 1 certolizumab pegol $200 \mathrm{mg} \mathrm{sc}$.

Analysis 7.1. Comparison 7 Mean HAQ-DI from baseline at week 24, Outcome 1 certolizumab pegol $200 \mathrm{mg} \mathrm{sc.}$

Analysis 7.2. Comparison 7 Mean HAQ-DI from baseline at week 24, Outcome 2 certolizumab $400 \mathrm{mg} \mathrm{sc}$.

Analysis 8.1. Comparison $8 \mathrm{HAQ}-\mathrm{DI}$ at 24 weeks, any dose, Outcome 1 Change from baseline.

Analysis 9.1. Comparison 9 HAQ-DI at 52 weeks, any dose, Outcome 1 Change from baseline.

Analysis 10.1. Comparison 10 SF-36 Physical Component Summary (PCS), week 24, Outcome 1 certolizumab pegol $200 \mathrm{mg}$ sc. .

Analysis 10.2. Comparison 10 SF-36 Physical Component Summary (PCS), week 24, Outcome 2 certolizumab pegol $400 \mathrm{mg} \mathrm{sc.}$

Analysis 11.1. Comparison 11 SF-36 Mental Component Summary (MCS), week 24, Outcome 1 certolizumab pegol $200 \mathrm{mg}$ sc. ..

Analysis 11.2. Comparison 11 SF-36 Mental Component Summary (MCS), week 24, Outcome 2 certolizumab pegol 400 mg sc. ..

Analysis 12.1. Comparison 12 SF-36 Physical Component Summary (PCS), week 52, Outcome 1 certolizumab 200 mg sc. .......

Analysis 12.2. Comparison 12 SF-36 Physical Component Summary (PCS), week 52, Outcome 2 certolizumab 400 mg sc. .......

Analysis 13.1. Comparison 13 SF-36 Mental Component Summary (MCS), week 52, Outcome 1 certolizumab pegol 200 mg sc. ..

Analysis 13.2. Comparison 13 SF-36 Mental Component Summary (MCS), week 52, Outcome 2 certolizumab pegol $400 \mathrm{mg} \mathrm{sc.} \mathrm{..}$

Analysis 14.1. Comparison 14 SF-36 Physical Component Summary (PCS) at week 24, any dose, Outcome 1 Change from baseline.

Analysis 15.1. Comparison 15 SF-36 Mental Component Summary (MCS) at week 24, any dose, Outcome 1 Change from baseline.

Analysis 16.1. Comparison 16 SF-36 Physical Component Summary (PCS) at week 52, any dose, Outcome 1 Change from baseline.

Analysis 17.1. Comparison 17 SF-36 Mental Component Summary (MCS) at week 52, any dose, Outcome 1 Change from baseline.

Analysis 18.1. Comparison 18 Disease Activity Score (DAS-28) (ESR) remission (<2.6), any doses, 12 weeks, Outcome 1 Proportion of participants achieving remission 12 weeks certolizumab $200 \mathrm{mg}$.

Analysis 19.1. Comparison 19 Disease Activity Score (DAS-28) (ESR) remission (<2.6), any dose, 24 weeks, Outcome 1 Proportion of participants achieving remission 24 weeks.

Analysis 20.1. Comparison 20 Disease Activity Score (DAS-28) (ESR) remission (<2.6), any dose, 52 weeks, Outcome 1 Proportion of participants achieving remission 52 weeks. 
Analysis 21.1. Comparison 21 Disease Activity Score (DAS-28) (ESR) remission (<2.6), any time, Outcome 1 Proportion of participants achieving remission 12 weeks certolizumab $200 \mathrm{mg}$.

Analysis 21.2. Comparison 21 Disease Activity Score (DAS-28) (ESR) remission (<2.6), any time, Outcome 2 Proportion of participants achieving remission 24 weeks certolizumab $200 \mathrm{mg}$.

Analysis 21.3. Comparison 21 Disease Activity Score (DAS-28) (ESR) remission $(<2.6)$, any time, Outcome 3 Proportion of participants achieving remission 24 weeks certolizumab $400 \mathrm{mg}$.

Analysis 21.4. Comparison 21 Disease Activity Score (DAS-28) (ESR) remission (<2.6), any time, Outcome 4 Proportion of participants achieving remission 52 weeks certolizumab $200 \mathrm{mg}$.

Analysis 21.5. Comparison 21 Disease Activity Score (DAS-28) (ESR) remission $(<2.6)$, any time, Outcome 5 Proportion of participants achieving remission 52 weeks certolizumab $400 \mathrm{mg}$.

Analysis 22.1. Comparison 22 DAS-28 at 12 weeks, $200 \mathrm{mg}$ certolizumab, Outcome 1 DAS 28 (ESR) change from baseline. ....... Analysis 23.1. Comparison 23 DAS-28 at 24 weeks, $400 \mathrm{mg}$ certolizumab, Outcome 1 DAS 28 (ESR) change from baseline. ....... Analysis 24.1. Comparison 24 DAS-28 at week 52, certolizumab $200 \mathrm{mg}$, Outcome 1 DAS 28 (ESR) Change from baseline. ........ Analysis 25.1. Comparison 25 DAS-28 at week 52, certolizumab $400 \mathrm{mg}$, Outcome 1 DAS 28 (ESR) Change from baseline. ........ Analysis 26.1. Comparison 26 DAS-28 at 24 weeks, any dose, Outcome 1 Change from baseline.

Analysis 27.1. Comparison 27 DAS-28 at 52 weeks, any dose, Outcome 1 Change from baseline.

Analysis 28.1. Comparison 28 DAS-28 at 24 weeks, $200 \mathrm{mg}$ certolizumab, Outcome 1 DAS 28 (ESR) change from baseline. ........ Analysis 29.1. Comparison 29 Erosion score (ES), Outcome 1 Change from the baseline mean ES at week 24, certolizumab pegol $200 \mathrm{mg}$.

Analysis 29.2. Comparison 29 Erosion score (ES), Outcome 2 Change from the baseline mean ES at week 24, certolizumab pegol $400 \mathrm{mg}$.

Analysis 29.3. Comparison 29 Erosion score (ES), Outcome 3 Change from the baseline mean ES at week 52, certolizumab pegol $200 \mathrm{mg}$.

Analysis 29.4. Comparison 29 Erosion score (ES), Outcome 4 Change from the baseline mean ES at week 52, certolizumab pegol $400 \mathrm{mg}$.

Analysis 30.1. Comparison 30 Erosion score (ES) at 24 weeks, any dose, Outcome 1 Change from baseline.

Analysis 31.1. Comparison 31 Erosion score (ES) at 52 weeks, any dose, Outcome 1 Change from baseline.

Analysis 32.1. Comparison 32 Joint space narrowing (JSN), Outcome 1 Change from the baseline mean JSN 24 weeks, certolizumab pegol $200 \mathrm{mg}$.

Analysis 32.2. Comparison 32 Joint space narrowing (JSN), Outcome 2 Change from the baseline mean JSN 24 weeks,certolizumab pegol $400 \mathrm{mg}$.

Analysis 32.3. Comparison 32 Joint space narrowing (JSN), Outcome 3 Change from the baseline mean JSN 52 weeks, certolizumab pegol $200 \mathrm{mg}$.

Analysis 32.4. Comparison 32 Joint space narrowing (JSN), Outcome 4 Change from the baseline mean JSN 52 weeks, certolizumab pegol $400 \mathrm{mg}$.

Analysis 33.1. Comparison 33 Joint space narrowing (JSN) at 24 weeks, any dose, Outcome 1 Change from baseline.

Analysis 34.1. Comparison 34 Joint space narrowing (JSN) at 52 weeks, any dose, Outcome 1 Change from baseline.

Analysis 35.1. Comparison 35 Modified Total Sharp Scores (mTSS) at 24 weeks, any dose, Outcome 1 Change from baseline. ... Analysis 36.1. Comparison 36 Modified Total Sharp Scores (mTSS) at 52 weeks, any dose, Outcome 1 Change from baseline. ... Analysis 37.1. Comparison 37 Modified total Sharp scores (mTSS), Outcome 1 Change from the baseline mean mTSS 24 weeks, certolizumab pegol $200 \mathrm{mg}$.

Analysis 37.2. Comparison 37 Modified total Sharp scores (mTSS), Outcome 2 Change from the baseline mean mTSS 24 weeks, certolizumab $400 \mathrm{mg}$.

Analysis 37.3. Comparison 37 Modified total Sharp scores (mTSS), Outcome 3 Change from the baseline mean mTSS 52 weeks, certolizumab pegol $200 \mathrm{mg}$.

Analysis 37.4. Comparison 37 Modified total Sharp scores (mTSS), Outcome 4 Change from the baseline mean mTSS 52 weeks, certolizumab pegol $400 \mathrm{mg}$.

Analysis 38.1. Comparison 38 Certolizumab pegol $1 \mathrm{mg} / \mathrm{kg} /$ day sc, Outcome 1 Headache.

Analysis 38.2. Comparison 38 Certolizumab pegol $1 \mathrm{mg} / \mathrm{kg} /$ day sc, Outcome 2 Lower respiratory tract infection.

Analysis 38.3. Comparison 38 Certolizumab pegol $1 \mathrm{mg} / \mathrm{kg} /$ day sc, Outcome 3 Adverse events Intensity severe.

Analysis 38.4. Comparison 38 Certolizumab pegol $1 \mathrm{mg} / \mathrm{kg} /$ day sc, Outcome 4 Antinuclear antibodies (ANA).

Analysis 38.5. Comparison 38 Certolizumab pegol $1 \mathrm{mg} / \mathrm{kg} /$ day sc, Outcome 5 Urinary tract infection.

Analysis 39.1. Comparison 39 Certolizumab $5 \mathrm{mg} / \mathrm{kg} /$ day sc, Outcome 1 Lower respiratory tract infection.

Analysis 39.2. Comparison 39 Certolizumab $5 \mathrm{mg} / \mathrm{kg} /$ day sc, Outcome 2 Urinary tract infection.

Analysis 40.1. Comparison 40 Certolizumab $20 \mathrm{mg} / \mathrm{kg} /$ day sc, Outcome 1 Headache. 
Analysis 40.2. Comparison 40 Certolizumab $20 \mathrm{mg} / \mathrm{kg} /$ day sc, Outcome 2 Lower respiratory tract infection.

Analysis 40.3. Comparison 40 Certolizumab $20 \mathrm{mg} / \mathrm{kg} /$ day sc, Outcome 3 Death.

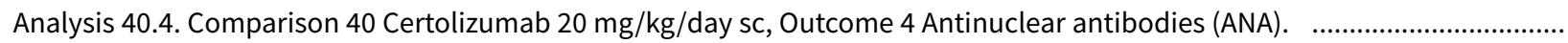

Analysis 40.5. Comparison 40 Certolizumab $20 \mathrm{mg} / \mathrm{kg} /$ day sc, Outcome 5 Urinary tract infection.

Analysis 41.1. Comparison 41 Safety, SAE certolizumab 200 mg, Outcome 1 Serious Adverse Events (SAE).

Analysis 42.1. Comparison 42 Safety, SAE certolizumab $400 \mathrm{mg}$, Outcome 1 Serious Adverse Events (SAEs).

Analysis 43.1. Comparison 43 Withdrawals, Outcome 1 All Withdrawn: any doses any follow-up.

Analysis 43.2. Comparison 43 Withdrawals, Outcome 2 Withdrawals due to adverse events.

Analysis 44.1. Comparison 44 ACR at 24 weeks, any dose, Outcome 1 ACR20.

Analysis 44.2. Comparison 44 ACR at 24 weeks, any dose, Outcome 2 ACR50.

Analysis 44.3. Comparison 44 ACR at 24 weeks, any dose, Outcome 3 ACR70.

Analysis 45.1. Comparison 45 ACR at 52 weeks, any dose, Outcome 1 ACR20.

Analysis 45.2. Comparison 45 ACR at 52 weeks, any dose, Outcome 2 ACR50.

Analysis 45.3. Comparison 45 ACR at 52 weeks, any dose, Outcome 3 ACR70.

Analysis 46.1. Comparison 46 ACR20-ACR70, 24 weeks, $200 \mathrm{mg}$ certolizumab pegol, Outcome 1 ACR 20.

Analysis 46.2. Comparison 46 ACR20-ACR70, 24 weeks, $200 \mathrm{mg}$ certolizumab pegol, Outcome 2 ACR 70.

Analysis 47.1. Comparison 47 ACR20-ACR70 at 24 weeks, $400 \mathrm{mg}$ certolizumab, Outcome 1 ACR 20.

Analysis 47.2. Comparison 47 ACR20-ACR70 at 24 weeks, $400 \mathrm{mg}$ certolizumab, Outcome 2 ACR 70.

Analysis 48.1. Comparison 48 ACR20-ACR70 at 52 weeks, $200 \mathrm{mg}$ certolizumab, Outcome 1 ACR 20.

Analysis 48.2. Comparison 48 ACR20-ACR70 at 52 weeks, $200 \mathrm{mg}$ certolizumab, Outcome 2 ACR 70.

Analysis 49.1. Comparison 49 ACR20-ACR70 at 52 weeks, $400 \mathrm{mg}$ certolizumab, Outcome 1 ACR 20

Analysis 49.2. Comparison 49 ACR20-ACR70 at 52 weeks, 400 mg certolizumab, Outcome 2 ACR 70.

Analysis 50.1. Comparison 50 Safety, Outcome 1 Any adverse event certolizumab $200 \mathrm{mg}$.

Analysis 50.2. Comparison 50 Safety, Outcome 2 Any adverse events certolizumab $400 \mathrm{mg}$.

Analysis 50.3. Comparison 50 Safety, Outcome 3 Adverse events: Intensity mild certolizumab $200 \mathrm{mg}$.

Analysis 50.4. Comparison 50 Safety, Outcome 4 Adverse events: Intensity mild certolizumab $400 \mathrm{mg}$.

Analysis 50.5. Comparison 50 Safety, Outcome 5 Adverse events: Intensity moderate certolizumab $200 \mathrm{mg}$

Analysis 50.6. Comparison 50 Safety, Outcome 6 Adverse events: Intensity moderate certolizumab $400 \mathrm{mg}$.

Analysis 50.7. Comparison 50 Safety, Outcome 7 Adverse events: Intensity severe certolizumab $200 \mathrm{mg}$.

Analysis 50.8. Comparison 50 Safety, Outcome 8 Adverse events: Intensity severe certolizumab $400 \mathrm{mg}$.

Analysis 50.9. Comparison 50 Safety, Outcome 9 Adverse events related to study drug certolizumab $200 \mathrm{mg}$.

Analysis 50.10. Comparison 50 Safety, Outcome 10 Adverse events related to study drug certolizumab $400 \mathrm{mg}$

Analysis 50.11. Comparison 50 Safety, Outcome 11 Serious Infections certolizumab $200 \mathrm{mg}$.

Analysis 50.12. Comparison 50 Safety, Outcome 12 Serious infections certolizumab $400 \mathrm{mg}$.

Analysis 50.13. Comparison 50 Safety, Outcome 13 Adverse events leading to death certolizumab $200 \mathrm{mg}$.

Analysis 50.14. Comparison 50 Safety, Outcome 14 Adverse events leading to death certolizumab $400 \mathrm{mg}$.

Analysis 50.15. Comparison 50 Safety, Outcome 15 Adverse events leading to withdrawal certolizumab $200 \mathrm{mg}$.

Analysis 50.16. Comparison 50 Safety, Outcome 16 Adverse events leading to withdrawal certolizumab $400 \mathrm{mg}$.

Analysis 50.17. Comparison 50 Safety, Outcome 17 Death certolizumab $200 \mathrm{mg}$.

Analysis 50.18. Comparison 50 Safety, Outcome 18 Death certolizumab $400 \mathrm{mg}$.

Analysis 50.19. Comparison 50 Safety, Outcome 19 Deaths overall.

Analysis 50.20. Comparison 50 Safety, Outcome 20 Tuberculosis certolizumab $200 \mathrm{mg}$.

Analysis 50.21. Comparison 50 Safety, Outcome 21 Tuberculosis certolizumab $400 \mathrm{mg}$.

Analysis 50.22. Comparison 50 Safety, Outcome 22 Tuberculosis overall.

Analysis 50.23. Comparison 50 Safety, Outcome 23 Malignancies included lymphoma certolizumab $200 \mathrm{mg}$.

Analysis 50.24. Comparison 50 Safety, Outcome 24 Malignancies included lymphoma certolizumab $400 \mathrm{mg}$.

Analysis 50.25. Comparison 50 Safety, Outcome 25 Injection side reactions certolizumab $200 \mathrm{mg}$.

Analysis 50.26. Comparison 50 Safety, Outcome 26 Injection side reactions certolizumab $400 \mathrm{mg}$.

Analysis 50.27. Comparison 50 Safety, Outcome 27 Antinuclear antibodies (ANA) Anti-certolizumab pegol antibodies certolizumab $200 \mathrm{mg}$.

Analysis 50.28. Comparison 50 Safety, Outcome 28 Anti-certolizumab pegol antibodies certolizumab $400 \mathrm{mg}$. .......................

Analysis 50.29. Comparison 50 Safety, Outcome 29 Systemic lupus erythematosus certolizumab $200 \mathrm{mg}$. 
Analysis 50.30. Comparison 50 Safety, Outcome 30 Prolonged activated partial thromboplastin time (aPTT) certolizumab 200 mg.

Analysis 50.31. Comparison 50 Safety, Outcome 31 Prolonged activated partial thromboplastin time (aPTT) certolizumab 400 mg.

Analysis 50.32. Comparison 50 Safety, Outcome 32 Urinary tract infection certolizumab $200 \mathrm{mg}$.

Analysis 50.33. Comparison 50 Safety, Outcome 33 Urinary tract infection certolizumab $400 \mathrm{mg}$.

Analysis 50.34. Comparison 50 Safety, Outcome 34 Upper respiratory tract infection certolizumab $200 \mathrm{mg}$.

Analysis 50.35. Comparison 50 Safety, Outcome 35 Upper respiratory tract infection certolizumab $400 \mathrm{mg}$.

Analysis 50.36. Comparison 50 Safety, Outcome 36 Lower respiratory tract infection/ lung infection certolizumab $200 \mathrm{mg}$. ..... Analysis 50.37. Comparison 50 Safety, Outcome 37 Lower respiratory tract infection/ lung infection certolizumab $400 \mathrm{mg}$. ...... Analysis 50.38. Comparison 50 Safety, Outcome 38 Pneumonia certolizumab $200 \mathrm{mg}$. Analysis 50.39. Comparison 50 Safety, Outcome 39 Pneumonitis certolizumab $400 \mathrm{mg}$. Analysis 50.40. Comparison 50 Safety, Outcome 40 Headache certolizumab $200 \mathrm{mg}$. Analysis 50.41. Comparison 50 Safety, Outcome 41 Headache certolizumab $400 \mathrm{mg}$. Analysis 50.42. Comparison 50 Safety, Outcome 42 Bacteriuria certolizumab $200 \mathrm{mg}$. Analysis 50.43. Comparison 50 Safety, Outcome 43 Bacteriuria certolizumab $400 \mathrm{mg}$. Analysis 50.44. Comparison 50 Safety, Outcome 44 Nasopharyngitis/Pharyngitis certolizumab $200 \mathrm{mg}$. Analysis 50.45. Comparison 50 Safety, Outcome 45 Nasopharyngitis/Pharyngitis certolizumab $400 \mathrm{mg}$. Analysis 50.46. Comparison 50 Safety, Outcome 46 Injection site pain certolizumab $200 \mathrm{mg}$. Analysis 50.47. Comparison 50 Safety, Outcome 47 Injection site pain certolizumab $400 \mathrm{mg}$. Analysis 50.48. Comparison 50 Safety, Outcome 48 Hypertension certolizumab $200 \mathrm{mg}$. Analysis 50.49. Comparison 50 Safety, Outcome 49 Hypertension certolizumab $400 \mathrm{mg}$ Analysis 50.50. Comparison 50 Safety, Outcome 50 Hematuria certolizumab $200 \mathrm{mg}$. Analysis 50.51. Comparison 50 Safety, Outcome 51 Haematuria certolizumab $400 \mathrm{mg}$.

Analysis 50.52. Comparison 50 Safety, Outcome 52 Hepatic enzyme increased certolizumab $200 \mathrm{mg}$.

Analysis 50.53. Comparison 50 Safety, Outcome 53 Hepatic enzyme increased certolizumab $400 \mathrm{mg}$.

Analysis 50.54. Comparison 50 Safety, Outcome 54 AST increased certolizumab $200 \mathrm{mg}$.

Analysis 50.55. Comparison 50 Safety, Outcome 55 AST increased certolizumab $400 \mathrm{mg}$

Analysis 50.56. Comparison 50 Safety, Outcome 56 ALT increased certolizumab $200 \mathrm{mg}$.

Analysis 50.57. Comparison 50 Safety, Outcome 57 ALT increased certolizumab $400 \mathrm{mg}$

Analysis 50.58. Comparison 50 Safety, Outcome 58 Diarrhoea certolizumab $200 \mathrm{mg}$.

Analysis 50.59. Comparison 50 Safety, Outcome 59 Gastroenteritis certolizumab $200 \mathrm{mg}$.

Analysis 50.60. Comparison 50 Safety, Outcome 60 Gastrointestinal disorders certolizumab $400 \mathrm{mg}$.

Analysis 50.61. Comparison 50 Safety, Outcome 61 Back pain certolizumab $200 \mathrm{mg}$.

Analysis 50.62. Comparison 50 Safety, Outcome 62 Back pain certolizumab $400 \mathrm{mg}$.

Analysis 50.63. Comparison 50 Safety, Outcome 63 Hematologic abnormalities certolizumab $200 \mathrm{mg}$

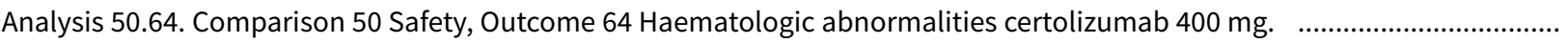

Analysis 50.65. Comparison 50 Safety, Outcome 65 Herpes viral infection certolizumab $200 \mathrm{mg}$.

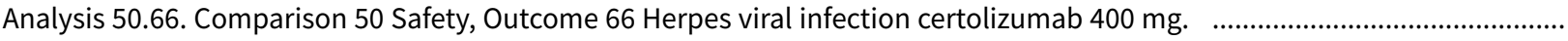

Analysis 50.67. Comparison 50 Safety, Outcome 67 Bacterial peritonitis certolizumab $200 \mathrm{mg}$.

Analysis 50.68. Comparison 50 Safety, Outcome 68 Bacterial peritonitis certolizumab $400 \mathrm{mg}$.

Analysis 50.69. Comparison 50 Safety, Outcome 69 Opportunistic infections certolizumab $200 \mathrm{mg}$.

Analysis 50.70. Comparison 50 Safety, Outcome 70 Opportunistic infections certolizumab $400 \mathrm{mg}$.

Analysis 50.71. Comparison 50 Safety, Outcome 71 Infections and infestations certolizumab $200 \mathrm{mg}$.

Analysis 50.72. Comparison 50 Safety, Outcome 72 Infections and infestations certolizumab $400 \mathrm{mg}$.

Analysis 50.73. Comparison 50 Safety, Outcome 73 Decreased haemoglobin certolizumab $200 \mathrm{mg}$.

Analysis 50.74. Comparison 50 Safety, Outcome 74 Decreased haemoglobin certolizumab $400 \mathrm{mg}$.

Analysis 50.75. Comparison 50 Safety, Outcome 75 Increased platelet count certolizumab $200 \mathrm{mg}$ 
Analysis 50.80. Comparison 50 Safety, Outcome 80 Vomiting certolizumab $400 \mathrm{mg}$.

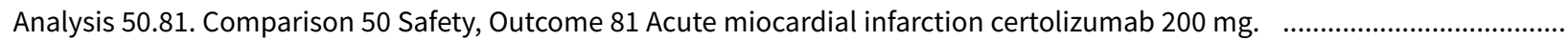

Analysis 50.82. Comparison 50 Safety, Outcome 82 Acute myocardial infarction certolizumab $400 \mathrm{mg}$. ..................................

Analysis 50.83. Comparison 50 Safety, Outcome 83 Abdominal pain/discomfort/dyspepsia certolizumab $200 \mathrm{mg}$. ..................

Analysis 50.84. Comparison 50 Safety, Outcome 84 Constipation certolizumab $200 \mathrm{mg}$.

Analysis 50.85. Comparison 50 Safety, Outcome $85 \mathrm{Skin}$ and subcutaneous tissue disorders certolizumab $200 \mathrm{mg}$. .................

Analysis 50.86. Comparison 50 Safety, Outcome 86 Skin and subcutaneous tissue disorders certolizumab $400 \mathrm{mg}$.

Analysis 50.87. Comparison 50 Safety, Outcome 87 Cough certolizumab $200 \mathrm{mg}$.

Analysis 50.88. Comparison 50 Safety, Outcome 88 Pruritus certolizumab $200 \mathrm{mg}$.

Analysis 50.89. Comparison 50 Safety, Outcome 89 Fatigue certolizumab $200 \mathrm{mg}$.

Analysis 50.90. Comparison 50 Safety, Outcome 90 Fatigue certolizumab $400 \mathrm{mg}$.

Analysis 50.91. Comparison 50 Safety, Outcome 91 Periodontitis certolizumab $200 \mathrm{mg}$.

Analysis 50.92. Comparison 50 Safety, Outcome 92 Arthritis bacterial certolizumab $400 \mathrm{mg}$.

Analysis 50.93. Comparison 50 Safety, Outcome 93 Mastitis certolizumab $400 \mathrm{mg}$.

Analysis 50.94. Comparison 50 Safety, Outcome 94 Benign tumour certolizumab $400 \mathrm{mg}$.

Analysis 50.95. Comparison 50 Safety, Outcome 95 Dizziness postural certolizumab $400 \mathrm{mg}$.

Analysis 50.96. Comparison 50 Safety, Outcome 96 Menorrhagia certolizumab $400 \mathrm{mg}$.

Analysis 50.97. Comparison 50 Safety, Outcome 97 Corneal perforation certolizumab $400 \mathrm{mg}$.

Analysis 50.98. Comparison 50 Safety, Outcome 98 Conjunctivitis allergic certolizumab $400 \mathrm{mg}$.

Analysis 50.99. Comparison 50 Safety, Outcome 99 Periodontitis certolizumab $400 \mathrm{mg}$.

Analysis 51.1. Comparison 51 Participant's assessment of arthritis pain (VAS score 0 to $100 \mathrm{~mm}$ ), Outcome 1 Mean change at 24 weeks certolizumab pegol $200 \mathrm{mg}$.

Analysis 51.2. Comparison 51 Participant's assessment of arthritis pain (VAS score 0 to $100 \mathrm{~mm}$ ), Outcome 2 Mean change at 24 weeks certolizumab pegol $400 \mathrm{mg}$.

Analysis 51.3. Comparison 51 Participant's assessment of arthritis pain (VAS score 0 to $100 \mathrm{~mm}$ ), Outcome 3 Mean change at 52 weeks certolizumab pegol $200 \mathrm{mg}$.

Analysis 51.4. Comparison 51 Participant's assessment of arthritis pain (VAS score 0 to $100 \mathrm{~mm}$ ), Outcome 4 Mean change at 52 weeks certolizumab pegol $400 \mathrm{mg}$.

Analysis 52.1. Comparison 52 Participant's assessment of arthritis pain (VAS score 0 to $100 \mathrm{~mm}$ ) at 24 weeks, any dose, Outcome 1 Change from baseline.

Analysis 53.1. Comparison 53 Participant's assessment of arthritis pain (VAS score 0 to $100 \mathrm{~mm}$ ) at 52 weeks, any dose, Outcome 1 Change from baseline.

Analysis 54.1. Comparison 54 Withdrawals Withdrawn due to lack of efficacy: any doses any follow-up, Outcome 1 Withdrawn due to lack of efficacy: any doses any follow-up.

Analysis 55.1. Comparison 55 Summary of findings: certolizumab (with or without MTX) versus placebo (with or without MTX), Outcome 1 ACR 50200 mg certolizumab 24 weeks.

Analysis 55.2. Comparison 55 Summary of findings: certolizumab (with or without MTX) versus placebo (with or without MTX), Outcome $2 \mathrm{HAQ}$ change from baseline $200 \mathrm{mg}$ certolizumab 24 weeks.

Analysis 55.3. Comparison 55 Summary of findings: certolizumab (with or without MTX) versus placebo (with or without MTX), Outcome 3 Serious adverse events certolizumab $200 \mathrm{mg} \mathrm{sc}$.

Analysis 55.4. Comparison 55 Summary of findings: certolizumab (with or without MTX) versus placebo (with or without MTX), Outcome 4 Proportion of participants achieving remission 24 weeks certolizumab $200 \mathrm{mg}$.

Analysis 55.5. Comparison 55 Summary of findings: certolizumab (with or without MTX) versus placebo (with or without MTX), Outcome 5 Radiological changes: Erosion Scores (ES) certolizumab $200 \mathrm{mg}$ sc.

Analysis 55.6. Comparison 55 Summary of findings: certolizumab (with or without MTX) versus placebo (with or without MTX), Outcome 6 All Withdrawals:.

Analysis 55.7. Comparison 55 Summary of findings: certolizumab (with or without MTX) versus placebo (with or without MTX), Outcome 7 Withdrawals due to adverse events.

Analysis 55.8. Comparison 55 Summary of findings: certolizumab (with or without MTX) versus placebo (with or without MTX), Outcome 8 Deaths.

Analysis 55.9. Comparison 55 Summary of findings: certolizumab (with or without MTX) versus placebo (with or without MTX), Outcome 9 Tuberculosis.

Analysis 55.10. Comparison 55 Summary of findings: certolizumab (with or without MTX) versus placebo (with or without MTX), Outcome 10 Upper respiratory tract infections. 
Analysis 55.11. Comparison 55 Summary of findings: certolizumab (with or without MTX) versus placebo (with or without MTX), 160 Outcome 11 Lower respiratory tract infections.

Analysis 55.12. Comparison 55 Summary of findings: certolizumab (with or without MTX) versus placebo (with or without MTX), 161 Outcome 12 Malignancies including lymphoma.

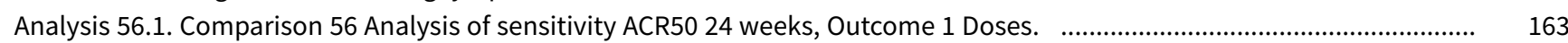

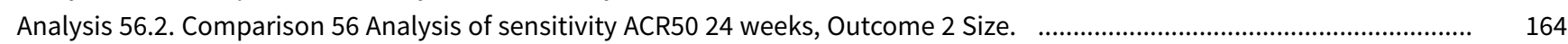

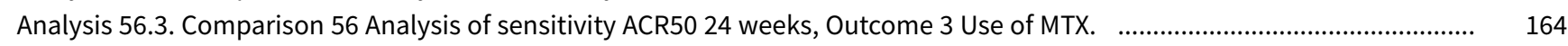

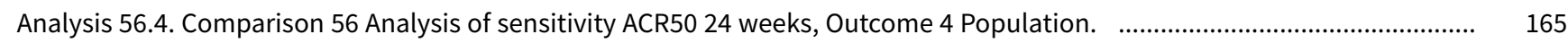

Analysis 56.5. Comparison 56 Analysis of sensitivity ACR50 24 weeks, Outcome 5 Duration of previous disease. ...................... 166

Analysis 56.6. Comparison 56 Analysis of sensitivity ACR50 24 weeks, Outcome 6 Published vs unpublished studies. .............. 167

Analysis 56.7. Comparison 56 Analysis of sensitivity ACR50 24 weeks, Outcome 7 Imputing to ACR50 200 mg from 24 missing 167 values with same proportion as reported outcomes.

Analysis 56.8. Comparison 56 Analysis of sensitivity ACR50 24 weeks, Outcome 8 Imputing to ACR50 200 mg from 24 weeks 50 $\%$ of missing outcomes.

Analysis 56.9. Comparison 56 Analysis of sensitivity ACR50 24 weeks, Outcome 9 Imputing to ACR50 200 mg from 24 weeks: the worst case.

Analysis 57.1. Comparison 57 Analysis of sensitivity ACR50 52 weeks, Outcome 1 Doses.

Analysis 57.2. Comparison 57 Analysis of sensitivity ACR50 52 weeks, Outcome 2 Size.

Analysis 57.3. Comparison 57 Analysis of sensitivity ACR50 52 weeks, Outcome 3 Use of MTX.

Analysis 57.4. Comparison 57 Analysis of sensitivity ACR50 52 weeks, Outcome 4 Population.

Analysis 57.5. Comparison 57 Analysis of sensitivity ACR50 52 weeks, Outcome 5 Duration of previous disease. 
[Intervention Review]

\title{
Certolizumab pegol (CDP870) for rheumatoid arthritis in adults
}

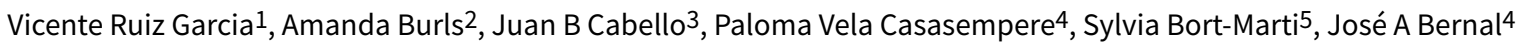

1Hospital at Home Unit, Tower C, Floor 1 Office 5 \& CASPe Spain, La Fe University Hospital, Valencia, Spain. 2School of Health Sciences, City University London, London, UK. ${ }^{3}$ Department of Cardiology \& CASP Spain, Hospital General Universitario de Alicante, Alicante, Spain. ${ }^{4}$ Department of Rheumatology, Hospital General Universitario Alicante, Alicante, Spain. ${ }^{5}$ Acella Incubator, Paterna, Spain

Contact address: Vicente Ruiz Garcia, Hospital at Home Unit, Tower C, Floor 1 Office 5 \& CASPe Spain, La Fe University Hospital, Av Fernando Abril Martorell n 106 , Valencia, 46026, Spain. vicenteruizgarcia@gmail.com.

Editorial group: Cochrane Musculoskeletal Group.

Publication status and date: New search for studies and content updated (no change to conclusions), published in Issue 9, 2017.

Citation: Ruiz Garcia V, Burls A, Cabello JB, Vela Casasempere P, Bort-Marti S, Bernal JA. Certolizumab pegol (CDP870) for rheumatoid arthritis in adults. Cochrane Database of Systematic Reviews 2017, Issue 9. Art. No.: CD007649. DOI: 10.1002/14651858.CD007649.pub4.

Copyright @ 2017 The Cochrane Collaboration. Published by John Wiley \& Sons, Ltd.

\section{A B S T R A C T}

\section{Background}

Tumour necrosis factor (TNF)-alpha inhibitors are beneficial for the treatment of rheumatoid arthritis (RA) for reducing the risk of joint damage, improving physical function and improving the quality of life. This review is an update of the 2014 Cochrane Review of the treatment of RA with certolizumab pegol.

\section{Objectives}

To assess the clinical benefits and harms of certolizumab pegol (CZP) in people with RA who have not responded well to conventional disease-modifying anti-rheumatic drugs (DMARDs).

\section{Search methods}

We searched the Cochrane Central Register of Controlled Trials (CENTRAL: Cochrane Library 2016, Issue 9), MEDLINE, Embase, Web of Knowledge, reference lists of articles, clinicaltrials.gov and ICTRP of WHO. The searches were updated from 2014 (date of the last search for the previous version) to 26 September 2016.

\section{Selection criteria}

Randomised controlled trials that compared certolizumab pegol with any other agent, including placebo or methotrexate (MTX), in adults with active RA, regardless of current or prior treatment with conventional disease-modifying anti-rheumatic drugs (DMARDs), such as MTX.

\section{Data collection and analysis}

Two review authors independently checked search results, extracted data and assessed trial quality. We resolved disagreements by discussion or referral to a third review author.

\section{Main results}

We included 14 trials in this update, three more than previously. Twelve trials (5422 participants) included measures of benefit. We pooled 11 of them, two more than previously. Thirteen trials included information on harms, (5273 participants). The duration of follow-up varied from 12 to 52 weeks and the range of doses of certolizumab pegol varied from 50 to $400 \mathrm{mg}$ given subcutaneously. In Phase III trials, the comparator was placebo plus MTX in seven trials and placebo in five. In the two Phase II trials the comparator was only placebo.

The approved dose of certolizumab pegol, $200 \mathrm{mg}$ every other week, produced clinically important improvements at 24 weeks for the following outcomes: 
- American College of Rheumatology (ACR) 50\% improvement (pain, function and other symptoms of RA): 25\% absolute improvement (95\% confidence interval (CI) 20\% to 33\%); number need to treat for an additional beneficial outcome (NNTB) of 4 (95\% Cl 3 to 5); risk ratio (RR) 3.80 ( $95 \% \mathrm{Cl} 2.42$ to 5.95$), 1445$ participants, 5 studies.

- The Health Assessment Questionnaire (HAQ): $-12 \%$ absolute improvement (95\% Cl $-9 \%$ to $-14 \%$ ); NNTB of 8 (95\% Cl 7 to 11$)$; mean difference (MD) - 0.35 (95\% Cl -0.43 to -0.26 ; 1268 participants, 4 studies) (scale 0 to 3; lower scores mean better function).

- Proportion of participants achieving remission (Disease Activity Score (DAS) <2.6) absolute improvement 10\% (95\% CI 8\% to 16\%); NNTB of 8 ( $95 \% \mathrm{Cl} 6$ to 12 ); risk ratio (RR) 2.94 (95\% Cl 1.64 to 5.28), 2420 participants, six studies.

- Radiological changes: erosion score (ES) absolute improvement $-0.29 \%$ (95\% Cl $-0.42 \%$ to $-0.17 \%$ ); NNTB of 6 (95\% Cl 4 to 10 ); MD -0.67 $(95 \% \mathrm{Cl}-0.96$ to -0.38$) ; 714$ participants, two studies (scale 0 to 230$)$, but not a clinically important difference.

-Serious adverse events (SAEs) were statistically but not clinically significantly more frequent for certolizumab pegol (200 mg every other week) with an absolute rate difference of $3 \%(95 \% \mathrm{Cl} 1 \%$ to $4 \%)$; number needed to treat for an additional harmful outcome (NNTH) of 33 (95\% $\mathrm{Cl} 25$ to 100$)$; Peto odds ratio (OR) 1.47 (95\% Cl 1.13 to 1.91 ); 3927 participants, nine studies.

There was a clinically significant increase in all withdrawals in the placebo groups (for all doses and at all follow-ups) with an absolute rate difference of $-29 \%(95 \% \mathrm{Cl}-16 \%$ to $-42 \%)$, NNTH of $3(95 \% \mathrm{Cl} 2$ to 6$)$, RR 0.47 (95\% $\mathrm{Cl} 0.39$ to 0.56$)$; and there was a clinically significant increase in withdrawals due to adverse events in the certolizumab groups (for all doses and at all follow-ups) with an absolute rate difference of $2 \%$ (95\% Cl 0\% to 3\%); NNTH of 58 (95\% Cl 28 to 329); Peto OR 1.45 (95\% Cl 1.09 to 1.94) 5236 participants Twelve studies.

We judged the quality of evidence to be high for ACR50, DAS remission, SAEs and withdrawals due to adverse events, and moderate for $\mathrm{HAQ}$ and radiological changes, due to concerns about attrition bias. For all withdrawals we judged the quality of evidence to be moderate, due to inconsistency.

\section{Authors' conclusions}

The results and conclusions did not change from the previous review. There is a moderate to high certainty of evidence from randomised controlled trials that certolizumab pegol, alone or combined with methotrexate, is beneficial in the treatment of RA for improved ACR50 and health-related quality of life, an increased chance of remission of RA, and reduced joint damage as seen on x-ray. Fewer people stopped taking their treatment, but most of these who did stopped due to serious adverse events. Adverse events were more frequent with active treatment. We found a clinically but not statistically significant risk of serious adverse events.

\section{PLAIN LANGUAGE SUMMARY}

\section{Certolizumab pegol for treating adults with rheumatoid arthritis}

We conducted an updated review of the benefits and harms of certolizumab pegol (CZP) for adults with active rheumatoid arthritis (RA). We searched for all relevant studies until September 2016 and found 14 trials with 5499 people.

The length of follow-up in most of the trials was 24 weeks; most participants were women.

\section{What is rheumatoid arthritis and what is certolizumab pegol?}

When you have RA, your immune system becomes overactive and attacks the lining of your joints. This makes your joints swollen, stiff and painful.

Certolizumab pegol is a biologic medication for the treatment of RA. It works by blocking a substance produced by the body known as tumour necrosis factor alpha (TNFa). Certolizumab pegol is given by injections under the skin. The approved dose is $200 \mathrm{mg}$.

\section{What happens to people with rheumatoid arthritis who take certolizumab pegol $200 \mathrm{mg}$ every other week after six months?}

\section{ACR50 (standard: a 50\% improvement in the number of tender or swollen joints and other outcomes such as pain and disability):}

- 25 more people out of 100 experienced improvements in the symptoms of their rheumatoid arthritis after six months with certolizumab pegol (absolute improvement 25\%).

- 36 people out of 100 who took certolizumab pegol experienced improvements compared to nine people out of 100 who took a placebo (a fake injection).

We rate the quality of evidence for ACR50 as high.

Health-related quality of life (Health Assessment Questionnaire, HAQ: 0 to 3 scale, where a lower score means improvement):

- people who took certolizumab pegol scored 0.35 points lower than people who took placebo (absolute improvement $12 \%$ ). 
- people on certolizumab pegol scored 0.48 points lower compared to 0.13 points lower for people who took a placebo.

We rate the quality of evidence for the HAQ as moderate, downgraded, due to concerns about the high number of people dropping out of the studies.

\section{Remission (absence of clinical signs of inflammation):}

- 10 people out of 100 experienced remission with certolizumab pegol (absolute improvement 10\%).

- 22 people out of 100 who took certolizumab pegol experienced remission compared to 12 people out of 100 who took a placebo.

We rate the quality of evidence for the remission as high.

\section{Radiological changes (x-rays of the joints, measured on a 0 to 230 unit scale):}

- the joint damage in people who took certolizumab pegol was 0.67 units less (absolute improvement -0.29\%).

- the damage to joints in people who took certolizumab pegol was 0.04 units less compared to people who took a placebo, whose joint damage was 0.7 units more.

We rate the quality of evidence for the findings in the radiological changes as moderate, downgraded, due to concerns about the high number of people dropping out of the studies.

\section{Serious adverse events:}

- three more people out of 100 experienced serious adverse events with certolizumab pegol (3\% absolute harm).

- nine people out of 100 who took certolizumab pegol experienced serious adverse events compared to six people out of 100 who took a placebo.

We rate the quality of evidence for serious adverse events as high.

\section{All Withdrawals}

- 29 fewer people out of 100 experienced withdrawals with certolizumab pegol (absolute harm 29\%).

- 23 people out of 100 who took certolizumab pegol experienced withdrawals compared to 52 people out of 100 who took a placebo.

We rate the quality of evidence for all withdrawals as moderate.

\section{Withdrawals due to adverse events}

- two more people out of 100 stopped treatment because of SAEs with certolizumab pegol ( $2 \%$ absolute harm).

- five people out of 100 who took certolizumab pegol estopped treatment because of SAEs compared to three people out of 100 who took a placebo.

We rate the quality of evidence for the withdrawals due to adverse events as high.

\section{In summary:}

- certolizumab pegol improves ACR50, health-related quality of life, and remission of RA.

- certolizumab pegol probably reduces joint damage as seen on $x$-ray.

- certolizumab pegol increases serious adverse events.

- with certolizumab pegol, fewer people stop taking their treatment, but those who stop do so because of serious adverse events. 
SUMMARY OF FINDINGS

Summary of findings for the main comparison. Certolizumab pegol $200 \mathrm{mg} \mathrm{sc}$ (with or without MTX) versus placebo (with or without MTX) for rheumatoid arthritis in adults

Certolizumab pegol $200 \mathrm{mg} \mathrm{sc}$ (with or without MTX) versus placebo (with or without MTX) for rheumatoid arthritis in adults

Patient or population: patients with rheumatoid arthritis in adults

Settings: adults (18 years old or more) who have persistent disease activity

Intervention: certolizumab pegol $200 \mathrm{mg}$ sc (with or without MTX) versus placebo (with or without MTX)

\begin{tabular}{|c|c|c|c|c|c|c|}
\hline \multirow[t]{3}{*}{ Outcomes } & \multicolumn{2}{|c|}{$\begin{array}{l}\text { Illustrative comparative risks* }(95 \% \\
\mathrm{CI})\end{array}$} & \multirow[t]{3}{*}{$\begin{array}{l}\text { Relative effect } \\
(95 \% \mathrm{CI})\end{array}$} & \multirow{3}{*}{$\begin{array}{l}\text { No of partici- } \\
\text { pants } \\
\text { (studies) }\end{array}$} & \multirow{3}{*}{$\begin{array}{l}\text { Quality of the } \\
\text { evidence } \\
\text { (GRADE) }\end{array}$} & \multirow[t]{3}{*}{ Comments } \\
\hline & Assumed risk & Corresponding risk & & & & \\
\hline & Control & $\begin{array}{l}\text { Summary of find- } \\
\text { ings certolizum- } \\
\text { ab pegol } 200 \text { mg sc } \\
\text { (with or without } \\
\text { MTX) versus place- } \\
\text { bo (with or without } \\
\text { MTX) }\end{array}$ & & & & \\
\hline $\begin{array}{l}\text { ACR } \mathbf{5 0 \%} \text { improvement } \\
\text { Follow-up: mean } 24 \text { weeks }\end{array}$ & \multirow[t]{3}{*}{87 per 1000} & \multirow[t]{3}{*}{$\begin{array}{l}\mathbf{3 5 9} \text { per } \mathbf{1 0 0 0} \\
\text { (328 to } 391)\end{array}$} & \multirow[t]{3}{*}{$\begin{array}{l}\text { RR } 3.80 \\
\text { (2.42 to } 5.95)\end{array}$} & \multirow[t]{3}{*}{$\begin{array}{l}1445 \\
\text { (5 studies) }\end{array}$} & \multirow[t]{3}{*}{$\begin{array}{l}\oplus \oplus \oplus \oplus \\
\text { high }\end{array}$} & $\begin{array}{l}\text { Absolute risk difference }=25 \%(95 \% \\
\mathrm{Cl} 20 \% \text { to } 33 \%) \text {. }\end{array}$ \\
\hline \multirow[t]{2}{*}{200 mg sc certolizumab pegol } & & & & & & $\begin{array}{l}\text { Relative per cent change }=280 \% \\
(142 \% \text { to } 495 \%)\end{array}$ \\
\hline & & & & & & NNTB $=4$ ( 3 to 5$)$ \\
\hline $\begin{array}{l}\text { HAQ change from baseline } \\
\text { Scale from: } 0 \text { to } 3 \text {. } \\
\text { Follow-up: mean } 24 \text { weeks }\end{array}$ & \multirow{3}{*}{$\begin{array}{l}\text { The mean HAQ } \\
\text { change from } \\
\text { baseline in the } \\
\text { control groups } \\
\text { was } \\
-\mathbf{0 . 1 3}\end{array}$} & \multirow{3}{*}{$\begin{array}{l}\text { The mean } \mathrm{HAQ} \\
\text { change from base- } \\
\text { line in the interven- } \\
\text { tion groups was } \\
\mathbf{0 . 3 5} \text { lower } \\
\text { ( } 0.43 \text { to } 0.26 \text { lower) }\end{array}$} & \multirow[t]{3}{*}{$\begin{array}{l}\text { MD }-0.35(-0.43 \\
\text { to }-0.26)\end{array}$} & \multirow[t]{3}{*}{$\begin{array}{l}1268 \\
\text { (4 studies) }\end{array}$} & \multirow[t]{3}{*}{$\begin{array}{l}\oplus \oplus \oplus \ominus \\
\text { moderate } 1\end{array}$} & $\begin{array}{l}\text { Absolute risk difference }=-12 \%(95 \% \\
\mathrm{Cl}-9 \% \text { to }-14 \%) \text {. }\end{array}$ \\
\hline $\begin{array}{l}\text { (lower scores means better } \\
\text { function) }\end{array}$ & & & & & & $\begin{array}{l}\text { Relative per cent change }=-21 \% \\
(-15 \% \text { to }-25 \%)\end{array}$ \\
\hline & & & & & & NNT $=8$ (7 to 11$)$ \\
\hline \multirow{2}{*}{$\begin{array}{l}\text { Proportion of patients } \\
\text { achieving DAS }<2.6 \text { (remis- } \\
\text { sion) } \\
\text { Follow-up: mean } 24 \text { weeks }\end{array}$} & \multirow[t]{2}{*}{123 per 1000} & \multirow[t]{2}{*}{$\begin{array}{l}\mathbf{2 1 6} \text { per } \mathbf{1 0 0 0} \\
(194 \text { to } 247)\end{array}$} & \multirow[t]{2}{*}{$\begin{array}{l}\text { RR } 2.94 \\
\text { (1.64 to } 5.28)\end{array}$} & \multirow[t]{2}{*}{$\begin{array}{l}2420 \\
\text { (6 studies) }\end{array}$} & \multirow[t]{2}{*}{$\begin{array}{l}\oplus \oplus \oplus \oplus \\
\text { high }\end{array}$} & $\begin{array}{l}\text { Absolute risk difference }=10 \%(95 \% \\
\mathrm{Cl} 8 \% \text { to } 16 \%) \text {. }\end{array}$ \\
\hline & & & & & & $\begin{array}{l}\text { Relative per cent change }=194 \% \\
(64 \% \text { to } 428 \%)\end{array}$ \\
\hline
\end{tabular}




\begin{tabular}{|c|c|c|c|c|c|c|}
\hline $\begin{array}{l}\text { Radiological changes: Ero- } \\
\text { sion Scores (ES) } \\
\text { Scale from: } 0 \text { to } 230 \\
\text { Follow-up: } 24 \text { weeks } \\
200 \text { mg sc certolizumab pegol }\end{array}$ & $\begin{array}{l}\text { The mean ra- } \\
\text { diological } \\
\text { changes: Ero- } \\
\text { sion Scores (ES) } \\
\text { in the control } \\
\text { groups was } \\
\mathbf{0 . 7}\end{array}$ & $\begin{array}{l}\text { The mean Radiologi- } \\
\text { cal changes: Erosion } \\
\text { Scores (ES) in the in- } \\
\text { tervention groups } \\
\text { was } \\
\mathbf{0 . 6 7} \text { lower } \\
\text { (0.96 to } 0.38 \text { lower) }\end{array}$ & $\begin{array}{l}\text { MD -0.67 }(-0.96 \\
\text { to }-0.28)\end{array}$ & $\begin{array}{l}714 \\
\text { ( } 2 \text { studies) }\end{array}$ & $\begin{array}{l}\oplus \oplus \oplus \ominus \\
\text { moderate } 1\end{array}$ & $\begin{array}{l}\text { Absolute risk difference }=-0.29 \% \\
(95 \% \mathrm{Cl}-0.42 \% \text { to }-0.17 \%) \\
\text { Relative per cent change }=-2.90 \% \\
(-4.16 \% \text { to }-1.65 \%) \\
\text { NNT }=6 \text { ( } 4 \text { to } 10)\end{array}$ \\
\hline $\begin{array}{l}\text { Serious adverse events } \\
\text { Follow-up: } 12 \text { to } 24 \text { weeks } \\
200 \text { mg sc certolizumab pegol }\end{array}$ & 58 per 1000 & $\begin{array}{l}85 \text { per } 1000 \\
(59 \text { to } 120)\end{array}$ & $\begin{array}{l}\text { Peto OR 1.47 } \\
\text { (1.13 to } 1.91 \text { ) }\end{array}$ & $\begin{array}{l}3927 \\
\text { (9 studies) }\end{array}$ & $\begin{array}{l}\oplus \oplus \oplus \oplus \\
\text { high }\end{array}$ & $\begin{array}{l}\text { Absolute risk difference }=3 \%(95 \% \mathrm{Cl} \\
1 \% \text { to } 4 \%) . \\
\text { Relative per cent change }=47 \%(13 \% \\
\text { to } 91 \%) . \mathrm{NNTH}=33 \text { ( } 25 \text { to } 100)\end{array}$ \\
\hline $\begin{array}{l}\text { All withdrawals: } \\
\text { All doses of certolizumab pe- } \\
\text { gol vs placebo } \\
\text { Follow-up: } 0 \text { to } 52 \text { weeks }\end{array}$ & 524 per 1000 & $\begin{array}{l}\mathbf{2 3 1} \text { per } \mathbf{1 0 0 0} \\
\text { (203 to } 291 \text { ) }\end{array}$ & $\begin{array}{l}\text { RR } 0.47 \\
(0.39 \text { to } 0.56)\end{array}$ & $\begin{array}{l}5200 \\
\text { (13 studies) }\end{array}$ & $\begin{array}{l}\oplus \oplus \oplus \ominus \\
\text { moderate }^{2}\end{array}$ & $\begin{array}{l}\text { Absolute risk difference }=-29 \%(95 \% \\
\mathrm{Cl}-16 \% \text { to }-42 \%) . \\
\text { Relative per cent change }=-53 \% \\
(-44 \% \text { to }-61 \%) . \text { NNTH }=3(2 \text { to } 6)\end{array}$ \\
\hline $\begin{array}{l}\text { Withdrawals due to adverse } \\
\text { events } \\
\text { All doses of certolizumab pe- } \\
\text { gol versus placebo } \\
\text { Follow-up: } 0 \text { to } 52 \text { weeks }\end{array}$ & 38 per 1000 & $\begin{array}{l}52 \text { per } 1000 \\
(40 \text { to } 73)\end{array}$ & $\begin{array}{l}\text { Peto OR } 1.45 \\
\text { (1.09 to } 1.94)\end{array}$ & $\begin{array}{l}5236 \\
\text { (12 studies) }\end{array}$ & $\begin{array}{l}\oplus \oplus \oplus \oplus \\
\text { high }\end{array}$ & $\begin{array}{l}\text { Absolute risk difference }=2 \%(95 \% \mathrm{Cl} \\
0 \% \text { to } 3 \%) \text {. } \\
\text { Relative per cent change }=45 \%(9 \% \\
\text { to } 94 \%) . \\
\text { NNTH }=58(28 \text { to } 329)\end{array}$ \\
\hline
\end{tabular}

*The basis for the assumed risk (e.g. the median control group risk across studies) is provided in footnotes. The corresponding risk (and its $95 \%$ confidence interval) is based on the assumed risk in the comparison group and the relative effect of the intervention (and its $95 \% \mathrm{Cl}$ ).

Cl: Confidence interval; RR: Risk ratio; OR: Odds ratio; NNTB: number needed to treat for an additional beneficial outcome

GRADE Working Group grades of evidence

High quality: Further research is very unlikely to change our confidence in the estimate of effect.

Moderate quality: Further research is likely to have an important impact on our confidence in the estimate of effect and may change the estimate.

Low quality: Further research is very likely to have an important impact on our confidence in the estimate of effect and is likely to change the estimate.

Very low quality: We are very uncertain about the estimate.

1 We downgraded the quality of evidence by one level for risk of bias due to attrition bias analysed per protocol. We have rated all the trials at low risk for attrition bias since reasons for attrition/exclusions were reported in most of them, and reasons were similar. However, for HAQ-DI and radiological changes we can only conduct a per protocol analysis, as these are continuous outcomes that count the average number of participants still in the trials. For DAS remission, ACR50, SAEs, all withdrawals and withdrawals due to AEs we conducted an ITT analysis, which is a more conservative approach, not requiring downgrading.

$\checkmark$ V 2 We downgraded the quality of evidence by one level for inconsistency, due to heterogeneity (not all the confidence intervals overlap, and 12 is $79 \%$ ). 


\section{B A C K G R O U N D}

\section{Description of the condition}

Rheumatoid arthritis (RA) is a chronic inflammatory disease characterised by synovial inflammation of joints and other structures such as tendon sheaths and bursas, autoantibody production (rheumatoid factor and anti-citrullinated protein antibody (ACPA)), with both cartilage and bone destruction. RA typically causes a symmetrical polyarticular arthritis with pain, swelling and stiffness of the affected joints. If the disease is not controlled early, damage may become permanent, leading to significant disability. People with RA commonly experience fatigue and show changes in the blood, such as anaemia due to chronic inflammation, and an acute phase reaction. In some people organs such as the skin (as rheumatoid nodules), lungs (pleural inflammation and alveolitis), heart (pericarditis), blood vessels (vasculitis) and the eyes (dry eyes or inflammation) may be affected (Tureson 2013). RA is also associated with reduced life expectancy; in a Spanish cohort, the standardised mortality ratio was 1.89 (Abasolo 2016), specifically due to cardiovascular disease (Meune 2009).

Despite progress in understanding the pathogenesis of RA, its cause remains unknown. Important genetic influences are recognised, with more than 100 RA risk loci identified (Okada 2014). Based on twin studies, heritability is approximately 60\% (MacGregor 2000), so environment also plays a key role in RA pathogenesis. Moreover, in recent years environmental factors have gained importance in explaining the development of RA: smoking has specifically been associated with the development of ACPA-positive RA (Lundberg 2013), and cumulative evidence from a large number of studies implicates the microbiome of the periodontium, lung, and gut in RA pathogenesis (Kharlamova 2016).

People of all ages are affected, but the disease begins most commonly between the ages of 40 and 70 years, with incidence rising with increasing age (Doran 2002). The global prevalence is $0.24 \%$, with twice as many women as men affected (Cross 2014). Significant functional limitations occur in $15 \%$ of sufferers five years after disease onset, with around a third of those in paid work experiencing work disability (Young 2000). In Finland, the risk of disability is seven times higher in people with RA compared with the general population (Sokka 2003). Rapid induction of remission translates to the maintenance of work capacity (Puolakka 2005).

\section{Description of the intervention}

The management of RA has undergone dramatic changes during the last 15 years. The latest updated recommendations of both the American College of Rheumatology (Singh 2016) and the European League Against Rheumatism (Smolen 2014) emphasise the importance of starting therapy with disease-modifying antirheumatic drugs (DMARDs) as soon as the diagnosis of RA is made; the search for remission or low disease activity using a treat-to-target approach; and close monitoring by using composite measures of disease activity and appropriate switching of drug treatment when the objectives are not reached. Methotrexate (MTX) remains the drug of choice at the start of treatment of RA (LopezOlivo 2014), although leflunomide or triple therapy are considered excellent alternatives (Singh 2012).
People sometimes do not respond to or are unable to tolerate DMARDs (Yee 2003). The newer biological drugs that have been introduced and approved for the treatment of RA in recent decades have been associated with clinical outcome improvement (Singh 2009), but also with higher rates of adverse events (Singh 2011).

\section{How the intervention might work}

RA is characterised by immunological activation of many cell types and a network of cytokines, particularly tumour necrosis factor alpha (TNFa) (Brennan 2008). Inhibitors of TNFa have been a major development in the treatment of RA. Randomised trials have shown that these drugs are highly beneficial in people with RA who have not responded well to conventional DMARDs. TNFa inhibitors have been shown to reduce the risk of joint damage, improve physical function and quality of life (Chen 2006). Five TNFa inhibitors are currently licensed for use against RA in Europe and the USA. These are adalimumab (Navarro-Sarabia 2005), etanercept (Lethaby 2013), golimumab (Singh 2010), infliximab (Blumenauer 2002) and certolizumab pegol (Ruiz Garcia 2014). Comparative efficacy studies to evaluate variations between anti-TNF and non-anti-TNF biologics have shown little difference between them (Navarro-Millán 2013). One pragmatic, open-label controlled trial (Jobanputra 2012) has directly compared etanercept and adalimumab, and reported similar persistence rates, efficacy and safety over two years of treatment. Similar results have been obtained with certolizumab pegol in extension studies, with the American College of Rheumatology ACR20 at 57\% and ACR50 at $27 \%$ at eight years (NCT00160693), and ACR20 at $81 \%$ and ACR50 at $58 \%$ at seven years (NCT00175877). An important limitation of the wider use of TNF inhibitors is the high cost, between USD 10,000 and USD 25,000 per person a year. However, the recent entry of bio similars is causing a significant drop in prices. Biosimilars are biological products that are copies of an approved innovator biopharmaceutical, developed after the expiration of the innovator's patent and submitted for separate marketing approval. The use of bio similars may dramatically increase in the near future, mainly due to cost savings (Dörner 2016).

A systematic review of infliximab and adalimumab has shown that the risks of malignancy and serious infection were increased, with odds ratios (ORs) of 3.3 (95\% confidence interval $(\mathrm{Cl}) 1.2$ to 9.1 ) and $2.0(95 \% \mathrm{Cl} 1.3$ to 3.1 ) respectively (Bongartz 2006). However, more recent data show that therapy with anti-TNF is not related to an increased risk of malignancies (skin cancer, melanoma, lymphoma or solid tumours) (Lopez-Olivo 2012). A second review of nine biologic drugs (the five TNF inhibitors etanercept, adalimumab, infliximab, golimumab and certolizumab pegol; the interleukin (IL)- 1 antagonist anakinra; the IL- 6 antagonist tocilizumab; the antiCD28 abatacept; and anti-B cell rituximab) showed that biologics as a group were associated with a statistically significantly higher rate of total adverse events (OR $1.28,95 \% \mathrm{Cl} 1.09$ to 1.50 ) and withdrawals due to adverse events (OR $1.47,95 \% \mathrm{Cl} 1.20$ to 1.86 ), and an increased risk of tuberculosis (TB) reactivation (OR 4.68, $95 \% \mathrm{Cl} 1.18$ to 18.60 ) compared to control (Singh 2011). Moreover, the risk of serious infection is increased in people with RA treated with biological therapies compared with conventional DMARDs (Singh 2015).

Certolizumab pegol (CZP) was approved by the US Food and Drug Administration (FDA) and the European Medicines Evaluation Agency (EMEA) in 2009 for adults suffering from moderate to severe RA. Certolizumab pegol is an anti-TNF consisting of a humanised 
immunoglobulin fragment (Fab) conjugated to polyethylene glycol (PEG), also termed pegylation. This unique molecular structure yields a longer half-life and reduces the need for frequent dosing (Choy 2002). Certolizumab pegol in combination with MTX is indicated for the treatment of moderate to severe active RA in adults when the response to conventional DMARDs, including MTX, has been inadequate. It is also indicated in severe, active and progressive RA not treated previously with conventional DMARDs. In the case of intolerance, side effects or contraindications to MTX it also can be given as monotherapy. The drug has been shown to reduce the rate of progression of joint damage, as measured by $x$-ray, and to improve physical function. Long-term follow-up studies of commercially-sponsored randomised controlled trials (RCTs) show persistence rates of $59.9 \%$ at week 232 (Smolen 2015), with $46.7 \%$ of participants having low disease activity at two years (Keystone 2012). Whether such rates can be replicated in routine care remains to be seen.

\section{Why it is important to do this review}

Biological treatment has led to a radical change in the prognosis and quality of life of people with RA. However, clinicians need to take into account the potential risks associated with their use. This review summarises the current data available on the benefits and harms of certolizumab pegol, on its own and in combination with MTX, for the treatment of RA. New evidence about efficacy, safety and long-term persistence has become available since our previous update. It is important to be sure that clinicians choose the treatment for people with RA appropriately, using the best medical evidence available (Emparanza 2015).

\section{O B JE C T IVES}

To assess the clinical benefits and harms of certolizumab pegol (CZP) in people with RA who have not responded well to conventional disease-modifying anti-rheumatic drugs (DMARDs).

\section{MET HOD S}

\section{Criteria for considering studies for this review \\ Types of studies}

Randomised controlled trials (RCTs).

\section{Types of participants}

Adults (18 years and older) with RA who have persistent disease activity.

People with RA were defined as those meeting the American College of Rheumatology (ACR) 1987 revised criteria (Arnett 1988) for RA. That is to say, they had to have an active form of the disease as demonstrated by at least two of the following symptoms:

1. Three or more tender joint areas as observed by a physician;

2. Three or more swollen joint areas as observed by a physician;

3. Early morning stiffness with a duration > 30 minutes;

4. Acute phase reactants such as a Westergren erythrocyte sedimentation rate (ESR) more than $30 \mathrm{~mm}$ /hour or C-reactive protein (CRP) more than $10 \mathrm{mg} / \mathrm{mL}$.

\section{Types of interventions}

Certolizumab pegol (CZP)) at any dose.
The comparators were placebo or any DMARD including other biologic agents used to treat RA.

\section{Types of outcome measures}

\section{Major outcomes}

- The proportion of participants achieving an ACR50

- Health-related quality of life, such as the Health Assessment Questionnaire (HAQ) or Short Form Health Survey (SF-36)

- Disease Activity Score (DAS28 or other versions of DAS)

- Radiological changes (erosion score (ES), modified total Sharp score, joint space narrowing)

- Serious adverse events (SAEs)

- All withdrawals

- Withdrawals due to adverse events

The ACR50 is defined as a $50 \%$ improvement in the number of tender and swollen joints and a 50\% improvement in at least three of the following items: observer evaluation of overall disease activity, patient evaluation of overall disease activity, patient evaluation of pain, a score of physical disability, or improvements in blood acute-phase responses.

Scores in the HAQ range from 0 to 3 , with 3 indicating a worse health state, so a negative change indicates improvement. The SF-36 is a scale from 0 to 100 where 0 is the worst and 100 the best health state.

Serious adverse events are defined as malignancies and all infections, especially tuberculosis, and death.

We sought all causes of withdrawals from the medication.

\section{Minor outcomes}

- ACR20 and ACR70 (a $20 \%$ or $70 \%$ improvement respectively in the parameters described above)

- Frequency of adverse events

- Withdrawals due to lack of efficacy

We sought reports of the following adverse events: headache, fever, blood disorders, laboratory disorders, abdominal pain, nasopharyngitis, nausea, respiratory tract infections, urinary tract infections, neck pain, congestive heart failure, pruritus and anaphylaxis.

\section{Search methods for identification of studies}

\section{Electronic searches}

The search strategy used the revision of the Cochrane highly sensitive search strategy (HSSS) for PubMed (Glanville 2006), the best sensitivity filter developed by the Hedges Team (Wong 2006a; Wong 2006b), and followed the Cochrane Musculoskeletal Review Group (CMSG) recommendations. Searches included both $\mathrm{MeSH}$ headings and text terms for CDP870 and rheumatoid arthritis. Tamara Rader, Information Scientist of the CMSG, conducted the searches. These included: MEDLINE (Appendix 1); Embase (Appendix 2); CINAHL (Appendix 3); Cochrane Database of Systematic Reviews (CDSR) and Cochrane Central Register of Controlled Trials (CENTRAL), HTA, DARE, NHS EED (the Cochrane Library) (Appendix 4); SCOPUS (Appendix 5); TOXLINE (TOXNET) (Appendix 6). 
Safety data were obtained from clinical trials.

We updated the searches in CENTRAL (the Cochrane Library 2014, Issue 5), MEDLINE (2009 to 5 June 2014), Embase (2009 to 5 June 2014), SCOPUS (2009 to 5 June 2014), TOXLINE (2009 to 5 June 2014), Web of Knowledge (2009 to 5 June 2014) and the websites of the FDA and EMEA (2009 to 5 June 2014).

For this updated review, we updated the searches of MEDLINE; Embase, Cochrane Database of Systematic Reviews (CDSR) and Cochrane Central Register of Controlled Trials (CENTRAL), HTA, DARE, NHS EED (the Cochrane Library), and WOK in January 2016 and again in September 2016 (see Appendix 10; Appendix 11; Appendix 12; Appendix 13).

\section{Searching other resources}

1. We examined the information made available by the main researchers and sponsors in ClinicalTrials.gov and the WHO International Clinical Trials Registry Platform (apps.who.int/ trialsearch/).

2. We reviewed information on the clinical trial meta-register database (www.controlled-trials.com/mrct/).

3. We inspected the reference lists of all identified studies for more trials.

4. When published data were missing, incomplete, or inconsistent with the trial protocols, we sought further information from the authors and manufacturers (UCB).

\section{Data collection and analysis}

\section{Selection of studies}

Two review authors independently checked the search results for studies that potentially met the inclusion criteria, resolving disagreements by discussion or by referral to a third review author.

\section{Inclusion criteria}

1. RCTs that compared certolizumab pegol with any other agent including placebo in adults with active RA despite current or prior treatment with DMARDs.

2. Trials that were fully published as a paper or available as a complete trial report. Where they were published only as abstracts, we requested the trial reports from the manufacturers.

3. Studies having at least three months of follow-up to assess benefits.

To assess harms we also sought studies having a suboptima length of follow-up, from eight weeks.

\section{Exclusion criteria}

1. Trials of certolizumab pegol for juvenile arthritis, Crohn's disease, psoriatic arthritis and other forms of spondyloarthritis.

2. Trials of certolizumab pegol comparing different doses or routes of administration without another active or placebo control group (except for assessing harm outcomes).

3. Studies reporting solely on laboratory measures aimed at investigating disease or treatment mechanisms and which did not report relevant clinical outcomes.

4. Observational studies of certolizumab pegol.

5. Interim results of trials.

\section{Data extraction and management}

Two review authors independently checked titles and abstracts of studies found by the search, to assess which studies might potentially meet the inclusion criteria; where there was doubt, we acquired the full article for further inspection. We then obtained studies identified by this process and two review authors independently screened them to see if they met the review criteria using a web interface.

We extracted data when possible for intention-to-treat populations, as raw numbers plus any summary measures with the standard deviations, confidence intervals and $P$ values of the outcomes reported. We compiled them in an Excel spreadsheet. We would have resolved any differences of opinion and data discrepancies by reference to a third review author (SB) but this proved to be unnecessary.

\section{Assessment of risk of bias in included studies}

According to the recommendations in the Cochrane Handbook for Systematic Reviews of Interventions (Higgins 2011), we assessed the risks of bias by creating a 'Risk of bias' table for each study. We present a summary below as a 'Risk of bias' graph.

The main criteria used to assess the risks of bias included: random sequence generation, allocation concealment, blinding of participants, incomplete outcome data, selective reporting of outcomes, and other potential biases (such as fraud or imbalance in the groups, or the sponsor either owning the data or needing to approve the manuscript). We rated the risk of bias in each study on the basis of each criterion as: low risk of bias, high risk of bias, unclear risk of bias (either lack of information or uncertainty over the potential bias). We included these criteria in the tables, resolving disagreements by discussion between the two review authors with recourse to a third review author if necessary, but in the event there were no disagreements.

\section{Measures of treatment effect}

We used the risk difference to quantify the number needed to treat for an additional beneficial outcome (NNTB) (Laupacis 1988). We calculated the NNTB from the risk ratio according to the formula NNTB $=1 / A^{*}{ }^{*}(1-R R)$, where ACR is the assumed control risk and $\mathrm{RR}$ the risk ratio. When events were very rare (fewer than 10\%) we used the Peto odds ratio (Peto OR). For continuous data we used mean differences (MDs) when the results were measured in the same way in the different studies. We used standardised mean differences (SMDs) when the results obtained were conceptually the same but used different measurement scales. We recorded the central estimate (mean) and standard deviation (SD). Where these were not directly stated we calculated them from the standard error or the different means and their respective confidence intervals (Cls) or $\mathrm{P}$ values. When medians and interquartile ranges were the only data provided, we used the median as a proxy measure of the mean and we considered the difference between the first and third interquartile to be equivalent to 1.35 of the SD.

\section{Unit of analysis issues}

Most of the clinical trials had a simple parallel-group design with participants individually randomised to one of two intervention groups. The unit of analysis was not an issue for this review. 


\section{Dealing with missing data}

We carried out an intention-to-treat analysis. Every individual allocated to the intervention was counted, whether they completed the follow-up or not. We have assumed that those who dropped out had no change in their outcome. This rule is conservative for the response to treatment because it assumes that those discontinuing the studies would not have responded. It is not conservative for adverse effects. However, assuming that all those leaving early had developed side effects could overestimate risk.

When published data were missing, incomplete or inconsistent with the RCT protocols or meeting abstracts, we asked for further information from the authors and manufacturers. We excluded abstracts of studies only if they were interim reports of studies that had not yet finished recruiting.

\section{Assessment of heterogeneity}

We have explored heterogeneity between the trials using the $\mathrm{Chi}^{2}$ test for heterogeneity, with a $10 \%$ level of significance, and the $1^{2}$ statistic. We interpreted the ranges of $1^{2}$ according to the recommendations in the Cochrane Handbook for Systematic Reviews of Interventions:

$0 \%$ to $40 \%$ might not be important;

$30 \%$ to $60 \%$ may represent moderate heterogeneity;

$50 \%$ to $90 \%$ may represent substantial heterogeneity;

$75 \%$ to $100 \%$ represents considerable heterogeneity (Higgins 2011).

\section{Assessment of reporting biases}

We planned to explore reporting bias using funnel plots when doing a meta-analysis for 10 or more studies.

\section{Data synthesis}

We explored the need to pool the results according to a fixedeffect or random-effects model analysis (Laird 1990). We planned to use the fixed-effect model to pool the data because statistical heterogeneity in our preview review was not high. However, we decided finally to perform a random-effects model, despite the $1^{2}$ values being low. Although it was the same drug, there was

Figure 1. Update: Records identified through the databases: $(n=559)$ Additional records identified through other sources (Clinicaltrials.gov, ICRTP) clear clinical heterogeneity (different doses, allowing MTX or not, different follow-up, different duration of RA, etc.).

\section{Subgroup analysis and investigation of heterogeneity}

We planned subgroup analyses for the duration of the illness (approximately three years evolution), participants' sex, drug dose and administration, and methodological quality. If we had detected heterogeneity then we would have conducted a subgroup analysis (Yusuf 1991), or a meta-regression (Thompson 1999) to see if it

\section{Sensitivity analysis}

We planned the following sensitivity analyses in order to explore effect size differences and the robustness of conclusions:

1. Effect of study quality, defined as random sequence generation, allocation concealment, blinding of participants, incomplete outcome data, selective outcome reporting and other potential sources of bias.

2. Effect of imputation, size of trials, use of concomitant methotrexate, and doses of certolizumab pegol.

\section{'Summary of findings' table}

We used the GRADE approach, developed by the GRADE working group, to provide an overall assessment of the quality of the evidence by outcome. The GRADE approach specifies four levels of quality, with the highest quality rating for RCTs. Review authors can, however, downgrade randomised trial evidence from 'high' to 'moderate', 'low' or even 'very low' quality evidence, depending on the presence of specific factors: design or implementation, imprecision, inconsistency, indirectness, or reporting bias (see Cochrane Handbook for Systematic Reviews of Interventions Chapter XII (section 12.2) (Higgins 2011)).

\section{RESULTS}

\section{Description of studies}

\section{Results of the search}

See the flow chart (Figure 1) and 'Results of searches' in Appendix 10; Appendix 11; Appendix 12; Appendix 13; Appendix 14; Appendix 15; Appendix 16; Appendix 17. could be explained. 


\section{( $n=98)$ Flow diagram.}

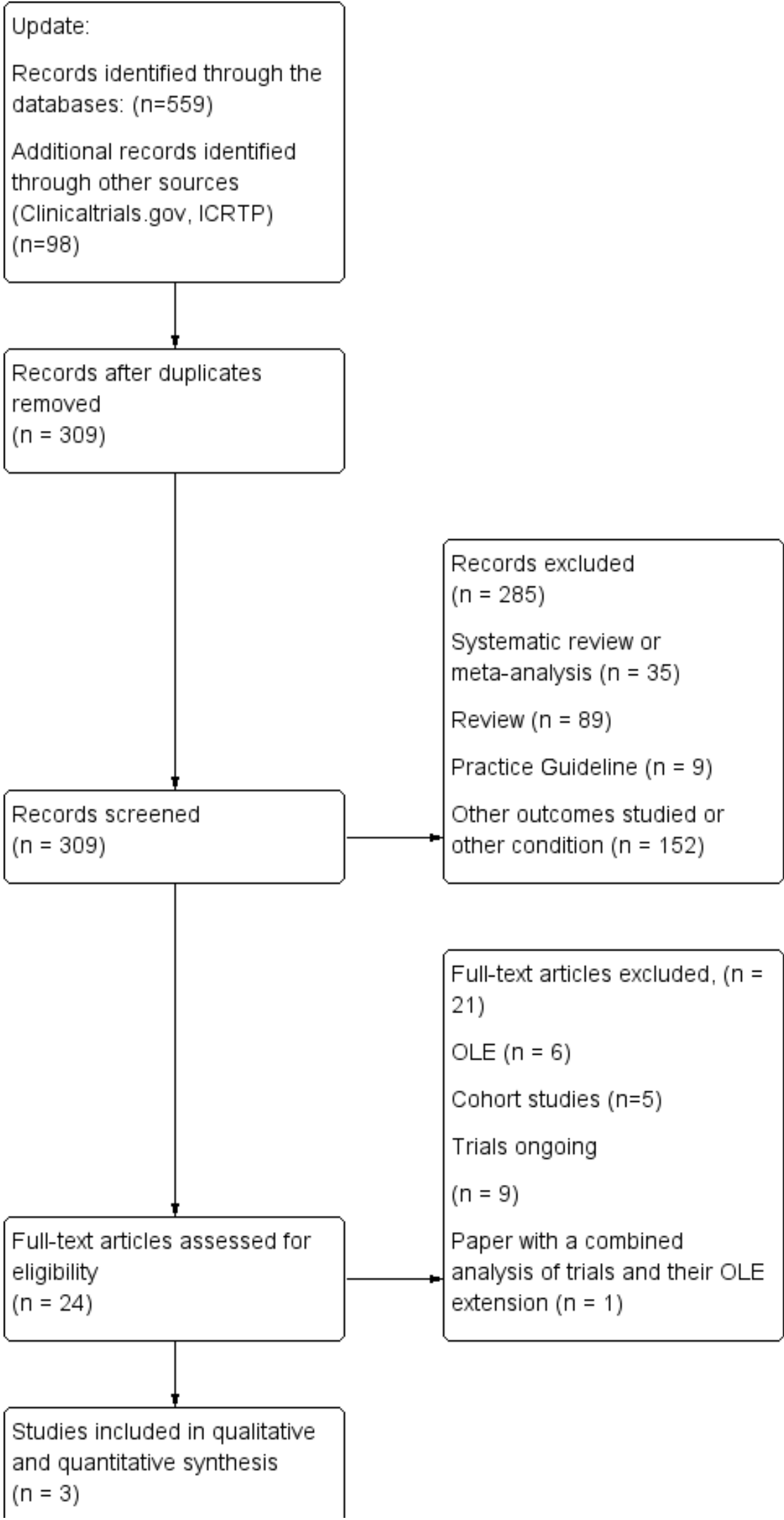

Records identified through the previous review $(n=11)$ 
Figure 1. (Continued)

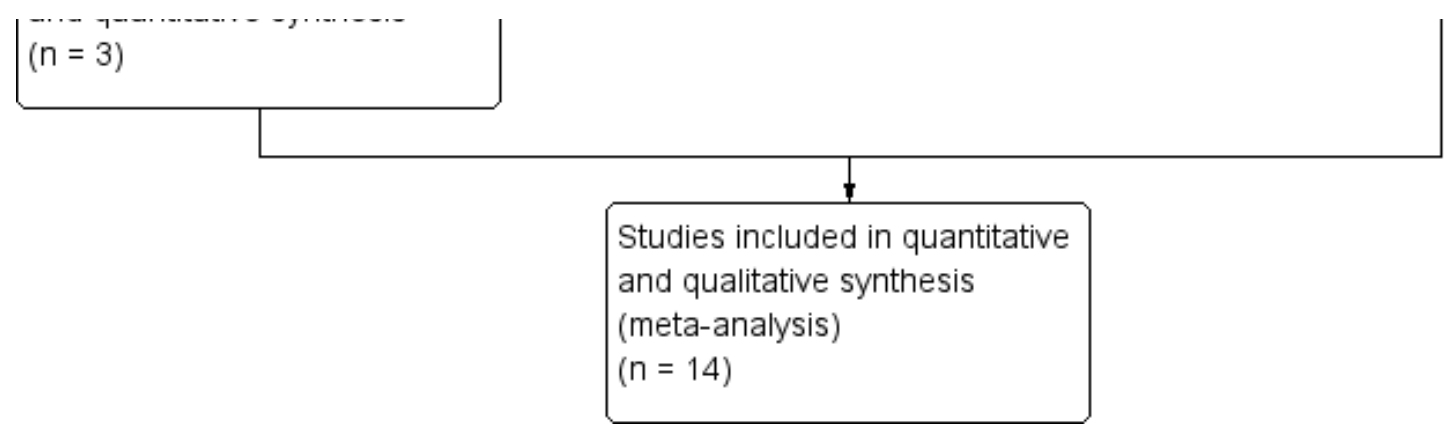

We include 14 trials in this update. Eleven (5422 participants) were included in the pooled analysis for benefits, two more than previously, and 13 (5273 participants) in the pooled analysis for safety. The duration of follow-up varied from 12 to 52 weeks and the range of doses of certolizumab pegol varied from 50 to $400 \mathrm{mg}$ given subcutaneously (sc). In Phase III trials, the control was placebo plus MTX in seven trials and placebo alone in five trials. In Phase II the comparator was placebo. So summarising 7 trials compared certolizumab plus MTX and 7 trials certolizumab compared with placebo.

In accord with Cochrane MECIR standards, the Cochrane Musculoskeletal Group (CMSG) updated the searches on 25 January 2016 and reran them on 27 September, 2016.

\section{Included studies}

We include 14 trials, 12 in the assessment of benefits (CDP870-004 2001; Choy 2012; Smolen 2015; Fleischmann 2009; Yamamoto (a) 2014; Yamamoto (b) 2014; NCT00993317; Atsumi 2016; Emery 2015; Keystone 2008; Smolen 2009; Weinblatt 2012) and 14 trials in the assessment of harms (CDP870-004 2001; Choy 2012; Smolen 2015; Choy 2002; Fleischmann 2009; Yamamoto (a) 2014; Yamamoto (b) 2014; NCT00993317; Østergaard 2015; Atsumi 2016; Emery 2015; Keystone 2008; Smolen 2009; Weinblatt 2012). See Table 1. See the Characteristics of included studies and the demographics and flow of participants in Table 2 and Table 4 for details. Only Choy 2002 and CDP870-004 2001 were Phase II studies. We found a third Phase II study (Kaushik 2005) but we were advised by UCB that: "this publication refers to the 2 previous phase II". We used all the Phase III studies to assess both benefits and harms. CDP870-004 2001 only contributed data on benefits, as it did not report any data on harms.
Due to the short follow-up for assessing benefits, we only included Choy 2002 for safety data. The data from the two Phase II studies (CDP870-004 2001; Choy 2002) were not pooled with the rest of the studies, due to the different follow-ups and doses used.

We retrieved 12 Phase III trials (Choy 2012; Smolen 2015; Fleischmann 2009; Yamamoto (a) 2014; Yamamoto (b) 2014; NCT00993317; Østergaard 2015; Atsumi 2016; Emery 2015; Keystone 2008; Smolen 2009; Weinblatt 2012). All the trials were funded by UCB. Data from Choy 2012 were provided by UCB from the clinical study summary (www.clinicalstudyresults.org/ documents/company-study_4348_0.pdf) and the EMA 2009 reports; they were finally published in 2012 (the study was completed in 2004).

Table 2 shows the demographic and baseline characteristics for the Phase III trials: age, gender, rheumatoid factor (RF) positivity, MTX concomitant dose, number of previous DMARDs, basal HAQ and basal DAS28, among other outcomes. Table 3 provides the flow chart of participants in the Phase III studies.

\section{Excluded studies}

The main reasons for exclusion were: 1) reviews; 2) different drugs; and 3) another outcome reported. See the Table Characteristics of excluded studies.

\section{Risk of bias in included studies}

We present the judgements about each 'Risk of bias' item as percentages across all included studies (Figure 2). We rated most of the trials at low risk of bias. The overall likelihood of bias seemed to be low. 
Figure 2. Risk of bias graph: review authors' judgements about each risk of bias item presented as percentages across all included studies.

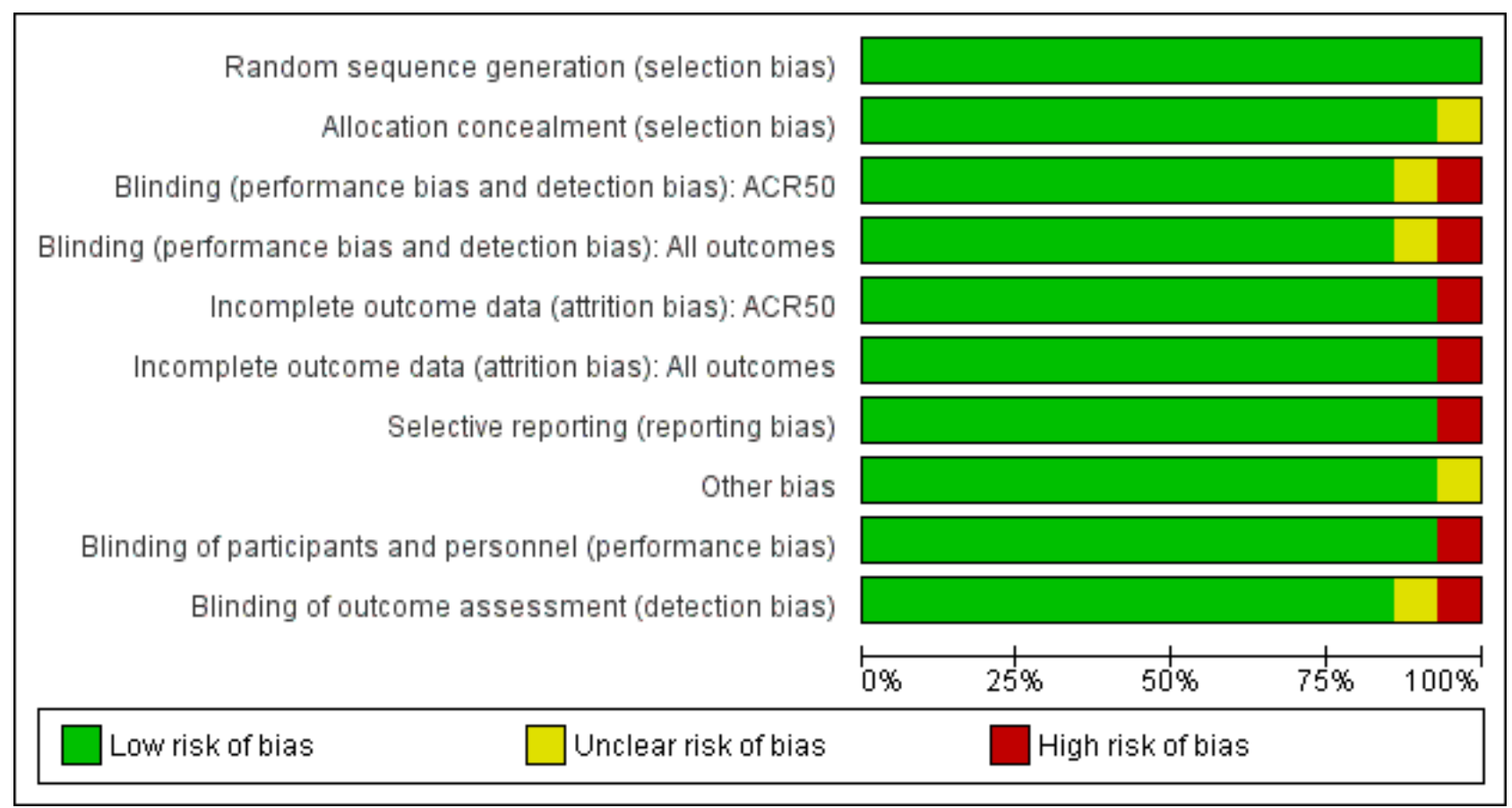

\section{Allocation}

All studies except CDP870-004 2001 reported adequate methods of randomisation and allocation concealment. Eight studies (Choy 2012; Smolen 2015; Fleischmann 2009; Atsumi 2016; Emery 2015; Keystone 2008; Smolen 2009; Weinblatt 2012) used the interactive voice response system (IVRS) method of allocation concealment. The Asian trials (Yamamoto (a) 2014; Yamamoto (b) 2014; NCT00993317) were described as: 'external randomisation' (NCT00993317) or randomisation by blocks (Yamamoto (a) 2014; Yamamoto (b) 2014), so the risk of bias seemed to be low.

\section{Blinding}

All studies except CDP870-004 2001 reported adequate blinding. Refer to Figure 3. 
Figure 3. Risk of bias summary: review authors' judgements about each risk of bias item for each included study.

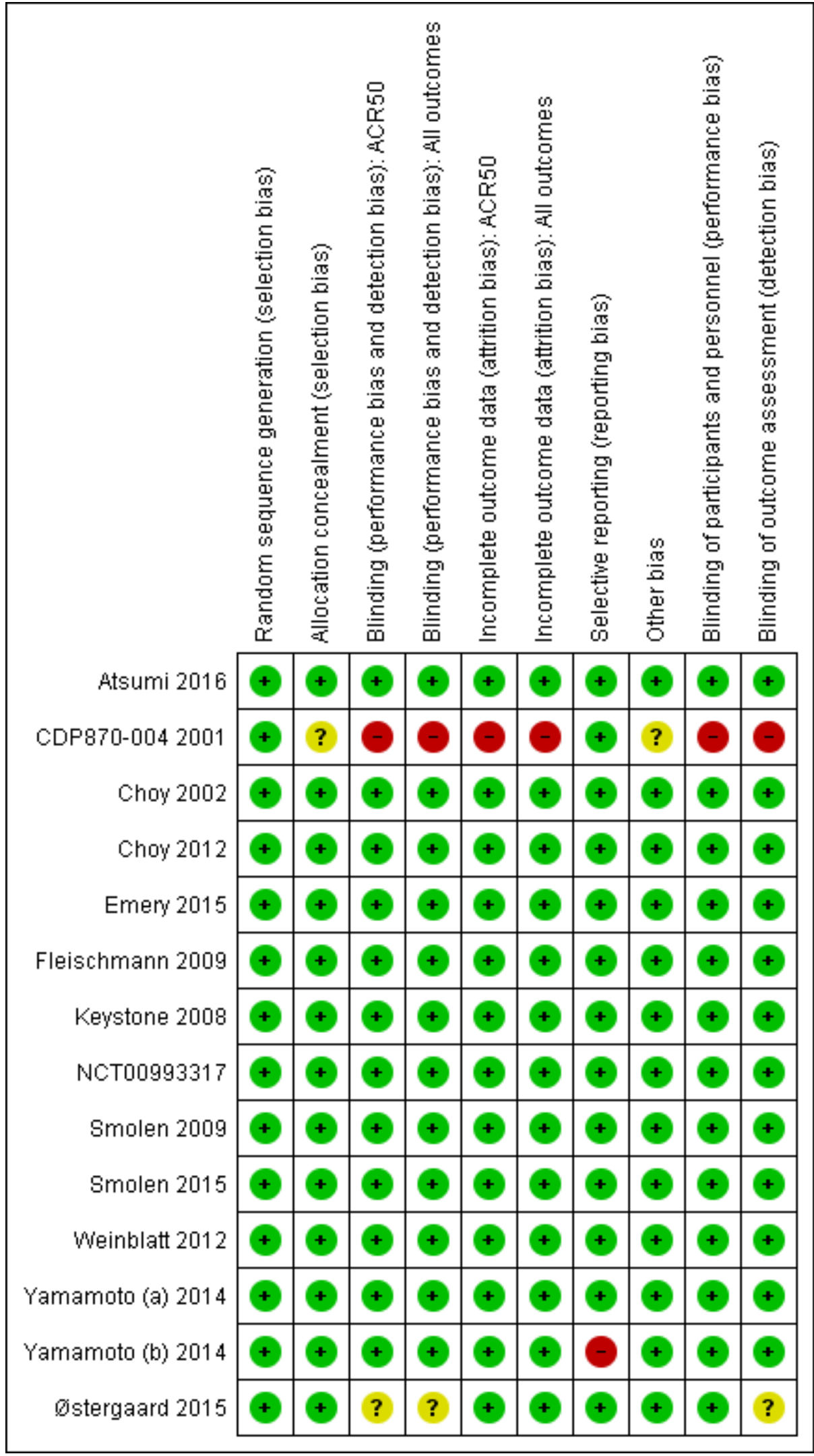

Phase II: 
- CDP870-004 2001 did not disclose the methods of blinding, and UCB explained to us: "CPD-870 and the placebo utilized in this study (saline) did not have the same viscosity therefore full blinding was not possible. Study drug was to be prepared by a pharmacist having no other involvement in the study; injections of study medications were given by a nurse or physician who had no other involvement in the study...";

- Choy 2002 disclosed the methods of blinding: "Placebo (sodium acetate buffer) was given similarly as a single intravenous infusion of $100 \mathrm{ml}$ over $60 \mathrm{~min}$ ". It was unlikely that the blinding could have been broken. UCB explained to us: "all data were entered and Database locked after completion of the clinical phase for the first study period and before ESR and CRP were entered into the database. ESR and CRP data were withheld from investigator and sponsor study personal during the course of the study because knowledge of patient's profile could potentially unblind the study..., auto AB, anti CZP level, TNFalpha, IL6 and IL1b were transferred into the database after Database lock."

Phase III:

- UCB told us, "in Fleischmann 2009, Choy 2012, Keystone 2008, Smolen 2009, Smolen 2015, Weinblatt 2012, all the study staff, with the exception of the unblinded dispenser, was blind to the treatment. Each study center was required to have a written blinding plan in place signed by the Principal Investigator, which detailed the study center's steps for ensuring that the double blind nature of the study was maintained. All the studies were monitored by two different independent teams from the sponsor, one devoted to blind data and one devoted to possibly unblinded information (such as study medications related topics) and completely separate documentation/filing systems were maintained for the duration of the trials";

- Keystone 2008: "Radiographs were read at a central location by 3 independent readers. Readers were blinded as to the patient's identity, clinical data, treatment, and time point (sequence) at which the radiograph was taken";

- Smolen 2009: "Radiographs were read centrally and blinded (for treatment, visit and patient identification) and independently by two experienced readers";

- Fleischmann 2009 disclosed methods of blinding: "Solutions of active drug or placebo were prepared by the pharmacist or other unblinded, qualified site personnel, before distributing to blinded study personnel for administration".

- in the Japanese and Korean trials (Yamamoto (a) 2014; Yamamoto (b) 2014; NCT00993317) "All study staff with the exception of the unblinded dispenser were blind to the treatment, ... These unblinded personnel were not allowed to engage in any other study activities".

- in Østergaard 2015: "The personnel administering the injections had no involvement in the study other than performing the erythrocyte sedimentation rate analysis"

- in Atsumi 2016: "Drug administration was performed by dedicated non blinded persons due to distinguish ability of CZP from PBO; however, these personnel were not permitted to engage in other study activities to maintain blinding. All investigators and healthcare professionals involved in safety/ efficacy assessments were blind to study medications"

- in Emery 2015: "Sponsor, investigator site and vendor staff involved will be blinded to the testaments assignment with the following exceptions: sponsor clinical study supplies coordinator and qualifier person unblinded site personnel involved in ESR determination" (UCB private files). We do not have any information about how the blinding was performed.

For these reasons, we rated the risk of bias for blinding as low.

\section{Incomplete outcome data}

All studies, except the small Phase II trial (CDP870-004 2001) reported adequate methods of handling missing outcome data. All other studies gave a full account of all withdrawals and reasons for withdrawals. Where possible, we extracted data to allow an intention-to-treat analysis in Choy 2012; Fleischmann 2009; NCT00993317. Eight out of 11 studies reported less than $80 \%$ completion rates. However, for ACR20, ACR50, ACR70 DAS remission, SAEs, withdrawals and withdrawals due to adverse events we conducted an ITT analysis. Only radiological scores and $\mathrm{HAQ}$ were analysed per protocol. In consequence for the overall estimation, we think the risk of bias is low. Refer to Figure 3.

The completion rates in the certolizumab pegol group ranged from 68\% in Fleischmann 2009 to $90 \%$ in Weinblatt 2012. In all trials, fewer participants in the placebo-treated group completed the trial compared to the treatment arm. More participants who were treated with placebo withdrew due to lack of efficacy. The percentage of those completing the trial in the placebo group ranged from 15\% in the 12-month results of Yamamoto (a) 2014 to $86 \%$ in the 12 -week results of Weinblatt 2012 . We imputed missing data using last observation carried forward (LOCF) in most trials. The new trials for this update (Atsumi 2016: Emery 2015) reported low rates of participants who finished the trials.

In the Atsumi 2016 trial, "Patients who did not achieve an improvement of RA symptoms (defined as the persistence of DAS28[ESR] $\geq 3.2$ for4 weeks or longer) after Week 24 were eligible to withdraw from trial and move to rescue treatment with open label trial of CZP” so, $22.6 \%$ in the certolizumab pegol group and $44.6 \%$ in placebo group were withdrawn. We did not find this assumption in the protocol in clinicaltrials.gov/ct2/ show/NCT01451203. Similary in Emery 2015 the participants "not achieving sufficient improvement defined as DAS 28 DAS28[ESR] $\geq 3.2$ and or $\geq 1.2$ point improvement in DAS28(ESR) from BL at weeks 20 and 24 were withdrawn to allow them to switch to a complementary medication". In this trial $15 \%$ of people withdrew from the placebo arm and $8 \%$ from the certolizumab pegol arm, but people also withdrew for lack of efficacy, adverse events, protocol violation and being lost to follow-up. Total withdrawals in the placebo group amounted to $34 \%$ of participants and $24 \%$ from the certolizumab pegol group. We did not find in the protocol hold in clinicaltrials.gov again this assumption clinicaltrials.gov/ ct2/show/NCT01519791?term=NCT01519791\&rank=1. In Keystone 2008 "certolizumab pegol or placebo patients who were ACR20 non-responders at both weeks 12 and 14 in RCT, were required to withdraw at week 16 ". One hundred-and-thirty-nine out of 199 left the placebo arm (70\%) and 181 out of 783 in the certolizumab pegol arm (23\%). In Østergaard 2015 three of 27 participants discontinued due to adverse events and lack of efficacy, while one of 17 in the placebo group discontinued for withdrawal of consent. Newly we did not find any assumption in the protocol. This trial was small (41 people) with very short follow-up of two weeks, focused only on radiological changes. In summary, higher rates of withdrawal in the certolizumab pegol arm with a long-term follow-up can introduce a serious bias into the interpretation of effectiveness of certolizumab 
pegol. Moreover, the assumption that people could be withdrawn if they did not achieve a good response was not prespecified in the protocols.

\section{Selective reporting}

All studies reported their prespecified outcomes, except for Yamamoto (b) 2014. UCB gave ACR20/50/70 as a figure as well as providing the DAS, but we could not pool DAS data and we had no information about the modified Total Sharp Score (mTTS) for radiographic progression.

We changed our previous assessment of the bias in Fleischmann 2009 , because all the primary outcomes were described in the paper.

In the previous version of the review Choy 2012 only reported ACR20, but the ACR50, HAQ disability index and acute-phase reactant (CRP) are now available, so we have revised our 'Risk of bias' assessment to low.

In summary, we think the risk of reporting bias in this update is low. Refer to Figure 3.

\section{Other potential sources of bias}

We did not detect potential threats to validity, such as fraud or imbalance in the groups (relating to the baseline characteristics). All studies included in this review were sponsored by the manufacturer of certolizumab pegol. There is evidence that industry-sponsored trials may overestimate the treatment effect (Bhandari 2004) and there is also evidence that most of the authors of published trials have a conflict of interest. However, there is a lack of consensus on whether these conflicts result in reduced quality of the trials and, in view of this, we have decided to rate the risk of bias for this domain as low.

We searched for more trials as well as for more information about unpublished trials (see Characteristics of ongoing studies table), but no information was available, either from the sponsors or from any publication.

In summary, we think the risk of other potential sources of bias is low for this update. Refer to Figure 3.

\section{Summary assessment of risk of bias by outcomes}

Figure 2 and Figure 3 provide a graphical summary of the results of the 'Risk of bias' assessments for the 14 included studies.

\section{The main major outcomes}

ACR 50 response at six months and 52 weeks: we rated six studies at six months and three studies at 52 weeks included in the metaanalysis at low risk for adequate allocation concealment, blinding and reporting of appropriate outcomes. Although there were high rates of withdrawals, we rated the trials at low risk of bias, since we were able to conduct an ITT analysis. Another concern was that all studies were sponsored by the manufacturer of certolizumab pegol.

$\mathrm{HAQ}$ change from baseline, response at six months and 52 weeks: we rated five studies at six months and two studies at 52 weeks included in the meta-analysis at low risk for adequate allocation concealment, blinding and reporting of appropriate outcomes. However, we had concerns about bias for incomplete outcome data due to the high dropout rates.This item was subject to a per protocol analysis, which we downgraded by one level. Another concern was that all studies were sponsored by the manufacturer of certolizumab pegol.

Proportion of participants achieving remission (DAS < 2.6) at 24 weeks: six studies. We rated them at low risk of bias for all the domains. Despite the rates of withdrawals, we conducted an ITT analysis for this outcome. Another concern was that all studies were sponsored by the manufacturer of certolizumab pegol.

Radiological changes (ES scores) at 24 weeks: two studies. We rated We rated all domains at low risk of bias. However, we had concerns about bias for incomplete outcome data, due to the dropout rates in both studies.This item was subject to per protocol analysis, and we downgraded it by one level. Another concern was that all studies were sponsored by the manufacturer of certolizumab pegol.

Serious adverse events with certolizumab pegol $200 \mathrm{mg}$ at any follow-up: we rated nine studies included in the meta-analysis at low risk of bias for adequate allocation concealment, blinding and reporting of appropriate outcomes. We analysed all of them on an ITT basis for all randomised participants who received at least one dose, but in two out of the nine studies the analysis was per protocol: in Smolen 2009 "two patients in the placebo group received certolizumab pegol $200 \mathrm{mg}$ and were included in the certolizumab pegol $200 \mathrm{mg}$ group for safety evaluations", and in Weinblatt 2012 nine participants fewer were analysed in the certolizumab pegol arm and three participants fewer in the placebo group. In Atsumi 2016, an ITT analysis was performed. However, in Emery 2015, the analysis was per protocol, with two participants fewer in the control group and one less in the (CZP) group. We performed an ITT analysis In Østergaard 2015 trial. Another concern was that all studies were sponsored by the manufacturer of certolizumab pegol.

Withdrawals for all doses and follow-up to 52 weeks: we rated 13 studies at low risk of bias in all the domains. We conducted an ITT analysis for all the trials. Another concern was that all studies were sponsored by the manufacturer of certolizumab pegol.

Withdrawals due to adverse events for all doses and follow-up to 52 weeks: we rated 12 studies at low risk of bias in all the domains. We conducted an ITT analysis for all the trials. Another concern was that all studies were sponsored by the manufacturer of certolizumab pegol.

\section{Effects of interventions}

See: Summary of findings for the main comparison Certolizumab pegol $200 \mathrm{mg}$ sc (with or without MTX) versus placebo (with or without MTX) for rheumatoid arthritis in adults

We conducted our analyses based on the doses used in the trials, i.e. the drug exposure time for subcutaneous (sc) doses of $200 \mathrm{mg}$ and $400 \mathrm{mg}$. For $400 \mathrm{mg}$ the most usual was at four-week intervals, and for $200 \mathrm{mg} s c$ the most frequently-used was every other week, but in some trials such as Keystone 2008 and Smolen 2009 the interval was every two weeks for the $400 \mathrm{mg}$ dose as well. As we had two periods of follow-up (six months and one year) in one study, we could not combine them, so we pooled each outcome at each follow-up. We also had studies with more than one dose, so we split the placebo arm to enable us to pool results. We did not find strong differences that could justify our not combining the results for benefits and harms. We decided to perform a random-effects 
model, in spite of the low values of 12 . Although it was the same drug, there is clear clinical heterogeneity (different doses, allowing MTX or not, different follow-up, different duration of RA, etc.).

\section{Major outcomes}

\section{ACR50}

We noted significant improvements for all doses at any given time point for the ACR50 compared to placebo (see 'Benefits' tables, ACR Table 4, Data and analyses).

The ACR50 with $200 \mathrm{mg}$ certolizumab pegol showed, at 24 weeks, a risk ratio (RR) of 3.80 (95\% confidence interval ( $\mathrm{Cl}) 2.42$ to 5.95), five studies, involving 1445 participants (Analysis 2.1); The ACR50 with $400 \mathrm{mg}$ certolizumab pegol showed, at 24 weeks, a RR of 4.65 (95\% $\mathrm{Cl} 3.09$ to 6.99), five studies, involving 1591 participants (Analysis 3.1). We judged the quality of evidence for ACR50 with 200 and 400 $\mathrm{mg}$ certolizumab pegol at 24 weeks to be high

The ACR50 with $200 \mathrm{mg}$ certolizumab pegol showed, at 52 weeks a RR of 1.54 (95\% Cl 1.38 to 1.73 ), three studies, involving 881 participants (Analysis 4.1). This analysis reported an High value of 12. We explained this due to that the results of RAPID1 showed a very high values RR 5.02 whereas the remaining trials showed lowest values around RR of 1.41 or 1.21). Moreover the Cl of RAPID1 did not overlap the remaining trials.

The ACR50 with $400 \mathrm{mg}$ certolizumab pegol showed, at 52 weeks, a RR of 5.27 (95\% Cl 3.19 to 8.71), one study, involving 589 participants (Analysis 5.1).

We judged the quality of evidence for ACR50 with 200 and $400 \mathrm{mg}$ certolizumab pegol at 52 weeks to be high.

The NNTB was close to 4 for all the sub analyses (Table 4).

\section{Health-related quality of life}

We found an improvement in physical function and quality of life measured with the HAQ and SF-36 (in the mental and physical components) at all follow-ups (see 'Health-related quality of life' tables, (Table 5)) with certolizumab pegol compared to placebo.

HAQ at 24 weeks, 200 mg: mean difference (MD) -0.35 (95\% Cl -0.43 to -0.26), four studies, involving 1268 participants (Analysis 7.1).

We judged the quality of evidence for HAQ at 24 weeks, $200 \mathrm{mg}$ to be moderate. We downgraded the quality of evidence by one level, due to a high risk of attrition bias (per protocol analysis).

HAQ disability index (HAQ-DI) at 24 weeks, $400 \mathrm{mg}$ : MD -0.38 (95\% $\mathrm{Cl}-0.48$ to -0.28 ), four studies, involving 1425 participants (Analysis 7.2).

We judged the quality of evidence for HAQ-DI, 24 weeks, $400 \mathrm{mg}$ to be moderate. We downgraded the quality of evidence by one level, due to a high risk of attrition bias (per protocol analysis).

HAQ-DI at 24 weeks, any dose: MD $-0.36(95 \% \mathrm{Cl}-0.43$ to -0.29$)$, five studies, involving 2246 participants (Analysis 8.1).

We judged the quality of evidence for HAQ-DI, 24 weeks any dose $200 \mathrm{mg}$ to be moderate. We downgraded the quality of evidence by one level, due to a high risk of attrition bias (per protocol analysis).
HAQ-Di, 52 weeks, any dose: MD $-0.32(95 \% \mathrm{Cl}-0.39$ to -0.26$)$, two studies, involving 1837 participants (Analysis 9.1).

We judged the quality of evidence for HAQ-DI at 24 weeks, $200 \mathrm{mg}$ to be moderate. We downgraded the quality of evidence by one level, due to a high risk of attrition bias (per protocol analysis).

We judged the quality of evidence for HAQ-DI at 52 weeks, any dose to be to be moderate. We downgraded the quality of evidence by one level, due to a high risk of attrition bias (per protocol analysis). This analysis reported a High value of $\mathrm{I}^{2}$. We explained this due to that the results of RAPID1 showed a very high values MD -0.42 whereas the remaining trial showed lowest values around MD of -0.18 . Moreover the $\mathrm{Cl}$ of RAPID1 did not overlap the remaining trial.

SF-36 physical component summary (PCS) at 24 weeks, any dose: MD $5.29(95 \% \mathrm{Cl} 4.37$ to 6.21$)$, three studies, involving 1765 participants (Analysis 14.1).

SF-36 mental component summary (MCS) at 24 weeks, any dose: MD $4.01(95 \% \mathrm{Cl} 2.94$ to 5.08), four studies, involving 2012 participants (Analysis 15.1);

We judged the quality of evidence for SF-36 PCS and SF-36 MCS at 24 weeks, any dose, to be moderate. We downgraded the quality of evidence by one level due to a high risk of attrition bias (per protocol analysis).

SF-36 PCS at 52 weeks, any dose: MD 6.47 (95\% Cl 5.13 to 7.81 ), one study, involving 982 participants (Analysis 16.1).

SF-36 MCS at 52 weeks, any dose: MD 4.30 ( $95 \% \mathrm{Cl} 2.57$ to 6.03 ), one study, involving 982 participants (Analysis 17.1).

We judged the quality of evidence for SF-36 PCS and SF-36 MCS at 52 weeks, any dose, to be moderate. We downgraded the quality of evidence by one level, due to a high risk of attrition bias (per protocol analysis).

\section{DAS-28}

We observed significant improvements for all doses and at any given time point compared to placebo.

At 24 weeks the proportion of participants achieving remission (DAS <2.6) was higher in the $200 \mathrm{mg}$ certolizumab pegol group than in the placebo group (RR $2.94,95 \% \mathrm{Cl} 1.64$ to 5.28 ), six studies, involving 2420 participants (Analysis 19.1.1); and RR of $1.71(95 \% \mathrm{Cl}$ 1.43 to 2.04 ) at 52 weeks, three studies, involving 1689 participants (Analysis 20.1.1.).

We judged the quality of evidence for DAS $<2.6,200 \mathrm{mg}$ at 24 and 52 weeks to be high.

The RR for participants achieving remission (DAS <2.6) with $200 \mathrm{mg}$ certolizumab pegol at 12 weeks was $1.99(95 \% \mathrm{Cl} 1.44$ to 2.76$)$, two studies, involving 1942 participants (Analysis 21.1).

We judged the quality of evidence for DAS $<2.6$ at 12 weeks, $200 \mathrm{mg}$ to be high.

The RR for participants achieving remission (DAS < 2.6) with 400 mg certolizumab pegol was $7.18(95 \% \mathrm{Cl} 3.12$ to 16.50$)$ at 24 weeks, three studies, involving 1201 participants (Analysis 21.3); and at 52 
weeks the RR was 12.49 (95\% Cl 3.99 to 39.12), one study, involving 583 patients (Analysis 21.5).

We judged the quality of evidence for DAS $<2.6,400 \mathrm{mg}$ at 24 and 52 weeks to be high.

\section{Radiological changes}

Radiological changes were expressed as modified Total Sharp Scores (mTSS), the erosion score (ES) and joint space narrowing (JSN). All certolizumab pegol groups showed improvements compared to placebo in the mean changes from baseline. There was a clear radiological benefit, regardless of the dose, associated with drug exposure time (see 'Radiological changes', Table 6).

ES at $200 \mathrm{mg}, 24$ weeks: MD $-0.35(95 \% \mathrm{Cl}-0.50$ to -0.21$)$, two studies, involving 859 participants (Analysis 29.1).

We judged the quality of evidence for ES at $200 \mathrm{mg}, 24$ weeks to be moderate. We downgraded the quality of evidence by one level, due to a high risk of attrition bias (per protocol analysis).

ES at $200 \mathrm{mg}, 52$ weeks: MD $-1.14(95 \% \mathrm{Cl}-1.54$ to -0.74$)$, two studies, involving 1235 participants (Analysis 29.3).

We judged the quality of evidence for ES at $200 \mathrm{mg}, 52$ weeks to be moderate. We downgraded the quality of evidence by one level, due to a high risk of attrition bias (per protocol analysis).

ES at any dose, 24 weeks: MD $-0.70(95 \% \mathrm{Cl}-0.98$ to -0.42$)$, two studies, involving 1437 participants (Analysis 30.1).

We judged the quality of evidence for ES at any dose, 24 weeks to be moderate. We downgraded the quality of evidence by one level, due to a high risk of attrition bias (per protocol analysis).

ES at any dose, 52 weeks: MD $-1.16(95 \% \mathrm{Cl}-1.56$ to -0.77$)$, two studies, involving 1599 participants (Analysis 31.1).

We judged the quality of evidence for ES at any dose, 52 weeks to be moderate. We downgraded the quality of evidence by one level, due to a high risk of attrition bias (per protocol analysis).

Joint space narrowing (JSN) at $200 \mathrm{mg}, 24$ weeks: MD -0.45 (95\% $\mathrm{Cl}-0.77$ to -0.13 ), two studies, involving 861 participants (Analysis 32.1).

We judged the quality of evidence for JSN at $200 \mathrm{mg}, 24$ weeks to be moderate. We downgraded the quality of evidence by one level, due to a high risk of attrition bias (per protocol analysis).

JSN at $200 \mathrm{mg}, 52$ weeks: MD $-0.67(95 \% \mathrm{Cl}-1.02$ to -0.32$)$, two studies, involving 1239 participants (Analysis 32.3).

We judged the quality of evidence for JSN at $200 \mathrm{mg}, 52$ weeks to be moderate. We downgraded the quality of evidence by one level, due to a high risk of attrition bias (per protocol analysis).

JSN at any dose, 24 weeks: MD $-0.50(95 \% \mathrm{Cl}-0.79$ to -0.21$)$, two studies, involving 1439 participants (Analysis 33.1).

We judged the quality of evidence for JSN at any dose, 24 weeks to be moderate. We downgraded the quality of evidence by one level, due to a high risk of attrition bias (per protocol analysis).
JSN at any dose, 52 weeks: MD $-0.70(95 \% \mathrm{Cl}-1.04$ to -0.36$)$, two studies, involving 1602 participants (Analysis 34.1).

We judged the quality of evidence for JSN at any dose, 52 weeks to be moderate. We downgraded the quality of evidence by one level, due to a high risk of attrition bias (per protocol analysis).

MTSS at any dose, 24 weeks: MD $-0.86(95 \% \mathrm{Cl}-1.19$ to -0.53$)$, three studies, involving 1753 participants (Analysis 35.1).

We judged the quality of evidence for mTSS at any dose, 24 weeks to be moderate. We downgraded the quality of evidence by one level, due to a high risk of attrition bias (per protocol analysis).

Modified Total Sharp Scores (mTSS) at $200 \mathrm{mg}, 24$ weeks: MD -0.74 (95\% Cl -1.11 to -0.37 ), three studies, involving 1029 participants (Analysis 35.1.1).

We judged the quality of evidence for mTSS at $200 \mathrm{mg}, 24$ weeks to be moderate. We downgraded the quality of evidence by one level, due to a high risk of attrition bias (per protocol analysis).

MTSS at any dose, 52 weeks: MD $-1.63(95 \% \mathrm{Cl}-2.13$ to -1.13$)$, three studies, involving 1915 participants (Analysis 36.1).

We judged the quality of evidence for mTSS at any dose, 52 weeks to be moderate. We downgraded the quality of evidence by one level, due to a high risk of attrition bias (per protocol analysis).

MTSS at $200 \mathrm{mg}, 52$ weeks: MD $-1.54(95 \% \mathrm{Cl}-2.06$ to -1.01$)$, three studies, involving 1462 participants (Analysis 36.1.1).

We judged the quality of evidence for mTSS $200 \mathrm{mg}, 52$ weeks to be moderate. We downgraded the quality of evidence by one level, due to a high risk of attrition bias (per protocol analysis).

\section{Serious adverse events (SAEs) as defined in the studies}

The clinical study summary of CDP870-004 2001 did not define SAEs. All the new trials that were added in this update reported on SAES.

We reported adverse events grouped by the dosages:

SAEs for certolizumab pegol $200 \mathrm{mg}$ and any follow-up time point: Peto OR 1.47 (95\% Cl 1.13 to 1.91), nine studies, involving 3927 participants (Analysis 41.1);

We judged the quality of evidence for SAEs for certolizumab pegol $200 \mathrm{mg}$ and any follow-up to be high.

SAEs for certolizumab pegol $400 \mathrm{mg}$ and any follow-up time point: RR 1.98 ( $95 \% \mathrm{Cl} 1.36$ to 2.90 ), six studies, involving 1624 participants (Analysis 42.1); 95 events were reported in the certolizumab pegol groups versus 31 events in the control groups.

We judged the quality of evidence for SAEs for certolizumab pegol $400 \mathrm{mg}$ at any follow-up time point to be high.

We decided to use Peto OR due to the low number of events in both 200 and $400 \mathrm{mg}$ of certolizumab pegol.

\section{All withdrawals}

There were more withdrawals "at any dose and at any follow-up" in placebo groups (53\%) versus the certolizumab pegol groups (23\%): 
RR 0.47 (95\% $\mathrm{Cl} 0.39$ to 0.56 ), 13 studies, involving 5200 participants (Analysis 43.1).

We judged the quality of evidence for all withdrawals "at any dose and at any follow-up" to be moderate. We downgraded the quality of evidence by one level for inconsistency due to heterogeneity (not all of the confidence intervals overlap, and $\mathrm{I}^{2}$ is $79 \%$ ).

\section{Withdrawals due to adverse events}

There were more withdrawals "at any dose and at any followup due to adverse events" in the certolizumab pegol groups (5\%) versus placebo groups (4\%).

Withdrawals at any dose and at any follow-up due to adverse events: Peto OR 1.45 (95\% Cl 1.09 to 1.94), 12 studies, involving 5236 participants (Analysis 43.2).

We judged the quality of evidence for withdrawals at any dose and at any follow-up due to adverse events for certolizumab pegol to be high.

We have included all results in Summary of findings for the main comparison.

\section{Minor outcomes}

\section{ACR20 and ACR70}

We saw an improvement in ACR20 and ACR70 compared to placebo for all doses and at any time point.

ACR20 for any dose at 24 weeks: RR 2.76 (95\% Cl 2.29 to 3.33), eight studies, involving 2935 participants (Analysis 44.1).

ACR70 for any dose at 24 weeks: RR 4.15 ( $95 \% \mathrm{Cl} 2.68$ to 6.42 ), seven studies, involving 2705 participants (Analysis 44.3).

We judged the quality of evidence for ACR20 and ACR70 for any dose at 24 weeks for certolizumab pegol to be high.

ACR20 for any dose at 52 weeks: RR 1.46 (95\% $\mathrm{Cl} 1.11$ to 1.93), three studies, involving 2180 participants (Analysis 45.1).

We judged the quality of evidence for ACR20 for any dose at 52 weeks for certolizumab pegol to be moderate. We downgraded the quality of evidence one level for inconsistency due to heterogeneity (not all the confidence intervals overlap and $\mathrm{l} 2$ is $88 \%$ ).

ACR70 for any dose at 52 weeks: RR 1.89 (95\% $\mathrm{Cl} 1.44$ to 2.48), three studies, involving 2180 participants (Analysis 45.3).

We judged the quality of evidence for ACR70 for any dose at 52 weeks for certolizumab pegol to be high.

\section{Adverse events}

We reported all adverse events in Data and analyses but we have not commented on all of them in this section, but only those that we thought were noteworthy (see Table 7).

\section{Any adverse event}

We pooled the data for any adverse event from nine trials: $200 \mathrm{mg}$ certolizumab pegol: RR $1.16(95 \% \mathrm{Cl} 1.03$ to 1.31$)$, nine studies, involving 3927 participants (Analysis 50.1).
We judged the quality evidence for any adverse event for $200 \mathrm{mg}$ certolizumab pegol to bemoderate. We downgraded the quality of evidence one level for inconsistency due to heterogeneity (not all the confidence intervals overlap and $\mathrm{I} 2$ is $74 \%$ ).

Safety, any adverse event at $400 \mathrm{mg}$ certolizumab pegol: RR 1.19 $(95 \% \mathrm{Cl} 1.05$ to 1.34$)$, six studies, involving 1624 participants (Analysis 50.2).

We judged the quality of evidence for any adverse event for $400 \mathrm{mg}$ certolizumab pegol to be high.

We excluded Choy 2002 because it showed more events than participants in the certolizumab pegol group (62 events in 24 participants) as well as in the placebo group (19 events in 12 participants). We therefore could not calculate the RR.

\section{Adverse events: severe intensity as defined in the studies}

There were no differences in the number of SAEs between participants treated with $200 \mathrm{mg}$ : Peto OR 1.14 (95\% Cl 0.78 to 1.65), four studies, involving 2249 participants Analysis 50.7).

We judged the quality of evidence for adverse events with severe intensity for $200 \mathrm{mg}$ certolizumab pegol to be moderate. We downgraded the quality of evidence one level for imprecision due to the $95 \%$ confidence interval around the pooled effect including both harm and no harm.

Participants treated with $400 \mathrm{mg}$ of certolizumab pegol: Peto OR $1.23(95 \% \mathrm{Cl} 0.83$ to 1.81$)$, five studies involving 1462 participants (Analysis 50.8).

We judged the quality of evidence for adverse events with severe intensity for $400 \mathrm{mg}$ certolizumab pegol to be moderate. We downgraded the quality of evidence one level for imprecision, due to the $95 \%$ confidence interval around the pooled effect including both harm and no harm.

\section{Serious adverse infections (SAIs)}

This composite outcome included any severe events of infections, infestations and tuberculous (disseminated tuberculosis, peritoneal tuberculosis, pulmonary tuberculosis, lymph node tuberculosis, tuberculosis), lower respiratory tract infection, and obstructive chronic bronchitis with acute exacerbation. More SAls were reported in the $200 \mathrm{mg}$ certolizumab pegol-treated group (Peto OR 1.94, 95\% Cl 0.99 to 3.80), three studies, involving 1283 participants; and in the $400 \mathrm{mg}$ certolizumab pegol-treated group (Peto OR 3.25, 95\% Cl 1.65 to 6.39), four studies, involving 1422 participants; 63 events were reported in the certolizumab pegol groups versus 13 events in the control groups. There were no differences between the rates of SAls in the $200 \mathrm{mg}$ and $400 \mathrm{mg}$ certolizumab pegol groups. See more details in (Analysis 50.11; Analysis 50.12)

We judged the quality of evidence for SAls for $200 \mathrm{mg}$ certolizumab pegol to be moderate. We downgraded the quality of evidence one level for imprecision due to the $95 \%$ confidence interval around the pooled effect including both harm and no harm.

We judged the quality of evidence for SAls for $400 \mathrm{mg}$ certolizumab pegol to be high. 


\section{Adverse events leading to death as defined in the studies}

We did not find statistically significant differences in the number of adverse events leading to death between the placebo and certolizumab pegol-treated groups. Eleven deaths due to adverse events in the certolizumab pegol groups were reported, versus one death in the control groups:

$200 \mathrm{mg}$ certolizumab pegol: Peto OR 1.63 ( $95 \% \mathrm{Cl} 0.41$ to 6.47 ), six studies involving 3322 participants (Analysis 50.13).

We judged the quality of evidence for adverse events leading to death for $200 \mathrm{mg}$ certolizumab pegol to be moderate. We downgraded the quality of evidence one level for imprecision due to the $95 \%$ confidence interval around the pooled effect including both harm and no harm.

$400 \mathrm{mg}$ certolizumab pegol: Peto OR 2.16 (95\% Cl 0.40 to 11.79 ), three studies, involving 1179 participants ( Analysis 50.14).

We judged the quality of evidence for adverse events leading to death for $400 \mathrm{mg}$ certolizumab pegol to be moderate. We downgraded the quality of evidence one level for imprecision due to the $95 \%$ confidence interval around the pooled effect including both harm and no harm.

\section{Death}

In Keystone 2008, in the placebo-treated group one participant died of myocardial infarction. In the $200 \mathrm{mg}$ certolizumab pegoltreated group one participant died of hepatic neoplasm, another died of peritonitis and cirrhosis, and one died during the posttreatment period (more than 84 days after the last injection). In the $400 \mathrm{mg}$ certolizumab pegol-treated group one died of cerebral stroke, one of myocardial necrosis, one of cardiac arrest and one of atrial fibrillation.
In Smolen 2009, in the 200 mg certolizumab pegol-treated group one participant died of myocardial infarction; one died during the study in the $400 \mathrm{mg}$ certolizumab pegol-treated group (fracture, shock), which was assessed as unlikely to be related to the study medication.

In Choy 2002, in the open phase one participant in the certolizumab pegol-treated group ( $20 \mathrm{mg} / \mathrm{kg}$ CDP870) died from complications following rapid drainage of a large, chronic rheumatoid pericardial effusion. In the opinion of the investigator, this event was unrelated to treatment with CDP870.

In Weinblatt 2012, one participant died of sigmoid diverticulitis and one of necrotising pneumonia; both deaths were ruled out as possibly related to certolizumab pegol.

In Yamamoto (a) 2014, one participant died of a rupture of a dissecting aortic aneurysm in the thoracic region, but UCB considered this unlikely to have been related to the study medication.

In Emery 2015 "The single CZP-related death in this study occurred in a 65-year-old patient of Indian origin, with hypertension and diabetes mellitus. The patient died of cardiorespiratory failure and acute respiratory distress syndrome, secondary to septic shock caused by bowel perforations. Acid-fast bacillus stains of the gut and saliva were positive. This, in conjunction with the gut pathology, led to a diagnosis of disseminated, non-characterised, mycobacterium infection; the QuantiFERON test was negative and there was no PCR confirmation of TB".

Choy 2012; Smolen 2015; Fleischmann 2009; Yamamoto (b) 2014; Østergaard 2015; Atsumi 2016 did not report any deaths.

Overall certolizumab pegol deaths: Peto OR $2.63(95 \% \mathrm{Cl} 0.78$ to 8.91), 10 studies, involving 4745 participants (Analysis 50.19) and Figure 4. 
Figure 4. Forest plot of comparison 49: Summary of findings: certolizumab (with or without MTX) versus placebo (with or without MTX), outcome: 49.8 Deaths.

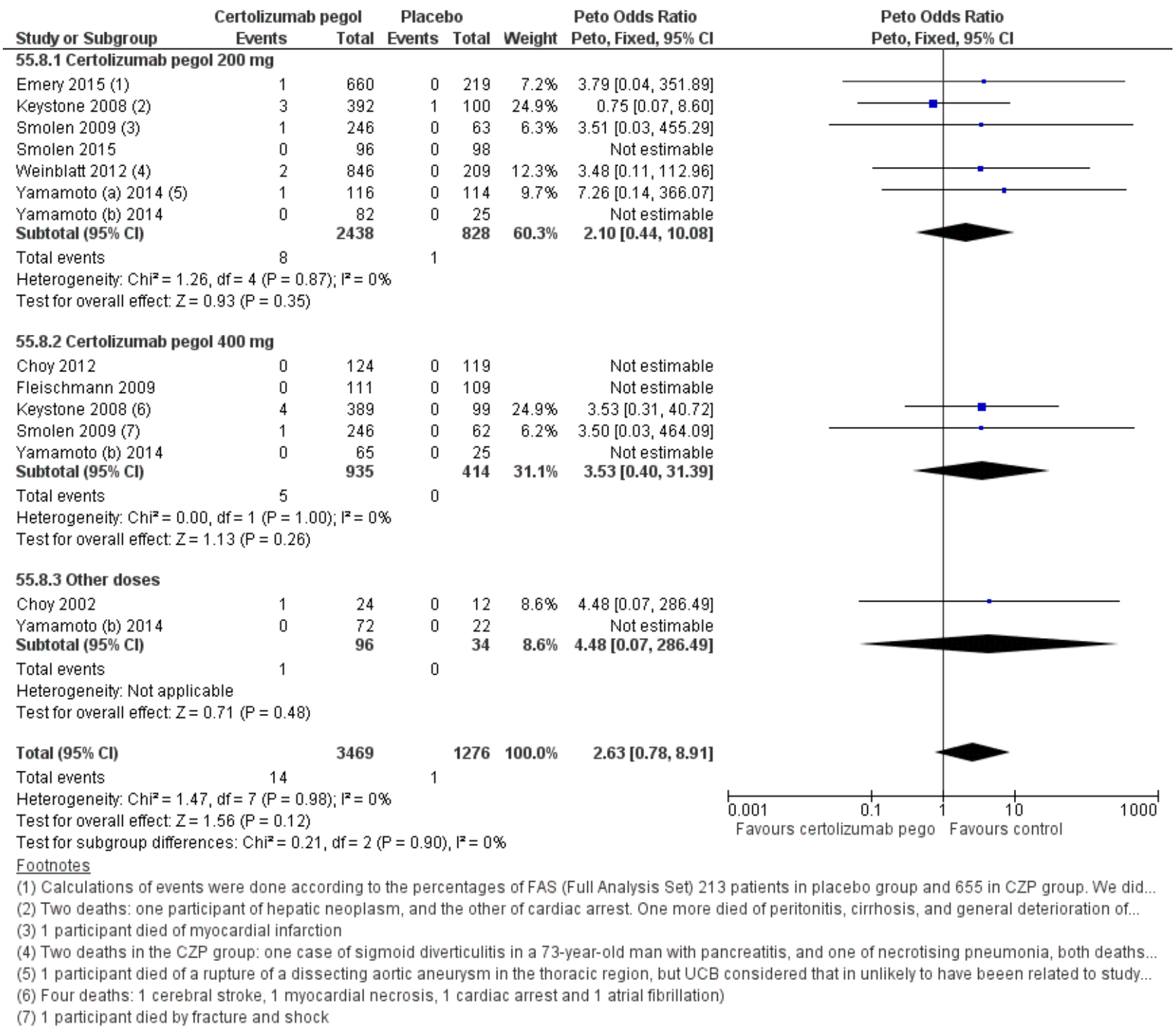

We judged the quality of evidence for deaths at any dose of certolizumab pegol to be moderate. We downgraded the quality of evidence one level for imprecision due to the 95\% confidence interval around the pooled effect including both harm and no harm.

\section{Tuberculosis}

We noted a significant increase in the number of cases of tuberculosis in both certolizumab pegol-treated groups: 10 participants $(0.4 \%)$ in the certolizumab pegol $200 \mathrm{mg}$ group and five $(0.7 \%)$ in the certolizumab pegol $400 \mathrm{mg}$ group, versus two and no cases in their respective placebo groups: $200 \mathrm{mg}$ certolizumab pegol Peto OR 1.90 ( $95 \% \mathrm{Cl} 0.55$ to 6.58 ), seven studies, involving 3538 participants (Analysis 50.20;); $400 \mathrm{mg}$ certolizumab pegol Peto OR 4.55 (95\% Cl 0.71 to 29.11$)$, three studies, involving 1179 participants (Analysis 50.21). The overall analysis with both doses (200 and $400 \mathrm{mg}$ ) did not reach statistical significance: Peto OR 1.91 (95\% Cl 0.61 to 5.96), seven studies, involving 4074 participants (Analysis 50.22). In Smolen 2009, five participants in the certolizumab pegol arms (three in certolizumab pegol $200 \mathrm{mg}$ and two in $400 \mathrm{mg}$ ) developed tuberculosis (three from Russia, one each from Poland and Latvia). In NCT00993317 (200 mg certolizumab pegol) two participants developed tuberculosis. For this update, only five participants developed tuberculosis in the Emery 2015 study, three in the certolizumab pegol group and two in the placebo group.

We judged the quality of evidence for tuberculosis for $200 \mathrm{mg}$ and $400 \mathrm{mg}$ of certolizumab pegol to be to be moderate. We downgraded the quality of evidence one level for imprecision, due to the $95 \%$ confidence interval around the pooled effect including both harm and no harm.

\section{Other infections}

The types of different infections reported (pneumonitis, bacterial arthritis, mastitis, urinary tract infection, herpes viral, bacterial 
peritonitis, and opportunistic infection) are presented in Data and analyses.

Upper respiratory tract infection was more frequent with $200 \mathrm{mg}$ certolizumab pegol than in the placebo group (Peto OR 1.68, 95\% $\mathrm{Cl} 1.28$ to 2.20), eight studies, involving 3608 participants (Analysis 50.34); and $400 \mathrm{mg}$ certolizumab pegol (Peto OR 1.42, 95\% Cl 0.77 to 2.61), four studies, involving 1364 participants (Analysis 50.35).

We judged the quality of evidence for upper respiratory tract infection for $200 \mathrm{mg}$ certolizumab pegol to be high.

We judged the quality of evidence for upper respiratory tract infection for $400 \mathrm{mg}$ certolizumab pegol to be moderate. We downgraded the quality of evidence one level for imprecision, due to the $95 \%$ confidence interval around the pooled effect including both harm and no harm.

Nasopharyngitis was more frequent with both doses of certolizumab pegol than in the placebo group: $200 \mathrm{mg}$ certolizumab pegol Peto OR 1.37 (95\% $\mathrm{Cl} 1.01$ to 1.84$)$ seven studies, involving 2553 participants (Analysis 50.44)); and $400 \mathrm{mg}$ certolizumab pegol Peto OR 1.98 ( $95 \% \mathrm{Cl} 1.26$ to 3.11), four studies, involving 1364 participants (Analysis 9.41). ( Analysis 50.45)

We judged the quality of evidence for nasopharyngitis for $200 \mathrm{mg}$ and $400 \mathrm{mg}$ of certolizumab pegol to be moderate. We downgraded the quality of evidence one level for imprecision, due to the $95 \%$ confidence interval around the pooled effect including both harm and no harm.

\section{Pain at the site of injection}

Pain at the site of injection was not statistically significant compared with placebo: in the $200 \mathrm{mg}$ certolizumab pegol-treated group (Peto OR $1.85,95 \% \mathrm{Cl} 0.49$ to 6.92 ), three studies, involving 1091 participants (Analysis 50.46); This analysis reported a High value of $\mathrm{I}^{2}$. We explained this due to that the results of RAPID1 showed a very high values RR 4.60 whereas the remaining trial showed lowest values around RR of 0.05 . Moreover the $\mathrm{Cl}$ of RAPID1 did not overlap the remaining trials.

When we studied $400 \mathrm{mg}$ certolizumab pegol-treated group we found (Peto OR $1.74,95 \% \mathrm{Cl} 0.41$ to 7.42 ), three studies, involving 1179 participants (Analysis 50.47). The wide Cls were due to the fact that, surprisingly, pain was not observed in any placebo group. Similar data were observed for local reactions at the injection site. We judged the quality of evidence pain for $200 \mathrm{mg}$ and $400 \mathrm{mg}$ of certolizumab pegol to be high.

\section{Other adverse events}

Hypertension was more frequent with both doses of certolizumab pegol than with placebo: $200 \mathrm{mg}$ certolizumab pegol Peto OR 3.09 (95\% Cl 1.64 to 5.84$)$, four studies, involving 1353 participants (Analysis 50.48); 400 mg certolizumab pegol: Peto OR $3.35(95 \% \mathrm{Cl}$ 1.80 to 6.20 ), three studies, involving 1121 participants (Analysis 50.49).

We judged the quality of evidence for other adverse events for 200 $\mathrm{mg}$ and $400 \mathrm{mg}$ of certolizumab pegol to be high.

The secondary events for headache, blood disorders, laboratory disorders, back pain, nausea/vomiting, urinary tract infections, pruritus and cough and others are described in detail in Data and analyses.

Despite the report from the EMA (www.ema.europa.eu/docs/ en_GB/document_library/EPAR_-_Public_assessment_report/ human/001037/WC500069735.pdf), we could not extract more data on adverse events, because the information was disclosed as combined data without the number of events in each trial. Moreover, the adverse events were grouped by 'primary system organ class': cardiac disorders, endocrine disorders, neoplasms benign, malignant and unspecified (excluding cysts and polyps).

\section{Pain (VAS assessment)}

Participants' assessment of arthritis pain with a visual analogue scale (VAS) score (0 to $100 \mathrm{~mm}$ ) improved at all doses and at all time points. At week 24, the overall mean difference (MD) was - 21.07 (95\% Cl -23.59 to -18.55), four studies, involving 2064 participants (Analysis 52.1); and at week 52 the MD was -23.48 (95\% Cl -27.09 to -19.88), one study, involving 982 participants (Analysis 53.1).

We judged the quality of evidence for patients' assessment of arthritis pain with a VAS for $200 \mathrm{mg}$ and $400 \mathrm{mg}$ of certolizumab pegol to be high.

\section{Withdrawals due to lack of efficacy}

There were more withdrawals "due to lack of efficacy" in placebo groups (39\%) versus the certolizumab pegol groups (13\%)

Withdrawals at any dose and at any follow-up due to lack of efficacy: RR 0.31 ( $95 \% \mathrm{Cl} 0.26$ to 0.37 ), eight studies, involving 3433 participants(Analysis 54.1).

We judged the quality of evidence for withdrawals due to lack of efficacy at any dose and at any follow-up for certolizumab pegol to be high.

\section{Assessment of heterogeneity}

When we analysed the ACR50 at 24 weeks (Analysis 44.2) we found a low probability of statistical heterogeneity $\left(1^{2}=0 \%\right)$. When we reviewed the demographics of Phase III studies (Table 2) we found similar proportions of men and women, similar mean ages, and similar baseline HAQ-Di. We only found differences in the mean disease duration in Fleischmann 2009 and Choy 2012, around 9.4 years compared with around six years in most arms of the other studies where data were available (with low heterogeneity, $\left.1^{2}=13 \%\right)$. Disease duration was not available for Smolen 2015; Yamamoto (a) 2014; Yamamoto (b) 2014 ( ${ }^{2}=6 \%$, and an overall $\mathrm{I}^{2}=7 \%$ ) (Analysis 56.5 ). Rheumatoid factor (RF) positivity varied from around $74 \%$ in the certolizumab pegol-treated participants in Weinblatt 2012 up to $100 \%$ in Fleischmann 2009. Similarly disease activity measures such as CRP and swollen joint counts, but not DAS-28 and HAQ-D1, were generally lower in Weinblatt 2012.

When we analysed the ACR50 at 52 weeks (Analysis 45.2) we found a high probability of statistical heterogeneity $\left(I^{2}=84 \%\right)$. When we compared the new trials Atsumi 2016 and Emery 2015 with the previous trial Keystone 2008, we observed that the average period of persistent disease in the new trials is around four months, whereas for Keystone 2008 it is 6.1 years. Baseline HAQ-Di in Keystone 2008 and Emery 2015 is around 1.6 whereas in Atsumi 2016 it is around 1.1. Participants in Atsumi 2016 are MTX-naïve, 
participants in Emery 2015 are DMARDS-naïve, whereas in Keystone 2008 participants were treated on average with 1.3 DMARDS.

However, despite these differences there were no compelling reasons for not combining the trial data for the most important variables.

Although we include 14 trials in this update, no more than seven trials were analysed in each forest plot, so we did not produce a funnel plot.

\section{Subgroup analysis}

We had planned subgroup analyses for the duration of the illness (approximately three years evolution), participants' sex, drug dose, administration and methodological quality, but only subgroup analysis of the dose of certolizumab pegol was performed. All Phase III trials were conducted in participants with a high mean duration of RA (from 6.1 to 9.5 years) and we could not obtain any data categorised by sex. All Phase III trials allowed previous DMARD treatment (mean from 1.2 to two years). All Phase III trials included in the meta-analysis were rated as high quality, and so we did not perform more subgroup analysis.

\section{Sensitivity analysis}

We have done a sensitivity analysis with the major outcome ACR50. In the previous version of this review we re-analysed quality (adequate sequence generation, good allocation concealment, adequate blinding, etc.) and did not show any changes. For this update we have more information about the quality of the trials from UCB, and we rated most trials as high quality, so we did not perform a sensitivity analysis based on quality. However, we sought heterogeneity by analysing for doses of certolizumab pegol, size, use of concomitant MTX, different populations (Japanese and Korean trials versus other populations) and by published versus unpublished trials, but found no statistical heterogeneity (Analysis 56.1; Analysis 56.2; Analysis 56.3; Analysis 56.4; Analysis 56.6). These analysis were performed for 24 weeks in our previous review and remain unchanged because the new trials included in this update were conducted to 52 weeks. When we analysed for the same categories we did find heterogeneity from the Keystone 2008 in all the issues that were tested (Analysis 57.1; Analysis 57.2; Analysis 57.3; Analysis 57.4; Analysis 57.5).

Finnally we analysed imputing missing values in the same proportion as reported ACR50\%, imputing the $50 \%$ of ACR $50 \%$ and the results are robust for ACR50 $200 \mathrm{mg}$ to 24 weeks RR $3.34(95 \%$ $\mathrm{Cl} 2.68$ to 4.17 ) and RR 1.17 (95\% Cl 1.04 to 1.32$)$. Only when we checked the worst case (all the missing values did not reach ACR50 in certolizumab pegol) and did ACR50 in placebo the results were favouring to placebo RR 0.47 (95\% Cl 0.43 to 0.52 ). Analysis 56.7; Analysis 56.8; Analysis 56.9.

\section{DISCUSSION}

\section{Summary of main results}

This review evaluates the benefits and harms of certolizumab pegol for the treatment of people with RA when compared to placebo, using RCTs with at least three months of follow-up.

The results and conclusions did not change from the previous version of the review. There is low-level evidence from randomised controlled trials that certolizumab pegol, alone or combined with methotrexate, is beneficial in the treatment of RA: it improved the American College of Rheumatology ACR50 (pain, function and other symptoms of RA), health-related quality of life, and the chance of remission of RA, reduced joint damage as seen on the $x$-ray, and increased serious adverse events. Fewer people stopped taking their treatment, but most of them stopped due to serious adverse events. Adverse events were more frequent with active treatment. We found a potential risk of serious adverse events.

We found 14 studies, three more than in the previous version of the review. The duration of follow-up was from 12 to 52 weeks and the range of doses of certolizumab pegol varied from 50 to $400 \mathrm{mg}$ given subcutaneously.

Certolizumab pegol at the standard dose $(200 \mathrm{mg}$ ) was shown to be clinically effective at 12, 24 and 52 weeks. However the data from 52 weeks should be interpreted with caution, because a large number of participants deemed not to be achieving a sufficient response were withdrawn at week 24 .

Important clinical differences between placebo and certolizumab pegol were observed for measures of disease activity, in favour of certolizumab pegol. The differences were both statistically significant and clinically important for the participant-reported outcomes ACR50, HAQ, and SF-36 (physical (PCS) and mental (MCS) component summary scores), and for structural damage measures. Changes in $\mathrm{HAQ}$ at 24 weeks with $200 \mathrm{mg}$ certolizumab pegol were -0.35 (mean changes in $\mathrm{HAQ}$ greater than -0.22 are clinically meaningful). In addition, the results with SF-36 (physical and mental components) can be considered relevant because in people with RA improvements in the SF-36 PCS and HAQ-DI are associated with improved work productivity and reduced long-term disability, healthcare use, costs and mortality (Hazes 2010).

All certolizumab pegol groups showed improvements in radiological outcomes compared to placebo, measured as the mean changes from baseline. There was a clear radiological benefit, although it should be borne in mind that radiographic changes occur in a relatively small proportion of people with RA over the duration of research studies, and the changes did not represent a clinically meaningful benefit for participants.

Serious adverse events were more frequent in the certolizumab pegol groups.

We observed more withdrawals in participants treated with certolizumab pegol. Participants in the placebo group were more likely to discontinue treatment, due to lack of beneficial effect, but more participants withdrew from the certolizumab pegol group, due to adverse reactions. The most frequent side effects were infections and nasopharyngitis. Unfortunately, the newer clinical trials do not provide data on hypertension. However, as reported in the previous version, hypertension is increased in the certolizumab pegol group.

In the previous version we stated we would compare our data with data from the EMA documents. We requested access to the drug company submissions to the EMA for marketing authorisation of certolizumab pegol. Our request was denied, despite an appeal. The EMA stated that "...in the course of emerging legal proceedings before the General Court of the European Union, the Agency has been ordered to suspend the implementation of the certain 
decisions granting access to documents submitted by marketing authorisation holders of medicinal products".

Mortality was increased with certolizumab pegol. These differences did not achieve statistical significance but it should be noted that there was only one death in the placebo group compared with 14 in the certolizumab pegol group. Death was primarily related to cardiovascular events, as reported by Bykerk 2013. However treatment with anti-TNF has been shown to reduce cardiovascular events in people with RA (Roubille 2015).

We found an increased risk of serious infections with certolizumab pegol. This risk is recognised with anti-TNFs, both in randomised trials and in observational studies (FDA 2013).

Contrary to the findings of Lopez-Olivo 2012, we did not find an increased risk of malignancies or lymphoma, for $200 \mathrm{mg}$ or for 400 $\mathrm{mg}$ of certolizumab pegol.

We have found discordance between the number of cases of tuberculosis reported in ClinicalTrials.org and the one instance reported in Emery 2015. Despite the difference, the frequency of tuberculosis has decreased in recent clinical trials. This could be due to several reasons. In 2007 the WHO introduced stricter tuberculosis screening guidelines, considering a positive purified protein derivative (PPD) test $5 \mathrm{~mm}$ or more (previously between 10 and $20 \mathrm{~mm}$ according to each national guideline), and tuberculosis prophylaxis was recommended if active tuberculosis was ruled out. Furthermore, fewer participants from areas of high tuberculosis prevalence have been recruited, and latent tuberculosis is generally an exclusion criterion.

The results and conclusions did not change from the previous review.

\section{Overall completeness and applicability of evidence}

We have included all available RCTs for certolizumab pegol in people with RA, with a September 2016 search date. This updated review provides confirmatory evidence of the benefit of certolizumab pegol for people with RA.

It is important to state that three studies had a follow-up of 52 weeks, and in two of them non-responders were withdrawn at week 24. Thus there are important uncertainties about sustained effects in a disease with a lifelong course and the need for therapy over many years. An additional note of caution relates to the population selection in terms of significant co morbidities and exclusion of people with previous malignancy, for example.

In all trials except the Smolen 2015 trial (without a clear definition of its inclusion and exclusion criteria in ClinicalTrials.org), people with previous neoplasia, any risk of infectious disease, previous tuberculosis, or prior treatment with any TNFa inhibitor were excluded. In the Yamamoto (a) 2014, Yamamoto (b) 2014 and NCT00993317) trials, people with New York Heart Association (NYHA) class III or IV heart failure were also excluded. Moreover, in the Keystone 2008 trial "Patients who, in the investigator's opinion, were at a high risk of infection" were excluded, as were those who had a history of malignancy, demyelinating disease, blood dyscrasias, or severe, progressive, and/or uncontrolled renal, hepatic, haematologic, gastrointestinal, endocrine, pulmonary, cardiac, neurologic, or cerebral disease". Thus, whilst it is clear that certolizumab pegol is beneficial and has an acceptable safety profile in people selected for clinical trials, careful clinical judgement is needed to ensure benefits in routine care, particularly in people susceptible to infections such as those with chronic respiratory diseases.

We only have information about the comparison between certolizumab pegol and placebo. There is no head-to-head comparison between certolizumab pegol and other anti-TNFs. For this reason current evidence does not support the use of certolizumab pegol over another anti-TNF.

\section{Quality of the evidence}

The quality of the evidence found in the trials included in this review was high to moderate. Studies had high standards for treatment allocation, concealment, blinding, and attrition bias. Other GRADE considerations for downgrading are: imprecision, indirectness and inconsistency or other bias.

Despite differences in the importance of the outcomes (higher for ACR50, HAQ and DAS remission, and lower for radiological changes), we rated the quality of the evidence as high for all the outcomes except for the $\mathrm{HAQ}$, radiological changes and all withdrawals, which we rated as moderate quality.

Outcome measures in favour of certolizumab pegol were statistically significant in both random-effects and fixed-effect models. We chose to apply a random-effects model, although statistical heterogeneity was low. Clinical heterogeneity, however, was substantial (for example, with varying follow-up times, doses, use of methotrexate) and, as expected, pooling resulted in wide confidence intervals.

\section{Major outcomes}

Summary of findings for the main comparison for certolizumab pegol $200 \mathrm{mg}$, structured according to the GRADE system (GRADE Handbook), showed:

1) We judged the quality of evidence for the primary outcome $\mathbf{A C R}$ $\mathbf{5 0} \%$ improvement at 24 weeks to be high.

2) We judged the quality of evidence for the primary outcome HAQ at 24 weeks to be moderate. We downgraded the quality of evidence by one level, due to a high risk of attrition bias (per protocol analysis).

3) We judged the quality of evidence for the primary outcome Proportion of participants achieving DAS $<2.6$ (remission) at 24 weeks to be high.

4) We judged the quality of evidence for the primary outcome Erosion score (ES), at 24 weeks to be moderate. We downgraded the quality of evidence by one level, due to a high risk of attrition bias (per protocol analysis).

5) We judged the quality of evidence for the primary outcome Serious adverse events at 24 weeks to be high.

6) We judged the quality of evidence for the primary outcome Withdrawals, at 24 weeks to be moderate. We downgraded the quality of evidence

one level for inconsistency, due to heterogeneity (not all the confidence intervals overlap and $\mathrm{I} 2$ is $79 \%$ ). 
7) We judged the quality of evidence for the primary outcome Withdrawals due to adverse events at 24 weeks to be high.

\section{Minor outcomes}

8) We judged the quality of evidence for the secondary outcome ACR20 at 24 weeks to be high.

9) We judged the quality of evidence for the secondary outcome ACR70 at 24 weeks to be high.

10) We judged the quality of evidence for Tuberculosis for 200 $\mathrm{mg}$ and 400 of certolizumab pegol to be to be moderate. We downgraded the quality of evidence one level for imprecision, due to the $95 \%$ confidence interval around the pooled effect including both harm and no harm.

11) We judged the quality of evidence for Death for any dose of certolizumab pegol to be moderate. We downgraded the quality of evidence one level for imprecision, due to the $95 \%$ confidence interval around the pooled effect including both harm and no harm.

12) We judged the quality of evidence for the secondary outcome Withdrawals due to lack of efficacy to be high.

\section{Potential biases in the review process}

This updated review has fewer limitations than the earlier version, primarily because key data from a greater number of studies, including key study quality data, were available either as published reports or directly from the pharmaceutical company. From 14 included trials, 12 with over 5400 participants reported benefits and 14 trials reported safety, providing a substantial evidence base. We lacked detail that may have been available in submissions to the EMA as part of this drug's marketing authorisation and we also did not have access to study protocols, so we were not able to judge whether there was a concern about selective reporting. Lack of availability of detailed study reports with individual patient data denied us the opportunity of presenting a richer description of adverse events, particularly serious adverse reactions.

\section{Agreements and disagreements with other studies or reviews}

The NICE 2009 and EMA 2009 reports, performed as systematic reviews, have shown results quite similar to those in our review.

The meta-analysis by Singh 2011 described the adverse effects of nine biologics and included RCTs, controlled clinical trials (CCTs) and open-label extensions (OLEs), showing similar overall results. Moreover, Singh 2011 found similar results with certolizumab pegol for serious adverse events and serious infections, but failed to find an increased rate of withdrawals due to adverse events. In this study the risk of serious infections was about four times higher for certolizumab pegol and the authors performed sensitivity analyses using different models to explain the results. However, the significant differences between certolizumab pegol and five other biologics as determined in the standard dose model (main model) persisted in the unadjusted and dose-adjusted models for each comparison, with the minor exception of certolizumab pegol versus golimumab.

Zhou 2014 did not find differences in adverse events in a metaanalysis of nine RCTs of certolizumab pegol in RA. Only six trials for adverse events were included in this systematic review. The reason for the difference from our results is that Zhou 2014 only include adverse events until week 24 . However, there was agreement in ACR response rate at 24 weeks.

\section{AUTHORS' CONCLUSIONS}

\section{Implications for practice}

This review confirms that certolizumab pegol compared with placebo is clinically beneficial, improving ACR50, quality of life and increasing the chance of remission. In addition certolizumab pegol compared with placebo reduces the risk of radiographic damage. There is a potential risk of serious adverse events, including hypertension and tuberculosis in susceptible individuals, which should be borne in mind when considering certolizumab pegol. There was no direct evidence comparing certolizumab with other TNF inhibitors.

There is a moderate to high certainty of evidence, obtained from randomised controlled trials, that certolizumab pegol, alone or combined with methotrexate, is beneficial in the treatment of RA. It improved ACR50 (pain, function and other symptoms of RA), healthrelated quality of life, and the chance of remission of RA, reduced joint damage as seen on the $x$-ray, but increased serious adverse events. Fewer people stopped taking their treatment, but most of those who did stopped because of serious adverse events. Adverse events were more frequent with active treatment. We found a clinically but not statistically significant risk of serious adverse events.

\section{Implications for research}

Treatment options for RA have expanded considerably in recent years and include biologic agents targeting a variety of elements of the inflammatory process. It is important that we undertake studies to compare the new drugs that have been shown to be effective in clinically-relevant populations.

We must emphasize that complete remission is the major target in clinical practice, and it should be considered as an outcome for future clinical trials using ACR/EULAR remission criteria ( Felson 2011 .

New agents continue to target people who have failed to respond to methotrexate. Given that there are a number of biologics that have been found to be effective in this patient group, ethics review boards need to consider whether it is justifiable to undertake studies of new agents for this population that compare the effectiveness to placebo or to background methotrexate.

Longer-term studies and observational data are important for the assessment of longer-term drug toxicity and rarer adverse events.

\section{ACKN OWLEDGEMEN TS}

2016 update: Thanks to Maria del Mar Ubeda and Eukene Ansuategui, information specialist in the Hospital of San Sebastian, who kindly performed the searches. Thanks to Jordi Pardo, a good friend who is always taking care of us from Canada. Thanks also to Marc de Longueville and Pablo Talavera from UCB who sent us additional data and explanations about how the trials were done. 


\section{RE F E R E N C E S}

\section{References to studies included in this review}

\section{Atsumi 2016 \{published data only\}}

Atsumi T, Yamamoto K, Takeuchi T, Yamanaka H, Ishiguro N, Tanaka Y, et al. Baseline parameters identified in early, methotrexate-naive rheumatoid arthritis patients with better outcomes with certolizumab pegol+methotrexate compared to placebo+methotrexate: Post-hoc analyses of copera, a randomized, controlled, phase 3 study. Annals of the Rheumatic Diseases.Conference: Annual European Congress of Rheumatology of the European League Against Rheumatism, EULAR 2015 Rome Italy. 10 - 13 June 2015. 2015; Vol. 74:716-7.

Atsumi T, Yamamoto K, Takeuchi T, Yamanaka H, Ishiguro N, Tanaka Y, et al. Clinical benefit of 1-year certolizumab pegol treatment in MTX-naïve, early rheumatoid arthritis patients is maintained after discontinuation up to 1 year. Arthritis and Rheumatology.Conference: American College of Rheumatology/ Association of Rheumatology Health Professionals Annual Scientific Meeting, ACR/ARHP 2015 San Francisco, CA United States. 6 - 11 November 2015. 2015; Vol. 67.

* Atsumi T, Yamamoto K, Takeuchi T, Yamanaka H, Ishiguro N, Tanaka Y, et al. The first double-blind, randomised, parallelgroup certolizumab pegol study in methotrexate-naive early rheumatoid arthritis patients with poor prognostic factors, COPERA, shows inhibition of radiographic progression. Annals of the Rheumatic Diseases 2016;75(1):75-83.

Atsumi T, Yamamoto K, Takeuchi T, Yamanaka H, Ishiguro N, Tanaka Y, et al. The first early rheumatoid arthritis, certolizumab pegol, multicenter, double-blind, randomized, parallel-group study: C-Opera, in patients fulfilling the 2010 ACR/EULAR classification criteria, demonstrates inhibition of joint damage progression. Annals of the Rheumatic Diseases.Conference: Annual European Congress of Rheumatology of the European League Against Rheumatism, EULAR 2014 Paris France. 11 - 14 June 2014. 2014; Vol. 73.

Atsumi T, Yamamoto K, Takeuchi T, Yamanaka H, Ishiguro N, Tanaka Y, et al. The first, multicenter, double-blind, randomized, parallel-group study of certolizumab pegol in early rheumatoid arthritis demonstrates inhibition of joint damage progression. Arthritis and Rheumatology.Conference: 2014 ACR/ARHP Annual Meeting Boston, MA United States. 14 - 19 November 2014. 2014; Vol. 66:S1078-9.

\section{CDP870-004 2001 \{published and unpublished data\}}

Emery P, Smolen J, Choy E, et al. CDP870 a novel, humanised tumour necrosis factor alpha inhibitor improves HRQOL. Late breaking abstract. European League Against Rheumatism Annual Conference. 2002

* European Medicines Agency. Assesment report for Cimzia. Procedure No EMEA/H/C/001037. www.ema.europa.eu/docs/ en_GB/document_library/EPAR_-_Public_assessment_report/ human/001037/WC500069735.pdf. London, 2009 (accessed 3rd July 2017):1-47. [Procedure No.EMEA/H/C/001037]

Keystone E, Choy E, Kalden J, Klareskog, Sany J, Smolen J, et al. CDP870, A novel pegylated, humanized TNF-alpha inhibitor, is effective in treating the signs and symptoms of rheumatoid arthritis (RA). Abstract to Rheumatology annual scientific meeting [abstract \# LB-3]. 2001.

\section{Choy 2002 \{published data only\}}

* Choy EH, Hazleman B, Smith M, Moss K, Lisi L, Scott DG, et al. Efficacy of a novel pegylated humanized anti-TNF fragment (CDP870) in patients with rheumatoid arthritis: a phase II double-blinded, randomized, dose-escalating trial. Rheumatology (Oxford) 2002;41(10):1133-7.

\section{Choy 2012 \{published data only\}}

* Choy E, McKenna F, Vencovsky J, Valente R, Goel N, Vanlunen B, et al. Certolizumab pegol plus MTX administered every 4 weeks is effective in patients with RA who are partial responders to MTX. Rheumatology (Oxford) 2012;51(7):1226-34

UCB. Clinical Study Summary Study No.: CDP870-014. www.ucb.com/_up/ucb_com_patients/documents/C87032 CSS_20080608.pdf 2008 (accessed 3rd July 2017).

\section{Emery 2015 \{published data only\}}

Emery P, Bingham C, Burmester GR, Bykerk VP, Furst D, Mariette $X$, et al. Improvements in workplace and household productivity following 52 weeks of treatment with certolizumab pegol in combination with methotrexate in DMARD-naive patients with severe, active and progressive rheumatoid arthritis: Results from the c-early randomized, double-blind, controlled phase 3 study. Value in Health.Conference: ISPOR 18th Annual European Congress Milan, Italy.Conference. 7 - 11 November 2015. 2015; Vol. 18:7.

Emery P, Bingham CO, Burmester G-R, Bykerk VP, Furst DE, Mariette $X$, et al. Improvements in patient-reported outcomes and workplace and household productivity following 52 weeks of treatment with certolizumab pegol in combination with methotrexate in DMARD-naive early rheumatoid arthritis patients: Results from the C-early randomized, double-blind, controlled phase 3 study. Annals of the Rheumatic Diseases.Conference: Annual European Congress of Rheumatology of the European League Against Rheumatism, EULAR 2015 Rome Italy. 10 - 13 June 2015. 2015; Vol. 74:712-3.

Emery P, Bingham CO, Burmester G-R, Bykerk VP, Furst DE, Mariette X, et al. SAT0165 Improvements in patient-reported outcomes and workplace and household productivity following 52 weeks of treatment with certolizumab pegol in combination with methotrexate in dmard-naïve early rheumatoid arthritis patients: results from the C-Early randomized, double-blind, controlled phase 3 study. Annals of the Rheumatic Diseases 2015;74(Suppl 2):712-3.

* Emery P, Bingham CO, Burmester G-R, Bykerk VP, Furst DE, Mariette X, et al. sSAT0164 The first study of certolizumab pegol in combination with methotrexate in dmard-naïve early rheumatoid arthritis patients led to Sustained clinical response and inhibition of radiographic progression at 52 weeks: The C-Early randomized, double-blind, controlled phase 3 study. Annals of the Rheumatic Diseases 2015;74(Suppl 2):712. 
Weinblatt M, Bingham C, Burmester G, Bykerk V, Furst DE, Mariette $X$, et al. Early response as a predictor of long-term remission in DMARD-naive patients with severe, active and progressive rheumatoid arthritis treated with certolizumab pegol in combination with methotrexate. Arthritis and Rheumatology.Conference: American College of Rheumatology/ Association of Rheumatology Health Professionals Annual Scientific Meeting, ACR/ARHP 2015 San Francisco, CA United States. 6 - 11 November 2015. 2015; Vol. 67.

Weinblatt M, Bingham C, Burmester G, Bykerk VP, Furst DE, Mariette $\mathrm{X}$, et al. Certolizumab pegol in combination with methotrexate in DMARD-naive patients with active, severe, progressive rheumatoid arthritis: Results from a randomized, double-blind, controlled phase 3 study. Arthritis and Rheumatology.Conference: American College of Rheumatology/ Association of Rheumatology Health Professionals Annual Scientific Meeting, ACR/ARHP 2015 San Francisco, CA United States. 6 - 11 November 2015. 2015; Vol. 67.

\section{Fleischmann 2009 \{published data only\}}

Fleischmann D, Mason D, Cohen S. Efficacy and safety of certolizumab pegol monotherapy in patients with rheumatoid arthritis failing previous DMARD therapy. Annals of the Rheumatic Diseases 2007;66 Suppl II:169.

Fleischmann R, Keininger DL, Tahiri-Fitzgerald E, Mease P. Certolizumab pegol monotherapy 400mg every 4 weeks improves physical functioning and reduces pain in patients with rheumatoid arthritis Who have previously failed DMARD therapy. Program and abstracts of the European League Against Rheumatism (EULAR) Annual Meeting; Barcelona, Spain 13-16 June [Abstract \#0148]. 2007.

* Fleischmann R, Vencovsky J, Van Vollenhoven RF, Borenstein D, Box J, Coteur G, et al. Efficacy and safety of certolizumab pegol monotherapy every 4 weeks in patients with rheumatoid arthritis failing previous disease modifying antirheumatic therapy: the FAST4WARD study. Annals of the Rheumatic Diseases 2009;68(6):805-11.

Keystone E, Mason D, Fleischmann R. Certolizumab pegol 400 mg every 4 weeks as monotherapy rapidly reduces disease activity in active rheumatoid arthritis. Program and abstracts of the American College of Rheumatology (ACR) 71st Annual Meeting; November 6 - 11; Boston, Massachusetts. [Abstract \#277]. 2007.

Strand V, Brown M, Purcaru O, Richard L. Certolizumab pegol monotherapy improves productivity in patients with active rheumatoid arthritis: results from a phase III randomized controlled trial. Program and abstracts of the European League Against Rheumatism (EULAR) Annual Meeting; Barcelona, Spain 13 - 16 June [Abstract \#0478]. 2007.

Strand V, Keininger D, Tahiri-Fitzgerald E, Fleischmann R. Certolizumab pegol monotherapy $400 \mathrm{mg}$ every 4 weeks improves health-related quality of life and relieves fatigue in patients with rheumatoid arthritis who have previously failed DMARD therapy. Program and abstracts of the European League Against Rheumatism (EULAR) Annual Meeting; Barcelona, Spain 13 - 16 June [Abstract \#0205]. 2007.

\section{Keystone 2008 \{published data only\}}

Curtis JR, Chen L, Luijtens K, Navarro-Millan I, Goel N, Gervitz L, et al. Dose escalation of certolizumab pegol from $200 \mathrm{mg}$ to $400 \mathrm{mg}$ every other week provides no additional efficacy in rheumatoid arthritis: an analysis of individual patient-level data. Arthritis and Rheumatism 2011;63(8):2203-8.

Curtis JR, Luijtens K, Kavanaugh A. Predicting future response to certolizumab pegol in rheumatoid arthritis patients: features at 12 weeks associated with low disease activity at 1 year. Arthritis Care Research (Hoboken) 2012;64(5):658-67.

Haraoui B, Bykerk VP, Van Vollenhoven R, De Longueville M, Luijtens K, Ralston P, et al. Analysis of pooled data from two randomized controlled trials and their open-label extensions: Long-term safety in rheumatoid arthritis before and after certolizumab pegol dose increase/decrease. Arthritis and Rheumatology.Conference: 2014 ACR/ARHP Annual Meeting Boston, MA United States 14 - 19 NOvember 2014. 2014; Vol. 66:S199.

* Keystone E, Heijde D, Mason D Jr, Landewé R, Vollenhoven RV, Combe B, et al. Certolizumab pegol plus methotrexate is significantly more effective than placebo plus methotrexate in active rheumatoid arthritis: findings of a fifty-two-week, phase III, multicenter, randomized, double-blind, placebocontrolled, parallel-group study. Arthritis and Rheumatism 2008;58(11):3319-29.

Keystone E, Landewé R, Van Vollenhoven R, Combe B, Strand V, Mease P, et al. 5-Year results from the RAPID 1 trial and openlabel extension: long-term safety and efficacy of certolizumab pegol in combination with methotrexate in the treatment of rheumatoid arthritis. Annals of the Rheumatic Diseases 2013;72(12):A228-9. [10.1136/annrheumdis-2013-203695. [Epub ahead of print]]

Keystone E, Landewé R, Van Vollenhoven R, Combe B, Strand V, Mease $P$, et al. Long-term safety and efficacy of certolizumab pegol in combination with methotrexate in the treatment of rheumatoid arthritis: 5-year results from a 52-week randomized controlled trial and open-label extension study. Journal of Rheumatology Conference: 69th Annual Meeting of the Canadian Rheumatology Association, CRA 2014 Whistler, BC Canada. 24 February - 1 March 2014. 2014; Vol. 41:7.

Keystone E, Landewé R, Van Vollenhoven R, Combe B, Strand V, Mease $P$, et al. Long-term safety and efficacy of certolizumab pegol in combination with methotrexate in the treatment of rheumatoid arthritis: 5-year results from the RAPID 1 trial and open-label extension. Annals of the Rheumatic Diseases 2013;65:S988-9. [PUBMED: 23918037]

Keystone E, Mason D, Combe B. The anti-TNF certolizumab pegol in combination with methotrexate is significantly more effective than methotrexate alone in the treatment of patients with active rheumatoid arthritis: 1-year results from the RAPID 1 study. Program and abstracts of the American College of Rheumatology (ACR) 71st Annual Meeting; 6 - 11 November 2007; Boston, Massachusetts [Abstract \#700]. 2007.

Keystone EC, Combe B, Smolen J, Strand V, Goel N, van Vollenhoven R, et al. Sustained efficacy of certolizumab 
pegol added to methotrexate in the treatment of rheumatoid arthritis: 2-year results from the RAPID 1 trial. Rheumatology (Oxford) 2012;51:1628-38.

NCT00175877. A study of the safety and effectiveness of lyophilized certolizumab pegol in the treatment of signs and symptoms of rheumatoid arthritis and in prevention of joint damage in patients with active rheumatoid arthritis. clinicaltrials.gov/ct2/show/NCT00175877 (first received 9th September 2005).

Strand V, Keininger DL, Tahiri-Fizgerald E. Certolizumab pegol results in clinically meaningful improvements in physical function and health-related quality of life in patients with active rheumatoid arthritis despite treatment with methotrexate. Program and abstracts of the American College of Rheumatology (ACR) 71st Annual Meeting; 6 - 11 November 2007; Boston, Massachusetts. [Abstract \#946]. 2007.

Strand V, Mease P, Burmester G, Nikaï E, Coteur G, Vollenhoven R, et al. Rapid and sustained improvements in health-related quality of life, fatigue, and other patientreported outcomes in rheumatoid arthritis patients treated with certolizumab pegol plus methotrexate over 1 year: results from the RAPID 1 randomized controlled trial. Arthritis Research \& Therapy 2009; Vol. 11, issue 6:R170.

UCB. Preliminary results suggest certolizumab pegol plus methotrexate is effective in reducing signs and symptoms of rheumatoid arthritis in patients refractory to methotrexate. Results from the RAPID 1 Study. Abstract number: OPO016. EULAR 2007. 2007.

Van der Heijde D, Keystone EC, Curtis JR, Landewé RB, Schiff MH, Khanna D, et al. Timing and magnitude of initial change in disease activity score 28 predicts the likelihood of achieving low disease activity at 1 year in rheumatoid arthritis patients treated with certolizumab pegol: a post-hoc analysis of the RAPID 1 trial. Journal of Rheumatology 2012;39(7):1326-33.

Van der Heijde D, Strand V, Keystone E, Landewé R. Inhibition of radiographic progression by lyophilized certolizumab pegol added to methotrexate in comparison with methotrexate alone in patients with rheumatoid arthritis: the RAPID 1 trial. Program and abstracts of the American College of Rheumatology (ACR) 71st Annual Meeting; November 6-11; Boston, Massachusetts. [Abstract \#940]. 2007.

Van der Heijde D, Weinblatt M, Landewé R, Goel N, Wells A, Fleischmann R. Inhibition of progression of structural damage by week 16 with certolizumab pegol: Results from the RAPID trials. Arthritis and Rheumatism 2008;58(9 Suppl):529-30 .

\section{NCT00993317 \{published data only\}}

* NCT00993317. A study of CDP870 as add-on medication to methotrexate (MTX) in patients with rheumatoid arthritis. clinicaltrials.gov/ct2/show/study/NCT00993317 (firs treceived 9th October 2009).

\section{Smolen 2009 \{published data only\}}

Haraoui B, Bykerk VP, Van Vollenhoven R, De Longueville M, Luijtens K, Ralston P, et al. Analysis of pooled data from two randomized controlled trials and their open-label extensions:
Long-term safety in rheumatoid arthritis before and after certolizumab pegol dose increase/decrease. Arthritis and Rheumatology.Conference: 2014 ACR/ARHP Annual Meeting Boston, MA United States 14 - 19 November 2014. 2014; Vol. 66:S199.

Kavanaugh A, Smolen JS, Emery P, Purcaru O, Keystone E, Richard L, et al. Effect of certolizumab pegol with methotrexate on home and work place productivity and social activities in patients with active rheumatoid arthritis. Arthritis and Rheumatism 2009;61(11):1592-600.

Landewé R, Strand V, Smolen J, Van der Heijde D. Liquid formulation certolizumab pegol with methotrexate decreases progression of structural joint damage in rheumatoid arthritis patients: the RAPID 2 study. Program and abstracts of the American College of Rheumatology (ACR) 71st Annual Meeting; 6 - 11 November 2007; Boston, Massachusetts. [Abstract \#696]. 2007.

Mease P, Mason D, Kavanaugh A, Smolen J. Efficacy and rapid response of certolizumab pegol liquid formulation in combination with methotrexate (MTX) in patients with active rheumatoid arthritis despite MTX therapy: results from the RAPID 2 study. Program and abstracts of the American College of Rheumatology (ACR) 71st Annual Meeting; 6 - 11 November 2007; Boston, Massachusetts. [Abstract \#941]. 2007.

Schiff M, Keininger DL, Tahiri-Fitzgerald E. Certolizumab pegol added onto methotrexate improves physical functioning and reduces pain in patients with rheumatoid arthritis who have an incomplete response to methotrexate: data from rapid 2. Program and abstracts of the European League Against Rheumatism (EULAR) Annual Meeting; Barcelona, Spain 13 - 16 June [Abstract \#0200]. 2007.

Smolen J, Brzezicki J, Mason D, Kavanaugh A. Efficacy and safety of certolizumab pegol in combination with methotrexate $(\mathrm{mtx})$ in patients with active rheumatoid arthritis despite MTX therapy: results from the Rapid 2 study. Program and abstracts of the European League Against Rheumatism (EULAR) Annual Meeting; Barcelona, Spain 13 - 16 June [Abstract \#0202]. 2007.

* Smolen JS, Landewe RB, Mease PJ, BrzezickiJ, Mason D, Luijtens K, et al. Efficacy and safety of certolizumab pegol plus methotrexate in active rheumatoid arthritis: the RAPID 2 study. A randomised controlled trial. Annals of the Rheumatic Diseases 2009;68:797-804.

Smolen JS, Van Vollenhoven R, Kavanaugh A, Strand V, Vencovsky J, Schiff M, et al. Certolizumab pegol plus methotrexate 5-year results from the rheumatoid arthritis prevention of structural damage (RAPID) 2 randomized controlled trial and long-term extension in rheumatoid arthritis patients. Arthritis Research \& Therapy 2015;17:245.

Strand V, Keininger DL, Tahiri-Fizgerald E. Certolizumab pegol results in clinically meaningful improvements in physical function and health-related quality of life in patients with active rheumatoid arthritis despite treatment with methotrexate. Program and abstracts of the European League Against Rheumatism (EULAR) Annual Meeting; Barcelona, Spain 13 - 16 June 2007 [Abstract \#0335]. 2007. 
Strand V, Smolen JS, Van Vollenhoven RF, Mease P, Burmester GR, Hiepe F, et al. Certolizumab pegol plus methotrexate provides broad relief from the burden of rheumatoid arthritis: analysis of patient-reported outcomes from the RAPID 2 trial. Annals of the Rheumatic Diseases 2011;70(6):996-1002.

\section{Smolen 2015 \{published data only\}}

NCT00674362. Rheumatoid arthritis (RA) moderate to low disease activity study (CERTAIN). clinicaltrials.gov/ct2/show/ NCT00674362 (first received 5th May 2008).

Smolen JS, Emery P, Ferraccioli G, Samborski W, Berenbaum F, Davies $\mathrm{O}$, et al. Maintenance of remission in rheumatoid arthritis patients with low-moderate disease activity following withdrawal of certolizumab PEGOL treatment: Week 52 results from the certain study. Annals of the Rheumatic Disease 2013;71 Suppl 3:361.

* Smolen JS, Emery P, Ferraccioli GF, Samborski W, Berenbaum F, Davies OR, et al. Certolizumab pegol in rheumatoid arthritis patients with low to moderate activity: the CERTAIN double-blind, randomised, placebo-controlled trial. Annals of the Rheumatic Diseases 2015; Vol. 74, issue 5:843-50. [DOI: 10.1136/annrheumdis-2013-204632]

Smolen JS, Van Vollenhoven R, Kavanaugh A, Strand V, Vencovsky J, Schiff M, et al. Certolizumab pegol plus methotrexate 5-year results from the rheumatoid arthritis prevention of structural damage (RAPID) 2 randomized controlled trial and long-term extension in rheumatoid arthritis patients. Arthritis Research \& Therapy 2015;17:245.

\section{Weinblatt 2012 \{published data only\}}

Pope J, Bingham CO 3rd, Fleischmann RM, Dougados M, Massarotti EM, Wollenhaupt J, et al. Impact of certolizumab pegol on patient-reported outcomes in rheumatoid arthritis and correlation with clinical measures of disease activity. Arthritis Research \& Therapy 2015;17:343.

* Weinblatt ME, Fleischmann R, Huizinga TW, Emery P, Pope J, Massarotti EM, et al. Efficacy and safety of certolizumab pegol in a broad population of patients with active rheumatoid arthritis: results from the REALISTIC phase IIIb study. Rheumatology (Oxford) 2012;51:2204-14.

Weinblatt ME, Fleischmann R, Van Vollenhoven RF, Emery P, Huizinga TW, Cutolo $M$, et al. Twenty-eight-week results from the REALISTIC phase IIIb randomized trial: efficacy, safety and predictability of response to certolizumab pegol in a diverse rheumatoid arthritis population. Arthritis Research \& Therapy 2015;17:325. [DOI: 10.1186/s13075-015-0841-9]

\section{Yamamoto (a) 2014 \{unpublished data only\}}

NCT00791921. Efficacy confirmation trial of CDP870 without coadministration of methotrexate (MTX) in Japanese rheumatoid arthritis (RA). clinicaltrials.gov/show/NCT00791921 (first received 14 November 2008).

NCT00850343. Long-term treatment study of certolizumab pegol without coadministration of methotrexate in Japanese rheumatoid arthritis (RA) patients. clinicaltrials.gov/ct2/show/
NCT00850343?term=NCT00850343\&rank=1 (first received 23rd February 2009).

Takeuchi T, Yamamoto K, Yamanaka H, Ishiguro N, Tanaka Y, Eguchi K, et al. Better clinical responses seen early with the loading dose of certolizumab pegol are maintained until one year. Annals of the Rheumatic Diseases.Conference: Annual European Congress of Rheumatology of the European League Against Rheumatism, EULAR 2014 Paris France. 11 - 14 June 2014. 2014; Vol. 73.

Tanaka Y, Yamamoto K, Takeuchi T, Yamanaka H, Ishiguro N, Eguchi K, et al. Long-term efficacy and safety of certolizumab pegol in Japanese rheumatoid arthritis patients who could not receive methotrexate: 52 -week results from an open-label extension of the HIKARI study. Modern Rheumatology 2013; Vol. 24, issue 5:725-33. [DOI: 10.3109/14397595.2013.865822]

* Yamamoto, Kazuhiko, Takeuchi, Tsutomu, Yamanaka, Hisashi, et al. Efficacy and safety of certolizumab pegol without methotrexate co-administration in Japanese patients with active rheumatoid arthritis: The HIKARI randomized, placebocontrolled trial. Modern Rheumatology 2014;24:552-560.

Yamanaka H, Yamamoto K, Takeuchi T, Ishiguro N, Tanaka Y, Eguchi K, et al. AB0469. Improved physical function, pain, and health related quality of life with certolizumab pegol in Japanese rheumatoid arthritis patients without methotrexate co-administration:results from the Hikari study. Annals of the Rheumaic Diseases 2013;71 Suppl 3:664.

Yamanaka H, Yamamoto K, Takeuchi T, Ishiguro N, Tanaka Y, Eguchi K, et al. Certolizumab pegol improved physical function and heath related quality of life in patients with active rheumatoid arthritis who could not be treated with methotrexate: results form HIKARI study. Modern Rheumatology 2012;22 Suppl II:S83.

\section{Yamamoto (b) 2014 \{published data only\}}

NCT00791999. Efficacy confirmation trial of CDP870 as addon medication to methotrexate (MTX) in Japanese rheumatoid arthritis (RA). clinicaltrials.gov/ct2/show/study/NCT00791999? term $=$ NCT00791999\&rank $=1$ (first received 14th November 2008).

Takeuchi T, Yamamoto K, Yamanaka H, Ishiguro N, Tanaka Y, Eguchi K, et al. Better clinical responses seen early with the loading dose of certolizumab pegol are maintained until one year. Annals of the Rheumatic Diseases.Conference: Annual European Congress of Rheumatology of the European League Against Rheumatism, EULAR 2014 Paris France. 11 - 14 June 2014. 2014; Vol. 73.

Tanaka Y, Yamamoto K, Takeuchi T, Yamanaka H, Ishiguro N, Eguchi K, et al. Long-term efficacy and safety of certolizumab pegol in Japanese rheumatoid arthritis patients with an inadequate response to methotrexate: 52 -week results from an open-label extension of the J-RAPID study. Modern Rheumatology 2014;24(5):734-43.

* Yamamoto K, Takeuchi T, Yamanaka H, Ishiguro N, Tanaka Y, Eguchi K, et al. Efficacy and safety of certolizumab pegol plus methotrexate in Japanese rheumatoid arthritis patients 
with an inadequate response to methotrexate: the J-RAPID randomized, placebo-controlled trial. Modern Rheumatology 2014;24(5):715-24. [DOI: 10.3109/14397595.2013.864224]

Yamanaka H, Yamamoto K, Takeuchi T, Ishiguro N, Tanaka Y, Eguchi K, et al. AB0468 Improved physical function, pain, and health related quality of life with certolizumab pegol in Japanese rheumatoid arthritis patients with an inadequate response to methotrexate: Results from the JRapid study. Annals of the Rheumatic Diseases 2013;71 Suppl 3:664. [DOI: 10.1136/annrheumdis-2012-eular.468]

Yamanaka H, Yamamoto K, Takeuchi T, Ishiguro N, Tanaka Y, Eguchi K, et al. Certolizumab pegol improved physical function and heath related quality of life in patients with active Rheumatoid Arthritis despite treatment with methotrexate: results form the JRAPID study. Modern Rheumatology 2012;22 Suppl Il:S83.

\section{Østergaard 2015 \{published data only\}}

Østergaard M, Axelsen MB, Jacobsson LTH, Schaufelberger C, Hansen MS, Bijlsma JWJ, et al. Dynamic magnetic resonance imaging in the assessment of the response to certolizumab pegol in rheumatoid arthritis patients: Results from a phase IIIB randomized study. Arthritis and Rheumatology.Conference: 2014 ACR/ARHP Annual Meeting Boston, MA United States.Conference Start: 20141114 Conference End: 20141119. Conference Publication: (var.pagings) 2014;66(pp S518-S519):October.

* Østergaard M, Jacobsson LTH, Schaufelberger C, Hansen MS, Bijlsma JWJ, Dudek A, et al. MRI assessment of early response to certolizumab pegol in rheumatoid arthritis: a randomised, double-blind, placebo-controlled phase IIIb study applying MRI at weeks $0,1,2,4,8$ and 16. Annals of the Rheumatic Diseases 2015;74(6):1156-63.

\section{References to studies excluded from this review}

\section{Alten 2013 \{published and unpublished data\}}

Alten R, Fleischmann R, van Vollenhoven R, Vencovsky J, Davies O, Stach C, et al. Long term tolerability and efficacy of a 4-week-administration of certolizumab pegol as monotherapy and combination therapy in rheumatoid arthritis; 5-year-data of an open extension study. Zeitschrift Für Rheumatologie 2013;72:111.

\section{Bykerk 2015 \{published data only\}}

Bykerk VP, Bingham C, Burmester G, Furst DE, Mariette X, Purcaru O, et al. Reduction of disease burden on workplace and household productivity following 52 weeks of treatment with certolizumab pegol in combination with methotrexate in DMARD-naive patients with active, severe, progressive rheumatoid arthritis. Arthritis and Rheumatology.Conference: American College of Rheumatology/Association of Rheumatology Health Professionals Annual Scientific Meeting, ACR/ARHP 2015 San Francisco, CA United States. Conference Start: 20151106 Conference End: 20151111. Confer 2015;67(no pagination):October.

\section{Curtis 2014 \{published data only\}}

Curtis JR, Churchill M, Kivitz A, Samad A, Gauer L, Coteur G, et al. Randomization to patient-reported RAPID3 versus physician- based CDAl tools for prediction of treatment response and assessment of patient-reported outcomes in rheumatoid arthritis patients receiving certolizumab pegol: Results from the predict study. Annals of the Rheumatic Diseases.Conference: Annual European Congress of Rheumatology of the European League Against Rheumatism, EULAR 2014 Paris France. 11 -14 June 2014. 2014; Vol. 73.

\section{Curtis 2015a \{published data only\}}

Curtis JR, Churchill M, Kivitz A, Samad A, Gauer L, Gervitz L, et al. A randomized trial comparing disease activity measures for the assessment and prediction of response in rheumatoid arthritis patients initiating certolizumab pegol. Arthritis \& Rheumatology 2015;67(12):3104-12.

\section{Curtis 2015b \{published data only\}}

Curtis JR, De Longueville M, O'Brien C, Haraoui B. Improvement in disease activity and the long-term risk of serious infectious events in rheumatoid arthritis patients treated with certolizumab pegol. Arthritis and Rheumatology.Conference: American College of Rheumatology/Association of Rheumatology Health Professionals Annual Scientific Meeting, ACR/ARHP 2015 San Francisco, CA United States. 6 - 11 November 2015. 2015; Vol. 67.

\section{Dose Flex 2007 \{published data only\}}

* Furst DE, Shaikh SA, Greenwald M, Bennett B, Davies O, Luijtens $\mathrm{K}$, et al. Two dosing regimens of certolizumab pegol in patients with active rheumatoid arthritis. Arthritis Care \& Research 2015;67(2):151-60.

NCT00580840. Dosing flexibility study in patients with rheumatoid arthritis (DoseFlex). clinicaltrials.gov/ct2/show/ results/NCT00580840 (first received 21st December 2007).

\section{Fleischmann 2013 \{published data only\}}

Fleischmann R, Van Vollenhoven R, Vencovsky J, Alten R, Davies O, Stach C, et al. Long-term safety and efficacy of 4weekly certolizumab pegol monotherapy and combination therapy in rheumatoid arthritis: 5-year results from an openlabel extension study. Annals of the Rheumatic Diseases 2013;72:435.

\section{Kavanaugh 2013 \{published data only\}}

Kavanaugh A, Smolen JS, Emery P, Keystone E, Strand V, Purcaru O, et al. Long-term benefits over more than 4 years of certolizumab pegol combination therapy on workplace and household productivity, and participation in social activities in rheumatoid arthritis: Results from the open-label extension study. Value in Health 2013;16:A570.

\section{Kavanaugh 2014 \{published data only\}}

Kavanaugh A, Mease PJ, Strand V, Purcaru O, Curtis JR. PMS66 - Effect of certolizumab pegol on workplace And household productivity In United States patients with rheumatoid arthritis with or without prior anti-Tnf exposure: results from the Predict study. Value in Health 2014;17(3):A53.

\section{Kivitz 2014 \{published data only\}}

Kivitz AJ, Schechtman J, Texter M, Fichtner A, De Longueville M, Chartash EK. Vaccine responses in patients with rheumatoid 
arthritis treated with certolizumab pegol: results from a singleblind randomized phase IV trial. Journal of Rheumatology 2014;41(4):648-57.

\section{NCT00160641 \{published data only\}}

NCT00160641. A study of the safety and effectiveness of liquid certolizumab pegol in the treatment of signs and symptoms of rheumatoid arthritis and in prevention of joint damage in patients with active rheumatoid arthritis. clinicaltrials.gov/ct2/ show/NCT00160641?term=NCT00160641\&rank=1 (first received 8th September 2005).

\section{NCT00160693 \{published data only\}}

NCT00160693. Open label long-term safety study of certolizumab pegol (CZP) for patients with rheumatoid arthritis. clinicaltrials.gov/ct2/show/NCT00160693 (first received 6th September 2005).

\section{NCT00753454 \{published data only\}}

NCT00753454. Open label extension for patients coming from the dosing flexibility study in patients with rheumatoid arthritis (RA) (Dose Flex II). clinicaltrials.gov/ct2/show/NCT00753454? term=NCT00753454\&rank=1 (first received 5th June 2008).

\section{NCT00843778 \{published data only\}}

NCT00843778. Follow-up of rheumatoid arthritis (RA) moderate to low disease activity study (CERTAIN 2). clinicaltrials.gov/ct2/ show/NCT00843778?term $=$ NCT00843778\&rank=1 (first received 5th January 2009).

\section{NCT00851318 \{published data only\}}

NCT00851318. Long-term treatment study of certolizumab pegol (CDP870) as add-on medication to methotrexate in Japanese rheumatoid arthritis (RA) Patients. clinicaltrials.gov/ ct2/show/NCT00851318?term=certolizumab+and +arthritis\&lup_s=01\%2F01\%2F2013\&lup_e=03\%2F01\%2F2016\&rank=1 (first received 23rd February 2009).

NCT00993668 \{unpublished data only\}

NCT00993668. Assessing the use of certolizumab pegol in adult subjects with rheumatoid arthritis on the antibody response when receiving influenza virus and pneumococcal vaccines. clinicaltrials.gov/ct2/show/results/NCT00993668? term=NCT00993668\&rank=1 (first received 9th October 2009).

NCT01197066 \{published data only\}

NCT01197066. Open-label, extension study of CDP870 in patients with rheumatoid arthritis. clinicaltrials.gov/ct2/show/ NCT01197066? (first received 17th March 2010).

\section{NCT01255761 PREDICT \{published data only\}}

NCT01255761. A comparison of two assessment tools in predicting treatment success of cimzia in rheumatoid arthritis subjects (PREDICT). clinicaltrials.gov/ct2/show/NCT01255761? term=NCT01255761\&rank=1 (first received 6th December 2010).

\section{NCT01292265 \{published data only\}}

NCT01292265. A 12 week study to assess changes in joint inflammation using ultrasonography in patients with rheumatoid arthritis (RA). clinicaltrials.gov/ ct2/show/NCT01292265?term=certolizumab+and
+arthritis\&lup_s=01\%2F01\%2F0201\&lup_e=03\%2F01\%2F2016\&rank $=25$ (first received 7th February 2011).

NCT01374971 \{published data only\}

NCT01374971. Rheumatoid arthritis treatment and biopsy study assessing certolizumab pegol (Cimzia). clinicaltrials.gov/ct2/ show/results/NCT01374971?term=NCT01374971\&rank=1 (first received 14th June 2011).

\section{NCT01443364 \{published data only\}}

NCT01443364. Open label study to assess the predictability of early response to certolizumab pegol in patients with rheumatoid arthritis (SPEED). clinicaltrials.gov/ ct2/show/NCT01443364?term=certolizumab+and +arthritis\&lup_s=01\%2F01\%2F2013\&lup_e=03\%2F01\%2F2016\&rank=13 (first received 27th September 2011).

\section{NCT01526434 \{published data only\}}

NCT01526434. Health-related quality of life and patientreported outcomes in rheumatoid arthritis patients treated with certolizumab pegol. clinicaltrials.gov/ ct2/show/NCT01526434?term=certolizumab+and +arthritis\&lup_s=01\%2F01\%2F0201\&lup_e=03\%2F01\%2F2016\&rank=28 (first received 1st February 2012).

\section{NCT02319642 \{published data only\}}

NCT02319642. An open-label extension study of certolizumab pegol in Chinese patients with rheumatoid arthritis who enrolled in RA0044 (RAPID-C OLE). clinicaltrials.gov/ ct2/show/NCT02319642?term=certolizumab+and +arthritis\&lup_s=01\%2F01\%2F2013\&lup_e=03\%2F01\%2F2016\&rank=4 (first received 15th December 2014).

\section{NCT02586246 \{published data only\}}

NCT02586246. Long-term treatment study of CDP870 selfiifjection in patients with active rheumatoid arthritis who are participating in the long-term treatment studies (Study 275-08-002 or Study 275-08-004) of CDP870. clinicaltrials.gov/ ct2/show/NCT02586246?term=certolizumab+and +arthritis\&lup_s=01\%2F01\%2F0201\&lup_e=03\%2F01\%2F2016\&rank=24 (first received 23rd October 2015).

\section{References to ongoing studies}

\section{NCT01295151 \{published data only\}}

NCT01295151. SWITCH clinical trial for patients with rheumatoid arthritis who have failed an initial TNF-blocking drug (SWITCH). clinicaltrials.gov/ct2/show/NCT01295151 (first received 11th February 2011)

* Navarro C, Nuria C, Brown S, Bosworth A, et al. The 'Switch' study protocol: a randomised-controlled trial of switching to an alternative tumour-necrosis factor (TNF)-inhibitor drug or abatacept or rituximab in patients with rheumatoid arthritis who have failed an initial TNF-inhibitor drug. BMC Musculoskeletal Disorders 2014; Vol. 15:452. [DOI: 10.1186/1471-2474-15-452]

\section{NCT01489384 \{published data only\}}

NCT01489384. Cimzia treatment in rheumatoid arthritis: randomizing to stop versus continue disease- 
modifying anti-rheumatic drug(s). clinicaltrials.gov/ ct2/show/NCT01489384?term=certolizumab+and +arthritis\&lup_s=01\%2F01\%2F0201\&lup_e=03\%2F01\%2F2016\&rank Ab (first received 7th December 2011).

\section{NCT01491815 \{published data only\}}

NCT01491815. Active conventional therapy compared to three different biologic treatments in early rheumatoid arthritis with subsequent dose reduction. clinicaltrials.gov/ ct2/show/NCT01491815?term=certolizumab+and +arthritis\&lup_s=01\%2F01\%2F0201\&lup_e=03\%2F01\%2F2016\&rank=5Arnett FC, Edworthy SM, Bloch DA, McShane DJ, Fries JF, (first received 8th December 2011).

\section{NCT01500278 \{published data only\}}

NCT01500278. Study to assess the short- and longterm efficacy of certolizumab pegol plus methotrexate compared to adalimumab plus methotrexate in subjects with moderate to severe rheumatoid arthritis (RA) inadequately responding to methotrexate. clinicaltrials.gov/ ct2/show/NCT01500278?term=certolizumab+and +arthritis\&lup_s=01\%2F01\%2F2013\&lup_e=03\%2F01\%2F2016\&rank= (first received 22nd December 2011).

\section{NCT01602302 \{published data only\}}

NCT01602302. Ultrasound and withdrawal of biological DMARDs in rheumatoid arthritis. clinicaltrials.gov/ ct2/show/NCT01602302?term=certolizumab+and +arthritis\&lup_s=01\%2F01\%2F0201\&lup_e=03\%2F01\%2F2016\&rank=48 (first received 16th May 2012).

\section{NCT02151851 \{published data only\}}

NCT02151851. A study of certolizumab pegol as additional therapy in Chinese patients with active rheumatoid arthritis (RAPID-C). clinicaltrials.gov/ ct2/show/NCT02151851?term=certolizumab+and +arthritis\&lup_s=01\%2F01\%2F2013\&lup_e=03\%2F01\%2F2016\&rank Bre 18 nnan 2008

(first received 28th May 2014).

\section{NCT02293590 \{published data only\}}

NCT02293590. RICE: Remission by Intra-articular Injection Plus Certolizumab. clinicaltrials.gov/ ct2/show/NCT02293590?term=certolizumab+and +arthritis\&lup_s=01\%2F01\%2F0201\&lup_e=03\%2F01\%2F2016\&rank= (first received 30th October 2014).

\section{NCT02430909 \{published data only\}}

NCT02430909. Multiple dose study of UCB4940 as add-on to certolizumab pegol in subjects with rheumatoid arthritis. clinicaltrials.gov/ct2/ show/NCT02430909?term=certolizumab+and +arthritis\&lup_s=01\%2F01\%2F0201\&lup_e=03\%2F01\%2F2016\&rank= (first received 27th April 2015).

\section{NCT02466581 \{published data only\}}

NCT02466581. Dose reduction for early rheumatoid arthritis patients with low disease activity. clinicaltrials.gov/ ct2/show/NCT02466581?term=certolizumab+and +arthritis\&lup_s=01\%2F01\%2F0201\&lup_e=03\%2F01\%2F2016\&rank= (first received 29th May 2015).

\section{Additional references}

\section{5 solo 2016} Gutierrez B, Rodriguez-Rodriguez L. Influence of demographic and clinical factors on the mortality rate of a rheumatoid arthritis cohort: A 20-year survival study. Seminars in Arthritis and Rheumatism 2016;45(5):533-8.

\section{Arnett 1988} Cooper NS, et al. The American Rheumatism Association 1987 revised criteria for the classification of rheumatoid arthritis. Arthritis and Rheumatism 1988;31(3):315-24.

\section{Bhandari 2004}

Bhandari M, Busse JW, Jackowski D, Montori VM, Schünemann H, Sprague S, et al. Association between industry funding and statistically significant pro-industry findings in medical and surgical randomized trials. Canadian Medical

\section{Blumenauer 2002}

Blumenauer BT, Judd M, Wells GA, Burls A, Cranney A, Hochberg MC, et al. Infliximab for the treatment of rheumatoid arthritis. Cochrane Database of Systematic Reviews 2002, Issue 3. [DOI: 10.1002/14651858.CD003785]

\section{Bongartz 2006}

Bongartz T, Sutton AJ, Sweeting MJ, Buchan I, Matteson EL, Montori V. Anti-TNF antibody therapy in rheumatoid arthritis and the risk of serious infections and malignancies: systematic review and meta-analysis of rare harmful effects in randomized controlled trials. JAMA 2006;295(19):2275-85.

Brennan FM, McInnes IB. Evidence that cytokines play a role in rheumatoid arthritis. The Journal of Clinical Investigation 2008;118(11):3537-45.

\section{Bykerk 2013}

Bykerk VP, Cush J, Winthrop K, Calabrese L, Lortholary O, 5de Longueville $\mathrm{M}$, et al. Update on the safety profile of certolizumab pegol in rheumatoid arthritis: an integrated analysis from clinical trials. Annals of the Rheumatic Diseases 2015;74(1):96-103. [DOI: 10.1136/annrheumdis-2013-203660]

\section{Chen 2006}

Chen YF, Jobanputra P, Barton P, Jowett S, Bryan S, Clark W, et al. A systematic review of the effectiveness of adalimumab, 3etanercept and infliximab for the treatment of rheumatoid arthritis in adults and an economic evaluation of their costeffectiveness. Health Technology Assessment 2006; Vol. 10, issue 42:1-235.

\section{Cross 2014}

Cross M, Smith E, Hoy D, Carmona L, Wolfe F, Vos T, et al. The 4global burden of rheumatoid arthritis: estimates from the global burden of disease 2010 study. Annals of the Rheumatic Diseases 2014;73(7):1316-22.
Abasolo L, Ivorra-Cortes J, Leon L, Jover JA, Fernandez- 


\section{Doran 2002}

Doran MF, Pond GR, Crowson CS, O'Fallon WM, Gabriel SE. Trends in incidence and mortality in rheumatoid arthritis in Rochester, Minnesota, over a forty-year period. Arthritis and Rheumatism 2002;46:625-31.

\section{Dörner 2016}

Dörner T, Strand V, Cornes P, Gonçalves J, Gulácsi L, Kay J, et al. The changing landscape of biosimilars in rheumatology. Annals of the Rheumatic Diseases 2016;75(6):974-82.

\section{EMA 2009}

European Medical Agency. Assesment report for Cimzia. Procedure No EMEA/H/C/001037. www.ema.europa.eu/docs/ en_GB/document_library/EPAR_-_Public_assessment_report/ human/001037/WC500069735.pdf. London, 2009 (accessed 4th July 2017):1-47. [Procedure No.EMEA/H/C/001037]

\section{Emparanza 2015}

Emparanza JI, Cabello JB, Burls AJE. Does evidence-based practice improve patient outcomes? An analysis of a natural experiment in a Spanish hospital. Journal of Evaluation in Clinical Practice 2015;21(6):1059-65.

\section{FDA 2013}

Center for Drug Evaluation and Research. Postmarket Drug Safety Information for Patients and Providers Information for Healthcare Professionals: Cimzia (certolizumab pegol), Enbrel (etanercept), Humira (adalimumab), and Remicade (infliximab). www.fda.gov/drugs/drugsafety/ postmarketdrugsafetyinformationforpatientsandproviders/ ucm124185.htm (accessed 7 Jul 2014).

\section{Felson 2011}

Felson DT, Smolen JS, Wells G, Zhang B, Van Tuyl LHD, Funovits J, et al. American College of Rheumatology/European League Against Rheumatism provisional definition of remission in rheumatoid arthritis for clinical trials. Arthritis and Rheumatism 2011;63(3):573-86.

\section{Glanville 2006}

Glanville JM, Lefebvre C, Miles JN, Camosso-Stefinovic J. How to identify randomized controlled trials in MEDLINE: ten years on. Journal of the Medical Library Association 2006;94(2):130-6.

\section{Hazes 2010}

Hazes JM, Taylor P, Strand V, Purcaru O, Coteur G, Mease P. Physical function improvements and relief from fatigue and pain are associated with increased productivity at work and at home in rheumatoid arthritis patients treated with certolizumab pegol. Rheumatology (Oxford) 2010;49(10):1900-10.

\section{Higgins 2011}

Higgins JP, Green S, editor(s). Cochrane Handbook for Systematic Reviews of Interventions Version 5.1.0 (updated March 2011). The Cochrane Collaboration, 2011. Available from www.handbook.cochrane.org.

\section{Jobanputra 2012}

Jobanputra P, Maggs F, Deeming A, Carruthers D, Rankin E, Jordan AC, et al. A randomised efficacy and discontinuation study of etanercept versus adalimumab (RED SEA) for rheumatoid arthritis: a pragmatic, unblinded, non inferiority study of first TNF inhibitor use: outcomes over 2 years. BMJ Open 2012;2(6):e001395.

\section{Keystone 2012}

Keystone EC, Combe B, Smolen J, Strand V, Goel N, van Vollenhoven R, et al. Sustained efficacy of certolizumab pegol added to methotrexate in the treatment of rheumatoid arthritis: 2-year results from the RAPID 1 trial. Rheumatology (Oxford) 2012;51(9):1628-38.

\section{Kharlamova 2016}

Kharlamova N, Jiang X, Sherina N, Potempa B, Israelsson L, Quirke AM, et al. Antibodies to Porphyromonas gingivalis Indicate Interaction Between Oral Infection, Smoking, and Risk Genes in Rheumatoid Arthritis Etiology. Arthritis \& Rheumatology 2016;68(3):604-13.

\section{Laird 1990}

Laird NM, Wang F. Estimating rates of change in randomized clinical trials. Controlled Clinical Trials 1990;11(6):405-19.

\section{Laupacis 1988}

Laupacis A, Sackett DL, Roberts RS. An assessment of clinically useful measures of the consequences of treatment. New England Journal of Medicine 1988;318(26):1728-33.

\section{Lethaby 2013}

Lethaby A, Lopez-Olivo MA, Maxwell L, Burls A, Tugwell P, Wells GA. Etanercept for the treatment of rheumatoid arthritis. Cochrane Database of Systematic Reviews 2013, Issue 5. [DOI: 10.1002/14651858.CD004525.pub2]

\section{Lopez-Olivo 2014}

Lopez-Olivo MA, Siddhanamatha HR, Shea B, Tugwell P, Wells GA, Suarez-Almazor ME. Methotrexate for treating rheumatoid arthritis. Cochrane Database of Systematic Reviews 2014, Issue 6. [DOI: 10.1002/14651858.CD000957.pub2]

\section{Lundberg 2013}

Lundberg K, Bengtsson C, Kharlamova N, Reed E, Jiang X, Kallberg $\mathrm{H}$, et al. Genetic and environmental determinants for disease risk in subsets of rheumatoid arthritis defined by the anticitrullinated protein/peptide antibody fine specificity profile. Annals of the Rheumatic Diseases 2013;72(5):652-8.

\section{MacGregor 2000}

MacGregor AJ, Snieder H, Rigby AS, Koskenvuo M, Kaprio J, Aho $\mathrm{K}$, et al. Characterizing the quantitative genetic contribution to rheumatoid arthritis using data from twins. Arthritis and Rheumatism 2000;43(1):30-7.

\section{Meune 2009}

Meune C, Touze E, Trinquart L, Allanmore Y. Trends in cardiovascular mortality in patients with rheumatoid arthritis over 50 years: a systematic review and meta-analysis of cohort studies. Rheumatology 2009;48:1309-13. 


\section{Navarro-Millán 2013}

Navarro-Millán I, Curtis JR. Newest clinical trial results with antitumor necrosis factor and non antitumor necrosis factor biologics for rheumatoid arthritis. Current Opinion in Rheumatology 2013;25(3):384-90.

\section{Navarro-Sarabia 2005}

Navarro-Sarabia F, Ariza-Ariza R, Hernandez-Cruz B, Villanueva I. Adalimumab for treating rheumatoid arthritis. Cochrane Database of Systematic Reviews 2005, Issue 3. [DOI: 10.1002/14651858.CD005113.pub2]

\section{NICE 2009}

UCB. Certolizumab pegol (CIMZIAC) for the treatment of rheumatoid arthritis. Single technology appraisal (STA) manufacturer submission to NICE. NICE 2009:1-180.

\section{Okada 2014}

Okada Y, Wu D, Trynka G, Raj T, Terao C, Ikari K, et al. Genetics of rheumatoid arthritis contributes to biology and drug discovery. Nature 2014;506(7488):376-81.

\section{Puolakka 2005}

Puolakka K, Kautiainen H, Möttönen T, Hannonen P, Korpela M, Hakala M, et al. FIN-RACo Trial Group. Early suppression of disease activity is essential for maintenance of work capacity in patients with recent-onset rheumatoid arthritis: five-year experience from the FIN-RACo trial. Arthritis and Rheumatology 2005;52(1):36-4.

\section{Roubille 2015}

Roubille C, Richer V, Starnino T, McCourt C, McFarlane A, Fleming $\mathrm{P}$, et al. The effects of tumour necrosis factor inhibitors, methotrexate, non-steroidal anti-inflammatory drugs and corticosteroids on cardiovascular events in rheumatoid arthritis, psoriasis and psoriatic arthritis: a systematic review and meta-analysis. Annals of the Rheumatic Diseases 2015;74(3):480-9.

\section{Singh 2009}

Singh JA, Christensen R, Wells GA, Suarez-Almazor ME, Buchbinder R, Lopez-Olivo MA, et al. Biologics for rheumatoid arthritis: an overview of Cochrane reviews. Cochrane Database of Systematic Reviews 2009, Issue 4. [DOI: 10.1002/14651858.CD007848.pub2]

\section{Singh 2010}

Singh JA, Noorbaloochi S, Singh G. Golimumab for rheumatoid arthritis. Cochrane Database of Systematic Reviews 2010, Issue 1. [DOI: 10.1002/14651858.CD008341]

\section{Singh 2011}

Singh JA, Wells GA, Christensen R, Tanjong Ghogomu E, Maxwell L, MacDonald JK, et al. Adverse effects of biologics: a network meta-analysis and Cochrane overview. Cochrane Database of Systematic Reviews 2011, Issue 2. [DOI: 10.1002/14651858.CD008794.pub2]

\section{Singh 2012}

Singh JA, Furst DE, Bharat A, Curtis JR, Kavanaugh AF, Kremer JM, et al. 2012 update of the 2008 American College of Rheumatology recommendations for the use of diseasemodifying antirheumatic drugs and biologic agents in the treatment of rheumatoid arthritis. Arthritis Care and Research 2012;64:625-39.

\section{Singh 2015}

Singh JA, Cameron C, Noorbaloochi S, Cullis T, Tucker M, Christensen $\mathrm{R}$, et al. Risk of serious infection in biological treatment of patients with rheumatoid arthritis: a systematic review and meta-analysis. Lancet 2015;386(9990):258-65.

\section{Singh 2016}

Singh A, Saag KG, Bridges SL, Akl A, Bannuru RR, Sullivan MC, et al. 2015 American College of Rheumatology guideline for the treatment of rheumatoid arthritis. Arthritis Care \& Research 2016;68(1):1-25.

\section{Smolen 2014}

Smolen JS, Landewé R, Breedveld FC, Buch M, Burmester G, Dougados $\mathrm{M}$, et al. EULAR recommendations for the management of rheumatoid arthritis with synthetic and biological disease-modifying antirheumatic drugs: 2013 update. Annals of the Rheumatic Diseases 2014;73(3):492-509.

\section{Sokka 2003}

Sokka T, Krishnan E, Hakkinen A, Hannonen P. Functional disability in rheumatoid arthritis patients compared with a community population in Finland. Arthritis and Rheumatism 2003;48(1):59-63. [PUBMED: 12528104]

\section{Thompson 1999}

Thompson SG, Sharp SJ. Explaining heterogeneity in metaanalysis: a comparison of methods. Statistics in Medicine 1999;18(20):2693-708.

\section{Tureson 2013}

Turesson C. Extra-articular rheumatoid arthritis. Current Opinion in Rheumatology 2013;23(3):360-6.

\section{Wong 2006a}

Wong SS, Wilczynski NL, Haynes RB. Developing optimal search strategies for detecting clinically sound treatment studies in EMBASE. Journal of the Medical Library Association 2006;94(1):41-7.

\section{Wong 2006b}

Wong SS, Wilczynski NL, Haynes RB. Optimal CINAHL search strategies for identifying therapy studies and review articles. Journal of Nursing Scholarship 2006;38(2):194-9.

\section{Yee 2003}

Yee CS, Filer A, Pace A, Douglas K, Situnayake D, Rowe IF. West Midlands Rheumatology Services and Training Committee. The prevalence of patients with rheumatoid arthritis in the West Midlands fulfilling the BSR criteria for anti-tumour necrosis factor therapy: an out-patient study. Rheumatology (Oxford) 2003;42:856-9.

\section{Young 2000}

Young A, Dixey J, Cox N, Davies P, Devlin J, Emery P, et al. How does functional disability in early rheumatoid arthritis affect 
patients and their lives? Results of 5 years of follow-up in 732 patients from the early RA study (ERAS). Rheumatology 2000;39(6):603-11.

\section{Yusuf 1991}

Yusuf S, Wittes J, Probstfield J, Tyroler HA. Analysis and interpretation of treatment effects in subgroups of patients in randomized clinical trials. JAMA 1991;266(1):93-8.

\section{Zhou 2014}

Zhou Q, Zhou Y, Chen H, Wang Z, Tang Z, Liu J. The efficacy and safety of certolizumab pegol (CZP) in the treatment of active rheumatoid arthritis (RA): a meta-analysis from nine randomized controlled trials. International Journal of Clinical and Experimental Medicine 2014;7(11):3870-80.

\section{References to other published versions of this review}

\section{Ruiz Garcia 2009}

Ruiz Garcia V, Burls A, Cabello López JCL, Fry-Smith AFS, GalvezMuñoz JG, Jobanputra P, et al. Certolizumab pegol (CDP870) for rheumatoid arthritis in adults. Cochrane Database of Systematic Reviews 2009, Issue 1. [DOI: 10.1002/14651858.CD007649]

\section{Ruiz Garcia 2011}

Ruiz Garcia V, Jobanputra P, Burls A, Cabello JB, Gálvez Muñoz JG, Saiz Cuenca ESC, Fry-Smith A. Certolizumab pegol (CDP870) for rheumatoid arthritis in adults. Cochrane Database of Systematic Reviews 2011, Issue 2. [DOI: 10.1002/14651858.CD007649.pub2]

\section{Ruiz Garcia 2014}

Ruiz Garcia V, Jobanputra P, Burls A, Cabello JB, Vela Casasempere P, Bort-Marti S, et al. Certolizumab pegol (CDP870) for rheumatoid arthritis in adults. Cochrane Database of Systematic Reviews 2014, Issue 9. [DOI: 10.1002/14651858.CD007649.pub3]

* Indicates the major publication for the study

\section{CHARACTERISTICS OF STUDIES}

Characteristics of included studies [ordered by study ID]

\section{Atsumi 2016}

\begin{tabular}{ll}
\hline Methods & Randomised clinical trial, double-blind \\
\hline Participants & Eligible patients were $20-64$ years old with RA fulfilling the 2010 ACR/EULAR classification criteria. \\
\hline Interventions & $\begin{array}{l}1.400 \mathrm{mg} \text { of CDP870 plus MTX given at week } 0,2.4 \text {, and thereafter } 200 \text { mg CDP870 given every } 2 \text { weeks } \\
(\mathrm{n}=159)\end{array}$ \\
2. Placebo plus MTX given every 2 weeks $(\mathrm{n}=157)$
\end{tabular}

Outcomes

Primary outcome measures: Inhibition of radiographic progression at week 52

Secondary outcomes measures: Inhibition of radiographic progression at week 24;

Clinical remission rate at week 24 and week 52

\section{Notes}

\section{C-OPERA Trial}

Countries/Cities: 73 sites in Japan

Dates conducted: from October 2011 to August 2013

Eligibility criteria: Eligible patients were 20-64 years old with RA fulfilling the 2010 ACR/EULAR classification criteria. Patients had $\leq 12$ months of persistent arthritic symptoms, at least moderate disease activity (Disease Activity Score 28-joint assessment (DAS28) with erythrocyte sedimentation rate (ESR) 23.2) and were MTX-naive. In addition, patients had poor prognostic factors: high anti-cyclic citrullinated peptide (anti-CCP) anti- body ( $\geq 3 \times$ upper limit of normal (ULN)) and either positive rheumatoid factor (RF) and/or presence of bone erosions (based on radiographs of hands/feet, assessed by the investigator at each study site).

Adverse events as a specified outcome: adverse events and serious adverse events were reported

Funding sources: Astellas Pharma Inc 
Conflict of interest: Principal Investigators are NOT employed by the organization sponsoring the study.

Restriction Description: Institute and/or Principal Investigator may publish trial data generated at their specific study site after Sponsor publication of the multi-center data. Sponsor must receive a site's manuscript prior to publication to ensure that no confidential information of Sponsor is included in the document. Sponsor may delay the publication for to seek patent protection.

TA has taken part in speakers' bureaus for Astellas, Bristol-Myers, Chugai and Mitsubishi-Tanabe; KY has received consultancy fees from Abbott, BMS, Chugai, Eisai, Mitsubishi-Tanabe, Pfizer, Roche and UCB Pharma, and has received research grants from Abbott, Eisai, Mitsubishi-Tanabe, Pfizer, Santen and UCB Pharma;

TT has received consultancy fees from AstraZeneca, Asahi Kasei, Eli Lilly, Mitsubishi-Tanabe and Novartis, research grants from Abbott, Astellas, BMS, Chugai, Daiichi-Sankyo, Eisai, Janssen, Mitsubishi-Tanabe, Nippon Shinyaku, Otsuka, Pfizer, Sanofi-Aventis, Santen, Takeda and Teijin, and has taken part in speakers' bureaus for Abbott, BMS, Chugai, Eisai, Janssen, Mitsubishi-Tanabe, Pfizer and Takeda and UCB Pharma; HY has received consultancy fees from Abbott, Astellas, BMS, Chugai, Eisai, Janssen, Mitsubishi-Tanabe, Pfizer, Takeda and UCB Pharma, and has received research grants from Abbott, Astellas, BMS, Chugai, Eisai, Janssen, Mitsubishi-Tanabe, Pfizer, Takeda and UCB Pharma; NI has received research grants from Abbott, Astellas, BMS, Takeda, Chugai, Eisai, Janssen, Kaken Mitsubishi-Tanabe and Pfizer, and has taken part in speakers' bureaus for Abbott, Astellas, BMS, Chugai, Eisai, Janssen, Kaken, Mitsubishi-Tanabe, Otsuka, Pfizer, Taisho-Toyama and Takeda;

YT has received research grants from Astellas, AbbVie, BMS, Chugai, Daiichi-Sankyo, Mitsubishi-Tanabe, MSD, has received consultancy fees from Abbott, AbbVie, Asahi Kasei, Astellas, AstraZeneca, Chugai, Daiichi-Sankyo, Eisai, Eli Lilly, GSK, Janssen, Mitsubishi-Tanabe, MSD, Pfizer, Quintiles, Takeda and UCB Pharma, and has taken part in speakers' bureaus for Abbott, AbbVie, Asahi Kasei, Astellas, AstraZeneca, Chugai, Daiichi-Sankyo, Eisai, Eli Lilly, GSK, Janssen, Mitsubishi-Tanabe, MSD, Pfizer, Quintiles, Takeda and UCB Pharma; KE has received consultancy fees from UCB Pharma; AW has received research grants from Daiichi-Sankyo, Dainippon-Sumitomo, Kyorin, Meiji Seika; Shionogi, Taiho, Taisho and Toyama Chemical, and has taken part in speakers' bureaus for Daiichi-Sankyo, Dainippon-Sumitomo, GSK, Mitsubishi-Tanabe, MSD, Pfizer, Shionogi and Taisho-Toyama;

HO has received consultancy fees from Astellas and UCB Pharma; SY has received research grant from BMS and taken part in speakers' bureaus for AbbVie, Astellas, Chugai, Eizai, Pfizer, Mitsubishi-Tanabe and Takeda; YY has no competing interests to disclose; YK has received speakers' bureau from Astellas, Chugai, and Ono; TM has received speaker honoraria from Pfizer Japan, Janssen Pharmaceutical Co. and Astellas Pharma; and research grants form Quintiles Transnational Japan K.K, Janssen Pharmaceutical Co., Takeda Chemical Industries, Daiichi Sankyo Co., Astellas Pharma, Eli Lilly Japan K.K., MSD Co., Nippon Kayaku Co., Parexel International Corp., Pfizer Japan and Bristol-Myers Squibb; MI has received payment for lectures from Astellas, Chugai, Ono and Tanabe-Mitsubishi, has received research grants from Pfizer and a royalty fee from Chugai; TS is an employee of UCB Pharma;

TO is an employee of Astellas;

DvdH has received consultancy fees from AbbVie, Amgen, AstraZeneca, Augurex, BMS, Celgene, Centocor, Chugai, Covagen, Daiichi, Eli-Lilly, Galapagos, GSK, Janssen Biologics, Merck, Novartis, Novo-Nordisk, Otsuka, Pfizer, Roche, Sanofi-Aventis, Schering-Plough, UCB Pharma and Vertex; and is the Director of Imaging Rheumatology bv;

NM has received research grants from Abbott, Astellas, Chugai, Eisai, Mitsubishi-Tanabe, Pfizer and Takeda;

TK has received consultancy fees from AbbVie, Astellas, BMS, Chugai, Daiichi-Sankyo, Eisai, Mitsubishi-Tanabe, Pfizer, Santen, Taisho-Toyama, Takeda, Teijin and UCB Pharma, and has taken part in speakers' bureaus for Abbott, Astellas, BMS, Chugai, Daiichi-Sankyo, Eisai, Mitsubishi-Tanabe, Pfizer, Santen, Taisho-Toyama, Takeda, Teijin and UCB Pharma.

\section{Risk of bias}


Atsumi 2016 (Continued)

Random sequence genera- $\quad$ Low risk $\quad$ "Patients were randomised 1:1"
tion (selection bias)

Allocation concealment Low risk "via an interactive web-response system"
(selection bias)

\begin{tabular}{|c|c|c|}
\hline $\begin{array}{l}\text { Blinding (performance } \\
\text { bias and detection bias) } \\
\text { ACR50 }\end{array}$ & Low risk & $\begin{array}{l}\text { ACR50 is a clinical outcome determined by healthcare professionals who were } \\
\text { blinded to study medications. }\end{array}$ \\
\hline $\begin{array}{l}\text { Blinding (performance } \\
\text { bias and detection bias) } \\
\text { All outcomes }\end{array}$ & Low risk & As above \\
\hline
\end{tabular}

\begin{tabular}{|c|c|c|}
\hline $\begin{array}{l}\text { Incomplete outcome data } \\
\text { (attrition bias) } \\
\text { ACR50 }\end{array}$ & Low risk & $\begin{array}{l}\text { Participants who did not achieve an improvement of symptoms at or after } \\
\text { week } 24 \text {, i.e. if moderate or higher disease activity (DAS28 (ESR) } \geq 3.2 \text { ) persist- } \\
\text { ed } \geq 4 \text { weeks in either treatment arm, were eligible to receive rescue treatment } \\
\text { with open-label certolizumab pegol after discontinuing D-B period. As a conse- } \\
\text { quence, the withdrawal rate in CTZ arm was } 22.6 \% \text {; withdrawal rate in Placebo } \\
\text { arm was } 44.6 \%\end{array}$ \\
\hline
\end{tabular}

\begin{tabular}{l}
\hline Incomplete outcome data Low risk As above \\
(attrition bias) \\
All outcomes
\end{tabular}

\begin{tabular}{|c|c|c|}
\hline $\begin{array}{l}\text { Selective reporting (re- } \\
\text { porting bias) }\end{array}$ & Low risk & $\begin{array}{l}\text { Data from all radiological (except for JSN outcome), clinical and safety out- } \\
\text { comes were provided }\end{array}$ \\
\hline Other bias & Low risk & The study appears to be free of other sources of bias \\
\hline $\begin{array}{l}\text { Blinding of participants } \\
\text { and personnel (perfor- } \\
\text { mance bias) } \\
\text { All outcomes }\end{array}$ & Low risk & $\begin{array}{l}\text { Study did not report blinding of participants. Drug administration was per- } \\
\text { formed by dedicated non-blinded persons, because obvious differences be- } \\
\text { tween certolizumab pegol and Placebo; however, these personnel were not } \\
\text { permitted to engage in other study activities, to maintain blinding. All investi- } \\
\text { gators and healthcare professionals involved in safety/efficacy assessments } \\
\text { were blind to study medications }\end{array}$ \\
\hline $\begin{array}{l}\text { Blinding of outcome as- } \\
\text { sessment (detection bias) } \\
\text { All outcomes }\end{array}$ & Low risk & $\begin{array}{l}\text { All investigators and healthcare professionals involved in safety/efficacy as- } \\
\text { sessments were blind to study medications. mTSS as main outcome assessed } \\
\text { by radiologist (namely, healthcare professionals) }\end{array}$ \\
\hline
\end{tabular}

CDP870-004 2001

\begin{tabular}{ll}
\hline Methods & Double-blind, multiple dose, 12-week, placebo-controlled dose-ranging study \\
\hline Participants & $\begin{array}{l}326 \text { participants with a history of inadequate response or intolerance to at least } 1 \text { DMARD and active RA } \\
\text { at screening }\end{array}$ \\
\hline Interventions & $\begin{array}{l}\text { 1. Placebo } \\
\text { 2. } 50,100,200,400,600 \text { and } 800 \mathrm{mg} \mathrm{sc} \\
\text { Given every } 4 \text { weeks in } 2 \text { dose groups, panel } 1 \text { and panel } 2 \\
\text { "Placebo: } 40 ; \text { active: } 40-41 / \text { arm); Panel 2: } 122 \text { (Placebo 44, active: } 39 / \text { arm). PP: } 186, \text { and } 113 \text { pts." }\end{array}$ \\
\hline
\end{tabular}

\section{Outcomes}

ACR20, ACR50, ACR70, subset of the ACR criterion, DAS responder rates at week 12 


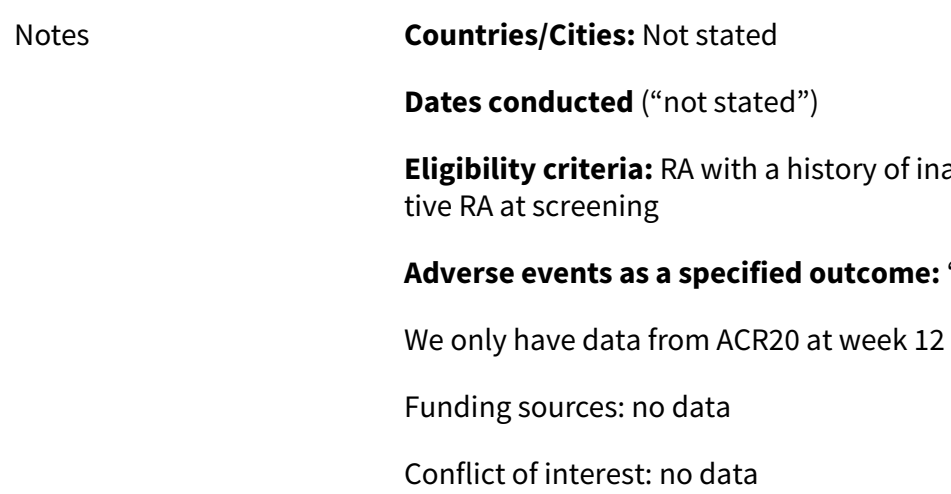

\section{Risk of bias}

\begin{tabular}{lll}
\hline Bias & Authors' judgement & Support for judgement \\
\hline $\begin{array}{l}\text { Random sequence genera- } \\
\text { tion (selection bias) }\end{array}$ & Low risk & $\begin{array}{l}\text { UCB reported: "Randomized code generated by Pharmaceutical Packaging } \\
\text { Service and based on instruction of the randomisation procedure prepared by } \\
\text { Celltech R\&D statistic" }\end{array}$
\end{tabular}

\begin{tabular}{|c|c|c|}
\hline $\begin{array}{l}\text { Allocation concealment } \\
\text { (selection bias) }\end{array}$ & Unclear risk & $\begin{array}{l}\text { UCB reported: "Patients were randomly assigned to treatment groups during } \\
\text { the DB phase (week 0_12) and received either placebo or CDP- } 870 \text { SC" }\end{array}$ \\
\hline
\end{tabular}

\begin{tabular}{|c|c|c|}
\hline $\begin{array}{l}\text { Blinding (performance } \\
\text { bias and detection bias) } \\
\text { ACR50 }\end{array}$ & High risk & $\begin{array}{l}\text { UCB reported as blinded but stated: "CPD- } 870 \text { and the placebo utilized in this } \\
\text { study (saline) did not have the same viscosity therefore full blinding was not } \\
\text { possible. Study drug was to be prepared by a pharmacist having no other in- } \\
\text { volvement in the study; injections of study medications were given by a nurse } \\
\text { or physician who had no other involvement in the study..." }\end{array}$ \\
\hline
\end{tabular}

\begin{tabular}{ll}
\hline Blinding (performance & High risk \\
bias and detection bias) & \\
All outcomes & \\
\hline
\end{tabular}

\begin{tabular}{|c|c|c|}
\hline $\begin{array}{l}\text { Incomplete outcome data } \\
\text { (attrition bias) } \\
\text { ACR50 }\end{array}$ & High risk & Data were not available \\
\hline $\begin{array}{l}\text { Incomplete outcome data } \\
\text { (attrition bias) } \\
\text { All outcomes }\end{array}$ & High risk & Data were not available \\
\hline $\begin{array}{l}\text { Selective reporting (re- } \\
\text { porting bias) }\end{array}$ & Low risk & $\begin{array}{l}\text { Efficacy was defined as ACR improvement in disease activity at week } 12 \text { and } \\
\text { was described }\end{array}$ \\
\hline Other bias & Unclear risk & There were so few data that was impossible to judge \\
\hline $\begin{array}{l}\text { Blinding of participants } \\
\text { and personnel (perfor- } \\
\text { mance bias) } \\
\text { All outcomes }\end{array}$ & High risk & See above \\
\hline $\begin{array}{l}\text { Blinding of outcome as- } \\
\text { sessment (detection bias) } \\
\text { All outcomes }\end{array}$ & High risk & See above \\
\hline
\end{tabular}


Choy 2002

\begin{tabular}{ll}
\hline Methods & Randomised double-blind placebo-controlled trial \\
\hline Participants & 36 people with RA defined by ACR classification criteria. People with active diseased defined as having \\
& $\begin{array}{l}\text { cor the following } 4 \text { criteria: tender joint count (TJC) } \geq 6, \text { swollen joint count }(\mathrm{SJC}) \geq 3 \text { (based on } 28 \text { joint } \\
\text { counts), morning stiffness of } \geq 45 \text { minutes, and ESR } \geq 28 \mathrm{~mm} / \mathrm{H} \text {. Participants had to have failed treat- } \\
\text { ment with at least } 1 \text { DMARD and have been off treatment for at least } 4 \text { weeks }\end{array}$ \\
\hline
\end{tabular}

\begin{tabular}{ll}
\hline Interventions & 1. Single intravenous infusion of placebo $(n=12)$ \\
& 2. 1,5 or $20 \mathrm{mg} / \mathrm{kg}$ of certolizumab pegol $($ each $n=8)$ for 8 weeks
\end{tabular}

Outcomes ACR20, ACR50, ACR70, pain score $(0-10 \mathrm{~cm})$, DAS, TJC, SJC, Health Assessment Questionnaire (HAQ), C
reactive protein $(C R P)$

Follow-up 8 weeks

Notes This study was only considered to assess safety because follow-up was less than 12 weeks

In the open-label phase, 1 participant who received $20 \mathrm{mg} / \mathrm{kg}$ died from complications following rapid drainage of a large, chronic rheumatoid pericardial effusion. No infective agent was isolated from either the pericardial fluid or peripheral blood. In the opinion of the investigator, this event was unrelated to treatment.

Countries/Cities: patients recruited from out-patient rheumatology clinics in London, Cambridge, Norfolk and Norwich (UK).

Dates conducted: not reported

Eligibility criteria: Patients aged 18-75 yr who satisfied the 1987 revised American College of Rheumatology (ACR) diagnostic criteria for RA

Adverse events: were reported

Funding sources: not stated, but UCB had all the data and sent us details of how was done

Conflict of interest: DA Isenberg, worked for Celltech Research and Development, Slough, UK

\section{Risk of bias}

\begin{tabular}{|c|c|c|}
\hline Bias & Authors' judgement & Support for judgement \\
\hline $\begin{array}{l}\text { Random sequence genera- } \\
\text { tion (selection bias) }\end{array}$ & Low risk & $\begin{array}{l}\text { Participants were divided into } 4 \text { groups. In each group of } 12 \text { patients } 8 \text { received } \\
\text { active treatment and } 4 \text { received placebo. UCB explain to us: "Methods for se- } \\
\text { quence generation was randomised, DB, sequential ascending dose" }\end{array}$ \\
\hline $\begin{array}{l}\text { Allocation concealment } \\
\text { (selection bias) }\end{array}$ & Low risk & Central allocation \\
\hline $\begin{array}{l}\text { Blinding (performance } \\
\text { bias and detection bias) } \\
\text { ACR50 }\end{array}$ & Low risk & $\begin{array}{l}\text { The study was blinded and UCB stated: "all data were entered and Database } \\
\text { locked after completion of the clinical phase for the first study period and be- } \\
\text { fore ESR and CRP were entered into the database. ESR and CRP data were } \\
\text { withheld from investigator and sponsor study personal during the course of } \\
\text { the study because knowledge of patient's profile could potentially unblind the } \\
\text { study..., auto AB, anti certolizumab pegol level, TNFalpha, IL6 and IL1b were } \\
\text { transferred into the database after DB lock" }\end{array}$ \\
\hline $\begin{array}{l}\text { Blinding (performance } \\
\text { bias and detection bias) } \\
\text { All outcomes }\end{array}$ & Low risk & See above \\
\hline
\end{tabular}


Choy 2002 (Continued)

$\begin{array}{ll}\begin{array}{l}\text { Incomplete outcome data } \\ \text { (attrition bias) }\end{array} & \text { Reasons for withdrawals were disclosed } \\ \text { ACR50 } & 92 \% \text { of certolizumab pegol group and } 50 \% \text { of placebo completed } 8 \text { weeks of } \\ & \text { treatment. We imputed missing data for analysis }\end{array}$

\begin{tabular}{|c|c|c|}
\hline $\begin{array}{l}\text { Incomplete outcome data } \\
\text { (attrition bias) } \\
\text { All outcomes }\end{array}$ & Low risk & Safety analysis also imputed missing data \\
\hline $\begin{array}{l}\text { Selective reporting (re- } \\
\text { porting bias) }\end{array}$ & Low risk & All the outcomes were available in the clinical study report as figures \\
\hline $\begin{array}{l}\text { Blinding of participants } \\
\text { and personnel (perfor- } \\
\text { mance bias) } \\
\text { All outcomes }\end{array}$ & Low risk & $\begin{array}{l}\text { UCB stated: " the study pharmacist prepared for infusion the study medication } \\
\text { and diluent, the pharmacy covered the solution with an opaque material and } \\
\text { labelled it with "130mL CDP } 870 \text { Engineered Fab' Conjugated to PEG or sodium } \\
\text { acetate placebo diluent" "For IV use only", administration details, the patient } \\
\text { number, patient initials, date and time to use the medication by and name of } \\
\text { investigator." }\end{array}$ \\
\hline
\end{tabular}

$\begin{array}{ll}\text { Blinding of outcome as- } & \text { Low risk }\end{array}$

sessment (detection bias)

All outcomes

\section{Choy 2012}

\begin{tabular}{ll}
\hline Methods & Phase III, randomised double-blind placebo-controlled multicentre trial \\
The primary objective of this study was to compare the efficacy of certolizumab pegol (CDP870 or CZP) \\
in combination with methotrexate (MTX) to MTX alone in treating the signs and symptoms of subjects \\
with rheumatoid arthritis (RA) who are partial responders to MTX.
\end{tabular}

Participants $\quad$ People with RA who are partial responders to MTX.

250 participants with RA, aged 18+ years, were randomised to 1 of 2 regimens of sc certolizumab pegol $400 \mathrm{mg}$ or placebo sc every 4 weeks for a total of 6 injections. Methotrexate treatment continue during the study taken prior to enrolment in the study.Participants who completed the current study or who withdrew on or after the Week 12 visit were eligible to participate in the open-label safety study (CDP870-015).

Inclusion and exclusion criteria were identical to Keystone 2008, but discontinued all DMARDs at least 28 days or 5 half-lives prior to first dose of study drug

\begin{tabular}{ll}
\hline Interventions & 1. Certolizumab pegol $400 \mathrm{mg}$ plus MTX $(\mathrm{n}=125)$ \\
2. Placebo sc plus MTX $(\mathrm{n}=125)$ \\
Every 4 weeks for a total of 6 injections
\end{tabular}

Outcomes Primary: ACR20 and safety at 24 weeks

Secondary endpoints: Participant's assessment of pain (VAS), participant's global assessment of arthritis, physician's global assessment of arthritis, participant's assessment of physical function by HAQ-DI, acute phase reactant value (only CRP for this study)

Follow-up 24 weeks 
Dates conducted: between October 2002 and January 2004.

Eligibility criteria: patients were aged 18-75 years, with adult-onset RA of at least 6 months' duration as defined by the 1987 ACR criteria and active disease defined as nine or more tender joints, nine or more swollen joints and at least one of the three following criteria: $\geq 45 \mathrm{~min}$ of morning stiffness, ESR $\geq 28 \mathrm{~mm} / \mathrm{h}$ (Westergren) or CRP $>10 \mathrm{mg} / \mathrm{l}$. Patients were required to have been receiving MTX for at least 6 months and on a stable dosage of $15-25 \mathrm{mg} /$ week for at least 8 weeks before the first dose of study medication (10-15 mg/week was deemed acceptable in cases where a dosage reduction had been necessary because of toxicity). All other DMARDs were to have been discontinued at least 28 days before the first study medication dose

Adverse events as a specified outcome: AEs were reported at each study visit. Treatment-emergent AEs were those reported after the first dose of study medication, including worsening of pre-existing conditions. Serious AEs (SAEs) were those that resulted in death or were life-threatening, caused or prolonged hospitalizations, required parenteral antibiotics, and/or that resulted in persistent or significant disability, incapacity or congenital abnormality/birth defect.

\section{Funding sources: UCB}

Conflict of interest: J.V. was a speaker at the meeting organized by UCB and is a member of a UCB advisory board. E.C. has received grants/research support from Abbott Laboratories, Allergan, Boehringer Ingelheim, Chelsea Therapeutics, GSK, Jazz Pharmaceuticals, Merrimack Pharmaceutical, MSD, Pfizer, Pierre Fabre Medicament, Roche, Chugai and Wyeth and UCB Pharma.

E.C. has also received consultancy fees from Abbott Laboratories, Allergan, Boehringer Ingelheim, Chelsea Therapeutics, Eli Lilly, GSK, Jazz Pharmaceuticals, Merrimack Pharmaceutical, MSD, Pfizer, Pierre Fabre Medicament, Roche, Schering Plough, Synovate, Chugai, MedImmune and Wyeth and UCB Pharma. E.C. is a member of a Speaker's Bureau for Abbott Laboratories, Allergan, Boehringer Ingelheim, Chelsea Therapeutics, Eli Lilly, GSK, Jazz Pharmaceuticals, Merrimack Pharmaceutical, MSD, Pfizer, Pierre Fabre Medicament, Roche, Schering Plough, Chugai and Wyeth and UCB Pharma.

B.V. is a UCB Pharma employee and has been granted UCB Pharma stock appreciation rights.

N.G. is a former employee of UCB Pharma, and is currently an employee of Array Biopharma, Inc. N.G. owns UCB Pharma stock.

O.D. is an employee of UCB Pharma and holds stock options.

R.A. has received research grants from Abbott, BMS, Merck Pharma GmbH, Novartis, Pfizer, Roche and UCB Pharma. R.A. is a member of a speaker's bureau for Abbott Laboratories, BMS, Horizon Pharma, Merck Pharma GmbH, Novartis, Roche, and has received consulting fees from Abbott Laboratories, Horizon Pharma, Merck Pharma GmbH, Novartis and Roche. R.A. has held non-remunerative positions of influence for Abbott Laboratories, BMS, Novartis Pharmaceuticals Corporation and Roche. All other authors have declared no conflicts of interest.

\section{Risk of bias}

\begin{tabular}{lll}
\hline Bias & Authors' judgement & Support for judgement \\
\hline $\begin{array}{l}\text { Random sequence genera- } \\
\text { tion (selection bias) }\end{array}$ & Low risk & $\begin{array}{l}\text { The randomisation code was generated by an independent group following } \\
\text { instruction of the randomisation procedures, prepared by the project statisti- } \\
\text { cian (EMEA report for the Phase III trial) }\end{array}$ \\
\hline $\begin{array}{l}\text { Allocation concealment } \\
\text { (selection bias) }\end{array}$ & Low risk & Via IVRS \\
\hline $\begin{array}{l}\text { Blinding (performance } \\
\text { bias and detection bias) } \\
\text { ACR50 }\end{array}$ & Low risk & $\begin{array}{l}\text { UCB: "All the study staff with the exception of the unblinded dispenser, was } \\
\text { blind to the treatment". "Each study center was required to have a written } \\
\text { blinding plan in place signed by the principal investigator, which detailed the }\end{array}$
\end{tabular}


Choy 2012 (Continued)

study center's steps for ensuring that the double blind nature of the study was maintained"

Blinding (performance $\quad$ Low risk $\quad$ See above
bias and detection bias)
All outcomes

\begin{tabular}{ll}
\hline $\begin{array}{l}\text { Incomplete outcome data } \\
\text { (attrition bias) }\end{array}$ & Low risk \\
ACR50 & $\begin{array}{l}77.8 \% \text { of certolizumab pegol group and } 53.7 \% \text { of placebo completed } 6 \text { months } \\
\text { of treatment. We imputed missing data for analysis }\end{array}$
\end{tabular}

\begin{tabular}{|c|c|c|}
\hline $\begin{array}{l}\text { Incomplete outcome data } \\
\text { (attrition bias) } \\
\text { All outcomes }\end{array}$ & Low risk & $\begin{array}{l}\text { Analysis per protocol for HAQ and safety } \\
\text { "Of the } 247 \text { patients randomised, } 124 \text { patients in the certolizumab pegol plus } \\
\text { MTX group (98\%) and } 119 \text { in the placebo plus MTX group }(98 \%) \text { received at } \\
\text { least one injection ( } 243 \text { total)" }\end{array}$ \\
\hline $\begin{array}{l}\text { Selective reporting (re- } \\
\text { porting bias) }\end{array}$ & Low risk & All the prespecified outcomes were reported \\
\hline Other bias & Low risk & The study appears to be free of other sources of bias \\
\hline $\begin{array}{l}\text { Blinding of participants } \\
\text { and personnel (perfor- } \\
\text { mance bias) } \\
\text { All outcomes }\end{array}$ & Low risk & $\begin{array}{l}\text { See above } \\
\text { "To preserve the blind to clinical research staff, the study site pharmacist la- } \\
\text { belled clinical supplies (study medication syringes), and a sorbitol placebo } \\
\text { was used to match the viscosity of certolizumab pegol" }\end{array}$ \\
\hline $\begin{array}{l}\text { Blinding of outcome as- } \\
\text { sessment (detection bias) } \\
\text { All outcomes }\end{array}$ & Low risk & See above \\
\hline
\end{tabular}

Emery 2015

\begin{tabular}{|c|c|}
\hline Methods & Randomised clinical trial, double-blind \\
\hline Participants & $\begin{array}{l}880 \text { participants were randomised. } 3 \text { were randomised in error, were not dosed, and were withdrawn } \\
\text { shortly afterwards as screen failures. } 2 \text { were included in the randomised Set } 1 \text { (RS1) only, and } 1 \text { of the } 3 \\
\text { was conservatively excluded from any output. Therefore, } 879 \text { subjects are in RS1. }\end{array}$ \\
\hline \multirow[t]{2}{*}{ Interventions } & 1. Placebo + MTX ( $n=219)$ \\
\hline & $\begin{array}{l}\text { 2. MTX + certolizumab pegol } 400 \mathrm{mg} \text { at } 0,2,4 \text { weeks, followed by a maintenance dose of certolizumab } \\
\text { pegol } 200 \mathrm{mg} \text { until week } 50(\mathrm{n}=660)\end{array}$ \\
\hline \multirow[t]{2}{*}{ Outcomes } & Primary: Percentage of participants in sustained remission at week 52 \\
\hline & $\begin{array}{l}\text { Secondary: Radiographic changes (mTTs, JNS, JE), ACR20, ACR50 and ACR70 at } 52 \text { weeks; Percentage } \\
\text { of participants with clinical remission (ACR/EULAR) at week } 52 \text { DAS } 28<2.6 \text { at week } 52 \text { Change in CDAI } \\
\text { SDAl at week } 52 \text { HAQ-DI week } 52 \text { Work product survey at week } 52 \text {. Serious adverse events; other ad- } \\
\text { verse events }\end{array}$ \\
\hline
\end{tabular}

Notes

\section{C-EARLY trial}

Countries/Cities: Europe, Australia, North America and Latin America at 181 sites

Dates conducted: from January 2012 to September 2015

Eligibility criteria: Eligible patients were DMARD-naïve, diagnosed with RA $\leq 1$ year prior to randomisation, fulfilled the 2010 American College of Rheumatology (ACR)/European League Against Rheumatism 
(EULAR) classification criteria and had poor prognostic factors for severe disease progression (positive for rheumatoid factor (RF) or anticitrullinated peptide antibody (ACPA) at screening).

Adverse events as a specified outcome: adverse events and serious adverse events were reported

Funding sources: UCB Pharma SA

Conflict of interest: Principal Investigators are NOTemployed by the organization sponsoring the study.The only disclosure restriction on the $\mathrm{PI}$ is that the sponsor can review results communications prior to public release and can embargo communications regarding trial results for a period that is more than $\mathbf{6 0}$ days but less than or equal to $\mathbf{1 8 0}$ days. The sponsor cannot require changes to the communication and cannot extend the embargo.

PE received consultancy and speaker's fee from Pfizer, MSD, AbbVie, UCB Pharma, Roche, Bristol-Myers Squibb, Schering-Plough, Novartis and Samsung. COBIII received consultancy fees from UCB Pharma. GRB received consultancy fees from AbbVie, MSD, Pfizer, Roche and UCB Pharma. DEF received research grants from Abbott, Actelion, Amgen, Bristol-Myers Squibb, Gilead, GlaxoSmithKline, NIH, Novartis, Pfizer, Roche/Genentech and UCB Pharma; consultancy fees from Abbott, Actelion, Amgen, Bristol-Myers Squibb, Biogen IDEC, Janssen, Gilead, GlaxoSmithKline, NIH, Novartis, Pfizer, Roche/Genentech and UCB Pharma and other fees from Abbott, Actelion, Amgen, Bristol-Myers Squibb, Biogen, IDEC, Janssen, Gilead, NIH, Roche/Genentech, Abbott, Actelion and UCB Pharma.

XM received research grants from Pfizer, GlaxoSmithKline and Roche and consultancy fees from Bristol-Myers Squibb, GlaxoSmithKline, Pfizer, Roche, UCB Pharma and Sanofi-Aventis. DvdH received consultancy fees from AbbVie, Amgen, AstraZeneca, Augurex, Bristol-Myers Squibb, Boehringer Ingelheim, Celgene, Centocor, Chugai, Covagen, Daiichi, Eli-Lilly, Galapagos, GlaxoSmithKline, Janssen, Merck, Novo-Nordisk, Otsuka, Pfizer, Roche, Sanofi-Aventis, UCB Pharma and Vertex; research grants from AbbVie, Amgen, AstraZeneca, Augurex, Bristol-Myers Squibb, Boehringer Ingelheim, Celgene, Centocor, Chugai, Covagen, Daiichi, Eli-Lilly, Galapagos, GlaxoSmithKline, Janssen, Merck, Novo-Nordisk, Otsuka, Pfizer, Roche, Sanofi-Aventis, UCB Pharma and Vertex and is Director of Imaging at Rheumatology BV.

RvV received research support from AbbVie, Bristol-Myers Squibb, GlaxoSmithKline, Pfizer, Roche and UCB Pharma and consultancy fees from AbbVie, Biotest, Bristol-Myers Squibb, GlaxoSmithKline, Janssen, Eli-Lilly, Merck, Pfizer, Roche, UCB Pharma and Vertex.

CA is an employee of UCB Pharma.

IM is an employee of UCB Pharma. OP is an employee of UCB Pharma.

DT is an employee of UCB Pharma.

$\mathrm{BV}$ is an employee of UCB Pharma.

MEW received research grants from Amgen, Bristol-Myers Squibb, Crescendo Bioscience and UCB Pharma and consultancy fees from AbbVie, Amgen, AstraZeneca, Bristol-Myers Squibb, Crescendo Bioscience, Eli-Lilly, Medlmmune, Merck, Novartis, Pfizer, Roche and UCB Pharma.

\section{Risk of bias}

\begin{tabular}{lll}
\hline Bias & Authors' judgement & Support for judgement \\
\hline $\begin{array}{l}\text { Random sequence genera- } \\
\text { tion (selection bias) }\end{array}$ & Low risk & UCB Pharma explained to us that was a external central of randomisation \\
\hline $\begin{array}{l}\text { Allocation concealment } \\
\text { (selection bias) }\end{array}$ & Low risk & $\begin{array}{l}\text { UCB private files: "An IXRS (interactive voice/web response system) is used for } \\
\text { subject registration as well as randomisation and treatment allocation". The } \\
\text { system stratified by disease duration of more or less than } 4 \text { months }\end{array}$ \\
\hline $\begin{array}{l}\text { Blinding (performance } \\
\text { bias and detection bias) } \\
\text { ACR50 }\end{array}$ & Low risk & $\begin{array}{l}\text { UCB private files; "Sponsor, investigator site and vendor staff involved will be } \\
\text { blinded to the testaments assignment with the following exceptions: sponsor } \\
\text { clinical study supplies coordinator and qualifier person unblinded site person- } \\
\text { nel involved in ESR determination" }\end{array}$ \\
\hline
\end{tabular}


Emery 2015 (Continued)

Blinding (performance Low risk UCB private files: "Sponsor, investigator site and vendor staff involved will be bias and detection bias) blinded to the testaments assignment with the following exceptions: sponsor All outcomes clinical study supplies coordinator and qualifier person unblinded site personnel involved in ESR determination"

Incomplete outcome data Low risk
(attrition bias)

Participants not achieving sufficient improvement (defined as DAS (ESR) $<3.2$ (attrition bias) and/or $>1.2$ point improvement in DAS 28 (ESR)) from baseline at weeks 20 ACR50 and 24 were withdrawn to allow them to switch to a complementary medication. There were $34 \%$ of withdrawals in placebo group and $24 \%$ in certolizumab pegol group at week 52

Incomplete outcome data Low risk See above

(attrition bias)

All outcomes

\begin{tabular}{|c|c|c|}
\hline $\begin{array}{l}\text { Selective reporting (re- } \\
\text { porting bias) }\end{array}$ & Low risk & All the outcomes in the protocol in www.clinicaltrials.gov were available \\
\hline Other bias & Low risk & The study appears to be free of other sources of bias \\
\hline $\begin{array}{l}\text { Blinding of participants } \\
\text { and personnel (perfor- } \\
\text { mance bias) } \\
\text { All outcomes }\end{array}$ & Low risk & $\begin{array}{l}\text { UCB private files: "Sponsor, investigator site and vendor staff involved will be } \\
\text { blinded to the testaments assignment with the following exceptions: sponsor } \\
\text { clinical study supplies coordinator and qualifier person unblinded site person- } \\
\text { nel involved in ESR determination" }\end{array}$ \\
\hline $\begin{array}{l}\text { Blinding of outcome as- } \\
\text { sessment (detection bias) } \\
\text { All outcomes }\end{array}$ & Low risk & $\begin{array}{l}\text { UCB private files: "Sponsor, investigator site and vendor staff involved will be } \\
\text { blinded to the testaments assignment with the following exceptions: sponsor } \\
\text { clinical study supplies coordinator and qualifier person unblinded site person- } \\
\text { nel involved in ESR determination" }\end{array}$ \\
\hline
\end{tabular}

\section{Fleischmann 2009}

\begin{tabular}{ll}
\hline Methods & Randomised double-blind trial \\
\hline Participants & 220 people aged $18-75$ years \\
\hline Interventions & $\begin{array}{l}\text { 1. Certolizumab pegol } 400 \text { mg sc every } 4 \text { weeks }(n=111) \\
\text { 2. Placebo }(n=109) \text { for } 24 \text { weeks }\end{array}$ \\
\hline
\end{tabular}

Outcomes ACR20, 50, 70, HAQ-DI, pain (VAS and mBPI), DAS-28, fatigue, and SF-36

Follow-up 24 weeks

Notes CPD870-011

\section{FAST4WARD}

Countries/Cities: conducted at 36 sites in Austria, Czech Republic and the USA.

Dates conducted: June 2003 to July 2004

Eligibility criteria: with RA defined by the ACR classification criteria who had previously failed at least 1 DMARD were included. Those previously treated with a TNF inhibitor were excluded. Participants had to have a TJC of $\geq 9$ (out of 68 ), SJC of $\geq 9$ (out of 66 ) and 1 of the following: morning stiffness of $\geq 45$ minutes; ESR $\geq 28 \mathrm{~mm} / \mathrm{H}$; or CRP $>10 \mathrm{mg} / \mathrm{L}$. People with a previous history of a serious or life-threatening infection were excluded. People with a history of TB, or evidence of TB on a chest radiograph, or those with a positive reaction to PPD reaction were also excluded. Patients on concurrent corticos- 
teroids were allowed entry provided the dose was the equivalent of $10 \mathrm{mg}$ or less of prednisolone. Parenteral corticosteroids were not permitted

Adverse events as a specified outcome:safety were assessed at baseline and weeks 1, 2, 4, 8, 12, 16, 20 and 24, with additional safety assessments at 4 and 12 weeks post final dose. Additional plasma samples were taken at weeks 21 and 22 .

Funding sources: UCB

Conflict of interest: JV has received a fee from UCB for speaking at a National Congress; RFVV has received consulting fees from $U C B$; DB has received reimbursement from UCB for attending a symposium and funds for research; JB has received reimbursement from UCB for attending a symposium and funds for research; GC is a full time employee of and holds stocks in UCB; Al is a full time employee at UCB and has shares in the company; NG is a full time employee of UCB and has shares and stock options in the company; VS has worked as an independent biopharmaceutical consultant in clinical development and regulatory affairs since September 1991 and is currently a consultant to various companies, but has not and does not now hold stock in any company. RF has received consulting fees and funds for clinical research from UCB.

JV has received a fee from UCB for speaking at a National Congress;

RFVV has received consulting fees from UCB; DB has received reimbursement from $U C B$ for attending a symposium and funds for research; JB has received reimbursement from UCB for attending a symposium and funds for research;

GC is a full time employee of and holds stocks in UCB; Al is a full time employee at UCB and has shares in the company;

NG is a full time employee of UCB and has shares and stock options in the company;

VS has worked as an independent biopharmaceutical consultant in clinical development and regulatory affairs since September 1991 and is currently a consultant to various companies, but has not and does not now hold stock in any company.

RF has received consulting fees and funds for clinical research from UCB.

\section{Risk of bias}

\begin{tabular}{lll}
\hline Bias & Authors' judgement & Support for judgement \\
\hline $\begin{array}{l}\text { Random sequence genera- } \\
\text { tion (selection bias) }\end{array}$ & Low risk & Code list prepared by independent group \\
\hline $\begin{array}{l}\text { Allocation concealment } \\
\text { (selection bias) }\end{array}$ & Low risk & Via IVRS \\
\hline $\begin{array}{l}\text { Blinding (performance } \\
\text { bias and detection bias) } \\
\text { ACR50 }\end{array}$ & Low risk & $\begin{array}{l}\text { UCB stated: "All the study staff with the exception of the unblinded dispenser, } \\
\text { was blind to the treatment". "Each study center was required to have a written } \\
\text { blinding plan in place signed by the principal investigator, which detailed the } \\
\text { study center's steps for ensuring that the double blind nature of the study was } \\
\text { maintained" }\end{array}$ \\
\hline
\end{tabular}

\begin{tabular}{lll}
$\begin{array}{l}\text { Blinding (performance } \\
\text { bias and detection bias) } \\
\text { All outcomes }\end{array}$ & Low risk & See above \\
\hline $\begin{array}{l}\text { Incomplete outcome data } \\
\text { (attrition bias) }\end{array}$ & Low risk & $\begin{array}{l}68.5 \% \text { of certolizumab pegol group and } 25.7 \% \text { of placebo completed } 6 \text { months } \\
\text { of treatment. We imputed missing data for analysis }\end{array}$ \\
\hline ACR50 & &
\end{tabular}

Incomplete outcome data Low risk

Full account of all withdrawals and reasons for withdrawals (attrition bias) 
Fleischmann 2009 (Continued) All outcomes
Quote: "All efficacy analyses were performed on the modified intent to treat (mITT) population (all randomised patients who had taken $>1$ dose of study medication). The actual number of subjects in the summaries varies slightly from the MITT numbers due to non-imputable missing data for each parameter. For the primary analysis, patients were considered "responders" if they achieved an ACR20 response vs baseline at week 24. Patients who withdrew for any reason were considered non responders."

The safety analysis was based on the 'last observation carried forward' approach

\begin{tabular}{lll}
\hline $\begin{array}{l}\text { Selective reporting (re- } \\
\text { porting bias) }\end{array}$ & Low risk & All the outcomes were available \\
\hline Other bias & Low risk & The study appears to be free of other sources of bias \\
\hline $\begin{array}{l}\text { Blinding of participants } \\
\begin{array}{l}\text { and personnel (perfor- } \\
\text { mance bias) }\end{array}\end{array}$ & Low risk \\
$\begin{array}{ll}\text { All outcomes } \\
\text { Blinding of outcome as- } \\
\text { sessment (detection bias) } \\
\text { All outcomes }\end{array}$ & Low risk & See above \\
\hline
\end{tabular}

Keystone 2008

\begin{tabular}{ll}
\hline Methods & Randomised double-blind trial \\
\hline Participants & 982 participants aged $>18$ years \\
& Participants were randomised 2:2:1 \\
\hline
\end{tabular}

Interventions

1. Certolizumab pegol sc at an initial dosage of $400 \mathrm{mg}$ given at weeks 0,2 , and 4 , with a subsequent dosage of $200 \mathrm{mg}(\mathrm{n}=393)$ or $400 \mathrm{mg}$ given every 2 weeks, plus MTX ( $n=390)$

2. Placebo plus MTX, same regimen ( $n=199)$

\section{Outcomes}

Co-primary endpoints: ACR20 at week 24 and the mean change from baseline in the mTSS at week 52

Major secondary end points: Change from baseline in mTSS at week 24

Change from baseline in the HAQ-DI at weeks 24 and 52

ACR20 responder rate at week 52

ACR50 and ACR70 responder rates at weeks 24 and 52

Follow-up 24 - 52 weeks

\section{RAPID1 Trial}

Countries/Cities:79 sites from EEUU, Argentina, Australia, Belgium, Bulgaria, Canada, Chile, Croatia, Czech Republic, Israel, Latvia, Russian Federation,Ukraine

Dates conducted: from February 2005 to October 2006

Eligibility criteria: patients were aged 18 years or older with active RA (according to the 1987 ACR RA classification criteria with an inadequate response to MTX therapy ( $\geq 10 \mathrm{mg}$ weekly for $\geq 6$ months with stable doses for $\geq 2$ months prior to baseline). Patients were ineligible if they had previously failed to respond to treatment with a TNF inhibitor. People with a history of TB or a chest radiograph showing active or latent TR or those with a nositive reaction o PPD were als ox exded 
Funding sources: UCB Pharma

Conflict of interest: Dr. Keystone has received consulting fees, speaking fees, and/or honoraria from Abbott, Amgen, Wyeth, Centocor, UCB, Roche, Genentech, Schering-Plough, and Bristol-Myers Squibb (less than USD 10,000 each).

Dr. van der Heijde has received consulting fees, speaking fees, and/or honoraria from Abbott, Amgen, Centocor, UCB, Roche, Schering-Plough, and Bristol-Myers Squibb (less than USD 10,000 each). Dr. Landewe' has received consulting fees, speaking fees, and/or honoraria from Abbott, Amgen, Bristol-Myers Squibb, Centocor, Schering-Plough, UCB, and Wyeth (less than USD 10,000 each).

Dr. van Vollenhoven has received consulting fees, speaking fees, and/or honoraria from UCB (more than USD 10,000).

Dr. Combe has received consulting fees, speaking fees, and/or honoraria from Abbott, Bristol-Myers Squibb, Merck, Sharp, \& Dohme, Roche, Schering, UCB, and Wyeth (less than USD 10,000 each).

Dr. Emery has received consulting fees from UCB (less than USD 10,000). Dr. Strand receives consulting fees (her primary source of income) from Abbott Immunology, Allergan, Almirall, AlPharma, Amgen, AstraZeneca, Bayhill, Bexel, Biogen Idec, Can-Fite, Centocor, Chelsea, Cypress Bioscience, Dianippon Sumitomo, Euro-Diagnostica, FibroGen, Forest, Genelabs, Genentech, Human Genome Sciences, Idera, Incyte, Jazz, Lexicon Genetics Lux Biosciences, Merck Serono, Novartis, Novo Nordisk, Noxxon Pharma, Nuon, Ono Pharmaceutical, Pfizer, Procter \& Gamble, Rigel, RiGEN, Roche, Sanofi-Aventis, Savient, Schering-Plough, Scios, SKK, UCB, VLST, Wyeth, XDx, and Zelos Therapeutics (less than USD 10,000 each) and receives fees as a member of the advisory board for Abbott, Amgen, Biogen Idec, Bioseek, Bristol-Myers Squibb, Can-Fite, Centocor, Chelsea, Cypress, Euro-Diagnostica, Forest, Idera, Incyte, Jazz, Novartis, Pfizer, Rigel, RiGEN, Roche, Savient, Schering-Plough, UCB, XDx, and Wyeth (less than USD 10,000 each).

Dr. Mease has received consulting fees, speaking fees, and/or honoraria from UCB (less than USD 10,000).

Mr. Desai owns stock or stock options in UCB

\section{Risk of bias}

\begin{tabular}{|c|c|c|}
\hline Bias & Authors' judgement & Support for judgement \\
\hline $\begin{array}{l}\text { Random sequence genera- } \\
\text { tion (selection bias) }\end{array}$ & Low risk & Code list prepared by independent group \\
\hline $\begin{array}{l}\text { Allocation concealment } \\
\text { (selection bias) }\end{array}$ & Low risk & IVRS used to allocate participant to treatment group (2:2:1 ratio) \\
\hline $\begin{array}{l}\text { Blinding (performance } \\
\text { bias and detection bias) } \\
\text { ACR50 }\end{array}$ & Low risk & $\begin{array}{l}\text { UCB stated: "All the study staff with the exception of the unblinded dispenser, } \\
\text { was blind to the treatment. Each study center was required to have a written } \\
\text { blinding plan in place signed by the principal investigator, which detailed the } \\
\text { study center's steps for ensuring that the double blind nature of the study was } \\
\text { maintained" }\end{array}$ \\
\hline $\begin{array}{l}\text { Blinding (performance } \\
\text { bias and detection bias) } \\
\text { All outcomes }\end{array}$ & Low risk & See above \\
\hline $\begin{array}{l}\text { Incomplete outcome data } \\
\text { (attrition bias) } \\
\text { ACR50 }\end{array}$ & Low risk & $\begin{array}{l}65 \% \text { of certolizumab } 200 \mathrm{mg} \text { and } 70.3 \% \text { certolizumab } 400 \mathrm{mg} \text { of group and } \\
22 \% \text { of placebo completed } 12 \text { months of treatment. We imputed missing data } \\
\text { for analysis }\end{array}$ \\
\hline
\end{tabular}


Keystone 2008 (Continued)

All outcomes (attrition bias)

Full account of all withdrawals and reasons for withdrawals

$\mathrm{HAQ}$, quote: "Analyses were performed using the last observation carried forward (LOCF) method for imputation of missing scores in the total ITT population and the actual scores (observed) in those who withdrew at week 16"

Safety: ITT analysis

$\begin{array}{ll}\begin{array}{l}\text { Selective reporting (re- } \\ \text { porting bias) }\end{array} & \text { Low risk } \\ \text { prespecified way }\end{array}$

\begin{tabular}{lll}
\hline Other bias & Low risk & The study appears to be free of other sources of bias \\
\hline $\begin{array}{l}\text { Blinding of participants } \\
\text { and personnel (perfor- }\end{array}$ & Low risk & See above \\
mance bias) & \\
All outcomes & \\
\hline
\end{tabular}
sessment (detection bias)

Methods Randomised, double-blind (participant, investigator, outcomes assessor), placebo-controlled, parallel-assignment, safety/efficacy study

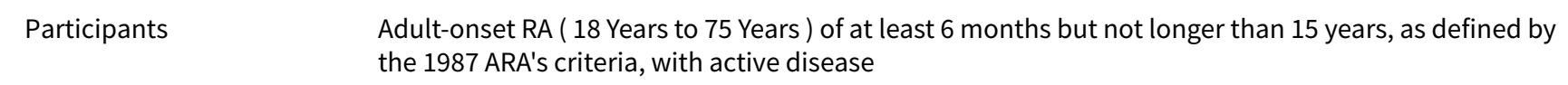

Interventions 1. CDP870 $200 \mathrm{mg}, 400 \mathrm{mg}$ CDP870 given at weeks 0, 2, 4, and thereafter $200 \mathrm{mg}$ CDP870 given every 2 weeks until week 22 (sc) plus MTX ( $\mathrm{n}=85$ )

2. Placebo plus MTX, same regimen ( $n=42$ )

Outcomes ACR20, ACR50, ACR70 responder rate; changes in HAQ-Di

Follow-up 24 weeks

Notes See clinicaltrials.gov/ct2/show/study/NCT00993317

Countries/Cities: 15 hospital in Korea

Dates conducted: from October 2009 to August 2011

\section{Eligibility criteria:}

- Adult-onset RA of at least 6 months but not longer than 15 years in duration as defined by the 1987 American College of Rheumatology classification criteria

- Active RA disease as defined by at least 9 tender joints and 9 swollen joints, ESR of $30 \mathrm{~mm} /$ hour or CRP of $1.5 \mathrm{mg} / \mathrm{dL}$

- MTX (with or without folic acid) for at least 24 weeks prior to the Baseline visit, The dose of MTX and route of administration must have been stable for at least 8 weeks prior to the baseline visit. The minimum stable dose of MTX allowed is $10 \mathrm{mg}$ weekly.

Adverse events as a specified outcome: adverse events and serious adverse events were reported

Funding sources: Korea Otsuka Pharmaceutical Co Ltd 
NCT00993317 (Continued)

Conflict of interest: "Principal Investigators are NOT employed by the organization sponsoring the study". "There is NOT an agreement between Principal Investigators and the Sponsor (or its agents) that restricts the PI's rights to discuss or publish trial results after the trial is completed".

\section{Risk of bias}

\begin{tabular}{lll}
\hline Bias & Authors' judgement & Support for judgement \\
\hline $\begin{array}{l}\text { Random sequence genera- } \\
\text { tion (selection bias) }\end{array}$ & Low risk & External central randomisation \\
\hline $\begin{array}{l}\text { Allocation concealment } \\
\text { (selection bias) }\end{array}$ & Low risk & $\begin{array}{l}\text { The allocation sequence was generate using uniform random numbers from } \\
\text { SAS RANUNI function }\end{array}$ \\
\hline $\begin{array}{l}\text { Blinding (performance } \\
\text { bias and detection bias) }\end{array}$ & Low risk & $\begin{array}{l}\text { "All study staff with the exception of the unblinded dispenser were blind to the } \\
\text { treatment, ... These unblinded personnel were not allowed to engage in any } \\
\text { other study activities" }\end{array}$ \\
\hline $\begin{array}{l}\text { Blinding (performance } \\
\text { bias and detection bias) } \\
\begin{array}{l}\text { All outcomes } \\
\hline\end{array}\end{array}$ & Low risk & See above \\
\hline
\end{tabular}

$\begin{array}{ll}\text { Incomplete outcome data } & \text { Low risk } \\ \text { (attrition bias) } & \begin{array}{l}70 \% \text { of certolizumab pegol group and } 50 \% \text { of placebo completed } 6 \text { months of } \\ \text { treatment. We imputed missing data for analysis }\end{array}\end{array}$
ACR50

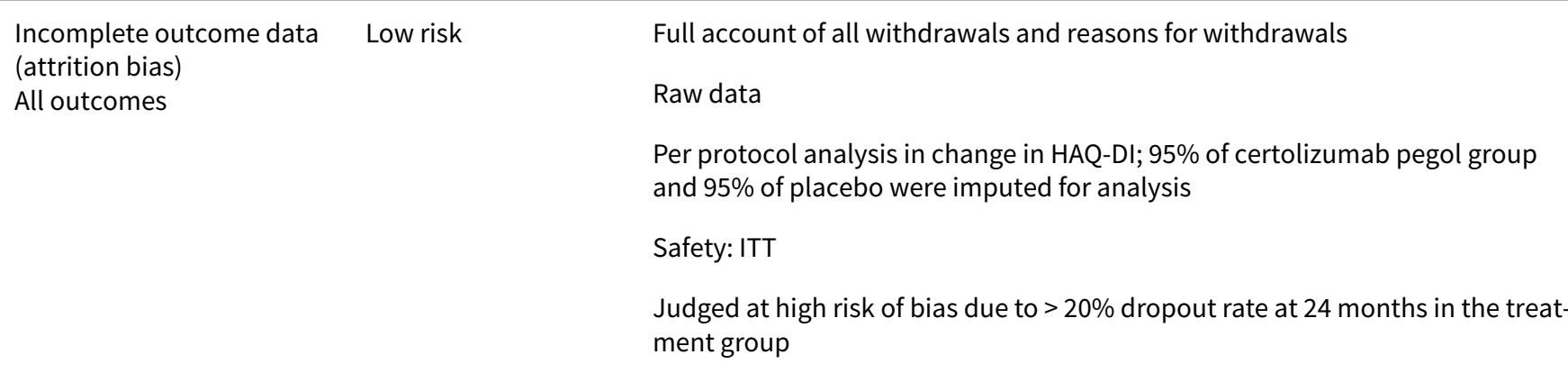

\begin{tabular}{|c|c|c|}
\hline $\begin{array}{l}\text { Selective reporting (re- } \\
\text { porting bias) }\end{array}$ & Low risk & $\begin{array}{l}\text { The study protocol is available and all of the study's prespecified (primary and } \\
\text { secondary) outcomes that are of interest in the review have been reported in } \\
\text { the prespecified way }\end{array}$ \\
\hline Other bias & Low risk & The study appears to be free of other sources of bias \\
\hline $\begin{array}{l}\text { Blinding of participants } \\
\text { and personnel (perfor- } \\
\text { mance bias) } \\
\text { All outcomes }\end{array}$ & Low risk & See above \\
\hline $\begin{array}{l}\text { Blinding of outcome as- } \\
\text { sessment (detection bias) } \\
\text { All outcomes }\end{array}$ & Low risk & See above \\
\hline
\end{tabular}

Smolen 2009

Methods Randomised double-blind trial


Smolen 2009 (Continued)
Participants
619 participants aged $>18$ years
Participants were randomised 2:2:1

Interventions

1. Certolizumab pegol sc, $400 \mathrm{mg}$ at weeks 0,2 and 4, followed by $200(n=246)$ or $400 \mathrm{mg}$ every 2 weeks, plus MTX $(n=246)$

2. Placebo (saline) plus MTX $(n=127)$

Outcomes

Primary endpoints: ACR20 response at week 24, and physician's global assessment of disease activity, participant's assessment of pain, HAQ-DI and serum CRP or ESR

Secondary endpoints: ACR50, ACR70, mean change from baseline in van der Heijde mTSS, SF-36 Health Survey, and individual ACR core set variables. Disease activity was assessed using the DAS-28 (ESR)

Follow-up 24 weeks

Countries/Cities: 121 sites from EEUU, Argentina, Australia, Belgium, Bulgaria, Canada, Chile, Croatia, Czech Republic, Estonia, Finland, France, Hungary, Israel, Latvia, Lithuania, Mexico, New Zealand, Russian Federation, Serbia, Slovakia, Ukraine

Dates conducted: from June 2005 to February 2012

Eligibility criteria: RA of at least 6 months and defined by the ACR classification criteria who had received MTX for $\geq 6$ months at a stable dose of $\geq 10 \mathrm{mg} /$ week for at least 2 months before baseline were included. At inclusion, participants had to have active disease as defined by: TJC and SJC of $\geq 9$, ESR $\geq 30 \mathrm{~mm} / \mathrm{H}$, and a CRP of $\geq 15 \mathrm{mg} / \mathrm{L}$. People with a disease duration of $>15$ years were excluded. People previously treated with a TNF inhibitor were also excluded if they had previously failed to respond to treatment. Participants with history of, or positive chest x-ray findings for TB, or a PPD skin test (defined as positive indurations by local medical practice) were excluded. As per protocol, if a positive PPD skin test was assumed by the local investigators to be related to previous bacille Calmette-Guerin (BCG) vaccination and was not associated with clinical or radiographic suspicion of TB, the person could be enrolled at the discretion of the investigator. In total, 101 participants (16\%) were enrolled with a PPD test $>5 \mathrm{~mm}$ at baseline. Participants who did not show an ACR20 response at both weeks 12 and 14 were to be withdrawn from the study, designated ACR20 non-responders in the primary analysis and allowed to enter an open-label extension study at week 16 with certolizumab pegol $400 \mathrm{mg}$ every 2 weeks.

Adverse events as a specified outcome: adverse events and serious adverse events were reported

Funding sources: UCB Pharma

Conflict of interest: J Smolen, R B Landewé, P Mease, RF van Vollenhoven, A Kavanaugh, M Schiff, GR Burmester, $V$ Strand and $D$ van der Heijde serve as consultants to UCB, Inc.

RB Landewé, A Kavanaugh, M Schiff and D van der Heijde receive research funding from UCB, Inc and GR Burmester

J Vencovsky have received honorarium from UCB, Inc for speaking.

D Mason and K Luijtens are employees of UCB, Inc.

J Brzezicki has nothing to disclose

\section{Risk of bias}

\begin{tabular}{lll}
\hline Bias & Authors' judgement & Support for judgement \\
\hline $\begin{array}{l}\text { Random sequence genera- } \\
\text { tion (selection bias) }\end{array}$ & Low risk & Code list prepared by independent group \\
\hline \hline
\end{tabular}


Smolen 2009 (Continued)

$\begin{aligned} & \text { Allocation concealment } \\ & \text { (selection bias) }\end{aligned} \quad$ IVRS used to allocate participant to treatment group (2:2:1 ratio)

Blinding (performance Low risk

bias and detection bias)

ACR50
UCB stated: "All the study staff with the exception of the unblinded dispenser, was blind to the treatment. Each study center was required to have a written blinding plan in place signed by the principal investigator, which detailed the study center's steps for ensuring that the double blind nature of the study was maintained"

\begin{tabular}{lll}
\hline $\begin{array}{l}\text { Blinding (performance } \\
\text { bias and detection bias) } \\
\text { All outcomes }\end{array}$ & Low risk & See above \\
\hline $\begin{array}{l}\text { Incomplete outcome data } \\
\begin{array}{l}\text { (attrition bias) } \\
\text { ACR50 }\end{array}\end{array}$ & Low risk & $\begin{array}{l}71 \% \text { of certolizumab pegol } 200 \mathrm{mg} \text { and } 74 \% \text { of certolizumab pegol } 400 \mathrm{mg} \text { re- } \\
\text { spectively and } 13 \% \text { of placebo groups completed } 6 \text { months of treatment. We } \\
\text { imputed missing data for analysis }\end{array}$ \\
\hline
\end{tabular}

\begin{tabular}{|c|c|c|}
\hline $\begin{array}{l}\text { Incomplete outcome data } \\
\text { (attrition bias) } \\
\text { All outcomes }\end{array}$ & Low risk & $\begin{array}{l}\text { Full account of all withdrawals and reasons for withdrawals } \\
\text { Safety: ITT analysis. Quote: "two patients in the placebo group received cer- } \\
\text { tolizumab pegol } 200 \mathrm{mg} \text { and were included in the certolizumab pegol } 200 \mathrm{mg} \\
\text { group for safety evaluations" }\end{array}$ \\
\hline $\begin{array}{l}\text { Selective reporting (re- } \\
\text { porting bias) }\end{array}$ & Low risk & $\begin{array}{l}\text { All the outcomes that are of interest in the review have been reported in the } \\
\text { prespecified way }\end{array}$ \\
\hline Other bias & Low risk & The study appears to be free of other sources of bias \\
\hline $\begin{array}{l}\text { Blinding of participants } \\
\text { and personnel (perfor- } \\
\text { mance bias) } \\
\text { All outcomes }\end{array}$ & Low risk & See above \\
\hline $\begin{array}{l}\text { Blinding of outcome as- } \\
\text { sessment (detection bias) } \\
\text { All outcomes }\end{array}$ & Low risk & $\begin{array}{l}\text { Radiographs were read centrally and blinded (for treatment, visit and partici- } \\
\text { pant identification) and checked independently by } 2 \text { experienced readers }\end{array}$ \\
\hline
\end{tabular}

Smolen 2015

\begin{tabular}{|c|c|}
\hline Methods & $\begin{array}{l}\text { A Phase IIIB, multicentre, double-blind, placebo-controlled, parallel-group study to evaluate the safety } \\
\text { and efficacy of certolizumab pegol, administered with DMARD }\end{array}$ \\
\hline Participants & People with low to moderate disease activity RA on DMARDs therapy for at least 6 months. \\
\hline Interventions & $\begin{array}{l}\text { 1. } 2 \text { × } 200 \mathrm{mg} \text { certolizumab pegol sc injections at week 0, week 2, ( } 96 \text { patients)and week } 4 \text {, followed by } \\
200 \mathrm{mg} \text { injections every } 2 \text { weeks until the last drug administration (Week 22) } \\
\text { 2. Placebo ( } 98 \text { patients), same regimen }\end{array}$ \\
\hline Outcomes & $\begin{array}{l}\text { Efficacy evaluations were performed every } 4 \text { weeks from weeks } 0 \text { to } 52 \text {. Adverse events (AEs) were as- } \\
\text { sessed every two weeks. Primary efficacy endpoint was the proportion of patients in stable CDAl remis- } \\
\text { sion (CDAI } 2.8 \text { ) at both weeks } 20 \text { and 24. Secondary outcomes included: DAS remission, ACR20, ACR50, } \\
\text { ACR70, SDAI, HAQ-DI, SF-36, Change From Baseline in Patient's Global Assessment of Disease Activity - } \\
\text { Visual Analog Scale (PtGADA-VAS) and Change From Baseline in Fatigue Assessment Scale at Week } 24\end{array}$ \\
\hline & Follow-up 24 weeks \\
\hline
\end{tabular}




\section{http://clinicaltrials.gov/ct2/show/NCT00674362?term=NCT00674362\&rank=1}

Countries/Cities: All patients, recruited from centres in Austria, France, Germany, Italy and Poland

Dates conducted: conducted between June 2008 and December 2010.

Eligibility criteria: Eligible patients ( $\geq 18$ years of age) had a diagnosis of RA23 ( 6 months-10 years), LDA/MDA at screening and baseline (defined by CDAI $>6$ and $\leq 16, \geq 2$ tender joints (28-joint count, TJC), $\geq 2$ swollen joints (28-joint count, SJC) and either erythrocyte sedimentation rate (Westergren-ESR) $\geq 28 \mathrm{~mm} / \mathrm{h}$ or C-reactive protein (CRP) $>10 \mathrm{mg} / \mathrm{L}$ ). Patients must have received mono or combination DMARD therapy (MTX, leflunomide, sulfasalazine and/or hydroxychloroquine) for $\geq 6$ months (dose stable $\geq 2$ months) prior to baseline, with corticosteroid dose stable $>1$ month (for exclusion criteria, see online supplementary material).

Adverse events as a specified outcome: Safety analysis was performed up to week 52 plus 12-week safety follow-up

\section{Funding sources: UCB}

Conflict of interest: This study is not published. Despite this, the following statement was on the trials registry, "Principal Investigators are NOT employed by the organization sponsoring the study"

JS has received grants from and provided expert advice to UCB Pharma. PE has received grants and consultancy fees from UCB Pharma, Pfizer, Merck, Abbott, Roche and BMS. GF has received speaking fees from UCB Pharma.

WS has acted as a consultant for UCB Pharma.

FB has received consultancy fees for UCB Pharma.

$\mathrm{HB}$ is a consultant for UCB Pharma.

OD is an employee and a shareholder for UCB Pharma.

WK and OP are employees of UCB Pharma.

BB is a former employee of UCB Pharma and also holds stock options with UCB Pharma

\section{Risk of bias}

Bias Authors' judgement Support for judgement

Random sequence genera- Low risk tion (selection bias)
Patients were randomised in a 1:1 ratio; Randomisation was performed centrally using an interactive voice-response system.

Allocation concealment Low risk Allocation by IVRS; so done remotely and therefore concealment satisfactory
(selection bias)
Allocation conceal
(selection bias)

Blinding (performance Low risk

UCB stated: "All the study staff with the exception of the unblinded dispenser, ACR50 was blind to the treatment. Each study center was required to have a written blinding plan in place signed by the principal investigator, which detailed the study center's steps for ensuring that the double blind nature of the study was maintained"

\begin{tabular}{lll}
\hline $\begin{array}{l}\text { Blinding (performance } \\
\text { bias and detection bias) }\end{array}$ & Low risk & \\
$\begin{array}{l}\text { All outcomes } \\
\text { Incomplete outcome data }\end{array}$ & Low risk & Full account of all withdrawals and reasons for withdrawals \\
$\begin{array}{l}\text { (attrition bias) } \\
\text { ACR50 }\end{array}$ & $\begin{array}{l}87.5 \% \text { of certolizumab pegol group and } 81 \% \text { of placebo completed } 6 \text { months } \\
\text { of treatment. We imputed missing data for analysis }\end{array}$
\end{tabular}


Smolen 2015 (Continued)

Incomplete outcome data Low risk $\quad 85 \%$ in SF-36, 84\% in Pain VAS, and 94\% in HAQ of certolizumab pegol group (attrition bias) completed 24 months of treatment. We imputed missing data for analysis. ITT All outcomes in safety analysis

Selective reporting (re- Low risk All the prespecified outcomes were reported
porting bias)

\begin{tabular}{lll}
\hline Other bias & Low risk & The study appears to be free of other sources of bias \\
\hline $\begin{array}{l}\text { Blinding of participants } \\
\text { and personnel (perfor- }\end{array}$ & Low risk & "Subject, caregiver, investigator and outcome assessor" \\
mance bias) & \\
All outcomes & \\
\hline
\end{tabular}

Blinding of outcome as- Low risk $\quad$ "Subject, caregiver, investigator and outcome assessor"
sessment (detection bias)

All outcomes

Weinblatt 2012

\begin{tabular}{ll}
\hline Methods & Randomised, double-blind (subject, outcomes assessor), parallel-assignment, safety/efficacy study \\
\hline Participants & Adults with established moderate-to-severe rheumatoid arthritis \\
\hline Interventions & $\begin{array}{l}1.400 \mathrm{mg} \text { certolizumab pegol given as } 2 \times 200 \mathrm{mg} \text { sc injections at weeks } 0,2 \text {, and } 4 \text {, followed by } 200 \mathrm{mg} \\
\text { certolizumab pegol given as } 1 \mathrm{sc} \text { injection at weeks } 6,8 \text {, and } 10 . \text { At Week } 12 \text { participants enter the open- } \\
\text { label phase and receive } 200 \mathrm{mg} \text { of certolizumab pegol every other week for a minimum } 16 \text { additional } \\
\text { weeks until certolizumab pegol is commercially available ( } \mathrm{n}=851)\end{array}$ \\
$\begin{array}{l}\text { 2. Placebo }(0.9 \% \text { saline) given as } 2 \text { sc injections at weeks } 0,2 \text {, and } 4 \text {, followed by placebo given as } 1 \mathrm{sc} \\
\text { injection at weeks } 6,8, \text { and } 10 . \text { At week } 12 \text { participants enter the open-label phase and receive } 200 \mathrm{mg} \\
\text { of certolizumab pegol every other week for a minimum } 16 \text { additional weeks until certolizumab pegol is } \\
\text { commercially available }(n=212)\end{array}$
\end{tabular}

Outcomes $\quad$ Primary outcome: ACR20 response rate at week 12.

Other outcomes: responder rate, disease activity, fatigue, physical functioning. Time frame: week 12 and every 8 weeks thereafter, until study completion

Follow-up 12 weeks

Notes

clinicaltrials.gov/ct2/show/results/NCT00717236?term=NCT00717236\&rank=1

\section{REALISTIC Trial}

Countries/Cities: 181 sites in EEUU, Canada, Frannce, Italy, Netherlands and Spain

Dates conducted: from July 2008 to March 2011

Eligibility criteria: Eligible patients were $\geq 18$ years of age, had adult-onset RA as defined by the 1987 ACR criteria for at least 3 months and showed an unsatisfactory response or intolerance to at least one DMARD (MTX, LEF, SSZ, chloroquine or HCQ, AZA and/or gold). Subjects had active disease as defined by at least five tender and at least four swollen joints (28-joint count) and either $\geq 10 \mathrm{mg} / \mathrm{l} \mathrm{CRP} \mathrm{or} \geq 28$ $\mathrm{mm} / \mathrm{h}$ ESR (Westergren method) at screening.

Adverse events as a specified outcome: adverse events and serious adverse events were reported

Funding sources: UCB Pharma 
Conflict of interest: "Principal Investigators are NOT employed by the organization sponsoring the study.". " There IS an agreement between Principal Investigators and the Sponsor (or its agents) that restricts the PI's rights to discuss or publish trial results after the trial is completed." " Restriction Description: $U C B$ has $>60$ but $<=180$ days to review results communications prior to public release and may delete information that is confidential and compromises ongoing studies or is considered proprietary. This restriction is not intended to compromise the objective scientific integrity of the manuscript, it being understood that the results shall be published regardless of outcome"

M.D. has received research grants and consulting fees from Abbott Laboratories, Bristol-Myers Squibb, Pfizer, Roche and UCB Pharma.

T.W.J.H. has received consulting fees from UCB Pharma.

R.F.v.V. has received research grants and consulting fees from UCB Pharma. C.O.B. has served as an investigator and received consulting fees from UCB Pharma. J.P. has received research grants and consulting fees from UCB Pharma, Abbott Laboratories, Actelion, Amgen, AstraZeneca, Bristol-Myers Squibb, Genentech, GlaxoSmithKline, Johnson \& Johnson, Medlmmune, Merck, Novartis, Pfizer, Roche, Sanofi, Sorono, Teva and United Therapeutics.

N.G. is a former employee of UCB Pharma and is currently an employee of Quintiles. N.G. owns UCB Pharma stock.

R.F. has received research grants and consulting fees from UCB Pharma.

M.E.W. has received research grants from Abbott, Bristol-Myers Squibb, Roche, Biogen/Idec, Medimmune, Cresendo Bioscience and UCB Pharma, and consulting fees from UCB Pharma, Abbott Laboratories, Amgen, Bristol-Myers Squibb, Roche, Biogen/Idec, Medimmune, Cresendo Bioscience Pfizer and Centocor.

J.W. has received consultancy fees from, and participated in a speakers bureau for, UCB Pharma. O.D. is a UCB Pharma employee and has stocks, stock options or bond holdings in UCB Pharma.

P.E. has received research grants and consulting fees from Pfizer, Merck, Abbott Laboratories, Roche, Bristol-Myers Squibb and UCB Pharma. B.D. is a UCB Pharma employee and owns UCB Pharma stock.

E.M. has received consulting fees from UCB Pharma, Amplimmune, Constellation Pharmaceuticals and Wachovia; has worked as an investigator for Bristol-Myers Squibb and Roche; and has received honorarium from the ACR and Up to Date.

\section{Risk of bias}

\section{Bias \\ Authors' judgement Support for judgement}

\begin{tabular}{lll}
\hline $\begin{array}{l}\text { Random sequence genera- } \\
\text { tion (selection bias) }\end{array}$ & Low risk & "Patients were randomised 4:1 via an interactive voice response system" \\
\hline $\begin{array}{l}\text { Allocation concealment } \\
\text { (selection bias) }\end{array}$ & Low risk & "Patients were randomised 4:1 via an interactive voice response system" \\
\hline $\begin{array}{l}\text { Blinding (performance } \\
\text { bias and detection bias) }\end{array}$ & Low risk & $\begin{array}{l}\text { Blinding of participants and key study personnel ensured, and unlikely that } \\
\text { the blinding could have been broken. UCB stated: "All the study staff with the } \\
\text { exception of the unblinded dispenser, was blind to the treatment". "Each } \\
\text { study center was required to have a written blinding plan in place signed by } \\
\text { the principal investigator, which detailed the study center's steps for ensuring } \\
\text { that the double blind nature of the study was maintained" }\end{array}$ \\
\hline $\begin{array}{l}\text { Blinding (performance } \\
\text { bias and detection bias) }\end{array}$ & Low risk & $\begin{array}{l}\text { See above } \\
\text { All outcomes }\end{array}$ \\
$\begin{array}{l}\text { Incomplete outcome data } \\
\text { (attrition bias) }\end{array}$ & Low risk & $\begin{array}{l}90 \% \text { of certolizumab pegol group and } 86 \% \text { of placebo completed } 12 \text { weeks of } \\
\text { treatment }\end{array}$ \\
\hline
\end{tabular}


Weinblatt 2012 (Continued) ACR50

$\begin{array}{ll}\begin{array}{l}\text { Incomplete outcome data } \\ \text { (attrition bias) }\end{array} & \text { Fow risk } \\ \begin{array}{l}\text { All outcomes } \\ \text { ITT analysis for efficacy outcomes but per protocol analysis for safety: } 9 \text { partic- } \\ \text { ipants fewer in certolizumab pegol arm and } 3 \text { fewer in placebo group }\end{array}\end{array}$

\begin{tabular}{|c|c|c|}
\hline $\begin{array}{l}\text { Selective reporting (re- } \\
\text { porting bias) }\end{array}$ & Low risk & $\begin{array}{l}\text { All the outcomes that are of interest to this review have been reported in the } \\
\text { prespecified way }\end{array}$ \\
\hline
\end{tabular}

\begin{tabular}{lll}
\hline Other bias & Low risk & The study appears to be free of other sources of bias \\
\hline $\begin{array}{l}\text { Blinding of participants } \\
\begin{array}{l}\text { and personnel (perfor- } \\
\text { mance bias) }\end{array}\end{array}$ & Low risk & $\begin{array}{l}\text { Although blinding is not described, blinding of participants and key study per- } \\
\text { sonnel ensured, and unlikely that the blinding could have been broken }\end{array}$
\end{tabular}

mance bias)

All outcomes

$\begin{array}{lll}\text { Blinding of outcome as- } & \text { Low risk } & \text { Blinding of participants and key study personnel ensured, and unlikely that } \\ \text { sessment (detection bias) } & \text { the blinding could have been broken }\end{array}$

All outcomes

Yamamoto (a) 2014

\begin{tabular}{ll}
\hline Methods & Randomised, double-blind trial \\
\hline Participants & Eligible patients were aged 20-74 years, Certoluzimab pegol ( $=116)$ Placebo $(\mathrm{n}=114)$ \\
\hline Interventions & $\begin{array}{l}\text { 1. Induction dose of } 400 \mathrm{mg} \text { in weeks } 0,2 \text { and 4, and thereafter } 200 \text { mg CDP870 given sc every } 2 \text { weeks } \\
\text { until week } 22 \\
\text { 2. Placebo, same regimen }\end{array}$ \\
\hline Outcomes & $\begin{array}{l}\text { Primary outcome: ACR20 at week } 12 \\
\text { Secondary outcome: ACR20 at week } 24 \\
\text { Follow-up 24 weeks }\end{array}$ \\
\hline
\end{tabular}

Notes

clinicaltrials.gov/ct2/show/NCT00791921?term=00791921\&rank=1

\section{HIKARI Trial}

Countries/Cities: 66 centers across Japan

Dates conducted: between 19 November 2008 and 16 September 2010

Eligibility criteria: patients with active RA who could not receive MTX due to insufficient efficacy, safety concerns or previous discontinuation for safety reasons.

inclusion criteria:

- Must have a diagnosis of adult-onset RA of at least 6 months but not longer than 15 years as defined by the 1987 ACR classification criteria

- Must have active RA disease as defined by: at least 6 tender joints and 6 swollen joints; ESR of $28 \mathrm{~mm} /$ hour or CRP of $2.0 \mathrm{mg} / \mathrm{dL}$

- Have failed to respond or have been resistant to at least 1 DMARD (including MTX)

- MTX cannot be administered for any of the reasons: incomplete response/safety concerns

Exclusion criteria: 
- A diagnosis of any other inflammatory arthritis

- Have a secondary, non-inflammatory type of arthritis (e.g. osteoarthritis, fibromyalgia)

- Currently have, or who have a history of, a demyelinating or convulsive disease of the central nervous system (e.g. multiple sclerosis, epilepsy)

- Have NYHA Class III or IV congestive heart failure

- Have, or who have a history of, tuberculosis

- Have a high risk of infection (with a current infectious disease, a chronic infectious disease, a history of serious infectious disease)

- Currently have, or who have a history of, malignancy

- Women who are breastfeeding or pregnant, who are of childbearing potential

- Previously received treatment with 2 or more anti-TNFa drugs or who previously failed to respond to treatment with 1 or more anti-TNFa drugs

Fewer than $10 \%$ of the participants were exposed to a previous TNF with a wash-out period minimum of 3 months for etanercept or 6 months for other biologics

Adverse events as a specified outcome: Treatment-emergent AEs (TEAEs) included all events from after administration of study drug until the last evaluation visit (not including the safety follow-up visit). TEAEs were coded by system organ class and preferred term using Medical Dictionary for Regulatory Activities (MedDRA)

Funding sources: Otsuka Pharmaceutical Co., Ltd. and UCB Japan

Conflict of interest: This study is already not published. This statement was in the trials registry: "Principal Investigators are NOT employed by the organization sponsoring the study. There is NOT an agreement between Principal Investigators and the Sponsor (or its agents) that restricts the PI's rights to discuss or publish trial results after the trial is completed".

KY has served as a consultant for UCB Pharma, Pfizer, Abbott, BMS, Roche, Chugai, Mitsubishi-Tanabe and Eisai and has received research funding from UCB Pharma, Pfizer, Abbott, Santen, Mitsubishi-Tanabe and Eisai.

TT has served as a consultant for AstraZeneca, Eli Lilly, Novartis, Mitsubishi-Tanabe and Asahi Kasei, has received research support from Abott, Astellas, BMS, Chugai, Daiichi-Sankyo, Eisai, Janssen, Mitsubishi-Tanabe, Nippon Shinyaku, Otsuka, Pfizer, Sanofi-Aventis, Santen, Takeda and Teijin, and has served on speaker bureaus for Abbott, BMS, Chugai, Eisai, Janssen, Mitsubishi-Tanabe, Pfizer and Takeda.

HY has served as a consultant for, and received research funding from, UCB Pharma, Abbott, Astellas, BMS, Chugai, Eisai, Janssen, Mitsubishi-Tanabe, Pfizer and Takeda.

$\mathrm{NI}$ has received research funding from Takeda, Mitsubishi- Tanabe, Astellas, Chugai, Abbott, BMS, Eisai, Janssen, Kaken and Pfizer and has served on speaker bureaus for Takeda, Mitsubishi-Tanabe, Astellas, Chugai, Abbott, BMS, Eisai, Janssen, Kaken, Pfizer, Taisho-Toyama and Otsuka.

YT has received research funding from BMS, MSD, Chugai, Mitsubishi-Tanabe, Astellas, Abbott, Eisai and Janssen and has served on speaker bureaus for UCB Pharma, Mitsubishi- Tanabe, Abbott, Eisai, Chugai, Janssen, Santen, Pfizer, Astellas, Daiichi-Sankyo, GSK, AstraZeneca, Otsuka, Actelion, Eli Lilly, Nippon Kayaku, Quintiles Transnational and Ono.

KE has served as a consultant for UCB Pharma

\section{Risk of bias}

\section{Bias}

Authors' judgement Support for judgement

Random sequence genera- Low risk

tion (selection bias)

External central of randomisation. Randomization by blocks 
Yamamoto (a) 2014 (Continued)

\begin{tabular}{|c|c|c|}
\hline $\begin{array}{l}\text { Allocation concealment } \\
\text { (selection bias) }\end{array}$ & Low risk & $\begin{array}{l}\text { The allocation sequence was generate using uniform random numbers from } \\
\text { SAS RANUNI function }\end{array}$ \\
\hline $\begin{array}{l}\text { Blinding (performance } \\
\text { bias and detection bias) } \\
\text { ACR50 }\end{array}$ & Low risk & $\begin{array}{l}\text { "All study staff with the exception of the unblinded dispenser were blind to the } \\
\text { treatment, ... These unblinded personnel were not allowed to engage in any } \\
\text { other study activities" }\end{array}$ \\
\hline $\begin{array}{l}\text { Blinding (performance } \\
\text { bias and detection bias) } \\
\text { All outcomes }\end{array}$ & Low risk & See above \\
\hline
\end{tabular}

$\begin{array}{ll}\begin{array}{l}\text { Incomplete outcome data } \\ \text { (attrition bias) }\end{array} & \begin{array}{l}71 \% \text { of certolizumab pegol group and } 15 \% \text { of placebo completed } 6 \text { months of } \\ \text { treatment. We imputed missing data for analysis }\end{array}\end{array}$
ACR50

$\begin{array}{ll}\begin{array}{l}\text { Incomplete outcome data Low risk } \\ \text { (attrition bias) }\end{array} & \text { Full account of all withdrawals and reasons for withdrawals } \\ \text { All outcomes } & \text { ITT analysis. } \\ & \text { Quote: "Of the } 230 \text { subjects in the Full Analysis Set (FAS), 230 are included in } \\ & \text { the adverse event reporting based upon the Safety Set (SS) population. The } \\ \text { Safety Set includes all subjects randomised who received at least } 1 \text { dosing" }\end{array}$

\begin{tabular}{lll}
\hline $\begin{array}{l}\text { Selective reporting (re- } \\
\text { porting bias) }\end{array}$ & Low risk & $\begin{array}{l}\text { The study protocol is available and all of the study's prespecified (primary and } \\
\text { secondary) outcomes that are of interest to this review have been reported in } \\
\text { the prespecified way }\end{array}$ \\
\hline Other bias & Low risk & The study appears to be free of other sources of bias \\
\hline $\begin{array}{l}\text { Blinding of participants } \\
\begin{array}{l}\text { and personnel (perfor- } \\
\text { mance bias) }\end{array}\end{array}$ & Low risk \\
$\begin{array}{ll}\text { All outcomes } \\
\text { Blinding of outcome as- } \\
\text { sessment (detection bias) } \\
\begin{array}{l}\text { All outcomes } \\
\hline\end{array}\end{array}$ & Low risk & See above \\
\hline
\end{tabular}

Yamamoto (b) 2014

\begin{tabular}{|c|c|}
\hline Methods & $\begin{array}{l}\text { Treatment, randomised, double-blind (participant, caregiver, investigator, outcomes assessor), dose- } \\
\text { comparison, parallel-assignment, safety/efficacy study }\end{array}$ \\
\hline Participants & $\begin{array}{l}\text { Eligible patients were aged from } 20-74 \text { years and had a diagnosis of RA defined by ACR (1987) criteria } \\
\text { for } 0.5-15 \text { years. }\end{array}$ \\
\hline \multirow[t]{5}{*}{ Interventions } & $\begin{array}{l}\text { Patients were randomised 1:1:1:1 to subcutaneous CZP 100, 200, or } 400 \text { mg plus MTX, or saline placebo } \\
\text { plus MTX, every } 2 \text { weeks (Q2W). }\end{array}$ \\
\hline & 1. Drug: CDP870 $400 \mathrm{mg}(\mathrm{n}=85)$ \\
\hline & 2. Drug: CDP870 $200 \mathrm{mg}(\mathrm{n}=82)$ \\
\hline & 3. Drug: CDP870 $100 \mathrm{mg}(\mathrm{n}=72$ ) \\
\hline & 4. Drug: placebo of CDP870 ( $n=77$ ) \\
\hline \multirow[t]{3}{*}{ Outcomes } & Primary outcome measures:ACR20 responder rate: week 12, 24 \\
\hline & $\begin{array}{l}\text { Secondary outcome measures:ACR20/50/70 responder rate: weeks } 1,2,4,6,8,12,14,16,20,24 \mathrm{DAS}-28 \\
\text { (ESR): weeks } 1,2,4,6,8,12,14,16,20,24\end{array}$ \\
\hline & Modified Total Sharp Score: week 24 \\
\hline
\end{tabular}




\section{JRAPID Trial}

Countries/Cities: 67 centers across Japan

Dates conducted: conducted between 19 November 2008 and 18 August 2010

Eligibility criteria: patients with active RA and an inadequate response to MTX received CZP or placebo while continuing to take their previous dosage of MTX. The MTX regimen could not be changed after initiation of the study treatment.

Adverse events as a specified outcome: Treatment-emergent AEs (TEAEs) included all events from after the administration of the study drug until the last evaluation visit (not including the safety follow-up visit). TEAEs were coded by system organ class and preferred term using MedDRA terminology (v11.1)

Funding sources: Otsuka Pharmaceutical Co., Ltd; UCB Japan Co. Ltd

Conflict of interest: "Principal Investigators are NOT employed by the organization sponsoring the study". "There is NOT an agreement between Principal Investigators and the Sponsor (or its agents) that restricts the PI's rights to discuss or publish trial results after the trial is completed".

The competing interests of all authors are provided below.

KY has served as a consultant for UCB Pharma, Pfizer, Abbott, BMS, Roche, Chugai, Mitsubishi-Tanabe and Eisai, and has received research funding from UCB Pharma, Pfizer, Abbott, Santen Mitsubishi-Tanabe, and Eisai.

TT has served as a consultant for AstraZeneca, Eli Lilly, Novartis, Mitsubishi-Tanabe and Asahi Kasei, and has received research support from Abott, Astellas, BMS, Chugai, Daiichi-Sankyo, Eisai, Janssen, Mitsubishi-Tanabe, Nippon Shinyaku, Otsuka, Pfizer, Sanofi-Aventis, Santen, Takeda and Teijin, and has served on speaker bureaus for Abbott, BMS, Chugai, Eisai, Janssen, Mitsubishi-Tanabe, Pfizer and Takeda.

HY has served as a consultant for, and received research funding from, UCB Pharma, Abbott, Astellas, BMS, Chugai, Eisai, Janssen, Mitsubishi-Tanabe, Pfizer and Takeda.

NI has received research funding from Takeda, Mitsubishi-Tanabe, Astellas, Chugai, Abbott, BMS, Eisai, Janssen, Kaken and Pfizer, and has served on speaker bureaus for Takeda, Mitsubishi-Tanabe, Astellas, Chugai, Abbott, BMS, Eisai, Janssen, Kaken, Pfizer, Taisho-Toyama and Otsuka.

YT has received research funding from BMS, MSD, Chugai, Mitsubishi-Tanabe, Astellas, Abbott, Eisai and Janssen, and has served on speaker bureaus for UCB Pharma, Mitsubishi-Tanabe, Abbott, Eisai, Chugai, Janssen, Santen, Pfizer, Astellas, Daiichi-Sankyo, GSK, AstraZeneca, Otsuka, Actelion, Eli Lilly, Nippon Kayaku, Quintiles Transnational and Ono.

KE has served as a consultant for UCB Pharma.

AW has received research support from Astellas, Daiichi- Sankyo, Kyorin, Shionogi, Taisho, Dainippon-Sumitomo, Taiho, Toyama Chemical and Meiji Seika, and has served on speaker bureaus for Abott, MSD, Otsuka, GSK, Shionogi, Daiichi-Sankyo, Taisho-Toyama, Dainippon-Sumitomo, Mitsubishi-Tanabe, Toyama Chemical, Bayer and Pfizer.

HO has served as a consultant for UCB Pharma and Astellas.

TS is an employee of Otsuka.

YS is an employee of UCB Pharma.

DvH has served as a consultant for, and received research support from, AbbVie, Amgen, AstraZeneca, BMS, Centocor, Chugai, Daiichi, Eli Lilly, GSK, Janssen, Merck, Novartis, Novo-Nordisk, Otsuka, Pfizer, Roche, Sanofi-Aventis, Schering-Plough, UCB Pharma and Vertex. DvH is also director of Imaging Rheumatology bv. 
Yamamoto (b) 2014 (Continued)

NM has received research support from Pfizer, Takeda, Mitsubishi-Tanabe, Chugai, Abbott, Eisai and Astellas.

TK has served on speaker bureaus for UCB Pharma, Pfizer, Chugai, Abbott, Mitsubishi-Tanabe, Takeda, Eisai, Santen, Astellas, Taisho-Toyama, BMS, Teijin and Daiichi-Sankyo.

\section{Risk of bias}

\begin{tabular}{lll}
\hline Bias & Authors' judgement & Support for judgement \\
\hline $\begin{array}{l}\text { Random sequence genera- } \\
\text { tion (selection bias) }\end{array}$ & Low risk & External central of randomisation. Randomization by blocks \\
\hline $\begin{array}{l}\text { Allocation concealment } \\
\text { (selection bias) }\end{array}$ & Low risk & $\begin{array}{l}\text { The allocation sequence was generate using uniform random numbers from } \\
\text { SAS RANUNI function }\end{array}$ \\
\hline $\begin{array}{l}\text { Blinding (performance } \\
\text { bias and detection bias) } \\
\text { ACR50 }\end{array}$ & Low risk & $\begin{array}{l}\text { "All study staff with the exception of the unblinded dispenser were blind to the } \\
\text { treatment, ... These unblinded personnel were not allowed to engage in any } \\
\text { other study activities" }\end{array}$ \\
\hline $\begin{array}{l}\text { Blinding (performance } \\
\text { bias and detection bias) }\end{array}$ & Low risk & See above \\
All outcomes & \\
\hline
\end{tabular}

$\begin{array}{ll}\text { Incomplete outcome data } & \text { Low risk } \\ \text { (attrition bias) } & 66 \% \text { of certolizumab pegol } 100 \mathrm{mg}, 80 \% \text { of certolizumab pegol } 200 \mathrm{mg}, \text { and } \\ \text { ACR50 } & 76 \% \text { of certolizumab pegol } 400 \mathrm{mg} \text { group (overall } 74 \% \text { in certolizumab pegol } \\ & \text { groups) and } 32 \% \text { of placebo completed } 6 \text { months of treatment. We imputed } \\ \text { missing data for analysis. }\end{array}$

Incomplete outcome data Low risk (attrition bias) Safety, quote: "Of the 316 subjects in the Full Analysis Set (FAS), 316 are includ-

All outcomes ed in the adverse event reporting based upon the Safety Set (SS) population. The Safety Set includes all subjects randomised who received at least 1 dosing"

Selective reporting (re- High risk
porting bias)

Participants were recruited in Japan between 2008 and 2010. In 2008, DAS28 (ESR) and Modified Total Sharp Score were secondary outcomes. In 2012 these outcomes were deleted from clinicaltrials.gov/ct2/show/record/ NCT00791999?term=NCT00791999\&rank=1\&sect=X0125

\begin{tabular}{lll}
\hline Other bias & Low risk & The study appears to be free of other sources of bias \\
\hline $\begin{array}{l}\text { Blinding of participants } \\
\begin{array}{l}\text { and personnel (perfor- } \\
\text { mance bias) }\end{array}\end{array}$ & Low risk & No details available \\
All outcomes & \\
\hline
\end{tabular}

\begin{tabular}{ll}
\hline Blinding of outcome as- & Low risk $\quad$ See above \\
sessment (detection bias) & \\
All outcomes & \\
\hline
\end{tabular}

\section{Østergaard 2015}

\begin{tabular}{ll}
\hline Methods & Randomised, double-blind, placebo-controlled \\
\hline Participants & 41 participants with active RA despite DMARD. Participants were randomised 2:1 \\
\hline
\end{tabular}


Østergaard 2015 (Continued)

Interventions
1. certolizumab pegol (loading dose $400 \mathrm{mg}$ every 2 weeks at weeks 0 - 4; certolizumab pegol $200 \mathrm{mg}$ every 2 weeks at weeks $6-16)(n=27)$

2. Placebo, then certolizumab pegol ( placebo at weeks 0 - 2; certolizumab pegol loading dose at weeks

2 - 6; certolizumab pegol $200 \mathrm{mg}$ every 2 weeks at weeks $8-16)(n=13)$

Outcomes Primary: Change in synovitis measured by Outcome Measures in Rheumatoid Arthritis Clinical Trials (OMERACT), Rheumatoid Arthritis Magnetic Resonance Image Scoring System (RAMRIS) score at weeks $1,2,4,8$ and 16

Secondary: Change From Baseline to Week 16 in the Dynamic Magnetic Resonance Image (MRI) Parameter, Initiation Rate of Enhancement (IRE);

Change from baseline to week 16 in the dynamic MRI parameter, Maximal Enhancement (ME);

Change from baseline to week 16 in the dynamic MRI parameter, number of voxels (Nvox) with plateau and washout pattern; Percentage of participants achieving a good European League Against Rheumatism (EULAR) response at week 16; Percentage of participants meeting the ACR 20\% criteria at week 16

\section{MARVELOUS Trial}

Only the data obtained at week 2 were useful. After week 2 both arms were treated with certolizumab pegol. Out of all the primary and secondary outcomes studied, only DAS and ACR20 measured at week 2 were reported. However since they are shown as a figure we are unable to use them. Only adverse event data were reported at week 2

Countries/Cities: Denmark, Polland, Netherlands, Sweden

\section{Dates conducted: From NOvember 2010 to September 2013}

Eligibility criteria: The study population was $\geq 18$ years of age with adult-onset RA of between 3 months and 15 years duration, as defined by the 1987 American College of Rheumatology (ACR) classification criteria

Adverse events as a specified outcome: adverse events and serious adverse events were reported

\section{Funding sources: $U C B$}

Conflict of interest: Principal Investigators are NOTemployed by the organization sponsoring the study.The only disclosure restriction on the $\mathrm{PI}$ is that the sponsor can review results communications prior to public release and can embargo communications regarding trial results for a period that is more than $\mathbf{6 0}$ days but less than or equal to $\mathbf{1 8 0}$ days. The sponsor cannot require changes to the communication and cannot extend the embargo.

\section{Competing interests}

$M \emptyset$ has received grant/research support from Abbott, Pfizer and Centocor, has acted as a consultant for Abbott, Pfizer, Merck, Roche, and UCB Pharma and has taken part in speakers bureaus for Abbott, Pfizer, Merck, BMS, UCB Pharma, and Mundipharma;

LTHJ has received grant/research support from Pfizer and has acted as a paid instructor for Abbvie, BMS, MSD, Pfizer and UCB Pharma;

MSH has acted as sponsored investigator for UCB Pharma and participated as an advisory board member for Roche;

JWJB has received grant/research support from Roche, UCB, Pfizer, MSD and BMS and has received consultancy fees from Roche, UCB, Pfizer, MSD, BMS and Jansen;

FS, RH and BS-E are employees of UCB Pharma;

HB has received consulting fees, honoraria, research or institutional support, educational grants, equipment, services or expenses from Abbott, Amgen, AstraZeneca, Aventis, Bristol Myers Squibb, Cambridge Nutritional Foods, Dansk Droge, Eurovita, Ferrosan, GlaxoSmithKline, Hoechst, LEO, Lundbeck, MSD, Mundipharma, Norpharma, NutriCare, Nycomed, Pfizer, Pharmacia, Pierre-Fabre, Proc- 


\section{Risk of bias}

\begin{tabular}{|c|c|c|}
\hline Bias & Authors' judgement & Support for judgement \\
\hline $\begin{array}{l}\text { Random sequence genera- } \\
\text { tion (selection bias) }\end{array}$ & Low risk & External central of randomisation \\
\hline $\begin{array}{l}\text { Allocation concealment } \\
\text { (selection bias) }\end{array}$ & Low risk & IVRS \\
\hline $\begin{array}{l}\text { Blinding (performance } \\
\text { bias and detection bias) } \\
\text { ACR50 }\end{array}$ & Unclear risk & Not measured at 2 weeks. Not applicable \\
\hline $\begin{array}{l}\text { Blinding (performance } \\
\text { bias and detection bias) } \\
\text { All outcomes }\end{array}$ & Unclear risk & $\begin{array}{l}\text { Due to differences in the presentation and viscosity of certolizumab pegol and } \\
\text { placebo, all study treatments (certolizumab pegol and placebo) were admin- } \\
\text { istered by unblinded study centre personnel to maintain study blinding. The } \\
\text { personnel administering the injections had no involvement in the study other } \\
\text { than performing the ESR analysis }\end{array}$ \\
\hline $\begin{array}{l}\text { Incomplete outcome data } \\
\text { (attrition bias) } \\
\text { ACR50 }\end{array}$ & Low risk & Not measured. Not applicable \\
\hline $\begin{array}{l}\text { Incomplete outcome data } \\
\text { (attrition bias) } \\
\text { All outcomes }\end{array}$ & Low risk & $\begin{array}{l}1 \text { participant withdrew prior to treatment and was not included in the Full } \\
\text { Analysis Set (FAS), but it is not clear from which arm the participant withdrew. } \\
\text { The FAS comprised } 27 \text { participants in the certolizumab pegol group and } 13 \\
\text { in the placebo } \rightarrow \text { certolizumab pegol group. During the double-blind phase, } 4 \\
\text { participants discontinued treatment: } 1 \text { from the placebo } \rightarrow \text { certolizumab pegol } \\
\text { group due to withdrawal of consent, and } 3 \text { from the certolizumab pegol group, } \\
2 \text { due to AEs and } 1 \text { due to lack of efficacy. Since it is not clear at which point of } \\
\text { the double-blind phase the withdrawals occurred, we did not input these data } \\
\text { to the analysis }\end{array}$ \\
\hline
\end{tabular}

\begin{tabular}{|c|c|c|}
\hline $\begin{array}{l}\text { Selective reporting (re- } \\
\text { porting bias) }\end{array}$ & Low risk & $\begin{array}{l}\text { All the outcomes listed in the protocol are reported in www.ClinicalTrial.gov. } \\
\text { However, the data were measured at week } 16 \text { and so cannot be used }\end{array}$ \\
\hline Other bias & Low risk & The study appears to be free of other sources of bias \\
\hline $\begin{array}{l}\text { Blinding of participants } \\
\text { and personnel (perfor- } \\
\text { mance bias) } \\
\text { All outcomes }\end{array}$ & Low risk & "The personnel administering the injections had no involvement in the study" \\
\hline $\begin{array}{l}\text { Blinding of outcome as- } \\
\text { sessment (detection bias) } \\
\text { All outcomes }\end{array}$ & Unclear risk & $\begin{array}{l}\text { "Due to differences in the presentation and viscosity of certolizumab pegol } \\
\text { and placebo, all study treatments (certolizumab pegol and placebo) were ad- } \\
\text { ministered by unblinded study centre personnel to maintain study blinding. } \\
\text { The personnel administering the injections had no involvement in the study } \\
\text { other than performing the erythrocyte sedimentation rate analysis" }\end{array}$ \\
\hline
\end{tabular}

ACR: American College of Rheumatology

ARA: American Rheumatology Association

CDAl: coronary diffuse atheromatous index

CRP: C-reactive protein

DAS: disease activity score 
DMARD: disease-modifying anti-rheumatic drug

ESR: erythrocyte sedimentation rate

HAQ-DI: health assessment questionnaire - disability index

ITT: intention-to-treat

IVRS: Interactive voice recognition system

$\mathrm{mBPI}$ : modified brief pain inventory

mTSS: modified total sharp score

MTX: methotrexate

NYHA: New York Heart Association

PPD: purified protein derivative

Q2W every two weeks

RA: rheumatoid arthritis

sc: subcutaneous

SDAI: Simplified Disease Activity Index

SF-36: short form 36

SJC: swollen joint count

TB: tuberculosis

TJC: tender joint count

VAS: visual analogue scale

Characteristics of excluded studies [ordered by study ID]

\begin{tabular}{|c|c|}
\hline Study & Reason for exclusion \\
\hline Alten 2013 & OLE \\
\hline Bykerk 2015 & $\begin{array}{l}\text { The outcomes reported (Disease Burden on Workplace and Household Productivity) are not cov- } \\
\text { ered in our review }\end{array}$ \\
\hline Curtis 2014 & There is only one arm without placebo or any comparator \\
\hline Curtis 2015a & There is only one arm without placebo or any comparator \\
\hline Curtis 2015b & There is only one arm without placebo or any comparator \\
\hline Dose Flex 2007 & $\begin{array}{l}\text { RCT that tested clinical efficacy of } 2 \text { dosing regimens of CZP ( } 200 \mathrm{mg} \text { every } 2 \text { weeks or } 400 \mathrm{mg} \text { every } \\
\text { four weeks + MTX) compared to MTX alone for maintenance of clinical response up to } 34 \text { weeks in } \\
\text { participants who have achieved ACR20 after a 16-week open-label run-in period of CZP treatment } \\
\text { (CZP } 200 \mathrm{mg} \text { every } 2 \text { weeks + MTX). Reason for exclusion is that participants do not have active dis- } \\
\text { ease at randomisation }\end{array}$ \\
\hline Fleischmann 2013 & OLE \\
\hline Kavanaugh 2013 & OLE \\
\hline Kavanaugh 2014 & There is only one arm without placebo or any comparator \\
\hline Kivitz 2014 & Phase IV clinical trial \\
\hline NCT00160641 & One simple group \\
\hline NCT00160693 & It is an OLE with just one simple group \\
\hline NCT00753454 & One simple group \\
\hline NCT00843778 & One simple group \\
\hline NCT00851318 & OLE \\
\hline
\end{tabular}




\begin{tabular}{|c|c|}
\hline Study & Reason for exclusion \\
\hline NCT00993668 & Excluded because adverse events were studied in the blinded period just at 4 weeks \\
\hline NCT01197066 & OLE \\
\hline NCT01255761 PREDICT & Phase IV. Both arms were treated with CZP $200 \mathrm{mg}$ \\
\hline NCT01292265 & Phase IV \\
\hline NCT01374971 & Phase IV \\
\hline NCT01443364 & OLE \\
\hline NCT01526434 & OLE \\
\hline NCT02319642 & OLE \\
\hline NCT02586246 & OLE \\
\hline
\end{tabular}

OLE: open-label extension

Characteristics of ongoing studies [ordered by study ID]

\begin{tabular}{ll}
\hline NCT01295151 & $\begin{array}{l}\text { SWITCH Clinical trial for patients with rheumatoid arthritis who have failed an initial TNF-blocking } \\
\text { drug (SWITCH) }\end{array}$ \\
\hline Methods & Randomised controlled trial \\
\hline Participants & People that have failed an anti-TNF therapy (the first of the biological therapies to be introduced) \\
\hline Interventions & Etanercept; abatacept; rituximab; adalimumab; certolizumab pegol; infliximab; golimumab \\
\hline Outcomes & Change in disease activity at 6 months; EULAR and ACR scores; CDAl; quality of life \\
\hline Starting date & 2011 \\
\hline Contact information & $\begin{array}{l}\text { Julia Brown, Director of Leeds Institute of Clinical Trials Research, University of Leeds } \\
\text { Notes }\end{array}$ \\
\hline $\begin{array}{l}\text { Only published the protocol : EXCLUDE } \\
\text { Infliximab, adalimumab, certolizumab or golimumab if initial failure to the receptor fusion protein }\end{array}$
\end{tabular}

\section{NCT01489384}

\section{Trial name or title}

\section{Methods}

Participants
Cimzia treatment in rheumatoid arthritis: randomising to stop versus continue disease-modifying anti-rheumatic drug(s) 
NCT01489384 (Continued)

\begin{tabular}{ll} 
Interventions & CZP plus DMRA vs CZP alone \\
\hline Outcomes & DAS28<3.2 at 18 months \\
\hline Starting date & 2011 \\
\hline Contact information & Janet Pope, MD (Pope Research Corporation) \\
\hline Notes & $\begin{array}{l}\text { The recruitment status of this study is unknown because the information has not been verified re- } \\
\text { Cently }\end{array}$
\end{tabular}

\section{NCT01491815}

\section{Trial name or title}

Active conventional therapy compared to three different biologic treatments in early rheumatoid arthritis with subsequent dose reduction: NORD-STAR trial

This is an international (Nordic) trial designed to compare the safety and efficacy of active conven-
tional therapy (ACT) and 3 biologic treatments in people with early rheumatoid arthritis (RA). The
global aim of this study is to assess and compare
$\begin{aligned} & \text { 1. the proportion of participants who achieve remission with ACT versus } 3 \text { different biologic thera- } \\ & \text { pies (Certolizumab pegol, abatacept or tocilizumab) } \\ & \text { 2. } 2 \text { alternative de-escalation strategies in participants who respond to first-line therapy. }\end{aligned}$

\begin{tabular}{ll}
\hline Participants & Estimated enrolment: 800 \\
\hline Interventions & Certolizumabl pegol, abatacept, tocilizumab \\
\hline Outcomes & . The proportion of participants in remission at week 24 from baseline according to CDAl. \\
& - The proportion of participants in remission at week 24 after dose-reduction according to CDAl. \\
\hline Starting date & 2012; estimated completion data: 2020 \\
\hline Contact information & Contact: Ronald van Vollenhoven, MD, Prof. +46(0)851776077 ronald.van.vollenhoven@ki.se \\
\hline Notes & \\
\hline
\end{tabular}

\section{NCT01500278}

Trial name or title Study to assess the short- and long-term efficacy of certolizumab pegol plus methotrexate compared to adalimumab plus methotrexate in subjects with moderate to severe rheumatoid arthritis (RA) inadequately responding to methotrexate

\begin{tabular}{ll}
\hline Methods & RCT \\
\hline Participants & 916 \\
\hline Interventions & CZP plus MTX vs adalimumab plus MTX \\
\hline Outcomes & ACR20 at 12 and 104 weeks \\
\hline
\end{tabular}


NCT01500278 (Continued)

\begin{tabular}{ll} 
Starting date & 2011 \\
\hline Contact information & UCB Pharma \\
\hline Notes & $\begin{array}{l}\text { Without results in clinicaltrials.gov/ct2/show } / \text { study } / \text { NCT01500278?term=certolizumab\&rank=34, } \\
\text { nor abstract of proceedings }\end{array}$
\end{tabular}

\section{NCT01602302}

\begin{tabular}{ll}
\hline Trial name or title & Ultrasound and withdrawal of biological DMARDs in rheumatoid arthritis (RA-BioStop) \\
\hline Methods & Phase IV \\
\hline Participants & Estimated enrolment: 110 \\
\hline Interventions & $\begin{array}{l}\text { Primary outcome measures: Active inflammation at the time of DMARD withdrawal indicated by } \\
\text { the presence of a PD-score } \geq 1 \text { in at least 1 joint out of a sonographic 14-joint count predicts relapse } \\
\text { rate at week } 16\end{array}$ \\
\hline Outcomes & Estimated completion data: September 2017 \\
\hline Starting date & Contact: Christian Dejaco, MD, PhD +43-316-80595 christian.dejaco@gmx.net \\
\hline Contact information & This study is currently recruiting participants \\
\hline Notes &
\end{tabular}

\section{NCT02151851}

\begin{tabular}{|c|c|}
\hline Trial name or title & $\begin{array}{l}\text { A study of certolizumab pegol as additional therapy in Chinese patients with active rheumatoid } \\
\text { arthritis (RAPID-C) }\end{array}$ \\
\hline Methods & Phase 3, multi centre, double-blind, placebo-controlled, parallel-group, randomised 24-week trial \\
\hline Participants & 400 participants ( 300 with CZP/100 placebo) \\
\hline Interventions & $\begin{array}{l}\text { CZP } 400 \mathrm{mg} \text { ( } 200 \mathrm{mg} \text { prefilled syringe [PFS], i.e. } 2 \text { injections) at baseline, and weeks } 2 \text { and } 4 \text {; then } \\
\text { CZP } 200 \mathrm{mg} \text { ( } 1 \text { injection) every } 2 \text { weeks until week } 22\end{array}$ \\
\hline Outcomes & ACR20 \\
\hline Starting date & June 2014; completion data: June 2016 \\
\hline Contact information & UCB Cares; UCB Pharma \\
\hline
\end{tabular}

\section{NCT02293590}

Trial name or title

Remission by Intra-articular injection plus CErtolizumab (RICE) 
NCT02293590 (Continued)

Methods

An open-label, randomised study to compare the efficacy of certolizumab pegol (CZP) plus a dynamic or fixed dose treatment strategy in patients with rheumatoid arthritis; a Phase II study

Participants 48

Interventions

48

after loading dose of $400 \mathrm{mg}$ at Weeks 0,2 and 4

Outcomes ACR50 at 24 weeks

\begin{tabular}{ll}
\hline Starting date & October 2014 \\
\hline Contact information & Rüdiger B. Müller, Cantonal Hospital of St. Gallen \\
\hline Notes & Recruiting participants \\
\hline
\end{tabular}

\section{NCT02430909} $\begin{array}{ll}\text { Trial name or title } & \begin{array}{l}\text { Multiple dose study of UCB4940 as add-on to certolizumab pegol in subjects with rheumatoid } \\ \text { arthritis }\end{array}\end{array}$

\begin{tabular}{|c|c|}
\hline Methods & Phase II double-blind, randomised, placebo-controlled study \\
\hline Participants & No data \\
\hline Interventions & $\begin{array}{l}\text { Certolizumab pegol ( } 400 \mathrm{mg} \text { at weeks } 0,2 \text {, and } 4 \text { followed by } 200 \mathrm{mg} \text { every } 2 \text { weeks) until week } 30+ \\
\text { placebo from week } 8 \text { to week } 18 \text { versus }\end{array}$ \\
\hline & $\begin{array}{l}\text { Certolizumab pegol ( } 400 \mathrm{mg} \text { at weeks } 0,2 \text {, and } 4 \text { followed by } 200 \mathrm{mg} \text { every } 2 \text { weeks) until week } 30+ \\
\text { UCB } 4940 \text { from week } 8 \text { until week } 18\end{array}$ \\
\hline
\end{tabular}

\begin{tabular}{ll}
\hline Outcomes & Adverse events; Change in DAS28 at week 20 \\
\hline Starting date & 2015 \\
\hline Contact information & UCB Cares +18878229493 (UCB)
\end{tabular}

\section{Notes}

\section{NCT02466581}

\begin{tabular}{ll}
\hline Trial name or title & Dose reduction for early rheumatoid arthritis patients with low disease activity \\
\hline Methods & Phase IV. This is an international (Nordic) trial designed to compare the safety and efficacy of active \\
& conventional therapy (ACT) and 3 biologic treatments (certolizumab pegol, abatacept or tocilizum- \\
ab) in people with early rheumatoid arthritis. The global aim of this study is to assess and compare & 2 alternative de-escalation strategies in participants who achieved low disease activity during first- \\
line therapy in the NORD-STAR study.
\end{tabular}

\section{Participants}

Interventions

Active Comparator: Arm 1 
Participants keep the intervention they had in the NORD-STAR-study (NCT01491815), i.e. 1 of the 4 below:

1. Sulphasalazine + hydroxychloroquine OR prednisolone plus methotrexate and steroids

2. Cimzia plus methotrexate and steroids

3. Orencia plus methotrexate and steroids

4. RoActemra plus methotrexate and steroids

Active Comparator: Arm 2

Participants keep the intervention they had in the NORD-STAR-study (NCT01491815), i.e. 1 of the 4 below:

1. Sulphasalazine + hydroxychloroquine OR prednisolone plus methotrexate and steroids

2. Cimzia plus methotrexate and steroids

3. Orencia plus methotrexate and steroids

3. RoActemra plus methotrexate and steroids.

This intervention is de-escalated starting 24 weeks after randomisation

Proportion of participants maintaining low disease activity after dose reduction
$\begin{aligned} & \text { The proportion of participants, with early dose reduction vs late dose reduction, who maintain low } \\ & \text { disease activity }(2.8<\mathrm{CDAI} \leq 10.0) \text { at } 24 \text { weeks after the dose was first reduced }\end{aligned}$

\begin{tabular}{ll}
\hline Starting date & May 2015 \\
\hline Contact information & Ronald van Vollenhoven \\
& $+46(0) 851776077$ ronald.van.vollenhoven@ki.se \\
\hline
\end{tabular}

Notes

This study is currently recruiting participants

CDAl: coronary diffuse atheromatous index

DMARD: disease-modifying anti-rheumatic drug

\section{DATA AND ANALYSES}

\section{Comparison 1. Efficacy at 12 weeks, any dose}

\begin{tabular}{lllll}
\hline $\begin{array}{l}\text { Outcome or subgroup ti- } \\
\text { tle }\end{array}$ & No. of studies & $\begin{array}{l}\text { No. of partici- } \\
\text { pants }\end{array}$ & Statistical method & Effect size \\
\hline 1 ACR20 & 6 & 2902 & Risk Ratio (M-H, Random, 95\% Cl) & $1.13[0.79,1.63]$ \\
\hline 1.1 certolizumab 50 mg sc & 1 & 47 & Risk Ratio (M-H, Random, 95\% Cl) & $0.27[0.13,0.57]$ \\
\hline $\begin{array}{l}1.2 \text { certolizumab } 100 \mathrm{mg} \\
\text { SC }\end{array}$ & 2 & 145 & Risk Ratio (M-H, Random, 95\% Cl) & $0.78[0.09,7.05]$ \\
\hline $\begin{array}{l}1.3 \text { certolizumab } 200 \mathrm{mg} \\
\text { SC }\end{array}$ & 6 & 2456 & Risk Ratio (M-H, Random, 95\% Cl) & $1.66[0.97,2.85]$ \\
\hline
\end{tabular}




\begin{tabular}{|c|c|c|c|c|}
\hline $\begin{array}{l}\text { Outcome or subgroup ti- } \\
\text { tle }\end{array}$ & No. of studies & $\begin{array}{l}\text { No. of partici- } \\
\text { pants }\end{array}$ & Statistical method & Effect size \\
\hline $\begin{array}{l}1.4 \text { certolizumab } 400 \mathrm{mg} \\
\text { sc }\end{array}$ & 2 & 161 & Risk Ratio (M-H, Random, 95\% Cl) & $1.40[0.38,5.23]$ \\
\hline $\begin{array}{l}1.5 \text { certolizumab } 600 \mathrm{mg} \\
\text { sc }\end{array}$ & 1 & 47 & Risk Ratio (M-H, Random, 95\% Cl) & $0.68[0.51,0.90]$ \\
\hline $\begin{array}{l}1.6 \text { certolizumab } 800 \mathrm{mg} \\
\text { sc }\end{array}$ & 1 & 46 & Risk Ratio (M-H, Random, 95\% Cl) & $0.83[0.66,1.04]$ \\
\hline 2 ACR50 & 4 & & Risk Ratio (M-H, Random, 95\% Cl) & Subtotals only \\
\hline 2.1 certolizumab $50 \mathrm{mg} \mathrm{sc}$ & 1 & 47 & Risk Ratio (M-H, Random, 95\% Cl) & $1.58[0.09,27.88]$ \\
\hline $\begin{array}{l}2.2 \text { certolizumab } 100 \mathrm{mg} \\
\text { sc }\end{array}$ & 1 & 48 & Risk Ratio (M-H, Random, 95\% Cl) & $1.10[0.06,20.96]$ \\
\hline $\begin{array}{l}2.3 \text { certolizumab } 200 \mathrm{mg} \\
\text { sc }\end{array}$ & 4 & 2118 & Risk Ratio (M-H, Random, 95\% Cl) & $1.89[1.06,3.37]$ \\
\hline $\begin{array}{l}2.4 \text { certolizumab } 400 \mathrm{mg} \\
\text { sc }\end{array}$ & 1 & 50 & Risk Ratio (M-H, Random, 95\% Cl) & $7.33[0.48,110.96]$ \\
\hline 3 ACR70 & 4 & & Risk Ratio (M-H, Random, 95\% Cl) & Subtotals only \\
\hline 3.1 certolizumab $50 \mathrm{mg} \mathrm{sc}$ & 1 & 47 & Risk Ratio (M-H, Random, 95\% Cl) & $1.13[0.06,21.47]$ \\
\hline $\begin{array}{l}3.2 \text { certolizumab } 100 \mathrm{mg} \\
\mathrm{sc}\end{array}$ & 1 & 48 & Risk Ratio (M-H, Random, 95\% Cl) & $0.66[0.03,14.89]$ \\
\hline $\begin{array}{l}3.3 \text { certolizumab } 200 \mathrm{mg} \\
\mathrm{sc}\end{array}$ & 4 & 2118 & Risk Ratio (M-H, Random, 95\% Cl) & $2.78[1.20,6.41]$ \\
\hline $\begin{array}{l}3.4 \text { certolizumab } 400 \mathrm{mg} \\
\text { sc }\end{array}$ & 1 & 50 & Risk Ratio (M-H, Random, 95\% Cl) & $5.23[0.34,80.54]$ \\
\hline
\end{tabular}

Analysis 1.1. Comparison 1 Efficacy at 12 weeks, any dose, Outcome 1 ACR20.

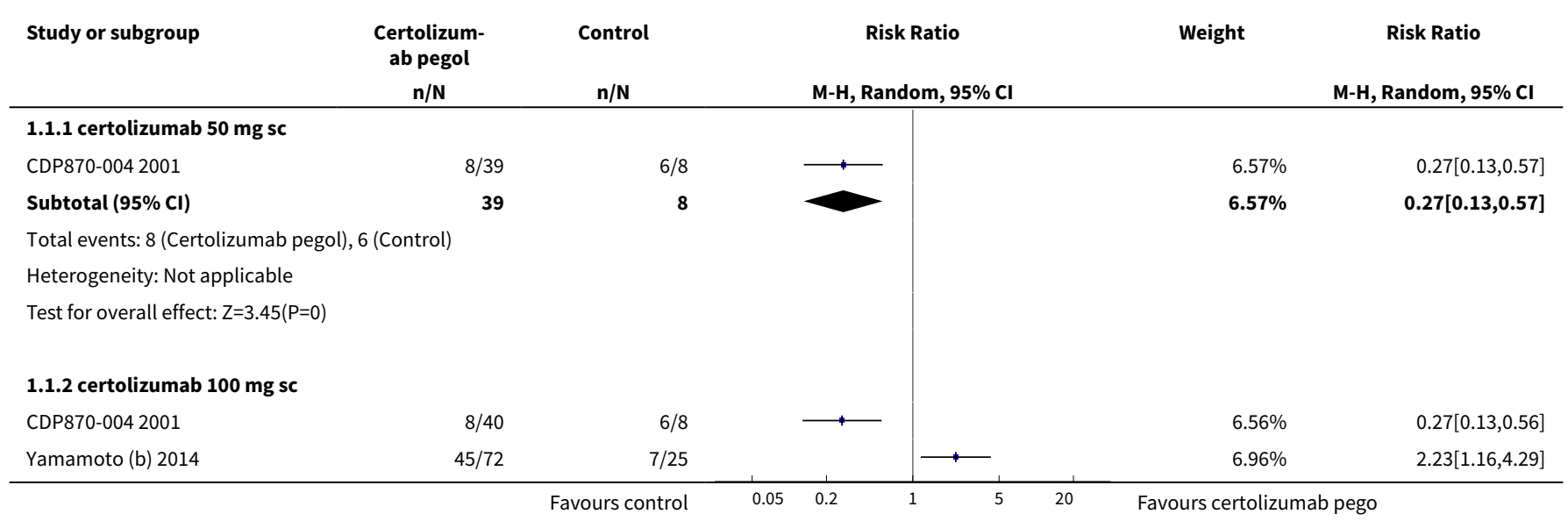




\begin{tabular}{|c|c|c|c|c|c|}
\hline Study or subgroup & $\begin{array}{c}\text { Certolizum- } \\
\text { ab pegol } \\
n / N\end{array}$ & $\begin{array}{c}\text { Control } \\
\mathbf{n} / \mathbf{N}\end{array}$ & $\begin{array}{c}\text { Risk Ratio } \\
\text { M-H, Random, } 95 \% \mathrm{Cl}\end{array}$ & Weight & $\begin{array}{c}\text { Risk Ratio } \\
\text { M-H, Random, 95\% CI }\end{array}$ \\
\hline Subtotal $(95 \% \mathrm{CI})$ & 112 & 33 & & $13.53 \%$ & $0.78[0.09,7.05]$ \\
\hline \multicolumn{6}{|c|}{ Total events: 53 (Certolizumab pegol), 13 (Control) } \\
\hline \multicolumn{6}{|c|}{ Heterogeneity: $\operatorname{Tau}^{2}=2.41 ; \mathrm{Chi}^{2}=20.04, \mathrm{df}=1(\mathrm{P}<0.0001) ; \mathrm{I}^{2}=95.01 \%$} \\
\hline \multicolumn{6}{|c|}{ 1.1.3 certolizumab $200 \mathrm{mg} \mathrm{sc}$} \\
\hline CDP870-004 2001 & $14 / 41$ & $6 / 8$ & $\longrightarrow$ & $7.29 \%$ & $0.46[0.25,0.82]$ \\
\hline Emery 2015 & $480 / 660$ & $148 / 219$ & * & $8.91 \%$ & $1.08[0.97,1.19]$ \\
\hline NCT00993317 & $52 / 85$ & $15 / 42$ & $\multimap$ & $7.94 \%$ & $1.71[1.1,2.66]$ \\
\hline Weinblatt 2012 & $435 / 851$ & $55 / 212$ & $\rightarrow-$ & $8.64 \%$ & $1.97[1.55,2.5]$ \\
\hline Yamamoto (b) 2014 & $63 / 82$ & $7 / 26$ & $\longrightarrow$ & $7.01 \%$ & $2.85[1.5,5.44]$ \\
\hline Subtotal $(95 \% \mathrm{Cl})$ & 1835 & 621 & & $47.65 \%$ & $1.66[0.97,2.85]$ \\
\hline \multicolumn{6}{|c|}{ Total events: 1122 (Certolizumab pegol), 248 (Control) } \\
\hline \multicolumn{6}{|c|}{ Heterogeneity: $\operatorname{Tau}^{2}=0.4 ; \mathrm{Chi}^{2}=89.85, \mathrm{df}=5(\mathrm{P}<0.0001) ; \mathrm{I}^{2}=94.44 \%$} \\
\hline \multicolumn{6}{|c|}{ Test for overall effect: $Z=1.85(P=0.06)$} \\
\hline \multicolumn{6}{|c|}{ 1.1.4 certolizumab $400 \mathrm{mg} \mathrm{sc}$} \\
\hline CDP870-004 2001 & $25 / 42$ & $6 / 8$ & $\rightarrow$ & $7.8 \%$ & $0.79[0.5,1.27]$ \\
\hline Yamamoto (b) 2014 & $66 / 85$ & $8 / 26$ & $\longrightarrow$ & $7.28 \%$ & $2.52[1.4,4.54]$ \\
\hline Subtotal $(95 \% \mathrm{Cl})$ & 127 & 34 & & $15.08 \%$ & $1.4[0.38,5.23]$ \\
\hline \multicolumn{6}{|c|}{ Total events: 91 (Certolizumab pegol), 14 (Control) } \\
\hline \multicolumn{6}{|c|}{ Heterogeneity: $\mathrm{Tau}^{2}=0.83 ; \mathrm{Chi}^{2}=12.22, \mathrm{df}=1(\mathrm{P}=0) ; \mathrm{I}^{2}=91.81 \%$} \\
\hline \multicolumn{6}{|c|}{ Test for overall effect: $Z=0.5(P=0.62)$} \\
\hline \multicolumn{6}{|c|}{ 1.1.5 certolizumab $600 \mathrm{mg} \mathrm{sc}$} \\
\hline CDP870-004 2001 & $25 / 39$ & $8 / 8$ & $\rightarrow$ & $8.51 \%$ & $0.68[0.51,0.9]$ \\
\hline Subtotal $(95 \% \mathrm{Cl})$ & 39 & 8 & 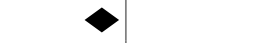 & $8.51 \%$ & $0.68[0.51,0.9]$ \\
\hline \multicolumn{6}{|c|}{ Total events: 25 (Certolizumab pegol), 8 (Control) } \\
\hline \multicolumn{6}{|c|}{ Heterogeneity: Not applicable } \\
\hline \multicolumn{6}{|c|}{ Test for overall effect: $Z=2.73(P=0.01)$} \\
\hline \multicolumn{6}{|c|}{ 1.1.6 certolizumab $800 \mathrm{mg} \mathrm{sc}$} \\
\hline CDP870-004 2001 & $30 / 38$ & $8 / 8$ & $*$ & $8.66 \%$ & $0.83[0.66,1.04]$ \\
\hline Subtotal $(95 \% \mathrm{Cl})$ & 38 & 8 & 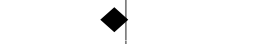 & $8.66 \%$ & $0.83[0.66,1.04]$ \\
\hline \multicolumn{6}{|c|}{ Total events: 30 (Certolizumab pegol), 8 (Control) } \\
\hline \multicolumn{6}{|c|}{ Heterogeneity: Not applicable } \\
\hline \multicolumn{6}{|c|}{ Test for overall effect: $Z=1.61(P=0.11)$} \\
\hline Total $(95 \% \mathrm{Cl})$ & 2190 & 712 & & $100 \%$ & $1.13[0.79,1.63]$ \\
\hline \multicolumn{6}{|c|}{ Total events: 1329 (Certolizumab pegol), 297 (Control) } \\
\hline \multicolumn{6}{|c|}{ Heterogeneity: Tau $^{2}=0.39 ; \mathrm{Chi}^{2}=183.28, \mathrm{df}=12(\mathrm{P}<0.0001) ; \mathrm{I}^{2}=93.45 \%$} \\
\hline \multicolumn{6}{|c|}{ Test for overall effect: $\mathrm{Z}=0.68(\mathrm{P}=0.5)$} \\
\hline Test for subgroup dif & $38, \mathrm{df}=1(\mathrm{P}=0), \mathrm{I}^{2}$ & & & & \\
\hline
\end{tabular}


Analysis 1.2. Comparison 1 Efficacy at 12 weeks, any dose, Outcome 2 ACR50.

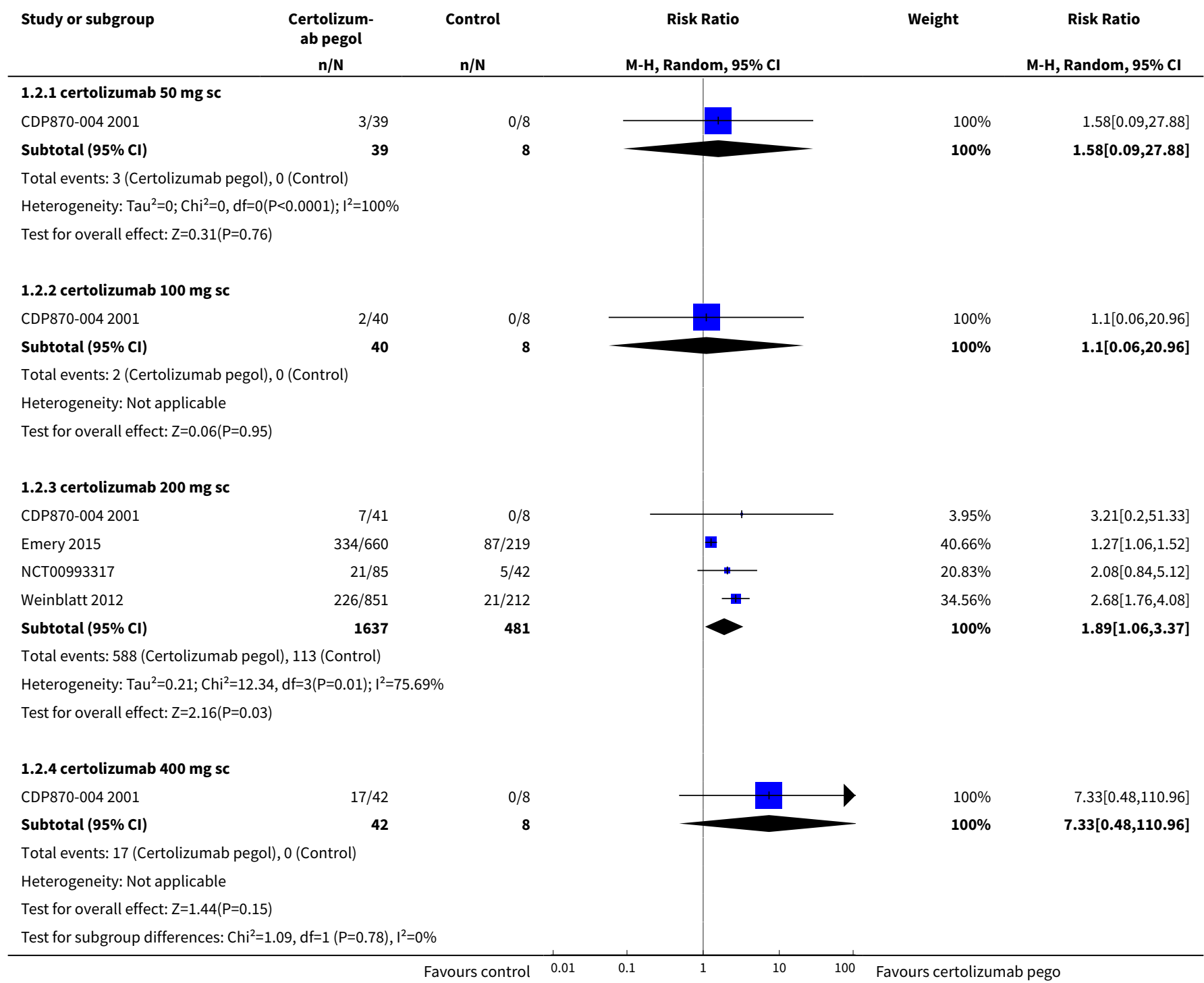

Analysis 1.3. Comparison 1 Efficacy at 12 weeks, any dose, Outcome 3 ACR70.

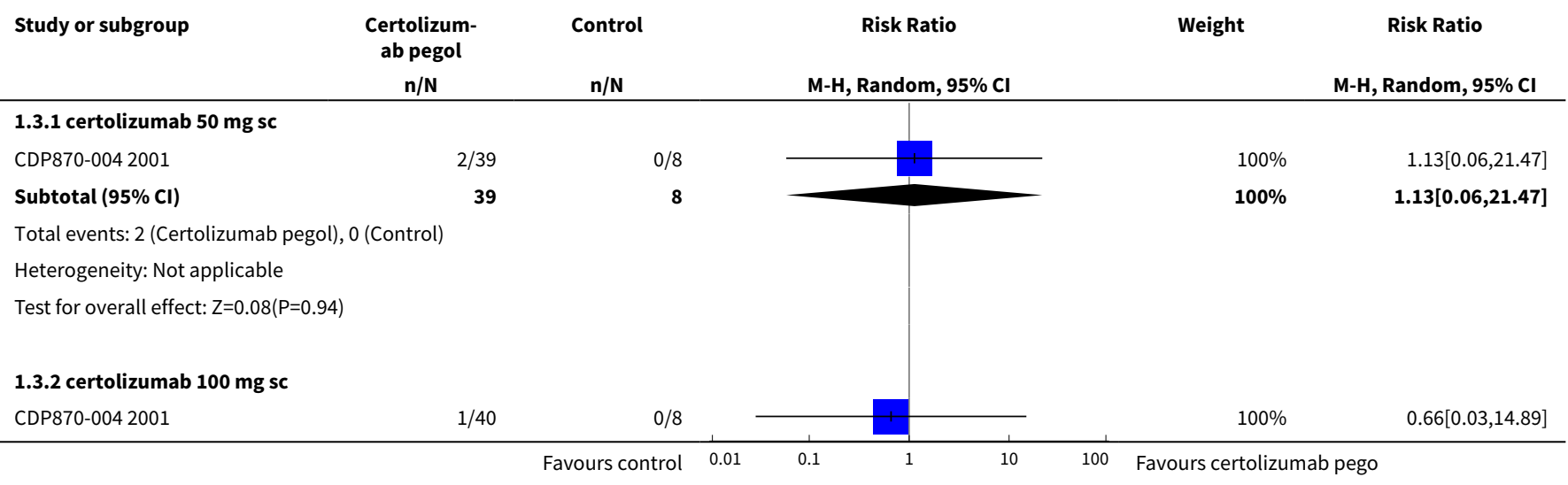




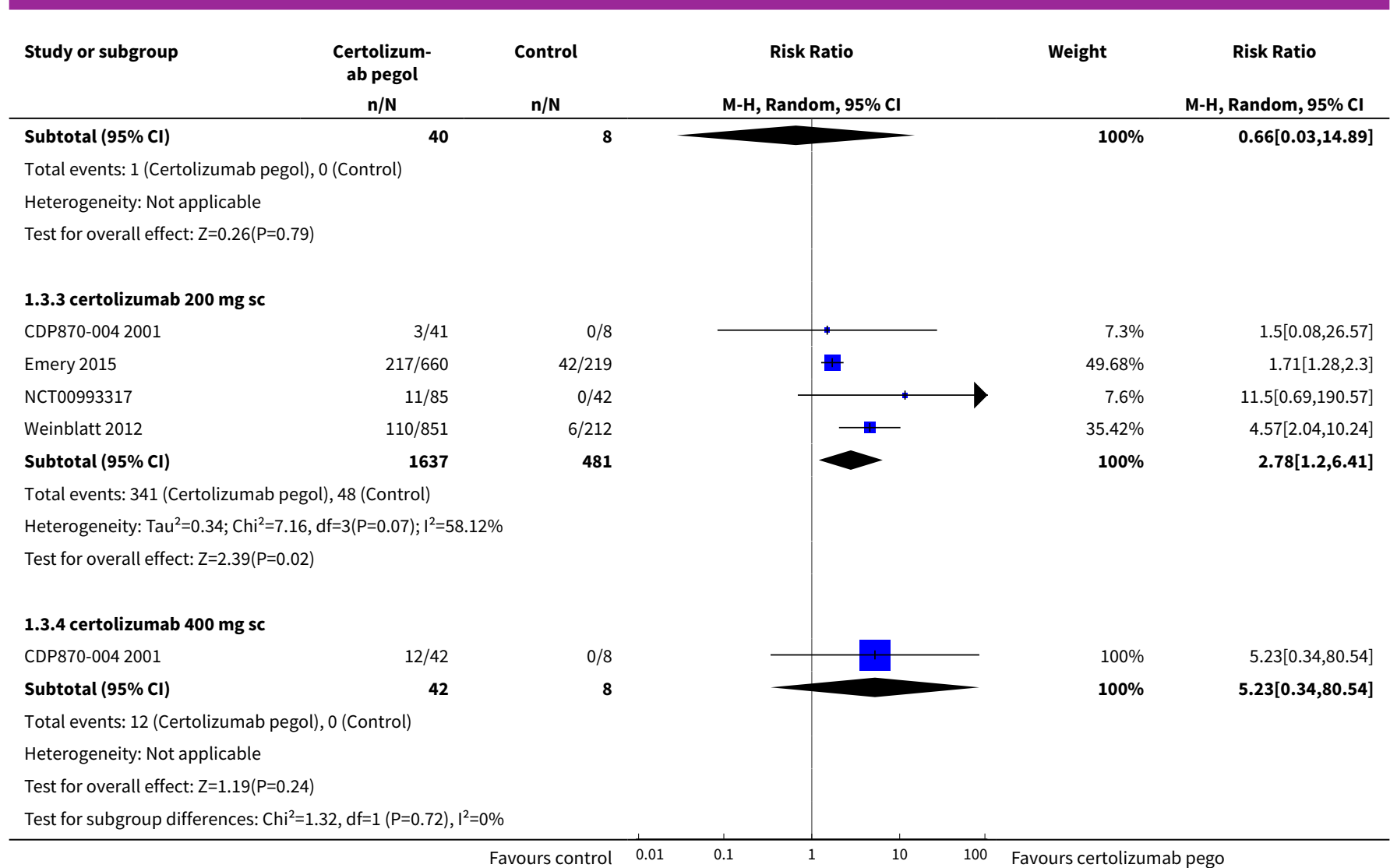

\section{Comparison 2. ACR50 24 weeks, $200 \mathrm{mg}$ certolizumab pegol}

\begin{tabular}{lllll}
\hline $\begin{array}{l}\text { Outcome or subgroup } \\
\text { title }\end{array}$ & No. of studies & $\begin{array}{l}\text { No. of partici- } \\
\text { pants }\end{array}$ & Statistical method & Effect size \\
\hline 1 ACR 50 & 5 & 1445 & Risk Ratio (M-H, Random, 95\% Cl) & $3.80[2.42,5.95]$ \\
\hline
\end{tabular}

Analysis 2.1. Comparison 2 ACR50 24 weeks, 200 mg certolizumab pegol, Outcome 1 ACR 50.

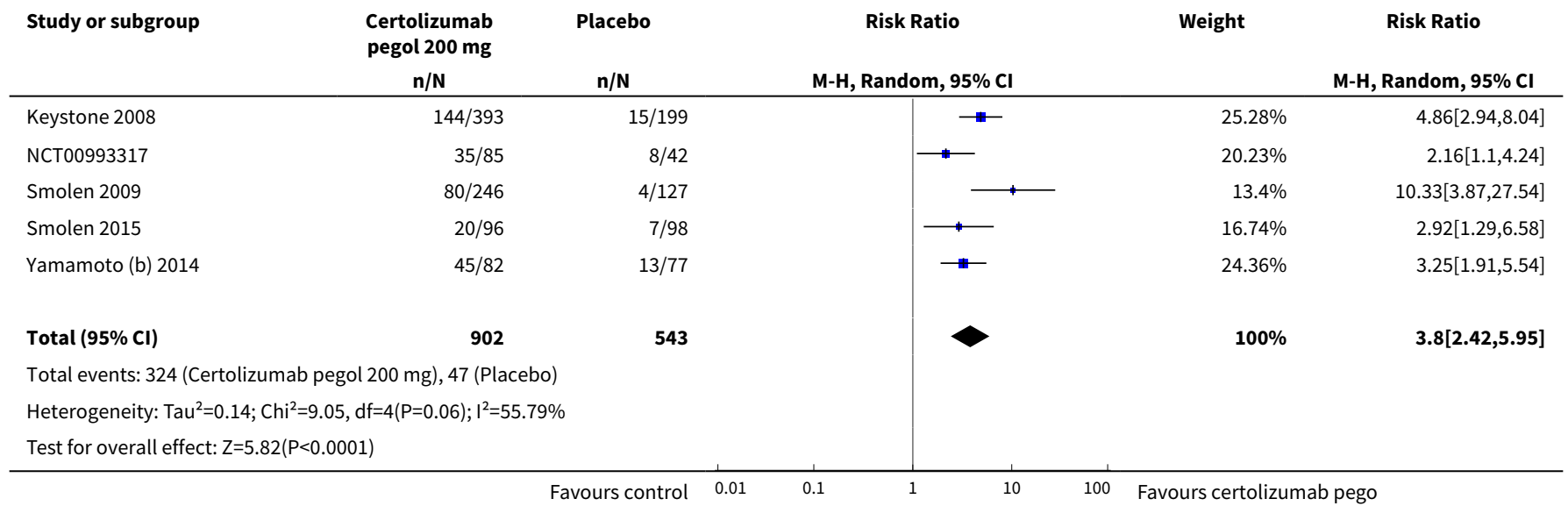


Comparison 3. ACR50 at 24 weeks, 400 mg certolizumab

\begin{tabular}{lllll}
\hline $\begin{array}{l}\text { Outcome or subgroup } \\
\text { title }\end{array}$ & No. of studies & $\begin{array}{l}\text { No. of partici- } \\
\text { pants }\end{array}$ & Statistical method & Effect size \\
\hline 1 ACR 50 & 5 & 1591 & Risk Ratio (M-H, Random, 95\% Cl) & $4.65[3.09,6.99]$ \\
\hline
\end{tabular}

\section{Analysis 3.1. Comparison 3 ACR50 at 24 weeks, 400 mg certolizumab, Outcome 1 ACR 50.}

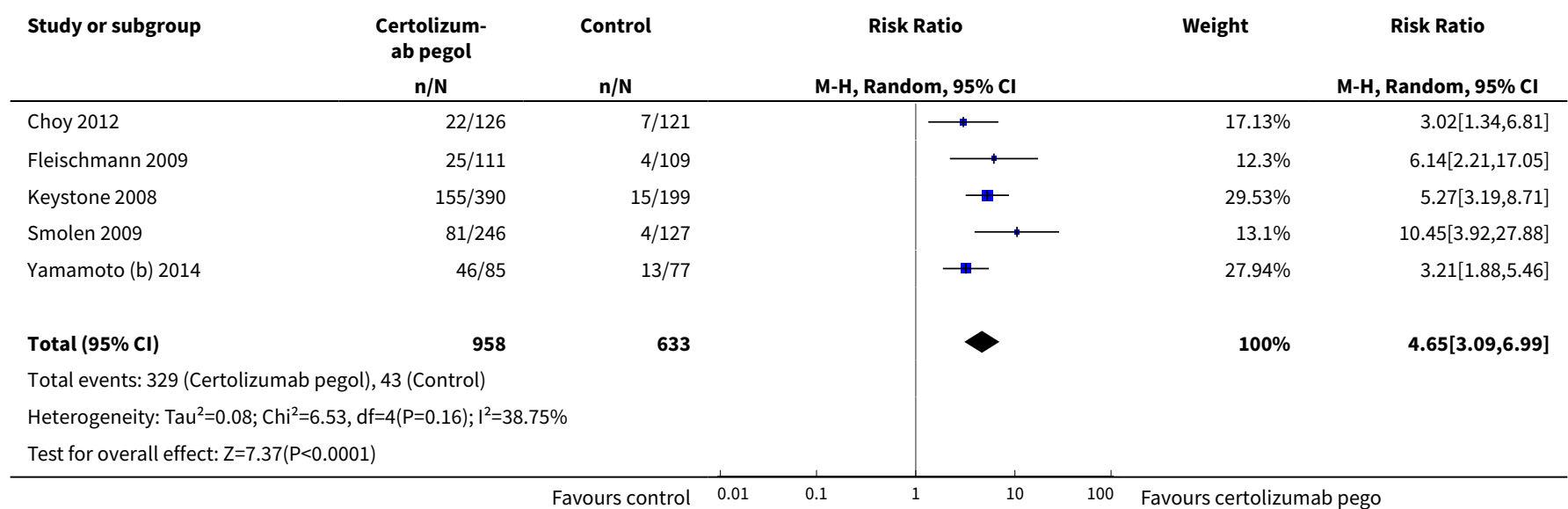

\section{Comparison 4. ACR50 at 52 weeks, $200 \mathrm{mg}$ certolizumab}

\begin{tabular}{lllll}
\hline $\begin{array}{l}\text { Outcome or subgroup } \\
\text { title }\end{array}$ & No. of studies & $\begin{array}{l}\text { No. of partici- } \\
\text { pants }\end{array}$ & Statistical method & Effect size \\
\hline 1 ACR 50 & 3 & 1790 & Risk Ratio (M-H, Fixed, 95\% Cl) & $1.54[1.38,1.73]$ \\
\hline
\end{tabular}

Analysis 4.1. Comparison 4 ACR50 at 52 weeks, 200 mg certolizumab, Outcome 1 ACR 50.

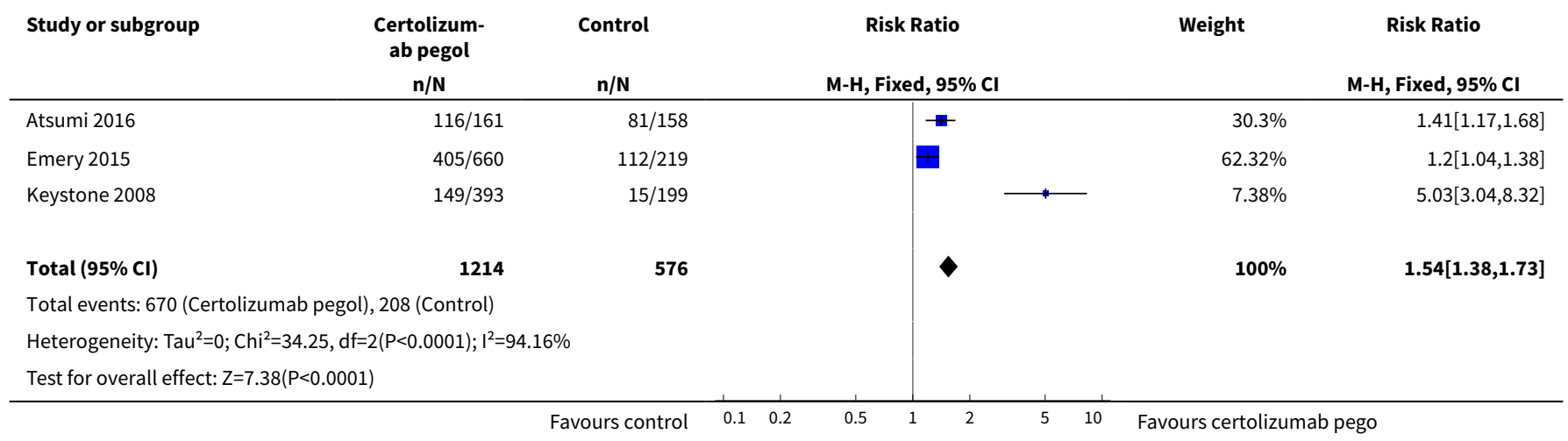


Comparison 5. ACR50 at 52 weeks, 400 mg certolizumab

\begin{tabular}{lllll}
\hline $\begin{array}{l}\text { Outcome or subgroup } \\
\text { title }\end{array}$ & No. of studies & $\begin{array}{l}\text { No. of partici- } \\
\text { pants }\end{array}$ & Statistical method & Effect size \\
\hline 1 ACR 50 & 1 & Risk Ratio (M-H, Fixed, 95\% Cl) & Totals not selected \\
\hline
\end{tabular}

Analysis 5.1. Comparison 5 ACR50 at 52 weeks, 400 mg certolizumab, Outcome 1 ACR 50.

\begin{tabular}{|c|c|c|c|c|c|c|c|}
\hline Study or subgroup & $\begin{array}{c}\text { Certolizumab pegol } \\
n / N\end{array}$ & $\begin{array}{c}\text { Control } \\
n / N\end{array}$ & & & $\begin{array}{l}\text { k Ratio } \\
\text { red, } 95 \% \mathrm{Cl}\end{array}$ & & $\begin{array}{c}\text { Risk Ratio } \\
\text { M-H, Fixed, 95\% Cl }\end{array}$ \\
\hline Keystone 2008 & $155 / 390$ & $15 / 199$ & & & 十 & & $5.27[3.19,8.71]$ \\
\hline
\end{tabular}

\section{Comparison 6. Mean HAQ-DI from baseline at week 12}

\begin{tabular}{lllll}
\hline Outcome or subgroup title & No. of studies & $\begin{array}{l}\text { No. of partici- } \\
\text { pants }\end{array}$ & Statistical method & Effect size \\
\hline 1 certolizumab pegol 200 mg sc & 1 & 1063 & $\begin{array}{l}\text { Mean Difference (IV, Fixed, 95\% } \\
\text { Cl) }\end{array}$ & $-0.22[-0.23,-0.21]$ \\
\hline
\end{tabular}

Analysis 6.1. Comparison 6 Mean HAQ-DI from baseline at week 12, Outcome 1 certolizumab pegol 200 mg sc.

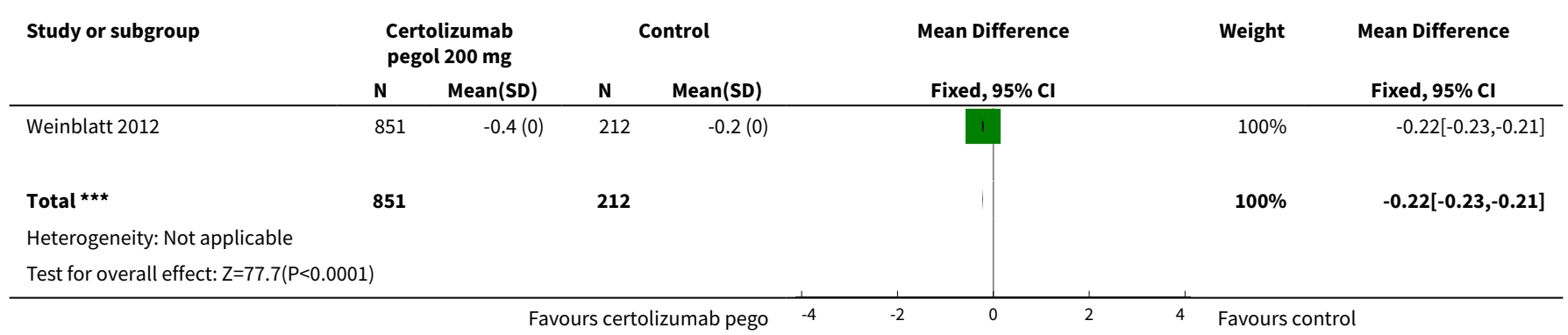

\section{Comparison 7. Mean HAQ-DI from baseline at week 24}

\begin{tabular}{llll}
\hline Outcome or subgroup title & No. of studies & $\begin{array}{l}\text { No. of partici- } \\
\text { pants }\end{array}$ & Statistical method \\
\hline $\begin{array}{l}1 \text { certolizumab pegol } 200 \mathrm{mg} \\
\text { SC }\end{array}$ & 4 & 1268 & Mean Difference (IV, Random, 95\% \\
\hline 2 certolizumab $400 \mathrm{mg} \mathrm{SC}$ & 4 & 1425 & $\mathrm{Cl})$ \\
\hline
\end{tabular}


Analysis 7.1. Comparison 7 Mean HAQ-DI from baseline at week 24, Outcome 1 certolizumab pegol $200 \mathrm{mg} \mathrm{sc}$.

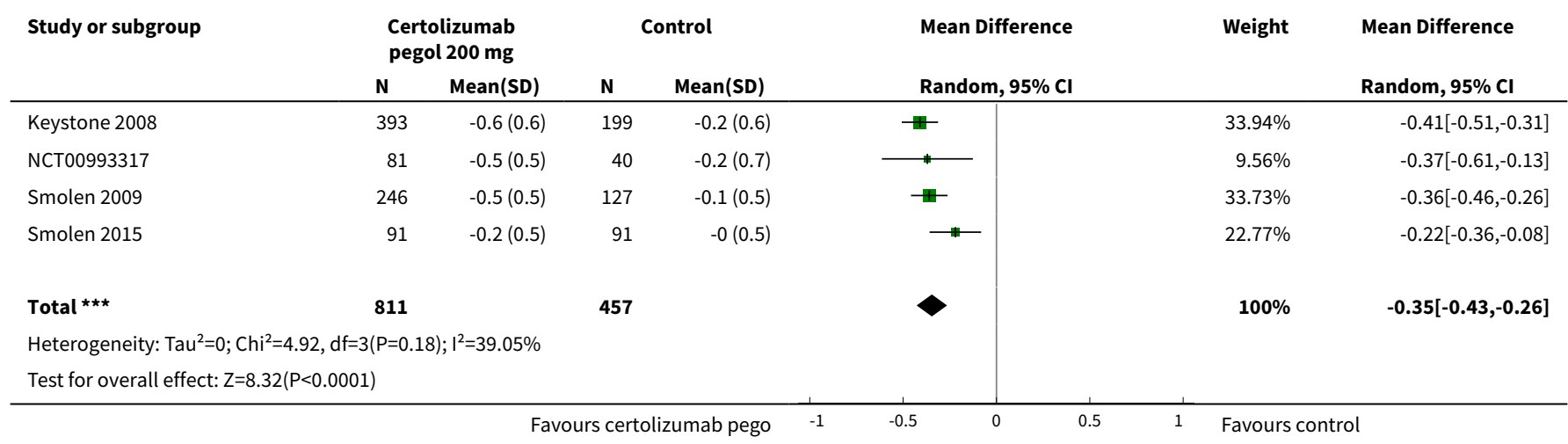

\section{Analysis 7.2. Comparison 7 Mean HAQ-DI from baseline at week 24, Outcome 2 certolizumab $400 \mathrm{mg} \mathrm{sc}$.}

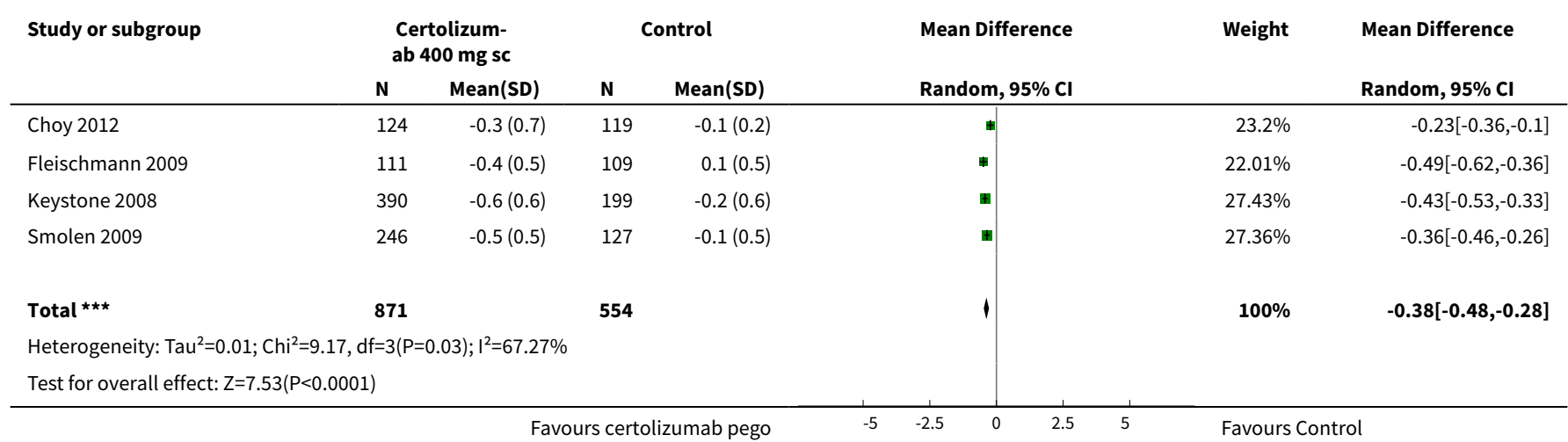

\section{Comparison 8. HAQ-DI at 24 weeks, any dose}

\begin{tabular}{|c|c|c|c|c|}
\hline Outcome or subgroup title & No. of studies & $\begin{array}{l}\text { No. of partici- } \\
\text { pants }\end{array}$ & Statistical method & Effect size \\
\hline 1 Change from baseline & 5 & 2246 & $\begin{array}{l}\text { Mean Difference (IV, Random, 95\% } \\
\mathrm{Cl} \text { ) }\end{array}$ & $-0.36[-0.43,-0.29]$ \\
\hline $\begin{array}{l}1.1 \text { certolizumab pegol } 200 \\
\mathrm{mg} \mathrm{sc}\end{array}$ & 3 & 985 & $\begin{array}{l}\text { Mean Difference (IV, Random, 95\% } \\
\mathrm{Cl} \text { ) }\end{array}$ & $-0.33[-0.44,-0.23]$ \\
\hline $\begin{array}{l}1.2 \text { certolizumab pegol } 400 \\
\mathrm{mg} \mathrm{sc}\end{array}$ & 4 & 1261 & $\begin{array}{l}\text { Mean Difference (IV, Random, 95\% } \\
\mathrm{Cl} \text { ) }\end{array}$ & $-0.38[-0.48,-0.27]$ \\
\hline
\end{tabular}


Analysis 8.1. Comparison $8 \mathrm{HAQ}$-DI at 24 weeks, any dose, Outcome 1 Change from baseline.

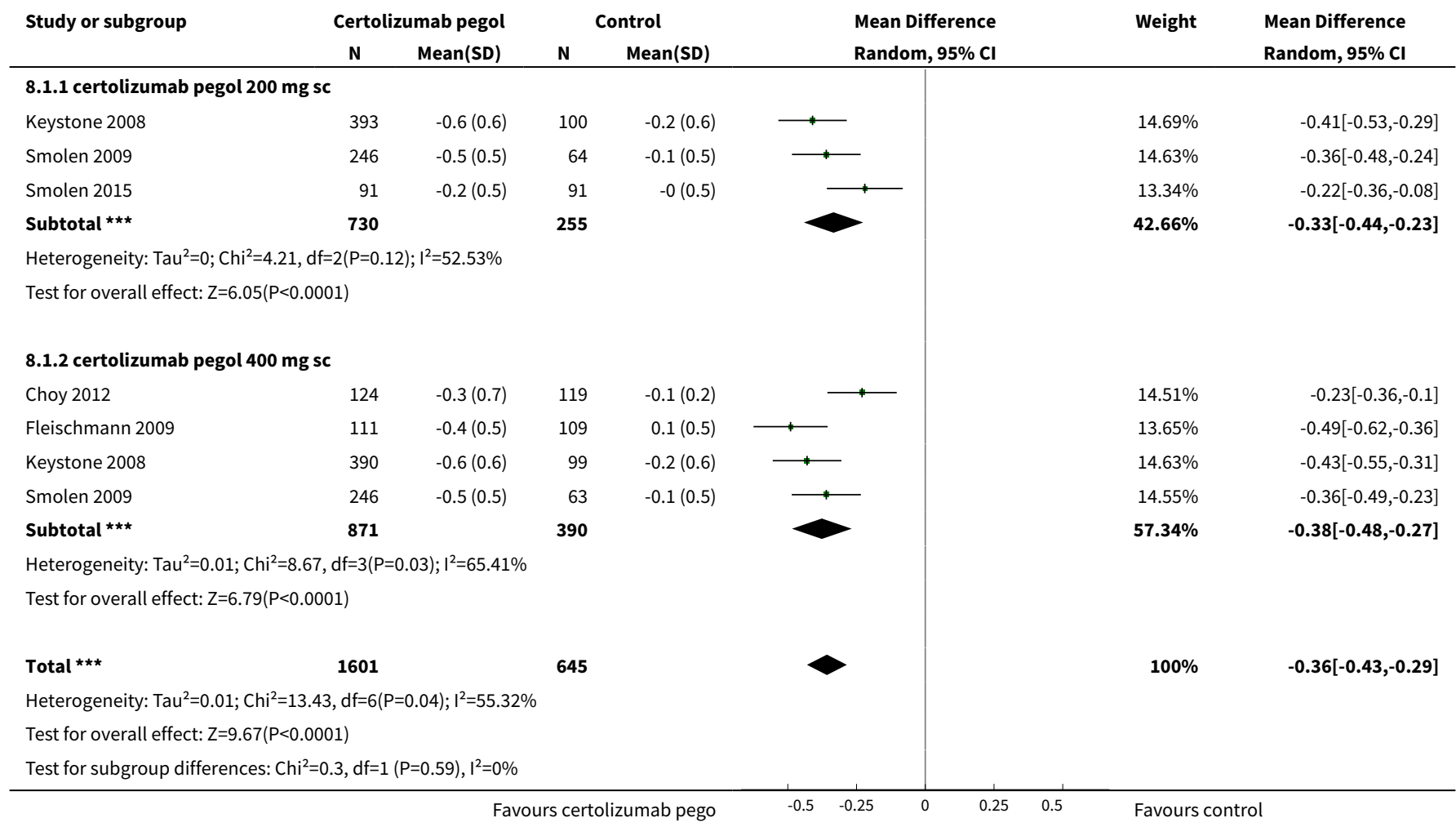

\section{Comparison 9. HAQ-DI at 52 weeks, any dose}

\begin{tabular}{|c|c|c|c|c|}
\hline Outcome or subgroup title & No. of studies & $\begin{array}{l}\text { No. of partici- } \\
\text { pants }\end{array}$ & Statistical method & Effect size \\
\hline 1 Change from baseline & 2 & 1837 & $\begin{array}{l}\text { Mean Difference (IV, Fixed, 95\% } \\
\mathrm{CI})\end{array}$ & $-0.32[-0.39,-0.26]$ \\
\hline $\begin{array}{l}1.1 \text { certolizumab pegol } 200 \mathrm{mg} \\
\text { sc }\end{array}$ & 2 & 1348 & $\begin{array}{l}\text { Mean Difference (IV, Fixed, 95\% } \\
\mathrm{CI})\end{array}$ & $-0.27[-0.35,-0.20]$ \\
\hline $\begin{array}{l}1.2 \text { certolizumab pegol } 400 \mathrm{mg} \\
\mathrm{sc}\end{array}$ & 1 & 489 & $\begin{array}{l}\text { Mean Difference (IV, Fixed, 95\% } \\
\mathrm{CI} \text { ) }\end{array}$ & $-0.45[-0.57,-0.33]$ \\
\hline
\end{tabular}

Analysis 9.1. Comparison $9 \mathrm{HAQ}-\mathrm{DI}$ at 52 weeks, any dose, Outcome 1 Change from baseline.

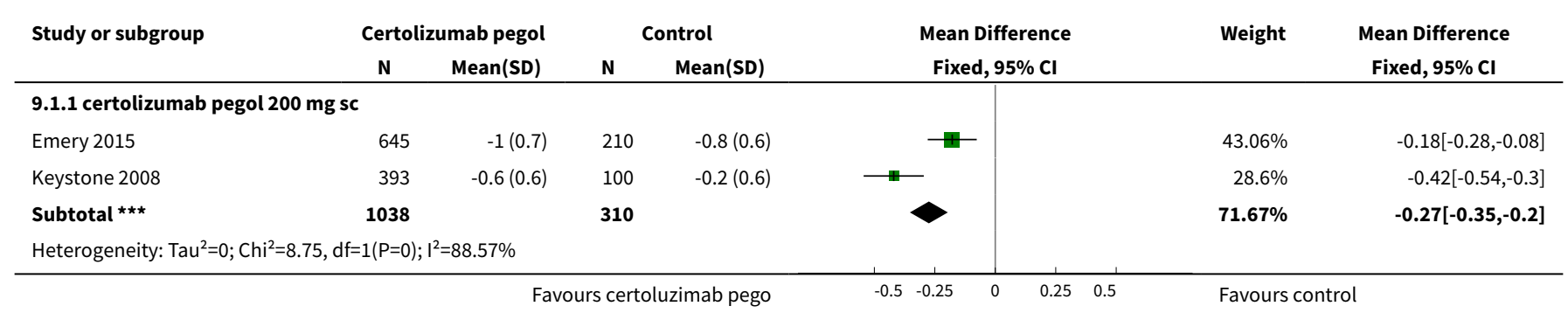




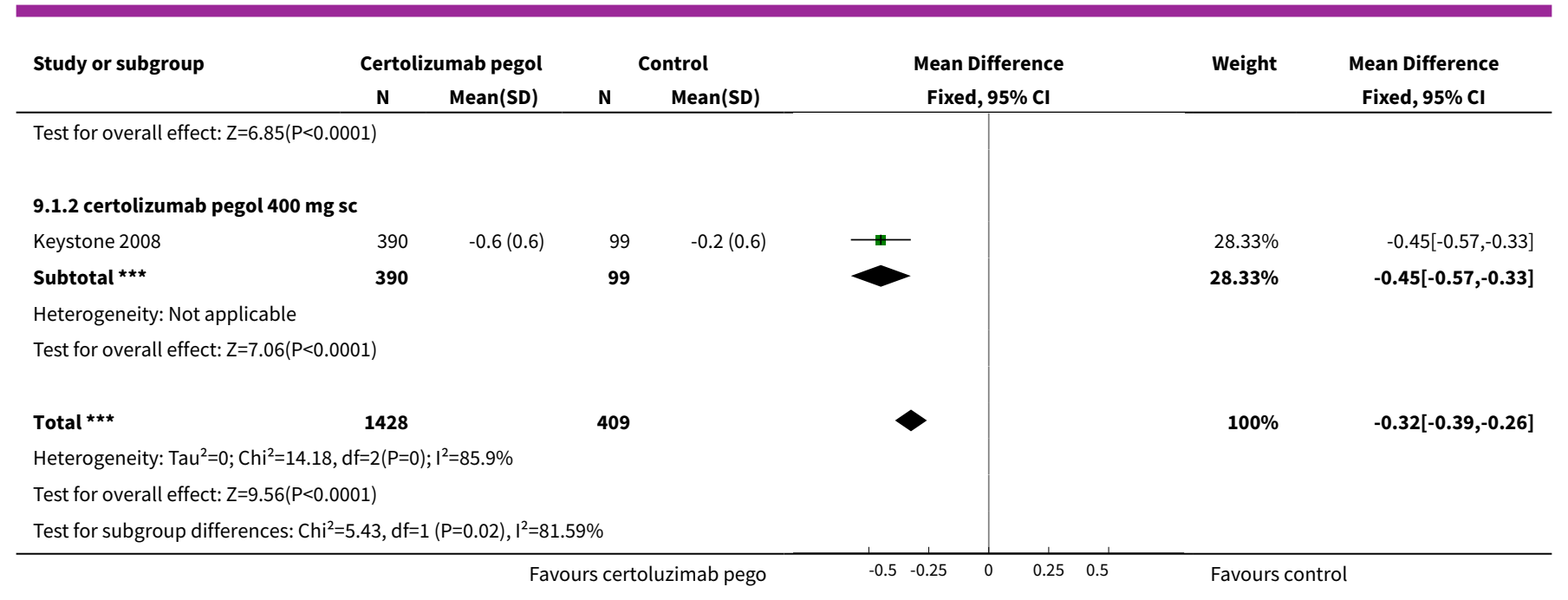

\section{Comparison 10. SF-36 Physical Component Summary (PCS), week 24}

\begin{tabular}{lllll}
\hline Outcome or subgroup title & No. of studies & $\begin{array}{l}\text { No. of partici- } \\
\text { pants }\end{array}$ & Statistical method & Effect size \\
\hline $\begin{array}{l}1 \text { certolizumab pegol } 200 \mathrm{mg} \\
\text { SC }\end{array}$ & 3 & 1129 & $\begin{array}{l}\text { Mean Difference (IV, Random, 95\% } \\
\text { CI) }\end{array}$ & 5.03 [3.90, 6.16] \\
\hline $\begin{array}{l}2 \text { certolizumab pegol } 400 \mathrm{mg} \\
\text { SC }\end{array}$ & 3 & 1205 & $\begin{array}{l}\text { Mean Difference (IV, Random, 95\% } \\
\text { CI) }\end{array}$ & $5.54[4.11,6.97]$ \\
\hline
\end{tabular}

Analysis 10.1. Comparison 10 SF-36 Physical Component Summary (PCS), week 24, Outcome 1 certolizumab pegol $200 \mathrm{mg} \mathrm{sc}$.

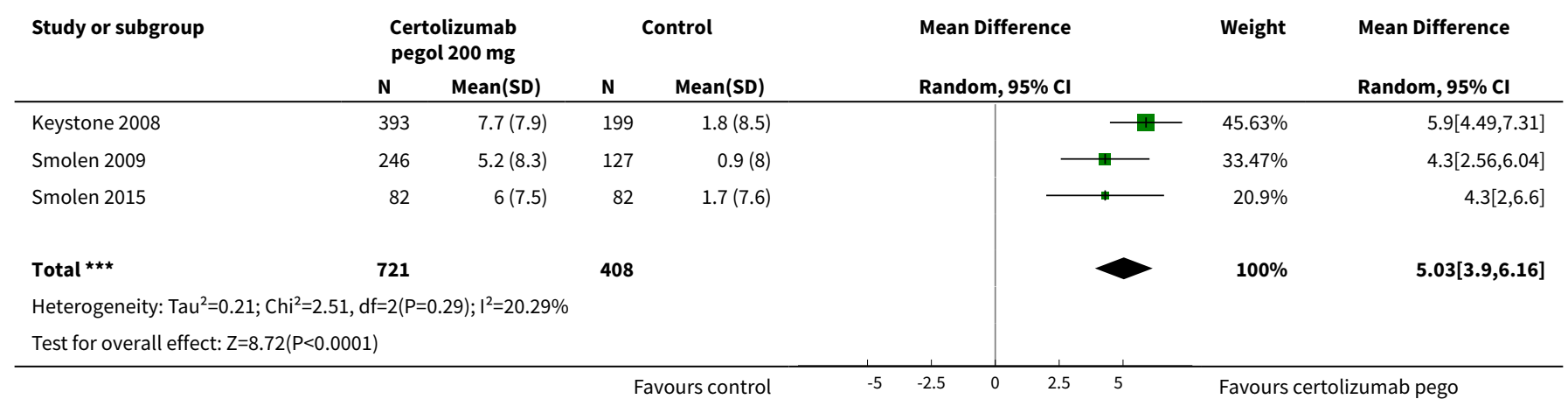




\section{Analysis 10.2. Comparison 10 SF-36 Physical Component Summary}

(PCS), week 24, Outcome 2 certolizumab pegol $400 \mathrm{mg} \mathrm{sc}$.

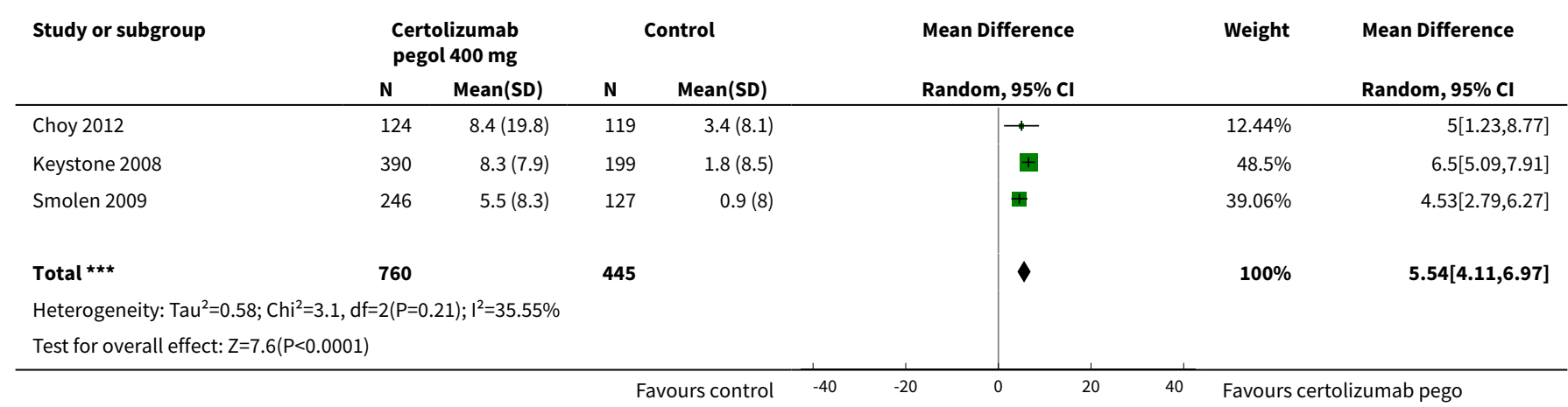

Comparison 11. SF-36 Mental Component Summary (MCS), week 24

\begin{tabular}{lllll}
\hline Outcome or subgroup title & No. of studies & $\begin{array}{l}\text { No. of partici- } \\
\text { pants }\end{array}$ & Statistical method & Effect size \\
\hline $\begin{array}{l}1 \text { certolizumab pegol } 200 \mathrm{mg} \\
\text { SC }\end{array}$ & 2 & 965 & $\begin{array}{l}\text { Mean Difference (IV, Random, 95\% } \\
\text { CI) }\end{array}$ & $4.18[2.70,5.66]$ \\
\hline $\begin{array}{l}2 \text { certolizumab pegol } 400 \mathrm{mg} \\
\text { SC }\end{array}$ & 3 & 1205 & $\begin{array}{l}\text { Mean Difference (IV, Random, 95\% } \\
\text { CI) }\end{array}$ & $4.05[2.77,5.34]$ \\
\hline
\end{tabular}

\section{Analysis 11.1. Comparison 11 SF-36 Mental Component Summary} (MCS), week 24, Outcome 1 certolizumab pegol $200 \mathrm{mg} \mathrm{sc}$.

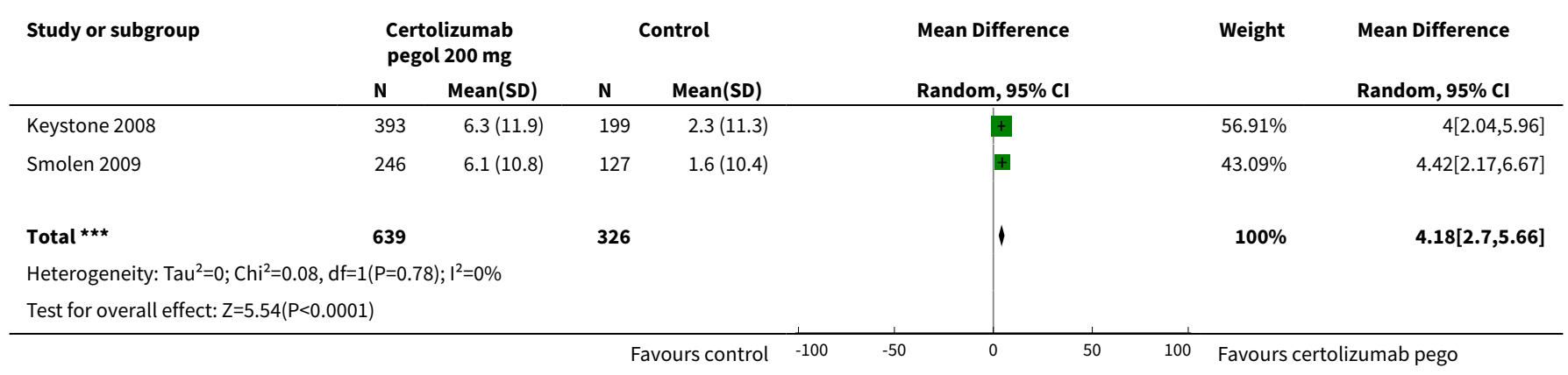

\section{Analysis 11.2. Comparison 11 SF-36 Mental Component Summary}

(MCS), week 24, Outcome 2 certolizumab pegol $400 \mathrm{mg} \mathrm{sc}$.

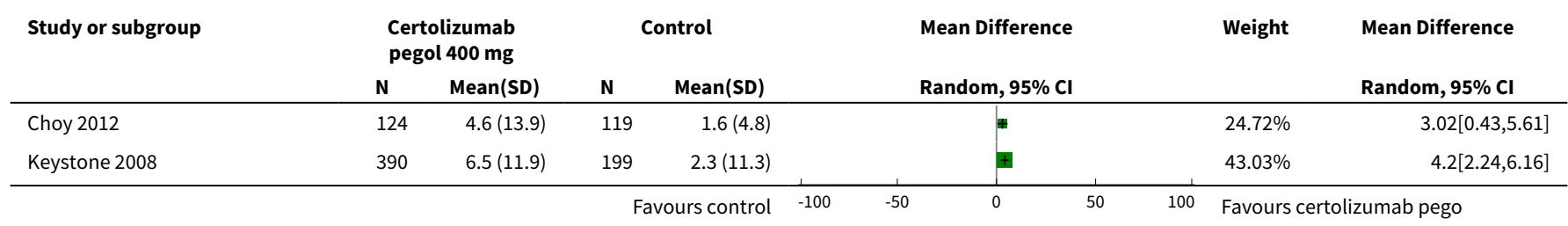




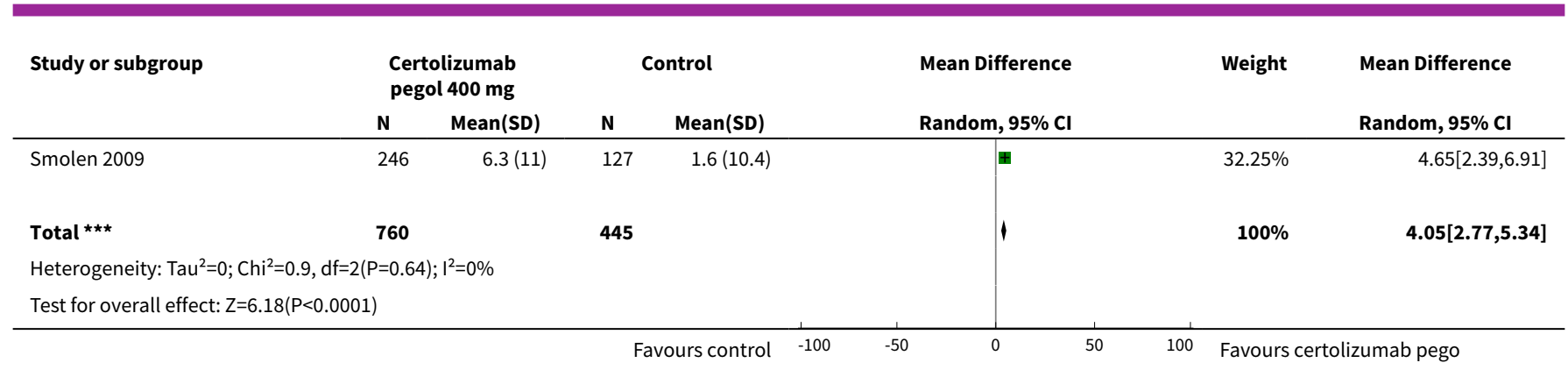

\section{Comparison 12. SF-36 Physical Component Summary (PCS), week 52}

\begin{tabular}{lllll}
\hline Outcome or subgroup title & No. of studies & $\begin{array}{l}\text { No. of partici- } \\
\text { pants }\end{array}$ & Statistical method & Effect size \\
\hline 1 certolizumab $200 \mathrm{mg} \mathrm{sc}$ & 1 & Mean Difference (IV, Fixed, 95\% Cl) & Totals not selected \\
\hline 2 certolizumab $400 \mathrm{mg} \mathrm{sc}$ & 1 & Mean Difference (IV, Fixed, 95\% Cl) & Totals not selected \\
\hline
\end{tabular}

Analysis 12.1. Comparison 12 SF-36 Physical Component Summary (PCS), week 52, Outcome 1 certolizumab 200 mg sc.

\begin{tabular}{|c|c|c|c|c|c|c|c|c|c|}
\hline \multirow[t]{2}{*}{ Study or subgroup } & \multicolumn{2}{|c|}{$\begin{array}{l}\text { Certolizumab } \\
\text { pegol } 200 \mathrm{mg}\end{array}$} & \multicolumn{2}{|c|}{ Control } & \multicolumn{3}{|c|}{ Mean Difference } & & \multirow{2}{*}{$\begin{array}{l}\text { Mean Difference } \\
\text { Fixed, } 95 \% \mathrm{Cl} \\
\end{array}$} \\
\hline & $\mathbf{N}$ & $\operatorname{Mean}(\mathrm{SD})$ & $\mathbf{N}$ & Mean(SD) & & & ,95\% Cl & & \\
\hline Keystone 2008 & 393 & $7.8(8.7)$ & 199 & $1.7(8.6)$ & & & + & & $6.06[4.59,7.53]$ \\
\hline
\end{tabular}

Analysis 12.2. Comparison 12 SF-36 Physical Component Summary (PCS), week 52, Outcome 2 certolizumab 400 mg sc.

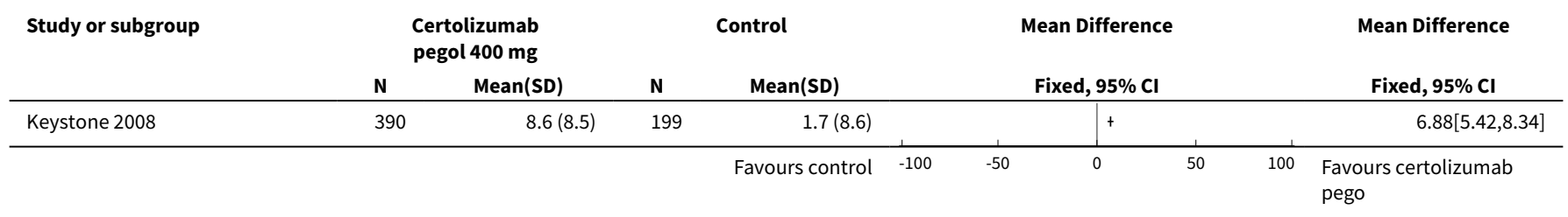

Comparison 13. SF-36 Mental Component Summary (MCS), week 52

\begin{tabular}{lllll}
\hline Outcome or subgroup title & No. of studies & $\begin{array}{l}\text { No. of partici- } \\
\text { pants }\end{array}$ & Statistical method & Effect size \\
\hline $\begin{array}{l}1 \text { certolizumab pegol } 200 \mathrm{mg} \\
\text { SC }\end{array}$ & 1 & Mean Difference (IV, Fixed, 95\% Cl) & Totals not selected \\
\hline
\end{tabular}




\begin{tabular}{lllll}
\hline Outcome or subgroup title & No. of studies & $\begin{array}{l}\text { No. of partici- } \\
\text { pants }\end{array}$ & Statistical method & Effect size \\
\hline $\begin{array}{l}2 \text { certolizumab pegol } 400 \mathrm{mg} \\
\text { SC }\end{array}$ & 1 & Mean Difference (IV, Fixed, 95\% CI) & Totals not selected \\
\hline
\end{tabular}

Analysis 13.1. Comparison 13 SF-36 Mental Component Summary

(MCS), week 52, Outcome 1 certolizumab pegol $200 \mathrm{mg} \mathrm{sc.}$

\begin{tabular}{|c|c|c|c|c|c|c|c|c|c|c|}
\hline \multirow[t]{2}{*}{ Study or subgroup } & \multicolumn{2}{|c|}{ Certolizumab 200 mg sc } & \multicolumn{2}{|c|}{ Control } & \multirow{2}{*}{\multicolumn{4}{|c|}{$\begin{array}{c}\text { Mean Difference } \\
\text { Fixed, } 95 \% \mathrm{Cl}\end{array}$}} & \multirow{2}{*}{\multicolumn{2}{|c|}{$\begin{array}{c}\text { Mean Difference } \\
\text { Fixed, } 95 \% \mathrm{Cl}\end{array}$}} \\
\hline & $\mathbf{N}$ & $\operatorname{Mean}(S D)$ & $\mathbf{N}$ & Mean(SD) & & & & & & \\
\hline \multirow[t]{2}{*}{ Keystone 2008} & 393 & $6.4(11.1)$ & 199 & $2.1(11.1)$ & & & + & & & $4.3[2.4,6.2]$ \\
\hline & & & & Favours control & -100 & -50 & 0 & 50 & 100 & $\begin{array}{l}\text { Favours certolizumab } \\
\text { pego }\end{array}$ \\
\hline
\end{tabular}

\section{Analysis 13.2. Comparison 13 SF-36 Mental Component Summary} (MCS), week 52, Outcome 2 certolizumab pegol 400 mg sc.

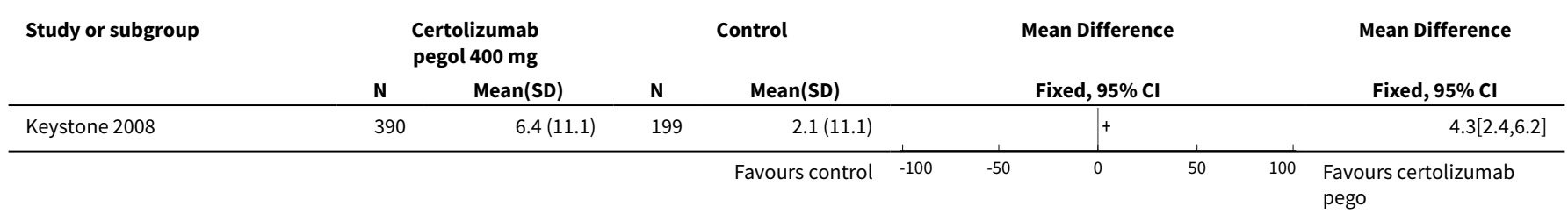

Comparison 14. SF-36 Physical Component Summary (PCS) at week 24, any dose

\begin{tabular}{lllll}
\hline Outcome or subgroup title & No. of studies & $\begin{array}{l}\text { No. of partici- } \\
\text { pants }\end{array}$ & Statistical method & Effect size \\
\hline $\begin{array}{l}1 \text { Change from baseline } \\
\text { p }\end{array}$ & 3 & 1765 & $\begin{array}{l}\text { Mean Difference (IV, Random, 95\% } \\
\text { Cl) }\end{array}$ & $5.29[4.37,6.21]$ \\
\hline $\begin{array}{l}1.1 \text { certolizumab pegol } 200 \mathrm{mg} \\
\text { sc }\end{array}$ & 3 & 967 & $\begin{array}{l}\text { Mean Difference (IV, Random, 95\% } \\
\text { Cl) }\end{array}$ & $4.99[3.79,6.20]$ \\
\hline $\begin{array}{l}1.2 \text { certolizumab pegol } 400 \mathrm{mg} \\
\text { sc }\end{array}$ & 2 & 798 & $\begin{array}{l}\text { Mean Difference (IV, Random, 95\% } \\
\text { Cl) }\end{array}$ & $5.62[3.70,7.54]$ \\
\hline
\end{tabular}

\section{Analysis 14.1. Comparison 14 SF-36 Physical Component Summary} (PCS) at week 24, any dose, Outcome 1 Change from baseline.

\begin{tabular}{|c|c|c|c|c|c|c|c|c|}
\hline \multirow[t]{2}{*}{ Study or subgroup } & \multicolumn{2}{|c|}{ Certolizumab pegol } & \multicolumn{2}{|c|}{ Control } & \multirow{2}{*}{\multicolumn{2}{|c|}{$\begin{array}{l}\text { Mean Difference } \\
\text { Random, } 95 \% \mathrm{Cl}\end{array}$}} & \multirow[t]{2}{*}{ Weight } & \multirow{2}{*}{$\begin{array}{l}\text { Mean Difference } \\
\text { Random, } 95 \% \mathrm{Cl}\end{array}$} \\
\hline & $\mathbf{N}$ & Mean(SD) & $\mathbf{N}$ & Mean(SD) & & & & \\
\hline \multicolumn{9}{|c|}{ 14.1.1 certolizumab pegol $200 \mathrm{mg} \mathrm{sc}$} \\
\hline Keystone 2008 & 393 & $7.7(7.9)$ & 100 & $1.8(8.5)$ & & $\longrightarrow$ & $25.1 \%$ & $5.9[4.07,7.73]$ \\
\hline Smolen 2009 & 246 & $5.2(8.3)$ & 64 & $0.9(8)$ & & $\longrightarrow$ & $17.16 \%$ & $4.3[2.08,6.52]$ \\
\hline
\end{tabular}




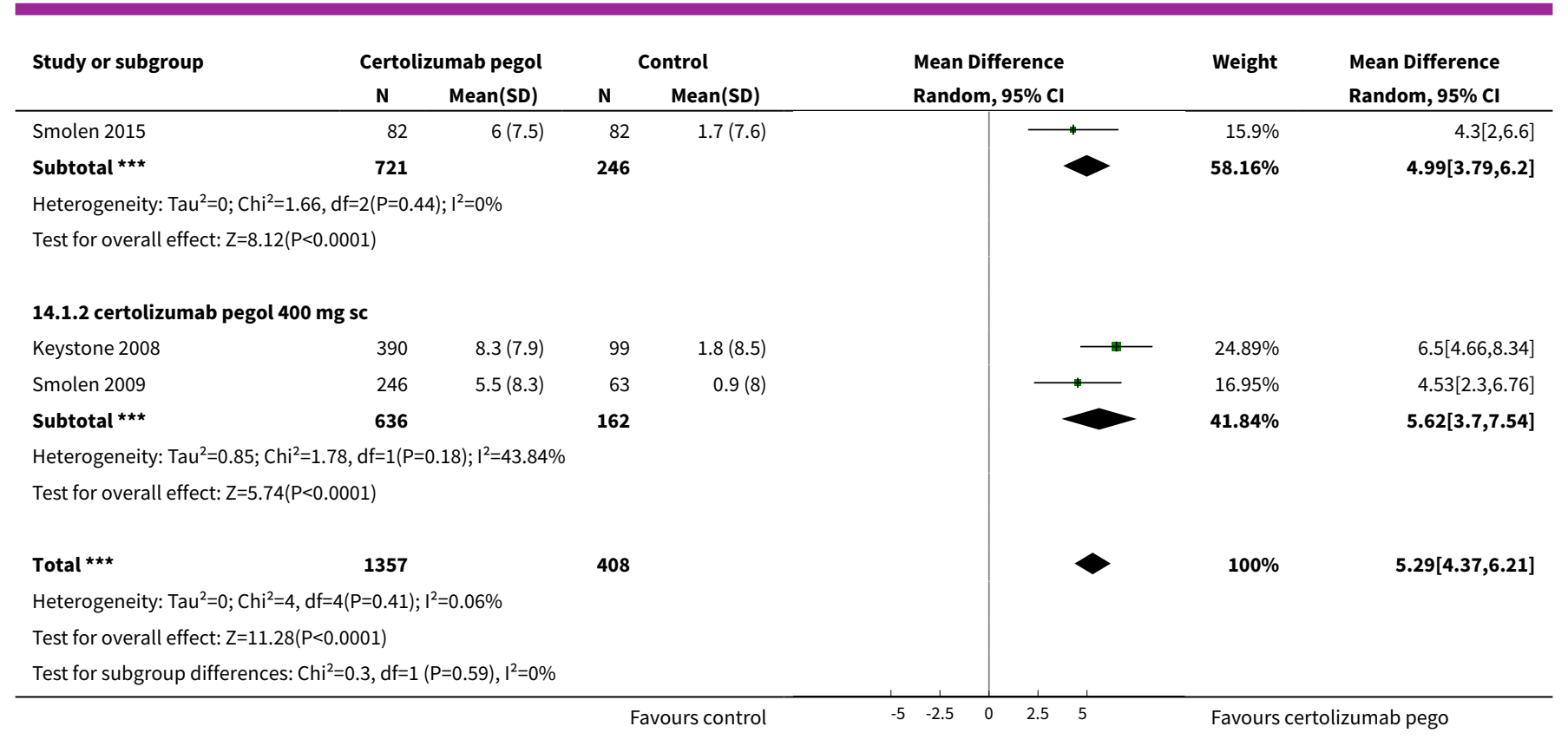

Comparison 15. SF-36 Mental Component Summary (MCS) at week 24, any dose

\begin{tabular}{|c|c|c|c|c|}
\hline Outcome or subgroup title & No. of studies & $\begin{array}{l}\text { No. of partici- } \\
\text { pants }\end{array}$ & Statistical method & Effect size \\
\hline 1 Change from baseline & 4 & 2012 & $\begin{array}{l}\text { Mean Difference (IV, Random, 95\% } \\
\mathrm{CI})\end{array}$ & $4.01[2.94,5.08]$ \\
\hline $\begin{array}{l}1.1 \text { certolizumab pegol } 200 \mathrm{mg} \\
\mathrm{sc}\end{array}$ & 3 & 971 & $\begin{array}{l}\text { Mean Difference (IV, Random, 95\% } \\
\mathrm{CI})\end{array}$ & $4.11[2.62,5.61]$ \\
\hline $\begin{array}{l}1.2 \text { certolizumab pegol } 400 \mathrm{mg} \\
\mathrm{sc}\end{array}$ & 3 & 1041 & $\begin{array}{l}\text { Mean Difference (IV, Random, 95\% } \\
\mathrm{Cl} \text { ) }\end{array}$ & $3.91[2.38,5.44]$ \\
\hline
\end{tabular}

\section{Analysis 15.1. Comparison 15 SF-36 Mental Component Summary} (MCS) at week 24, any dose, Outcome 1 Change from baseline.

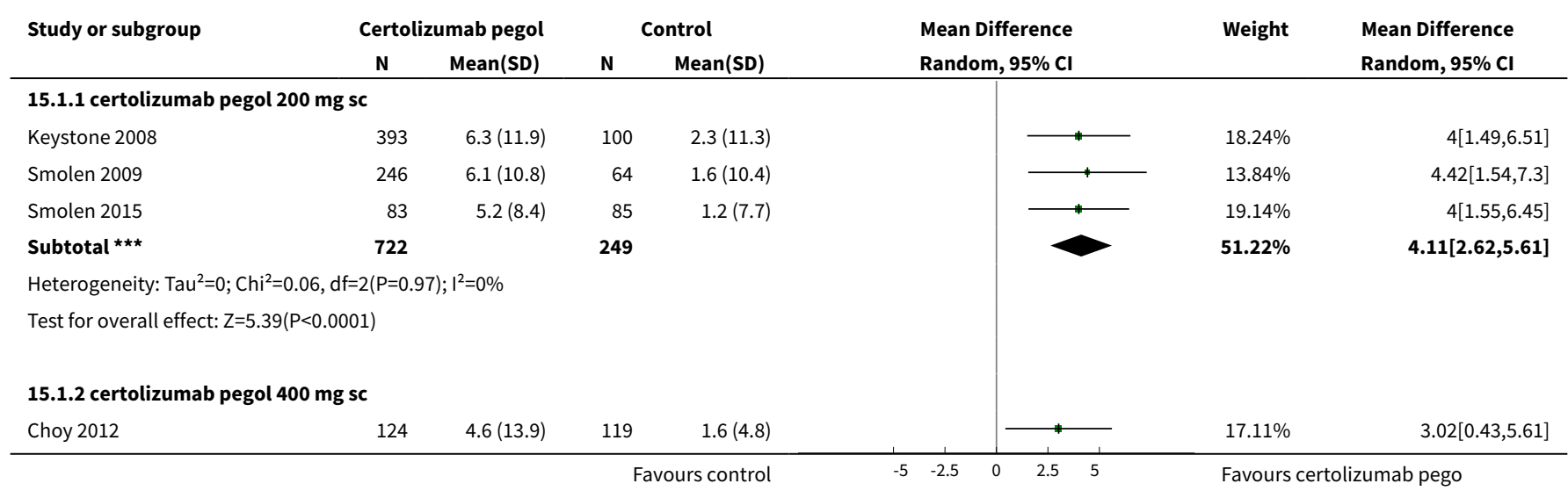




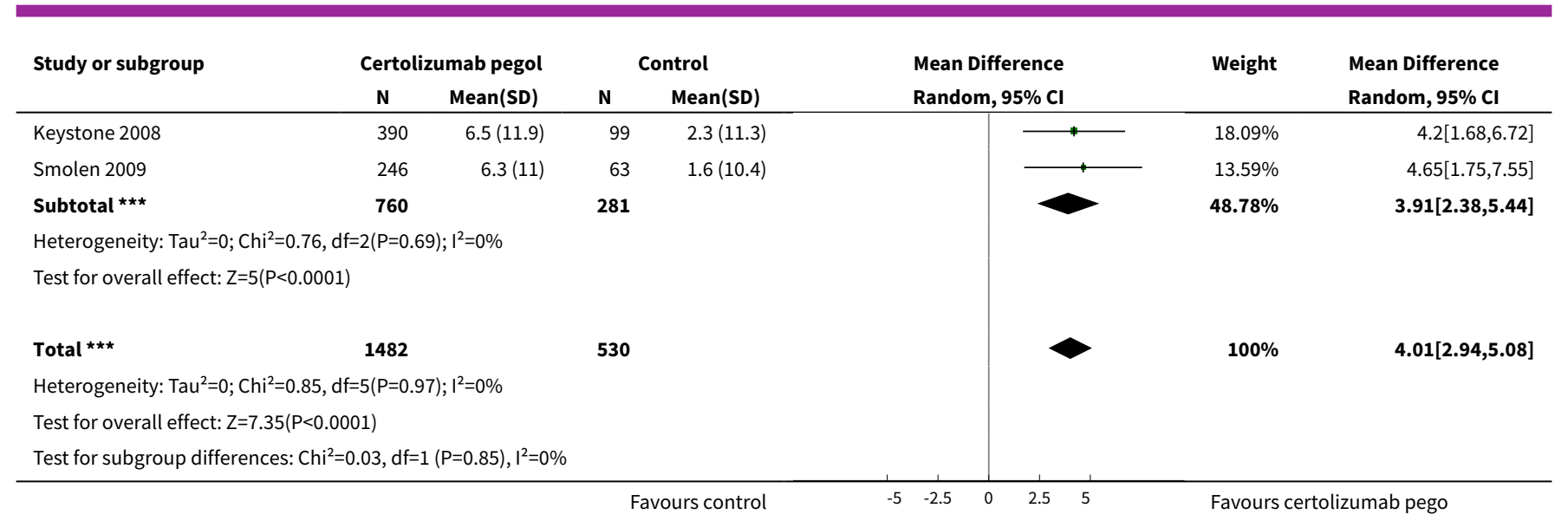

Comparison 16. SF-36 Physical Component Summary (PCS) at week 52, any dose

\begin{tabular}{|c|c|c|c|c|}
\hline Outcome or subgroup title & No. of studies & $\begin{array}{l}\text { No. of partici- } \\
\text { pants }\end{array}$ & Statistical method & Effect size \\
\hline 1 Change from baseline & 1 & & Mean Difference (IV, Fixed, 95\% CI) & $\begin{array}{l}\text { Totals not select- } \\
\text { ed }\end{array}$ \\
\hline $\begin{array}{l}1.1 \text { certolizumab pegol } 200 \mathrm{mg} \\
\text { sc }\end{array}$ & 1 & & Mean Difference (IV, Fixed, 95\% CI) & $0.0[0.0,0.0]$ \\
\hline $\begin{array}{l}1.2 \text { certolizumab pegol } 400 \mathrm{mg} \\
\text { sc }\end{array}$ & 1 & & Mean Difference (IV, Fixed, 95\% CI) & $0.0[0.0,0.0]$ \\
\hline
\end{tabular}

Analysis 16.1. Comparison 16 SF-36 Physical Component Summary (PCS) at week 52, any dose, Outcome 1 Change from baseline.

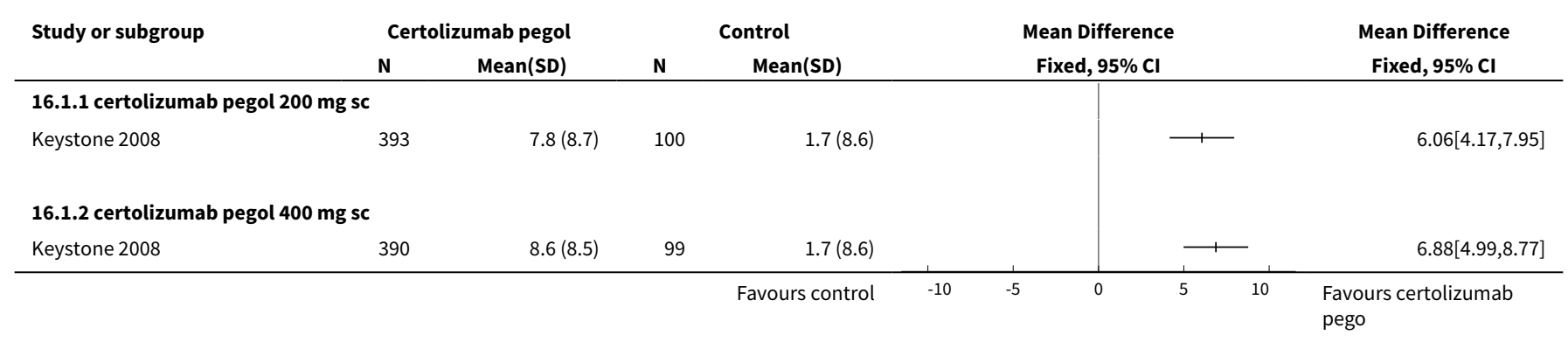

\section{Comparison 17. SF-36 Mental Component Summary (MCS) at week 52, any dose}

\begin{tabular}{lllll}
\hline Outcome or subgroup title & No. of studies & $\begin{array}{l}\text { No. of partici- } \\
\text { pants }\end{array}$ & Statistical method & Effect size \\
\hline 1 Change from baseline & 1 & & Mean Difference (IV, Fixed, 95\% Cl) & $\begin{array}{l}\text { Totals not select- } \\
\text { ed }\end{array}$ \\
\hline
\end{tabular}




\begin{tabular}{lllll}
\hline Outcome or subgroup title & No. of studies & $\begin{array}{l}\text { No. of partici- } \\
\text { pants }\end{array}$ & Statistical method & Effect size \\
\hline $\begin{array}{l}1.1 \text { certolizumab pegol } 200 \mathrm{mg} \\
\text { SC }\end{array}$ & 1 & Mean Difference (IV, Fixed, 95\% CI) & $0.0[0.0,0.0]$ \\
\hline $\begin{array}{l}1.2 \text { certolizumab pegol } 400 \mathrm{mg} \\
\text { SC }\end{array}$ & 1 & Mean Difference (IV, Fixed, $95 \% \mathrm{Cl})$ & $0.0[0.0,0.0]$ \\
\hline
\end{tabular}

\section{Analysis 17.1. Comparison 17 SF-36 Mental Component Summary} (MCS) at week 52, any dose, Outcome 1 Change from baseline.

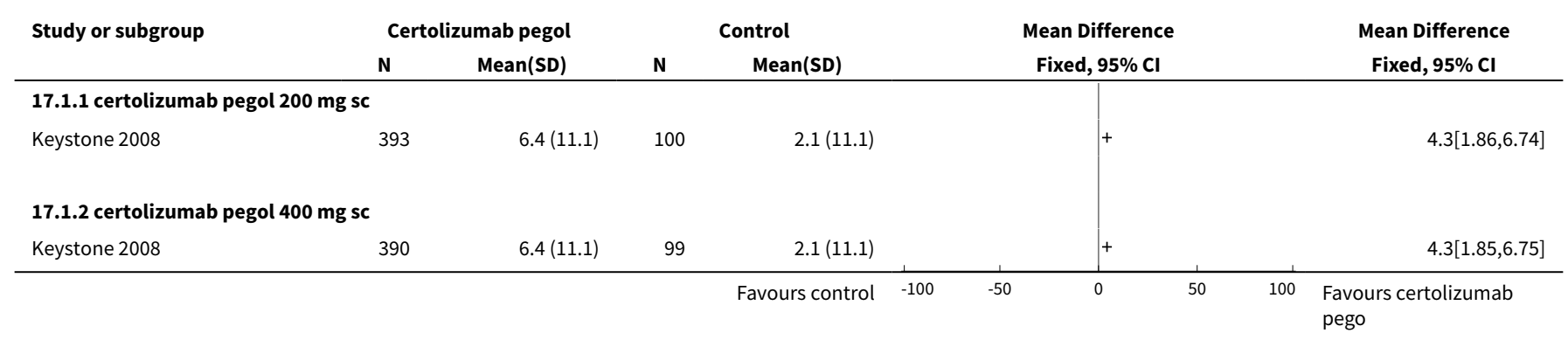

Comparison 18. Disease Activity Score (DAS-28) (ESR) remission (<2.6), any doses, 12 weeks

\begin{tabular}{lllll}
\hline Outcome or subgroup title & No. of studies & $\begin{array}{l}\text { No. of partici- } \\
\text { pants }\end{array}$ & Statistical method & Effect size \\
\hline $\begin{array}{l}1 \text { Proportion of participants achieving re- } \\
\text { mission } 12 \text { weeks certolizumab 200 mg }\end{array}$ & 2 & 1942 & $\begin{array}{l}\text { Peto Odds Ratio (Peto, } \\
\text { Fixed, 95\% Cl) }\end{array}$ & 1.94 [1.44, 2.61] \\
\hline
\end{tabular}

Analysis 18.1. Comparison 18 Disease Activity Score (DAS-28) (ESR) remission (<2.6), any doses, 12 weeks, Outcome 1 Proportion of participants achieving remission 12 weeks certolizumab 200 mg.

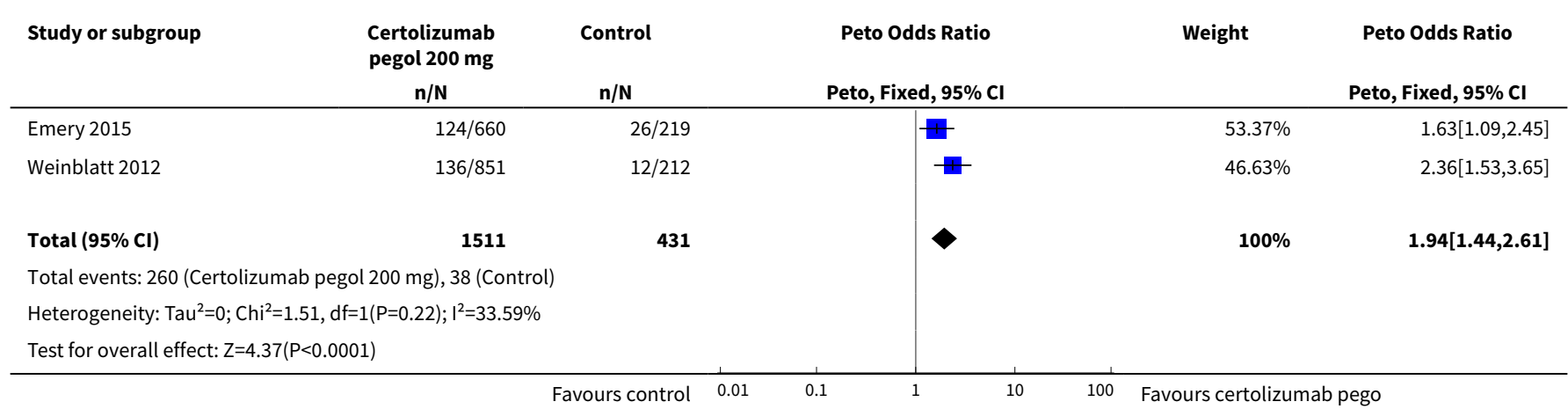


Comparison 19. Disease Activity Score (DAS-28) (ESR) remission (< 2.6), any dose, 24 weeks

\begin{tabular}{lllll}
\hline Outcome or subgroup title & No. of studies & $\begin{array}{l}\text { No. of partici- } \\
\text { pants }\end{array}$ & Statistical method & Effect size \\
\hline $\begin{array}{l}\text { 1 Proportion of participants achiev- } \\
\text { ing remission 24 weeks }\end{array}$ & 7 & 3462 & $\begin{array}{l}\text { Risk Ratio (M-H, Random, } \\
95 \% \mathrm{Cl})\end{array}$ & $3.27[1.96,5.46]$ \\
\hline 1.1 certolizumab pegol 200 mg sc & 6 & 2420 & $\begin{array}{l}\text { Risk Ratio (M-H, Random, } \\
95 \% \text { Cl) }\end{array}$ & $2.94[1.64,5.28]$ \\
\hline 1.2 certolizumab pegol $400 \mathrm{mg} \mathrm{sc}$ & 3 & 1042 & $\begin{array}{l}\text { Risk Ratio (M-H, Random, } \\
95 \% \text { Cl) }\end{array}$ & $4.46[1.95,10.21]$ \\
\hline
\end{tabular}

Analysis 19.1. Comparison 19 Disease Activity Score (DAS-28) (ESR) remission (<2.6), any dose, 24 weeks, Outcome 1 Proportion of participants achieving remission 24 weeks.

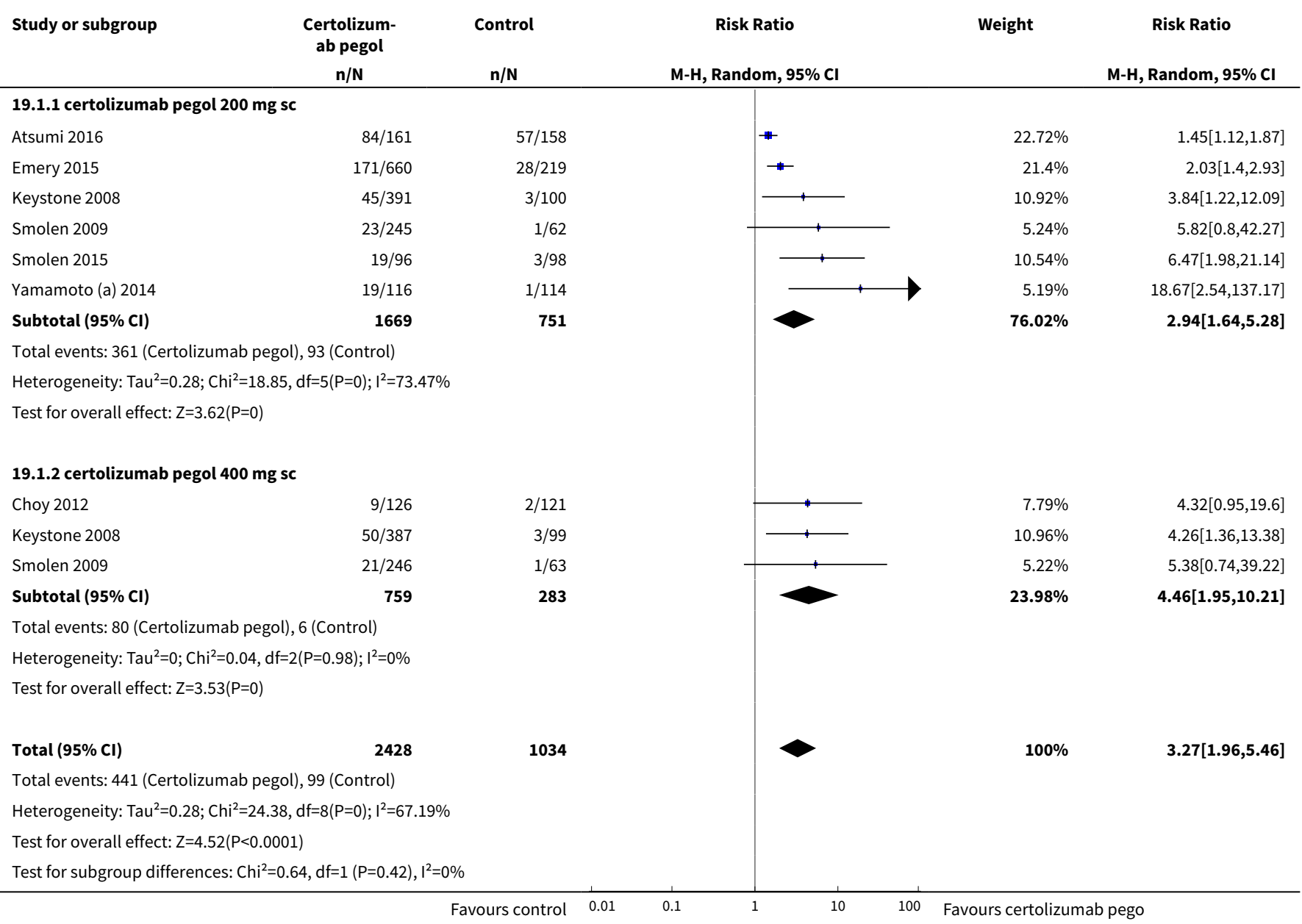


Comparison 20. Disease Activity Score (DAS-28) (ESR) remission (<2.6), any dose, 52 weeks

\begin{tabular}{|c|c|c|c|c|}
\hline Outcome or subgroup title & No. of studies & $\begin{array}{l}\text { No. of partici- } \\
\text { pants }\end{array}$ & Statistical method & Effect size \\
\hline $\begin{array}{l}1 \text { Proportion of participants achieving } \\
\text { remission } 52 \text { weeks }\end{array}$ & 3 & 2175 & $\begin{array}{l}\text { Risk Ratio (M-H, Fixed, 95\% } \\
\mathrm{Cl})\end{array}$ & $1.85[1.55,2.21]$ \\
\hline 1.1 certolizumab pegol 200 mg sc & 3 & 1689 & $\begin{array}{l}\text { Risk Ratio (M-H, Fixed, 95\% } \\
\mathrm{CI})\end{array}$ & $1.71[1.43,2.04]$ \\
\hline 1.2 certolizumab pegol 400 mg sc & 1 & 486 & $\begin{array}{l}\text { Risk Ratio (M-H, Fixed, 95\% } \\
\mathrm{Cl})\end{array}$ & $6.31[2.03,19.59]$ \\
\hline
\end{tabular}

Analysis 20.1. Comparison 20 Disease Activity Score (DAS-28) (ESR) remission (< 2.6), any dose, 52 weeks, Outcome 1 Proportion of participants achieving remission 52 weeks.

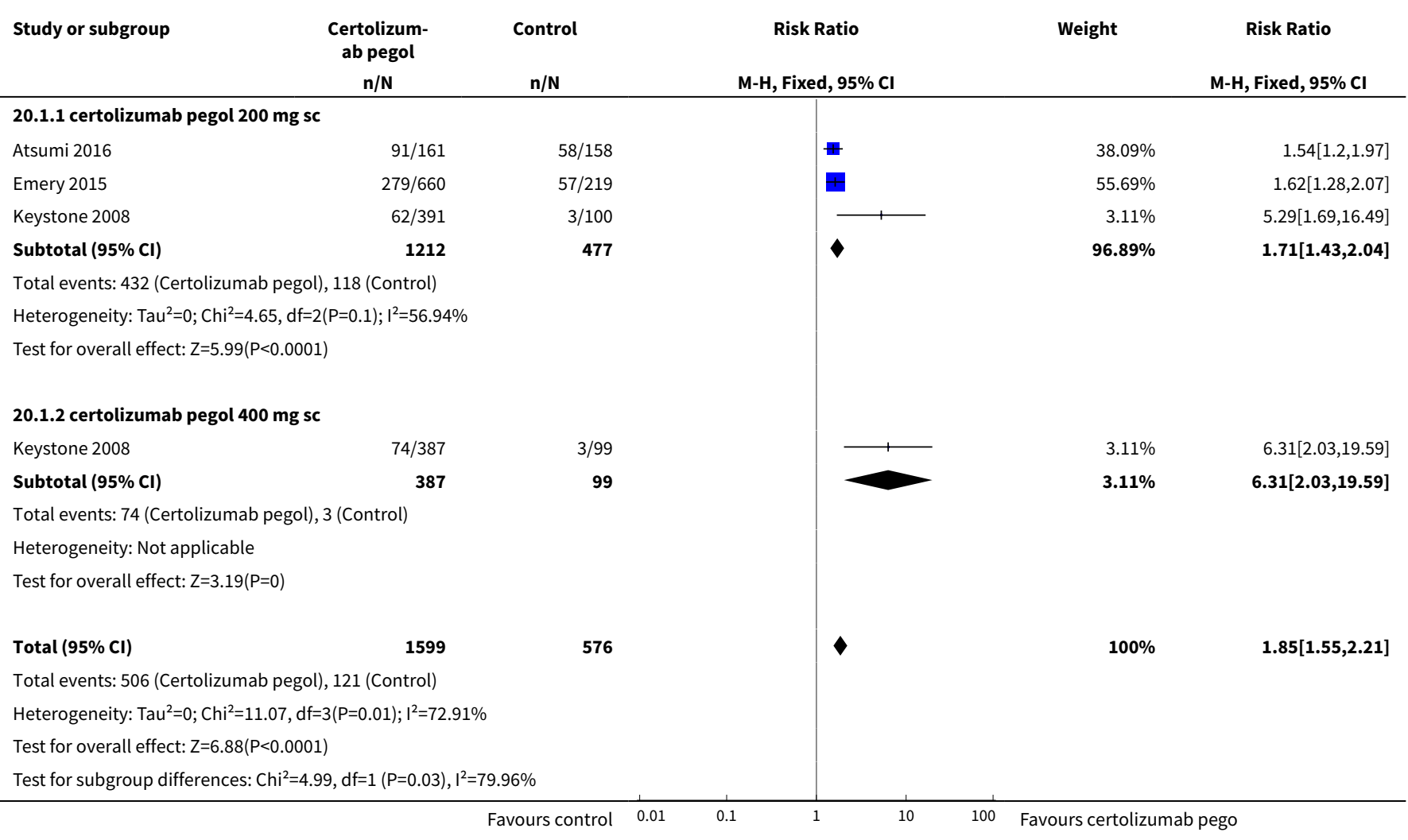

\section{Comparison 21. Disease Activity Score (DAS-28) (ESR) remission (<2.6), any time}

\begin{tabular}{lllll}
\hline Outcome or subgroup title & No. of studies & $\begin{array}{l}\text { No. of partici- } \\
\text { pants }\end{array}$ & Statistical method & Effect size \\
\hline $\begin{array}{l}1 \text { Proportion of participants achieving remis- } \\
\text { sion } 12 \text { weeks certolizumab } 200 \mathrm{mg}\end{array}$ & 2 & 1942 & $\begin{array}{l}\text { Risk Ratio (M-H, Fixed, } \\
95 \% \mathrm{Cl})\end{array}$ & $1.99[1.44,2.76]$ \\
\hline
\end{tabular}




\begin{tabular}{|c|c|c|c|c|}
\hline Outcome or subgroup title & No. of studies & $\begin{array}{l}\text { No. of partici- } \\
\text { pants }\end{array}$ & Statistical method & Effect size \\
\hline $\begin{array}{l}2 \text { Proportion of participants achieving remis- } \\
\text { sion } 24 \text { weeks certolizumab } 200 \text { mg }\end{array}$ & 6 & 2579 & $\begin{array}{l}\text { Risk Ratio (M-H, Ran- } \\
\text { dom, } 95 \% \mathrm{Cl})\end{array}$ & $3.79[1.90,7.56]$ \\
\hline $\begin{array}{l}3 \text { Proportion of participants achieving remis- } \\
\text { sion } 24 \text { weeks certolizumab } 400 \text { mg }\end{array}$ & 3 & 1201 & $\begin{array}{l}\text { Risk Ratio (M-H, Ran- } \\
\text { dom, } 95 \% \mathrm{Cl})\end{array}$ & $7.18[3.12,16.50]$ \\
\hline $\begin{array}{l}4 \text { Proportion of participants achieving remis- } \\
\text { sion } 52 \text { weeks certolizumab } 200 \text { mg }\end{array}$ & 3 & 1785 & $\begin{array}{l}\text { Risk Ratio (M-H, Fixed, } \\
95 \% \mathrm{Cl})\end{array}$ & $1.83[1.53,2.18]$ \\
\hline $\begin{array}{l}5 \text { Proportion of participants achieving remis- } \\
\text { sion } 52 \text { weeks certolizumab } 400 \mathrm{mg}\end{array}$ & 1 & & $\begin{array}{l}\text { Risk Ratio (M-H, Fixed, } \\
95 \% \mathrm{Cl})\end{array}$ & $\begin{array}{l}\text { Totals not select- } \\
\text { ed }\end{array}$ \\
\hline
\end{tabular}

\section{Analysis 21.1. Comparison 21 Disease Activity Score (DAS-28) (ESR) remission (<2.6), any time, Outcome 1 Proportion of participants achieving remission 12 weeks certolizumab $200 \mathrm{mg}$.}

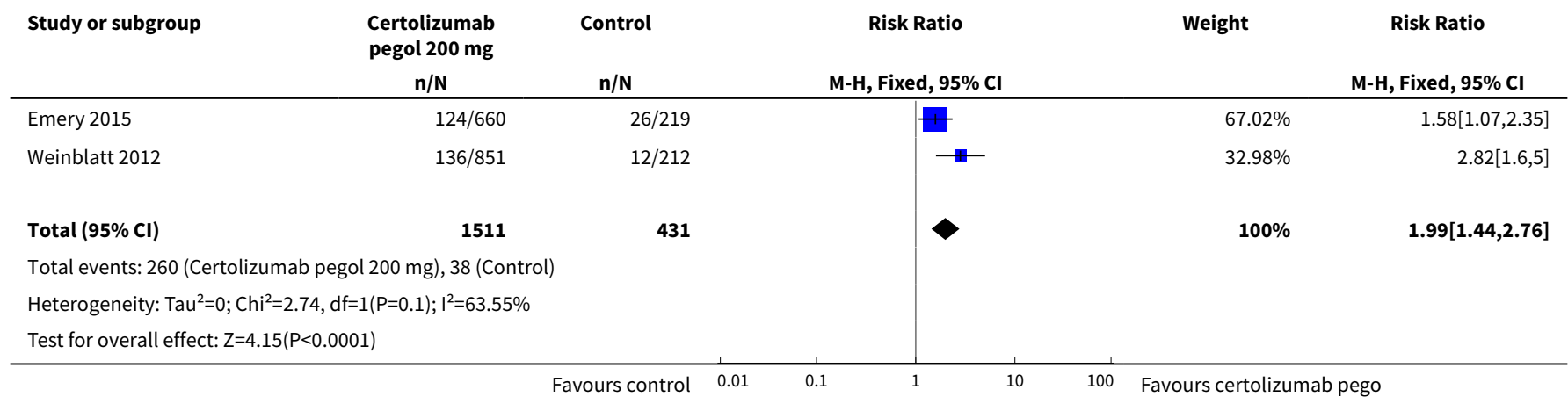

\section{Analysis 21.2. Comparison 21 Disease Activity Score (DAS-28) (ESR) remission (<2.6), any time, Outcome 2 Proportion of participants achieving remission 24 weeks certolizumab $200 \mathrm{mg}$.}

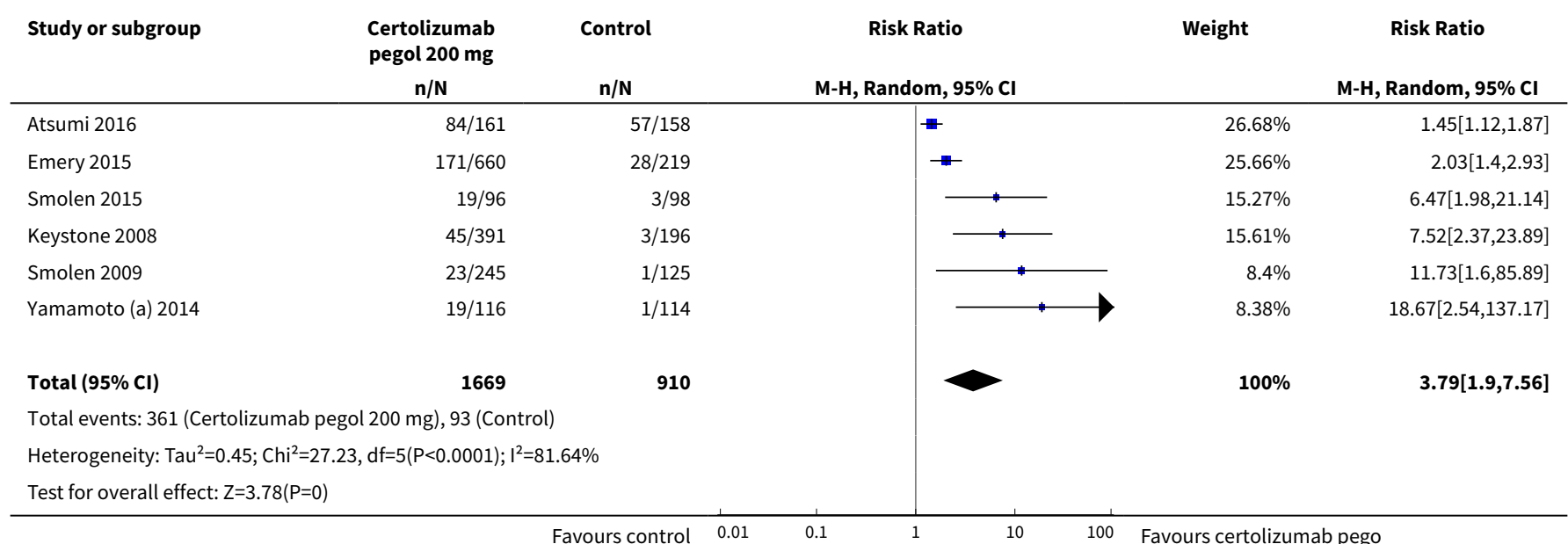


Analysis 21.3. Comparison 21 Disease Activity Score (DAS-28) (ESR) remission (<2.6), any time, Outcome 3 Proportion of participants achieving remission 24 weeks certolizumab $400 \mathrm{mg}$.

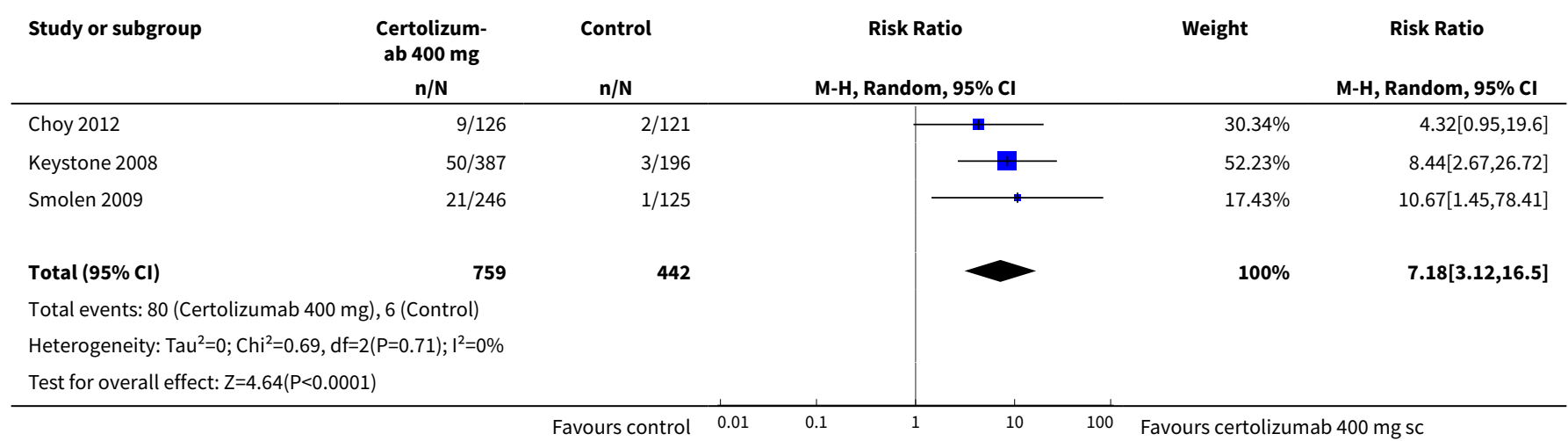

Analysis 21.4. Comparison 21 Disease Activity Score (DAS-28) (ESR) remission (<2.6), any time, Outcome 4 Proportion of participants achieving remission 52 weeks certolizumab $200 \mathrm{mg}$.

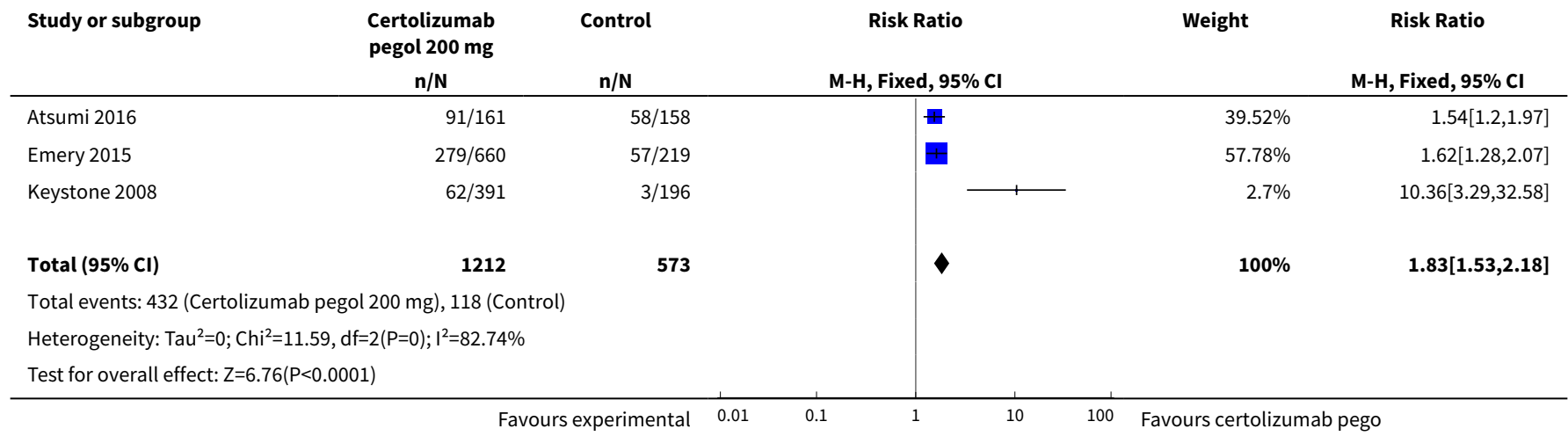

Analysis 21.5. Comparison 21 Disease Activity Score (DAS-28) (ESR) remission $(<2.6)$, any time, Outcome 5 Proportion of participants achieving remission 52 weeks certolizumab $400 \mathrm{mg}$.

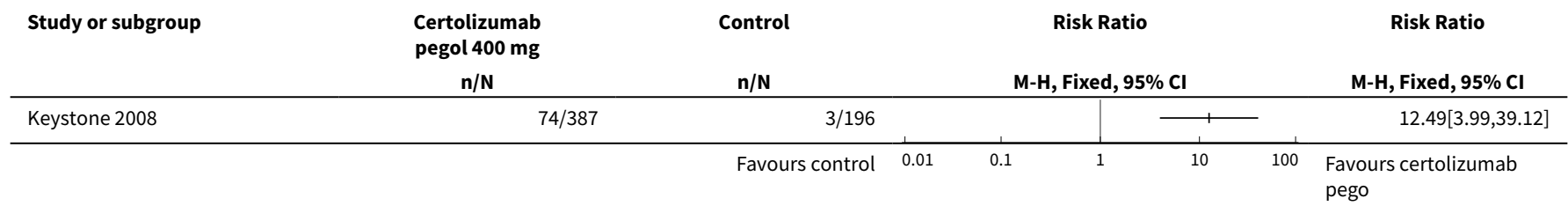

\section{Comparison 22. DAS-28 at 12 weeks, 200 mg certolizumab}

\begin{tabular}{lllll}
\hline Outcome or subgroup title & No. of studies & $\begin{array}{l}\text { No. of partici- } \\
\text { pants }\end{array}$ & Statistical method & Effect size \\
\hline 1 DAS 28 (ESR) change from baseline & 1 & & $\begin{array}{l}\text { Mean Difference (IV, Fixed, 95\% } \\
\text { Cl) }\end{array}$ & $\begin{array}{l}\text { Totals not select- } \\
\text { ed }\end{array}$ \\
\hline
\end{tabular}


Analysis 22.1. Comparison 22 DAS-28 at 12 weeks, $200 \mathrm{mg}$ certolizumab, Outcome 1 DAS 28 (ESR) change from baseline.

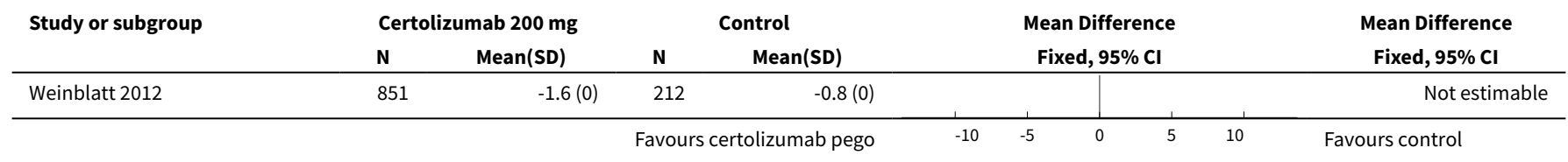

Comparison 23. DAS-28 at 24 weeks, 400 mg certolizumab

\begin{tabular}{lllll}
\hline Outcome or subgroup title & No. of studies & $\begin{array}{l}\text { No. of partici- } \\
\text { pants }\end{array}$ & Statistical method & Effect size \\
\hline 1 DAS 28 (ESR) change from baseline & 2 & 593 & $\begin{array}{l}\text { Mean Difference (IV, Random, } \\
95 \% \text { Cl) }\end{array}$ & $-1.46[-2.49,-0.42]$ \\
\hline
\end{tabular}

Analysis 23.1. Comparison 23 DAS-28 at 24 weeks, $400 \mathrm{mg}$ certolizumab, Outcome 1 DAS 28 (ESR) change from baseline.

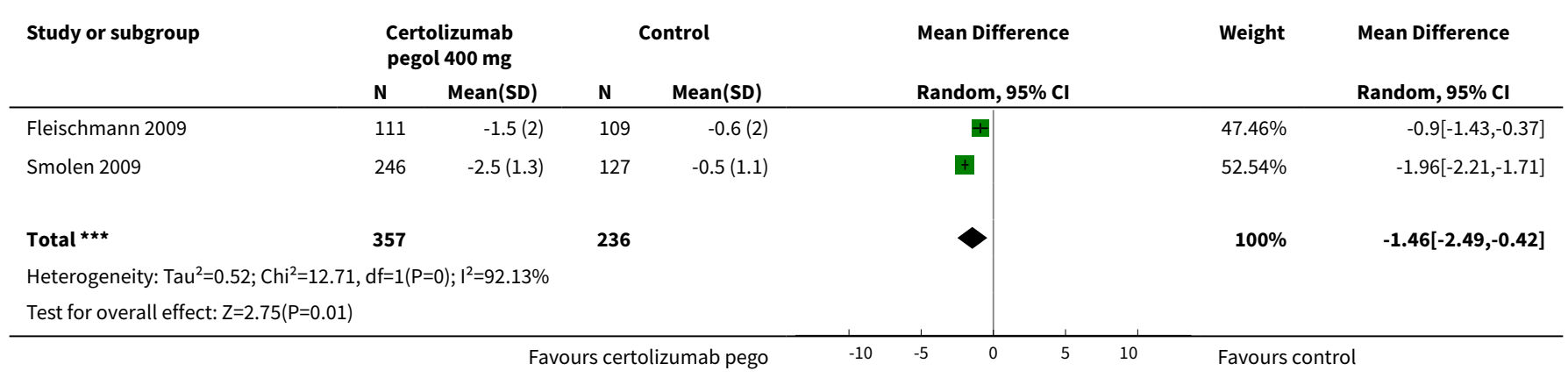

Comparison 24. DAS-28 at week 52, certolizumab $200 \mathrm{mg}$

\begin{tabular}{lllll}
\hline Outcome or subgroup title & No. of studies & $\begin{array}{l}\text { No. of partici- } \\
\text { pants }\end{array}$ & Statistical method & Effect size \\
\hline $\begin{array}{l}1 \text { DAS 28 (ESR) Change from base- } \\
\text { line }\end{array}$ & 1 & & $\begin{array}{l}\text { Mean Difference (IV, Fixed, 95\% } \\
\text { Cl) }\end{array}$ & $\begin{array}{l}\text { Totals not select- } \\
\text { ed }\end{array}$ \\
\hline
\end{tabular}


Analysis 24.1. Comparison 24 DAS-28 at week 52, certolizumab $200 \mathrm{mg}$, Outcome 1 DAS 28 (ESR) Change from baseline.

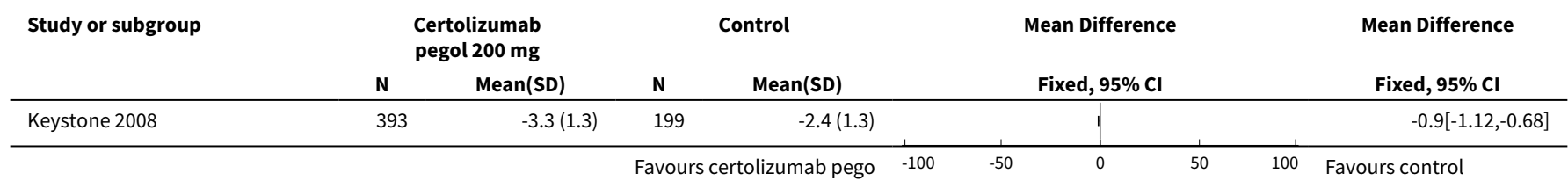

Comparison 25. DAS-28 at week 52, certolizumab $400 \mathrm{mg}$

\begin{tabular}{|c|c|c|c|c|}
\hline Outcome or subgroup title & No. of studies & $\begin{array}{l}\text { No. of partici- } \\
\text { pants }\end{array}$ & Statistical method & Effect size \\
\hline $\begin{array}{l}1 \text { DAS } 28 \text { (ESR) Change from base- } \\
\text { line }\end{array}$ & 1 & & $\begin{array}{l}\text { Mean Difference (IV, Fixed, 95\% } \\
\mathrm{CI} \text { ) }\end{array}$ & $\begin{array}{l}\text { Totals not select- } \\
\text { ed }\end{array}$ \\
\hline
\end{tabular}

Analysis 25.1. Comparison 25 DAS-28 at week 52, certolizumab $400 \mathrm{mg}$, Outcome 1 DAS 28 (ESR) Change from baseline.

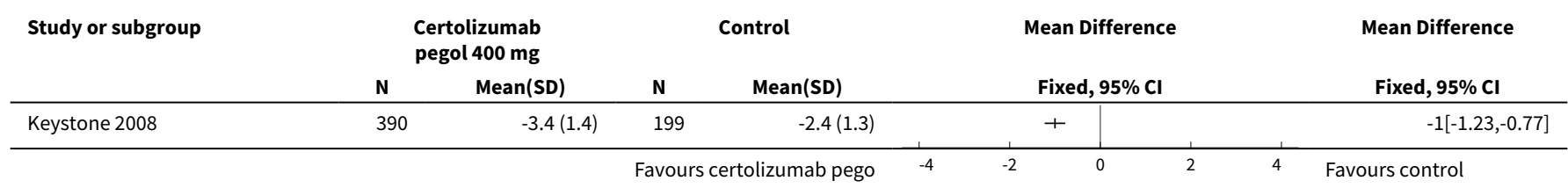

Comparison 26. DAS-28 at 24 weeks, any dose

\begin{tabular}{|c|c|c|c|c|}
\hline Outcome or subgroup title & No. of studies & $\begin{array}{l}\text { No. of partici- } \\
\text { pants }\end{array}$ & Statistical method & Effect size \\
\hline 1 Change from baseline & 2 & 839 & $\begin{array}{l}\text { Mean Difference (IV, Random, 95\% } \\
\mathrm{CI})\end{array}$ & $-1.59[-2.10,-1.08]$ \\
\hline $\begin{array}{l}1.1 \text { certolizumab pegol } 200 \\
\mathrm{mg} \mathrm{sc}\end{array}$ & 1 & 310 & $\begin{array}{l}\text { Mean Difference (IV, Random, 95\% } \\
\mathrm{CI})\end{array}$ & $-1.77[-2.08,-1.46]$ \\
\hline $\begin{array}{l}1.2 \text { certolizumab pegol } 400 \\
\mathrm{mg} \mathrm{sc}\end{array}$ & 2 & 529 & $\begin{array}{l}\text { Mean Difference (IV, Random, 95\% } \\
\mathrm{Cl} \text { ) }\end{array}$ & $-1.45[-2.49,-0.41]$ \\
\hline
\end{tabular}

Analysis 26.1. Comparison 26 DAS-28 at 24 weeks, any dose, Outcome 1 Change from baseline.

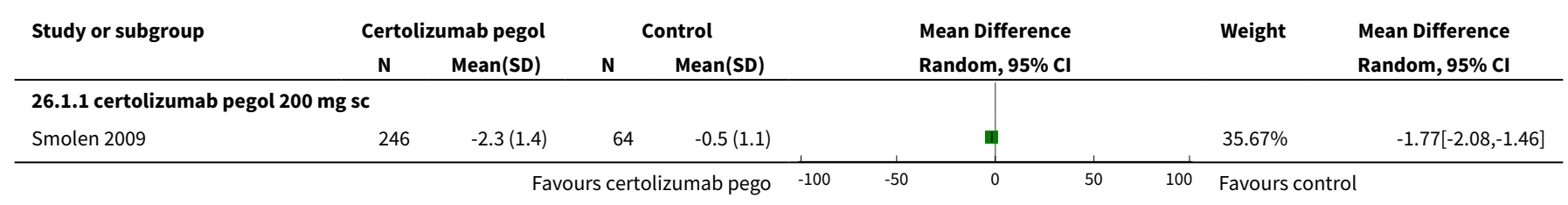




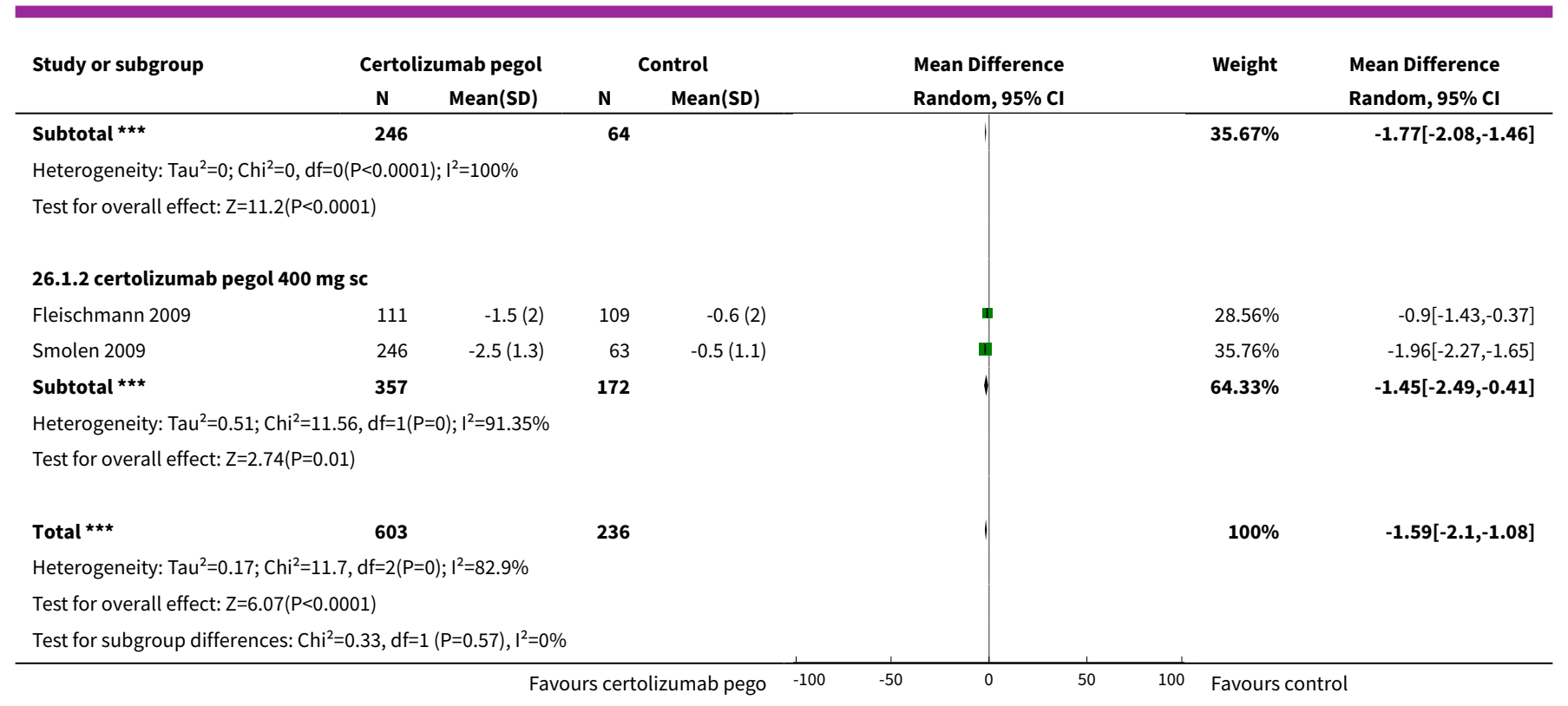

\section{Comparison 27. DAS-28 at 52 weeks, any dose}

\begin{tabular}{|c|c|c|c|c|}
\hline Outcome or subgroup title & No. of studies & $\begin{array}{l}\text { No. of partici- } \\
\text { pants }\end{array}$ & Statistical method & Effect size \\
\hline 1 Change from baseline & 2 & 1838 & $\begin{array}{l}\text { Mean Difference (IV, Fixed, 95\% } \\
\mathrm{CI} \text { ) }\end{array}$ & $-0.78[-0.93,-0.63]$ \\
\hline $\begin{array}{l}1.1 \text { certolizumab pegol } 200 \mathrm{mg} \\
\mathrm{sc}\end{array}$ & 2 & 1349 & $\begin{array}{l}\text { Mean Difference (IV, Fixed, 95\% } \\
\mathrm{CI} \text { ) }\end{array}$ & $-0.71[-0.88,-0.53]$ \\
\hline $\begin{array}{l}1.2 \text { certolizumab pegol } 400 \mathrm{mg} \\
\mathrm{sc}\end{array}$ & 1 & 489 & $\begin{array}{l}\text { Mean Difference (IV, Fixed, 95\% } \\
\mathrm{CI} \text { ) }\end{array}$ & $-1.0[-1.29,-0.71]$ \\
\hline
\end{tabular}

Analysis 27.1. Comparison 27 DAS-28 at 52 weeks, any dose, Outcome 1 Change from baseline.

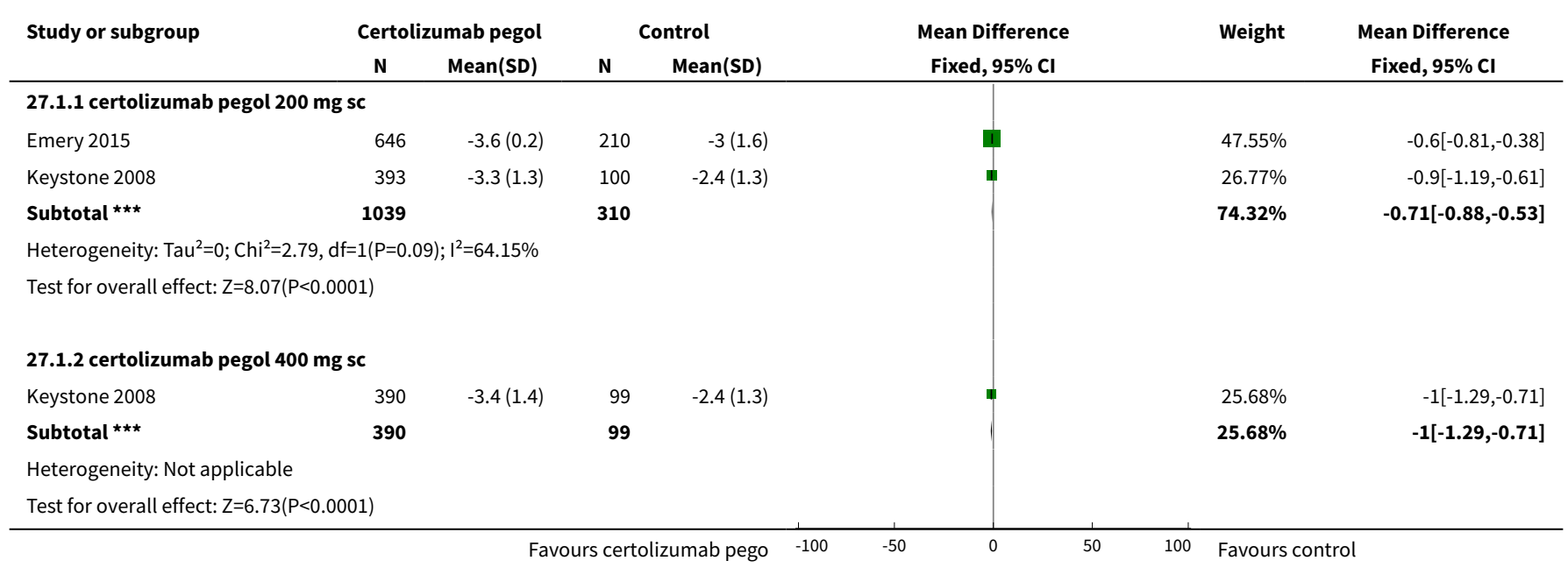




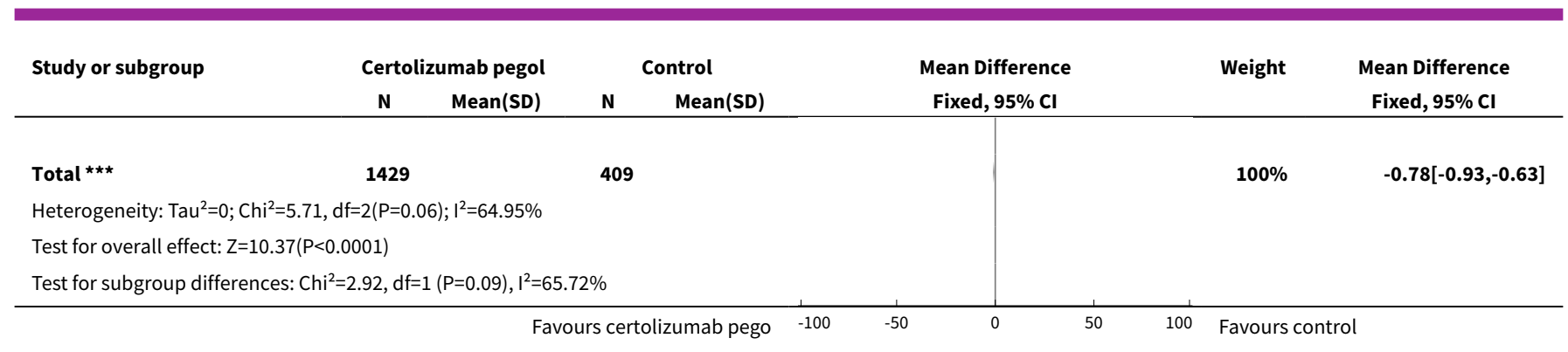

Comparison 28. DAS-28 at 24 weeks, $200 \mathrm{mg}$ certolizumab

\begin{tabular}{lllll}
\hline Outcome or subgroup title & No. of studies & $\begin{array}{l}\text { No. of partici- } \\
\text { pants }\end{array}$ & Statistical method & Effect size \\
\hline 1 DAS 28 (ESR) change from baseline & 1 & & $\begin{array}{l}\text { Mean Difference (IV, Fixed, 95\% } \\
\text { Cl) }\end{array}$ & $\begin{array}{l}\text { Totals not select- } \\
\text { ed }\end{array}$ \\
\hline
\end{tabular}

Analysis 28.1. Comparison 28 DAS-28 at 24 weeks, $200 \mathrm{mg}$ certolizumab, Outcome 1 DAS 28 (ESR) change from baseline.

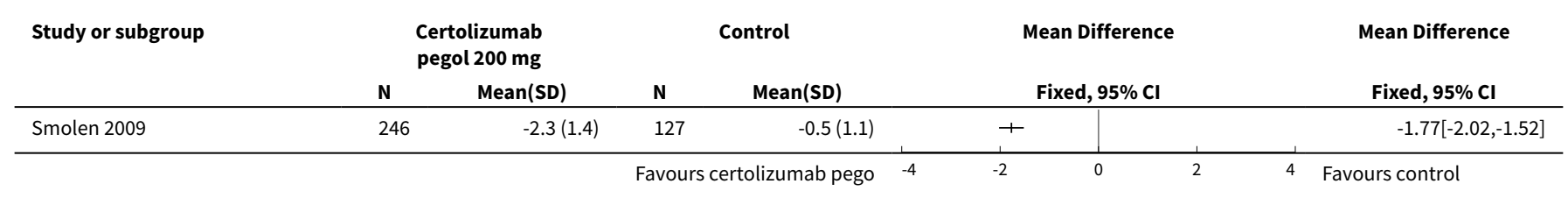

\section{Comparison 29. Erosion score (ES)}

\begin{tabular}{|c|c|c|c|c|}
\hline Outcome or subgroup title & No. of studies & $\begin{array}{l}\text { No. of partici- } \\
\text { pants }\end{array}$ & Statistical method & Effect size \\
\hline $\begin{array}{l}1 \text { Change from the baseline mean ES at } \\
\text { week } 24 \text {, certolizumab pegol } 200 \mathrm{mg}\end{array}$ & 2 & 859 & $\begin{array}{l}\text { Std. Mean Difference (IV, } \\
\text { Fixed, } 95 \% \mathrm{CI} \text { ) }\end{array}$ & $\begin{array}{l}-0.35[-0.50 \\
-0.21]\end{array}$ \\
\hline $\begin{array}{l}2 \text { Change from the baseline mean ES at } \\
\text { week } 24 \text {, certolizumab pegol } 400 \mathrm{mg}\end{array}$ & 2 & 869 & $\begin{array}{l}\text { Mean Difference (IV, Ran- } \\
\text { dom, } 95 \% \mathrm{CI} \text { ) }\end{array}$ & $\begin{array}{l}-0.76[-1.14 \\
-0.37]\end{array}$ \\
\hline $\begin{array}{l}3 \text { Change from the baseline mean ES at } \\
\text { week 52, certolizumab pegol } 200 \mathrm{mg}\end{array}$ & 2 & 1235 & $\begin{array}{l}\text { Mean Difference (IV, } \\
\text { Fixed, } 95 \% \mathrm{CI} \text { ) }\end{array}$ & $\begin{array}{l}-1.14[-1.54 \\
-0.74]\end{array}$ \\
\hline $\begin{array}{l}4 \text { Change from the baseline mean ES at } \\
\text { week } 52 \text {, certolizumab pegol } 400 \mathrm{mg}\end{array}$ & 1 & & $\begin{array}{l}\text { Mean Difference (IV, } \\
\text { Fixed, } 95 \% \mathrm{CI} \text { ) }\end{array}$ & $\begin{array}{l}\text { Totals not select- } \\
\text { ed }\end{array}$ \\
\hline
\end{tabular}


Analysis 29.1. Comparison 29 Erosion score (ES), Outcome 1 Change from the baseline mean ES at week 24, certolizumab pegol $200 \mathrm{mg}$.

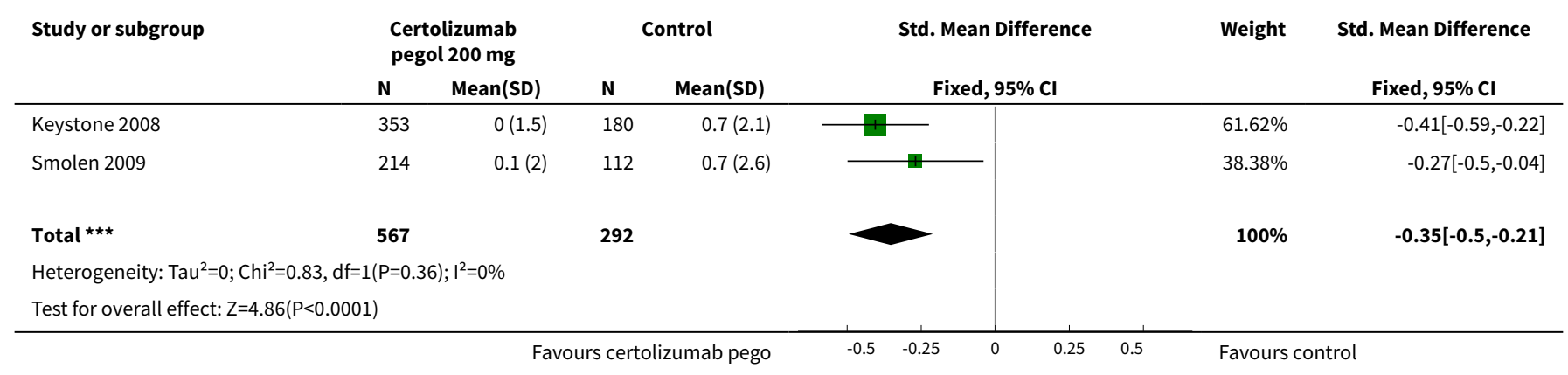

Analysis 29.2. Comparison 29 Erosion score (ES), Outcome 2 Change from the baseline mean ES at week 24, certolizumab pegol $400 \mathrm{mg}$.

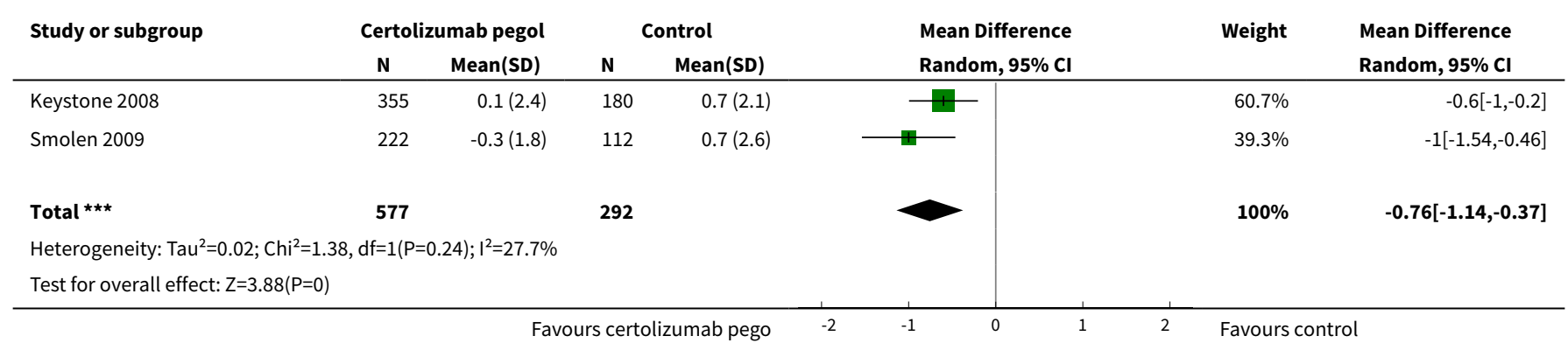

Analysis 29.3. Comparison 29 Erosion score (ES), Outcome 3 Change from the baseline mean ES at week 52, certolizumab pegol $200 \mathrm{mg}$.

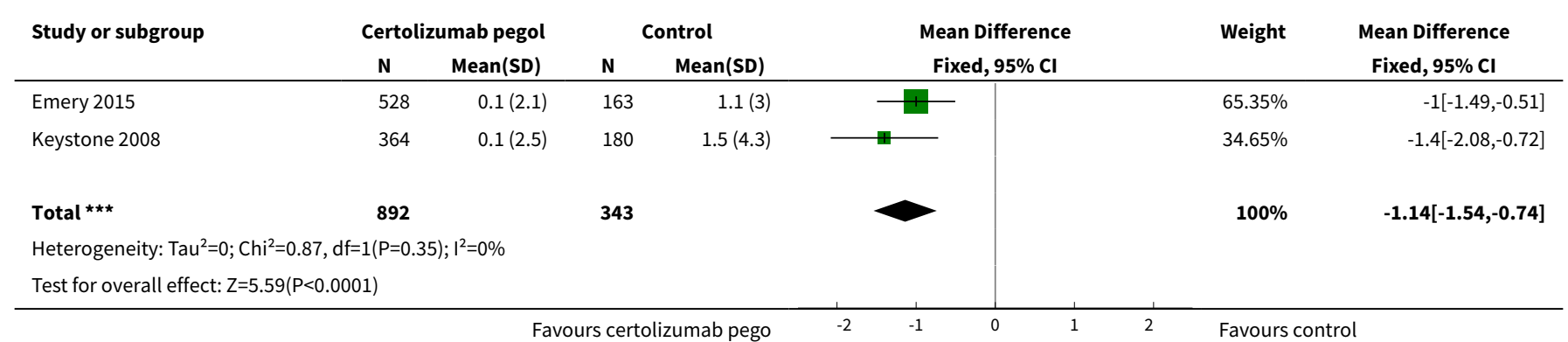

Analysis 29.4. Comparison 29 Erosion score (ES), Outcome 4 Change

from the baseline mean ES at week 52, certolizumab pegol $400 \mathrm{mg}$.

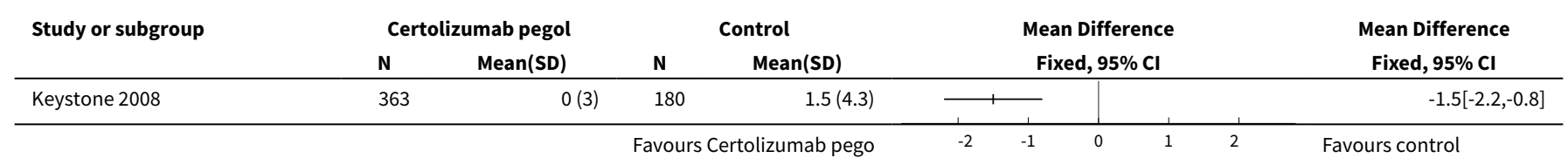


Comparison 30. Erosion score (ES) at 24 weeks, any dose

\begin{tabular}{lllll}
\hline Outcome or subgroup title & No. of studies & $\begin{array}{l}\text { No. of partici- } \\
\text { pants }\end{array}$ & Statistical method & Effect size \\
\hline $\begin{array}{llll}1 \text { Change from baseline } \\
\text { pan }\end{array}$ & 2 & 1437 & $\begin{array}{l}\text { Mean Difference (IV, Random, 95\% } \\
\text { Cl) }\end{array}$ & $-0.70[-0.98,-0.42]$ \\
\hline $\begin{array}{l}1.1 \text { certolizumab pegol 200 } \\
\text { mg sc }\end{array}$ & 2 & 714 & $\begin{array}{l}\text { Mean Difference (IV, Random, 95\% } \\
\text { Cl) }\end{array}$ & $-0.67[-1.06,-0.28]$ \\
\hline $\begin{array}{l}1.2 \text { certolizumab pegol 400 } \\
\text { mg sc }\end{array}$ & 2 & 723 & $\begin{array}{l}\text { Mean Difference (IV, Random, 95\% } \\
\text { Cl) }\end{array}$ & $-0.73[-1.14,-0.32]$ \\
\hline
\end{tabular}

Analysis 30.1. Comparison 30 Erosion score (ES) at 24 weeks, any dose, Outcome 1 Change from baseline.

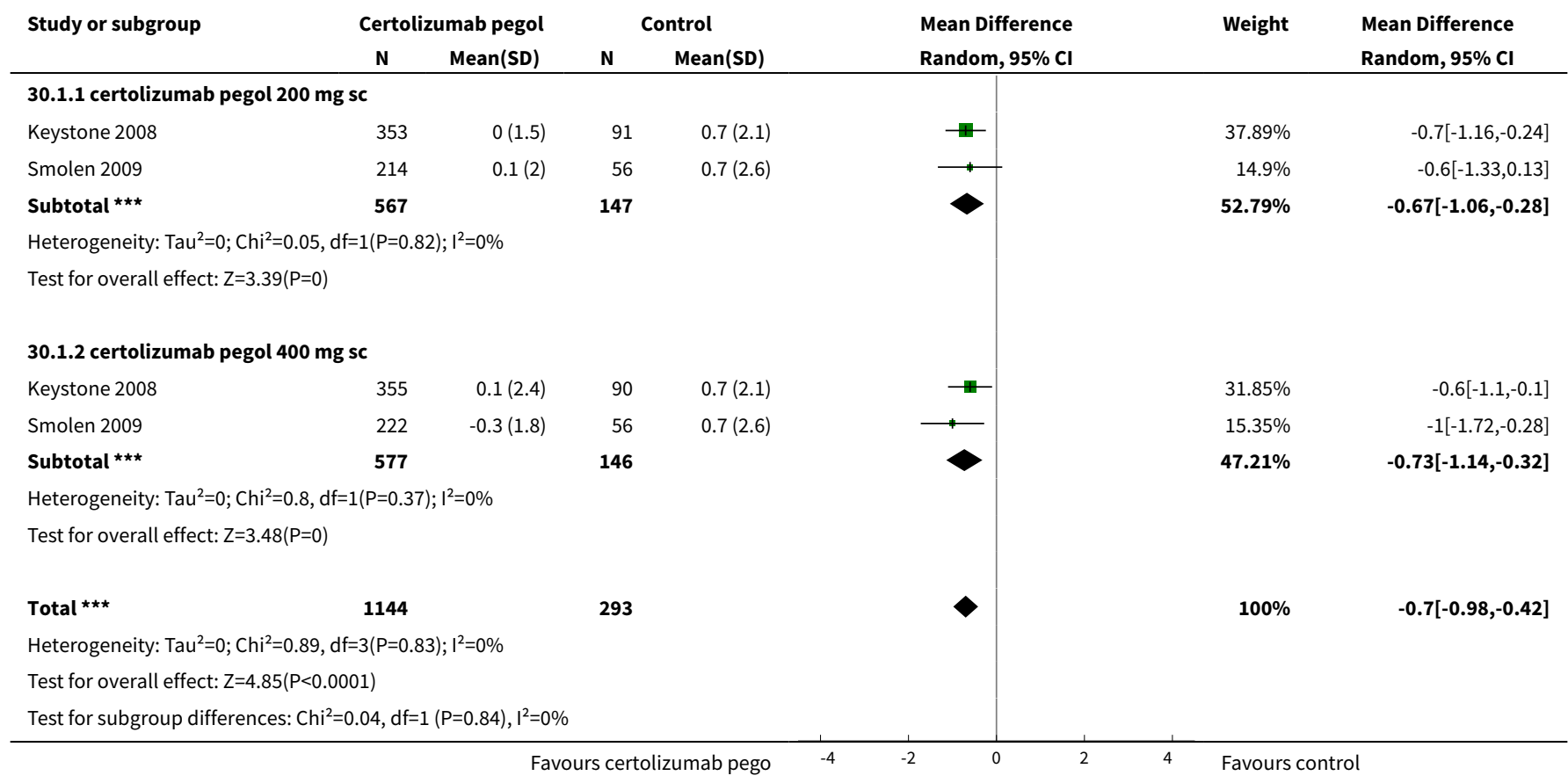

\section{Comparison 31. Erosion score (ES) at 52 weeks, any dose}

\begin{tabular}{lllll}
\hline Outcome or subgroup title & No. of studies & $\begin{array}{l}\text { No. of partici- } \\
\text { pants }\end{array}$ & Statistical method & Effect size \\
\hline $\begin{array}{l}1 \text { Change from baseline } \\
\text { PC }\end{array}$ & 2 & 1599 & $\begin{array}{l}\text { Mean Difference (IV, Fixed, 95\% } \\
\text { CI) }\end{array}$ & $-1.16[-1.56,-0.77]$ \\
\hline $\begin{array}{l}1.1 \text { certolizumab pegol 200 mg } \\
\text { SC }\end{array}$ & 2 & 1146 & $\begin{array}{l}\text { Mean Difference (IV, Fixed, 95\% } \\
\text { CI) }\end{array}$ & $-1.09[-1.52,-0.65]$ \\
\hline
\end{tabular}




\begin{tabular}{lllll}
\hline Outcome or subgroup title & No. of studies & $\begin{array}{l}\text { No. of partici- } \\
\text { pants }\end{array}$ & Statistical method & Effect size \\
\hline $\begin{array}{l}1.2 \text { certolizumab pegol } 400 \mathrm{mg} \\
\text { SC }\end{array}$ & 1 & 453 & $\begin{array}{l}\text { Mean Difference (IV, Fixed, } 95 \% \\
\text { CI) }\end{array}$ & $-1.5[-2.44,-0.56]$ \\
\hline
\end{tabular}

Analysis 31.1. Comparison 31 Erosion score (ES) at 52 weeks, any dose, Outcome 1 Change from baseline.

\begin{tabular}{|c|c|c|c|c|c|c|c|}
\hline \multirow[t]{2}{*}{ Study or subgroup } & \multicolumn{2}{|c|}{ Certolizumab pegol } & \multicolumn{2}{|c|}{ Control } & \multirow{2}{*}{$\begin{array}{c}\text { Mean Difference } \\
\text { Fixed, } 95 \% \mathrm{Cl}\end{array}$} & \multirow[t]{2}{*}{ Weight } & \multirow{2}{*}{$\begin{array}{c}\text { Mean Difference } \\
\text { Fixed, } 95 \% \mathrm{Cl}\end{array}$} \\
\hline & $\mathbf{N}$ & Mean(SD) & $\mathbf{N}$ & $\operatorname{Mean}(\mathrm{SD})$ & & & \\
\hline \multicolumn{8}{|c|}{ 31.1.1 certolizumab pegol $200 \mathrm{mg} \mathrm{sc}$} \\
\hline Emery 2015 & 528 & $0.1(2.1)$ & 163 & $1.1(3)$ & & $63.92 \%$ & $-1[-1.49,-0.51]$ \\
\hline Keystone 2008 & 364 & $0.1(2.5)$ & 91 & $1.5(4.3)$ & & $18.44 \%$ & $-1.4[-2.32,-0.48]$ \\
\hline 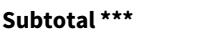 & 892 & & 254 & & & $82.35 \%$ & $-1.09[-1.52,-0.65]$ \\
\hline \multicolumn{8}{|c|}{ Heterogeneity: $\operatorname{Tau}^{2}=0 ; \mathrm{Chi}^{2}=0.56, \mathrm{df}=1(\mathrm{P}=0.45) ; \mathrm{I}^{2}=0 \%$} \\
\hline \multicolumn{8}{|c|}{ 31.1.2 certolizumab pegol $400 \mathrm{mg} \mathrm{sc}$} \\
\hline Keystone 2008 & 363 & $0(3)$ & 90 & $1.5(4.3)$ & - & $17.65 \%$ & $-1.5[-2.44,-0.56]$ \\
\hline 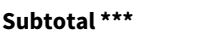 & 363 & & 90 & & & $17.65 \%$ & $-1.5[-2.44,-0.56]$ \\
\hline \multicolumn{8}{|c|}{ Heterogeneity: Not applicable } \\
\hline \multicolumn{8}{|c|}{ Test for overall effect: $Z=3.13(P=0)$} \\
\hline Total *** & 1255 & & 344 & & & $100 \%$ & $-1.16[-1.56,-0.77]$ \\
\hline \multicolumn{8}{|c|}{ Heterogeneity: $\operatorname{Tau}^{2}=0 ; \mathrm{Chi}^{2}=1.17, \mathrm{df}=2(\mathrm{P}=0.56) ; \mathrm{I}^{2}=0 \%$} \\
\hline \multicolumn{8}{|c|}{ Test for overall effect: $Z=5.76(P<0.0001)$} \\
\hline \multicolumn{8}{|c|}{ Test for subgroup differences: $\mathrm{Chi}^{2}=0.6, \mathrm{df}=1(\mathrm{P}=0.44), \mathrm{I}^{2}=0 \%$} \\
\hline
\end{tabular}

\section{Comparison 32. Joint space narrowing (JSN)}

\begin{tabular}{lllll}
\hline Outcome or subgroup title & No. of studies & $\begin{array}{l}\text { No. of partici- } \\
\text { pants }\end{array}$ & Statistical method & Effect size \\
\hline $\begin{array}{l}1 \text { Change from the baseline mean JSN 24 } \\
\text { weeks, certolizumab pegol 200 mg }\end{array}$ & 2 & 861 & Mean Difference (IV, Ran- & $\begin{array}{l}-0.45[-0.77, \\
\text { dom, 95\% Cl) }\end{array}$ \\
\hline $\begin{array}{l}\text { 2 Change from the baseline mean JSN 24 } \\
\text { weeks, certolizumab pegol 400 mg }\end{array}$ & 2 & 869 & Mean Difference (IV, Ran- & $-0.55[-0.86$, \\
\hline $\begin{array}{l}\text { 3 Change from the baseline mean JSN 52 } \\
\text { weeks, certolizumab pegol 200 mg }\end{array}$ & 2 & & Mean Difference (IV, & $-0.24]$ \\
\hline $\begin{array}{l}\text { 4 Change from the baseline mean JSN 52 } \\
\text { weeks, certolizumab pegol } 400 \text { mg }\end{array}$ & 1 & Fixed, 95\% Cl) & -0.67 [-1.02, \\
\hline
\end{tabular}


Analysis 32.1. Comparison 32 Joint space narrowing (JSN), Outcome 1

Change from the baseline mean JSN 24 weeks, certolizumab pegol $200 \mathrm{mg}$.

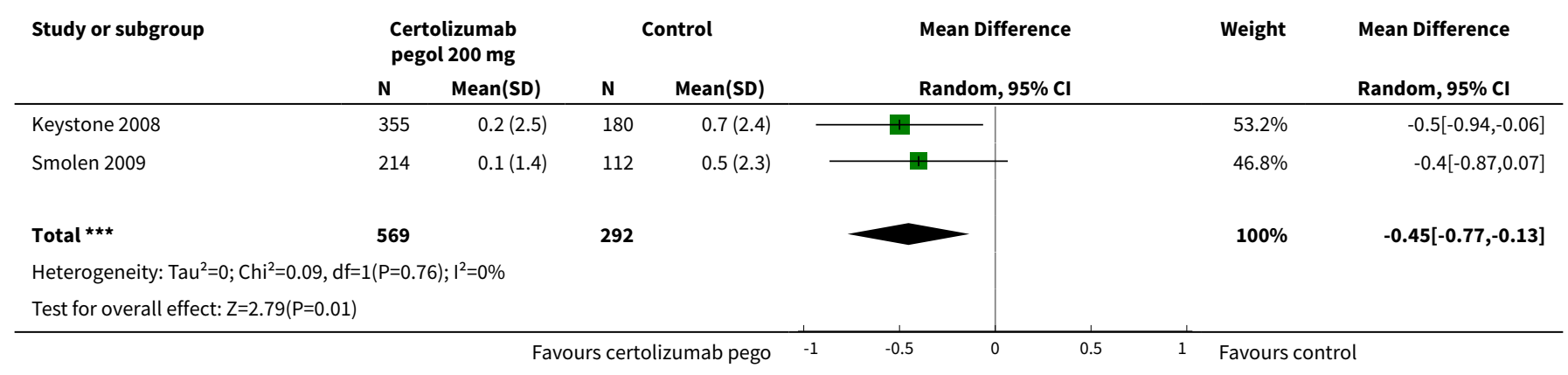

Analysis 32.2. Comparison 32 Joint space narrowing (JSN), Outcome 2 Change from the baseline mean JSN 24 weeks,certolizumab pegol $400 \mathrm{mg}$.

\begin{tabular}{|c|c|c|c|c|c|c|c|}
\hline \multirow[t]{2}{*}{ Study or subgroup } & \multicolumn{2}{|c|}{$\begin{array}{l}\text { Certolizumab } \\
\text { pegol } 400 \mathrm{mg}\end{array}$} & \multicolumn{2}{|c|}{ Control } & \multirow{2}{*}{$\begin{array}{l}\text { Mean Difference } \\
\text { Random, } 95 \% \mathrm{CI}\end{array}$} & \multirow[t]{2}{*}{ Weight } & \multirow{2}{*}{$\begin{array}{l}\text { Mean Difference } \\
\text { Random, } 95 \% \mathrm{Cl}\end{array}$} \\
\hline & $\mathbf{N}$ & Mean(SD) & $\mathbf{N}$ & Mean(SD) & & & \\
\hline Keystone 2008 & 355 & $0.2(2.4)$ & 180 & $0.7(2.4)$ & $\longrightarrow$ & $51.76 \%$ & $-0.5[-0.93,-0.07]$ \\
\hline Smolen 2009 & 222 & $-0.1(1)$ & 112 & $0.5(2.3)$ & $\longrightarrow$ & $48.24 \%$ & $-0.6[-1.05,-0.15]$ \\
\hline 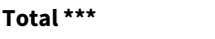 & 577 & & 292 & & & $100 \%$ & $-0.55[-0.86,-0.24]$ \\
\hline \multicolumn{8}{|c|}{ Heterogeneity: $\mathrm{Tau}^{2}=0 ; \mathrm{Chi}^{2}=0.1, \mathrm{df}=1(\mathrm{P}=0.75) ; \mathrm{I}^{2}=0 \%$} \\
\hline \multicolumn{8}{|c|}{ Test for overall effect: $Z=3.47(P=0)$} \\
\hline
\end{tabular}

Analysis 32.3. Comparison 32 Joint space narrowing (JSN), Outcome 3 Change from the baseline mean JSN 52 weeks,certolizumab pegol $200 \mathrm{mg}$.

\begin{tabular}{|c|c|c|c|c|c|c|c|}
\hline \multirow[t]{2}{*}{ Study or subgroup } & \multicolumn{2}{|c|}{ Certolizumab pegol } & \multicolumn{2}{|c|}{ Control } & \multirow{2}{*}{$\begin{array}{c}\text { Mean Difference } \\
\text { Fixed, } 95 \% \mathrm{Cl}\end{array}$} & \multirow[t]{2}{*}{ Weight } & \multirow{2}{*}{$\begin{array}{c}\text { Mean Difference } \\
\text { Fixed, } 95 \% \mathrm{Cl}\end{array}$} \\
\hline & $\mathbf{N}$ & Mean(SD) & $\mathbf{N}$ & Mean(SD) & & & \\
\hline Emery 2015 & 528 & $0.1(1.7)$ & 163 & $0.7(2.3)$ & & $83.08 \%$ & $-0.6[-0.98,-0.22]$ \\
\hline Keystone 2008 & 367 & $0.4(4.2)$ & 181 & $1.4(5)$ & $\longrightarrow$ & $16.92 \%$ & $-1[-1.85,-0.15]$ \\
\hline Total *** & 895 & & 344 & & & $100 \%$ & $-0.67[-1.02,-0.32]$ \\
\hline \multicolumn{8}{|c|}{ Heterogeneity: $\mathrm{Tau}^{2}=0 ; \mathrm{Chi}^{2}=0.71, \mathrm{df}=1(\mathrm{P}=0.4) ; \mathrm{I}^{2}=0 \%$} \\
\hline \multicolumn{3}{|c|}{ Test for overall effect: $Z=3.76(P=0)$} & & & & & \\
\hline
\end{tabular}

Analysis 32.4. Comparison 32 Joint space narrowing (JSN), Outcome 4 Change from the baseline mean JSN 52 weeks, certolizumab pegol $400 \mathrm{mg}$.

\begin{tabular}{|c|c|c|c|c|c|c|c|}
\hline \multirow{3}{*}{$\begin{array}{l}\text { Study or subgroup } \\
\text { Keystone } 2008\end{array}$} & \multicolumn{2}{|c|}{ Certolizumab } & \multicolumn{2}{|c|}{ Control } & \multirow{2}{*}{\multicolumn{2}{|c|}{$\begin{array}{c}\text { Mean Difference } \\
\text { Fixed, } 95 \% \mathrm{Cl}\end{array}$}} & \multirow{3}{*}{$\begin{array}{l}\text { Mean Difference } \\
\text { Fixed, } \mathbf{9 5 \%} \text { Cl } \\
-1.2[-1.98,-0.42]\end{array}$} \\
\hline & $\mathbf{N}$ & $\operatorname{Mean}(\mathrm{SD})$ & $\mathbf{N}$ & Mean(SD) & & & \\
\hline & 363 & $0.2(2.8)$ & 181 & $1.4(5)$ & 1 & & \\
\hline
\end{tabular}


Comparison 33. Joint space narrowing (JSN) at 24 weeks, any dose

\begin{tabular}{|c|c|c|c|c|}
\hline Outcome or subgroup title & No. of studies & $\begin{array}{l}\text { No. of partici- } \\
\text { pants }\end{array}$ & Statistical method & Effect size \\
\hline 1 Change from baseline & 2 & 1439 & $\begin{array}{l}\text { Mean Difference (IV, Random, 95\% } \\
\mathrm{Cl} \text { ) }\end{array}$ & $-0.50[-0.79,-0.21]$ \\
\hline $\begin{array}{l}1.1 \text { certolizumab pegol } 200 \\
\mathrm{mg} \mathrm{sc}\end{array}$ & 2 & 716 & $\begin{array}{l}\text { Mean Difference (IV, Random, 95\% } \\
\mathrm{Cl} \text { ) }\end{array}$ & $-0.46[-0.87,-0.04]$ \\
\hline $\begin{array}{l}1.2 \text { certolizumab pegol } 400 \\
\mathrm{mg} \mathrm{sc}\end{array}$ & 2 & 723 & $\begin{array}{l}\text { Mean Difference (IV, Random, 95\% } \\
\mathrm{Cl} \text { ) }\end{array}$ & $-0.54[-0.96,-0.13]$ \\
\hline
\end{tabular}

Analysis 33.1. Comparison 33 Joint space narrowing (JSN) at 24 weeks, any dose, Outcome 1 Change from baseline.

\begin{tabular}{|c|c|c|c|c|c|c|c|}
\hline \multirow[t]{2}{*}{ Study or subgroup } & \multicolumn{2}{|c|}{ Certolizumab pegol } & \multicolumn{2}{|c|}{ Control } & \multirow{2}{*}{$\begin{array}{l}\text { Mean Difference } \\
\text { Random, } 95 \% \mathrm{CI}\end{array}$} & \multirow[t]{2}{*}{ Weight } & \multirow{2}{*}{$\begin{array}{l}\text { Mean Difference } \\
\text { Random, } 95 \% \mathrm{Cl}\end{array}$} \\
\hline & $\mathbf{N}$ & $\operatorname{Mean}(S D)$ & $\mathbf{N}$ & Mean(SD) & & & \\
\hline \multicolumn{8}{|c|}{ 33.1.1 certolizumab pegol $200 \mathrm{mg} \mathrm{sc}$} \\
\hline Keystone 2008 & 355 & $0.2(2.5)$ & 91 & $0.7(2.4)$ & & $27.73 \%$ & $-0.5[-1.06,0.06]$ \\
\hline Smolen 2009 & 214 & $0.1(1.4)$ & 56 & $0.5(2.3)$ & - & $21.65 \%$ & $-0.4[-1.03,0.23]$ \\
\hline Subtotal ${ }^{\star \star \star}$ & 569 & & 147 & & & $49.37 \%$ & $-0.46[-0.87,-0.04]$ \\
\hline \multicolumn{8}{|c|}{ Heterogeneity: $\operatorname{Tau}^{2}=0 ; \mathrm{Chi}^{2}=0.05, \mathrm{df}=1(\mathrm{P}=0.82) ; \mathrm{I}^{2}=0 \%$} \\
\hline \multicolumn{8}{|c|}{ 33.1.2 certolizumab pegol $400 \mathrm{mg} \mathrm{sc}$} \\
\hline Keystone 2008 & 355 & $0.2(2.4)$ & 90 & $0.7(2.4)$ & - & $27.96 \%$ & $-0.5[-1.06,0.06]$ \\
\hline Smolen 2009 & 222 & $-0.1(1)$ & 56 & $0.5(2.3)$ & & $22.67 \%$ & $-0.6[-1.22,0.02]$ \\
\hline Subtotal $\star \star \star ~$ & 577 & & 146 & & & $50.63 \%$ & $-0.54[-0.96,-0.13]$ \\
\hline \multicolumn{8}{|c|}{ Heterogeneity: $\operatorname{Tau}^{2}=0 ; \mathrm{Chi}^{2}=0.06, \mathrm{df}=1(\mathrm{P}=0.81) ; \mathrm{I}^{2}=0 \%$} \\
\hline Total $\star \star \star ~$ & 1146 & & 293 & & & $100 \%$ & $-0.5[-0.79,-0.21]$ \\
\hline \multicolumn{8}{|c|}{ Heterogeneity: Tau $^{2}=0 ; \mathrm{Chi}^{2}=0.2, \mathrm{df}=3(\mathrm{P}=0.98) ; \mathrm{I}^{2}=0 \%$} \\
\hline \multicolumn{8}{|c|}{ Test for overall effect: $Z=3.35(P=0)$} \\
\hline \multicolumn{8}{|c|}{ Test for subgroup differences: $\mathrm{Chi}^{2}=0.09, \mathrm{df}=1(\mathrm{P}=0.77), \mathrm{I}^{2}=0 \%$} \\
\hline & & & cer & lab pego & -0.5 & Favours & \\
\hline
\end{tabular}

Comparison 34. Joint space narrowing (JSN) at 52 weeks, any dose

\begin{tabular}{lllll}
\hline Outcome or subgroup title & No. of studies & $\begin{array}{l}\text { No. of partici- } \\
\text { pants }\end{array}$ & Statistical method & Effect size \\
\hline $\begin{array}{l}1 \text { Change from baseline } \\
\text { PC }\end{array}$ & 2 & 1602 & $\begin{array}{l}\text { Mean Difference (IV, Fixed, 95\% } \\
\text { Cl) }\end{array}$ & $-0.70[-1.04,-0.36]$ \\
\hline $\begin{array}{l}1.1 \text { certolizumab pegol 200 mg } \\
\text { SC }\end{array}$ & 2 & 1149 & $\begin{array}{l}\text { Mean Difference (IV, Fixed, 95\% } \\
\text { CI) }\end{array}$ & $-0.64[-1.00,-0.28]$ \\
\hline
\end{tabular}




\begin{tabular}{lllll}
\hline Outcome or subgroup title & No. of studies & $\begin{array}{l}\text { No. of partici- } \\
\text { pants }\end{array}$ & Statistical method & Effect size \\
\hline $\begin{array}{l}1.2 \text { certolizumab pegol } 400 \mathrm{mg} \\
\text { SC }\end{array}$ & 1 & 453 & $\begin{array}{l}\text { Mean Difference (IV, Fixed, 95\% } \\
\text { CI) }\end{array}$ & $-1.2[-2.27,-0.13]$ \\
\hline
\end{tabular}

Analysis 34.1. Comparison 34 Joint space narrowing (JSN) at 52 weeks, any dose, Outcome 1 Change from baseline.

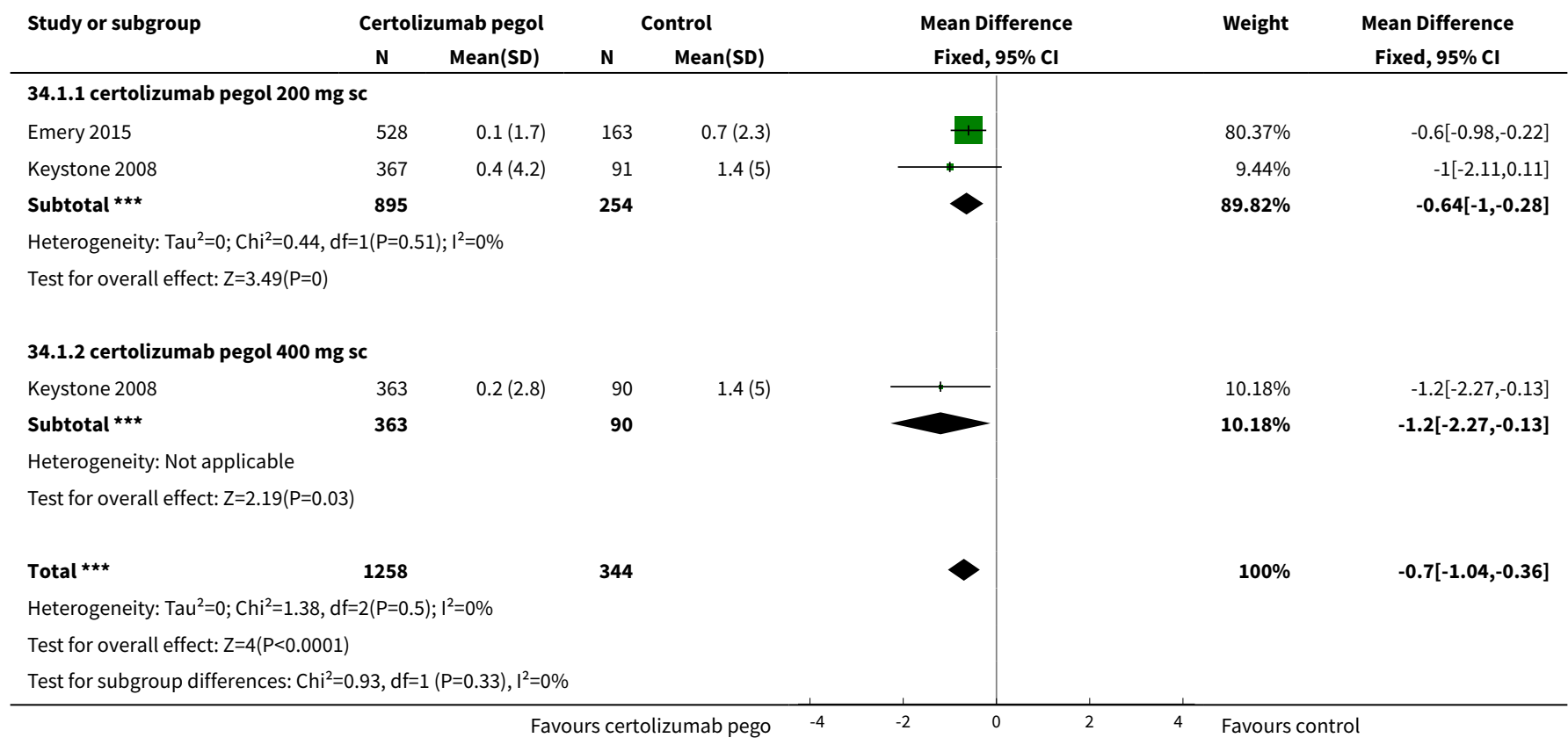

\section{Comparison 35. Modified Total Sharp Scores (mTSS) at 24 weeks, any dose}

\begin{tabular}{|c|c|c|c|c|}
\hline Outcome or subgroup title & No. of studies & $\begin{array}{l}\text { No. of partici- } \\
\text { pants }\end{array}$ & Statistical method & Effect size \\
\hline 1 Change from baseline & 3 & 1753 & $\begin{array}{l}\text { Mean Difference (IV, Random, 95\% } \\
\mathrm{CI})\end{array}$ & $-0.86[-1.19,-0.53]$ \\
\hline $\begin{array}{l}1.1 \text { certolizumab pegol } 200 \\
\mathrm{mg} \mathrm{sc}\end{array}$ & 3 & 1029 & $\begin{array}{l}\text { Mean Difference (IV, Random, 95\% } \\
\mathrm{CI})\end{array}$ & $-0.74[-1.11,-0.37]$ \\
\hline $\begin{array}{l}1.2 \text { certolizumab pegol } 400 \\
\mathrm{mg} \mathrm{sc}\end{array}$ & 2 & 724 & $\begin{array}{l}\text { Mean Difference (IV, Random, 95\% } \\
\mathrm{Cl} \text { ) }\end{array}$ & $-1.30[-1.99,-0.60]$ \\
\hline
\end{tabular}


Analysis 35.1. Comparison 35 Modified Total Sharp Scores (mTSS) at 24 weeks, any dose, Outcome 1 Change from baseline.

\begin{tabular}{|c|c|c|c|c|c|c|c|}
\hline \multirow[t]{2}{*}{ Study or subgroup } & \multicolumn{2}{|c|}{ Certolizumab pegol } & \multicolumn{2}{|c|}{ Control } & \multirow{2}{*}{$\begin{array}{l}\text { Mean Difference } \\
\text { Random, } 95 \% \mathrm{Cl}\end{array}$} & \multirow[t]{2}{*}{ Weight } & \multirow{2}{*}{$\begin{array}{l}\text { Mean Difference } \\
\text { Random, } 95 \% \mathrm{CI}\end{array}$} \\
\hline & $\mathbf{N}$ & Mean(SD) & $\mathbf{N}$ & Mean(SD) & & & \\
\hline \multicolumn{8}{|c|}{ 35.1.1 certolizumab pegol $200 \mathrm{mg} \mathrm{sc}$} \\
\hline Atsumi 2016 & 159 & $0.3(1.6)$ & 157 & $0.9(2.4)$ & & $54.82 \%$ & $-0.6[-1.04,-0.16]$ \\
\hline Keystone 2008 & 353 & $0.2(3.2)$ & 90 & $1.3(3.8)$ & & $14.73 \%$ & $-1.1[-1.95,-0.25]$ \\
\hline Smolen 2009 & 214 & $0.2(2.7)$ & 56 & $1.2(4.1)$ & & $8.35 \%$ & $-1[-2.13,0.13]$ \\
\hline Subtotal $\star \star \star$ & 726 & & 303 & & & $77.89 \%$ & $-0.74[-1.11,-0.37]$ \\
\hline \multicolumn{8}{|c|}{ Test for overall effect: $Z=3.9(P<0.0001)$} \\
\hline \multicolumn{8}{|c|}{ 35.1.2 certolizumab pegol $400 \mathrm{mg} \mathrm{sc}$} \\
\hline Keystone 2008 & 355 & $0.2(4.2)$ & 91 & $1.3(3.8)$ & & $13.39 \%$ & $-1.1[-1.99,-0.21]$ \\
\hline Smolen 2009 & 222 & $-0.4(2.1)$ & 56 & $1.2(4.1)$ & & $8.72 \%$ & $-1.6[-2.71,-0.49]$ \\
\hline Subtotal $\star \star \star$ & 577 & & 147 & & & $22.11 \%$ & $-1.3[-1.99,-0.6]$ \\
\hline \multicolumn{8}{|c|}{ Test for overall effect: $Z=3.65(P=0)$} \\
\hline Total $\star \star \star$ & 1303 & & 450 & & & $100 \%$ & $-0.86[-1.19,-0.53]$ \\
\hline \multicolumn{8}{|c|}{ Heterogeneity: $\mathrm{Tau}^{2}=0 ; \mathrm{Chi}^{2}=3.68, \mathrm{df}=4(\mathrm{P}=0.45) ; \mathrm{I}^{2}=0 \%$} \\
\hline \multicolumn{8}{|c|}{ Test for overall effect: $Z=5.16(P<0.0001)$} \\
\hline \multicolumn{8}{|c|}{ Test for subgroup differences: $\mathrm{Chi}^{2}=1.93, \mathrm{df}=1(\mathrm{P}=0.16), \mathrm{I}^{2}=48.3 \%$} \\
\hline
\end{tabular}

Comparison 36. Modified Total Sharp Scores (mTSS) at 52 weeks, any dose

\begin{tabular}{lllll}
\hline Outcome or subgroup title & No. of studies & $\begin{array}{l}\text { No. of partici- } \\
\text { pants }\end{array}$ & Statistical method & Effect size \\
\hline 1 Change from baseline & 3 & 1915 & $\begin{array}{l}\text { Mean Difference (IV, Fixed, 95\% } \\
\text { CI) }\end{array}$ & -1.63 [-2.13, -1.13] \\
\hline $\begin{array}{l}1.1 \text { certolizumab pegol 200 mg } \\
\text { sc }\end{array}$ & 3 & 1462 & $\begin{array}{l}\text { Mean Difference (IV, Fixed, 95\% } \\
\text { CI) }\end{array}$ & -1.54 [-2.06, -1.01] \\
\hline $\begin{array}{l}1.2 \text { certolizumab pegol 400 mg } \\
\text { sc }\end{array}$ & 1 & 453 & $\begin{array}{l}\text { Mean Difference (IV, Fixed, 95\% } \\
\text { CI) }\end{array}$ & $-2.60[-4.29,-0.91]$ \\
\hline
\end{tabular}

Analysis 36.1. Comparison 36 Modified Total Sharp Scores (mTSS) at 52 weeks, any dose, Outcome 1 Change from baseline.

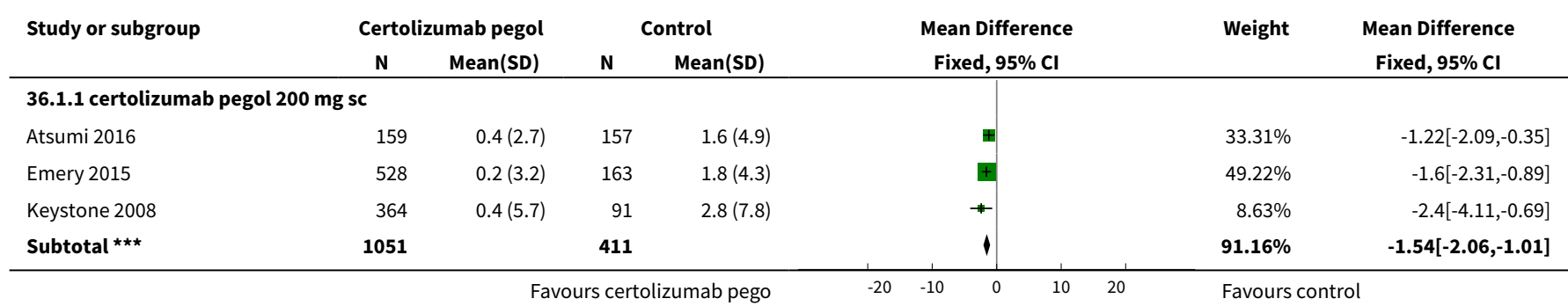




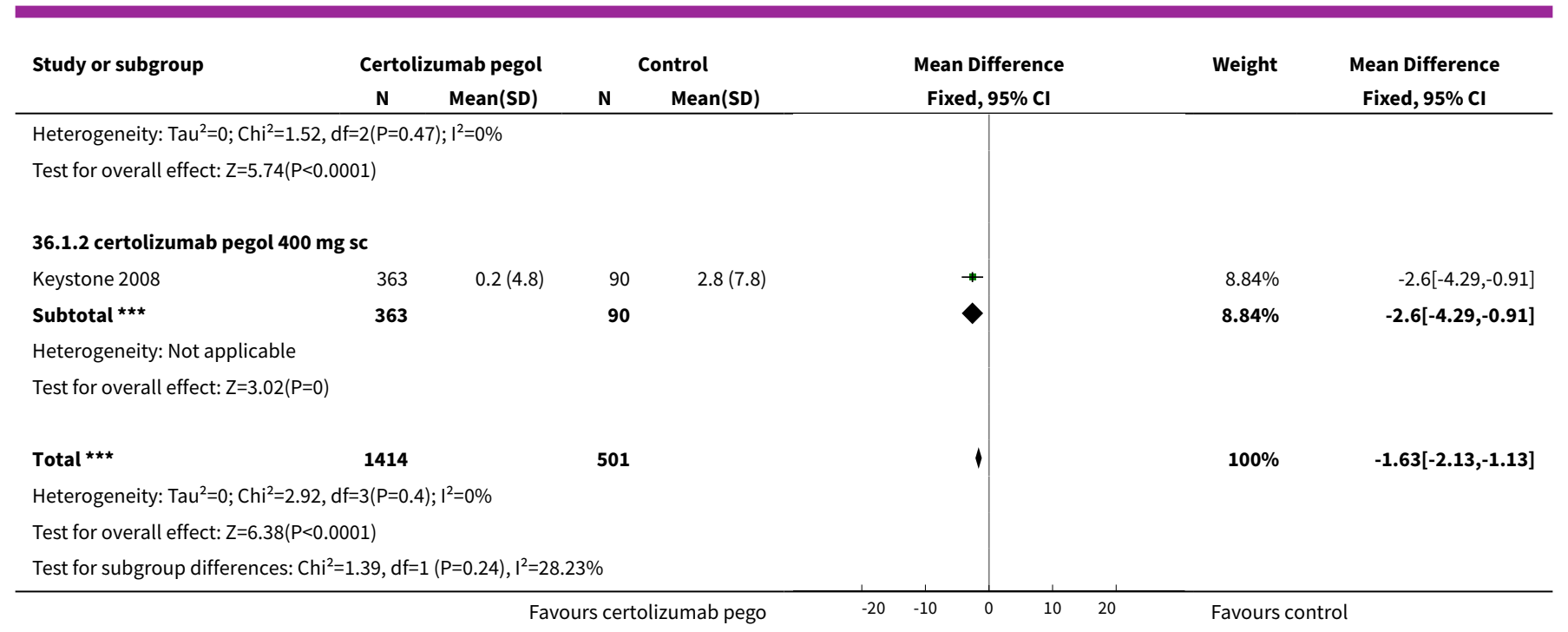

\section{Comparison 37. Modified total Sharp scores (mTSS)}

\begin{tabular}{lllll}
\hline Outcome or subgroup title & No. of studies & $\begin{array}{l}\text { No. of partici- } \\
\text { pants }\end{array}$ & Statistical method & Effect size \\
\hline $\begin{array}{l}1 \text { Change from the baseline mean mTSS 24 } \\
\text { weeks, certolizumab pegol 200 mg }\end{array}$ & 2 & 859 & $\begin{array}{l}\text { Mean Difference (IV, } \\
\text { Random, 95\% Cl) }\end{array}$ & $\begin{array}{l}-1.06[-1.58, \\
-0.55]\end{array}$ \\
\hline $\begin{array}{l}\text { 2 Change from the baseline mean mTSS 24 } \\
\text { weeks, certolizumab 400 mg }\end{array}$ & 2 & 869 & Mean Difference (IV, & -1.32 [-1.85, \\
\hline $\begin{array}{l}\text { 3 Change from the baseline mean mTSS 52 } \\
\text { weeks, certolizumab pegol 200 mg }\end{array}$ & 1 & 545 & Random, 95\% Cl) & $-0.78]$ \\
\hline $\begin{array}{l}\text { 4 Change from the baseline mean mTSS 52 } \\
\text { weeks, certolizumab pegol 400 mg }\end{array}$ & 1 & & Fixed, 95\% Cl) & $-2.4[-3.68,-1.12]$ \\
\hline
\end{tabular}

Analysis 37.1. Comparison 37 Modified total Sharp scores (mTSS), Outcome 1 Change from the baseline mean mTSS 24 weeks, certolizumab pegol $200 \mathrm{mg}$.

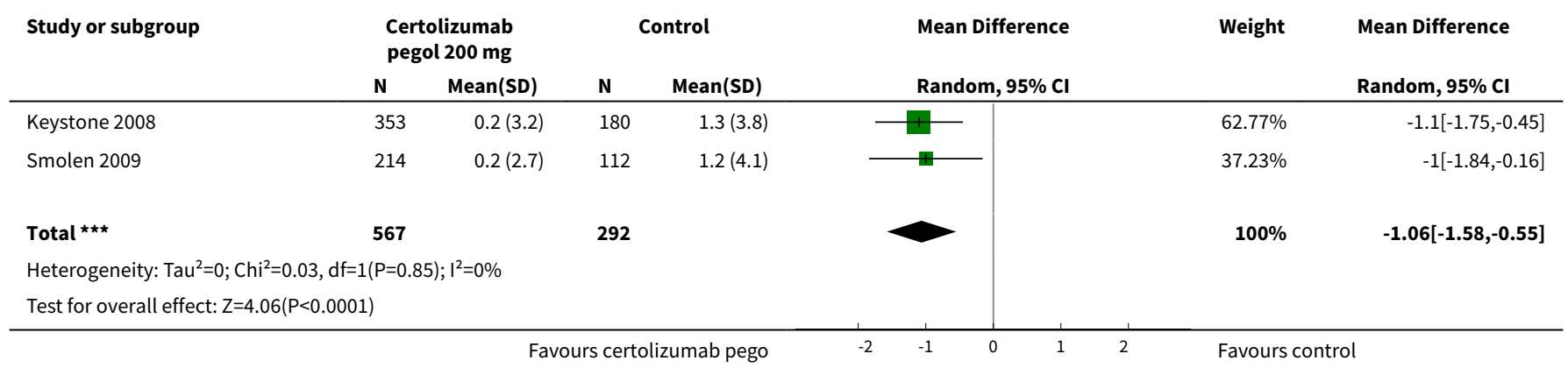


Analysis 37.2. Comparison 37 Modified total Sharp scores (mTSS), Outcome

2 Change from the baseline mean mTSS 24 weeks, certolizumab $400 \mathrm{mg}$.

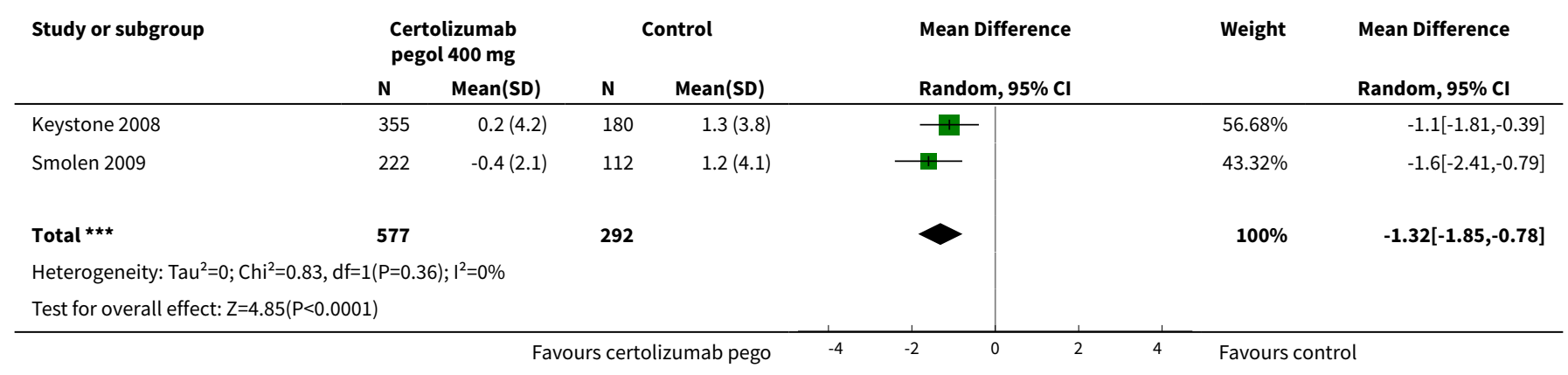

Analysis 37.3. Comparison 37 Modified total Sharp scores (mTSS), Outcome 3 Change from the baseline mean mTSS 52 weeks, certolizumab pegol $200 \mathrm{mg}$.

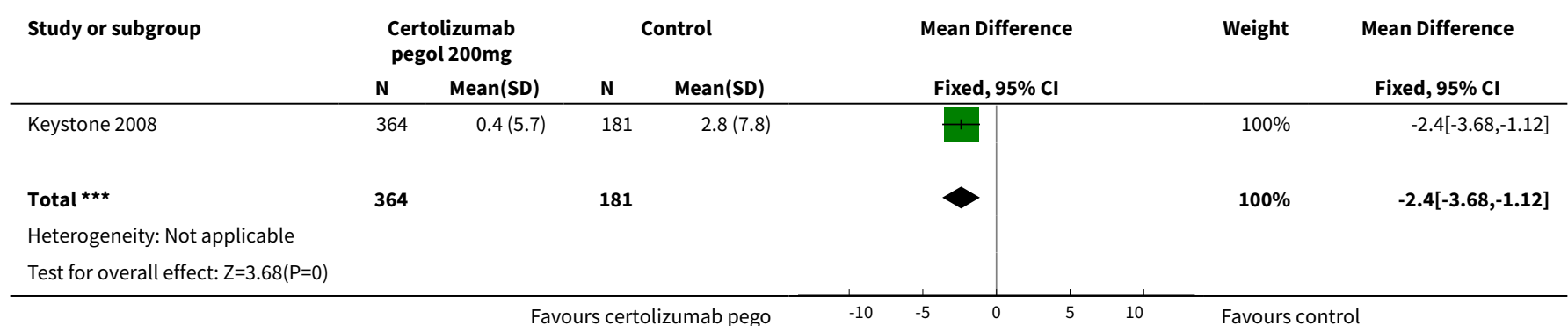

Analysis 37.4. Comparison 37 Modified total Sharp scores (mTSS), Outcome 4 Change from the baseline mean mTSS 52 weeks, certolizumab pegol $400 \mathrm{mg}$.

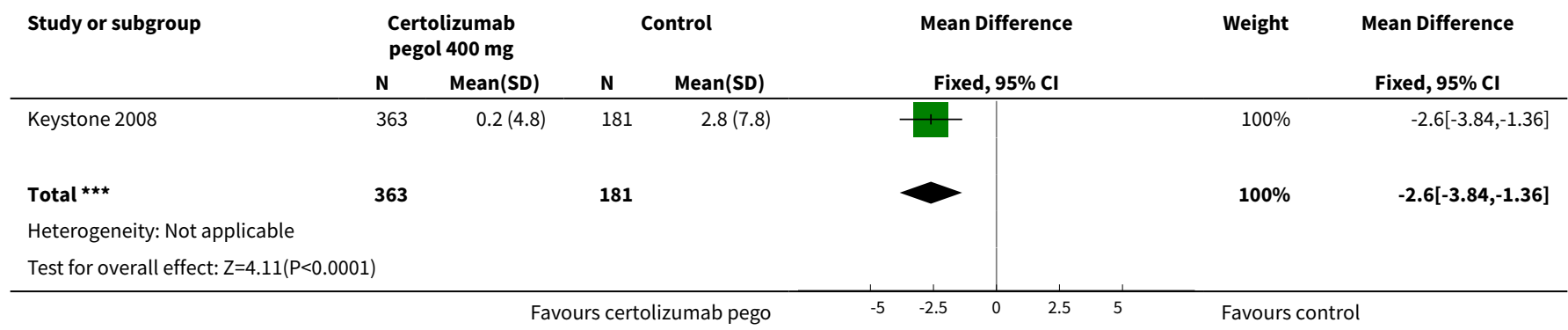

\section{Comparison 38. Certolizumab pegol $1 \mathrm{mg} / \mathrm{kg} / \mathrm{day}$ sc}

\begin{tabular}{lllll}
\hline Outcome or subgroup title & No. of studies & $\begin{array}{l}\text { No. of partici- } \\
\text { pants }\end{array}$ & Statistical method & Effect size \\
\hline 1 Headache & 1 & $\begin{array}{l}\text { Peto Odds Ratio (Peto, Fixed, 95\% } \\
\text { Cl) }\end{array}$ & Totals not selected \\
\hline
\end{tabular}




\begin{tabular}{lllll}
\hline Outcome or subgroup title & No. of studies & $\begin{array}{l}\text { No. of partici- } \\
\text { pants }\end{array}$ & Statistical method & Effect size \\
\hline $\begin{array}{l}\text { 2 Lower respiratory tract in- } \\
\text { fection }\end{array}$ & 1 & $\begin{array}{l}\text { Peto Odds Ratio (Peto, Fixed, 95\% } \\
\text { Cl) }\end{array}$ & Totals not selected \\
\hline $\begin{array}{l}\text { 3 Adverse events Intensity } \\
\text { severe }\end{array}$ & 1 & $\begin{array}{l}\text { Peto Odds Ratio (Peto, Fixed, 95\% } \\
\text { Cl) }\end{array}$ & Totals not selected \\
\hline $\begin{array}{l}\text { 4 Antinuclear antibodies } \\
\text { (ANA) }\end{array}$ & 1 & $\begin{array}{l}\text { Peto Odds Ratio (Peto, Fixed, 95\% } \\
\text { Cl) }\end{array}$ & Totals not selected \\
\hline 5 Urinary tract infection & 1 & $\begin{array}{l}\text { Peto Odds Ratio (Peto, Fixed, 95\% } \\
\text { Cl) }\end{array}$ & Totals not selected \\
\hline
\end{tabular}

Analysis 38.1. Comparison 38 Certolizumab pegol $1 \mathrm{mg} / \mathrm{kg} /$ day sc, Outcome 1 Headache.

\begin{tabular}{|c|c|c|c|c|c|c|c|}
\hline Study or subgroup & $\begin{array}{c}\text { certolizumab pegol } 1 \mathrm{mg} \\
\mathrm{n} / \mathrm{N}\end{array}$ & $\begin{array}{c}\text { control } \\
n / N\end{array}$ & & & $\begin{array}{l}\text { dds Ratio } \\
\text { xed, } 95 \% \mathrm{Cl}\end{array}$ & & $\begin{array}{c}\text { Peto Odds Ratio } \\
\text { Peto, Fixed, } 95 \% \mathrm{Cl}\end{array}$ \\
\hline Choy 2002 & $3 / 8$ & $1 / 12$ & & & 1 & & $5.65[0.64,49.98]$ \\
\hline
\end{tabular}

Analysis 38.2. Comparison 38 Certolizumab pegol $1 \mathrm{mg} / \mathrm{kg} /$ day sc, Outcome 2 Lower respiratory tract infection.

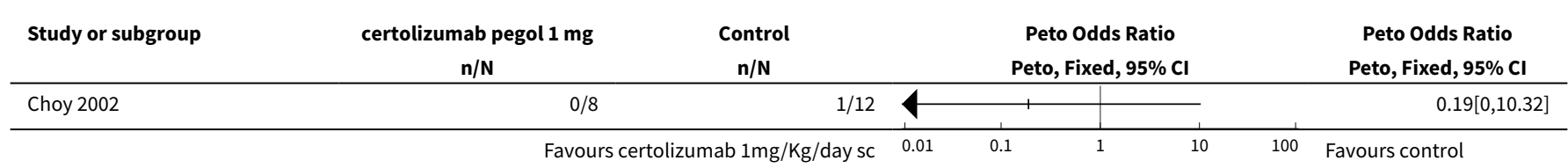

Analysis 38.3. Comparison 38 Certolizumab pegol $1 \mathrm{mg} / \mathrm{kg} /$ day sc, Outcome 3 Adverse events Intensity severe.

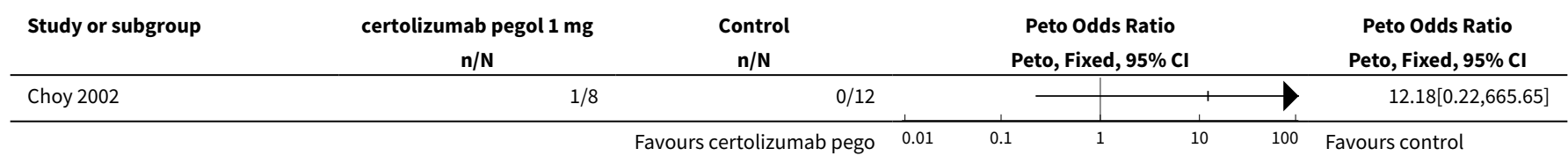

Analysis 38.4. Comparison 38 Certolizumab pegol $1 \mathrm{mg} / \mathrm{kg} /$ day sc, Outcome 4 Antinuclear antibodies (ANA).

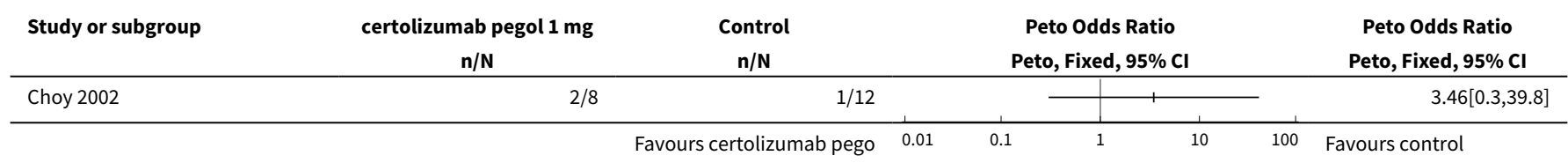


Analysis 38.5. Comparison 38 Certolizumab pegol $1 \mathrm{mg} / \mathrm{kg} /$ day sc, Outcome 5 Urinary tract infection.

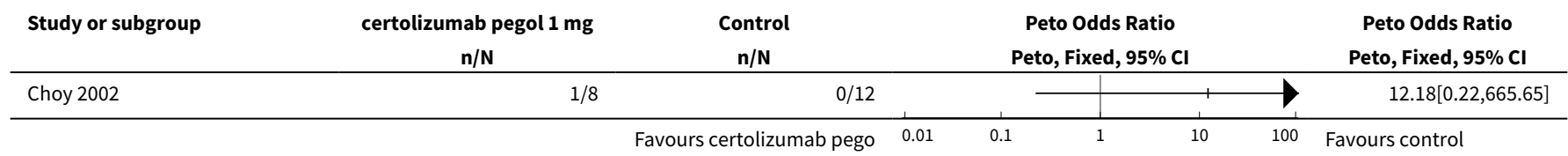

Comparison 39. Certolizumab $5 \mathrm{mg} / \mathrm{kg} / \mathrm{day} \mathrm{sc}$

\begin{tabular}{lllll}
\hline Outcome or subgroup title & No. of studies & $\begin{array}{l}\text { No. of partici- } \\
\text { pants }\end{array}$ & Statistical method & Effect size \\
\hline $\begin{array}{l}1 \text { Lower respiratory tract infec- } \\
\text { tion }\end{array}$ & 1 & $\begin{array}{l}\text { Peto Odds Ratio (Peto, Fixed, 95\% } \\
\text { Cl) }\end{array}$ & Totals not selected \\
\hline 2 Urinary tract infection & 1 & $\begin{array}{l}\text { Peto Odds Ratio (Peto, Fixed, 95\% } \\
\text { Cl) }\end{array}$ & Totals not selected \\
\hline
\end{tabular}

Analysis 39.1. Comparison 39 Certolizumab $5 \mathrm{mg} / \mathrm{kg} /$ day sc, Outcome 1 Lower respiratory tract infection.

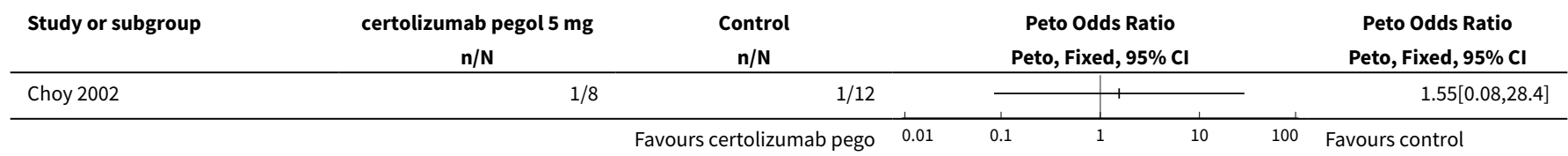

Analysis 39.2. Comparison 39 Certolizumab $5 \mathrm{mg} / \mathrm{kg} / \mathrm{day} \mathrm{sc}$, Outcome 2 Urinary tract infection.

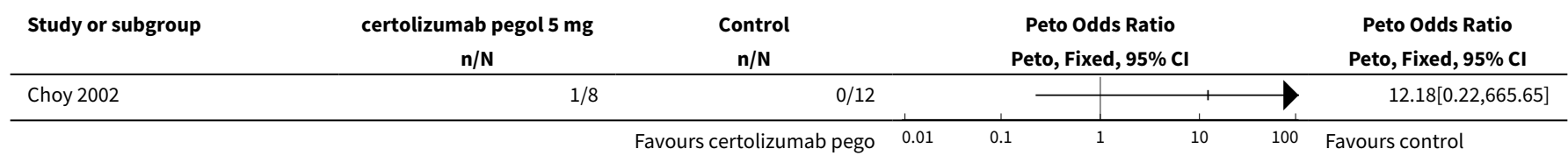

\section{Comparison 40. Certolizumab $20 \mathrm{mg} / \mathrm{kg} /$ day sc}

\begin{tabular}{lllll}
\hline $\begin{array}{l}\text { Outcome or subgroup ti- } \\
\text { tle }\end{array}$ & No. of studies & $\begin{array}{l}\text { No. of partici- } \\
\text { pants }\end{array}$ & Statistical method & Effect size \\
\hline 1 Headache & 1 & Risk Ratio (M-H, Fixed, 95\% Cl) & Totals not selected \\
\hline $\begin{array}{l}\text { L Lower respiratory tract } \\
\text { infection }\end{array}$ & 1 & 20 & Risk Ratio (M-H, Fixed, 95\% Cl) & $3.00[0.32,27.83]$ \\
\hline $\begin{array}{l}\text { 3 Death } \\
\text { 4 Antinuclear antibodies }\end{array}$ & 1 & Peto Odds Ratio (Peto, Fixed, 95\% Cl) & Totals not selected \\
\hline \begin{tabular}{l} 
(ANA) \\
\hline
\end{tabular}
\end{tabular}




\begin{tabular}{lllll}
\hline $\begin{array}{l}\text { Outcome or subgroup ti- } \\
\text { tle }\end{array}$ & No. of studies & $\begin{array}{l}\text { No. of partici- } \\
\text { pants }\end{array}$ & Statistical method & Effect size \\
\hline 5 Urinary tract infection & 1 & & Peto Odds Ratio (Peto, Fixed, 95\% Cl) & Totals not selected \\
\hline
\end{tabular}

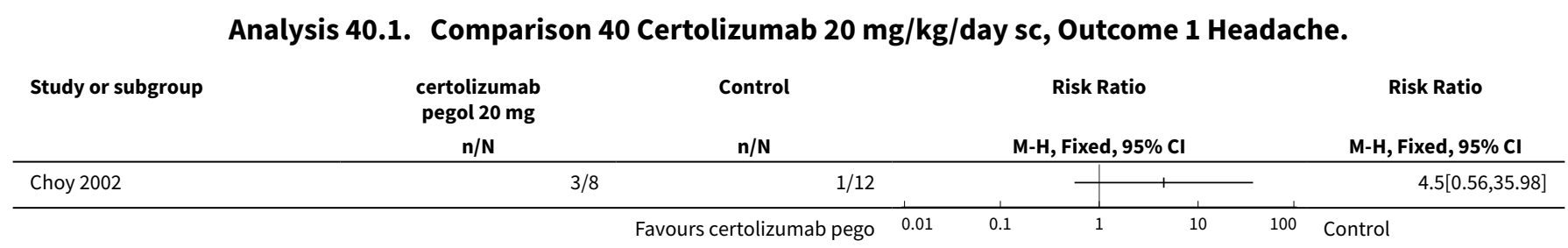

Analysis 40.2. Comparison 40 Certolizumab $20 \mathrm{mg} / \mathrm{kg} / \mathrm{day} \mathrm{sc}$, Outcome 2 Lower respiratory tract infection.

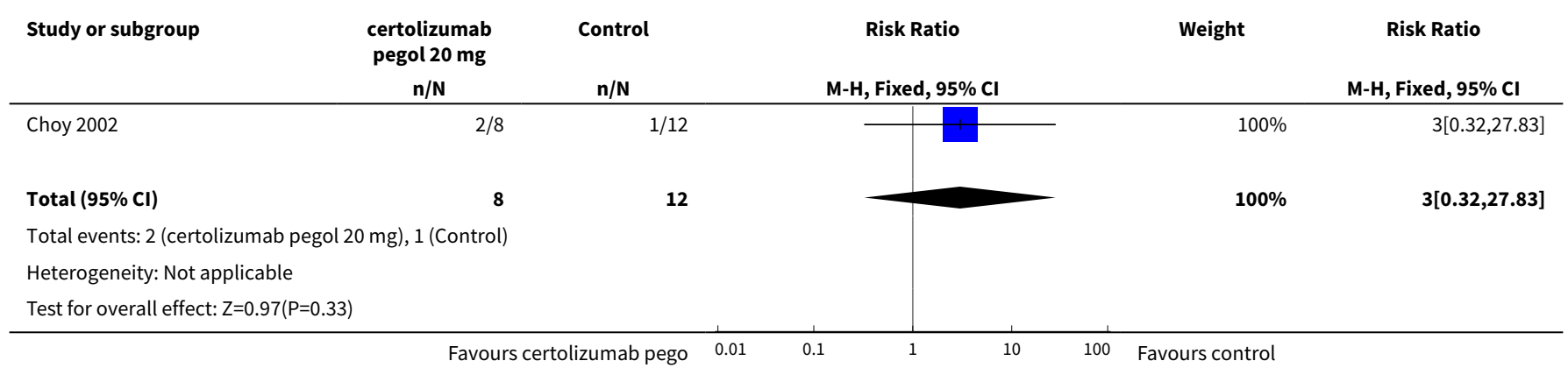

Analysis 40.3. Comparison 40 Certolizumab $20 \mathrm{mg} / \mathrm{kg} /$ day sc, Outcome 3 Death.

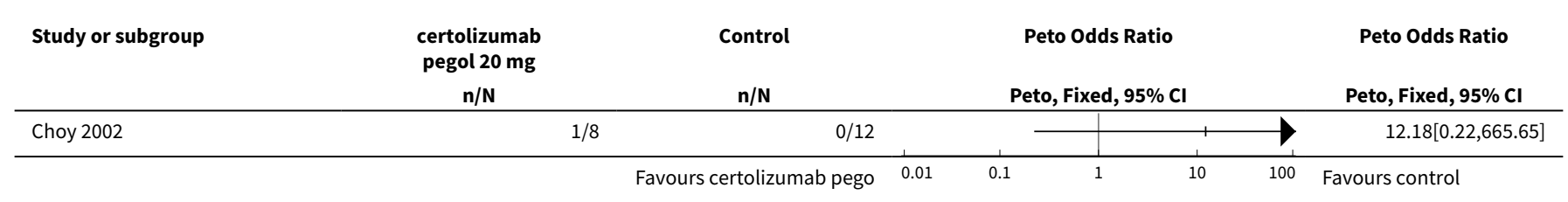

Analysis 40.4. Comparison 40 Certolizumab $20 \mathrm{mg} / \mathrm{kg} /$ day sc, Outcome 4 Antinuclear antibodies (ANA).

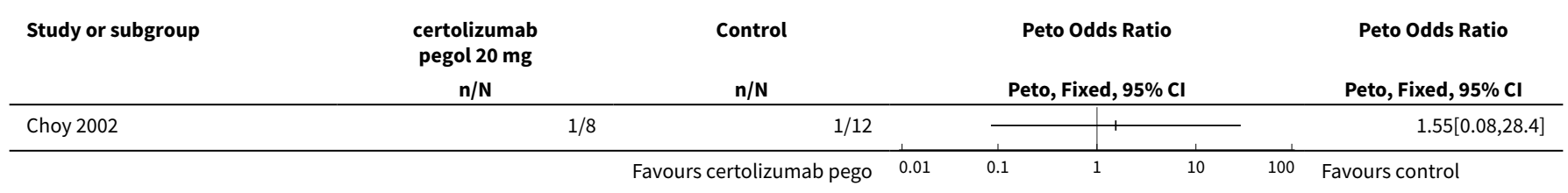


Analysis 40.5. Comparison 40 Certolizumab $20 \mathrm{mg} / \mathrm{kg} /$ day sc, Outcome 5 Urinary tract infection.

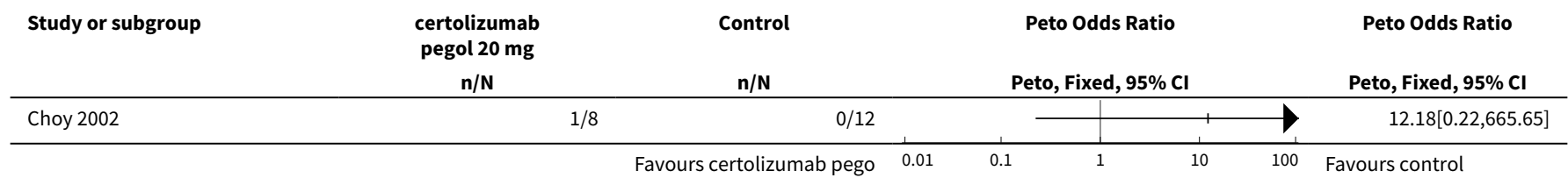

Comparison 41. Safety, SAE certolizumab $200 \mathrm{mg}$

\begin{tabular}{lllll}
\hline Outcome or subgroup title & No. of studies & $\begin{array}{l}\text { No. of partici- } \\
\text { pants }\end{array}$ & Statistical method & Effect size \\
\hline 1 Serious Adverse Events (SAE) & 9 & 3927 & $\begin{array}{l}\text { Peto Odds Ratio (Peto, Fixed, 95\% } \\
\text { Cl) }\end{array}$ & $1.47[1.13,1.91]$ \\
\hline
\end{tabular}

Analysis 41.1. Comparison 41 Safety, SAE certolizumab 200 mg, Outcome 1 Serious Adverse Events (SAE).

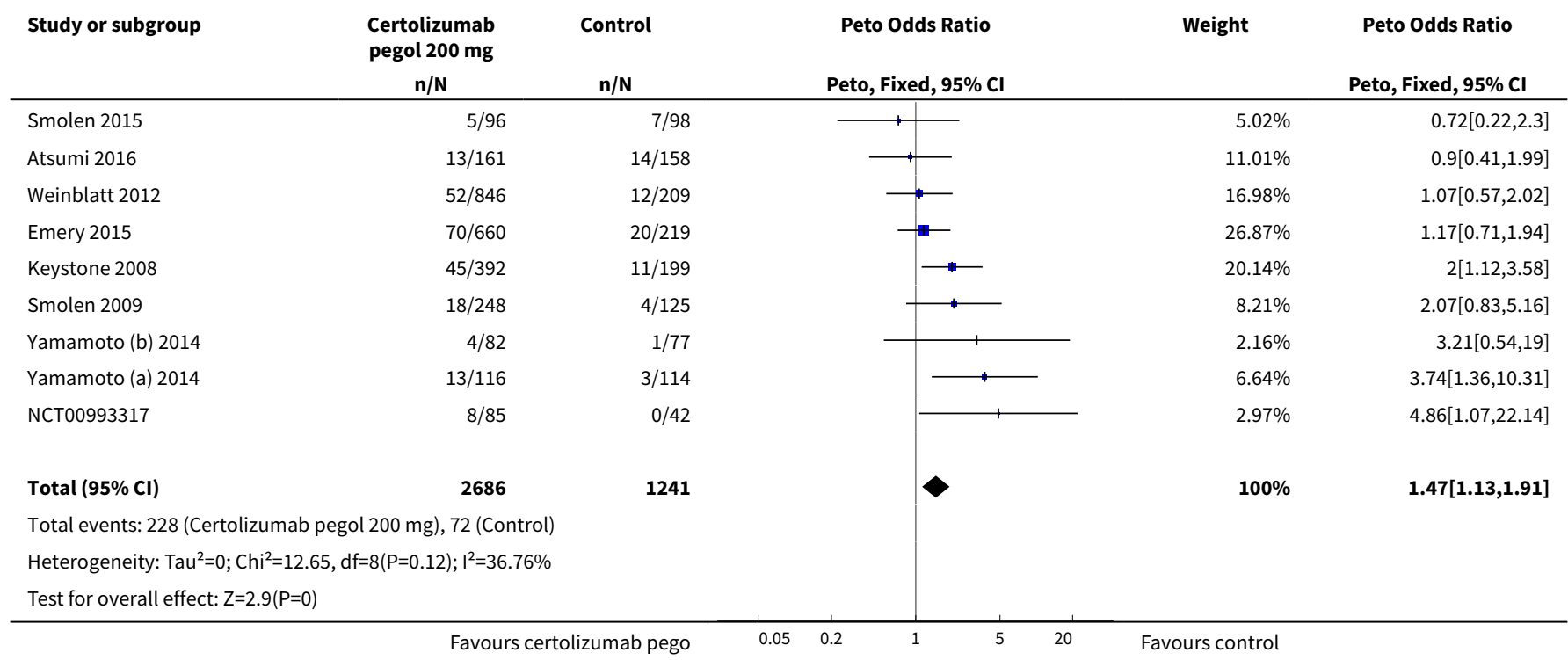

\section{Comparison 42. Safety, SAE certolizumab $400 \mathrm{mg}$}

\begin{tabular}{lllll}
\hline Outcome or subgroup title & No. of studies & $\begin{array}{l}\text { No. of partici- } \\
\text { pants }\end{array}$ & Statistical method & Effect size \\
\hline 1 Serious Adverse Events (SAEs) & 6 & 1624 & $\begin{array}{l}\text { Peto Odds Ratio (Peto, Fixed, 95\% } \\
\text { Cl) }\end{array}$ & $1.98[1.36,2.90]$ \\
\hline
\end{tabular}


Analysis 42.1. Comparison 42 Safety, SAE certolizumab 400 mg, Outcome 1 Serious Adverse Events (SAEs).

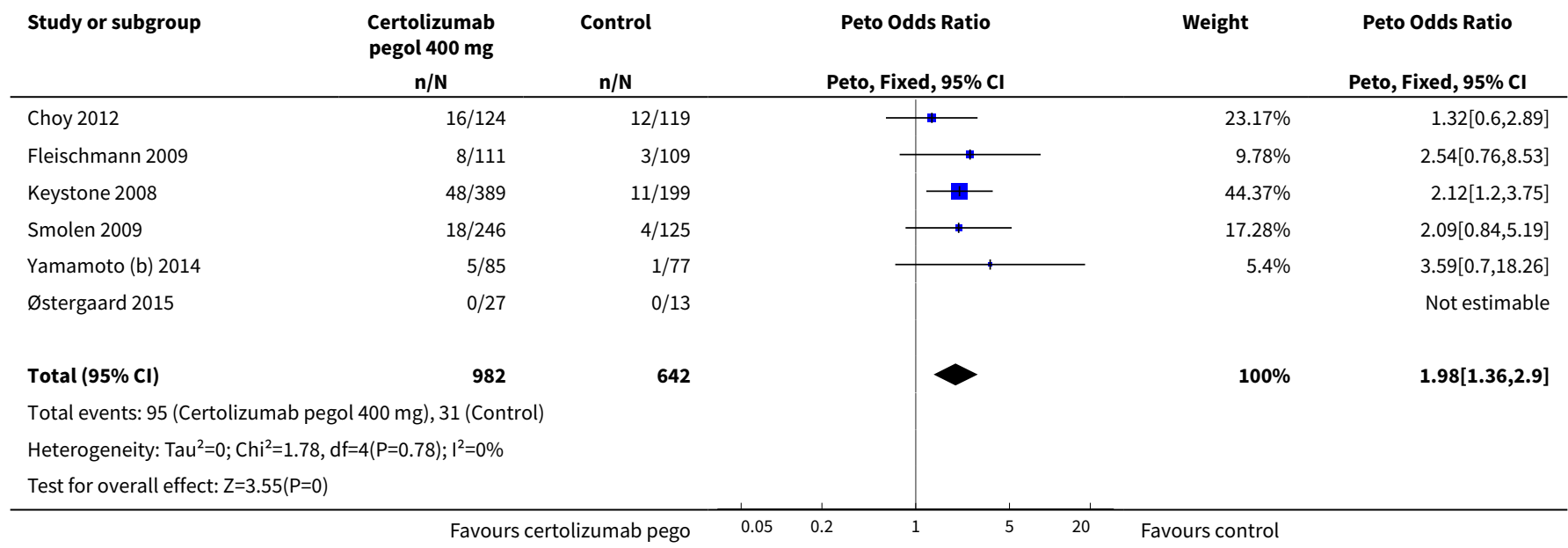

\section{Comparison 43. Withdrawals}

\begin{tabular}{lllll}
\hline Outcome or subgroup title & No. of studies & $\begin{array}{l}\text { No. of partici- } \\
\text { pants }\end{array}$ & Statistical method & Effect size \\
\hline $\begin{array}{l}\text { 1 All Withdrawn: any doses any fol- } \\
\text { low-up }\end{array}$ & 13 & 5200 & $\begin{array}{l}\text { Risk Ratio (M-H, Random, 95\% } \\
\text { Cl) }\end{array}$ & $0.47[0.39,0.56]$ \\
\hline 2 Withdrawals due to adverse events & 12 & 5236 & $\begin{array}{l}\text { Peto Odds Ratio (Peto, Fixed, } \\
95 \% \text { Cl) }\end{array}$ & $1.45[1.09,1.94]$ \\
\hline
\end{tabular}

Analysis 43.1. Comparison 43 Withdrawals, Outcome 1 All Withdrawn: any doses any follow-up.

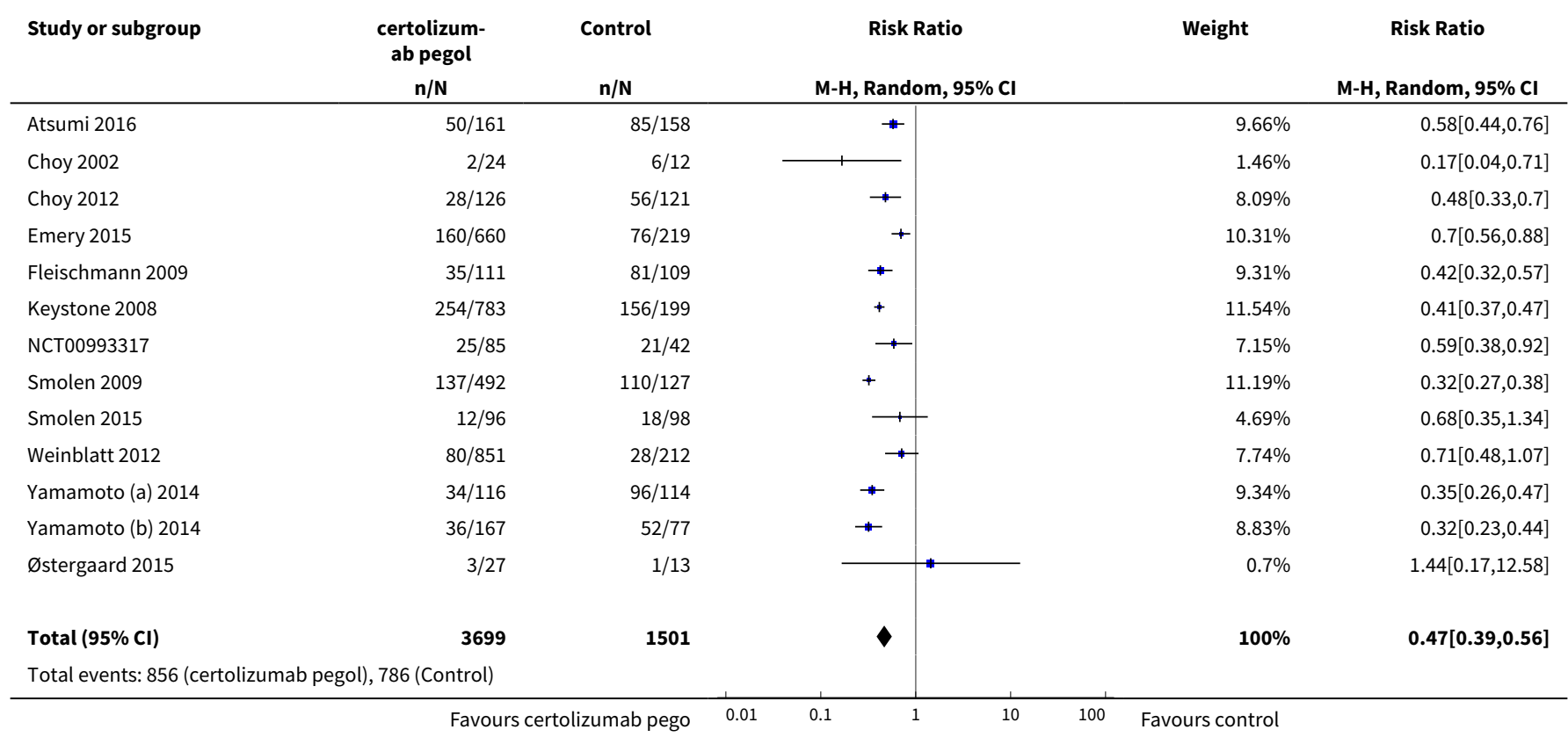




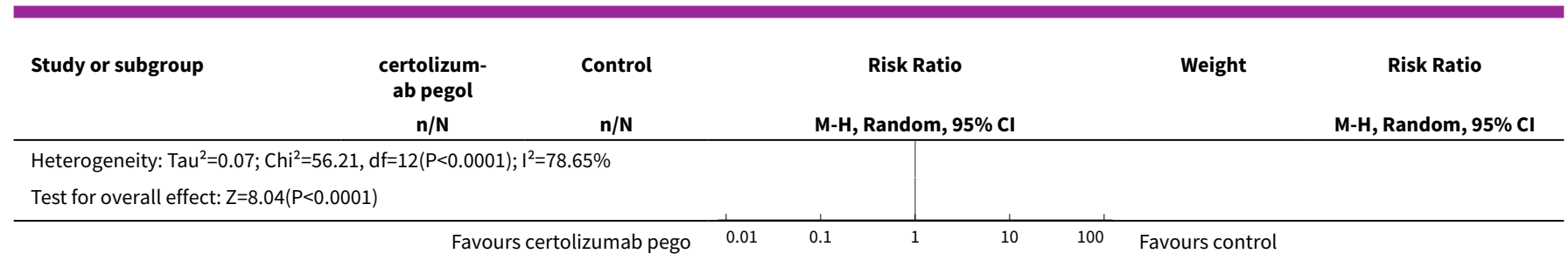

\section{Analysis 43.2. Comparison 43 Withdrawals, Outcome 2 Withdrawals due to adverse events.}

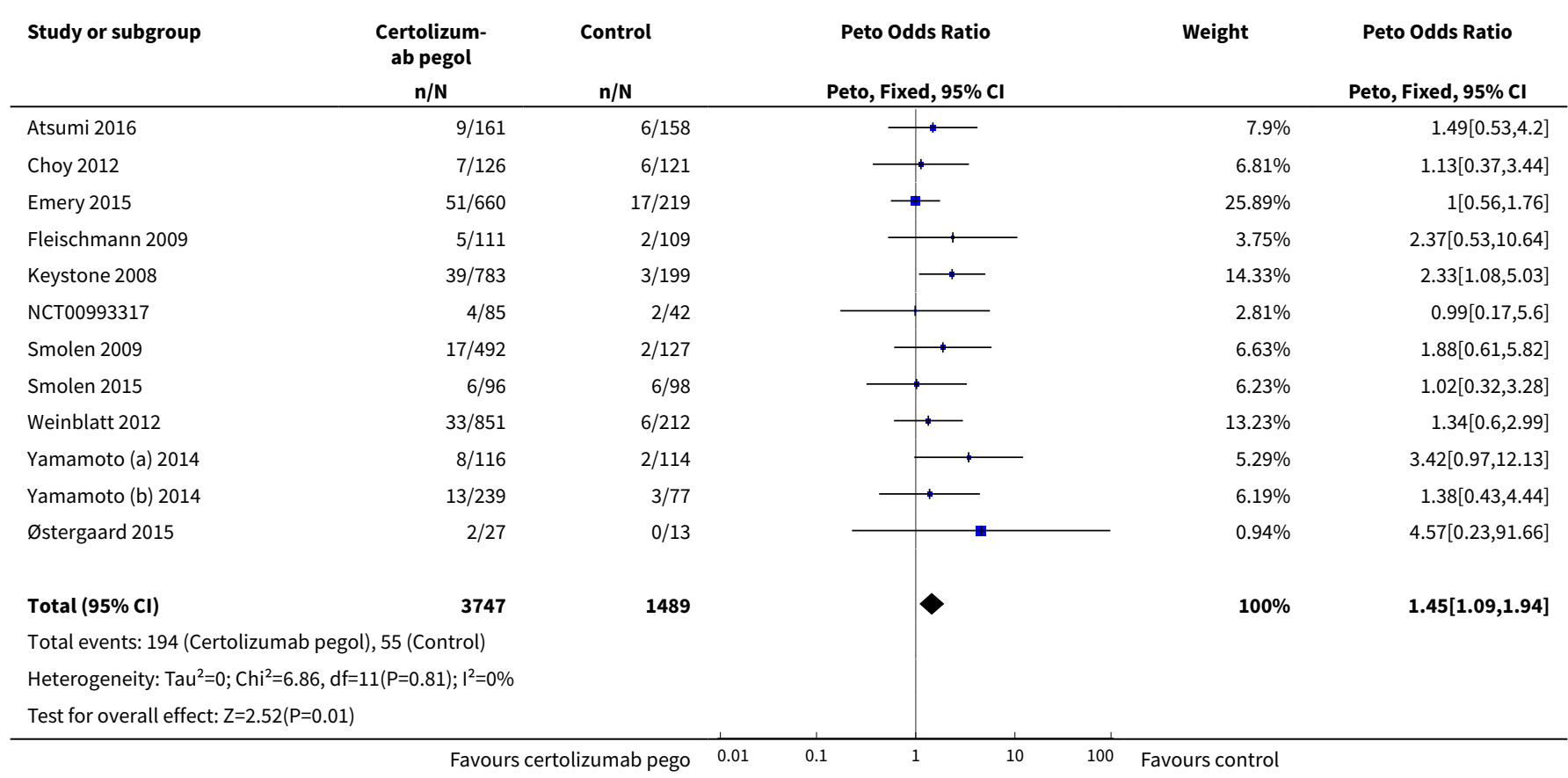

\section{Comparison 44. ACR at 24 weeks, any dose}

\begin{tabular}{|c|c|c|c|c|}
\hline $\begin{array}{l}\text { Outcome or subgroup ti- } \\
\text { tle }\end{array}$ & No. of studies & $\begin{array}{l}\text { No. of partici- } \\
\text { pants }\end{array}$ & Statistical method & Effect size \\
\hline $1 \mathrm{ACR} 20$ & 8 & 2935 & Risk Ratio (M-H, Random, 95\% Cl) & $2.76[2.29,3.33]$ \\
\hline $\begin{array}{l}1.1 \text { certolizumab } 100 \mathrm{mg} \\
\mathrm{sc}\end{array}$ & 1 & 98 & Risk Ratio (M-H, Random, 95\% Cl) & $2.65[1.28,5.47]$ \\
\hline $\begin{array}{l}1.2 \text { certolizumab } 200 \mathrm{mg} \\
\mathrm{sc}\end{array}$ & 6 & 1462 & Risk Ratio (M-H, Random, 95\% Cl) & $2.92[2.17,3.95]$ \\
\hline $\begin{array}{l}1.3 \text { certolizumab } 400 \mathrm{mg} \\
\mathrm{sc}\end{array}$ & 5 & 1375 & Risk Ratio (M-H, Random, 95\% Cl) & $2.65[1.98,3.56]$ \\
\hline 2 ACR50 & 7 & 2705 & Risk Ratio (M-H, Random, 95\% Cl) & $2.95[2.37,3.68]$ \\
\hline $\begin{array}{l}2.1 \text { certolizumab } 100 \mathrm{mg} \\
\text { sc }\end{array}$ & 1 & 98 & Risk Ratio (M-H, Random, 95\% Cl) & $2.89[1.13,7.38]$ \\
\hline
\end{tabular}




\begin{tabular}{|c|c|c|c|c|}
\hline $\begin{array}{l}\text { Outcome or subgroup ti- } \\
\text { tle }\end{array}$ & No. of studies & $\begin{array}{l}\text { No. of partici- } \\
\text { pants }\end{array}$ & Statistical method & Effect size \\
\hline $\begin{array}{l}2.2 \text { certolizumab } 200 \mathrm{mg} \\
\mathrm{sc}\end{array}$ & 5 & 1232 & Risk Ratio (M-H, Random, 95\% Cl) & $2.76[2.02,3.78]$ \\
\hline $\begin{array}{l}2.3 \text { certolizumab } 400 \mathrm{mg} \\
\mathrm{sc}\end{array}$ & 5 & 1375 & Risk Ratio (M-H, Random, 95\% Cl) & $3.18[2.29,4.41]$ \\
\hline 3 ACR70 & 7 & 2705 & Risk Ratio (M-H, Random, 95\% Cl) & $4.15[2.68,6.42]$ \\
\hline $\begin{array}{l}3.1 \text { certolizumab } 100 \mathrm{mg} \\
\text { sc }\end{array}$ & 1 & 98 & Risk Ratio (M-H, Random, 95\% Cl) & $6.86[0.97,48.72]$ \\
\hline $\begin{array}{l}3.2 \text { certolizumab } 200 \mathrm{mg} \\
\text { sc }\end{array}$ & 5 & 1232 & Risk Ratio (M-H, Random, 95\% Cl) & $4.29[2.36,7.77]$ \\
\hline $\begin{array}{l}3.3 \text { certolizumab } 400 \mathrm{mg} \\
\mathrm{sc}\end{array}$ & 5 & 1375 & Risk Ratio (M-H, Random, 95\% Cl) & $4.04[1.37,11.90]$ \\
\hline
\end{tabular}

Analysis 44.1. Comparison 44 ACR at 24 weeks, any dose, Outcome 1 ACR20.

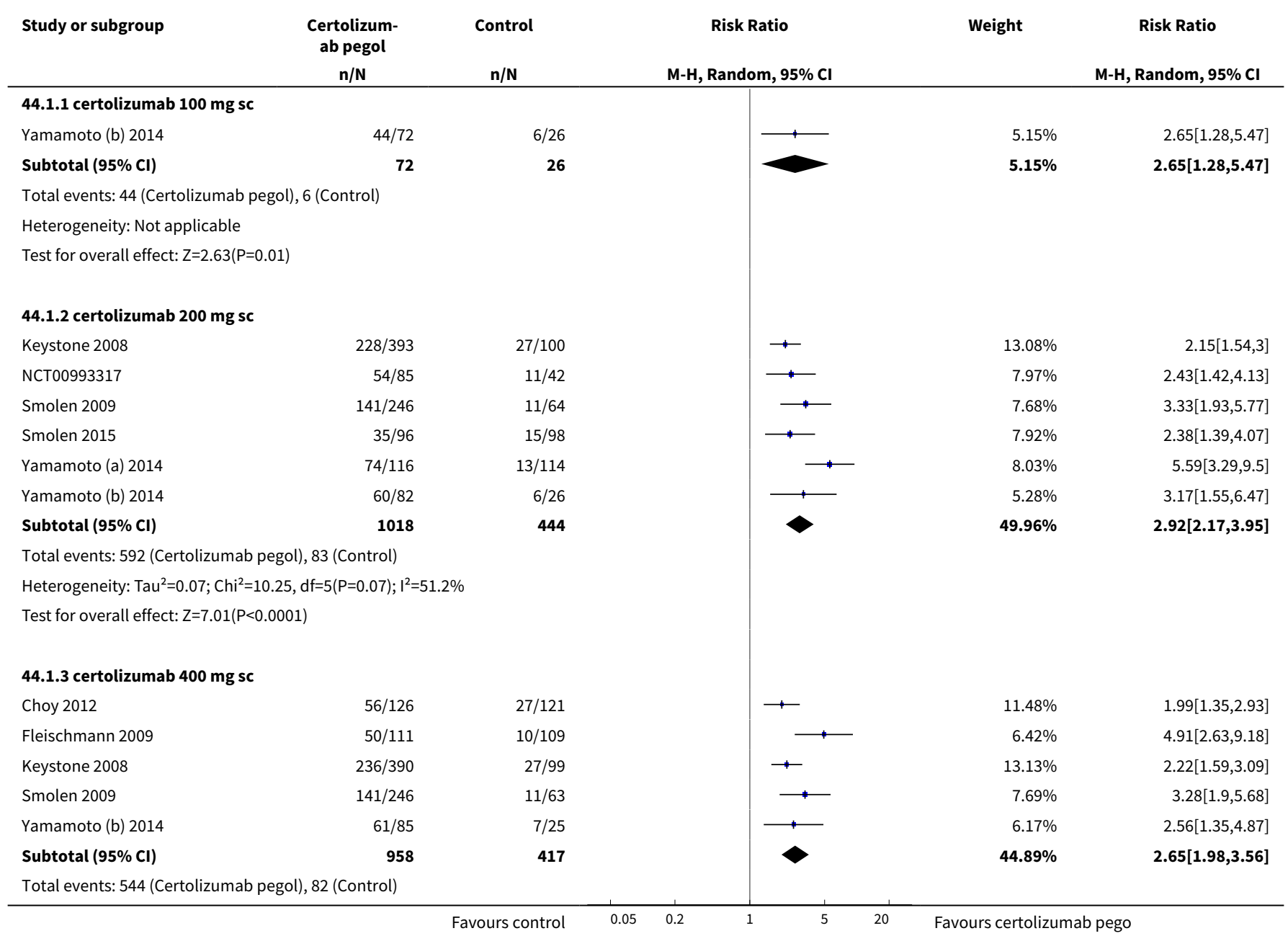




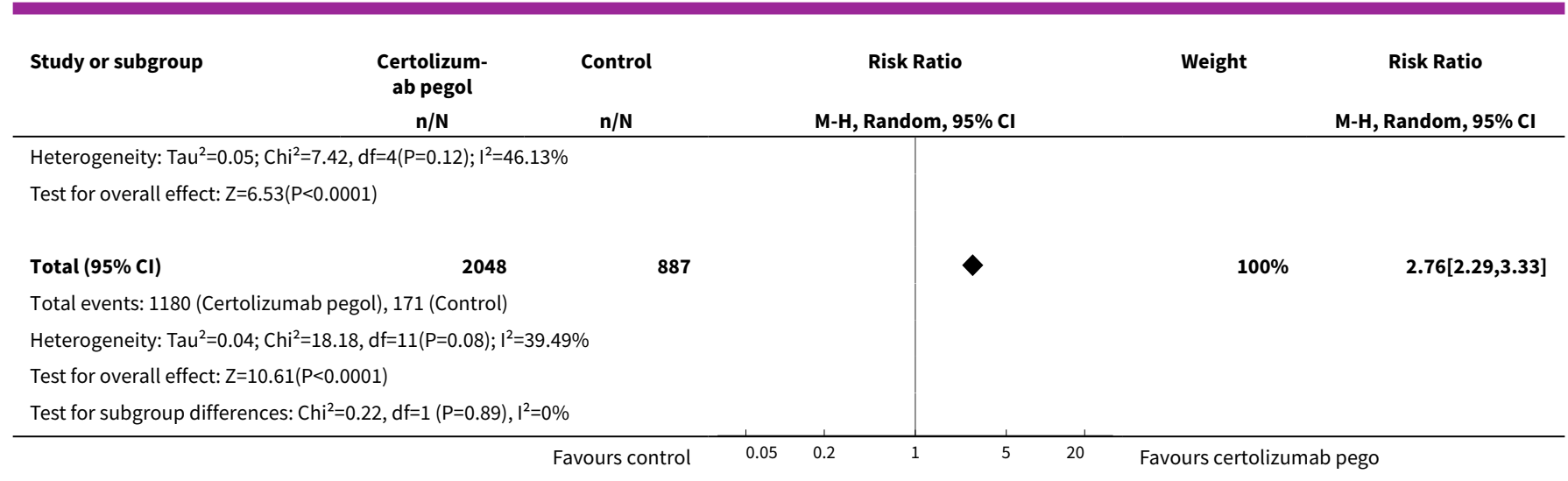

Analysis 44.2. Comparison 44 ACR at 24 weeks, any dose, Outcome 2 ACR50.

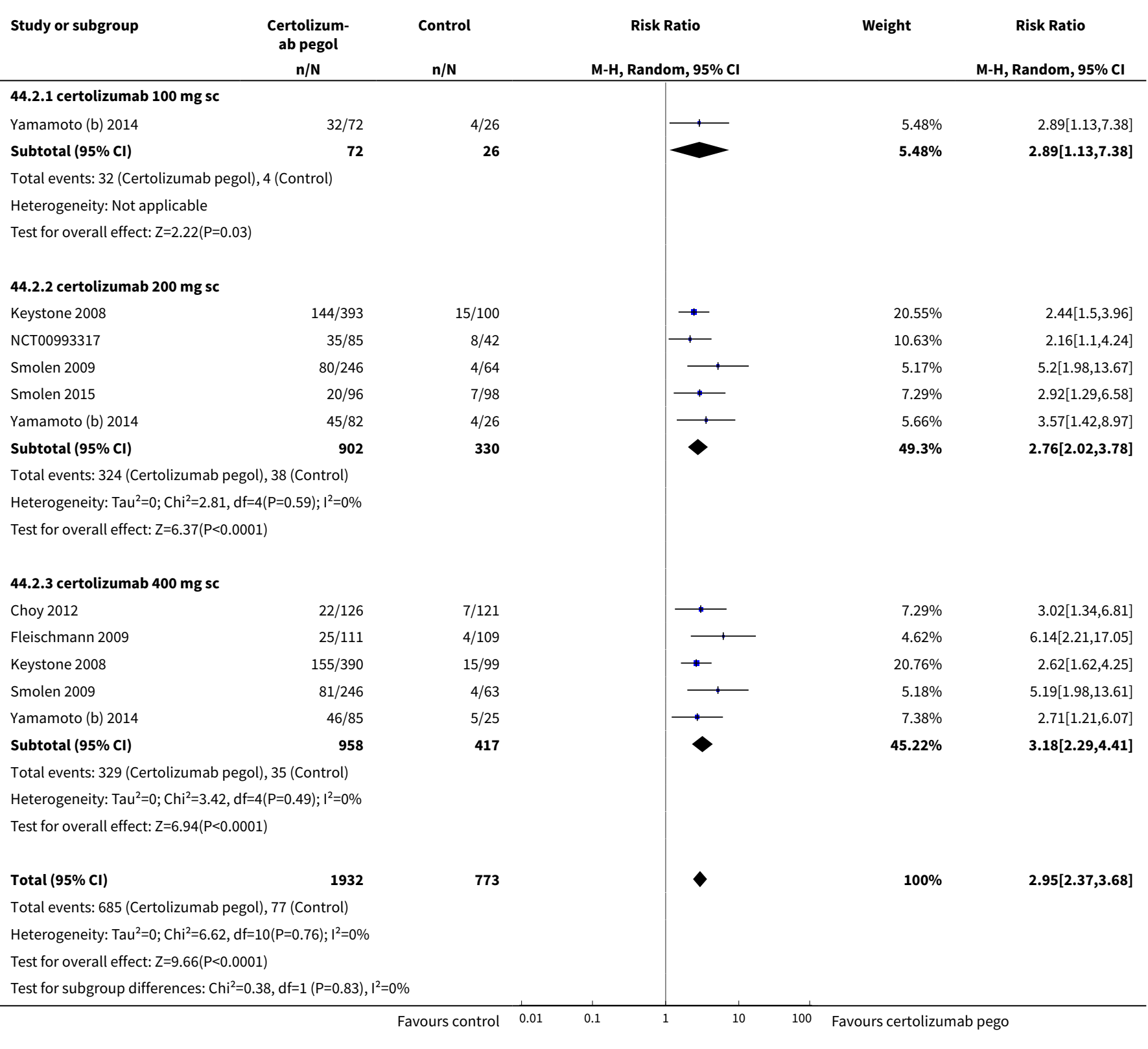


Analysis 44.3. Comparison 44 ACR at 24 weeks, any dose, Outcome 3 ACR70.

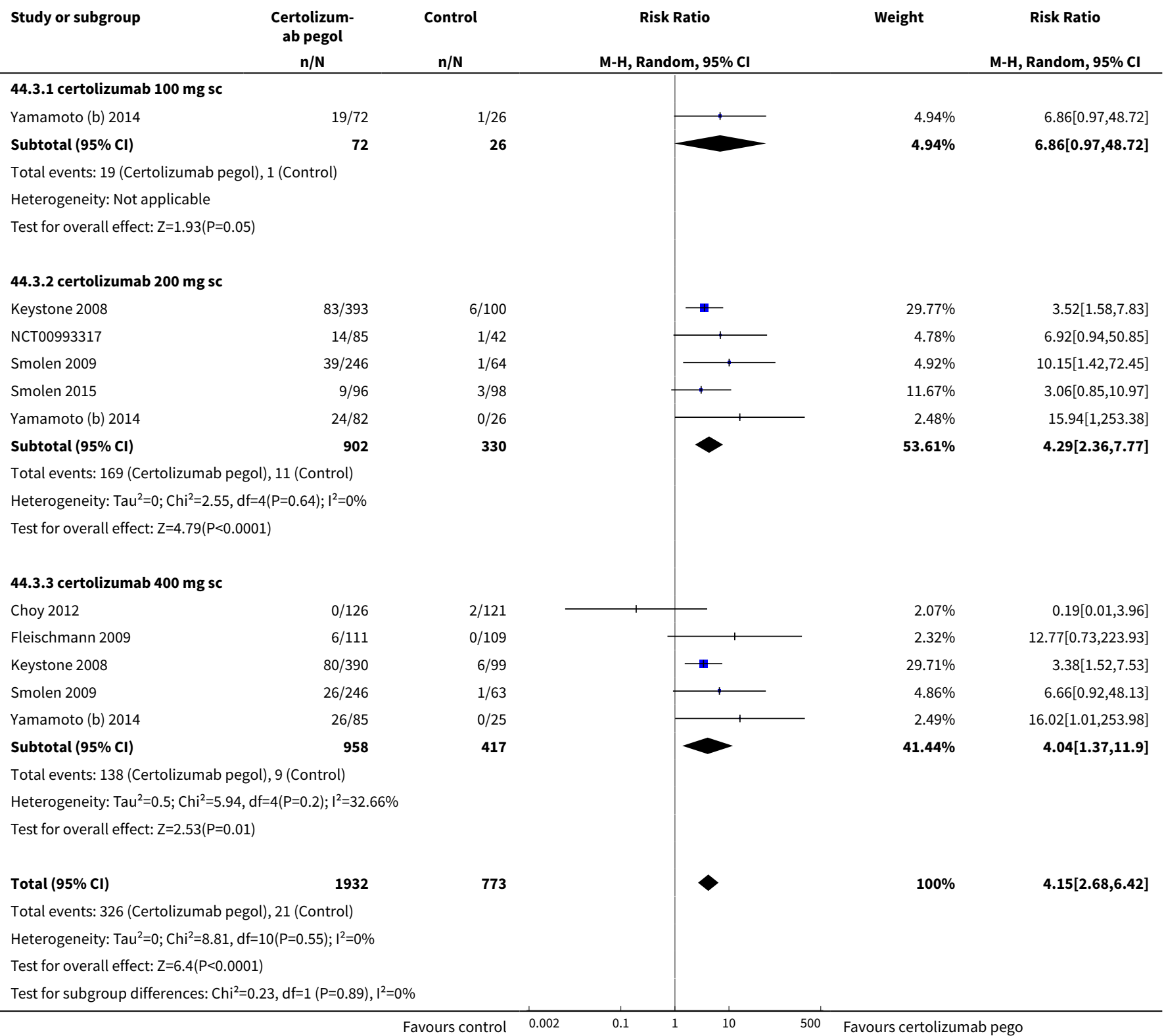

\section{Comparison 45. ACR at 52 weeks, any dose}

\begin{tabular}{lllll}
\hline $\begin{array}{l}\text { Outcome or subgroup } \\
\text { title }\end{array}$ & No. of studies & $\begin{array}{l}\text { No. of partici- } \\
\text { pants }\end{array}$ & Statistical method & Effect size \\
\hline 1 ACR20 & 3 & 2180 & Risk Ratio (M-H, Random, 95\% Cl) & $1.46[1.11,1.93]$ \\
\hline $\begin{array}{l}1.1 \text { certolizumab 200 mg } \\
\text { SC }\end{array}$ & 3 & 1691 & Risk Ratio (M-H, Random, 95\% Cl) & $1.30[1.03,1.65]$ \\
\hline
\end{tabular}




\begin{tabular}{|c|c|c|c|c|}
\hline $\begin{array}{l}\text { Outcome or subgroup } \\
\text { title }\end{array}$ & No. of studies & $\begin{array}{l}\text { No. of partici- } \\
\text { pants }\end{array}$ & Statistical method & Effect size \\
\hline $\begin{array}{l}1.2 \text { certolizumab } 400 \mathrm{mg} \\
\mathrm{sc}\end{array}$ & 1 & 489 & Risk Ratio (M-H, Random, 95\% Cl) & $2.08[1.48,2.93]$ \\
\hline 2 ACR50 & 3 & 2180 & Risk Ratio (M-H, Random, 95\% Cl) & $1.69[1.22,2.33]$ \\
\hline $\begin{array}{l}2.1 \text { certolizumab } 200 \mathrm{mg} \\
\mathrm{sc}\end{array}$ & 3 & 1691 & Risk Ratio (M-H, Random, 95\% Cl) & $1.48[1.11,1.96]$ \\
\hline $\begin{array}{l}2.2 \text { certolizumab } 400 \mathrm{mg} \\
\mathrm{sc}\end{array}$ & 1 & 489 & Risk Ratio (M-H, Random, 95\% Cl) & $2.62[1.62,4.25]$ \\
\hline 3 ACR70 & 3 & 2180 & Risk Ratio (M-H, Random, 95\% Cl) & $1.89[1.44,2.48]$ \\
\hline $\begin{array}{l}3.1 \text { certolizumab } 200 \mathrm{mg} \\
\mathrm{sc}\end{array}$ & 3 & 1691 & Risk Ratio (M-H, Random, 95\% Cl) & $1.71[1.39,2.11]$ \\
\hline $\begin{array}{l}3.2 \text { certolizumab } 400 \mathrm{mg} \\
\text { sc }\end{array}$ & 1 & 489 & Risk Ratio (M-H, Random, 95\% Cl) & $3.26[1.56,6.82]$ \\
\hline
\end{tabular}

Analysis 45.1. Comparison 45 ACR at 52 weeks, any dose, Outcome 1 ACR20.

\begin{tabular}{|c|c|c|c|c|c|}
\hline Study or subgroup & $\begin{array}{c}\text { Certolizum- } \\
\text { ab pegol } \\
n / N\end{array}$ & $\begin{array}{l}\text { Control } \\
\mathrm{n} / \mathrm{N}\end{array}$ & $\begin{array}{c}\text { Risk Ratio } \\
\text { M-H, Random, } 95 \% \mathrm{Cl}\end{array}$ & Weight & $\begin{array}{c}\text { Risk Ratio } \\
\text { M-H, Random, } 95 \% \mathrm{Cl}\end{array}$ \\
\hline \multicolumn{3}{|c|}{ 45.1.1 certolizumab $200 \mathrm{mg} \mathrm{sc}$} & & & \\
\hline Atsumi 2016 & $125 / 161$ & $108 / 158$ & - & $28.8 \%$ & $1.14[0.99,1.3]$ \\
\hline Emery 2015 & $452 / 660$ & $131 / 219$ & - & $29.2 \%$ & $1.14[1.02,1.29]$ \\
\hline Keystone 2008 & $208 / 393$ & $26 / 100$ & & $20.97 \%$ & $2.04[1.44,2.87]$ \\
\hline Subtotal $(95 \% \mathrm{Cl})$ & 1214 & 477 & 4 & $78.98 \%$ & $1.3[1.03,1.65]$ \\
\hline \multicolumn{6}{|c|}{ Total events: 785 (Certolizumab pegol), 265 (Control) } \\
\hline \multicolumn{6}{|c|}{ Heterogeneity: $\mathrm{Tau}^{2}=0.03 ; \mathrm{Chi}^{2}=11.66, \mathrm{df}=2(\mathrm{P}=0) ; 1^{2}=82.85 \%$} \\
\hline \multicolumn{6}{|c|}{ Test for overall effect: $Z=2.16(P=0.03)$} \\
\hline \multicolumn{6}{|c|}{ 45.1.2 certolizumab $400 \mathrm{mg} \mathrm{sc}$} \\
\hline Keystone 2008 & $213 / 390$ & 26/99 & $\rightarrow$ & $21.02 \%$ & $2.08[1.48,2.93]$ \\
\hline Subtotal $(95 \% \mathrm{Cl})$ & 390 & 99 & & $21.02 \%$ & $2.08[1.48,2.93]$ \\
\hline \multicolumn{6}{|c|}{ Total events: 213 (Certolizumab pegol), 26 (Control) } \\
\hline \multicolumn{6}{|c|}{ Heterogeneity: Not applicable } \\
\hline \multicolumn{6}{|c|}{ Test for overall effect: $Z=4.19(P<0.0001)$} \\
\hline Total $(95 \% \mathrm{Cl})$ & 1604 & 576 & & $100 \%$ & $1.46[1.11,1.93]$ \\
\hline \multicolumn{6}{|c|}{ Total events: 998 (Certolizumab pegol), 291 (Control) } \\
\hline \multicolumn{6}{|c|}{ Heterogeneity: $\mathrm{Tau}^{2}=0.06 ; \mathrm{Chi}^{2}=24.18, \mathrm{df}=3(\mathrm{P}<0.0001) ; 1^{2}=87.59 \%$} \\
\hline \multicolumn{6}{|c|}{ Test for overall effect: $Z=2.68(P=0.01)$} \\
\hline \multicolumn{6}{|c|}{ Test for subgroup differences: $\mathrm{Chi}^{2}=4.84, \mathrm{df}=1(\mathrm{P}=0.03), \mathrm{I}^{2}=79.32 \%$} \\
\hline
\end{tabular}


Analysis 45.2. Comparison 45 ACR at 52 weeks, any dose, Outcome 2 ACR50.

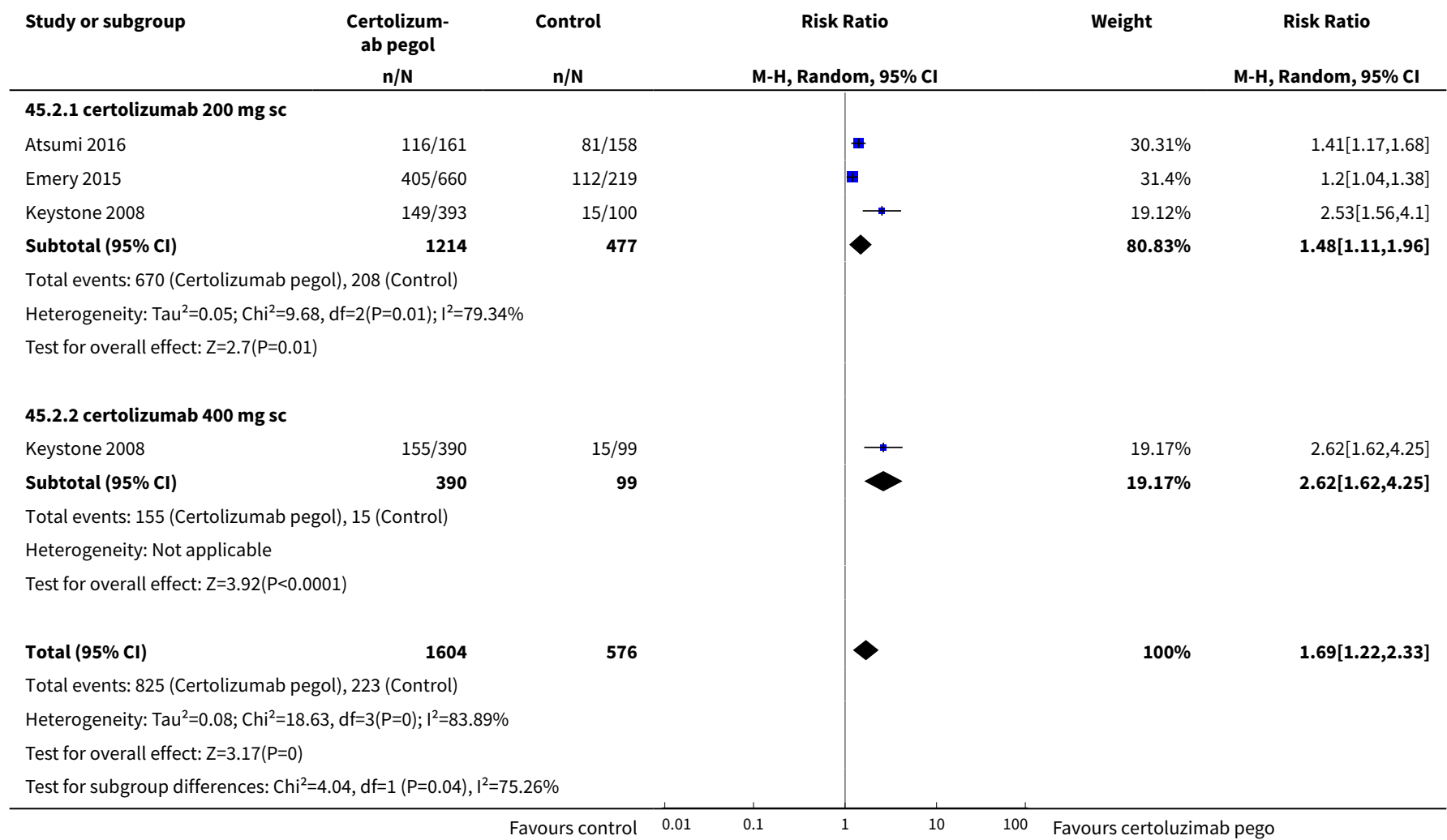

Analysis 45.3. Comparison 45 ACR at 52 weeks, any dose, Outcome 3 ACR70.

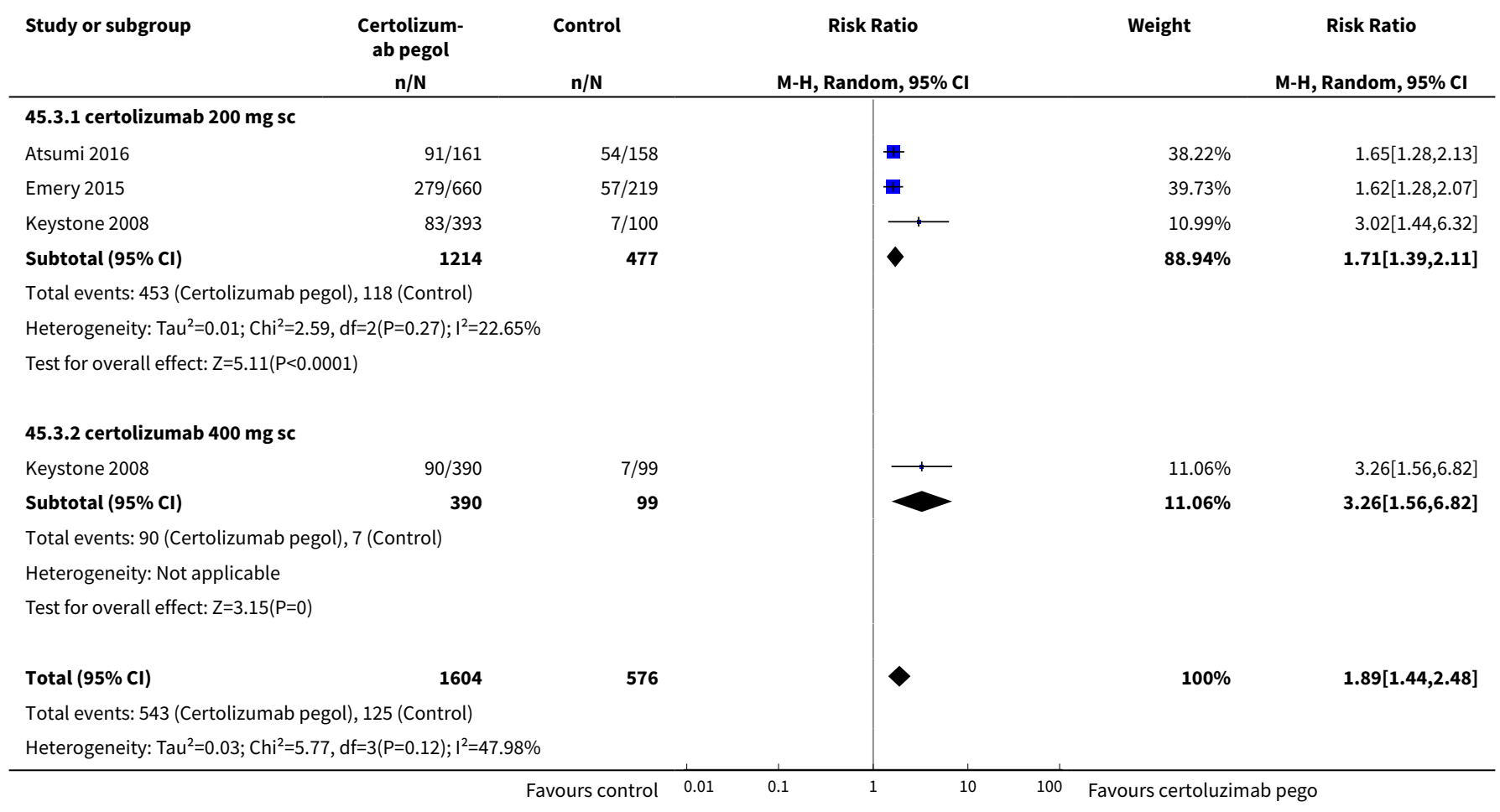




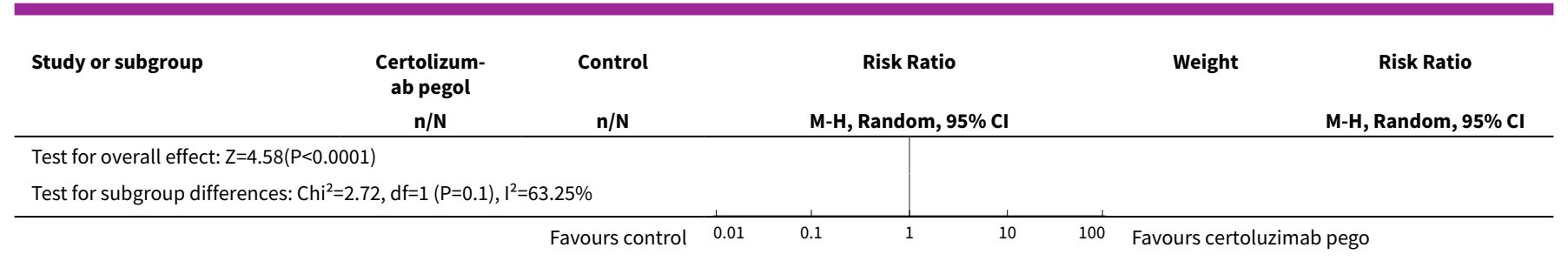

Comparison 46. ACR20-ACR70, 24 weeks, $200 \mathrm{mg}$ certolizumab pegol

\begin{tabular}{lllll}
\hline $\begin{array}{l}\text { Outcome or sub- } \\
\text { group title }\end{array}$ & No. of studies & $\begin{array}{l}\text { No. of partici- } \\
\text { pants }\end{array}$ & Statistical method & Effect size \\
\hline 1 ACR 20 & 6 & 1675 & Risk Ratio (M-H, Random, 95\% Cl) & $3.71[2.68,5.13]$ \\
\hline 2 ACR 70 & 5 & 1445 & Risk Ratio (M-H, Random, 95\% Cl) & $7.26[3.83,13.76]$ \\
\hline
\end{tabular}

Analysis 46.1. Comparison 46 ACR20-ACR70, 24 weeks, 200 mg certolizumab pegol, Outcome 1 ACR 20.

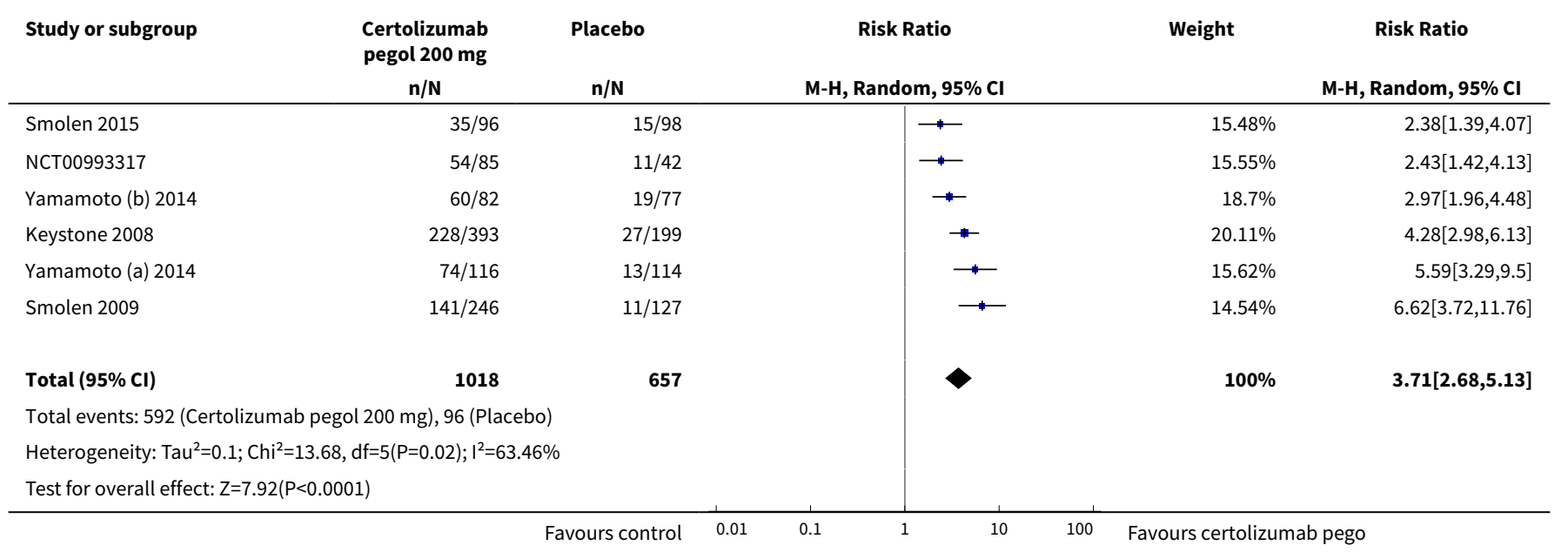

Analysis 46.2. Comparison 46 ACR20-ACR70, 24 weeks, 200 mg certolizumab pegol, Outcome 2 ACR 70.

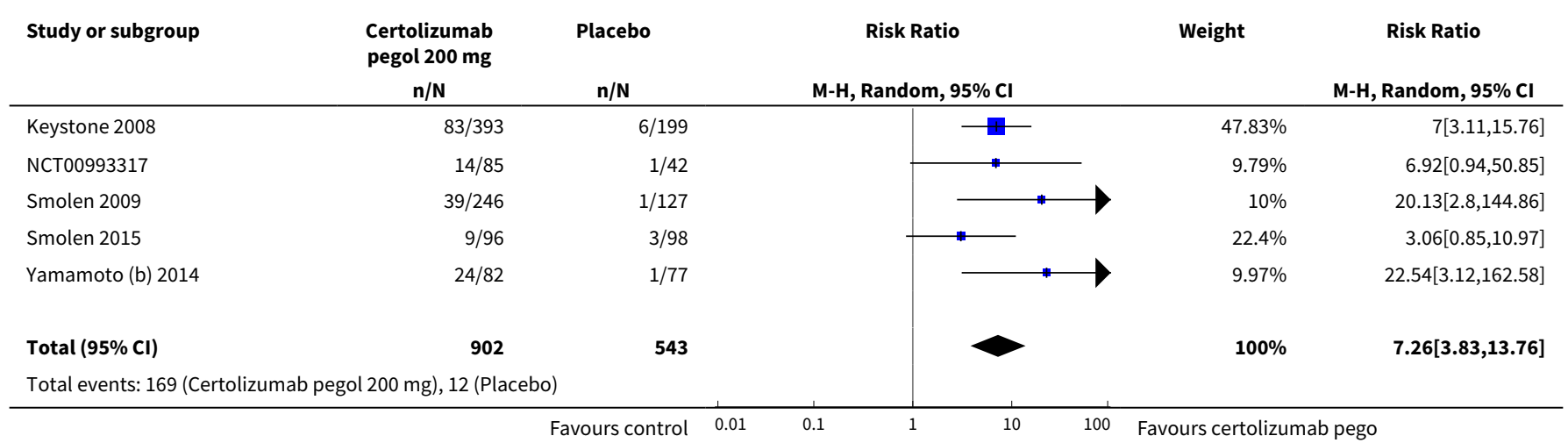




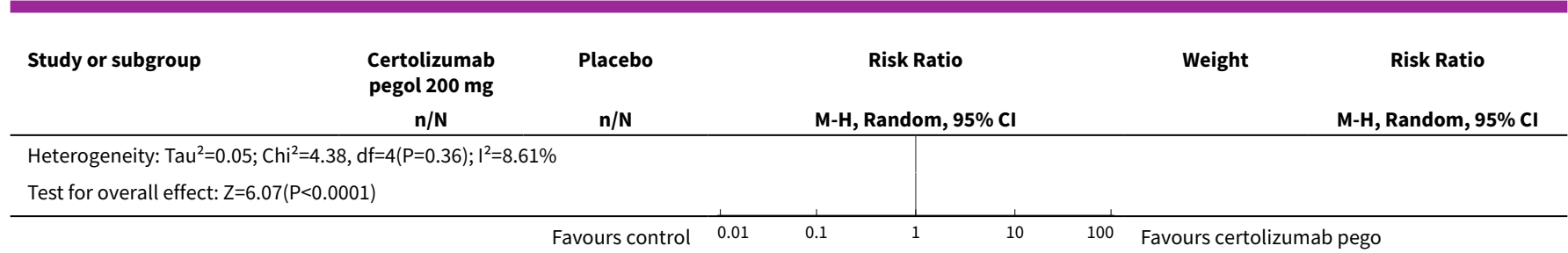

\section{Comparison 47. ACR20-ACR70 at 24 weeks, $400 \mathrm{mg}$ certolizumab}

\begin{tabular}{lllll}
\hline $\begin{array}{l}\text { Outcome or sub- } \\
\text { group title }\end{array}$ & No. of studies & $\begin{array}{l}\text { No. of partici- } \\
\text { pants }\end{array}$ & Statistical method & Effect size \\
\hline 1 ACR 20 & 5 & 1591 & Risk Ratio (M-H, Random, 95\% Cl) & $3.73[2.43,5.72]$ \\
\hline 2 ACR 70 & 5 & 1591 & Risk Ratio (M-H, Random, 95\% Cl) & $7.20[2.25,23.03]$ \\
\hline
\end{tabular}

Analysis 47.1. Comparison 47 ACR20-ACR70 at 24 weeks, $400 \mathrm{mg}$ certolizumab, Outcome 1 ACR 20.

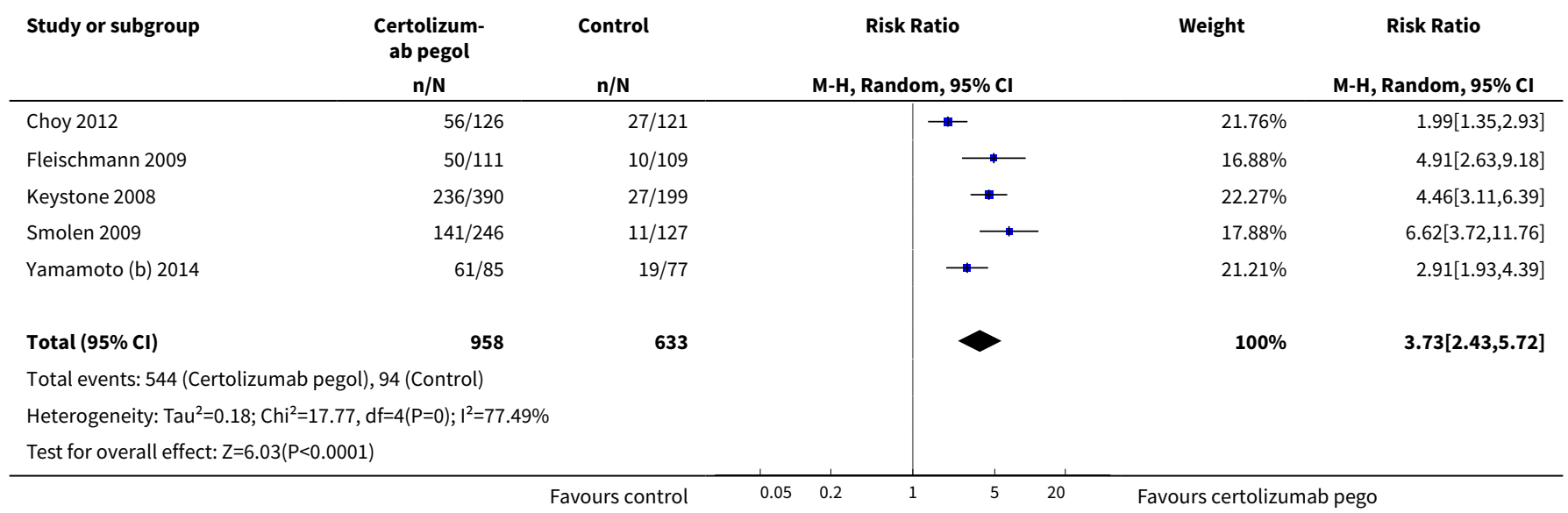

Analysis 47.2. Comparison 47 ACR20-ACR70 at 24 weeks, $400 \mathrm{mg}$ certolizumab, Outcome 2 ACR 70.

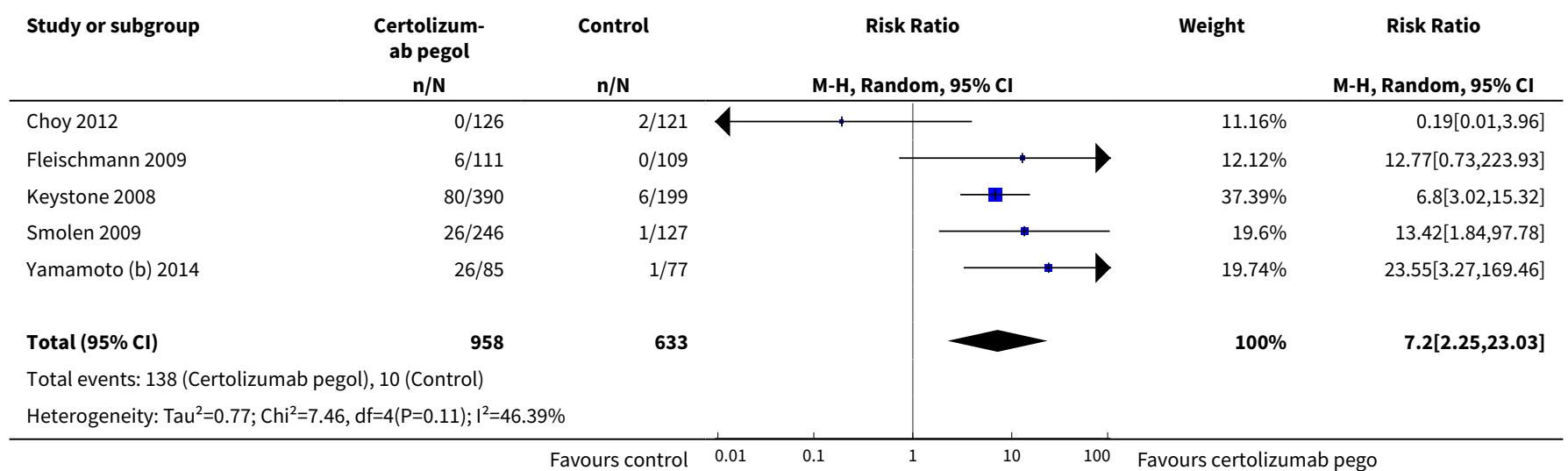




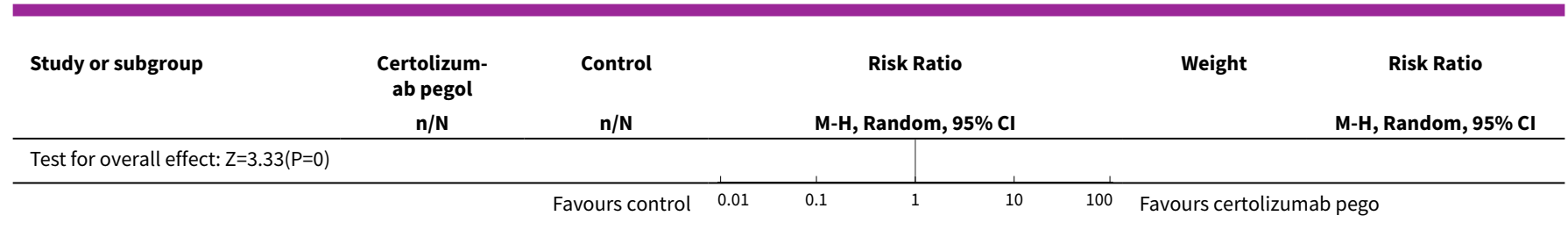

Comparison 48. ACR20-ACR70 at 52 weeks, $200 \mathrm{mg}$ certolizumab

\begin{tabular}{lllll}
\hline $\begin{array}{l}\text { Outcome or sub- } \\
\text { group title }\end{array}$ & No. of studies & $\begin{array}{l}\text { No. of partici- } \\
\text { pants }\end{array}$ & Statistical method & Effect size \\
\hline 1 ACR 20 & 3 & 1790 & Risk Ratio (M-H, Fixed, 95\% Cl) & $1.44[1.30,1.58]$ \\
\hline 2 ACR 70 & 3 & 1790 & Risk Ratio (M-H, Fixed, 95\% Cl) & $1.64[1.41,1.90]$ \\
\hline
\end{tabular}

Analysis 48.1. Comparison 48 ACR20-ACR70 at 52 weeks, $200 \mathrm{mg}$ certolizumab, Outcome 1 ACR 20.

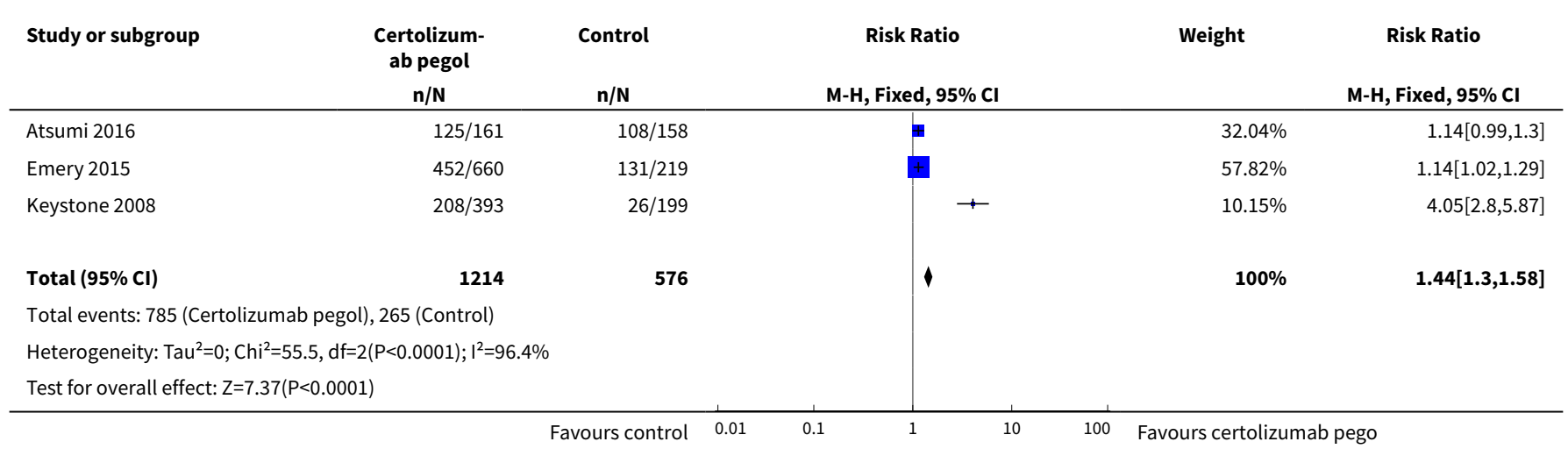

Analysis 48.2. Comparison 48 ACR20-ACR70 at 52 weeks, 200 mg certolizumab, Outcome 2 ACR 70.

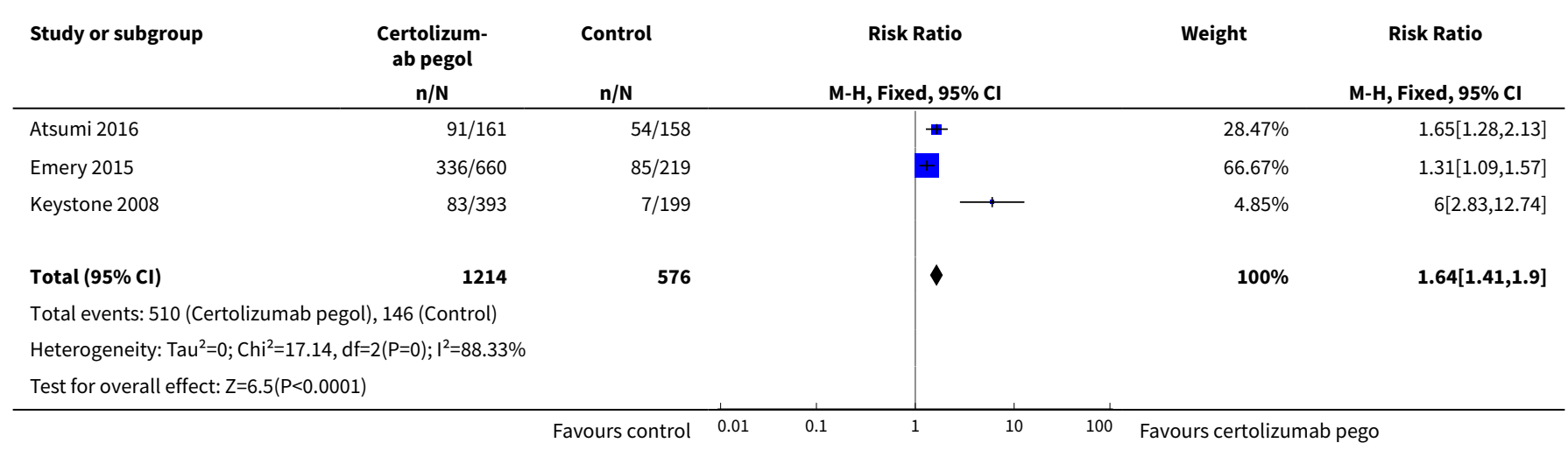


Comparison 49. ACR20-ACR70 at 52 weeks, $400 \mathrm{mg}$ certolizumab

\begin{tabular}{lllll}
\hline $\begin{array}{l}\text { Outcome or sub- } \\
\text { group title }\end{array}$ & No. of studies & $\begin{array}{l}\text { No. of partici- } \\
\text { pants }\end{array}$ & Statistical method & Effect size \\
\hline 1 ACR 20 & 1 & Risk Ratio (M-H, Fixed, 95\% Cl) & Totals not selected \\
\hline 2 ACR 70 & 1 & Risk Ratio (M-H, Fixed, 95\% Cl) & Totals not selected \\
\hline
\end{tabular}

Analysis 49.1. Comparison 49 ACR20-ACR70 at 52 weeks, 400 mg certolizumab, Outcome 1 ACR 20.

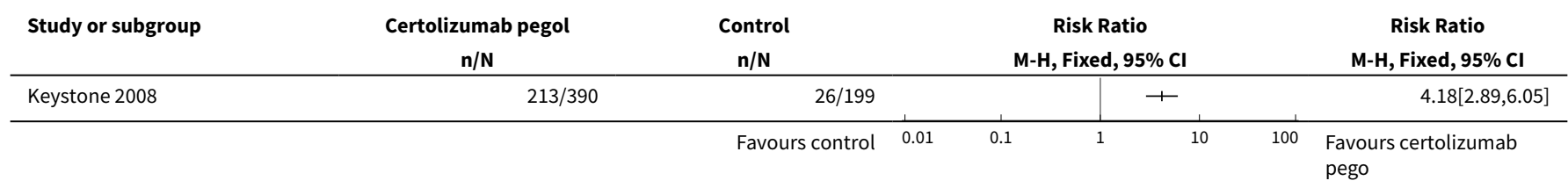

Analysis 49.2. Comparison 49 ACR20-ACR70 at 52 weeks, 400 mg certolizumab, Outcome 2 ACR 70.

\begin{tabular}{|c|c|c|c|c|c|c|c|}
\hline Study or subgroup & $\begin{array}{c}\text { Certolizumab pegol } \\
\mathrm{n} / \mathrm{N}\end{array}$ & $\begin{array}{c}\text { Control } \\
n / N\end{array}$ & & & $\begin{array}{l}\text { k Ratio } \\
\text { red, } 95 \% \mathrm{Cl}\end{array}$ & & $\begin{array}{c}\text { Risk Ratio } \\
\text { M-H, Fixed, 95\% Cl }\end{array}$ \\
\hline Keystone 2008 & $90 / 390$ & $7 / 199$ & & & 1 & & $6.56[3.1,13.89]$ \\
\hline
\end{tabular}

Comparison 50. Safety

\begin{tabular}{|c|c|c|c|c|}
\hline Outcome or subgroup title & No. of studies & $\begin{array}{l}\text { No. of partici- } \\
\text { pants }\end{array}$ & Statistical method & Effect size \\
\hline $\begin{array}{l}1 \text { Any adverse event certolizumab } \\
200 \mathrm{mg}\end{array}$ & 9 & 3927 & $\begin{array}{l}\text { Risk Ratio (M-H, Random, 95\% } \\
\mathrm{Cl} \text { ) }\end{array}$ & $1.16[1.03,1.31]$ \\
\hline $\begin{array}{l}2 \text { Any adverse events certolizumab } \\
400 \mathrm{mg}\end{array}$ & 6 & 1624 & $\begin{array}{l}\text { Risk Ratio (M-H, Random, 95\% } \\
\mathrm{Cl} \text { ) }\end{array}$ & $1.19[1.05,1.34]$ \\
\hline $\begin{array}{l}3 \text { Adverse events: Intensity mild cer- } \\
\text { tolizumab } 200 \mathrm{mg}\end{array}$ & 4 & 2249 & $\begin{array}{l}\text { Risk Ratio (M-H, Random, 95\% } \\
\mathrm{Cl} \text { ) }\end{array}$ & $1.18[1.00,1.41]$ \\
\hline $\begin{array}{l}4 \text { Adverse events: Intensity mild cer- } \\
\text { tolizumab } 400 \mathrm{mg}\end{array}$ & 5 & 1462 & $\begin{array}{l}\text { Risk Ratio (M-H, Random, 95\% } \\
\mathrm{Cl} \text { ) }\end{array}$ & $1.25[1.06,1.47]$ \\
\hline $\begin{array}{l}5 \text { Adverse events: Intensity moder- } \\
\text { ate certolizumab } 200 \mathrm{mg}\end{array}$ & 4 & 2249 & $\begin{array}{l}\text { Risk Ratio (M-H, Random, 95\% } \\
\mathrm{Cl} \text { ) }\end{array}$ & $1.07[0.86,1.32]$ \\
\hline $\begin{array}{l}6 \text { Adverse events: Intensity moder- } \\
\text { ate certolizumab } 400 \mathrm{mg}\end{array}$ & 5 & 1462 & $\begin{array}{l}\text { Risk Ratio (M-H, Random, 95\% } \\
\text { Cl) }\end{array}$ & $1.21[0.99,1.47]$ \\
\hline $\begin{array}{l}7 \text { Adverse events: Intensity severe } \\
\text { certolizumab } 200 \mathrm{mg}\end{array}$ & 4 & 2249 & $\begin{array}{l}\text { Peto Odds Ratio (Peto, Fixed, } \\
95 \% \mathrm{Cl} \text { ) }\end{array}$ & $1.14[0.78,1.65]$ \\
\hline
\end{tabular}




\begin{tabular}{|c|c|c|c|c|}
\hline Outcome or subgroup title & No. of studies & $\begin{array}{l}\text { No. of partici- } \\
\text { pants }\end{array}$ & Statistical method & Effect size \\
\hline $\begin{array}{l}8 \text { Adverse events: Intensity severe } \\
\text { certolizumab } 400 \mathrm{mg}\end{array}$ & 5 & 1462 & $\begin{array}{l}\text { Peto Odds Ratio (Peto, Fixed, } \\
95 \% \mathrm{Cl} \text { ) }\end{array}$ & $1.23[0.83,1.81]$ \\
\hline $\begin{array}{l}9 \text { Adverse events related to study } \\
\text { drug certolizumab } 200 \mathrm{mg}\end{array}$ & 2 & 964 & $\begin{array}{l}\text { Risk Ratio (M-H, Random, 95\% } \\
\text { Cl) }\end{array}$ & $1.59[1.27,1.99]$ \\
\hline $\begin{array}{l}10 \text { Adverse events related to study } \\
\text { drug certolizumab } 400 \mathrm{mg}\end{array}$ & 4 & 1219 & Risk Ratio (M-H, Fixed, 95\% Cl) & $1.47[1.20,1.80]$ \\
\hline $\begin{array}{l}11 \text { Serious Infections certolizumab } \\
200 \mathrm{mg}\end{array}$ & 3 & 1283 & $\begin{array}{l}\text { Peto Odds Ratio (Peto, Fixed, } \\
95 \% \mathrm{Cl} \text { ) }\end{array}$ & $1.94[0.99,3.80]$ \\
\hline $\begin{array}{l}12 \text { Serious infections certolizumab } \\
400 \mathrm{mg}\end{array}$ & 4 & 1422 & $\begin{array}{l}\text { Peto Odds Ratio (Peto, Fixed, } \\
95 \% \mathrm{Cl} \text { ) }\end{array}$ & $3.25[1.65,6.39]$ \\
\hline $\begin{array}{l}13 \text { Adverse events leading to death } \\
\text { certolizumab } 200 \mathrm{mg}\end{array}$ & 6 & 3322 & $\begin{array}{l}\text { Peto Odds Ratio (Peto, Fixed, } \\
95 \% \mathrm{Cl} \text { ) }\end{array}$ & $1.63[0.41,6.47]$ \\
\hline $\begin{array}{l}14 \text { Adverse events leading to death } \\
\text { certolizumab } 400 \mathrm{mg}\end{array}$ & 3 & 1179 & $\begin{array}{l}\text { Peto Odds Ratio (Peto, Fixed, } \\
95 \% \mathrm{Cl} \text { ) }\end{array}$ & $2.16[0.40,11.79]$ \\
\hline $\begin{array}{l}15 \text { Adverse events leading to with- } \\
\text { drawal certolizumab } 200 \mathrm{mg}\end{array}$ & 8 & 3608 & $\begin{array}{l}\text { Peto Odds Ratio (Peto, Fixed, } \\
95 \% \mathrm{Cl} \text { ) }\end{array}$ & $1.32[0.95,1.84]$ \\
\hline $\begin{array}{l}16 \text { Adverse events leading to with- } \\
\text { drawal certolizumab } 400 \mathrm{mg}\end{array}$ & 6 & 1624 & $\begin{array}{l}\text { Peto Odds Ratio (Peto, Fixed, } \\
95 \% \mathrm{Cl} \text { ) }\end{array}$ & $2.01[1.20,3.36]$ \\
\hline 17 Death certolizumab 200 mg & 6 & 3320 & $\begin{array}{l}\text { Peto Odds Ratio (Peto, Fixed, } \\
95 \% \mathrm{Cl} \text { ) }\end{array}$ & $2.66[0.63,11.16]$ \\
\hline 18 Death certolizumab $400 \mathrm{mg}$ & 5 & 1462 & Risk Ratio (M-H, Fixed, 95\% Cl) & $1.87[0.31,11.34]$ \\
\hline 19 Deaths overall & 10 & 4745 & $\begin{array}{l}\text { Peto Odds Ratio (Peto, Fixed, } \\
95 \% \mathrm{Cl} \text { ) }\end{array}$ & $2.63[0.78,8.91]$ \\
\hline 19.1 Certolizumab pegol $200 \mathrm{mg}$ & 7 & 3266 & $\begin{array}{l}\text { Peto Odds Ratio (Peto, Fixed, } \\
95 \% \mathrm{Cl} \text { ) }\end{array}$ & $2.10[0.44,10.08]$ \\
\hline 19.2 Certolizumab pegol $400 \mathrm{mg}$ & 5 & 1349 & $\begin{array}{l}\text { Peto Odds Ratio (Peto, Fixed, } \\
95 \% \mathrm{Cl} \text { ) }\end{array}$ & $3.53[0.40,31.39]$ \\
\hline 19.3 Other doses & 2 & 130 & $\begin{array}{l}\text { Peto Odds Ratio (Peto, Fixed, } \\
95 \% \mathrm{Cl} \text { ) }\end{array}$ & $4.48[0.07,286.49]$ \\
\hline $\begin{array}{l}20 \text { Tuberculosis certolizumab } 200 \\
\text { mg }\end{array}$ & 7 & 3538 & $\begin{array}{l}\text { Peto Odds Ratio (Peto, Fixed, } \\
95 \% \mathrm{Cl} \text { ) }\end{array}$ & $1.90[0.55,6.58]$ \\
\hline $\begin{array}{l}21 \text { Tuberculosis certolizumab } 400 \\
\text { mg }\end{array}$ & 3 & 1179 & $\begin{array}{l}\text { Peto Odds Ratio (Peto, Fixed, } \\
95 \% \mathrm{Cl} \text { ) }\end{array}$ & $4.55[0.71,29.11]$ \\
\hline 22 Tuberculosis overall & 7 & 4074 & $\begin{array}{l}\text { Peto Odds Ratio (Peto, Fixed, } \\
95 \% \mathrm{Cl} \text { ) }\end{array}$ & $1.91[0.61,5.96]$ \\
\hline 22.1 Certolizumab pegol $200 \mathrm{mg}$ & 6 & 3058 & $\begin{array}{l}\text { Peto Odds Ratio (Peto, Fixed, } \\
95 \% \mathrm{Cl} \text { ) }\end{array}$ & $1.53[0.40,5.77]$ \\
\hline
\end{tabular}




\begin{tabular}{|c|c|c|c|c|}
\hline Outcome or subgroup title & No. of studies & $\begin{array}{l}\text { No. of partici- } \\
\text { pants }\end{array}$ & Statistical method & Effect size \\
\hline 22.2 Certolizumab pegol 400 mg & 3 & 1016 & $\begin{array}{l}\text { Peto Odds Ratio (Peto, Fixed, } \\
95 \% \mathrm{Cl} \text { ) }\end{array}$ & $3.52[0.40,31.33]$ \\
\hline $\begin{array}{l}23 \text { Malignancies included lym- } \\
\text { phoma certolizumab } 200 \text { mg }\end{array}$ & 8 & 3768 & $\begin{array}{l}\text { Peto Odds Ratio (Peto, Fixed, } \\
95 \% \mathrm{Cl} \text { ) }\end{array}$ & $0.92[0.40,2.11]$ \\
\hline $\begin{array}{l}24 \text { Malignancies included lym- } \\
\text { phoma certolizumab } 400 \text { mg }\end{array}$ & 3 & 1179 & $\begin{array}{l}\text { Peto Odds Ratio (Peto, Fixed, } \\
95 \% \mathrm{Cl} \text { ) }\end{array}$ & $1.26[0.26,6.08]$ \\
\hline $\begin{array}{l}25 \text { Injection side reactions cer- } \\
\text { tolizumab } 200 \mathrm{mg}\end{array}$ & 5 & 2497 & $\begin{array}{l}\text { Peto Odds Ratio (Peto, Fixed, } \\
95 \% \mathrm{Cl} \text { ) }\end{array}$ & $3.34[1.85,6.06]$ \\
\hline $\begin{array}{l}26 \text { Injection side reactions cer- } \\
\text { tolizumab } 400 \mathrm{mg}\end{array}$ & 5 & 1584 & $\begin{array}{l}\text { Peto Odds Ratio (Peto, Fixed, } \\
95 \% \mathrm{Cl} \text { ) }\end{array}$ & $0.34[0.20,0.56]$ \\
\hline $\begin{array}{l}27 \text { Antinuclear antibodies (ANA) An- } \\
\text { ti-certolizumab pegol antibodies } \\
\text { certolizumab } 200 \mathrm{mg}\end{array}$ & 1 & & $\begin{array}{l}\text { Peto Odds Ratio (Peto, Fixed, } \\
95 \% \mathrm{Cl} \text { ) }\end{array}$ & $\begin{array}{l}\text { Totals not select- } \\
\text { ed }\end{array}$ \\
\hline $\begin{array}{l}28 \text { Anti-certolizumab pegol antibod- } \\
\text { ies certolizumab } 400 \text { mg }\end{array}$ & 2 & 591 & $\begin{array}{l}\text { Peto Odds Ratio (Peto, Fixed, } \\
95 \% \mathrm{Cl} \text { ) }\end{array}$ & $6.70[2.18,20.55]$ \\
\hline $\begin{array}{l}29 \text { Systemic lupus erythematosus } \\
\text { certolizumab } 200 \text { mg }\end{array}$ & 2 & 567 & $\begin{array}{l}\text { Peto Odds Ratio (Peto, Fixed, } \\
95 \% \mathrm{CI} \text { ) }\end{array}$ & $4.50[0.07,286.06]$ \\
\hline $\begin{array}{l}30 \text { Prolonged activated partial } \\
\text { thromboplastin time (aPTT) cer- } \\
\text { tolizumab } 200 \text { mg }\end{array}$ & 2 & 500 & $\begin{array}{l}\text { Peto Odds Ratio (Peto, Fixed, } \\
95 \% \mathrm{Cl} \text { ) }\end{array}$ & $2.73[0.98,7.61]$ \\
\hline $\begin{array}{l}31 \text { Prolonged activated partial } \\
\text { thromboplastin time (aPTT) cer- } \\
\text { tolizumab } 400 \mathrm{mg}\end{array}$ & 1 & & $\begin{array}{l}\text { Peto Odds Ratio (Peto, Fixed, } \\
95 \% \mathrm{Cl} \text { ) }\end{array}$ & $\begin{array}{l}\text { Totals not select- } \\
\text { ed }\end{array}$ \\
\hline $\begin{array}{l}32 \text { Urinary tract infection cer- } \\
\text { tolizumab } 200 \mathrm{mg}\end{array}$ & 6 & 3219 & $\begin{array}{l}\text { Peto Odds Ratio (Peto, Fixed, } \\
95 \% \mathrm{Cl} \text { ) }\end{array}$ & $0.98[0.68,1.40]$ \\
\hline $\begin{array}{l}33 \text { Urinary tract infection cer- } \\
\text { tolizumab } 400 \mathrm{mg}\end{array}$ & 2 & 959 & $\begin{array}{l}\text { Peto Odds Ratio (Peto, Fixed, } \\
95 \% \mathrm{Cl} \text { ) }\end{array}$ & $0.87[0.50,1.52]$ \\
\hline $\begin{array}{l}34 \text { Upper respiratory tract infection } \\
\text { certolizumab } 200 \mathrm{mg}\end{array}$ & 8 & 3608 & $\begin{array}{l}\text { Peto Odds Ratio (Peto, Fixed, } \\
95 \% \mathrm{Cl} \text { ) }\end{array}$ & $1.68[1.28,2.20]$ \\
\hline $\begin{array}{l}35 \text { Upper respiratory tract infection } \\
\text { certolizumab } 400 \mathrm{mg}\end{array}$ & 4 & 1364 & $\begin{array}{l}\text { Peto Odds Ratio (Peto, Fixed, } \\
95 \% \mathrm{Cl} \text { ) }\end{array}$ & $1.42[0.77,2.61]$ \\
\hline $\begin{array}{l}36 \text { Lower respiratory tract infection/ } \\
\text { lung infection certolizumab } 200 \text { mg }\end{array}$ & 6 & 2356 & $\begin{array}{l}\text { Peto Odds Ratio (Peto, Fixed, } \\
95 \% \mathrm{Cl} \text { ) }\end{array}$ & $2.12[0.76,5.95]$ \\
\hline $\begin{array}{l}37 \text { Lower respiratory tract infection/ } \\
\text { lung infection certolizumab } 400 \text { mg }\end{array}$ & 3 & 993 & $\begin{array}{l}\text { Peto Odds Ratio (Peto, Fixed, } \\
95 \% \mathrm{Cl} \text { ) }\end{array}$ & $2.11[0.75,5.95]$ \\
\hline 38 Pneumonia certolizumab 200 mg & 6 & 2804 & $\begin{array}{l}\text { Peto Odds Ratio (Peto, Fixed, } \\
95 \% \mathrm{Cl} \text { ) }\end{array}$ & $0.94[0.45,1.97]$ \\
\hline
\end{tabular}




\begin{tabular}{|c|c|c|c|c|}
\hline Outcome or subgroup title & No. of studies & $\begin{array}{l}\text { No. of partici- } \\
\text { pants }\end{array}$ & Statistical method & Effect size \\
\hline $\begin{array}{l}39 \text { Pneumonitis certolizumab } 400 \\
\text { mg }\end{array}$ & 1 & & $\begin{array}{l}\text { Peto Odds Ratio (Peto, Fixed, } \\
95 \% \mathrm{Cl} \text { ) }\end{array}$ & $\begin{array}{l}\text { Totals not select- } \\
\text { ed }\end{array}$ \\
\hline 40 Headache certolizumab 200 mg & 6 & 3251 & $\begin{array}{l}\text { Peto Odds Ratio (Peto, Fixed, } \\
95 \% \mathrm{Cl} \text { ) }\end{array}$ & $1.33[0.94,1.87]$ \\
\hline 41 Headache certolizumab 400 mg & 4 & 1364 & $\begin{array}{l}\text { Peto Odds Ratio (Peto, Fixed, } \\
95 \% \mathrm{Cl} \text { ) }\end{array}$ & $1.30[0.76,2.20]$ \\
\hline 42 Bacteriuria certolizumab 200 mg & 1 & & $\begin{array}{l}\text { Peto Odds Ratio (Peto, Fixed, } \\
95 \% \mathrm{Cl} \text { ) }\end{array}$ & $\begin{array}{l}\text { Totals not select- } \\
\text { ed }\end{array}$ \\
\hline 43 Bacteriuria certolizumab 400 mg & 1 & & $\begin{array}{l}\text { Peto Odds Ratio (Peto, Fixed, } \\
95 \% \mathrm{Cl} \text { ) }\end{array}$ & $\begin{array}{l}\text { Totals not select- } \\
\text { ed }\end{array}$ \\
\hline $\begin{array}{l}44 \text { Nasopharyngitis/Pharyngitis cer- } \\
\text { tolizumab } 200 \mathrm{mg}\end{array}$ & 7 & 2553 & $\begin{array}{l}\text { Peto Odds Ratio (Peto, Fixed, } \\
95 \% \mathrm{Cl} \text { ) }\end{array}$ & $1.37[1.01,1.84]$ \\
\hline $\begin{array}{l}45 \text { Nasopharyngitis/Pharyngitis cer- } \\
\text { tolizumab } 400 \mathrm{mg}\end{array}$ & 4 & 1364 & $\begin{array}{l}\text { Peto Odds Ratio (Peto, Fixed, } \\
95 \% \mathrm{Cl} \text { ) }\end{array}$ & $1.98[1.26,3.11]$ \\
\hline $\begin{array}{l}46 \text { Injection site pain certolizumab } \\
200 \mathrm{mg}\end{array}$ & 3 & 1091 & $\begin{array}{l}\text { Peto Odds Ratio (Peto, Fixed, } \\
95 \% \mathrm{Cl} \text { ) }\end{array}$ & $1.85[0.49,6.92]$ \\
\hline $\begin{array}{l}47 \text { Injection site pain certolizumab } \\
400 \mathrm{mg}\end{array}$ & 3 & 1179 & $\begin{array}{l}\text { Peto Odds Ratio (Peto, Fixed, } \\
95 \% \mathrm{Cl} \text { ) }\end{array}$ & $1.74[0.41,7.42]$ \\
\hline $\begin{array}{l}48 \text { Hypertension certolizumab } 200 \\
\text { mg }\end{array}$ & 4 & 1353 & $\begin{array}{l}\text { Peto Odds Ratio (Peto, Fixed, } \\
95 \% \mathrm{Cl} \text { ) }\end{array}$ & $3.09[1.64,5.84]$ \\
\hline $\begin{array}{l}49 \text { Hypertension certolizumab } 400 \\
\text { mg }\end{array}$ & 3 & 1121 & $\begin{array}{l}\text { Peto Odds Ratio (Peto, Fixed, } \\
95 \% \mathrm{Cl} \text { ) }\end{array}$ & $3.35[1.80,6.20]$ \\
\hline 50 Hematuria certolizumab 200 mg & 1 & & $\begin{array}{l}\text { Peto Odds Ratio (Peto, Fixed, } \\
95 \% \mathrm{Cl} \text { ) }\end{array}$ & $\begin{array}{l}\text { Totals not select- } \\
\text { ed }\end{array}$ \\
\hline 51 Haematuria certolizumab 400 mg & 1 & & $\begin{array}{l}\text { Peto Odds Ratio (Peto, Fixed, } \\
95 \% \mathrm{Cl} \text { ) }\end{array}$ & $\begin{array}{l}\text { Totals not select- } \\
\text { ed }\end{array}$ \\
\hline $\begin{array}{l}52 \text { Hepatic enzyme increased cer- } \\
\text { tolizumab } 200 \text { mg }\end{array}$ & 3 & 851 & $\begin{array}{l}\text { Peto Odds Ratio (Peto, Fixed, } \\
95 \% \mathrm{Cl} \text { ) }\end{array}$ & $0.84[0.56,1.27]$ \\
\hline $\begin{array}{l}53 \text { Hepatic enzyme increased cer- } \\
\text { tolizumab } 400 \mathrm{mg}\end{array}$ & 2 & 533 & $\begin{array}{l}\text { Peto Odds Ratio (Peto, Fixed, } \\
95 \% \mathrm{Cl} \text { ) }\end{array}$ & $0.69[0.25,1.92]$ \\
\hline $\begin{array}{l}54 \text { AST increased certolizumab } 200 \\
\text { mg }\end{array}$ & 1 & & $\begin{array}{l}\text { Peto Odds Ratio (Peto, Fixed, } \\
95 \% \mathrm{Cl} \text { ) }\end{array}$ & $\begin{array}{l}\text { Totals not select- } \\
\text { ed }\end{array}$ \\
\hline $\begin{array}{l}55 \text { AST increased certolizumab } 400 \\
\text { mg }\end{array}$ & 1 & & $\begin{array}{l}\text { Peto Odds Ratio (Peto, Fixed, } \\
95 \% \mathrm{Cl} \text { ) }\end{array}$ & $\begin{array}{l}\text { Totals not select- } \\
\text { ed }\end{array}$ \\
\hline $\begin{array}{l}56 \text { ALT increased certolizumab } 200 \\
\text { mg }\end{array}$ & 2 & 1252 & $\begin{array}{l}\text { Peto Odds Ratio (Peto, Fixed, } \\
95 \% \mathrm{Cl} \text { ) }\end{array}$ & $0.85[0.48,1.50]$ \\
\hline
\end{tabular}




\begin{tabular}{|c|c|c|c|c|}
\hline Outcome or subgroup title & No. of studies & $\begin{array}{l}\text { No. of partici- } \\
\text { pants }\end{array}$ & Statistical method & Effect size \\
\hline $\begin{array}{l}57 \text { ALT increased certolizumab } 400 \\
\text { mg }\end{array}$ & 1 & & $\begin{array}{l}\text { Peto Odds Ratio (Peto, Fixed, } \\
95 \% \mathrm{Cl} \text { ) }\end{array}$ & $\begin{array}{l}\text { Totals not select- } \\
\text { ed }\end{array}$ \\
\hline 58 Diarrhoea certolizumab 200 mg & 3 & 1200 & $\begin{array}{l}\text { Peto Odds Ratio (Peto, Fixed, } \\
95 \% \mathrm{Cl} \text { ) }\end{array}$ & $0.71[0.25,2.03]$ \\
\hline $\begin{array}{l}59 \text { Gastroenteritis certolizumab } 200 \\
\text { mg }\end{array}$ & 2 & 785 & $\begin{array}{l}\text { Peto Odds Ratio (Peto, Fixed, } \\
95 \% \mathrm{Cl} \text { ) }\end{array}$ & $0.97[0.33,2.87]$ \\
\hline $\begin{array}{l}60 \text { Gastrointestinal disorders cer- } \\
\text { tolizumab } 400 \mathrm{mg}\end{array}$ & 2 & 831 & $\begin{array}{l}\text { Peto Odds Ratio (Peto, Fixed, } \\
95 \% \mathrm{Cl} \text { ) }\end{array}$ & $1.05[0.54,2.03]$ \\
\hline 61 Back pain certolizumab 200 mg & 1 & & $\begin{array}{l}\text { Peto Odds Ratio (Peto, Fixed, } \\
95 \% \mathrm{Cl} \text { ) }\end{array}$ & $\begin{array}{l}\text { Totals not select- } \\
\text { ed }\end{array}$ \\
\hline 62 Back pain certolizumab $400 \mathrm{mg}$ & 2 & 831 & $\begin{array}{l}\text { Peto Odds Ratio (Peto, Fixed, } \\
95 \% \mathrm{Cl} \text { ) }\end{array}$ & $3.11[1.48,6.55]$ \\
\hline $\begin{array}{l}63 \text { Hematologic abnormalities cer- } \\
\text { tolizumab } 200 \text { mg }\end{array}$ & 2 & 821 & $\begin{array}{l}\text { Peto Odds Ratio (Peto, Fixed, } \\
95 \% \mathrm{Cl} \text { ) }\end{array}$ & $2.02[0.27,15.21]$ \\
\hline $\begin{array}{l}64 \text { Haematologic abnormalities cer- } \\
\text { tolizumab } 400 \text { mg }\end{array}$ & 2 & 750 & $\begin{array}{l}\text { Peto Odds Ratio (Peto, Fixed, } \\
95 \% \mathrm{Cl} \text { ) }\end{array}$ & $1.13[0.21,6.07]$ \\
\hline $\begin{array}{l}65 \text { Herpes viral infection certolizum- } \\
\text { ab } 200 \text { mg }\end{array}$ & 2 & 821 & $\begin{array}{l}\text { Peto Odds Ratio (Peto, Fixed, } \\
95 \% \mathrm{Cl} \text { ) }\end{array}$ & $5.80[0.34,100.23]$ \\
\hline $\begin{array}{l}66 \text { Herpes viral infection certolizum- } \\
\text { ab } 400 \mathrm{mg}\end{array}$ & 1 & & $\begin{array}{l}\text { Peto Odds Ratio (Peto, Fixed, } \\
95 \% \mathrm{Cl} \text { ) }\end{array}$ & $\begin{array}{l}\text { Totals not select- } \\
\text { ed }\end{array}$ \\
\hline $\begin{array}{l}67 \text { Bacterial peritonitis certolizum- } \\
\text { ab } 200 \text { mg }\end{array}$ & 1 & & $\begin{array}{l}\text { Peto Odds Ratio (Peto, Fixed, } \\
95 \% \mathrm{Cl} \text { ) }\end{array}$ & $\begin{array}{l}\text { Totals not select- } \\
\text { ed }\end{array}$ \\
\hline $\begin{array}{l}68 \text { Bacterial peritonitis certolizum- } \\
\text { ab } 400 \mathrm{mg}\end{array}$ & 1 & & $\begin{array}{l}\text { Peto Odds Ratio (Peto, Fixed, } \\
95 \% \mathrm{Cl} \text { ) }\end{array}$ & $\begin{array}{l}\text { Totals not select- } \\
\text { ed }\end{array}$ \\
\hline $\begin{array}{l}69 \text { Opportunistic infections cer- } \\
\text { tolizumab } 200 \mathrm{mg}\end{array}$ & 4 & 2070 & $\begin{array}{l}\text { Peto Odds Ratio (Peto, Fixed, } \\
95 \% \mathrm{Cl} \text { ) }\end{array}$ & $7.33[0.46,117.85]$ \\
\hline $\begin{array}{l}70 \text { Opportunistic infections cer- } \\
\text { tolizumab } 400 \mathrm{mg}\end{array}$ & 1 & & $\begin{array}{l}\text { Peto Odds Ratio (Peto, Fixed, } \\
95 \% \mathrm{Cl} \text { ) }\end{array}$ & $\begin{array}{l}\text { Totals not select- } \\
\text { ed }\end{array}$ \\
\hline $\begin{array}{l}71 \text { Infections and infestations cer- } \\
\text { tolizumab } 200 \mathrm{mg}\end{array}$ & 9 & 3910 & $\begin{array}{l}\text { Risk Ratio (M-H, Random, 95\% } \\
\mathrm{Cl} \text { ) }\end{array}$ & $1.27[1.10,1.46]$ \\
\hline $\begin{array}{l}72 \text { Infections and infestations cer- } \\
\text { tolizumab } 400 \mathrm{mg}\end{array}$ & 5 & 1404 & $\begin{array}{l}\text { Risk Ratio (M-H, Random, 95\% } \\
\text { Cl) }\end{array}$ & $1.43[1.03,1.98]$ \\
\hline $\begin{array}{l}73 \text { Decreased haemoglobin cer- } \\
\text { tolizumab } 200 \mathrm{mg}\end{array}$ & 1 & & $\begin{array}{l}\text { Peto Odds Ratio (Peto, Fixed, } \\
95 \% \mathrm{Cl} \text { ) }\end{array}$ & $\begin{array}{l}\text { Totals not select- } \\
\text { ed }\end{array}$ \\
\hline $\begin{array}{l}74 \text { Decreased haemoglobin cer- } \\
\text { tolizumab } 400 \mathrm{mg}\end{array}$ & 1 & & $\begin{array}{l}\text { Peto Odds Ratio (Peto, Fixed, } \\
95 \% \mathrm{Cl} \text { ) }\end{array}$ & $\begin{array}{l}\text { Totals not select- } \\
\text { ed }\end{array}$ \\
\hline
\end{tabular}




\begin{tabular}{|c|c|c|c|c|}
\hline Outcome or subgroup title & No. of studies & $\begin{array}{l}\text { No. of partici- } \\
\text { pants }\end{array}$ & Statistical method & Effect size \\
\hline $\begin{array}{l}75 \text { Increased platelet count cer- } \\
\text { tolizumab } 200 \mathrm{mg}\end{array}$ & 1 & & $\begin{array}{l}\text { Peto Odds Ratio (Peto, Fixed, } \\
95 \% \mathrm{Cl} \text { ) }\end{array}$ & $\begin{array}{l}\text { Totals not select- } \\
\text { ed }\end{array}$ \\
\hline $\begin{array}{l}76 \text { Increased platelet count cer- } \\
\text { tolizumab } 400 \mathrm{mg}\end{array}$ & 1 & & $\begin{array}{l}\text { Peto Odds Ratio (Peto, Fixed, } \\
95 \% \mathrm{Cl} \text { ) }\end{array}$ & $\begin{array}{l}\text { Totals not select- } \\
\text { ed }\end{array}$ \\
\hline $\begin{array}{l}77 \text { Cerebral haemorrhage including } \\
\text { subarachnoid certolizumab } 200 \mathrm{mg}\end{array}$ & 2 & 321 & $\begin{array}{l}\text { Peto Odds Ratio (Peto, Fixed, } \\
95 \% \mathrm{Cl} \text { ) }\end{array}$ & $1.27[0.12,13.50]$ \\
\hline $\begin{array}{l}78 \text { Ischaemic stroke certolizumab } \\
400 \mathrm{mg}\end{array}$ & 1 & & $\begin{array}{l}\text { Peto Odds Ratio (Peto, Fixed, } \\
95 \% \mathrm{Cl} \text { ) }\end{array}$ & $\begin{array}{l}\text { Totals not select- } \\
\text { ed }\end{array}$ \\
\hline $\begin{array}{l}79 \text { Nausea/vomiting certolizumab } \\
200 \mathrm{mg}\end{array}$ & 4 & 2447 & $\begin{array}{l}\text { Peto Odds Ratio (Peto, Fixed, } \\
95 \% \mathrm{Cl} \text { ) }\end{array}$ & $1.13[0.84,1.54]$ \\
\hline 80 Vomiting certolizumab 400 mg & 1 & & $\begin{array}{l}\text { Peto Odds Ratio (Peto, Fixed, } \\
95 \% \mathrm{Cl} \text { ) }\end{array}$ & $\begin{array}{l}\text { Totals not select- } \\
\text { ed }\end{array}$ \\
\hline $\begin{array}{l}81 \text { Acute miocardial infarction cer- } \\
\text { tolizumab } 200 \text { mg }\end{array}$ & 2 & 1073 & $\begin{array}{l}\text { Peto Odds Ratio (Peto, Fixed, } \\
95 \% \mathrm{Cl} \text { ) }\end{array}$ & $3.79[0.04,351.89]$ \\
\hline $\begin{array}{l}82 \text { Acute myocardial infarction cer- } \\
\text { tolizumab } 400 \mathrm{mg}\end{array}$ & 1 & & $\begin{array}{l}\text { Peto Odds Ratio (Peto, Fixed, } \\
95 \% \mathrm{Cl} \text { ) }\end{array}$ & $\begin{array}{l}\text { Totals not select- } \\
\text { ed }\end{array}$ \\
\hline $\begin{array}{l}83 \text { Abdominal pain/discomfort/dys- } \\
\text { pepsia certolizumab } 200 \mathrm{mg}\end{array}$ & 1 & & $\begin{array}{l}\text { Peto Odds Ratio (Peto, Fixed, } \\
95 \% \mathrm{Cl} \text { ) }\end{array}$ & $\begin{array}{l}\text { Totals not select- } \\
\text { ed }\end{array}$ \\
\hline $\begin{array}{l}84 \text { Constipation certolizumab } 200 \\
\text { mg }\end{array}$ & 1 & & $\begin{array}{l}\text { Peto Odds Ratio (Peto, Fixed, } \\
95 \% \mathrm{Cl} \text { ) }\end{array}$ & $\begin{array}{l}\text { Totals not select- } \\
\text { ed }\end{array}$ \\
\hline $\begin{array}{l}85 \text { Skin and subcutaneous tissue } \\
\text { disorders certolizumab } 200 \text { mg }\end{array}$ & 4 & 1395 & $\begin{array}{l}\text { Peto Odds Ratio (Peto, Fixed, } \\
95 \% \mathrm{Cl} \text { ) }\end{array}$ & $2.83[1.46,5.48]$ \\
\hline $\begin{array}{l}86 \text { Skin and subcutaneous tissue } \\
\text { disorders certolizumab } 400 \mathrm{mg}\end{array}$ & 1 & & $\begin{array}{l}\text { Peto Odds Ratio (Peto, Fixed, } \\
95 \% \mathrm{Cl} \text { ) }\end{array}$ & $\begin{array}{l}\text { Totals not select- } \\
\text { ed }\end{array}$ \\
\hline 87 Cough certolizumab 200 mg & 1 & & $\begin{array}{l}\text { Peto Odds Ratio (Peto, Fixed, } \\
95 \% \mathrm{Cl} \text { ) }\end{array}$ & $\begin{array}{l}\text { Totals not select- } \\
\text { ed }\end{array}$ \\
\hline 88 Pruritus certolizumab 200 mg & 1 & & $\begin{array}{l}\text { Peto Odds Ratio (Peto, Fixed, } \\
95 \% \mathrm{Cl} \text { ) }\end{array}$ & $\begin{array}{l}\text { Totals not select- } \\
\text { ed }\end{array}$ \\
\hline 89 Fatigue certolizumab 200 mg & 1 & & $\begin{array}{l}\text { Peto Odds Ratio (Peto, Fixed, } \\
95 \% \mathrm{Cl} \text { ) }\end{array}$ & $\begin{array}{l}\text { Totals not select- } \\
\text { ed }\end{array}$ \\
\hline 90 Fatigue certolizumab 400 mg & 1 & & $\begin{array}{l}\text { Peto Odds Ratio (Peto, Fixed, } \\
95 \% \mathrm{Cl} \text { ) }\end{array}$ & $\begin{array}{l}\text { Totals not select- } \\
\text { ed }\end{array}$ \\
\hline $\begin{array}{l}91 \text { Periodontitis certolizumab } 200 \\
\text { mg }\end{array}$ & 1 & & $\begin{array}{l}\text { Peto Odds Ratio (Peto, Fixed, } \\
95 \% \mathrm{Cl} \text { ) }\end{array}$ & $\begin{array}{l}\text { Totals not select- } \\
\text { ed }\end{array}$ \\
\hline $\begin{array}{l}92 \text { Arthritis bacterial certolizumab } \\
400 \mathrm{mg}\end{array}$ & 1 & & $\begin{array}{l}\text { Peto Odds Ratio (Peto, Fixed, } \\
95 \% \mathrm{Cl} \text { ) }\end{array}$ & $\begin{array}{l}\text { Totals not select- } \\
\text { ed }\end{array}$ \\
\hline
\end{tabular}




\begin{tabular}{|c|c|c|c|c|}
\hline Outcome or subgroup title & No. of studies & $\begin{array}{l}\text { No. of partici- } \\
\text { pants }\end{array}$ & Statistical method & Effect size \\
\hline 93 Mastitis certolizumab 400 mg & 1 & 220 & $\begin{array}{l}\text { Peto Odds Ratio (Peto, Fixed, } \\
95 \% \mathrm{Cl} \text { ) }\end{array}$ & $7.26[0.14,365.79]$ \\
\hline $\begin{array}{l}94 \text { Benign tumour certolizumab } 400 \\
\text { mg }\end{array}$ & 1 & & $\begin{array}{l}\text { Peto Odds Ratio (Peto, Fixed, } \\
95 \% \mathrm{Cl} \text { ) }\end{array}$ & $\begin{array}{l}\text { Totals not select- } \\
\text { ed }\end{array}$ \\
\hline $\begin{array}{l}95 \text { Dizziness postural certolizumab } \\
400 \mathrm{mg}\end{array}$ & 1 & & $\begin{array}{l}\text { Peto Odds Ratio (Peto, Fixed, } \\
95 \% \mathrm{Cl} \text { ) }\end{array}$ & $\begin{array}{l}\text { Totals not select- } \\
\text { ed }\end{array}$ \\
\hline $\begin{array}{l}96 \text { Menorrhagia certolizumab } 400 \\
\text { mg }\end{array}$ & 1 & & $\begin{array}{l}\text { Peto Odds Ratio (Peto, Fixed, } \\
95 \% \mathrm{Cl} \text { ) }\end{array}$ & $\begin{array}{l}\text { Totals not select- } \\
\text { ed }\end{array}$ \\
\hline $\begin{array}{l}97 \text { Corneal perforation certolizumab } \\
400 \mathrm{mg}\end{array}$ & 1 & & $\begin{array}{l}\text { Peto Odds Ratio (Peto, Fixed, } \\
95 \% \mathrm{Cl} \text { ) }\end{array}$ & $\begin{array}{l}\text { Totals not select- } \\
\text { ed }\end{array}$ \\
\hline $\begin{array}{l}98 \text { Conjunctivitis allergic cer- } \\
\text { tolizumab } 400 \mathrm{mg}\end{array}$ & 1 & & $\begin{array}{l}\text { Peto Odds Ratio (Peto, Fixed, } \\
95 \% \mathrm{Cl} \text { ) }\end{array}$ & $\begin{array}{l}\text { Totals not select- } \\
\text { ed }\end{array}$ \\
\hline $\begin{array}{l}99 \text { Periodontitis certolizumab } 400 \\
\text { mg }\end{array}$ & 1 & & Risk Ratio (M-H, Fixed, 95\% Cl) & $\begin{array}{l}\text { Totals not select- } \\
\text { ed }\end{array}$ \\
\hline
\end{tabular}

Analysis 50.1. Comparison 50 Safety, Outcome 1 Any adverse event certolizumab $200 \mathrm{mg}$.

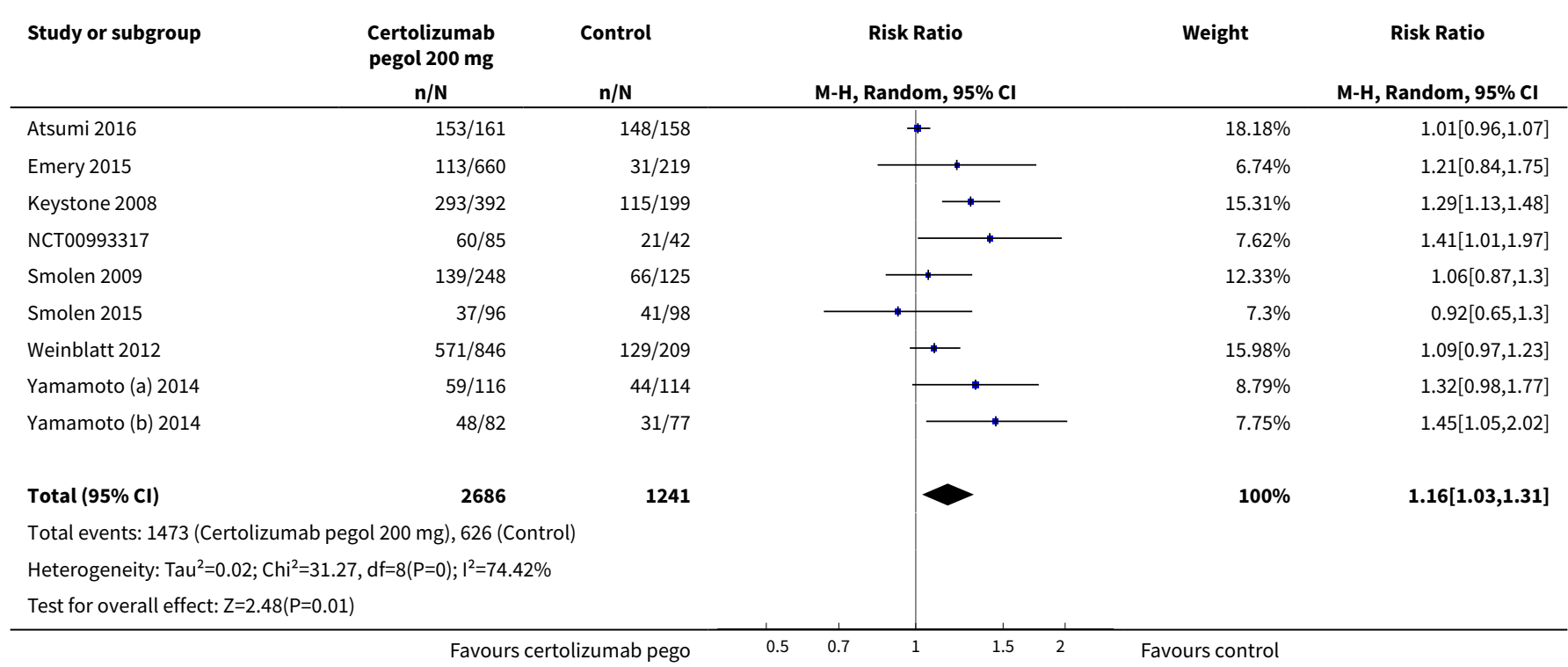

Analysis 50.2. Comparison 50 Safety, Outcome 2 Any adverse events certolizumab $400 \mathrm{mg}$.

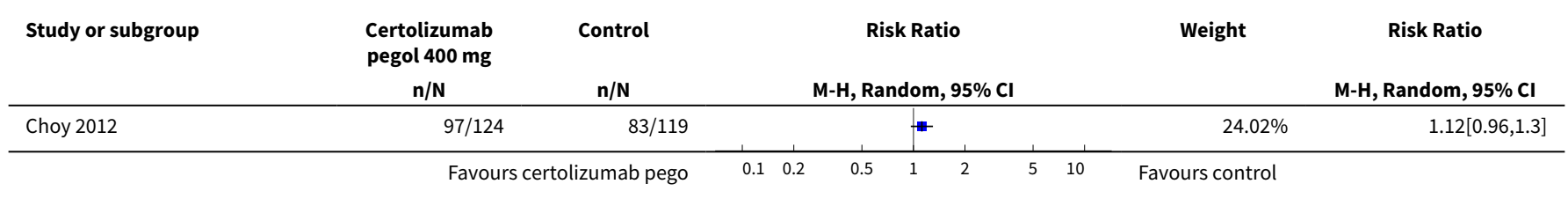




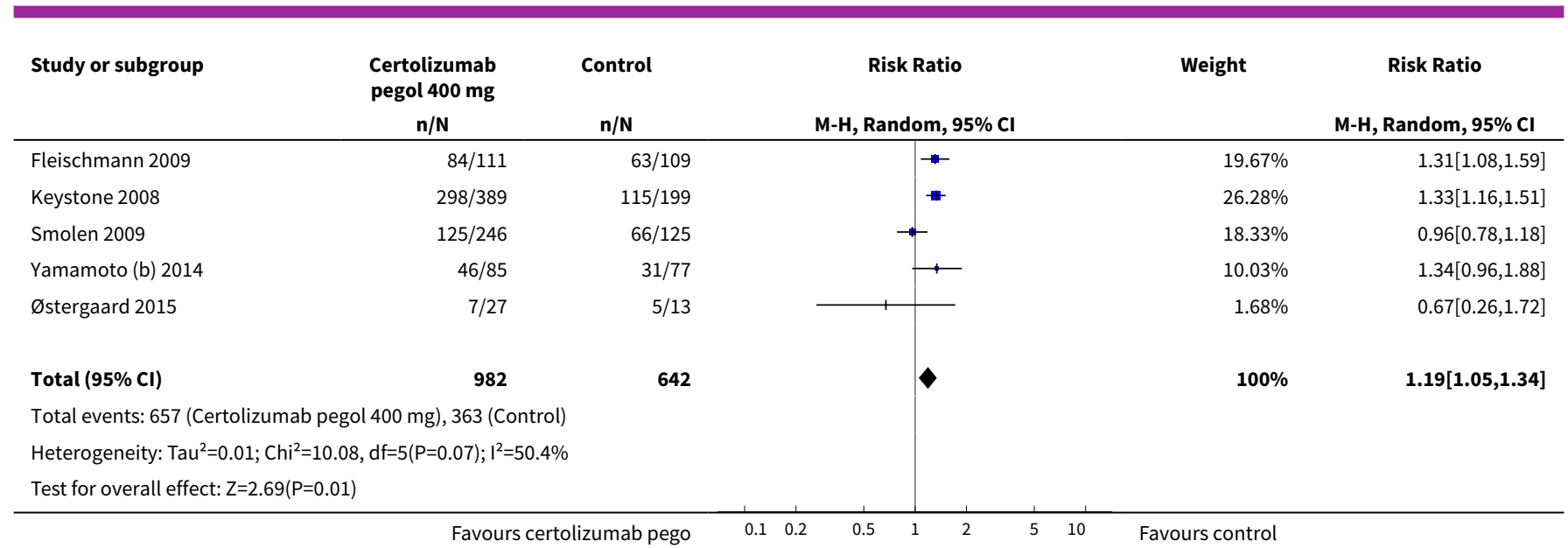

\section{Analysis 50.3. Comparison 50 Safety, Outcome 3 Adverse events: Intensity mild certolizumab 200 mg.}

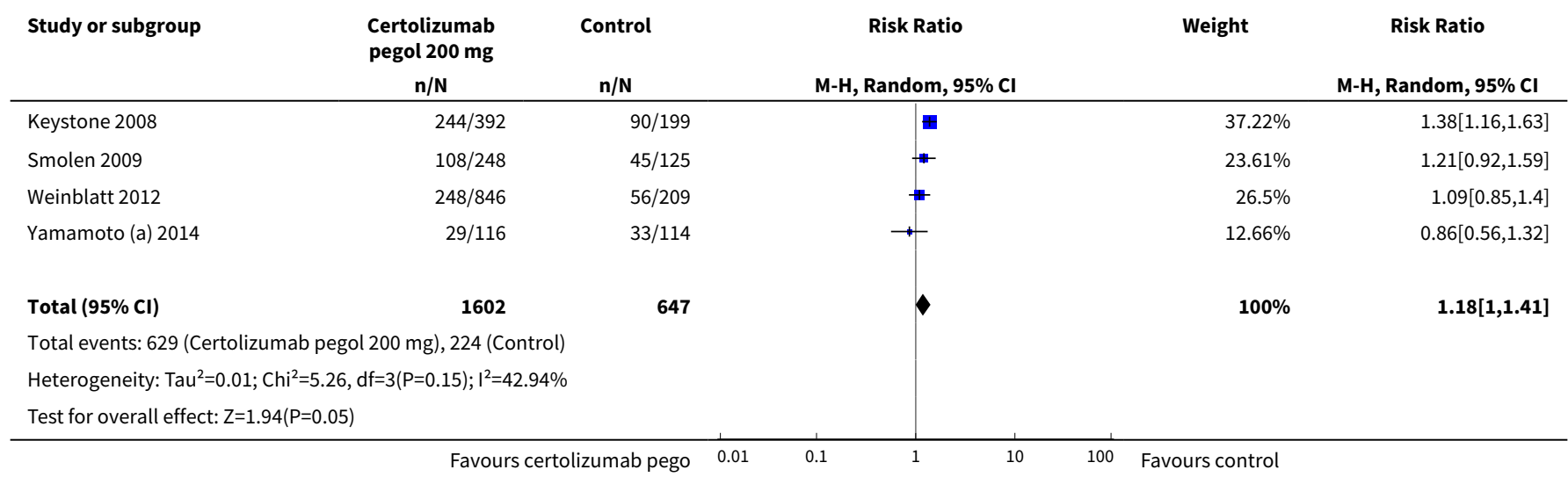

\section{Analysis 50.4. Comparison 50 Safety, Outcome 4 Adverse events: Intensity mild certolizumab 400 mg.}

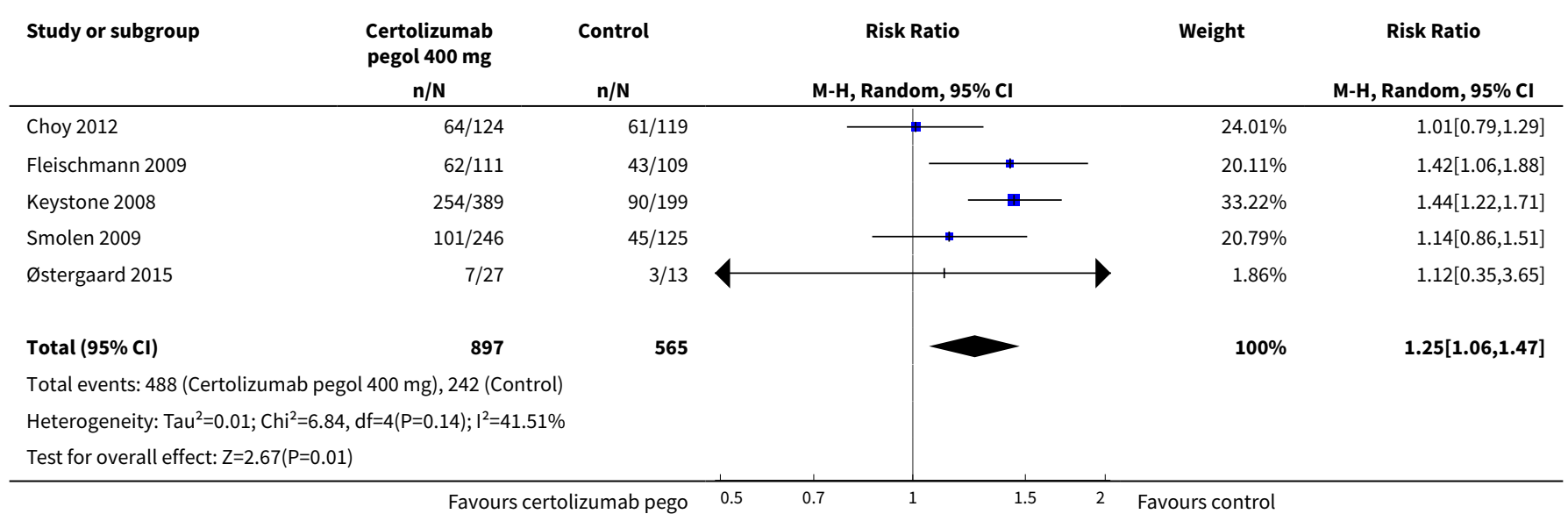


Analysis 50.5. Comparison 50 Safety, Outcome 5 Adverse events: Intensity moderate certolizumab 200 mg.

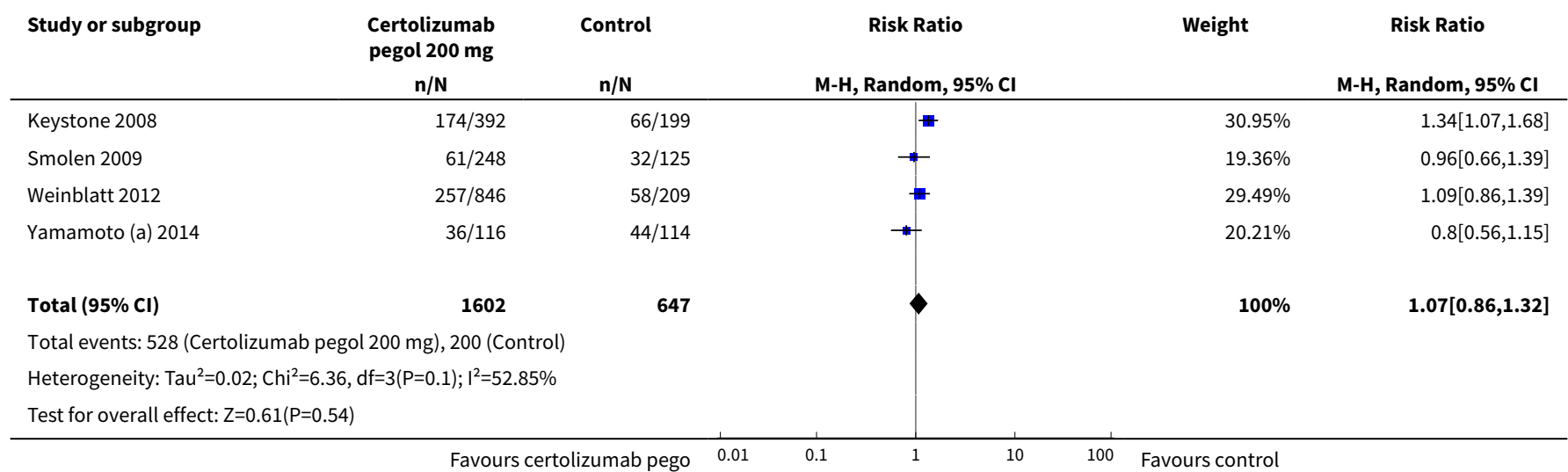

Analysis 50.6. Comparison 50 Safety, Outcome 6 Adverse events: Intensity moderate certolizumab 400 mg.

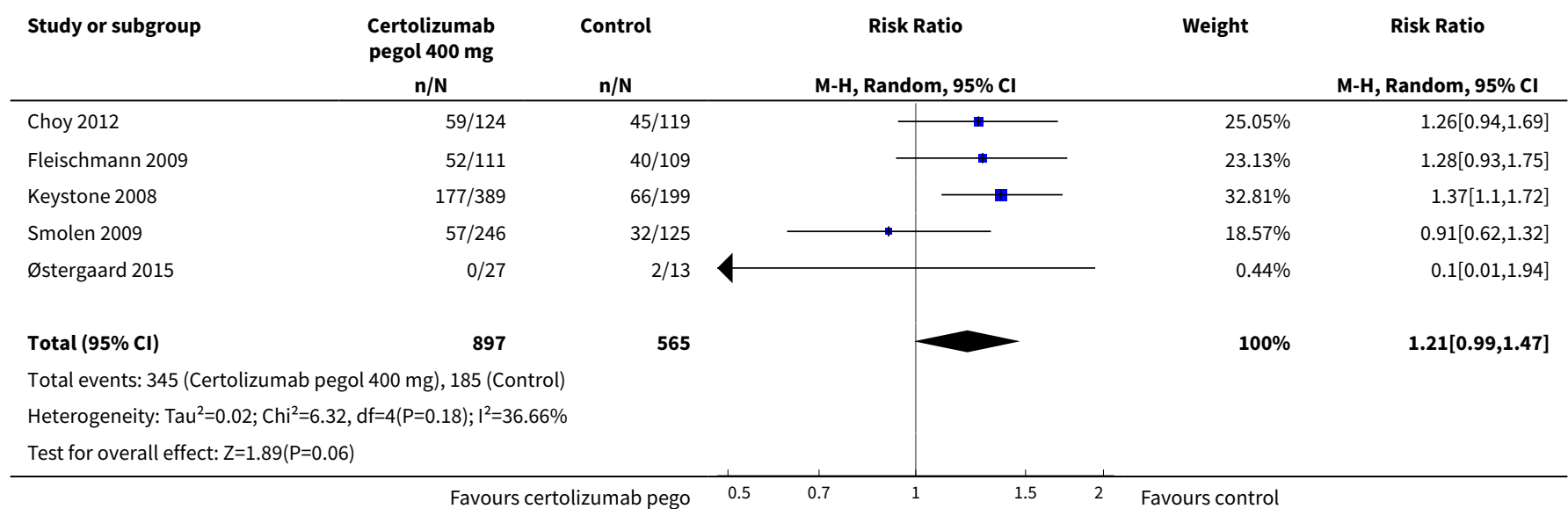

Analysis 50.7. Comparison 50 Safety, Outcome 7 Adverse events: Intensity severe certolizumab 200 mg.

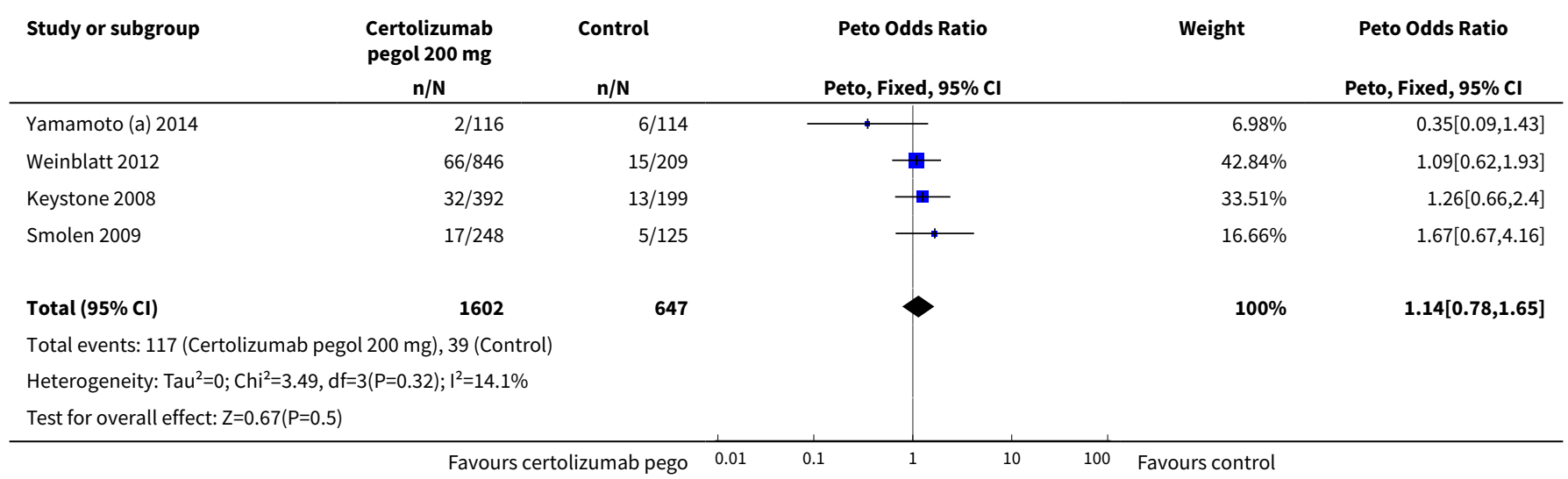


Analysis 50.8. Comparison 50 Safety, Outcome 8 Adverse events: Intensity severe certolizumab 400 mg.

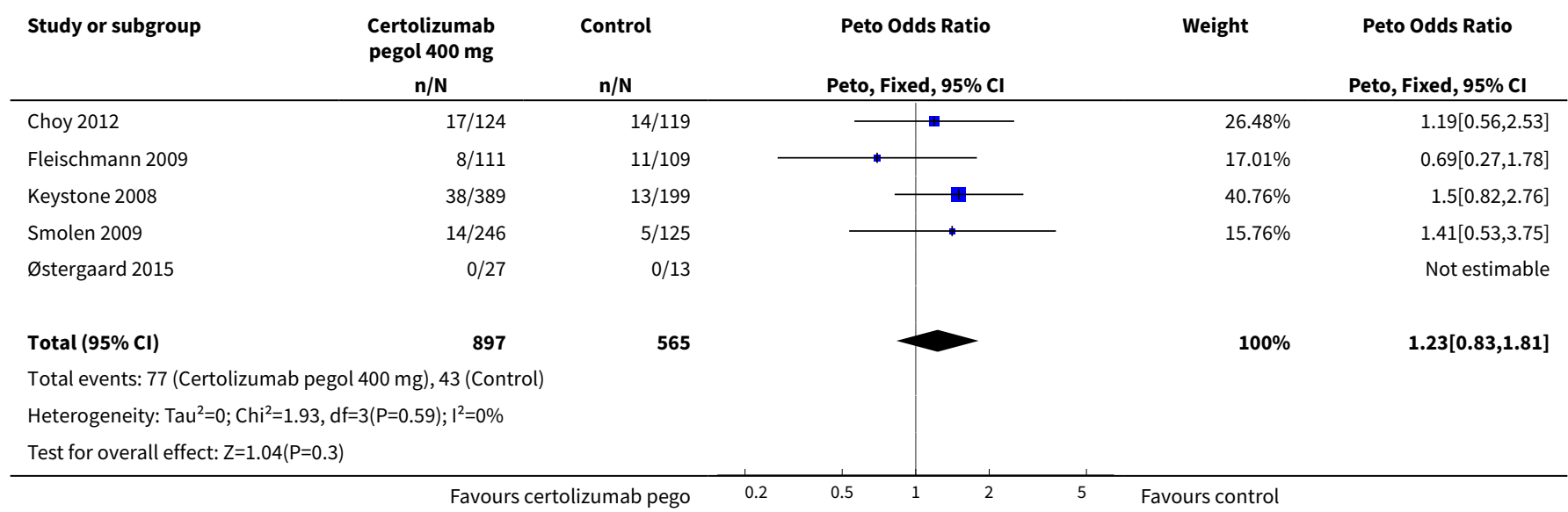

Analysis 50.9. Comparison 50 Safety, Outcome 9 Adverse events related to study drug certolizumab 200 mg.

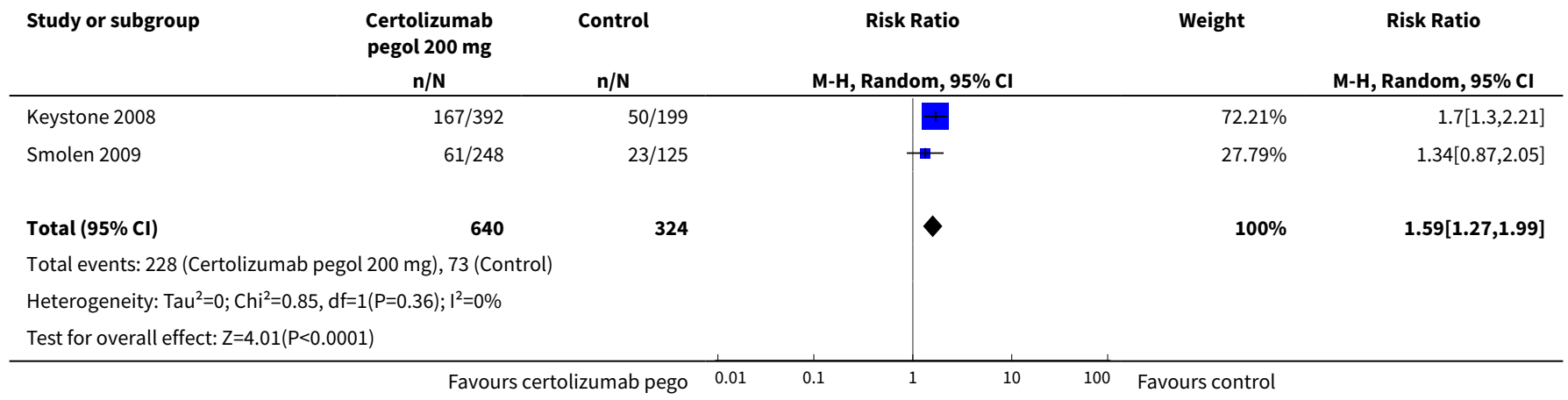

Analysis 50.10. Comparison 50 Safety, Outcome 10 Adverse events related to study drug certolizumab $400 \mathrm{mg}$.

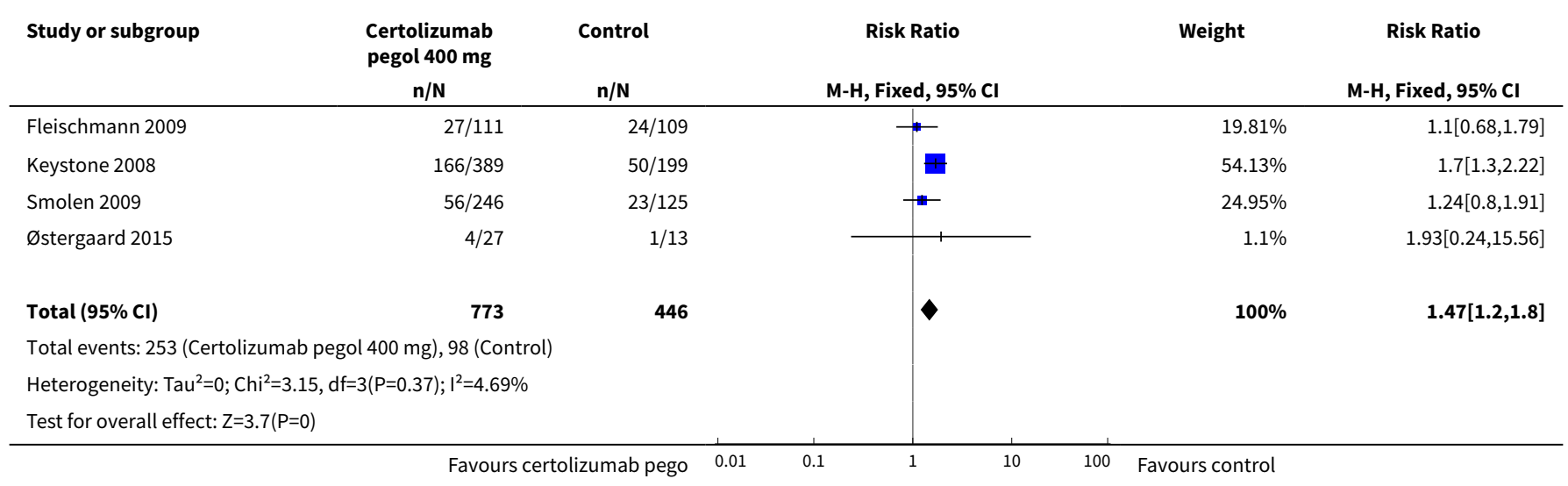




\section{Analysis 50.11. Comparison 50 Safety, Outcome 11 Serious Infections certolizumab 200 mg.}

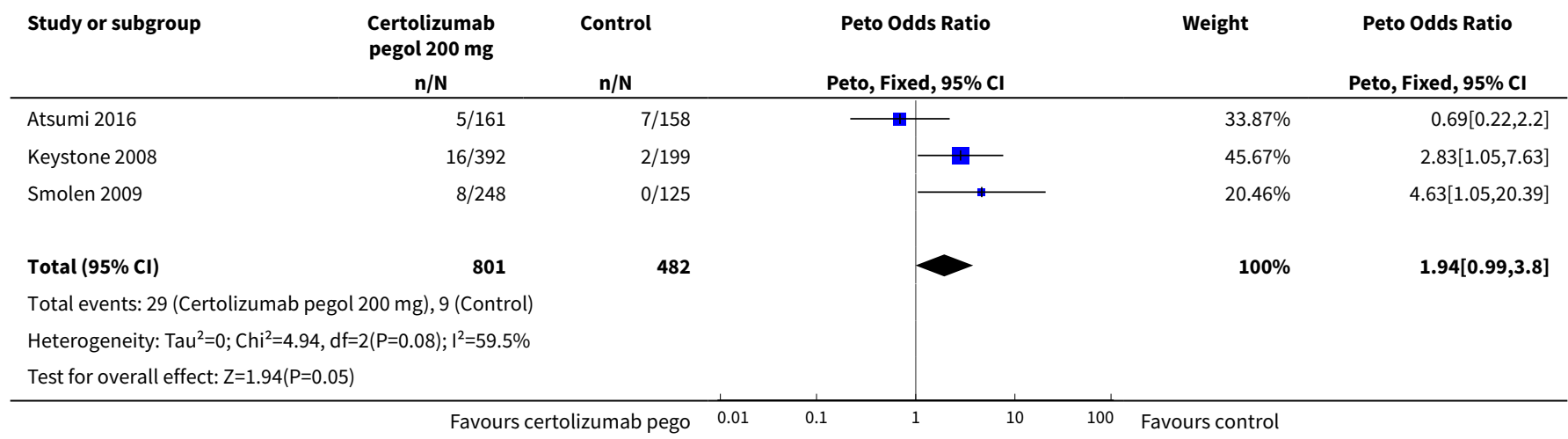

Analysis 50.12. Comparison 50 Safety, Outcome 12 Serious infections certolizumab 400 mg.

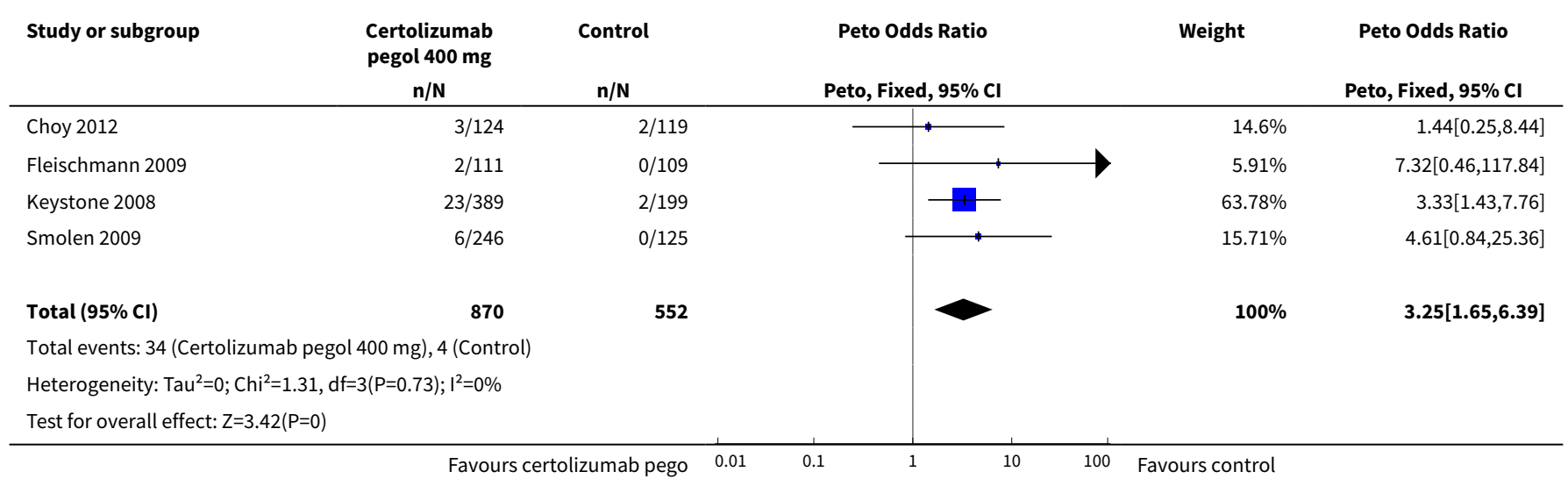

Analysis 50.13. Comparison 50 Safety, Outcome 13 Adverse events leading to death certolizumab $200 \mathrm{mg}$.

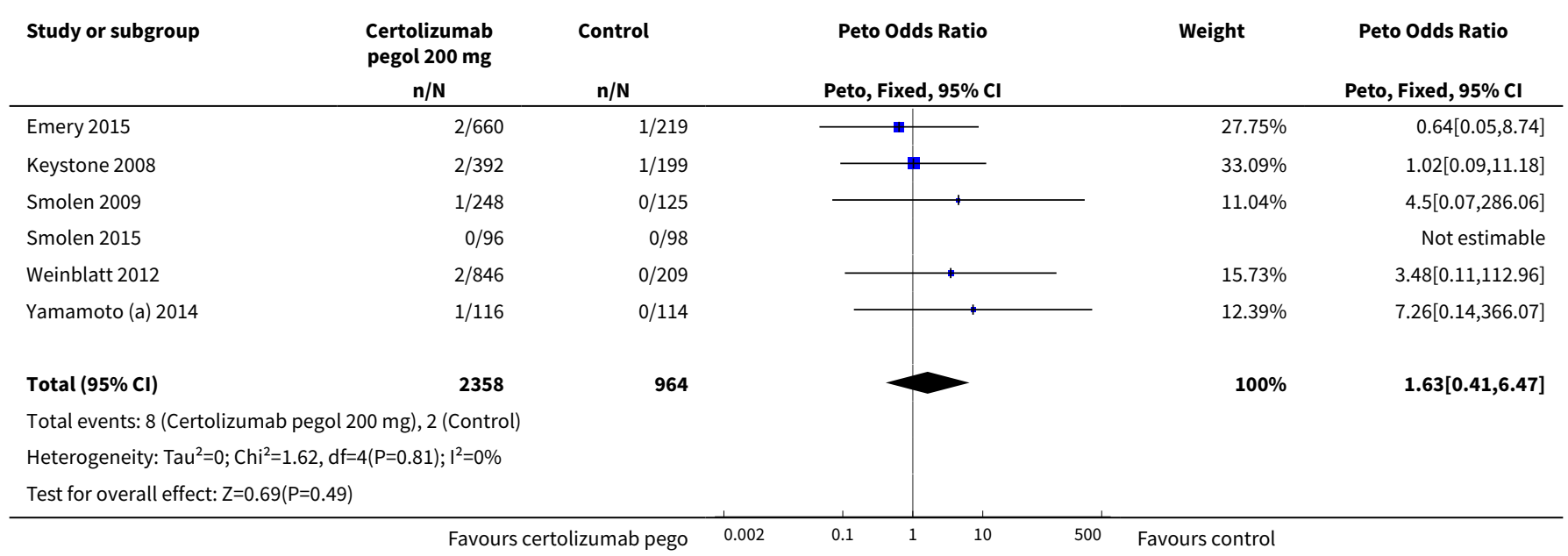


Analysis 50.14. Comparison 50 Safety, Outcome 14 Adverse events leading to death certolizumab $400 \mathrm{mg}$.

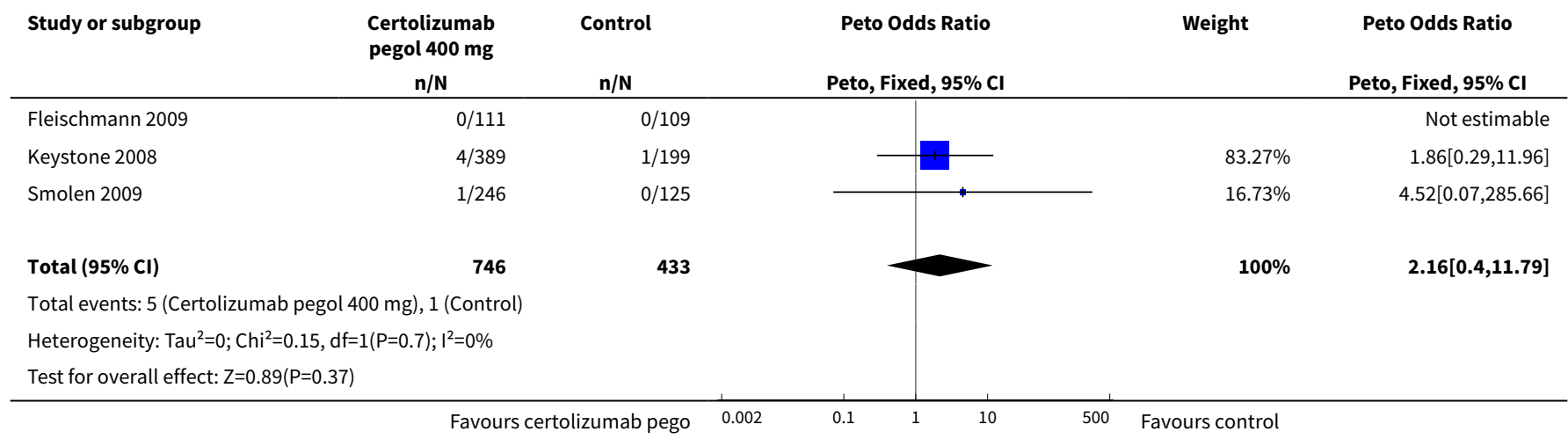

Analysis 50.15. Comparison 50 Safety, Outcome 15 Adverse events leading to withdrawal certolizumab 200 mg.

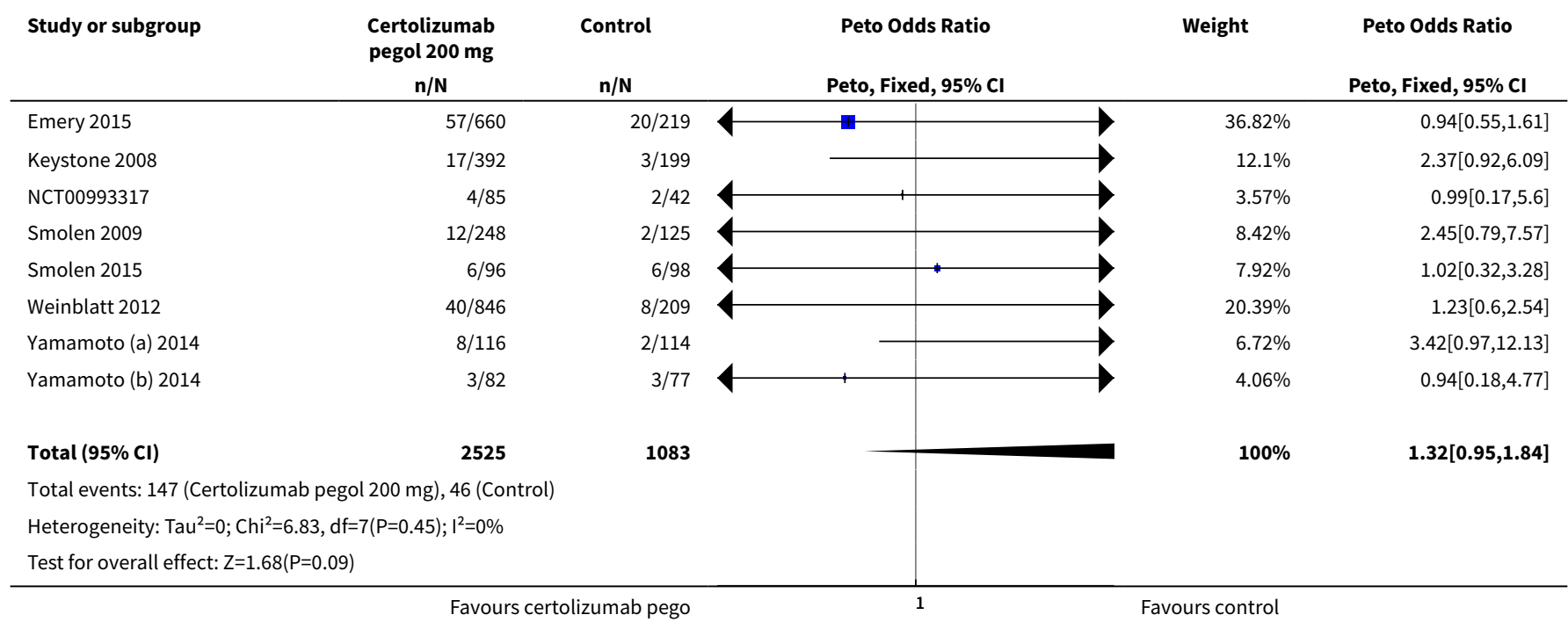

Analysis 50.16. Comparison 50 Safety, Outcome 16 Adverse events leading to withdrawal certolizumab $400 \mathrm{mg}$.

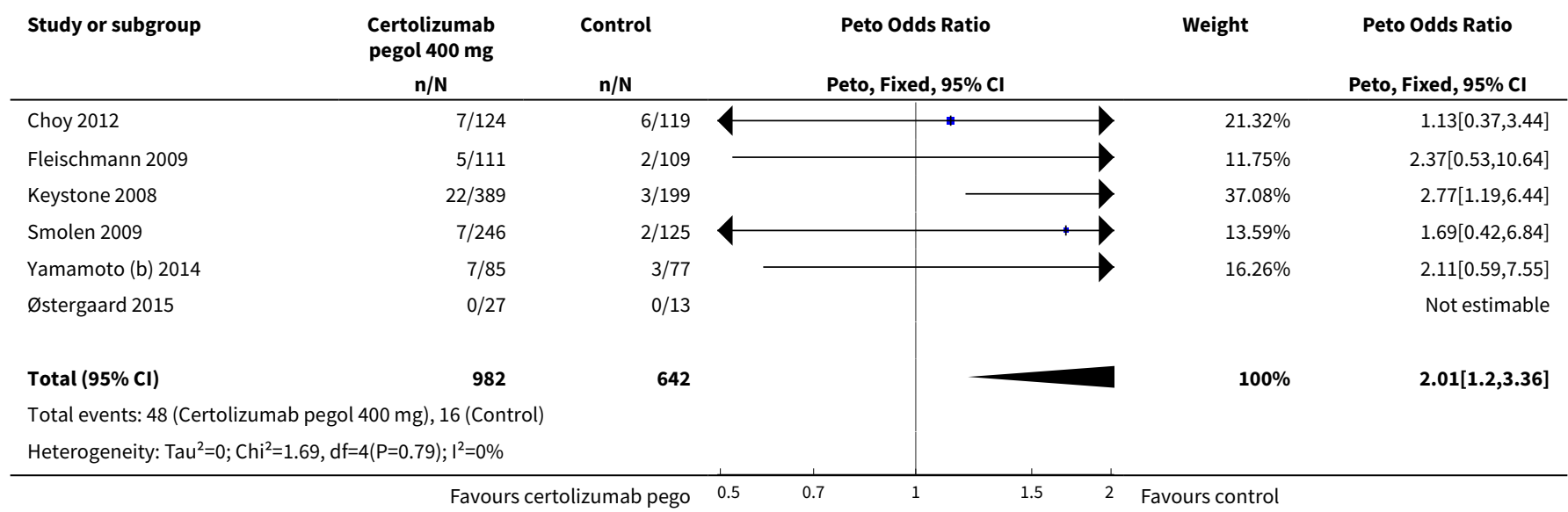




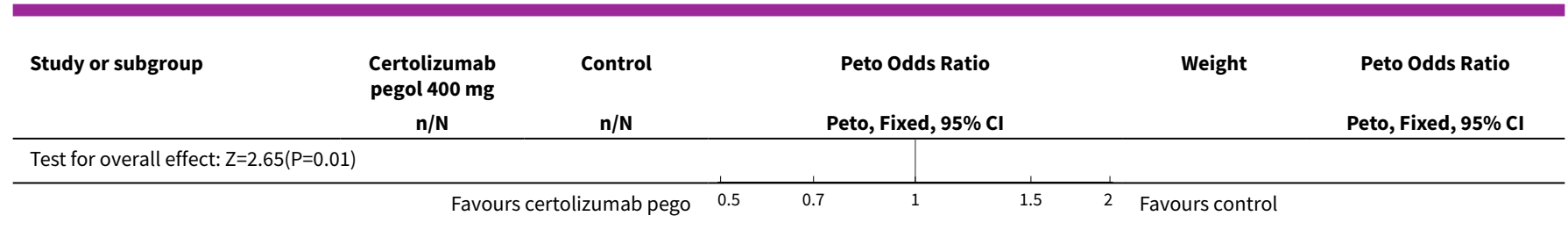

Analysis 50.17. Comparison 50 Safety, Outcome 17 Death certolizumab 200 mg.

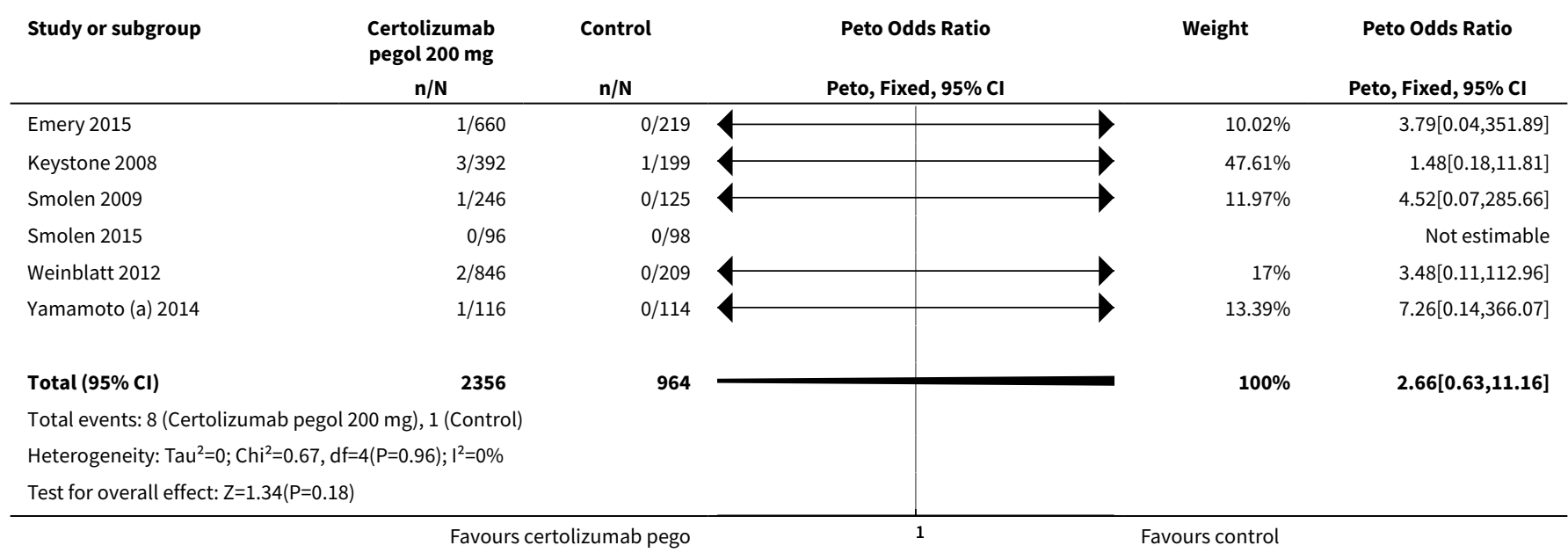

Analysis 50.18. Comparison 50 Safety, Outcome 18 Death certolizumab $400 \mathrm{mg}$.

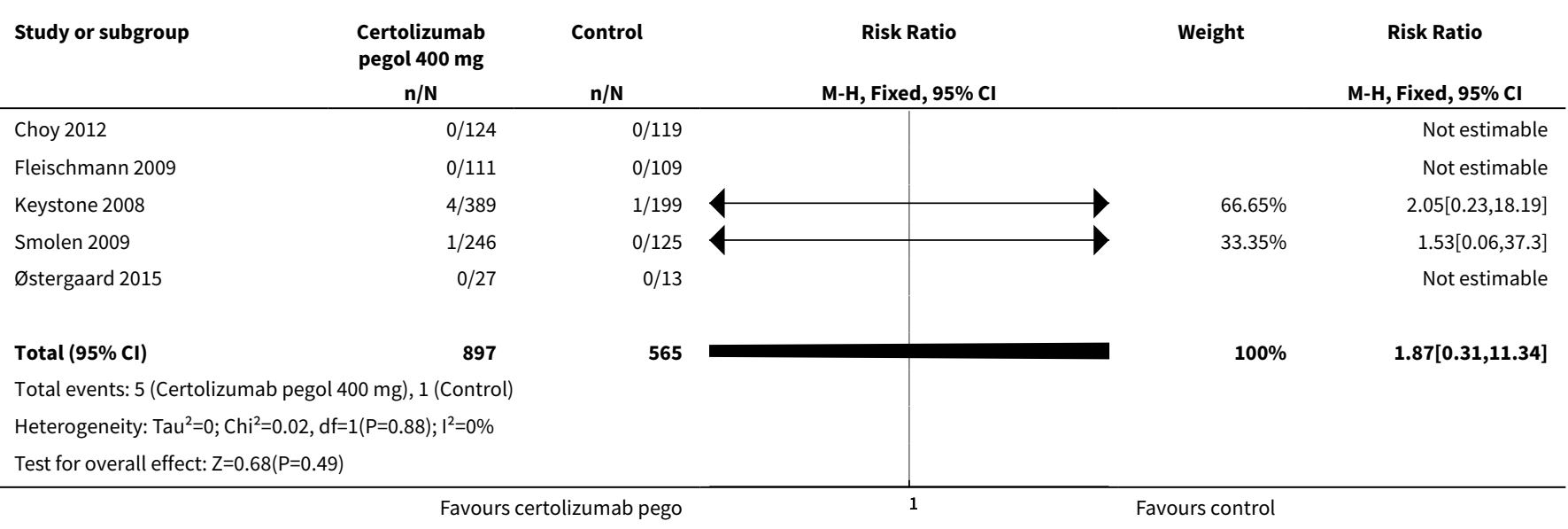

Analysis 50.19. Comparison 50 Safety, Outcome 19 Deaths overall.

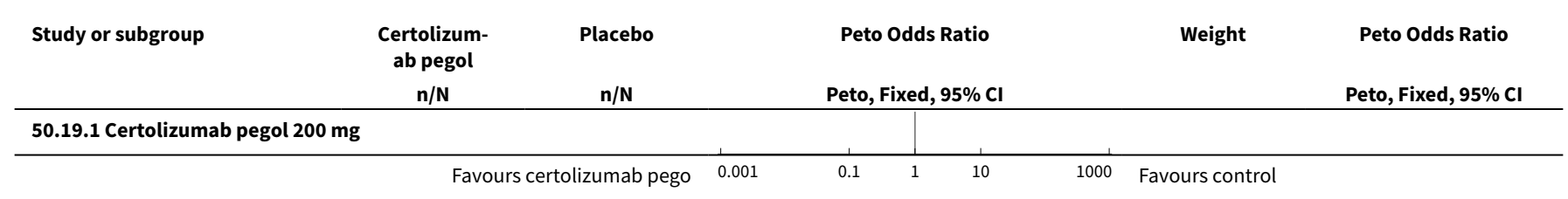




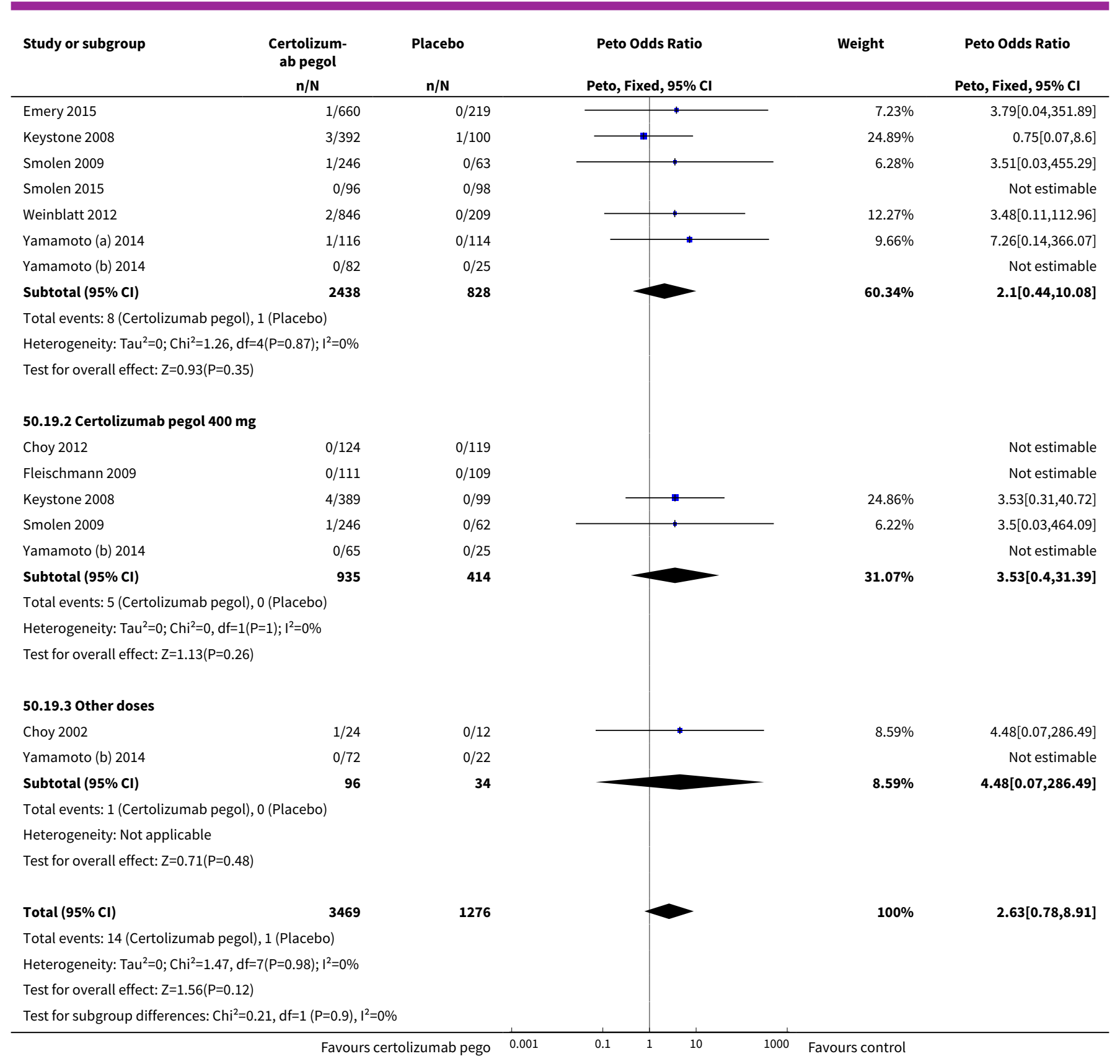

Analysis 50.20. Comparison 50 Safety, Outcome 20 Tuberculosis certolizumab $200 \mathrm{mg}$.

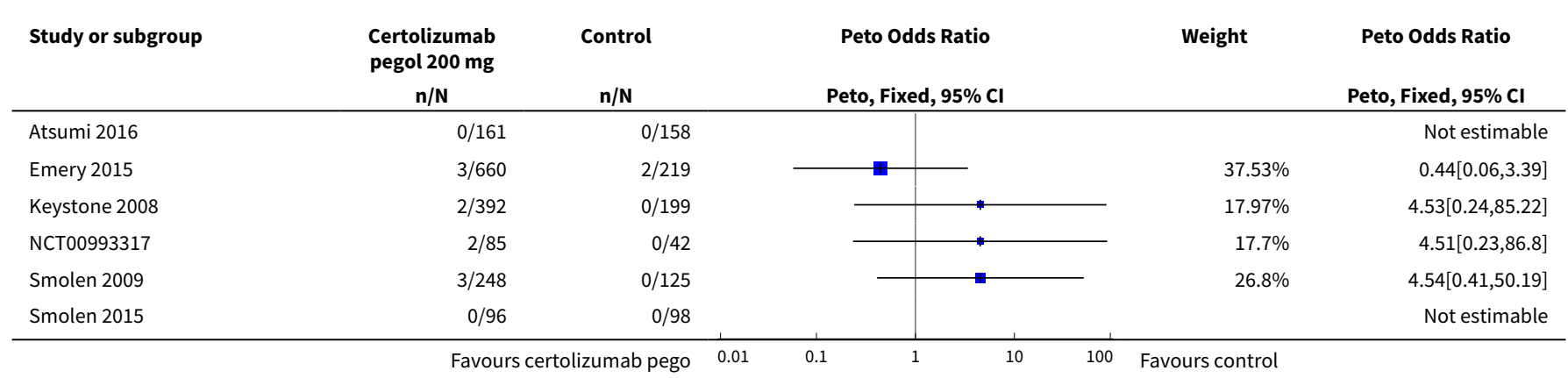




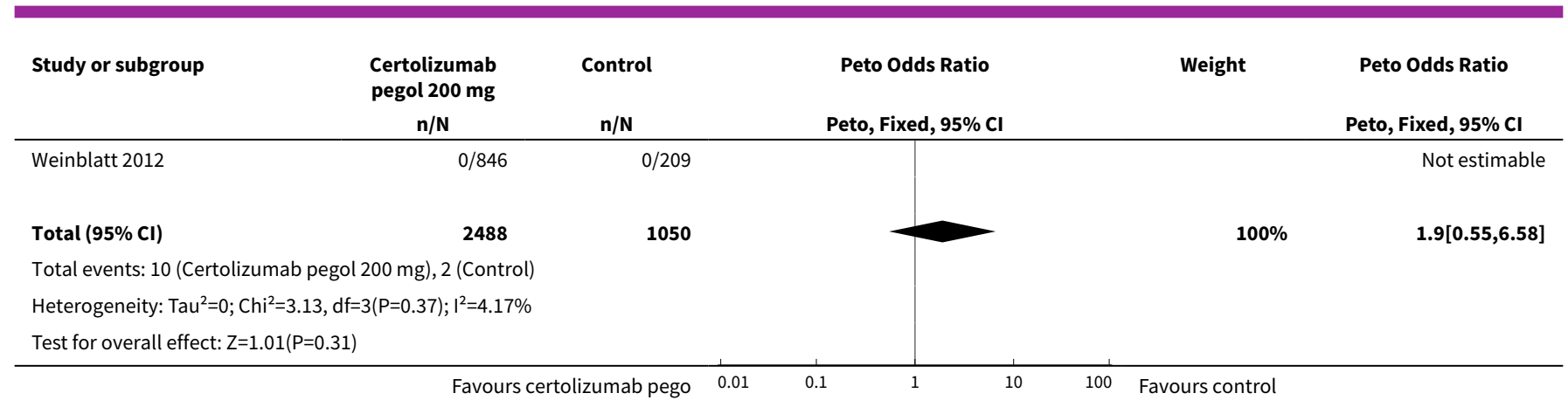

\section{Analysis 50.21. Comparison 50 Safety, Outcome 21 Tuberculosis certolizumab $400 \mathrm{mg}$.}

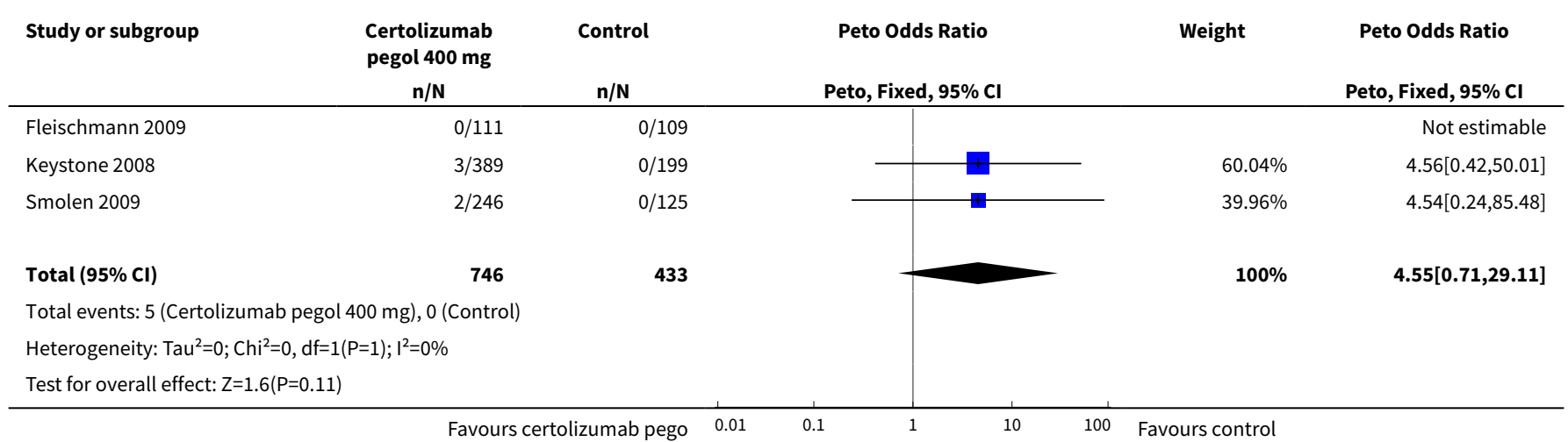

Analysis 50.22. Comparison 50 Safety, Outcome 22 Tuberculosis overall.

\begin{tabular}{|c|c|c|c|c|c|}
\hline Study or subgroup & $\begin{array}{c}\text { Certolizum- } \\
\text { ab pegol } \\
n / N\end{array}$ & $\begin{array}{l}\text { Placebo } \\
n / \mathbf{N}\end{array}$ & $\begin{array}{c}\text { Peto Odds Ratio } \\
\text { Peto, Fixed, 95\% Cl }\end{array}$ & Weight & $\begin{array}{c}\text { Peto Odds Ratio } \\
\text { Peto, Fixed, 95\% Cl }\end{array}$ \\
\hline \multicolumn{6}{|c|}{ 50.22.1 Certolizumab pegol $200 \mathrm{mg}$} \\
\hline Emery 2015 & $3 / 660$ & $2 / 219$ & 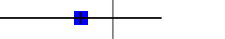 & $31.26 \%$ & $0.44[0.06,3.39]$ \\
\hline Keystone 2008 & $2 / 392$ & $0 / 100$ & & $10.85 \%$ & $3.52[0.11,110.51]$ \\
\hline NCT00993317 & $2 / 85$ & $0 / 42$ & & $14.74 \%$ & $4.51[0.23,86.8]$ \\
\hline Smolen 2015 & $0 / 96$ & $0 / 98$ & & & Not estimable \\
\hline Weinblatt 2012 & $0 / 846$ & $0 / 209$ & & & Not estimable \\
\hline Subtotal $(95 \% \mathrm{Cl})$ & 2327 & 731 & & $73.02 \%$ & $1.53[0.4,5.77]$ \\
\hline \multicolumn{6}{|c|}{ Total events: 10 (Certolizumab pegol), 2 (Placebo) } \\
\hline \multicolumn{6}{|c|}{ Heterogeneity: $\operatorname{Tau}^{2}=0 ; \mathrm{Chi}^{2}=2.5, \mathrm{df}=3(\mathrm{P}=0.48) ; \mathrm{I}^{2}=0 \%$} \\
\hline \multicolumn{6}{|c|}{ Test for overall effect: $Z=0.62(P=0.53)$} \\
\hline \multicolumn{6}{|c|}{ 50.22.2 Certolizumab pegol $400 \mathrm{mg}$} \\
\hline Fleischmann 2009 & $0 / 111$ & $0 / 109$ & & & Not estimable \\
\hline Keystone 2008 & $3 / 389$ & $0 / 99$ & & $16.22 \%$ & $3.52[0.21,59.11]$ \\
\hline Smolen 2009 & $2 / 246$ & $0 / 62$ & & $10.76 \%$ & $3.51[0.11,111.96]$ \\
\hline Subtotal $(95 \% \mathrm{Cl})$ & 746 & 270 & & $26.98 \%$ & $3.52[0.4,31.33]$ \\
\hline \multicolumn{6}{|c|}{ Total events: 5 (Certolizumab pegol), 0 (Placebo) } \\
\hline
\end{tabular}




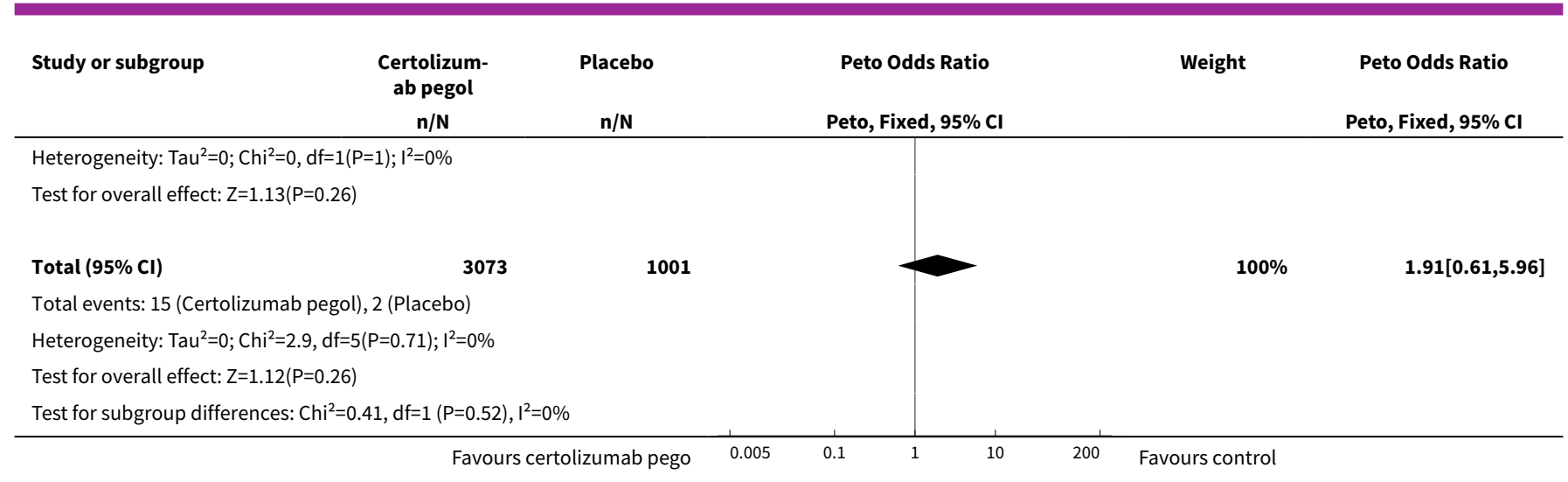

Analysis 50.23. Comparison 50 Safety, Outcome 23 Malignancies included lymphoma certolizumab $200 \mathrm{mg}$.

\begin{tabular}{|c|c|c|c|c|c|}
\hline Study or subgroup & $\begin{array}{c}\text { Certolizumab } \\
\text { pegol } 200 \mathrm{mg} \\
\mathrm{n} / \mathrm{N} \\
\end{array}$ & $\begin{array}{l}\text { Control } \\
n / N\end{array}$ & $\begin{array}{c}\text { Peto Odds Ratio } \\
\text { Peto, Fixed, 95\% Cl }\end{array}$ & Weight & $\begin{array}{c}\text { Peto Odds Ratio } \\
\text { Peto, Fixed, } 95 \% \mathrm{CI}\end{array}$ \\
\hline Atsumi 2016 & $1 / 161$ & $0 / 158$ & & $4.43 \%$ & $7.25[0.14,365.57]$ \\
\hline Emery 2015 & $6 / 660$ & $2 / 219$ & & $26.32 \%$ & $1[0.2,4.97]$ \\
\hline Keystone 2008 & $7 / 392$ & $1 / 199$ & & $31.3 \%$ & $2.61[0.6,11.41]$ \\
\hline NCT00993317 & $0 / 85$ & $0 / 42$ & & & Not estimable \\
\hline Smolen 2015 & $0 / 96$ & $2 / 98$ & & $8.82 \%$ & $0.14[0.01,2.2]$ \\
\hline Weinblatt 2012 & $4 / 846$ & $2 / 209$ & & $16.82 \%$ & $0.43[0.06,3.18]$ \\
\hline Yamamoto (a) 2014 & $0 / 116$ & $1 / 114$ & & $4.43 \%$ & $0.13[0,6.7]$ \\
\hline Total $(95 \% \mathrm{CI})$ & 2604 & 1164 & & $100 \%$ & $0.92[0.4,2.11]$ \\
\hline \multicolumn{6}{|c|}{ Total events: 19 (Certolizumab pegol $200 \mathrm{mg}$ ), 9 (Control) } \\
\hline Test for overall effect & & & & & \\
\hline
\end{tabular}

Analysis 50.24. Comparison 50 Safety, Outcome 24 Malignancies included lymphoma certolizumab 400 mg.

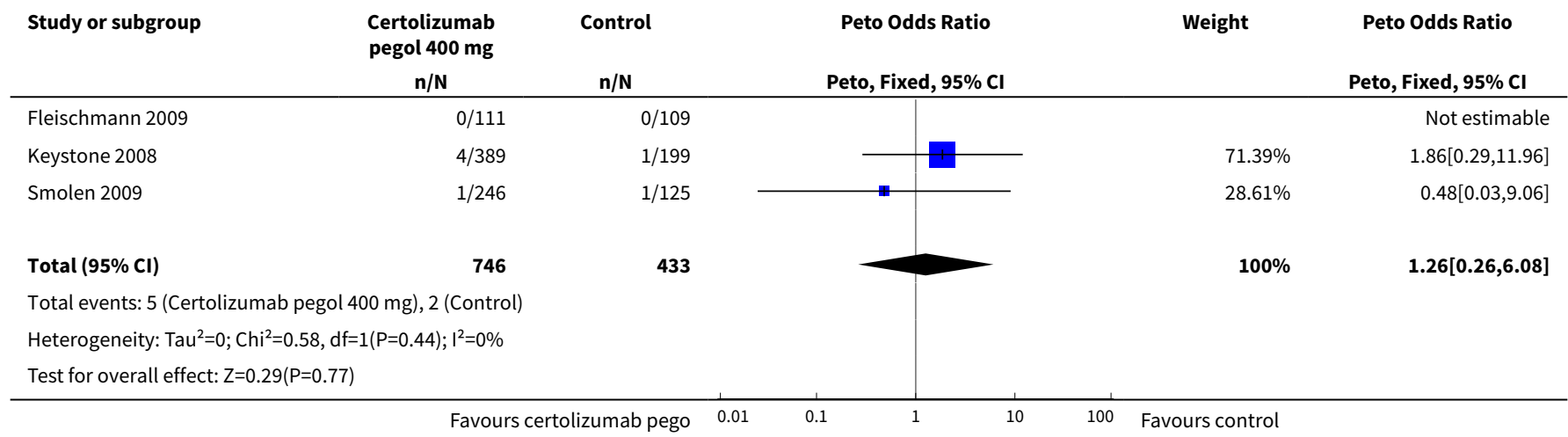


Analysis 50.25. Comparison 50 Safety, Outcome 25 Injection side reactions certolizumab $200 \mathrm{mg}$.

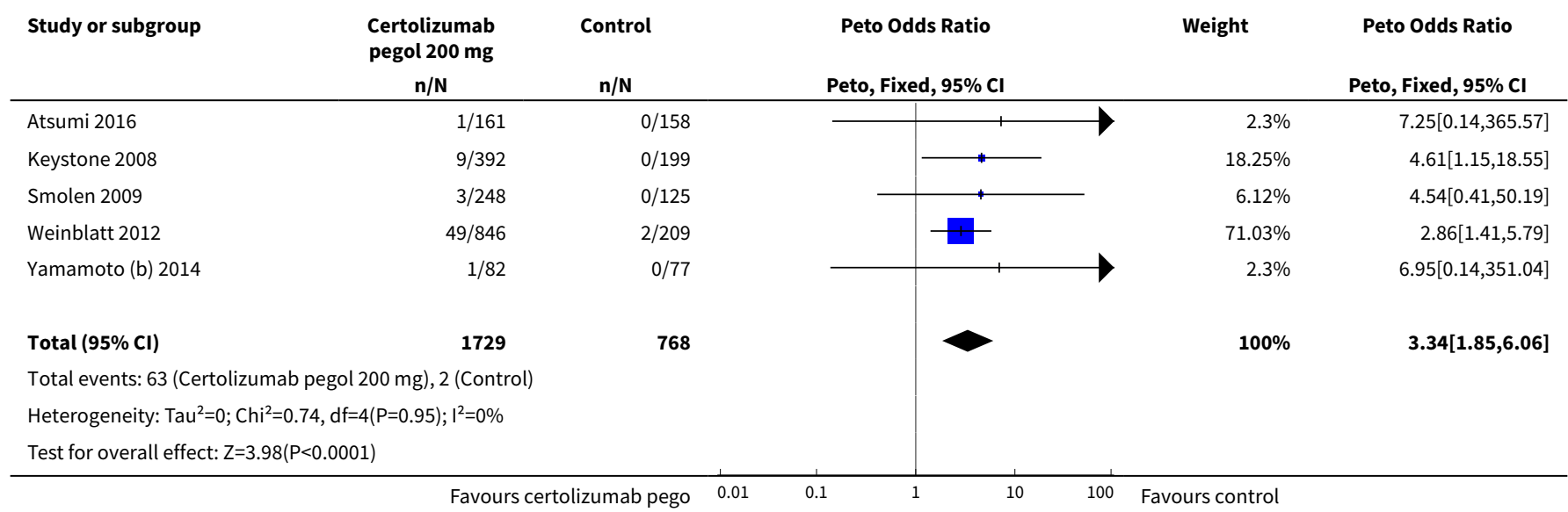

Analysis 50.26. Comparison 50 Safety, Outcome 26 Injection side reactions certolizumab $400 \mathrm{mg}$.

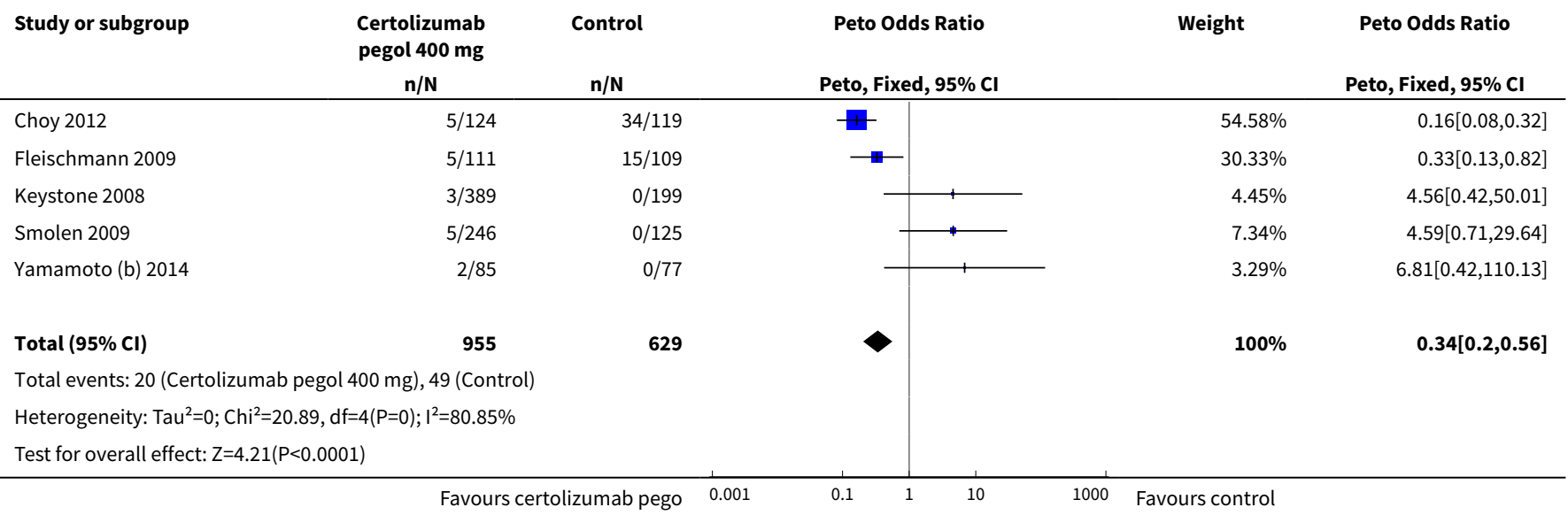

Analysis 50.27. Comparison 50 Safety, Outcome 27 Antinuclear antibodies (ANA) Anti-certolizumab pegol antibodies certolizumab $200 \mathrm{mg}$.

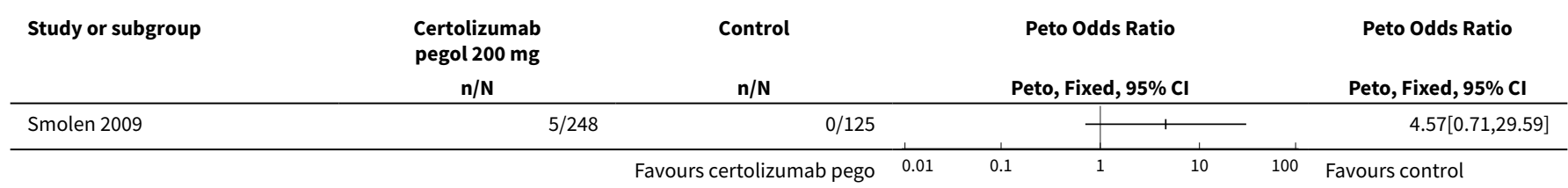


Analysis 50.28. Comparison 50 Safety, Outcome 28 Anti-certolizumab pegol antibodies certolizumab $400 \mathrm{mg}$.

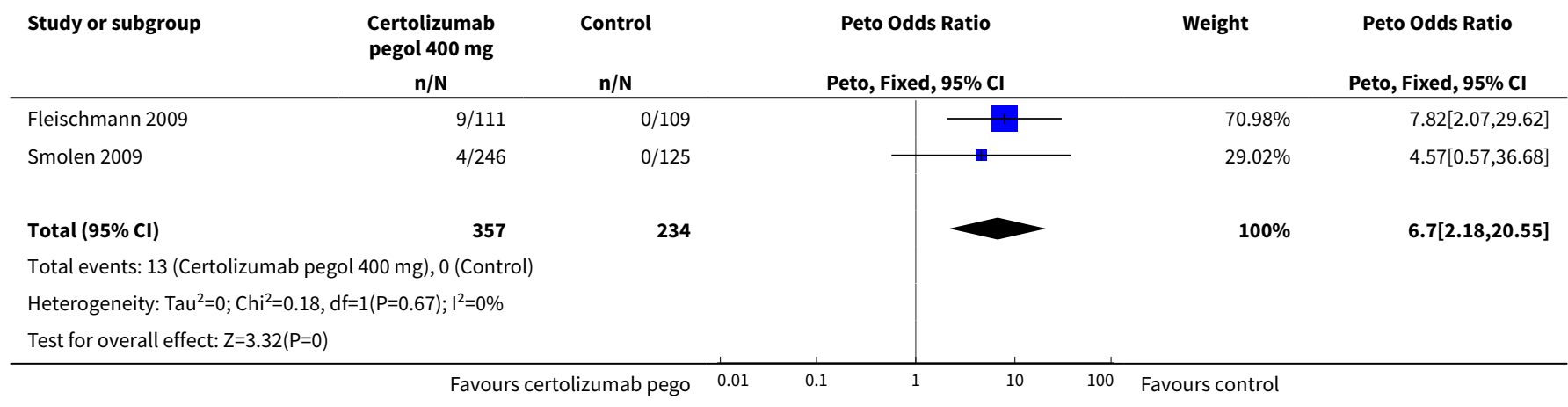

Analysis 50.29. Comparison 50 Safety, Outcome 29 Systemic lupus erythematosus certolizumab 200 mg.

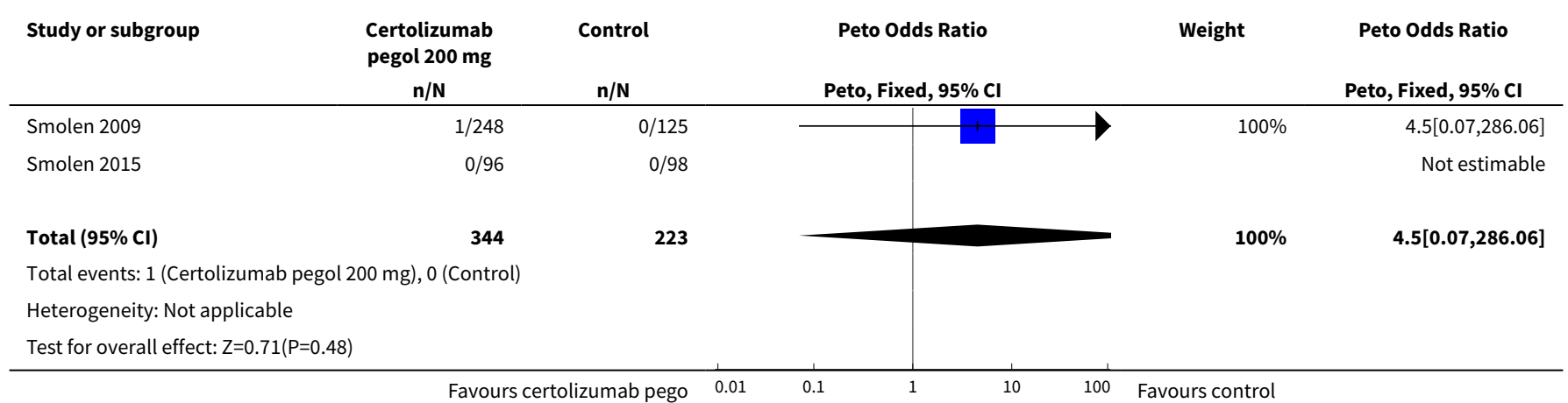

Analysis 50.30. Comparison 50 Safety, Outcome 30 Prolonged activated partial thromboplastin time (aPTT) certolizumab $200 \mathrm{mg}$.

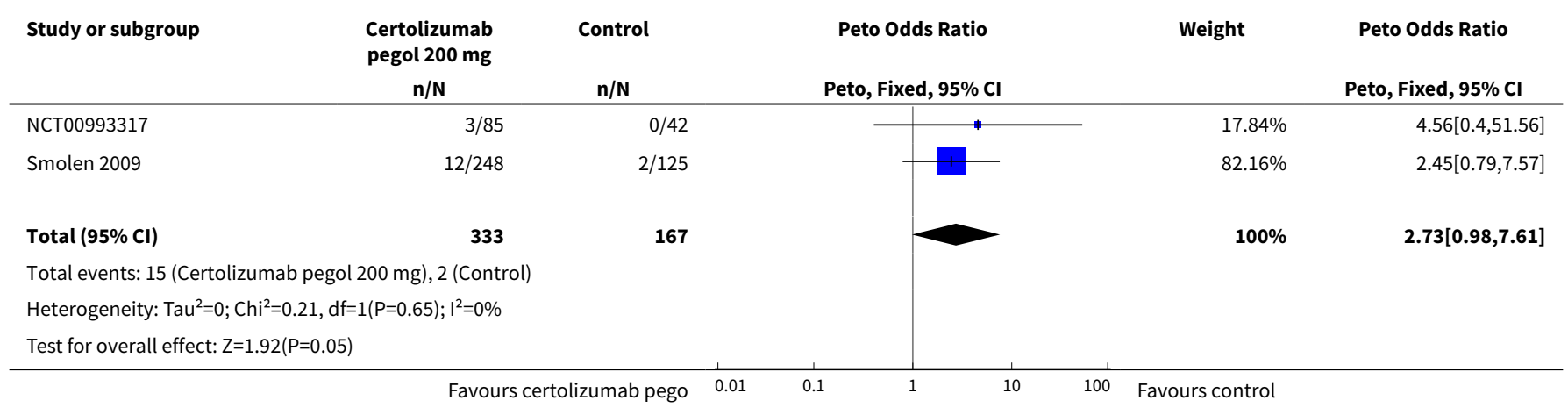


Analysis 50.31. Comparison 50 Safety, Outcome 31 Prolonged activated partial thromboplastin time (aPTT) certolizumab $400 \mathrm{mg}$.

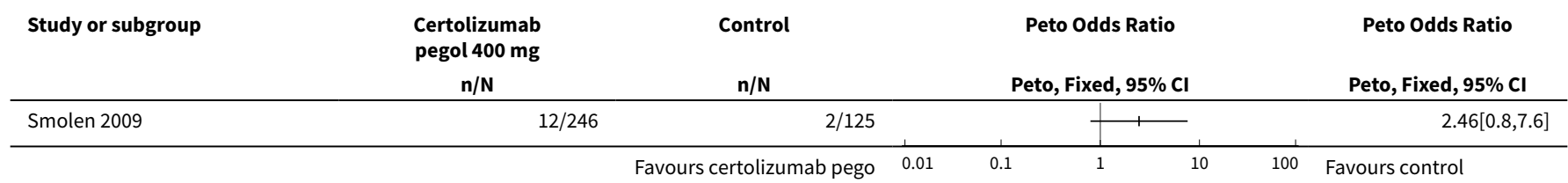

Analysis 50.32. Comparison 50 Safety, Outcome 32 Urinary tract infection certolizumab $200 \mathrm{mg}$.

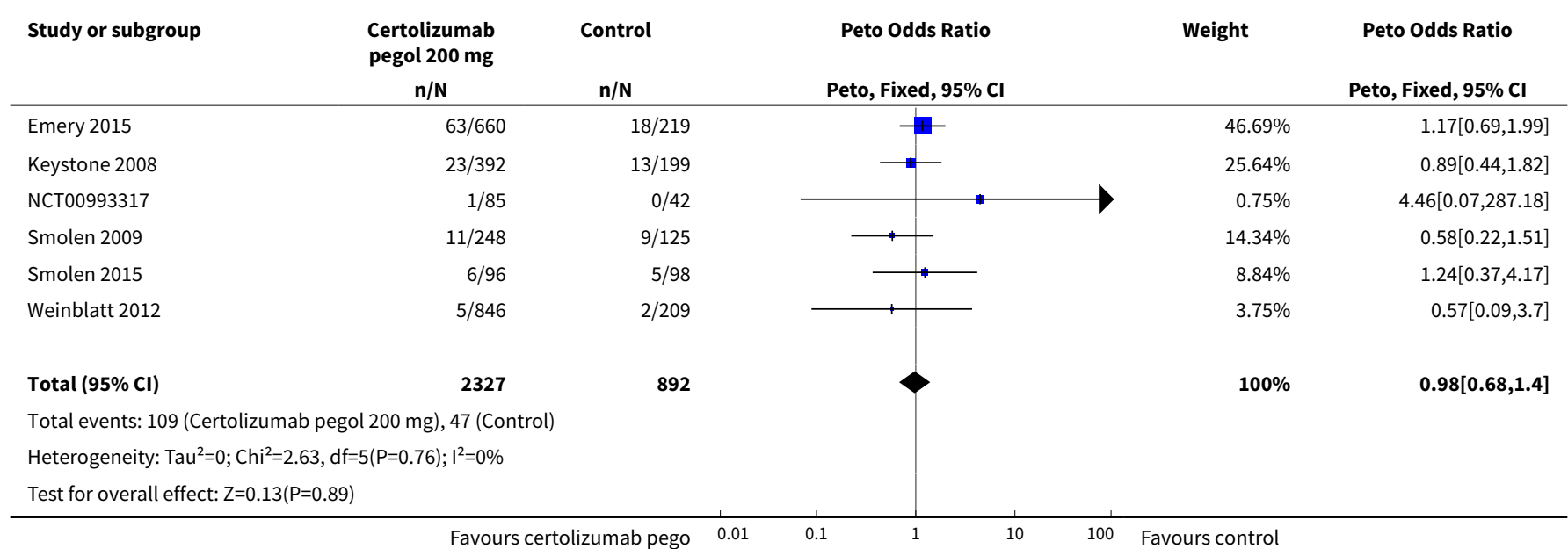

Analysis 50.33. Comparison 50 Safety, Outcome 33 Urinary tract infection certolizumab $400 \mathrm{mg}$.

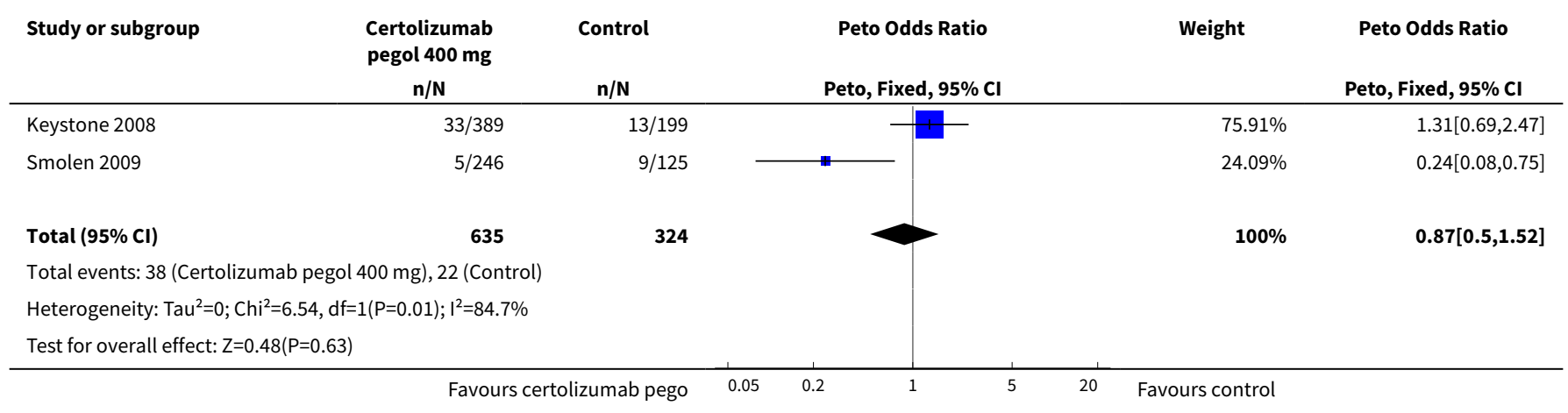

Analysis 50.34. Comparison 50 Safety, Outcome 34 Upper respiratory tract infection certolizumab $200 \mathrm{mg}$.

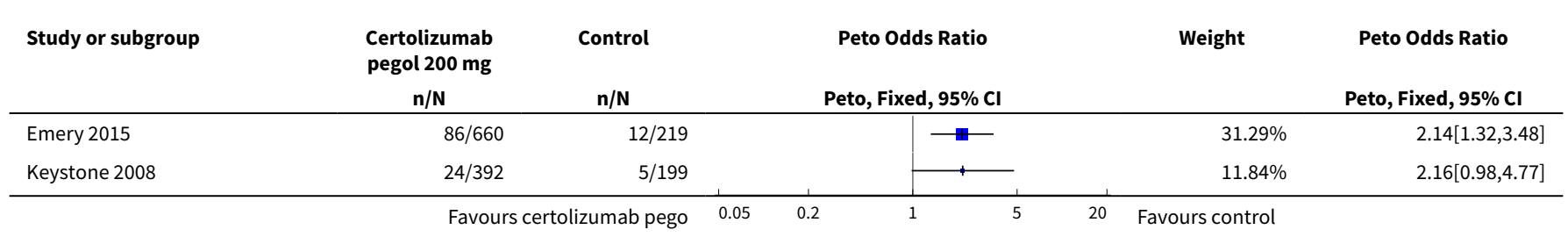




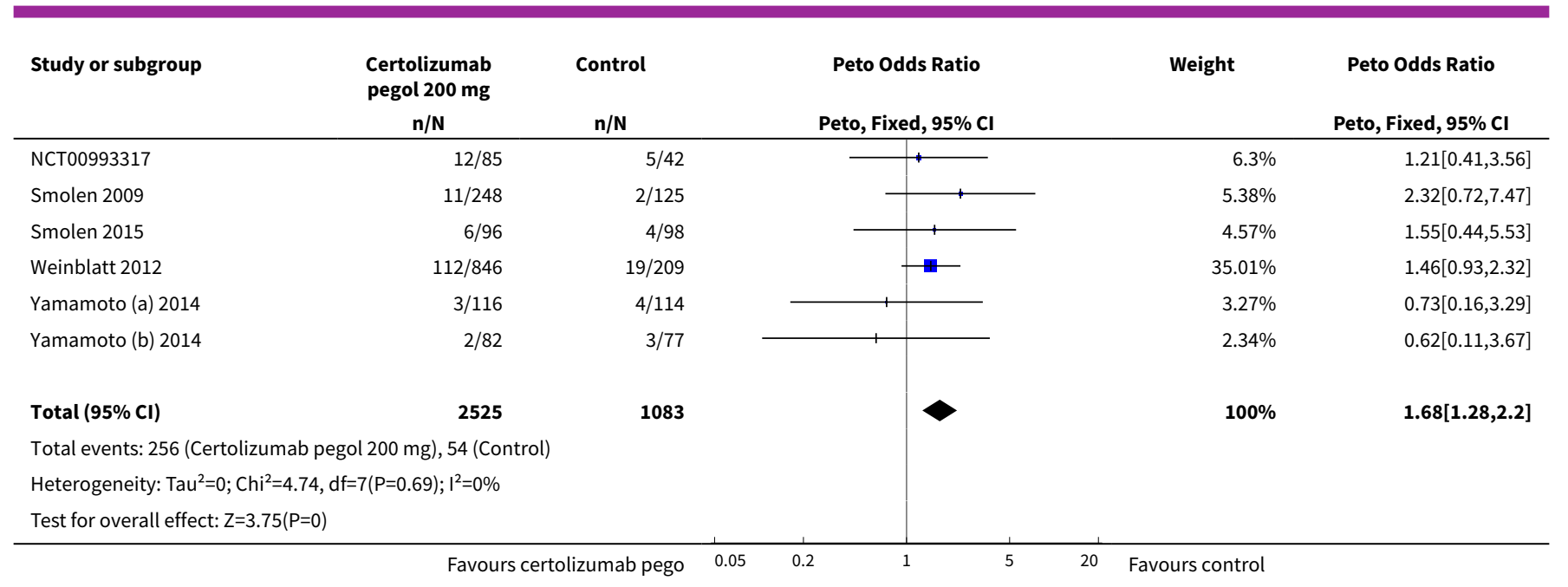

\section{Analysis 50.35. Comparison 50 Safety, Outcome 35 Upper respiratory tract infection certolizumab $400 \mathrm{mg}$.}

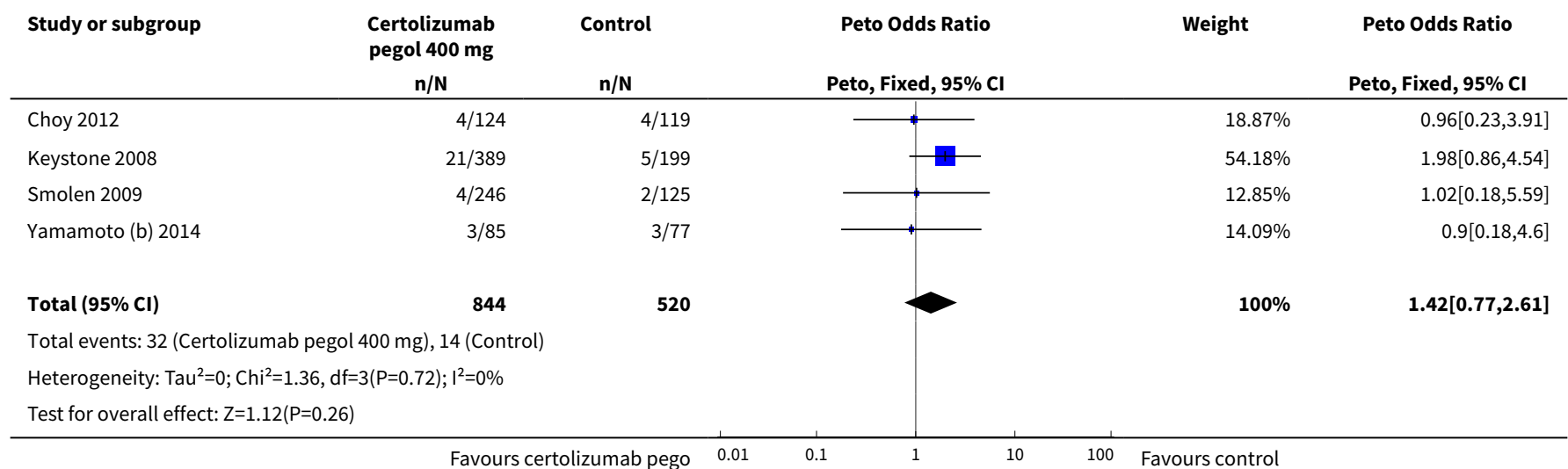

Analysis 50.36. Comparison 50 Safety, Outcome 36 Lower respiratory tract infection/ lung infection certolizumab $200 \mathrm{mg}$.

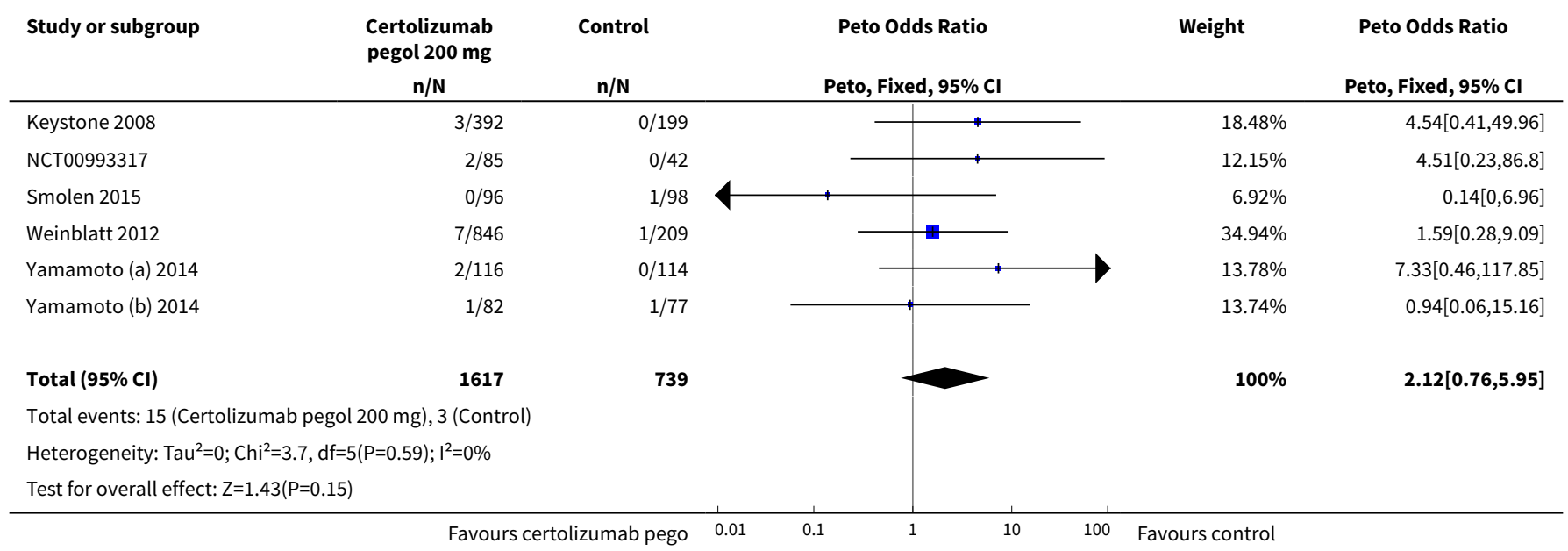


Analysis 50.37. Comparison 50 Safety, Outcome 37 Lower respiratory tract infection/ lung infection certolizumab $400 \mathrm{mg}$.

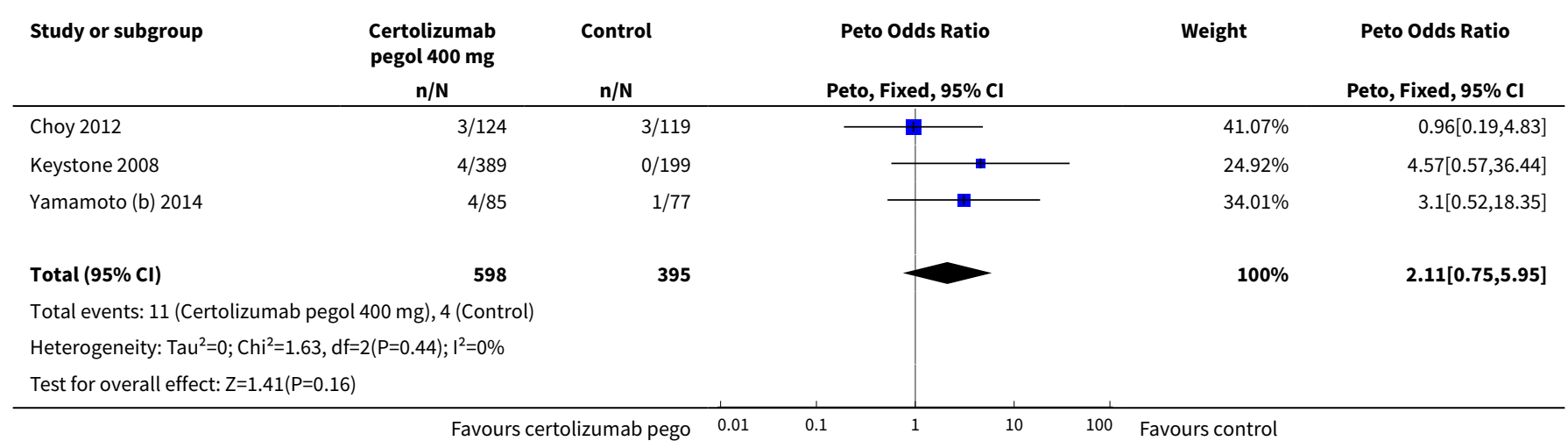

Analysis 50.38. Comparison 50 Safety, Outcome 38 Pneumonia certolizumab $200 \mathrm{mg}$.

\begin{tabular}{|c|c|c|c|c|c|}
\hline \multirow[t]{2}{*}{ Study or subgroup } & $\begin{array}{l}\text { Certolizumab } \\
200 \mathrm{mg} \text { pegol }\end{array}$ & Control & \multirow[b]{2}{*}{ Peto, Fixed, $95 \% \mathrm{Cl}$} & Weight & \multirow{2}{*}{$\begin{array}{c}\text { Peto Odds Ratio } \\
\text { Peto, Fixed, } 95 \% \text { CI }\end{array}$} \\
\hline & $\mathrm{n} / \mathrm{N}$ & $\mathrm{n} / \mathrm{N}$ & & & \\
\hline Smolen 2015 & $0 / 96$ & $1 / 98$ & & $3.51 \%$ & $0.14[0,6.96]$ \\
\hline Emery 2015 & $4 / 660$ & $3 / 219$ & 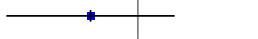 & $18.27 \%$ & $0.38[0.07,2.12]$ \\
\hline Atsumi 2016 & $7 / 161$ & $8 / 158$ & & $50.37 \%$ & $0.85[0.3,2.4]$ \\
\hline Weinblatt 2012 & $7 / 846$ & $1 / 209$ & $\rightarrow$ & $17.74 \%$ & $1.59[0.28,9.09]$ \\
\hline Yamamoto (a) 2014 & $2 / 116$ & $0 / 114$ & & $6.99 \%$ & $7.33[0.46,117.85]$ \\
\hline Total $(95 \% \mathrm{Cl})$ & 1964 & 840 & & $100 \%$ & $0.94[0.45,1.97]$ \\
\hline \multicolumn{6}{|c|}{ Total events: 21 (Certolizumab 200 mg pegol), 13 (Control) } \\
\hline \multicolumn{6}{|c|}{ Heterogeneity: $\mathrm{Tau}^{2}=0 ; \mathrm{Chi}^{2}=5, \mathrm{df}=5(\mathrm{P}=0.42) ; \mathrm{I}^{2}=0 \%$} \\
\hline Test for overall effect & & & & & \\
\hline
\end{tabular}

Analysis 50.39. Comparison 50 Safety, Outcome 39 Pneumonitis certolizumab 400 mg.

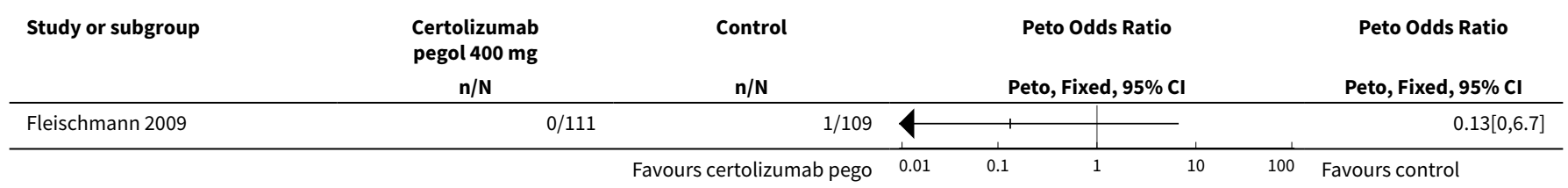


Analysis 50.40. Comparison 50 Safety, Outcome 40 Headache certolizumab 200 mg.

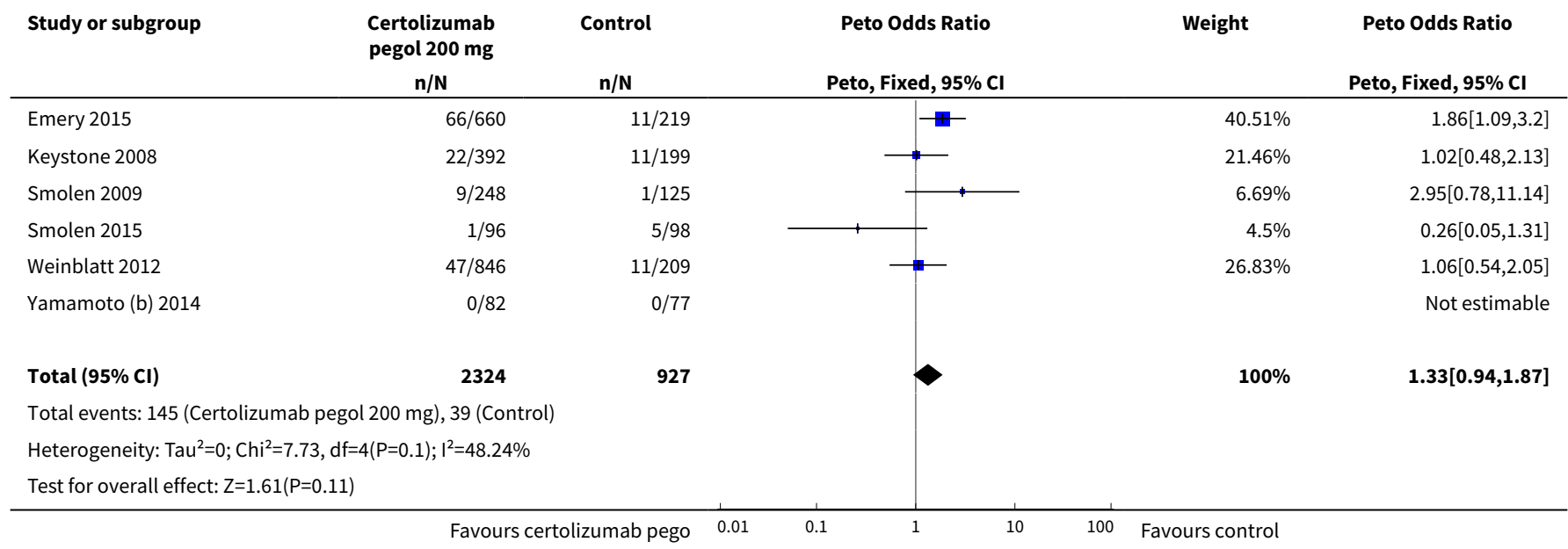

Analysis 50.41. Comparison 50 Safety, Outcome 41 Headache certolizumab $400 \mathrm{mg}$.

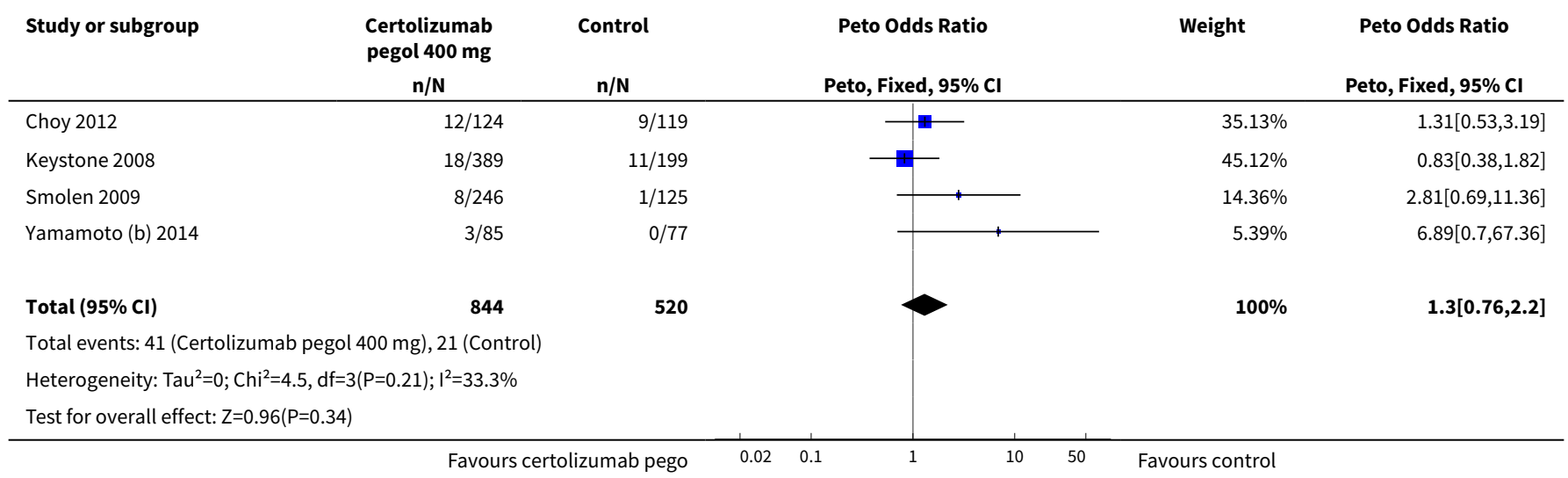

Analysis 50.42. Comparison 50 Safety, Outcome 42 Bacteriuria certolizumab $200 \mathrm{mg}$.

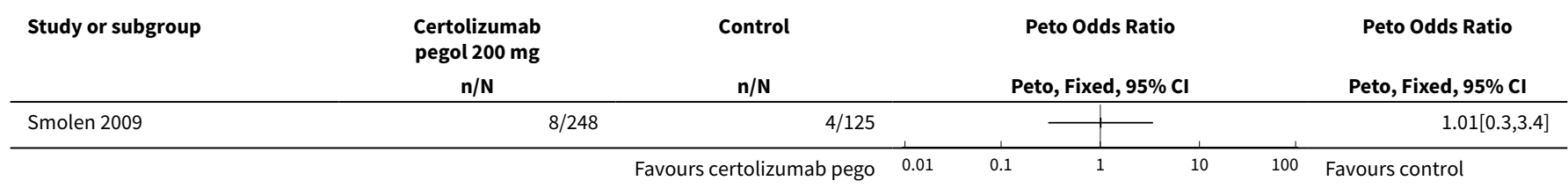

Analysis 50.43. Comparison 50 Safety, Outcome 43 Bacteriuria certolizumab 400 mg.

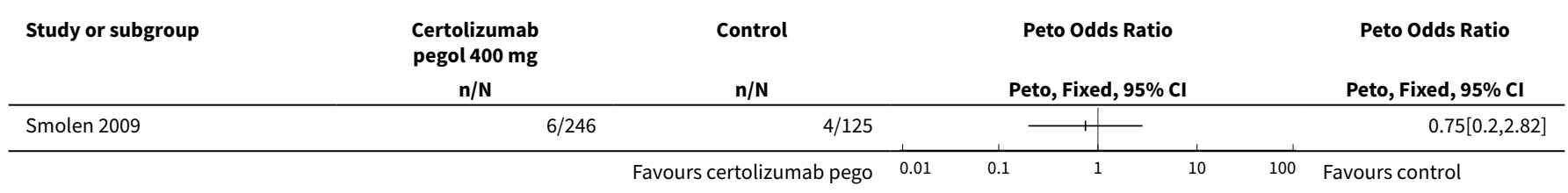


Analysis 50.44. Comparison 50 Safety, Outcome 44 Nasopharyngitis/Pharyngitis certolizumab 200 mg.

\begin{tabular}{|c|c|c|c|c|c|}
\hline Study or subgroup & $\begin{array}{c}\text { Certolizumab } \\
\text { pegol } 200 \mathrm{mg} \\
\mathrm{n} / \mathrm{N}\end{array}$ & $\begin{array}{l}\text { Control } \\
\mathrm{n} / \mathrm{N}\end{array}$ & Peto Odds Ratio & Weight & $\begin{array}{l}\text { Peto Odds Ratio } \\
\text { Peto, Fixed, } 95 \% \mathrm{Cl}\end{array}$ \\
\hline Emery 2015 & $60 / 660$ & $17 / 219$ & - & $30.64 \%$ & $1.18[0.69,2.03]$ \\
\hline Keystone 2008 & $21 / 392$ & $3 / 199$ & $\longrightarrow$ & $12 \%$ & $2.68[1.13,6.36]$ \\
\hline NCT00993317 & $10 / 85$ & $4 / 42$ & 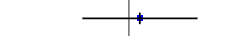 & $6.47 \%$ & $1.25[0.39,4.06]$ \\
\hline Smolen 2009 & $8 / 248$ & $1 / 125$ & & $4.57 \%$ & $2.79[0.69,11.32]$ \\
\hline Smolen 2015 & $10 / 96$ & $11 / 98$ & - & $10.96 \%$ & $0.92[0.37,2.27]$ \\
\hline Yamamoto (a) 2014 & $26 / 116$ & $21 / 114$ & - & $21.86 \%$ & $1.28[0.67,2.42]$ \\
\hline Yamamoto (b) 2014 & $16 / 82$ & $12 / 77$ & $\longrightarrow$ & $13.5 \%$ & $1.31[0.58,2.95]$ \\
\hline Total $(95 \% \mathrm{Cl})$ & 1679 & 874 & 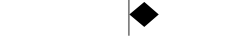 & $100 \%$ & $1.37[1.01,1.84]$ \\
\hline \multicolumn{6}{|c|}{ Total events: 151 (Certolizumab pegol 200 mg), 69 (Control) } \\
\hline \multicolumn{6}{|c|}{ Heterogeneity: $\mathrm{Tau}^{2}=0 ; \mathrm{Chi}^{2}=4.44, \mathrm{df}=6(\mathrm{P}=0.62) ; 1^{2}=0 \%$} \\
\hline \multicolumn{6}{|c|}{ Test for overall effect: $Z=2.04(P=0.04)$} \\
\hline
\end{tabular}

\section{Analysis 50.45. Comparison 50 Safety, Outcome 45 Nasopharyngitis/Pharyngitis certolizumab 400 mg.}

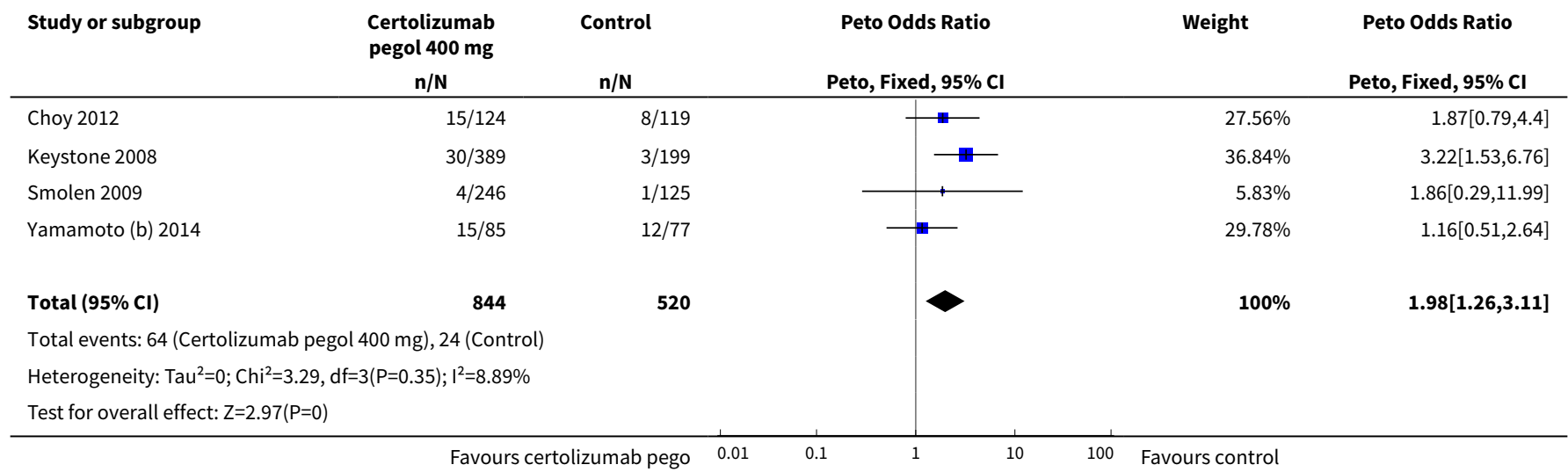

\section{Analysis 50.46. Comparison 50 Safety, Outcome 46 Injection site pain certolizumab 200 mg.}

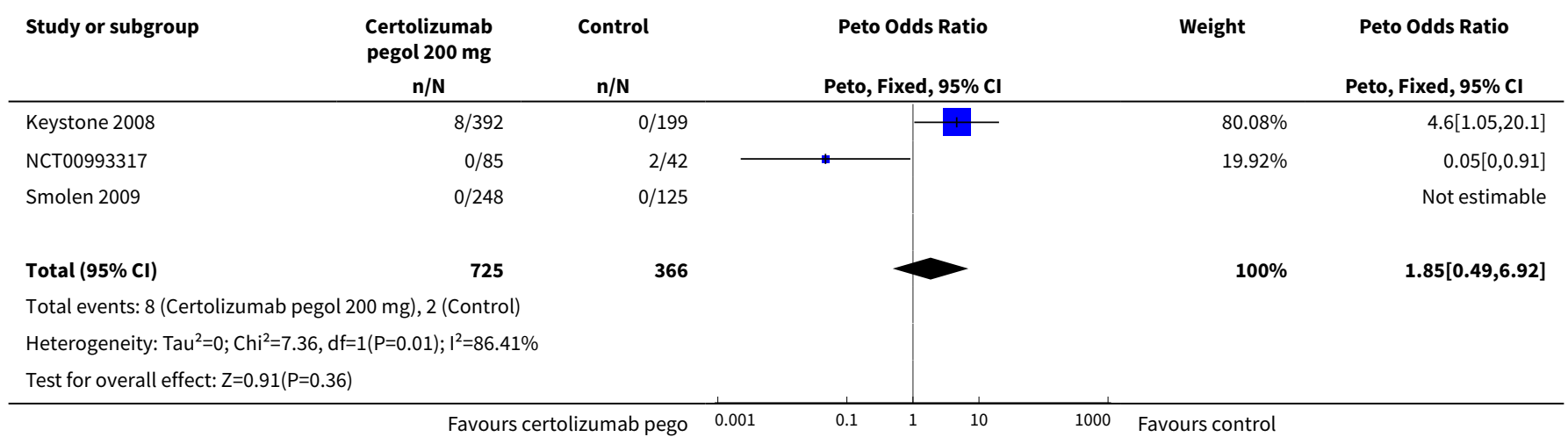


Analysis 50.47. Comparison 50 Safety, Outcome 47 Injection site pain certolizumab $400 \mathrm{mg}$.

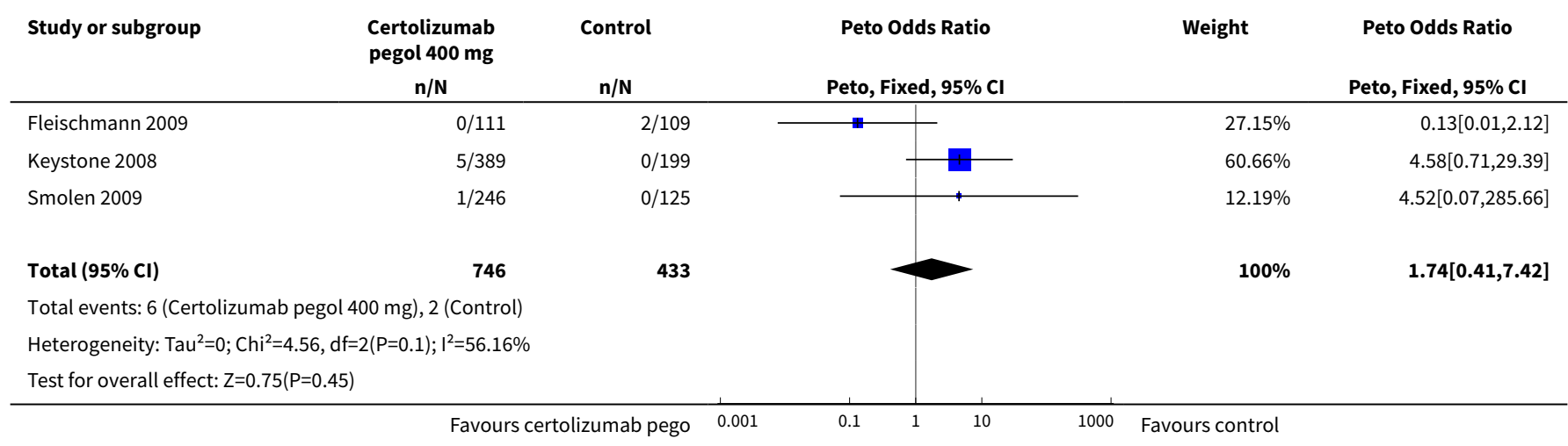

Analysis 50.48. Comparison 50 Safety, Outcome 48 Hypertension certolizumab $200 \mathrm{mg}$.

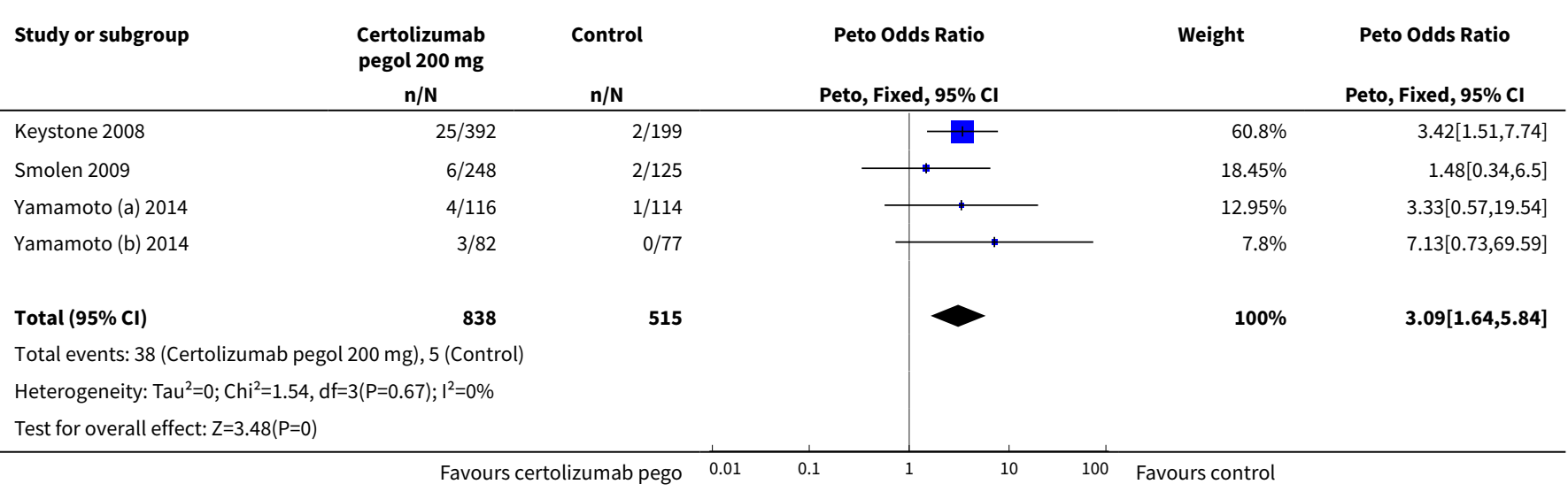

Analysis 50.49. Comparison 50 Safety, Outcome 49 Hypertension certolizumab 400 mg.

\begin{tabular}{|c|c|c|c|c|c|}
\hline \multirow[t]{2}{*}{ Study or subgroup } & \multirow[t]{2}{*}{$\begin{array}{c}\text { Certolizumab } \\
\text { pegol } 400 \mathrm{mg} \\
\mathrm{n} / \mathrm{N}\end{array}$} & \multirow[t]{2}{*}{$\begin{array}{l}\text { Control } \\
n / N\end{array}$} & \multirow[t]{2}{*}{$\begin{array}{c}\text { Peto Odds Ratio } \\
\text { Peto, Fixed, } 95 \% \mathrm{Cl} \\
\end{array}$} & \multirow[t]{2}{*}{ Weight } & \multirow[t]{2}{*}{$\begin{array}{c}\text { Peto Odds Ratio } \\
\text { Peto, Fixed, } 95 \% \mathrm{Cl}\end{array}$} \\
\hline & & & & & \\
\hline Keystone 2008 & $32 / 389$ & $2 / 199$ & & $71.34 \%$ & $3.76[1.81,7.8]$ \\
\hline Smolen 2009 & $9 / 246$ & $2 / 125$ & $\longrightarrow$ & $23.74 \%$ & $2.04[0.57,7.25]$ \\
\hline Yamamoto (b) 2014 & $2 / 85$ & $0 / 77$ & & $4.92 \%$ & $6.81[0.42,110.13]$ \\
\hline \multicolumn{6}{|c|}{ Total events: 43 (Certolizumab pegol $400 \mathrm{mg}$ ), 4 (Control) } \\
\hline \multicolumn{6}{|c|}{ Heterogeneity: $\mathrm{Tau}^{2}=0 ; \mathrm{Chi}^{2}=0.93, \mathrm{df}=2(\mathrm{P}=0.63) ; \mathrm{I}^{2}=0 \%$} \\
\hline Test for overall effect & & & & & \\
\hline
\end{tabular}


Analysis 50.50. Comparison 50 Safety, Outcome 50 Hematuria certolizumab 200 mg.

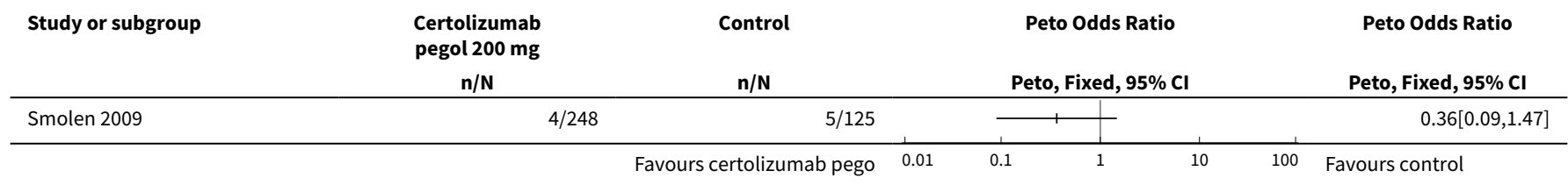

Analysis 50.51. Comparison 50 Safety, Outcome 51 Haematuria certolizumab $400 \mathrm{mg}$.

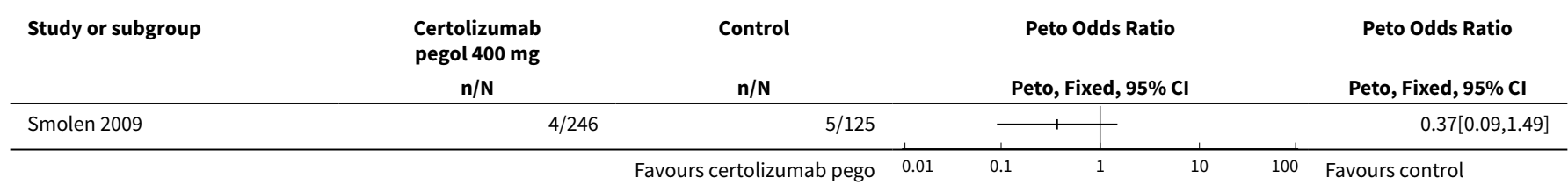

Analysis 50.52. Comparison 50 Safety, Outcome 52 Hepatic enzyme increased certolizumab 200 mg.

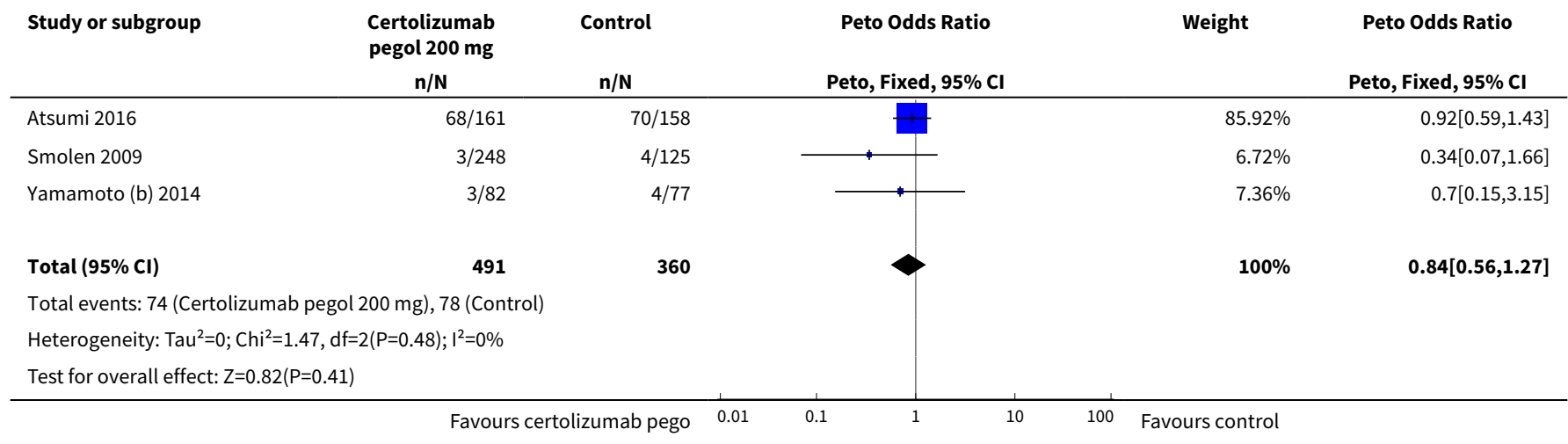

Analysis 50.53. Comparison 50 Safety, Outcome 53 Hepatic enzyme increased certolizumab 400 mg.

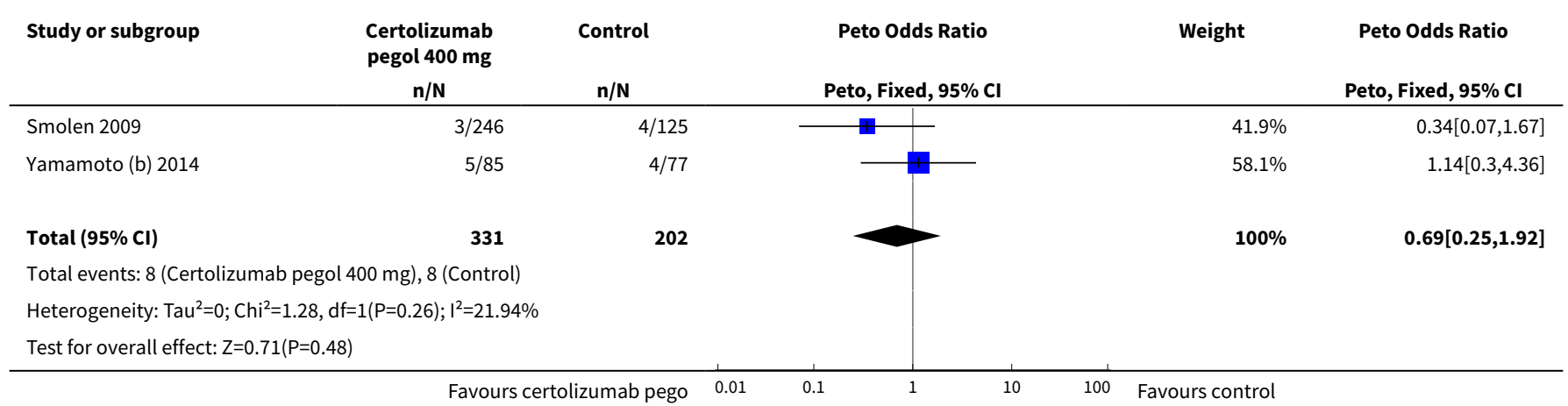


Analysis 50.54. Comparison 50 Safety, Outcome 54 AST increased certolizumab 200 mg.

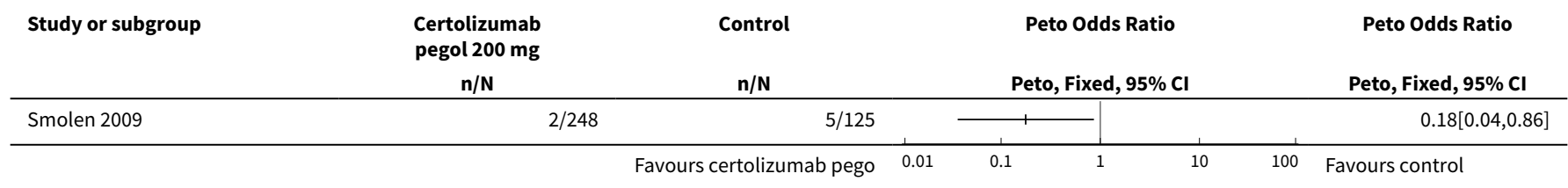

Analysis 50.55. Comparison 50 Safety, Outcome 55 AST increased certolizumab 400 mg.

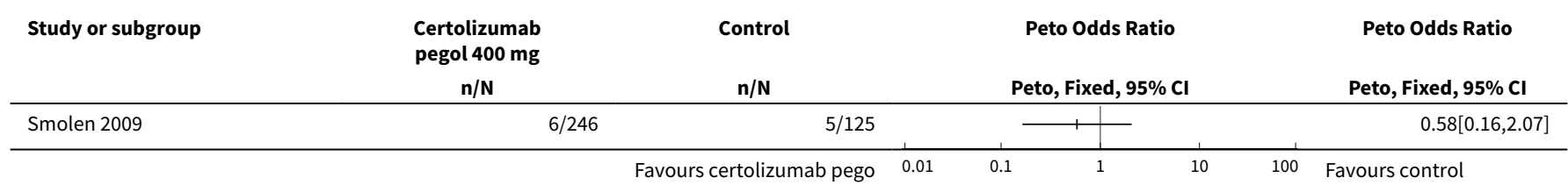

Analysis 50.56. Comparison 50 Safety, Outcome 56 ALT increased certolizumab 200 mg.

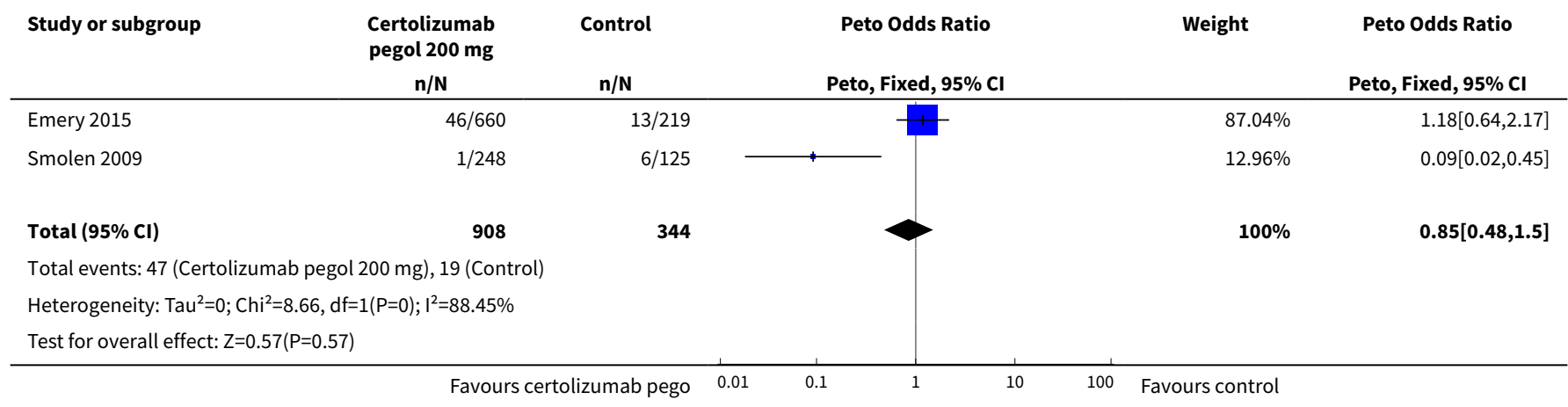

Analysis 50.57. Comparison 50 Safety, Outcome 57 ALT increased certolizumab 400 mg.

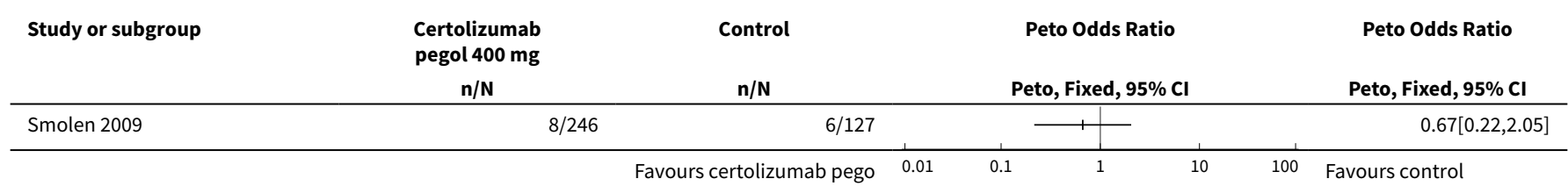

Analysis 50.58. Comparison 50 Safety, Outcome 58 Diarrhoea certolizumab $200 \mathrm{mg}$.

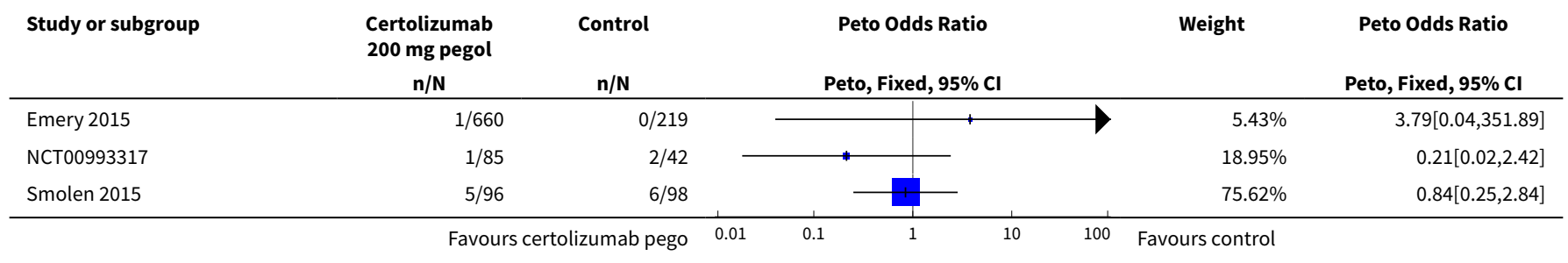




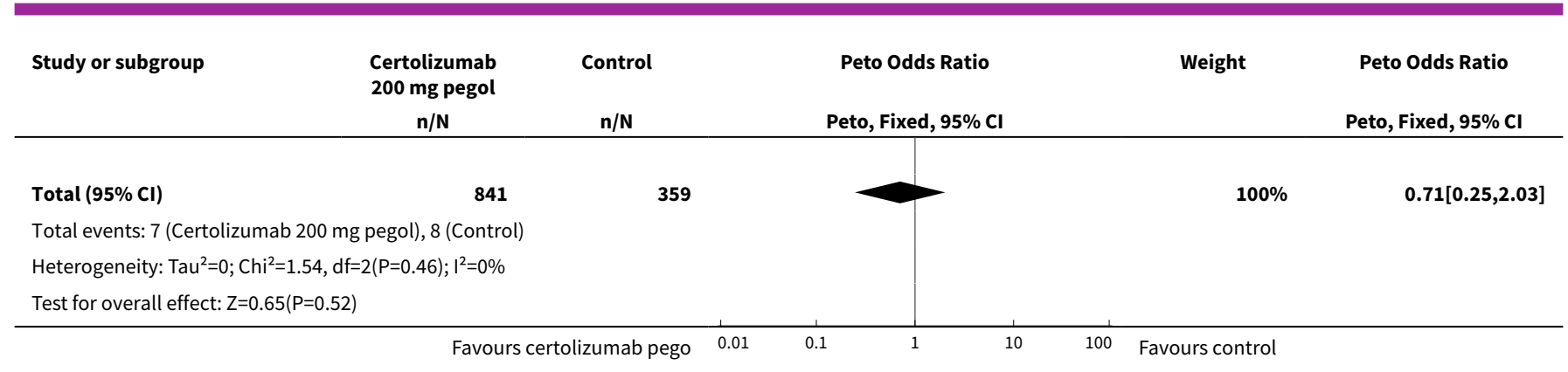

Analysis 50.59. Comparison 50 Safety, Outcome 59 Gastroenteritis certolizumab 200 mg.

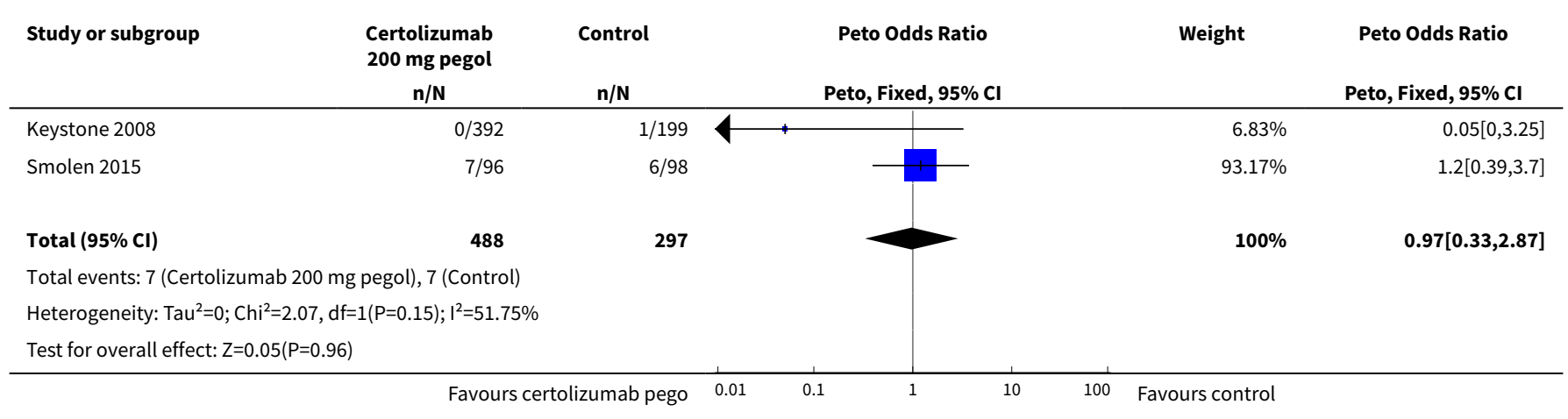

Analysis 50.60. Comparison 50 Safety, Outcome 60 Gastrointestinal disorders certolizumab $400 \mathrm{mg}$.

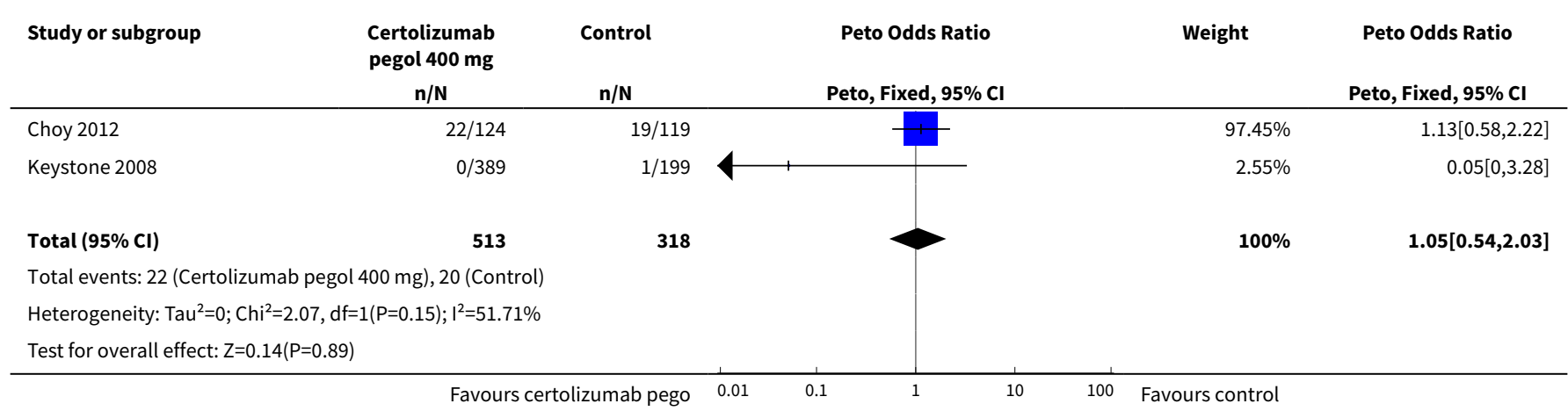

Analysis 50.61. Comparison 50 Safety, Outcome 61 Back pain certolizumab $200 \mathrm{mg}$.

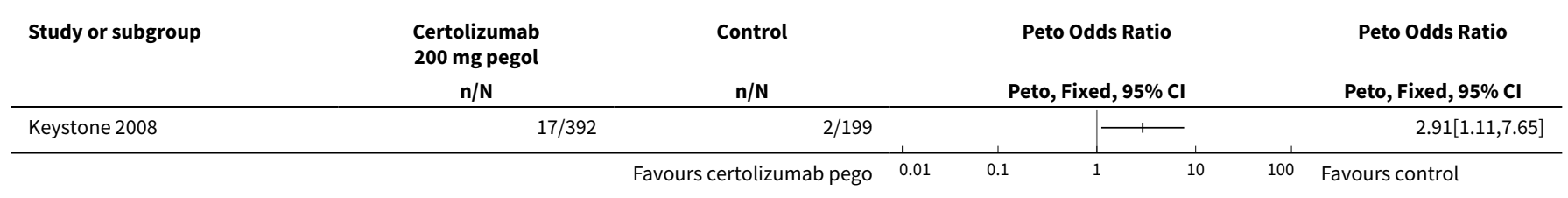


Analysis 50.62. Comparison 50 Safety, Outcome 62 Back pain certolizumab $400 \mathrm{mg}$.

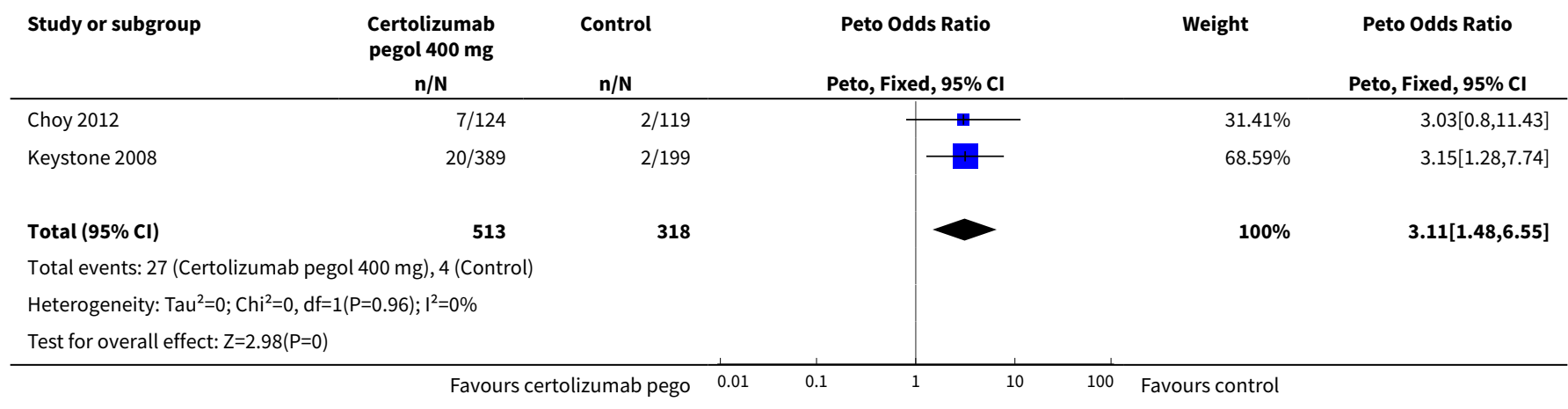

Analysis 50.63. Comparison 50 Safety, Outcome 63 Hematologic abnormalities certolizumab 200 mg.

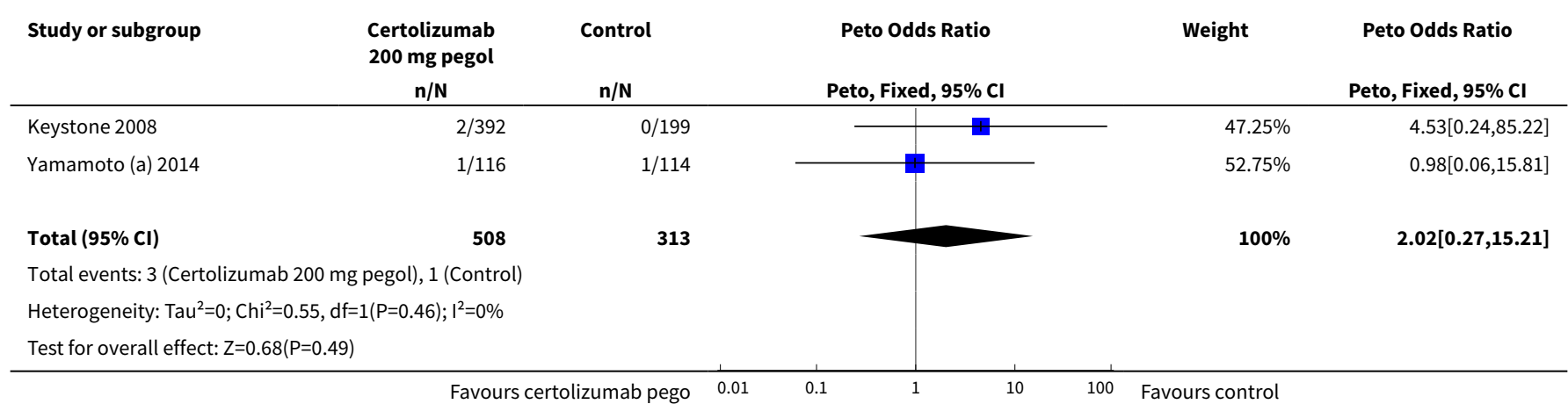

Analysis 50.64. Comparison 50 Safety, Outcome 64 Haematologic abnormalities certolizumab 400 mg.

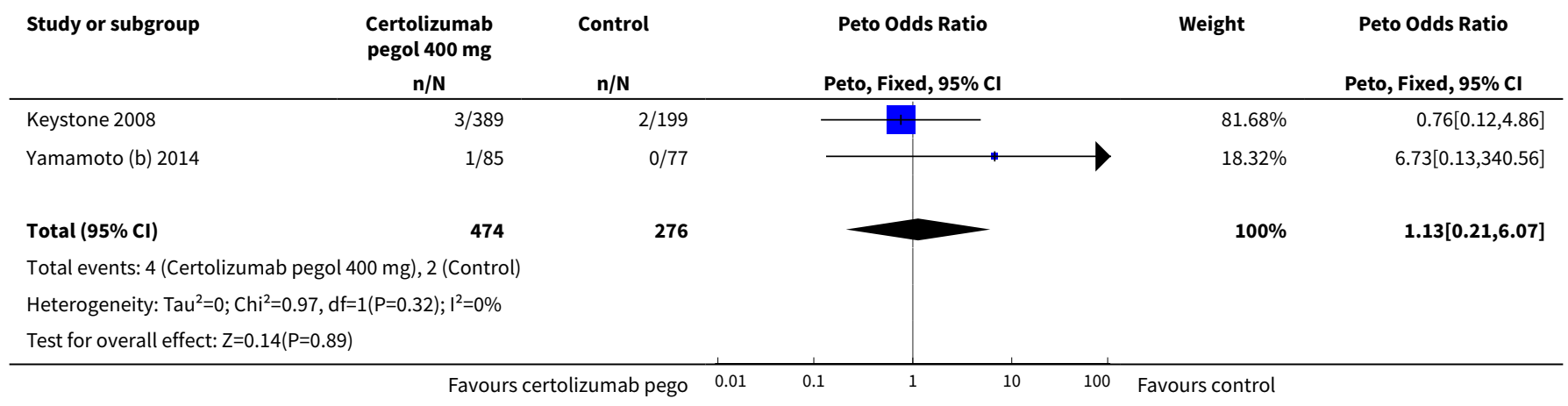

Analysis 50.65. Comparison 50 Safety, Outcome 65 Herpes viral infection certolizumab $200 \mathrm{mg}$.

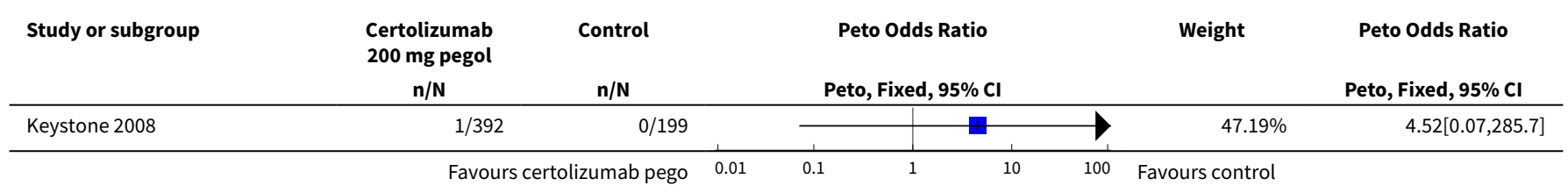




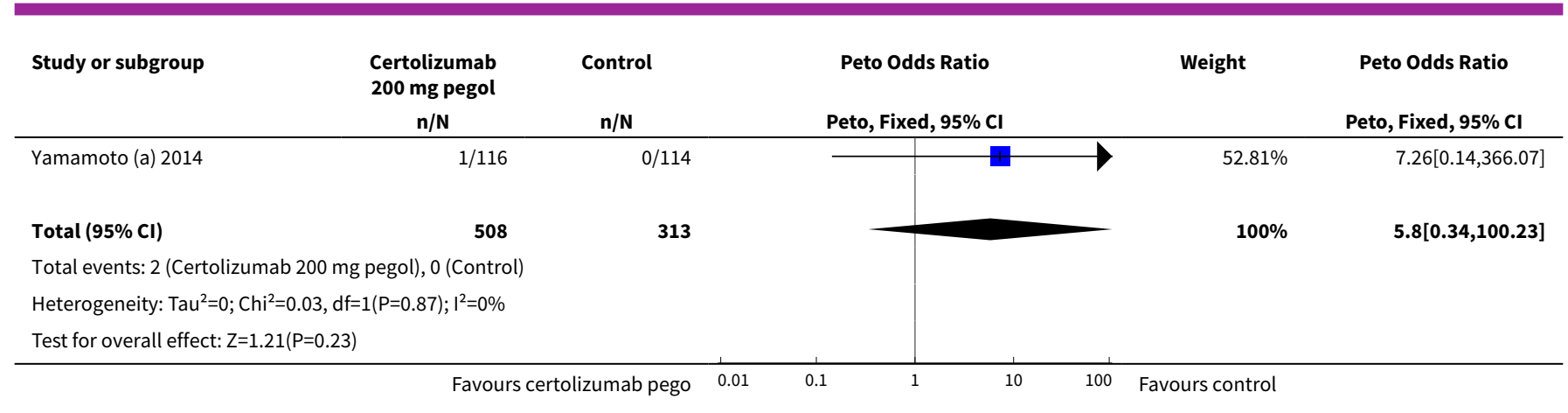

Analysis 50.66. Comparison 50 Safety, Outcome 66 Herpes viral infection certolizumab $400 \mathrm{mg}$.

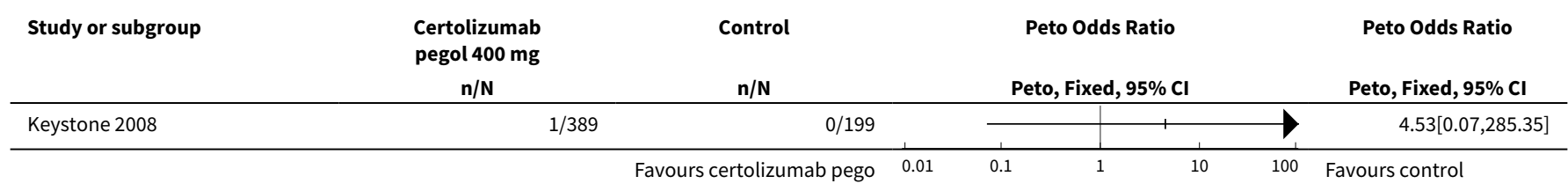

Analysis 50.67. Comparison 50 Safety, Outcome 67 Bacterial peritonitis certolizumab 200 mg.

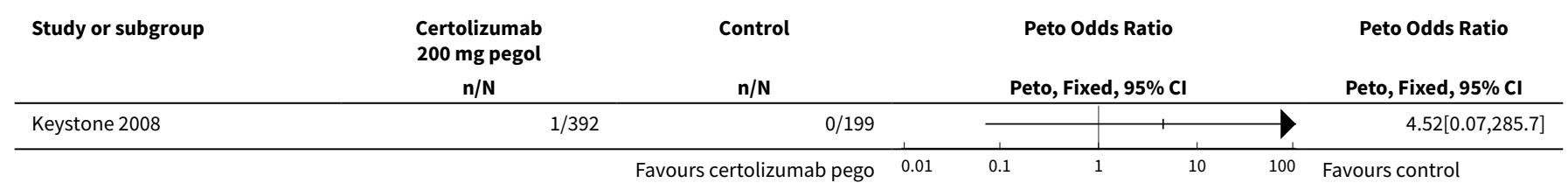

Analysis 50.68. Comparison 50 Safety, Outcome 68 Bacterial peritonitis certolizumab 400 mg.

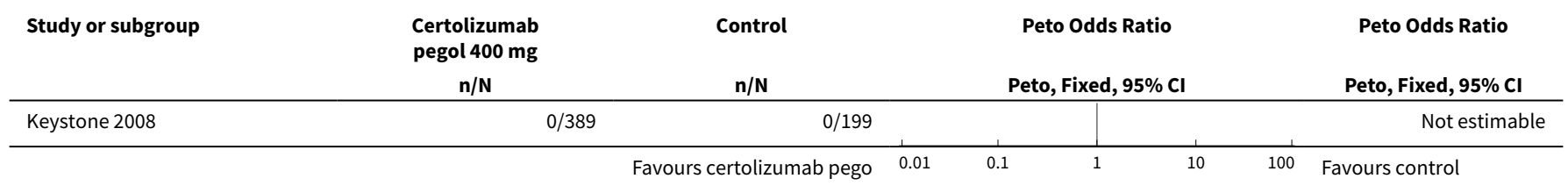

Analysis 50.69. Comparison 50 Safety, Outcome 69 Opportunistic infections certolizumab 200 mg.

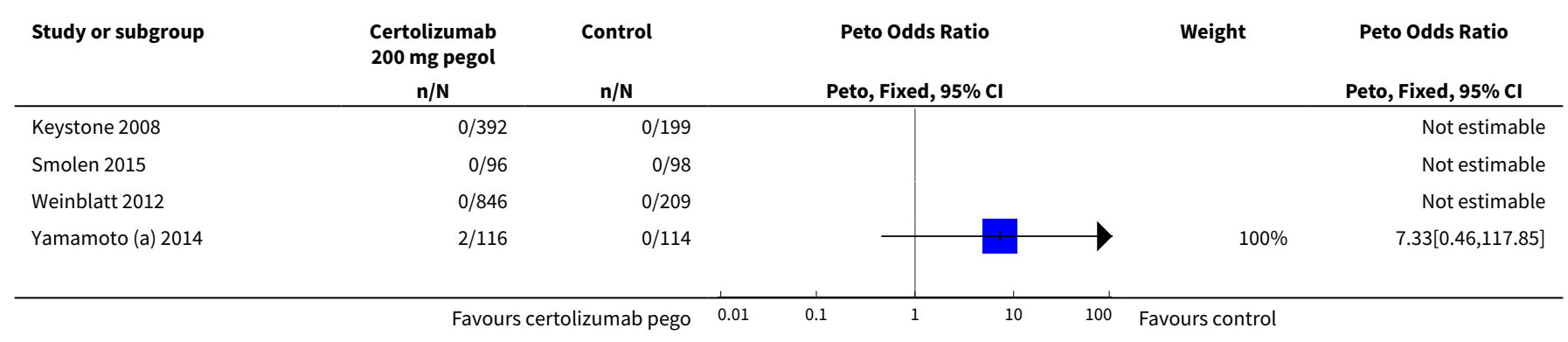




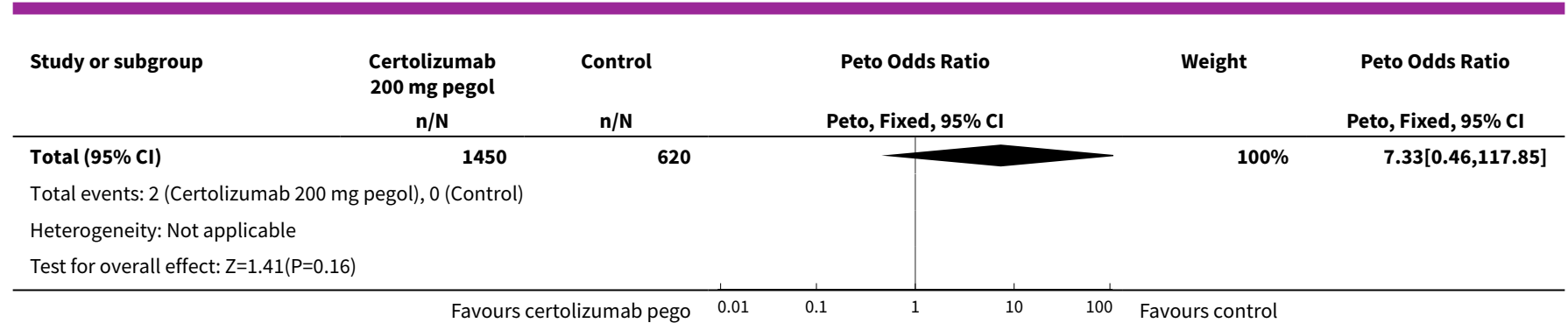

Analysis 50.70. Comparison 50 Safety, Outcome 70 Opportunistic infections certolizumab $400 \mathrm{mg}$.

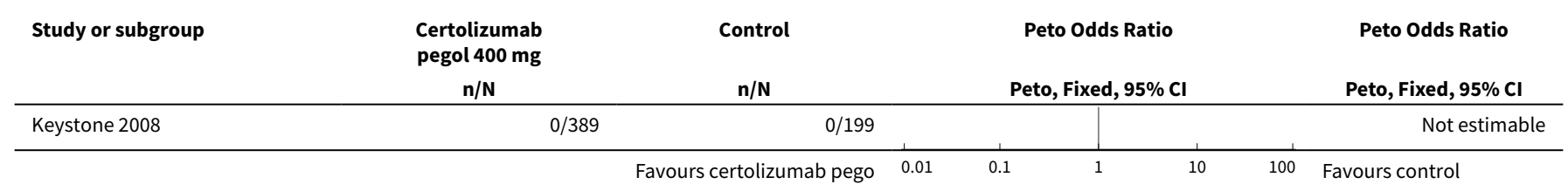

Analysis 50.71. Comparison 50 Safety, Outcome 71 Infections and infestations certolizumab $200 \mathrm{mg}$.

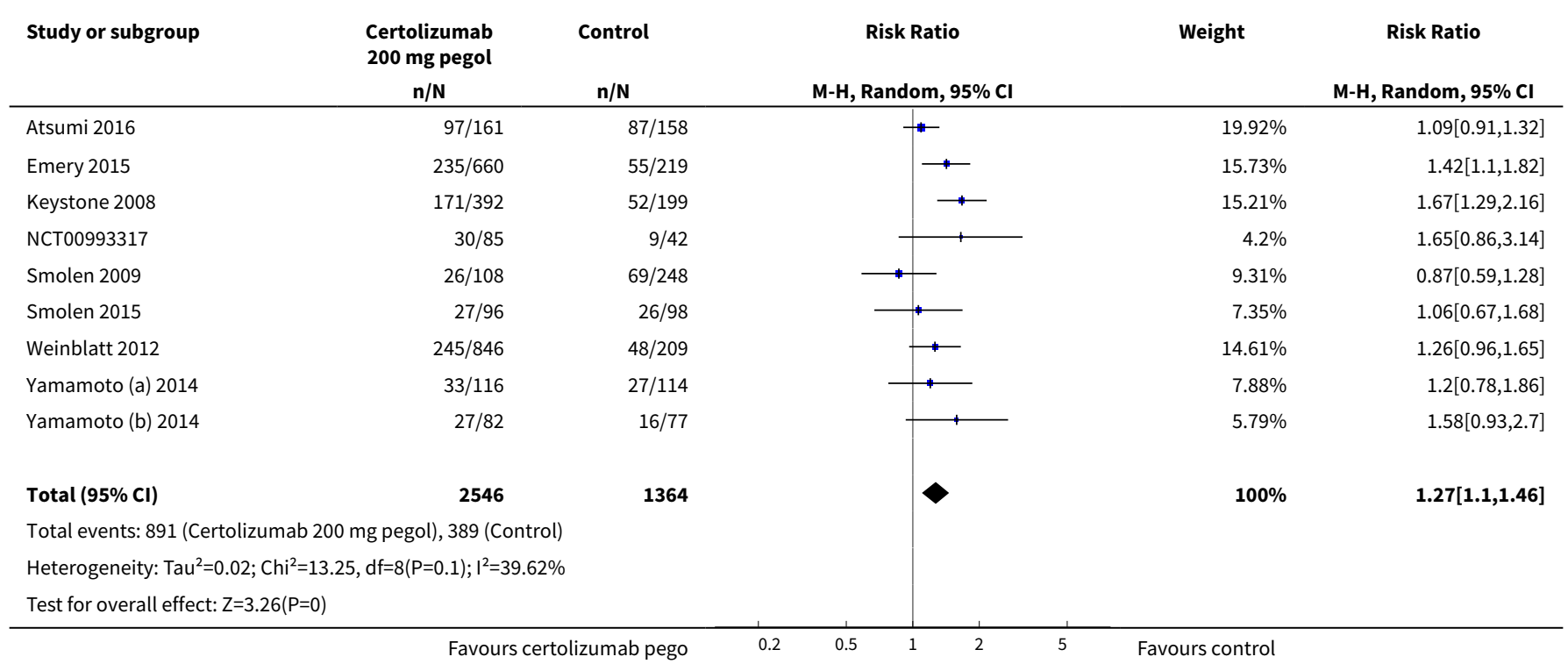

\section{Analysis 50.72. Comparison 50 Safety, Outcome 72 Infections and infestations certolizumab $400 \mathrm{mg}$.}

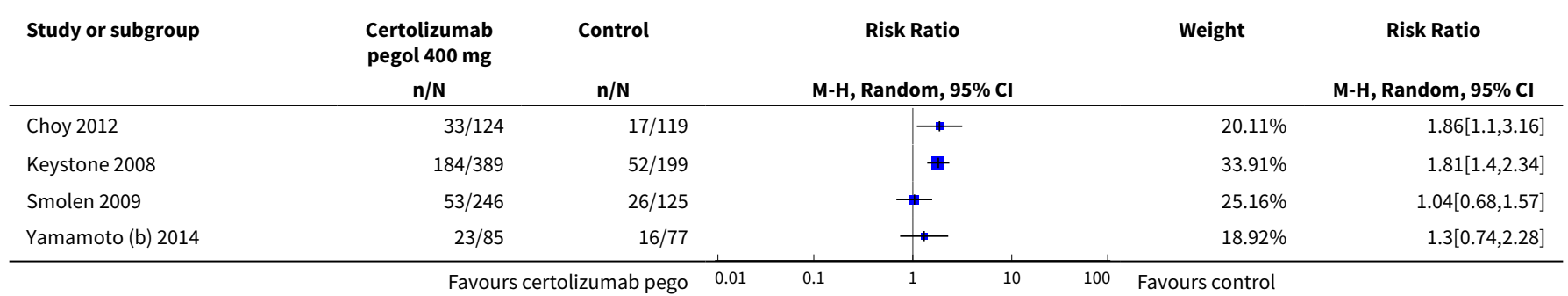




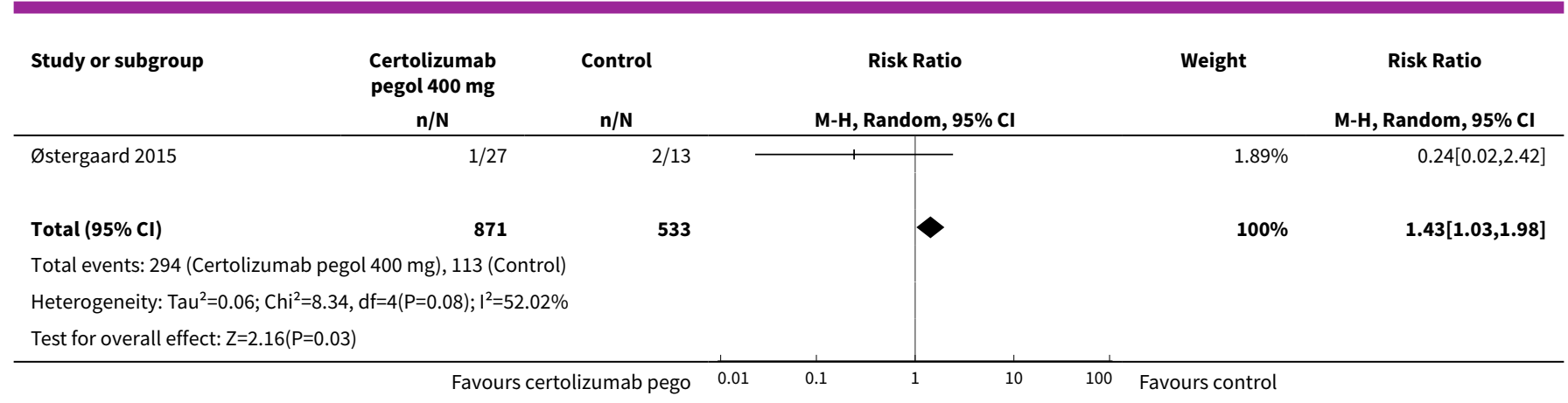

Analysis 50.73. Comparison 50 Safety, Outcome 73 Decreased haemoglobin certolizumab $200 \mathrm{mg}$.

\begin{tabular}{|c|c|c|c|c|c|c|c|}
\hline \multirow[t]{2}{*}{ Study or subgroup } & Certolizumab & Control & & \multicolumn{2}{|c|}{ Peto Odds Ratio } & & \multirow[t]{2}{*}{ Peto Odds Ratio } \\
\hline & $\mathrm{n} / \mathrm{N}$ & $\mathrm{n} / \mathrm{N}$ & & \multicolumn{2}{|c|}{ Peto, Fixed, $95 \% \mathrm{Cl}$} & & \\
\hline \multirow[t]{2}{*}{ Keystone 2008} & $2 / 392$ & $1 / 199$ & & & - & & $1.02[0.09,11.18]$ \\
\hline & & Favours control & 0.01 & 0.1 & 10 & 100 & $\begin{array}{l}\text { Favours certolizumab } \\
\text { pego }\end{array}$ \\
\hline
\end{tabular}

Analysis 50.74. Comparison 50 Safety, Outcome 74 Decreased haemoglobin certolizumab $400 \mathrm{mg}$.

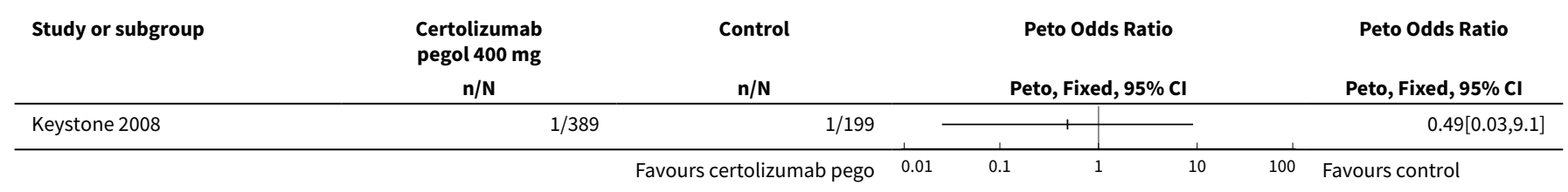

Analysis 50.75. Comparison 50 Safety, Outcome 75 Increased platelet count certolizumab 200 mg.

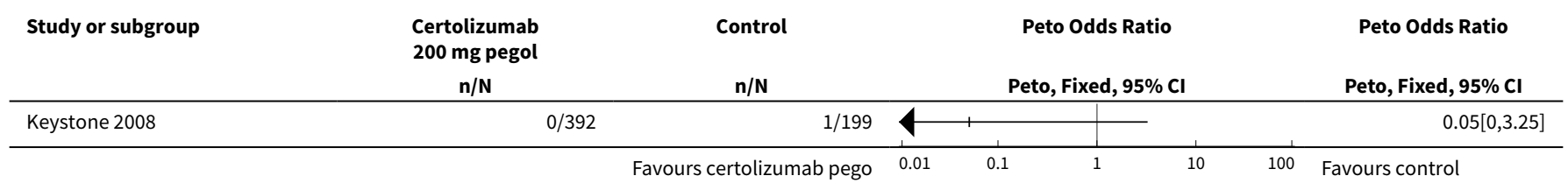

Analysis 50.76. Comparison 50 Safety, Outcome 76 Increased platelet count certolizumab 400 mg.

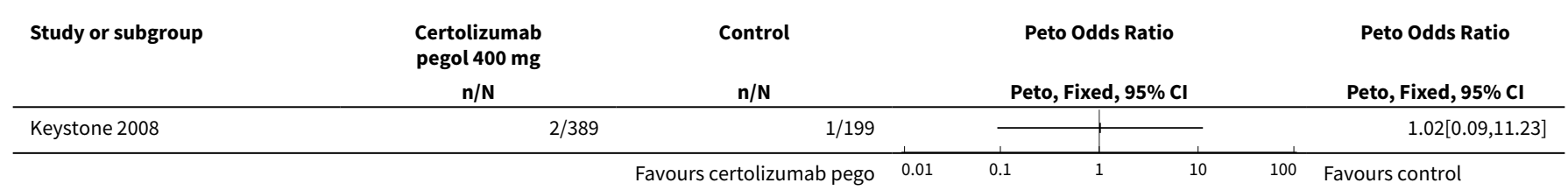


Analysis 50.77. Comparison 50 Safety, Outcome 77 Cerebral haemorrhage including subarachnoid certolizumab $200 \mathrm{mg}$.

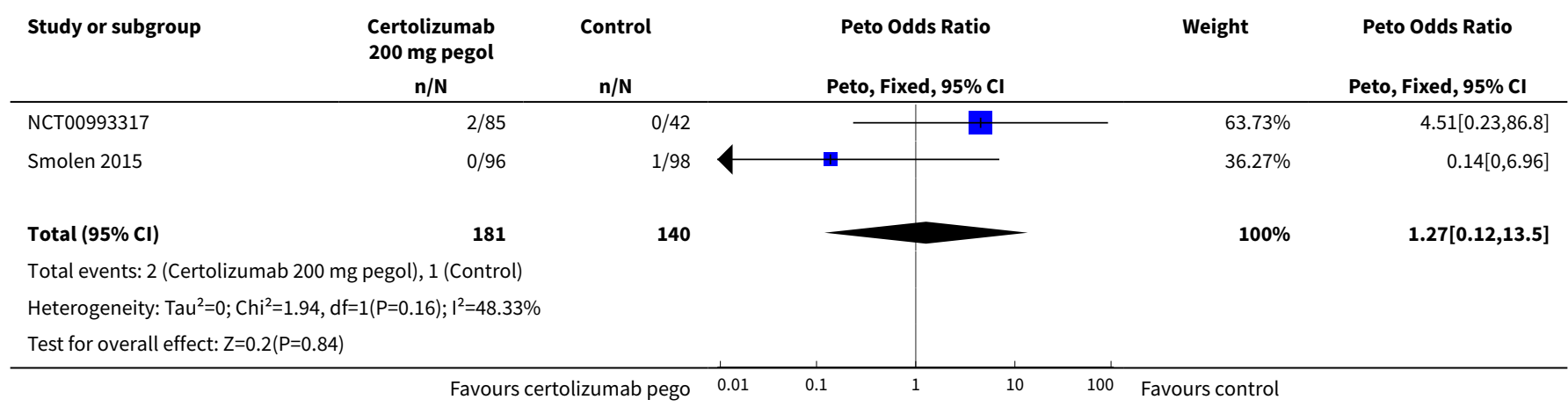

Analysis 50.78. Comparison 50 Safety, Outcome 78 Ischaemic stroke certolizumab 400 mg.

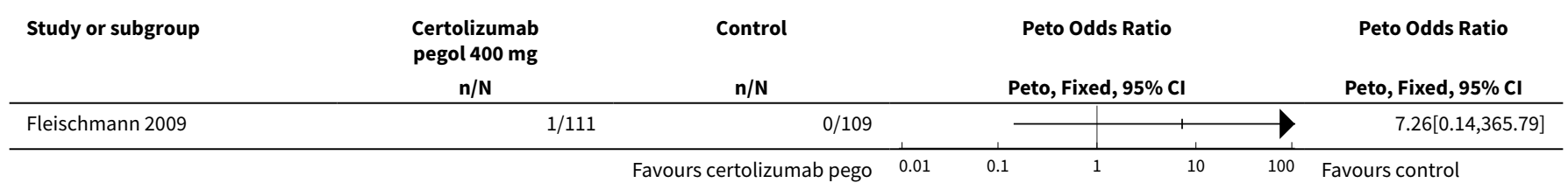

Analysis 50.79. Comparison 50 Safety, Outcome 79 Nausea/vomiting certolizumab 200 mg.

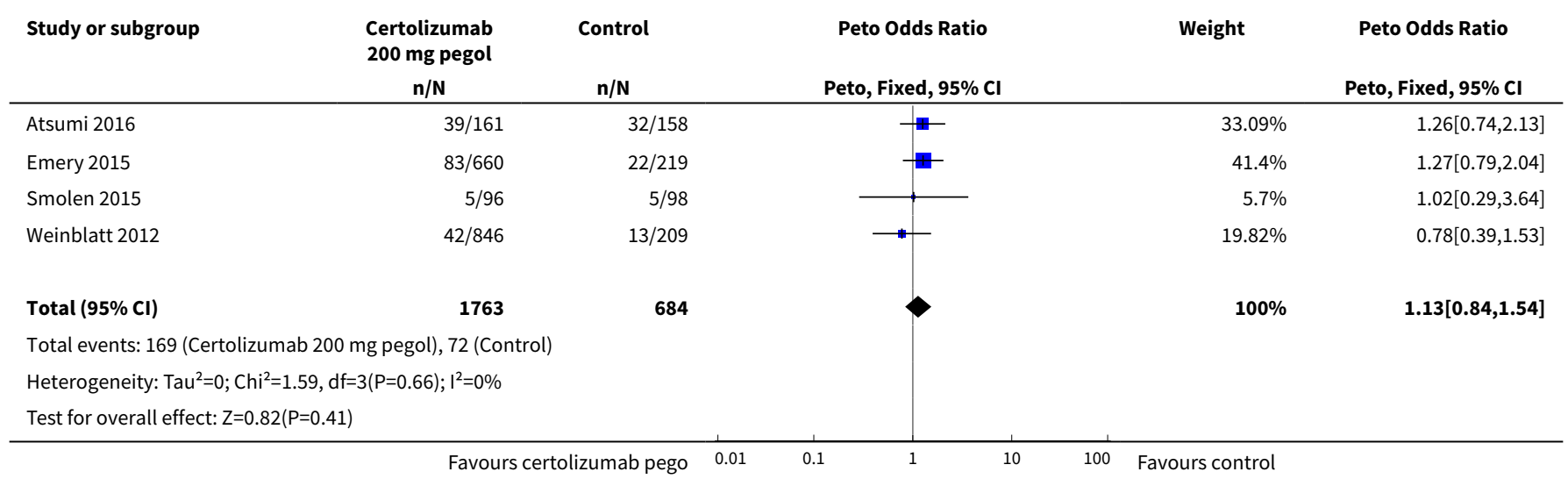

Analysis 50.80. Comparison 50 Safety, Outcome 80 Vomiting certolizumab $400 \mathrm{mg}$.

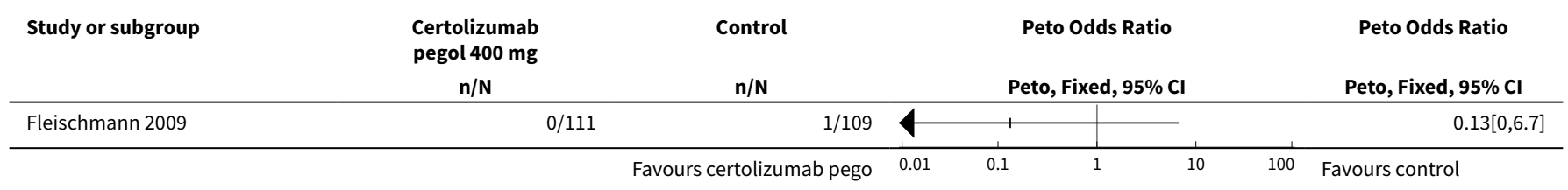


Analysis 50.81. Comparison 50 Safety, Outcome 81 Acute miocardial infarction certolizumab 200 mg.

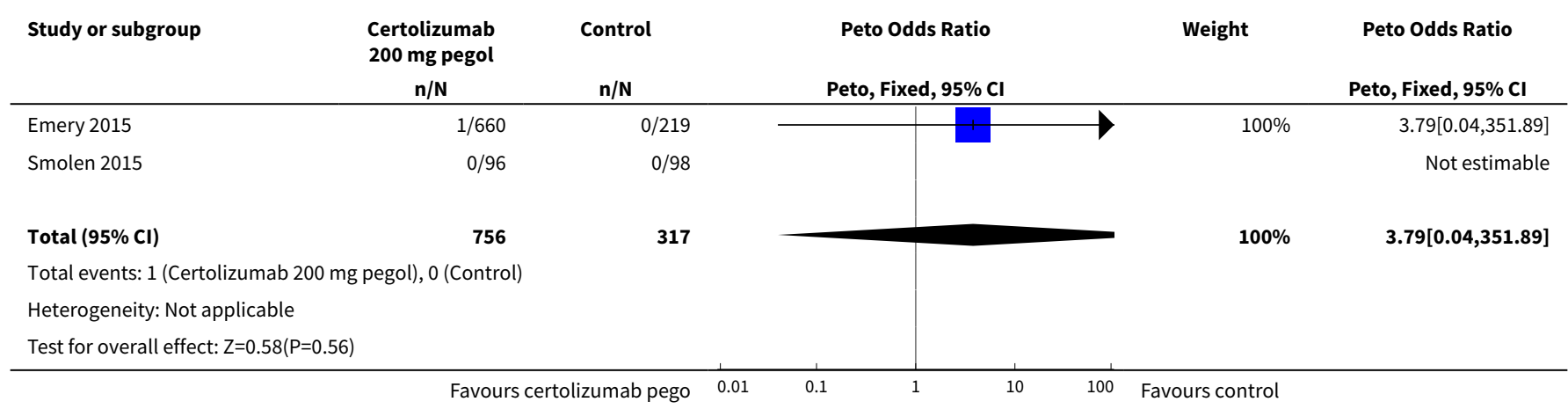

Analysis 50.82. Comparison 50 Safety, Outcome 82 Acute myocardial infarction certolizumab $400 \mathrm{mg}$.

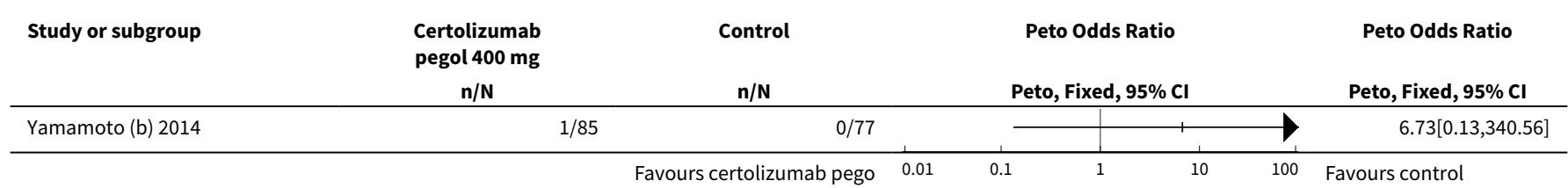

Analysis 50.83. Comparison 50 Safety, Outcome 83 Abdominal pain/discomfort/dyspepsia certolizumab $200 \mathrm{mg}$.

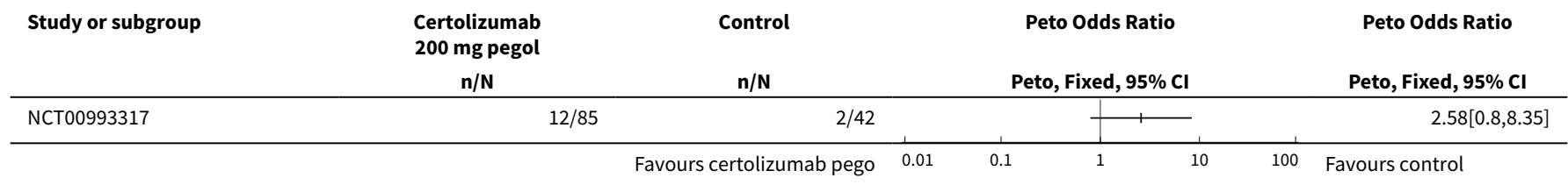

Analysis 50.84. Comparison 50 Safety, Outcome 84 Constipation certolizumab $200 \mathrm{mg}$.

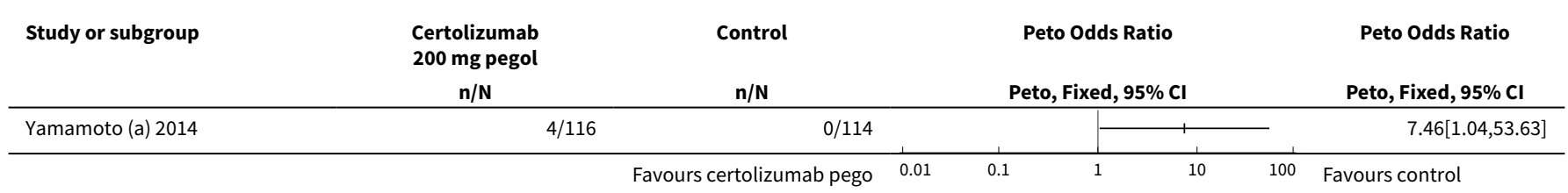

Analysis 50.85. Comparison 50 Safety, Outcome 85 Skin and subcutaneous tissue disorders certolizumab $200 \mathrm{mg}$.

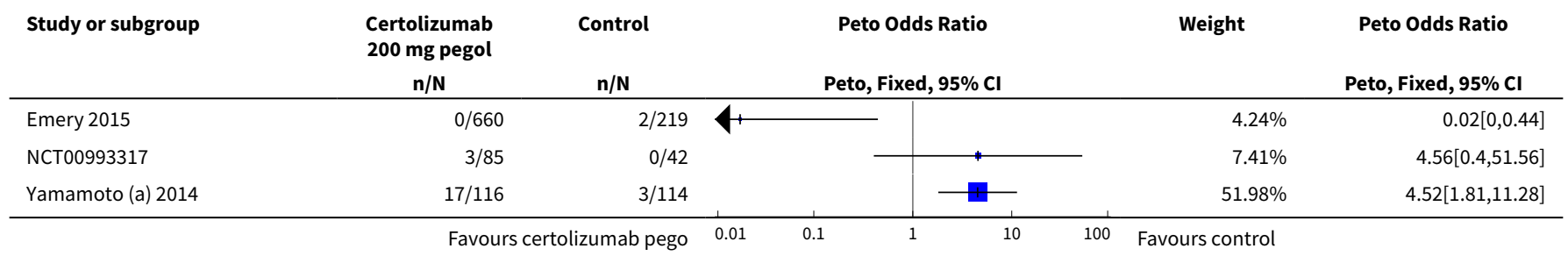




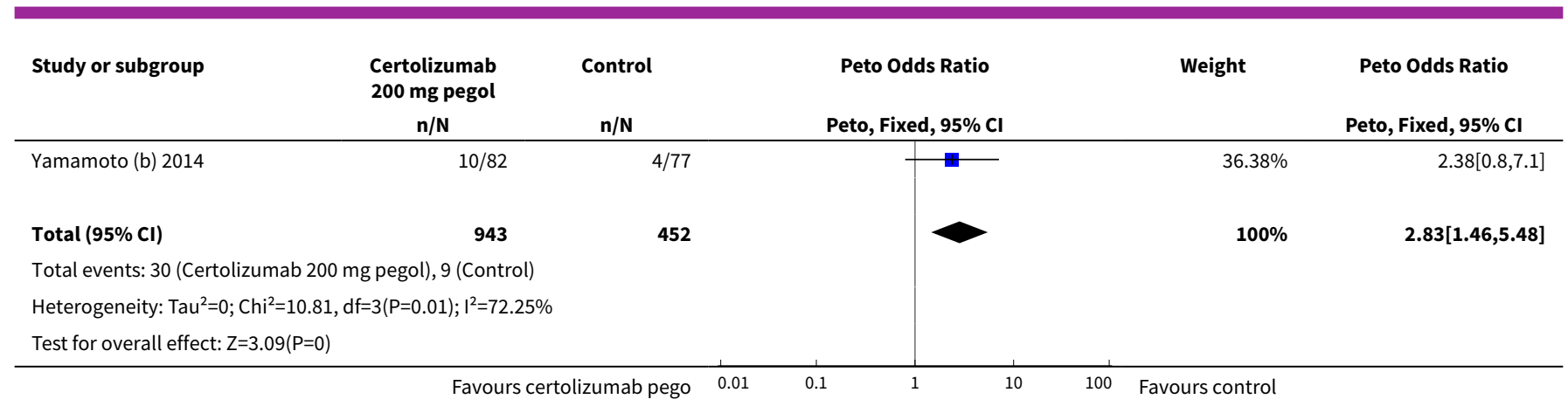

Analysis 50.86. Comparison 50 Safety, Outcome 86 Skin and subcutaneous tissue disorders certolizumab $400 \mathrm{mg}$.

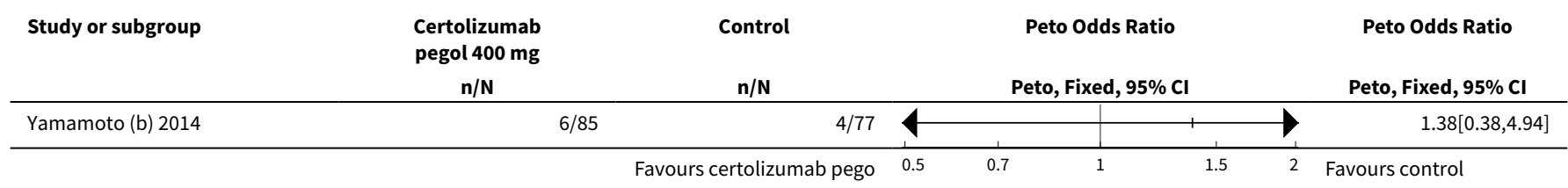

Analysis 50.87. Comparison 50 Safety, Outcome 87 Cough certolizumab $200 \mathrm{mg}$.

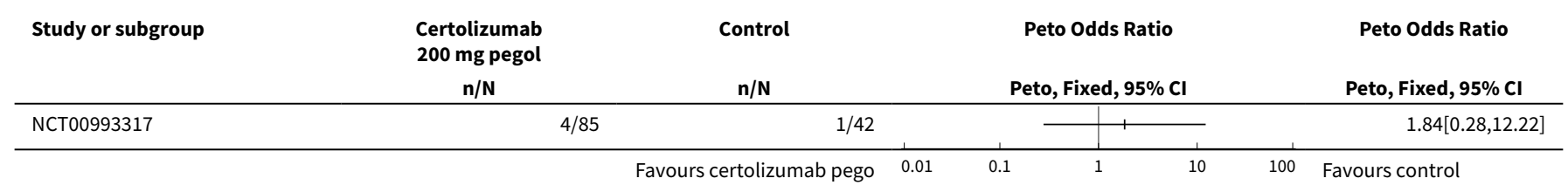

Analysis 50.88. Comparison 50 Safety, Outcome 88 Pruritus certolizumab 200 mg.

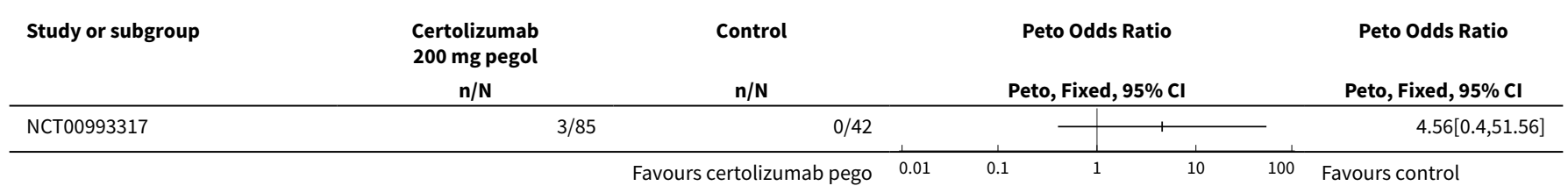

Analysis 50.89. Comparison 50 Safety, Outcome 89 Fatigue certolizumab 200 mg.

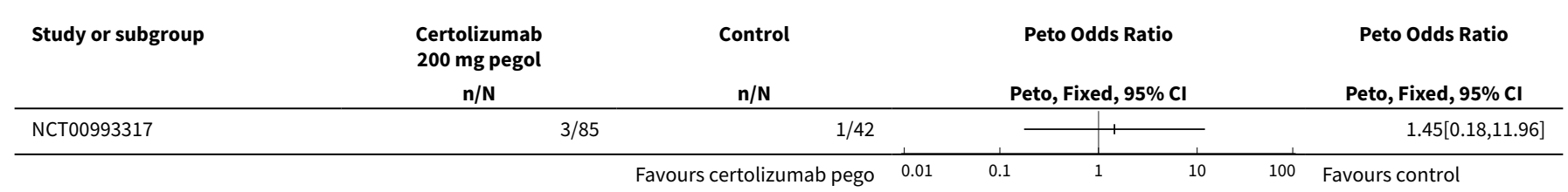


Analysis 50.90. Comparison 50 Safety, Outcome 90 Fatigue certolizumab 400 mg.

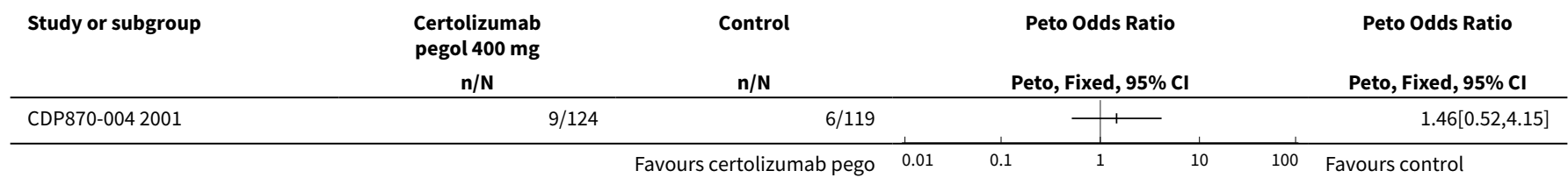

Analysis 50.91. Comparison 50 Safety, Outcome 91 Periodontitis certolizumab $200 \mathrm{mg}$.

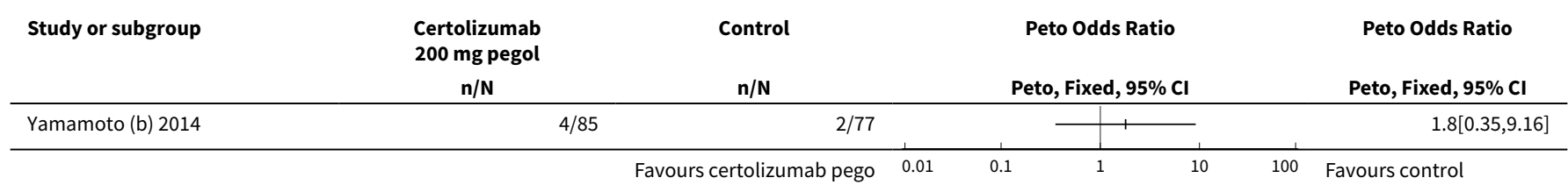

Analysis 50.92. Comparison 50 Safety, Outcome 92 Arthritis bacterial certolizumab $400 \mathrm{mg}$.

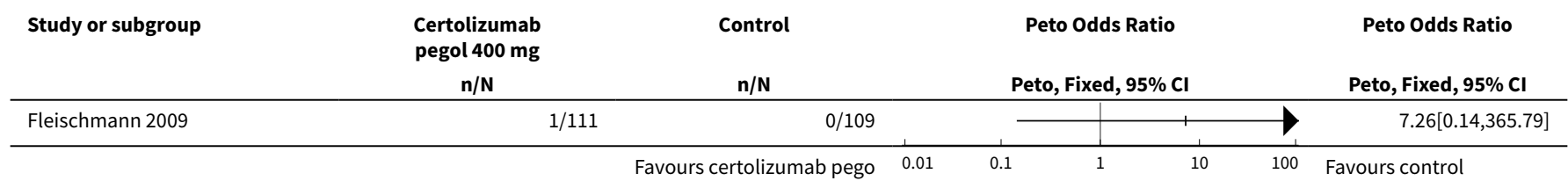

Analysis 50.93. Comparison 50 Safety, Outcome 93 Mastitis certolizumab 400 mg.

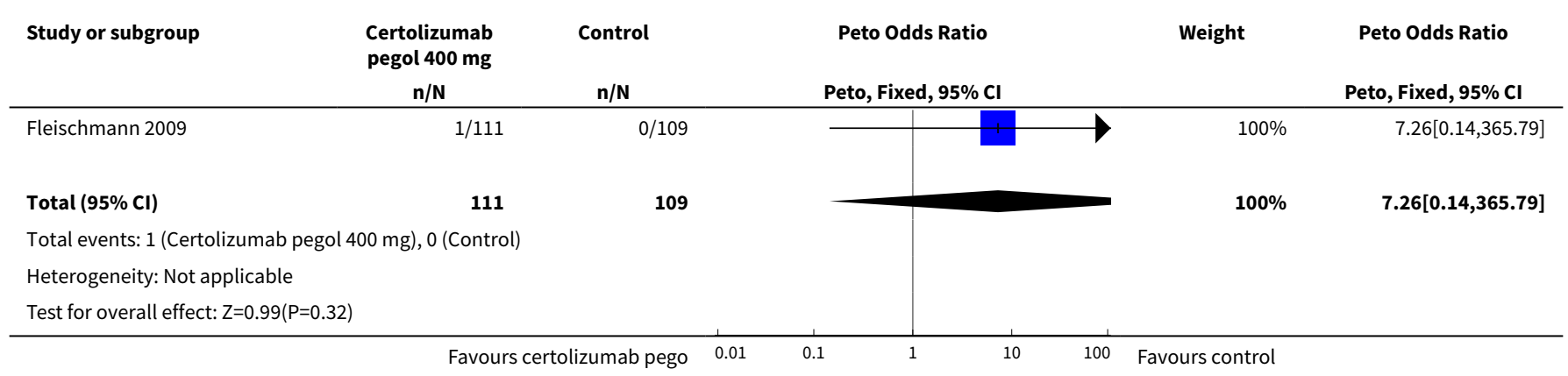

Analysis 50.94. Comparison 50 Safety, Outcome 94 Benign tumour certolizumab $400 \mathrm{mg}$.

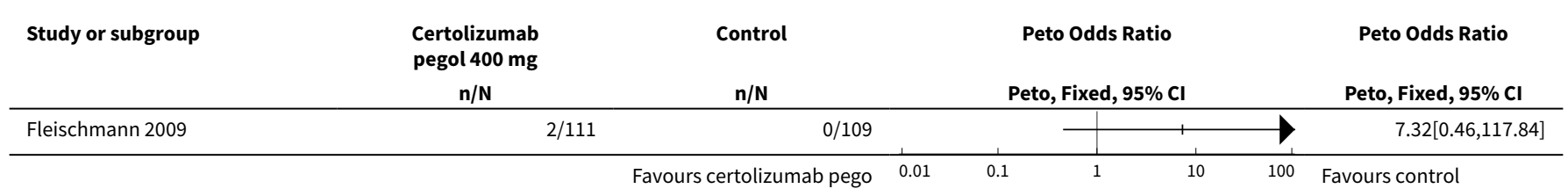


Analysis 50.95. Comparison 50 Safety, Outcome 95 Dizziness postural certolizumab $400 \mathrm{mg}$.

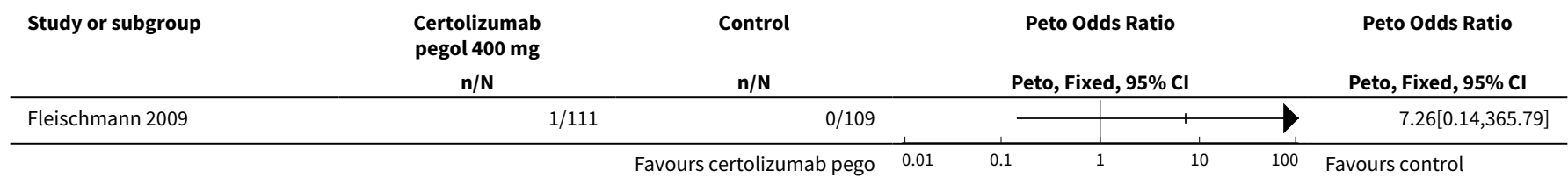

Analysis 50.96. Comparison 50 Safety, Outcome 96 Menorrhagia certolizumab $400 \mathrm{mg}$.

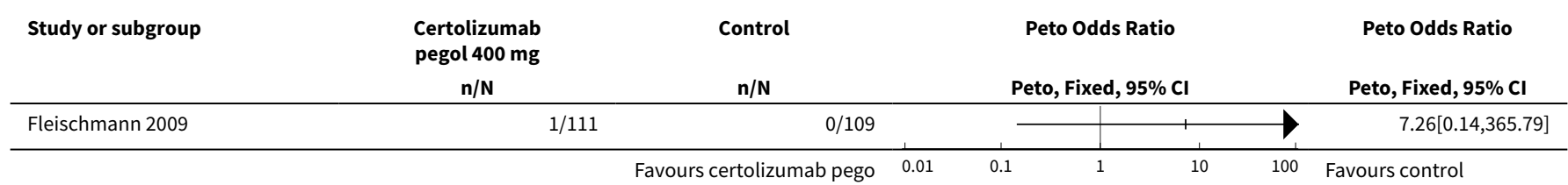

Analysis 50.97. Comparison 50 Safety, Outcome 97 Corneal perforation certolizumab 400 mg.

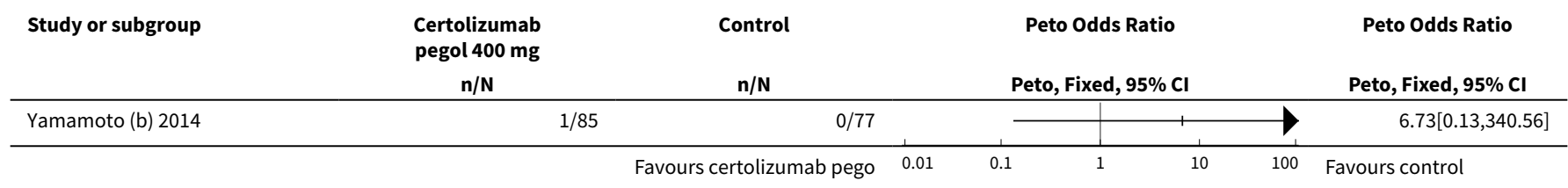

Analysis 50.98. Comparison 50 Safety, Outcome 98 Conjunctivitis allergic certolizumab $400 \mathrm{mg}$.

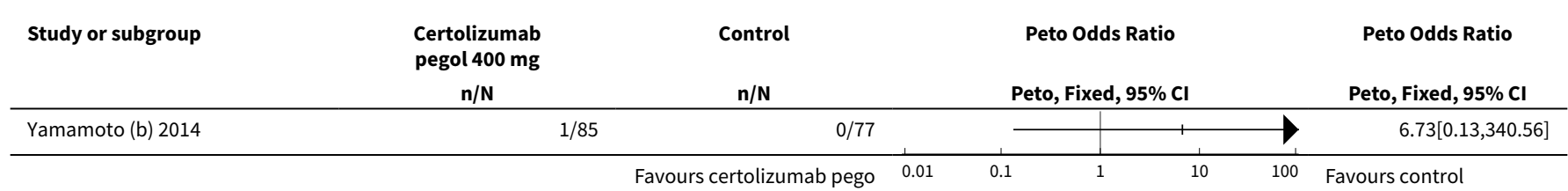

Analysis 50.99. Comparison 50 Safety, Outcome 99 Periodontitis certolizumab $400 \mathrm{mg}$.

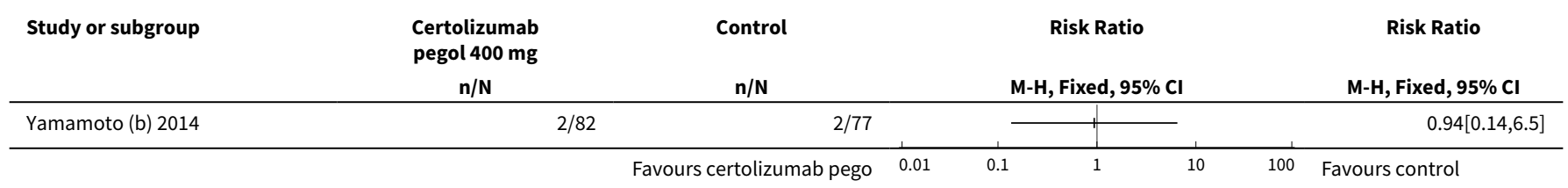


Comparison 51. Participant's assessment of arthritis pain (VAS score 0 to $100 \mathrm{~mm}$ )

\begin{tabular}{|c|c|c|c|c|}
\hline Outcome or subgroup title & No. of studies & $\begin{array}{l}\text { No. of partici- } \\
\text { pants }\end{array}$ & Statistical method & Effect size \\
\hline $\begin{array}{l}1 \text { Mean change at } 24 \text { weeks certolizum- } \\
\text { ab pegol } 200 \mathrm{mg}\end{array}$ & 2 & 965 & $\begin{array}{l}\text { Mean Difference (IV, Ran- } \\
\text { dom, } 95 \% \mathrm{Cl} \text { ) }\end{array}$ & $\begin{array}{l}-20.49[-23.43 \\
-17.55]\end{array}$ \\
\hline $\begin{array}{l}2 \text { Mean change at } 24 \text { weeks certolizum- } \\
\text { ab pegol } 400 \mathrm{mg}\end{array}$ & 3 & 1182 & $\begin{array}{l}\text { Mean Difference (IV, Ran- } \\
\text { dom, } 95 \% \mathrm{CI} \text { ) }\end{array}$ & $\begin{array}{l}-22.69[-25.53 \\
-19.84]\end{array}$ \\
\hline $\begin{array}{l}3 \text { Mean change at } 52 \text { weeks certolizum- } \\
\text { ab pegol } 200 \mathrm{mg}\end{array}$ & 1 & & $\begin{array}{l}\text { Mean Difference (IV, Fixed, } \\
95 \% \mathrm{CI})\end{array}$ & $\begin{array}{l}\text { Totals not select- } \\
\text { ed }\end{array}$ \\
\hline $\begin{array}{l}4 \text { Mean change at } 52 \text { weeks certolizum- } \\
\text { ab pegol } 400 \mathrm{mg}\end{array}$ & 1 & & $\begin{array}{l}\text { Mean Difference (IV, Fixed, } \\
95 \% \mathrm{CI} \text { ) }\end{array}$ & $\begin{array}{l}\text { Totals not select- } \\
\text { ed }\end{array}$ \\
\hline
\end{tabular}

Analysis 51.1. Comparison 51 Participant's assessment of arthritis pain (VAS score 0 to $100 \mathrm{~mm}$ ), Outcome 1 Mean change at 24 weeks certolizumab pegol $200 \mathrm{mg}$.

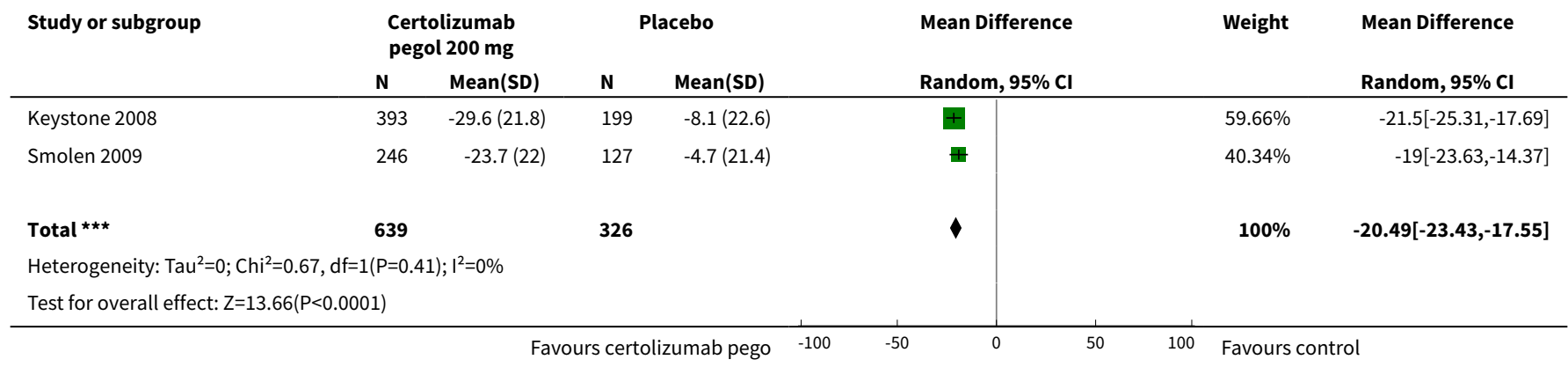

Analysis 51.2. Comparison 51 Participant's assessment of arthritis pain (VAS score 0 to $100 \mathrm{~mm}$ ), Outcome 2 Mean change at 24 weeks certolizumab pegol $400 \mathrm{mg}$.

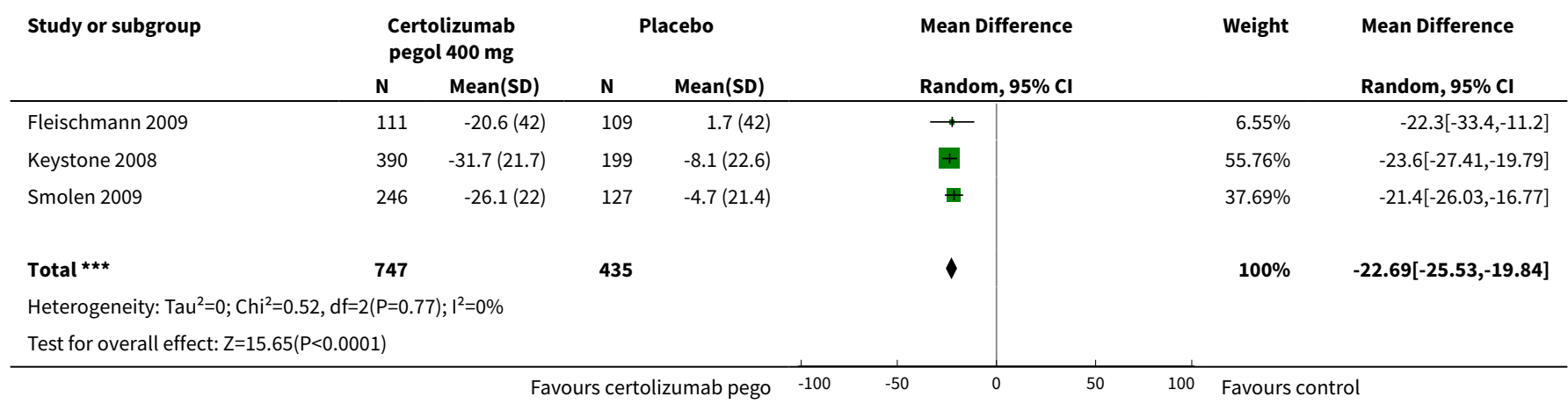


Analysis 51.3. Comparison 51 Participant's assessment of arthritis pain (VAS score

0 to $100 \mathrm{~mm}$ ), Outcome 3 Mean change at 52 weeks certolizumab pegol $200 \mathrm{mg}$.

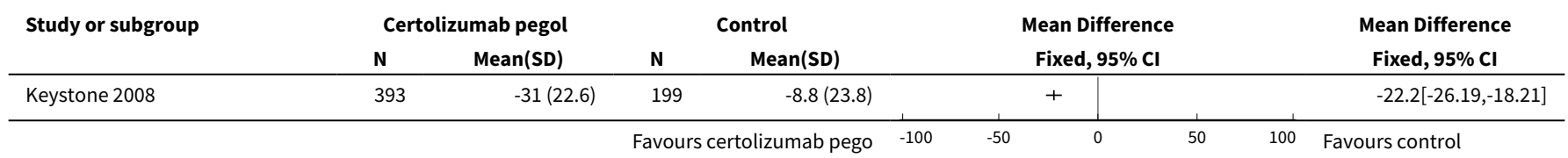

Analysis 51.4. Comparison 51 Participant's assessment of arthritis pain (VAS score

0 to $100 \mathrm{~mm}$ ), Outcome 4 Mean change at 52 weeks certolizumab pegol $400 \mathrm{mg}$.

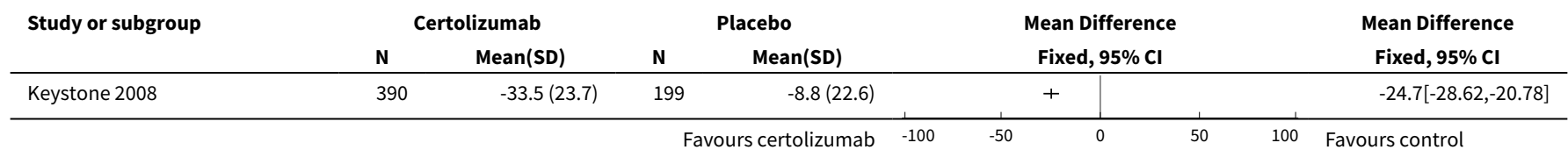

Comparison 52. Participant's assessment of arthritis pain (VAS score 0 to $100 \mathrm{~mm}$ ) at 24 weeks, any dose

\begin{tabular}{lllll}
\hline Outcome or subgroup title & No. of studies & $\begin{array}{l}\text { No. of partici- } \\
\text { pants }\end{array}$ & Statistical method & Effect size \\
\hline 1 Change from baseline & 4 & 2064 & $\begin{array}{l}\text { Mean Difference (IV, Random, } \\
95 \% \text { Cl) }\end{array}$ & -21.07 [-23.59, -18.55] \\
\hline $\begin{array}{l}1.1 \text { certolizumab pegol 200 } \\
\text { mg sc }\end{array}$ & 2 & 803 & $\begin{array}{l}\text { Mean Difference (IV, Random, } \\
95 \% \text { Cl) }\end{array}$ & $-20.48[-24.26,-16.69]$ \\
\hline $\begin{array}{l}1.2 \text { certolizumab pegol 400 } \\
\text { mg sc }\end{array}$ & 4 & 1261 & $\begin{array}{l}\text { Mean Difference (IV, Random, } \\
95 \% \text { Cl) }\end{array}$ & $-21.35[-25.08,-17.61]$ \\
\hline
\end{tabular}

Analysis 52.1. Comparison 52 Participant's assessment of arthritis pain (VAS score 0 to $100 \mathrm{~mm}$ ) at 24 weeks, any dose, Outcome 1 Change from baseline.

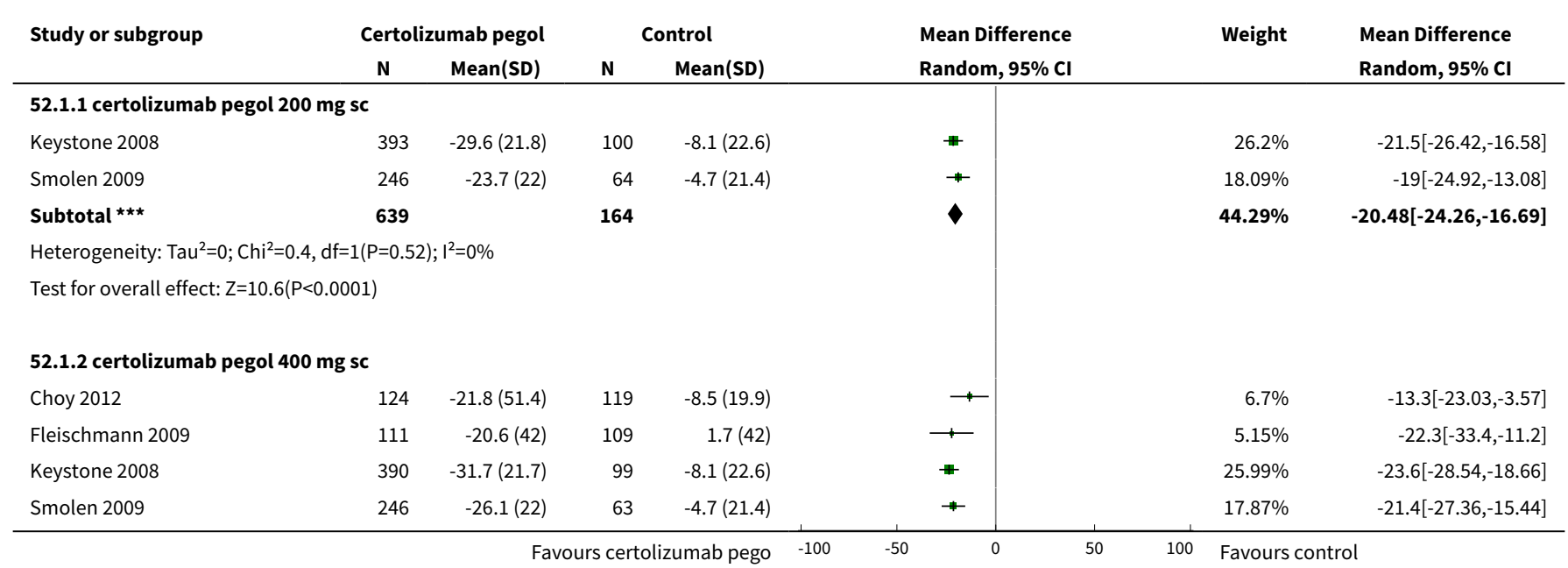




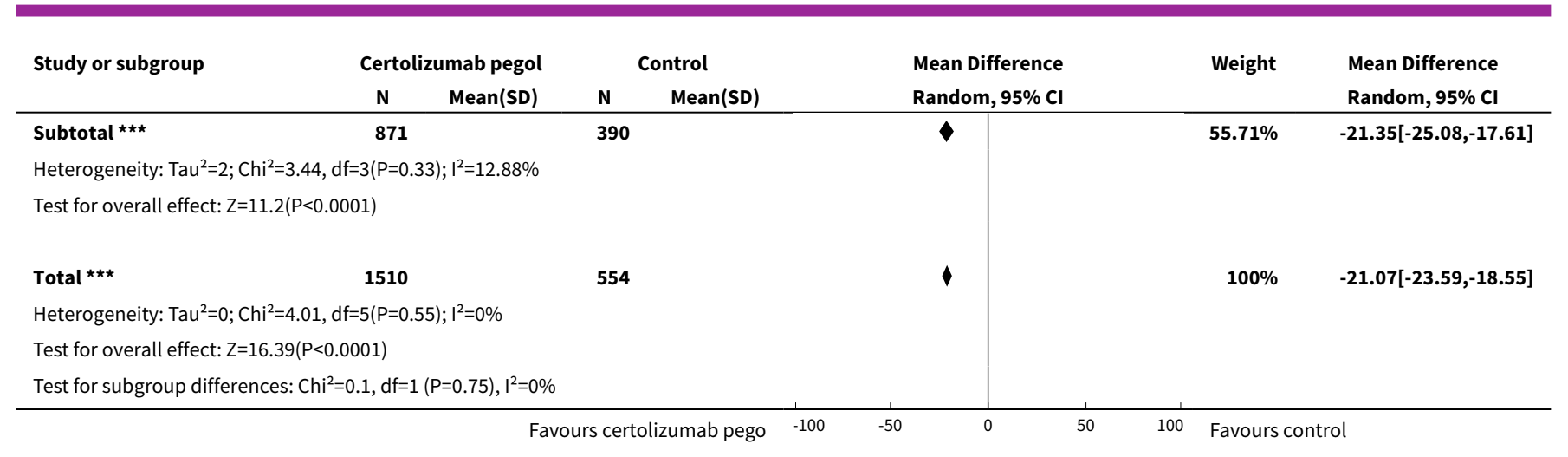

Comparison 53. Participant's assessment of arthritis pain (VAS score 0 to $100 \mathrm{~mm}$ ) at 52 weeks, any dose

\begin{tabular}{lllll}
\hline Outcome or subgroup title & No. of studies & $\begin{array}{l}\text { No. of partici- } \\
\text { pants }\end{array}$ & Statistical method & Effect size \\
\hline $\begin{array}{l}1 \text { Change from baseline } \\
\text { SC }\end{array}$ & 1 & Mean Difference (IV, Fixed, 95\% CI) & $\begin{array}{l}\text { Totals not select- } \\
\text { ed }\end{array}$ \\
\hline $\begin{array}{l}1.1 \text { certolizumab pegol } 200 \mathrm{mg} \\
\text { sc }\end{array}$ & 1 & Mean Difference (IV, Fixed, 95\% CI) & $0.0[0.0,0.0]$ \\
\hline $\begin{array}{l}1.2 \text { certolizumab pegol } 400 \mathrm{mg} \\
\text { sc }\end{array}$ & 1 & Mean Difference (IV, Fixed, 95\% Cl) & $0.0[0.0,0.0]$ \\
\hline
\end{tabular}

Analysis 53.1. Comparison 53 Participant's assessment of arthritis pain (VAS score 0 to $100 \mathrm{~mm}$ ) at 52 weeks, any dose, Outcome 1 Change from baseline.

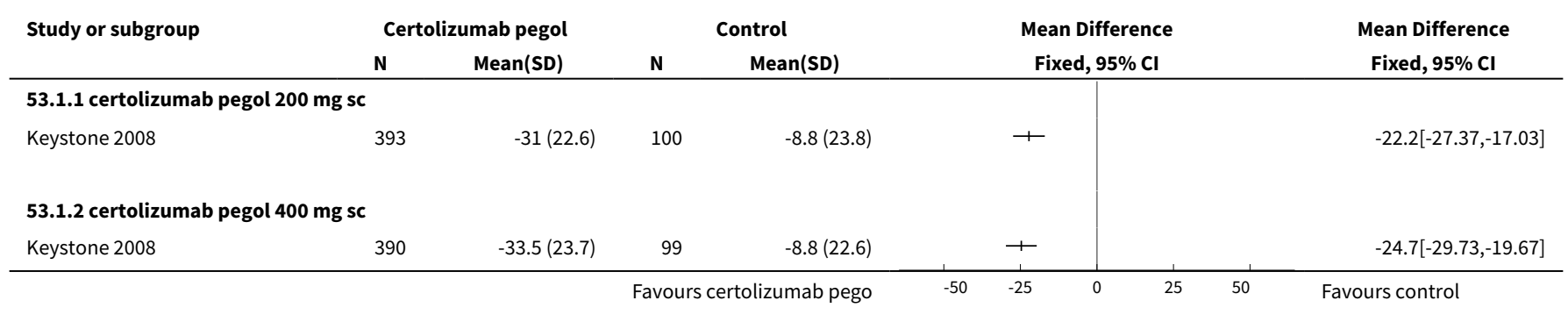

\section{Comparison 54. Withdrawals Withdrawn due to lack of efficacy: any doses any follow-up}

\begin{tabular}{lllll}
\hline Outcome or subgroup title & No. of studies & $\begin{array}{l}\text { No. of partici- } \\
\text { pants }\end{array}$ & Statistical method & Effect size \\
\hline $\begin{array}{l}1 \text { Withdrawn due to lack of efficacy: any } \\
\text { doses any follow-up }\end{array}$ & 8 & 3433 & $\begin{array}{l}\text { Risk Ratio (M-H, Random, } \\
95 \% \mathrm{Cl})\end{array}$ & $0.31[0.26,0.37]$ \\
\hline
\end{tabular}


Analysis 54.1. Comparison 54 Withdrawals Withdrawn due to lack of efficacy: any doses any follow-up, Outcome 1 Withdrawn due to lack of efficacy: any doses any follow-up.

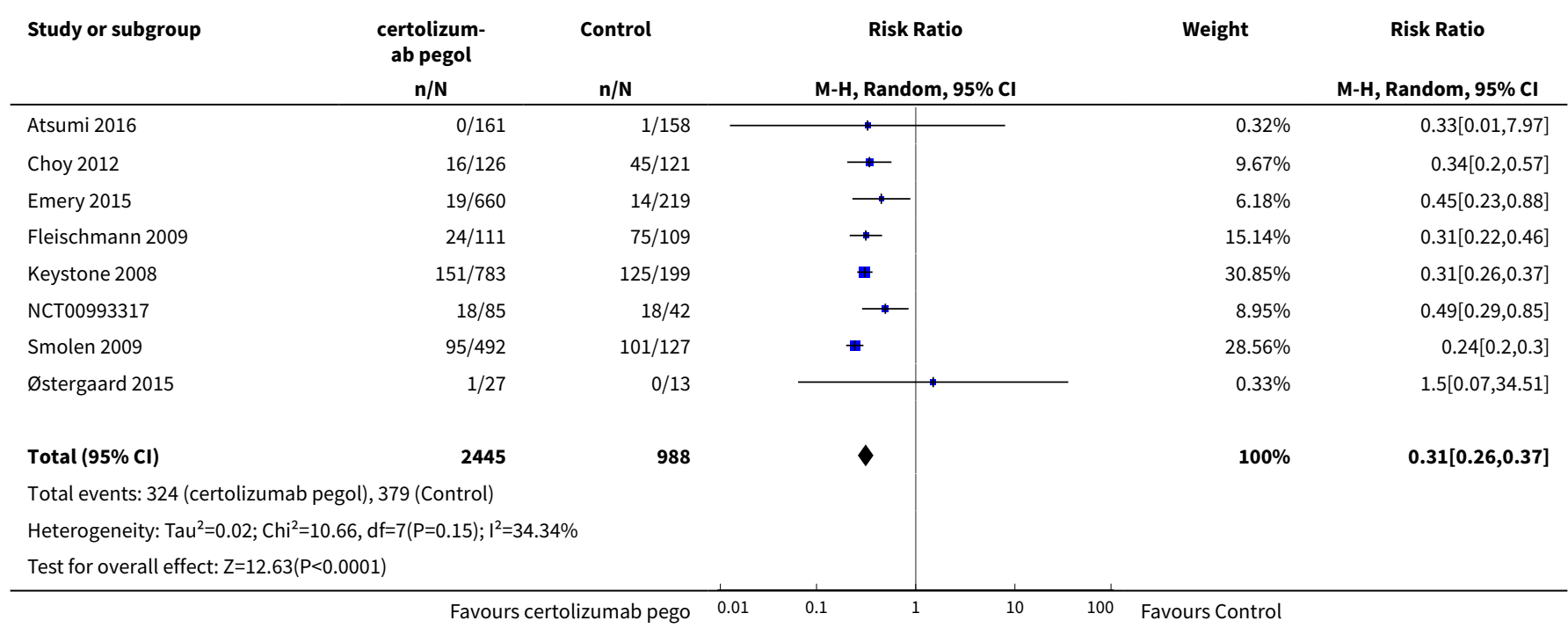

\section{Comparison 55. Summary of findings: certolizumab (with or without MTX) versus placebo (with or without MTX)}

\begin{tabular}{|c|c|c|c|c|}
\hline Outcome or subgroup title & No. of studies & $\begin{array}{l}\text { No. of partici- } \\
\text { pants }\end{array}$ & Statistical method & Effect size \\
\hline $\begin{array}{l}1 \text { ACR } 50200 \text { mg certolizumab } \\
24 \text { weeks }\end{array}$ & 5 & 1445 & Risk Ratio (M-H, Random, 95\% Cl) & $3.80[2.42,5.95]$ \\
\hline $\begin{array}{l}2 \mathrm{HAQ} \text { change from baseline } 200 \\
\text { mg certolizumab } 24 \text { weeks }\end{array}$ & 4 & 1268 & $\begin{array}{l}\text { Mean Difference (IV, Random, } \\
95 \% \mathrm{CI} \text { ) }\end{array}$ & $-0.35[-0.43,-0.26]$ \\
\hline $\begin{array}{l}3 \text { Serious adverse events cer- } \\
\text { tolizumab } 200 \mathrm{mg} \mathrm{sc}\end{array}$ & 9 & 3927 & $\begin{array}{l}\text { Peto Odds Ratio (Peto, Fixed, 95\% } \\
\mathrm{Cl} \text { ) }\end{array}$ & $1.47[1.13,1.91]$ \\
\hline $\begin{array}{l}4 \text { Proportion of participants } \\
\text { achieving remission } 24 \text { weeks } \\
\text { certolizumab } 200 \mathrm{mg}\end{array}$ & 4 & 1381 & Risk Ratio (M-H, Random, 95\% Cl) & $8.47[4.15,17.28]$ \\
\hline $\begin{array}{l}5 \text { Radiological changes: Erosion } \\
\text { Scores (ES) certolizumab } 200 \\
\text { mg sc }\end{array}$ & 2 & 859 & $\begin{array}{l}\text { Mean Difference (IV, Random, } \\
95 \% \mathrm{CI})\end{array}$ & $-0.67[-0.96,-0.38]$ \\
\hline $\begin{array}{l}5.1 \text { certolizumab } 200 \mathrm{mg} \mathrm{sc} 24 \\
\text { weeks }\end{array}$ & 2 & 859 & $\begin{array}{l}\text { Mean Difference (IV, Random, } \\
95 \% \mathrm{CI})\end{array}$ & $-0.67[-0.96,-0.38]$ \\
\hline 6 All Withdrawals: & 10 & 3962 & Risk Ratio (M-H, Random, 95\% Cl) & $0.42[0.36,0.50]$ \\
\hline $\begin{array}{l}7 \text { Withdrawals due to adverse } \\
\text { events }\end{array}$ & 9 & 3998 & $\begin{array}{l}\text { Peto Odds Ratio (Peto, Fixed, 95\% } \\
\mathrm{Cl} \text { ) }\end{array}$ & $1.66[1.15,2.37]$ \\
\hline 8 Deaths & 10 & 4745 & $\begin{array}{l}\text { Peto Odds Ratio (Peto, Fixed, 95\% } \\
\mathrm{Cl} \text { ) }\end{array}$ & $2.63[0.78,8.91]$ \\
\hline
\end{tabular}




\begin{tabular}{|c|c|c|c|c|}
\hline Outcome or subgroup title & No. of studies & $\begin{array}{l}\text { No. of partici- } \\
\text { pants }\end{array}$ & Statistical method & Effect size \\
\hline 8.1 Certolizumab pegol $200 \mathrm{mg}$ & 7 & 3266 & $\begin{array}{l}\text { Peto Odds Ratio (Peto, Fixed, 95\% } \\
\text { Cl) }\end{array}$ & $2.10[0.44,10.08]$ \\
\hline 8.2 Certolizumab pegol $400 \mathrm{mg}$ & 5 & 1349 & $\begin{array}{l}\text { Peto Odds Ratio (Peto, Fixed, 95\% } \\
\text { Cl) }\end{array}$ & $3.53[0.40,31.39]$ \\
\hline 8.3 Other doses & 2 & 130 & $\begin{array}{l}\text { Peto Odds Ratio (Peto, Fixed, 95\% } \\
\mathrm{Cl} \text { ) }\end{array}$ & $4.48[0.07,286.49]$ \\
\hline 9 Tuberculosis & 7 & 4074 & $\begin{array}{l}\text { Peto Odds Ratio (Peto, Fixed, 95\% } \\
\mathrm{Cl} \text { ) }\end{array}$ & $1.91[0.61,5.96]$ \\
\hline 9.1 Certolizumab pegol $200 \mathrm{mg}$ & 6 & 3058 & $\begin{array}{l}\text { Peto Odds Ratio (Peto, Fixed, 95\% } \\
\mathrm{Cl} \text { ) }\end{array}$ & $1.53[0.40,5.77]$ \\
\hline 9.2 Certolizumab pegol $400 \mathrm{mg}$ & 3 & 1016 & $\begin{array}{l}\text { Peto Odds Ratio (Peto, Fixed, 95\% } \\
\mathrm{Cl} \text { ) }\end{array}$ & $3.52[0.40,31.33]$ \\
\hline $\begin{array}{l}10 \text { Upper respiratory tract infec- } \\
\text { tions }\end{array}$ & 8 & 3692 & $\begin{array}{l}\text { Peto Odds Ratio (Peto, Fixed, 95\% } \\
\mathrm{Cl} \text { ) }\end{array}$ & $1.17[0.86,1.59]$ \\
\hline 10.1 Certolizumab pegol $200 \mathrm{mg}$ & 7 & 2528 & $\begin{array}{l}\text { Peto Odds Ratio (Peto, Fixed, 95\% } \\
\mathrm{Cl} \text { ) }\end{array}$ & $1.28[0.91,1.80]$ \\
\hline 10.2 Certolizumab pegol $400 \mathrm{mg}$ & 4 & 1164 & $\begin{array}{l}\text { Peto Odds Ratio (Peto, Fixed, 95\% } \\
\mathrm{Cl} \text { ) }\end{array}$ & $0.81[0.41,1.61]$ \\
\hline $\begin{array}{l}11 \text { Lower respiratory tract infec- } \\
\text { tions }\end{array}$ & 7 & 3073 & $\begin{array}{l}\text { Peto Odds Ratio (Peto, Fixed, 95\% } \\
\mathrm{Cl} \text { ) }\end{array}$ & $1.66[0.77,3.58]$ \\
\hline 11.1 Certolizumab pegol $200 \mathrm{mg}$ & 6 & 2218 & $\begin{array}{l}\text { Peto Odds Ratio (Peto, Fixed, 95\% } \\
\mathrm{Cl} \text { ) }\end{array}$ & $1.81[0.62,5.26]$ \\
\hline 11.2 Certolizumab pegol $400 \mathrm{mg}$ & 3 & 855 & $\begin{array}{l}\text { Peto Odds Ratio (Peto, Fixed, 95\% } \\
\mathrm{Cl} \text { ) }\end{array}$ & $1.52[0.50,4.59]$ \\
\hline $\begin{array}{l}12 \text { Malignancies including lym- } \\
\text { phoma }\end{array}$ & 7 & 3749 & $\begin{array}{l}\text { Peto Odds Ratio (Peto, Fixed, 95\% } \\
\mathrm{Cl} \text { ) }\end{array}$ & $0.90[0.39,2.08]$ \\
\hline 12.1 Certolizumab pegol $200 \mathrm{mg}$ & 6 & 2570 & $\begin{array}{l}\text { Peto Odds Ratio (Peto, Fixed, 95\% } \\
\mathrm{Cl} \text { ) }\end{array}$ & $0.79[0.29,2.12]$ \\
\hline 12.2 Certolizumab pegol $400 \mathrm{mg}$ & 3 & 1179 & $\begin{array}{l}\text { Peto Odds Ratio (Peto, Fixed, 95\% } \\
\mathrm{Cl} \text { ) }\end{array}$ & $1.26[0.26,6.08]$ \\
\hline
\end{tabular}


Analysis 55.1. Comparison 55 Summary of findings: certolizumab (with or without MTX) versus placebo (with or without MTX), Outcome 1 ACR 50200 mg certolizumab 24 weeks.

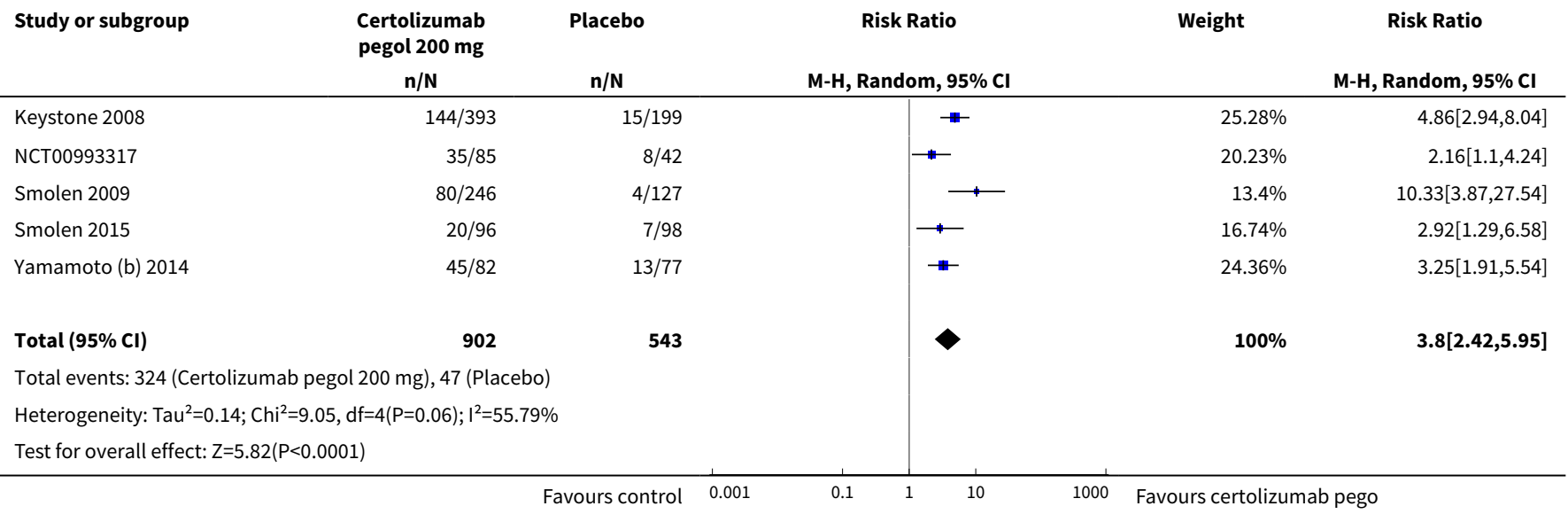

Analysis 55.2. Comparison 55 Summary of findings: certolizumab (with or without MTX) versus placebo (with or without MTX), Outcome $2 \mathrm{HAQ}$ change from baseline $200 \mathrm{mg}$ certolizumab 24 weeks.

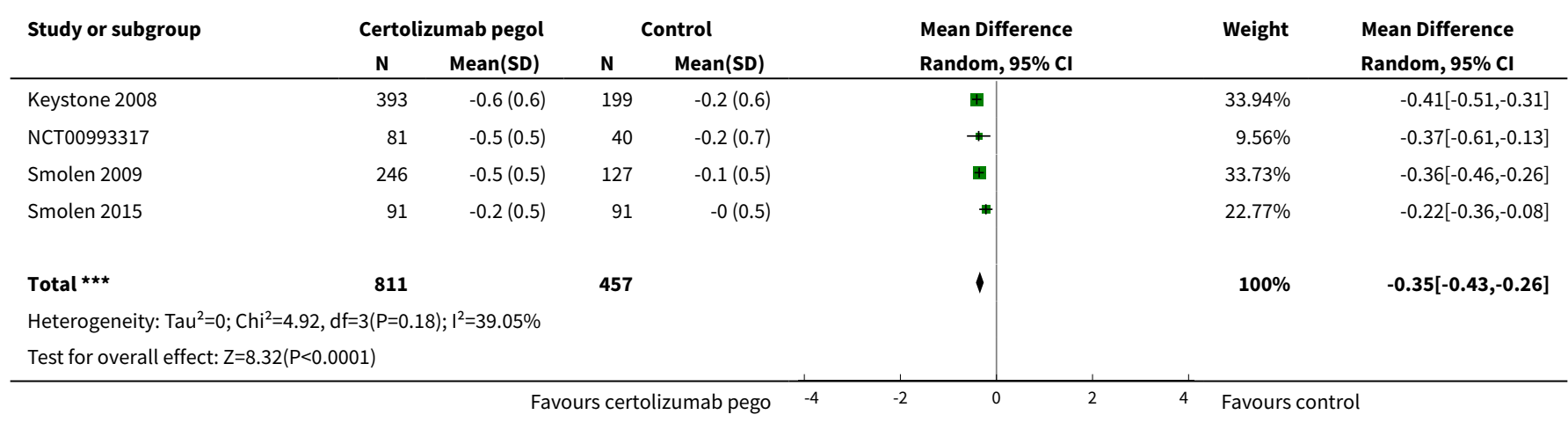

Analysis 55.3. Comparison 55 Summary of findings: certolizumab (with or without MTX) versus placebo (with or without MTX), Outcome 3 Serious adverse events certolizumab 200 mg sc.

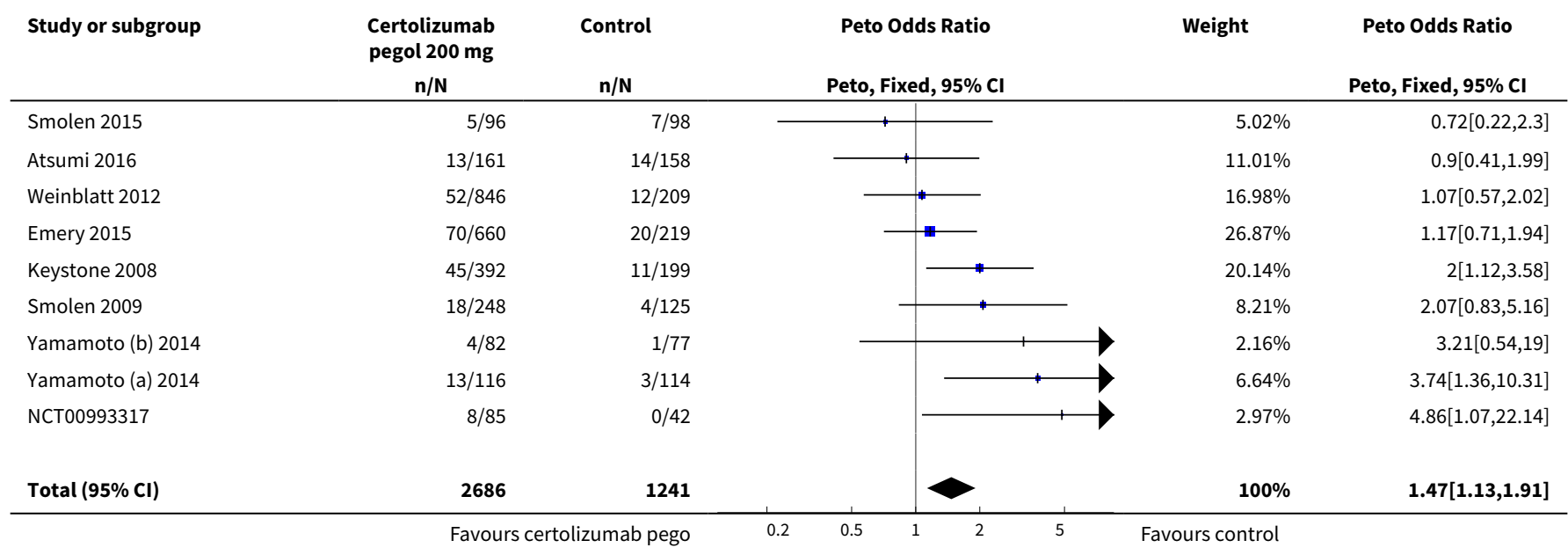




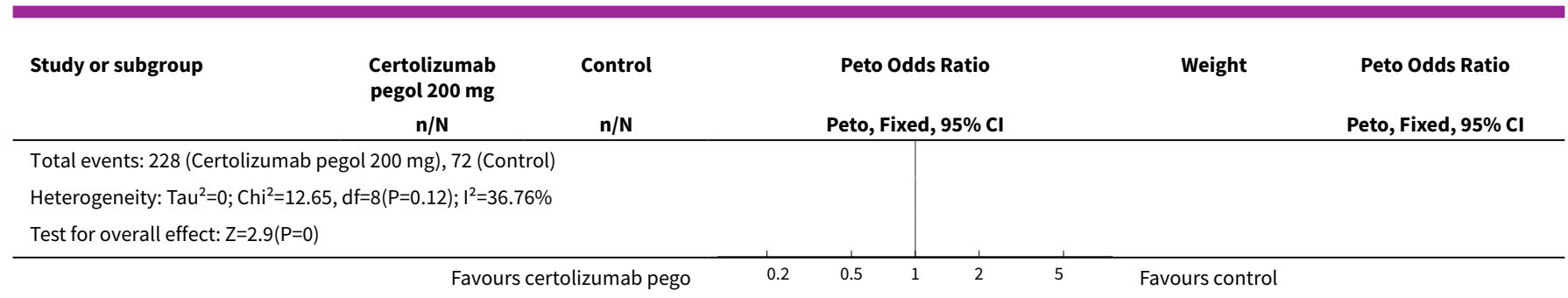

Analysis 55.4. Comparison 55 Summary of findings: certolizumab (with or without MTX) versus placebo (with or without MTX), Outcome 4 Proportion of participants achieving remission 24 weeks certolizumab $200 \mathrm{mg}$.

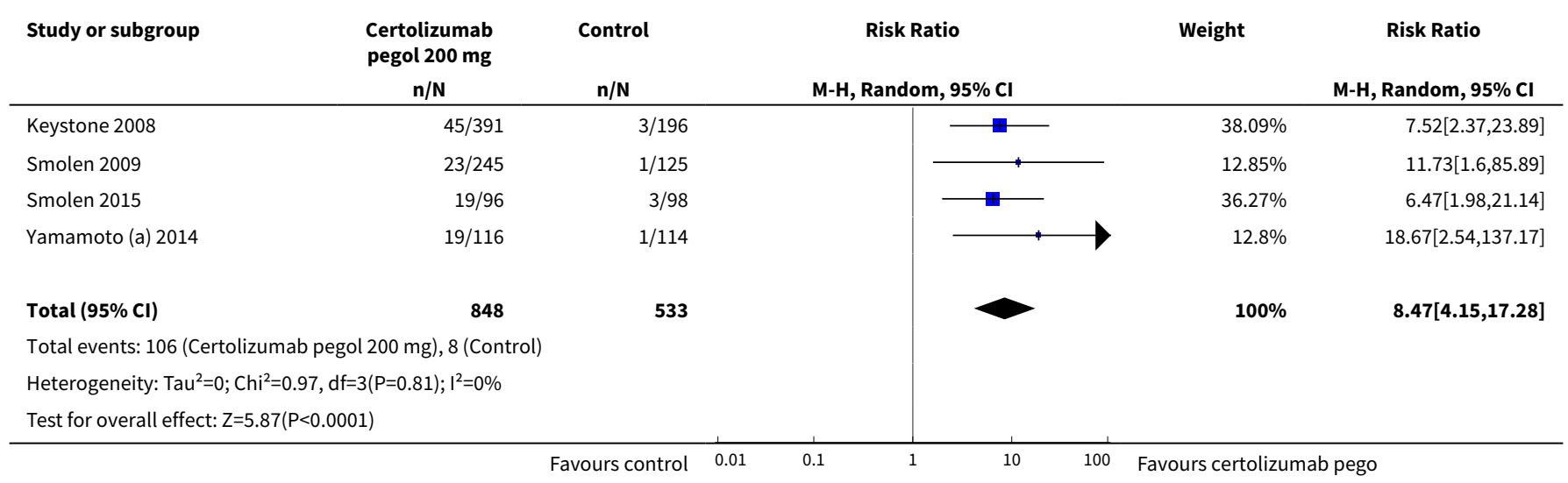

Analysis 55.5. Comparison 55 Summary of findings: certolizumab (with or without MTX) versus placebo (with or without MTX), Outcome 5 Radiological changes: Erosion Scores (ES) certolizumab 200 mg sc.

\begin{tabular}{|c|c|c|c|c|c|c|c|}
\hline \multirow[t]{2}{*}{ Study or subgroup } & \multicolumn{2}{|c|}{ Certolizumab pegol } & \multicolumn{2}{|c|}{ Control } & \multirow{2}{*}{$\begin{array}{l}\text { Mean Difference } \\
\text { Random, } 95 \% \mathrm{Cl}\end{array}$} & \multirow[t]{2}{*}{ Weight } & \multirow{2}{*}{$\begin{array}{l}\text { Mean Difference } \\
\text { Random, } 95 \% \mathrm{Cl}\end{array}$} \\
\hline & $\mathbf{N}$ & Mean(SD) & $\mathbf{N}$ & Mean(SD) & & & \\
\hline \multicolumn{8}{|c|}{ 55.5.1 certolizumab $200 \mathrm{mg}$ sc 24 weeks } \\
\hline Keystone 2008 & 353 & $0(1.5)$ & 180 & $0.7(2.1)$ & & $71.91 \%$ & $-0.7[-1.04,-0.36]$ \\
\hline Smolen 2009 & 214 & $0.1(2)$ & 112 & $0.7(2.6)$ & —- & $28.09 \%$ & $-0.6[-1.15,-0.05]$ \\
\hline Subtotal $\star \star \star$ & 567 & & 292 & & & $100 \%$ & $-0.67[-0.96,-0.38]$ \\
\hline \multicolumn{8}{|c|}{ Heterogeneity: $\mathrm{Tau}^{2}=0 ; \mathrm{Chi}^{2}=0.09, \mathrm{df}=1(\mathrm{P}=0.76) ; \mathrm{I}^{2}=0 \%$} \\
\hline \multicolumn{8}{|c|}{ Test for overall effect: $Z=4.51(P<0.0001)$} \\
\hline Total ${ }^{\star \star \star}$ & 567 & & 292 & & & $100 \%$ & $-0.67[-0.96,-0.38]$ \\
\hline \multicolumn{8}{|c|}{ Heterogeneity: $\mathrm{Tau}^{2}=0 ; \mathrm{Chi}^{2}=0.09, \mathrm{df}=1(\mathrm{P}=0.76) ; \mathrm{I}^{2}=0 \%$} \\
\hline \multicolumn{8}{|c|}{ Test for overall effect: $Z=4.51(P<0.0001)$} \\
\hline
\end{tabular}


Analysis 55.6. Comparison 55 Summary of findings: certolizumab (with or without MTX) versus placebo (with or without MTX), Outcome 6 All Withdrawals:.

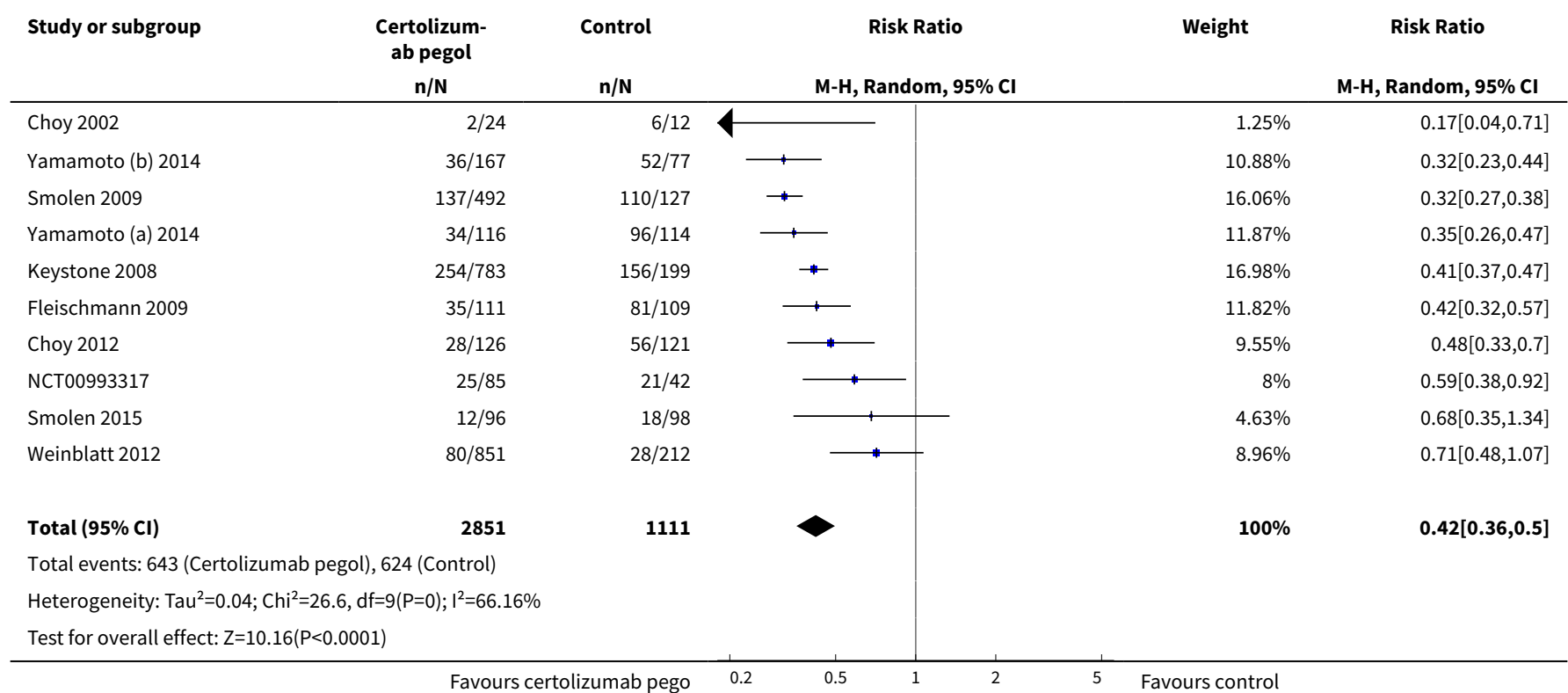

Analysis 55.7. Comparison 55 Summary of findings: certolizumab (with or without MTX) versus placebo (with or without MTX), Outcome 7 Withdrawals due to adverse events.

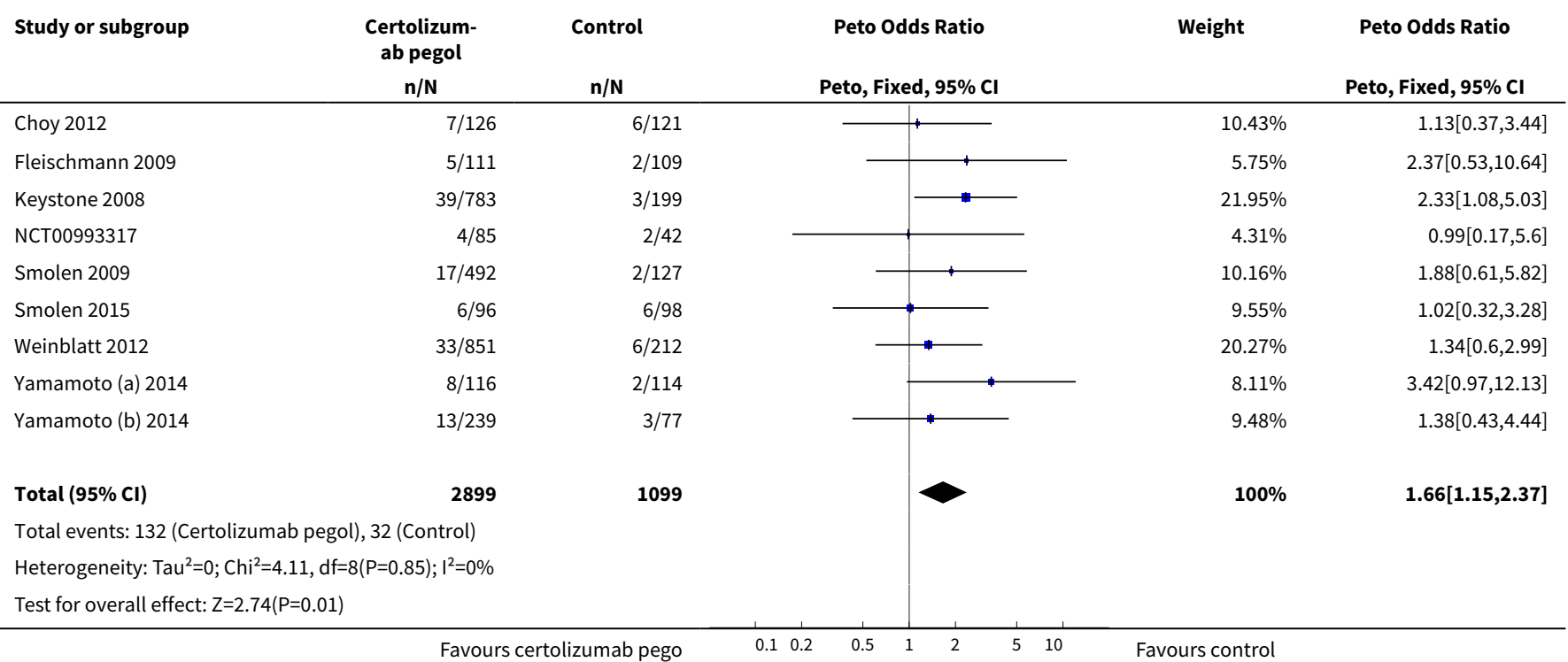


Analysis 55.8. Comparison 55 Summary of findings: certolizumab (with or without MTX) versus placebo (with or without MTX), Outcome 8 Deaths.

\begin{tabular}{|c|c|c|c|c|c|}
\hline Study or subgroup & $\begin{array}{c}\text { Certolizum- } \\
\text { ab pegol } \\
n / N\end{array}$ & $\begin{array}{l}\text { Placebo } \\
\text { n/N }\end{array}$ & $\begin{array}{c}\text { Peto Odds Ratio } \\
\text { Peto, Fixed, } 95 \% \mathrm{Cl}\end{array}$ & Weight & $\begin{array}{c}\text { Peto Odds Ratio } \\
\text { Peto, Fixed, 95\% Cl }\end{array}$ \\
\hline \multicolumn{3}{|c|}{ 55.8.1 Certolizumab pegol $200 \mathrm{mg}$} & & & \\
\hline Emery 2015 & $1 / 660$ & $0 / 219$ & & $7.23 \%$ & $3.79[0.04,351.89]$ \\
\hline Keystone 2008 & $3 / 392$ & $1 / 100$ & & $24.89 \%$ & $0.75[0.07,8.6]$ \\
\hline Smolen 2009 & $1 / 246$ & $0 / 63$ & & $6.28 \%$ & $3.51[0.03,455.29]$ \\
\hline Weinblatt 2012 & $2 / 846$ & $0 / 209$ & & $12.27 \%$ & $3.48[0.11,112.96]$ \\
\hline Yamamoto (a) 2014 & $1 / 116$ & $0 / 114$ & $\bullet$ & $9.66 \%$ & $7.26[0.14,366.07]$ \\
\hline Yamamoto (b) 2014 & $0 / 82$ & $0 / 25$ & & & Not estimable \\
\hline Subtotal $(95 \% \mathrm{CI})$ & 2438 & 828 & & $60.34 \%$ & $2.1[0.44,10.08]$ \\
\hline \multicolumn{6}{|c|}{ Total events: 8 (Certolizumab pegol), 1 (Placebo) } \\
\hline \multicolumn{6}{|c|}{ Heterogeneity: Tau $^{2}=0 ; \mathrm{Chi}^{2}=1.26, \mathrm{df}=4(\mathrm{P}=0.87) ; \mathrm{I}^{2}=0 \%$} \\
\hline \multicolumn{6}{|c|}{ 55.8.2 Certolizumab pegol $400 \mathrm{mg}$} \\
\hline Choy 2012 & $0 / 124$ & $0 / 119$ & & & Not estimable \\
\hline Fleischmann 2009 & $0 / 111$ & $0 / 109$ & & & Not estimable \\
\hline Keystone 2008 & $4 / 389$ & $0 / 99$ & & $24.86 \%$ & $3.53[0.31,40.72]$ \\
\hline Smolen 2009 & $1 / 246$ & $0 / 62$ & & $6.22 \%$ & $3.5[0.03,464.09]$ \\
\hline Yamamoto (b) 2014 & $0 / 65$ & $0 / 25$ & & & Not estimable \\
\hline Subtotal $(95 \% \mathrm{Cl})$ & 935 & 414 & & $31.07 \%$ & $3.53[0.4,31.39]$ \\
\hline \multicolumn{6}{|c|}{ Total events: 5 (Certolizumab pegol), 0 (Placebo) } \\
\hline \multicolumn{6}{|c|}{ Heterogeneity: Tau $^{2}=0 ; \mathrm{Chi}^{2}=0, \mathrm{df}=1(\mathrm{P}=1) ; \mathrm{I}^{2}=0 \%$} \\
\hline \multicolumn{6}{|c|}{ Test for overall effect: $Z=1.13(P=0.26)$} \\
\hline \multicolumn{6}{|l|}{ 55.8.3 Other doses } \\
\hline Subtotal $(95 \% \mathrm{CI})$ & 96 & 34 & & $8.59 \%$ & $4.48[0.07,286.49]$ \\
\hline \multicolumn{6}{|c|}{ Total events: 1 (Certolizumab pegol), 0 (Placebo) } \\
\hline \multicolumn{6}{|c|}{ Heterogeneity: Not applicable } \\
\hline \multicolumn{6}{|c|}{ Test for overall effect: $Z=0.71(P=0.48)$} \\
\hline Total $(95 \% \mathrm{Cl})$ & 3469 & 1276 & & $100 \%$ & $2.63[0.78,8.91]$ \\
\hline \multicolumn{6}{|c|}{ Total events: 14 (Certolizumab pegol), 1 (Placebo) } \\
\hline \multicolumn{6}{|c|}{ Heterogeneity: $\operatorname{Tau}^{2}=0 ; \mathrm{Chi}^{2}=1.47, \mathrm{df}=7(\mathrm{P}=0.98) ; \mathrm{I}^{2}=0 \%$} \\
\hline \multicolumn{6}{|c|}{ Test for overall effect: $Z=1.56(P=0.12)$} \\
\hline Test for subgroup dif & $1, d f=1(P=0.9), I$ & & & & \\
\hline
\end{tabular}

Analysis 55.9. Comparison 55 Summary of findings: certolizumab (with or without MTX) versus placebo (with or without MTX), Outcome 9 Tuberculosis.

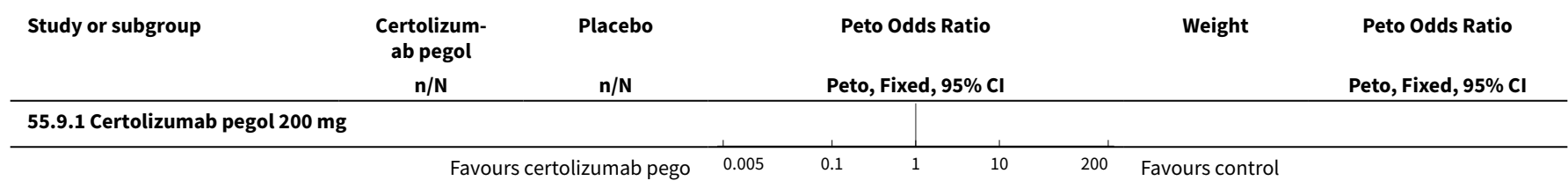




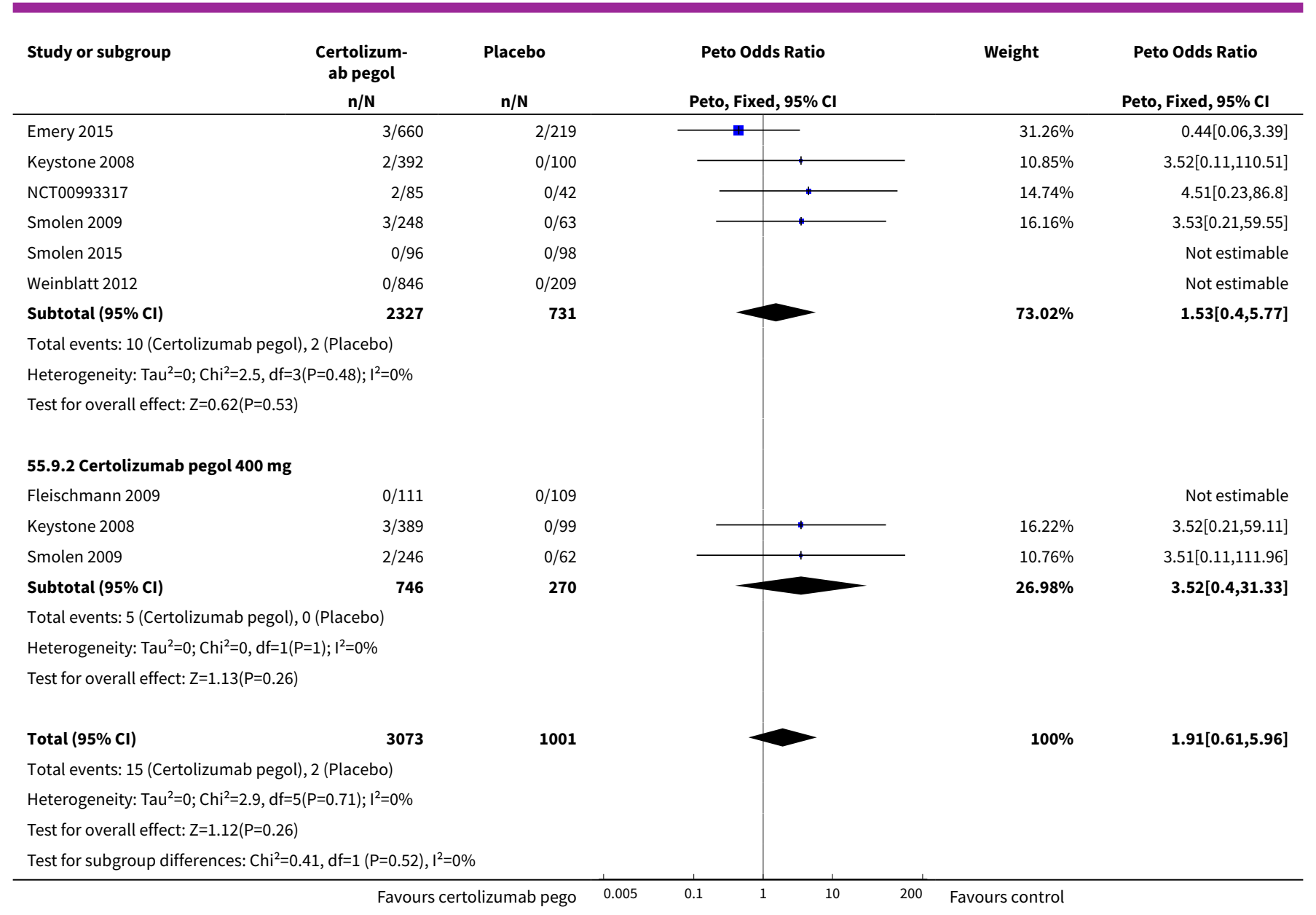

\section{Analysis 55.10. Comparison 55 Summary of findings: certolizumab (with or without MTX) versus placebo (with or without MTX), Outcome 10 Upper respiratory tract infections.}

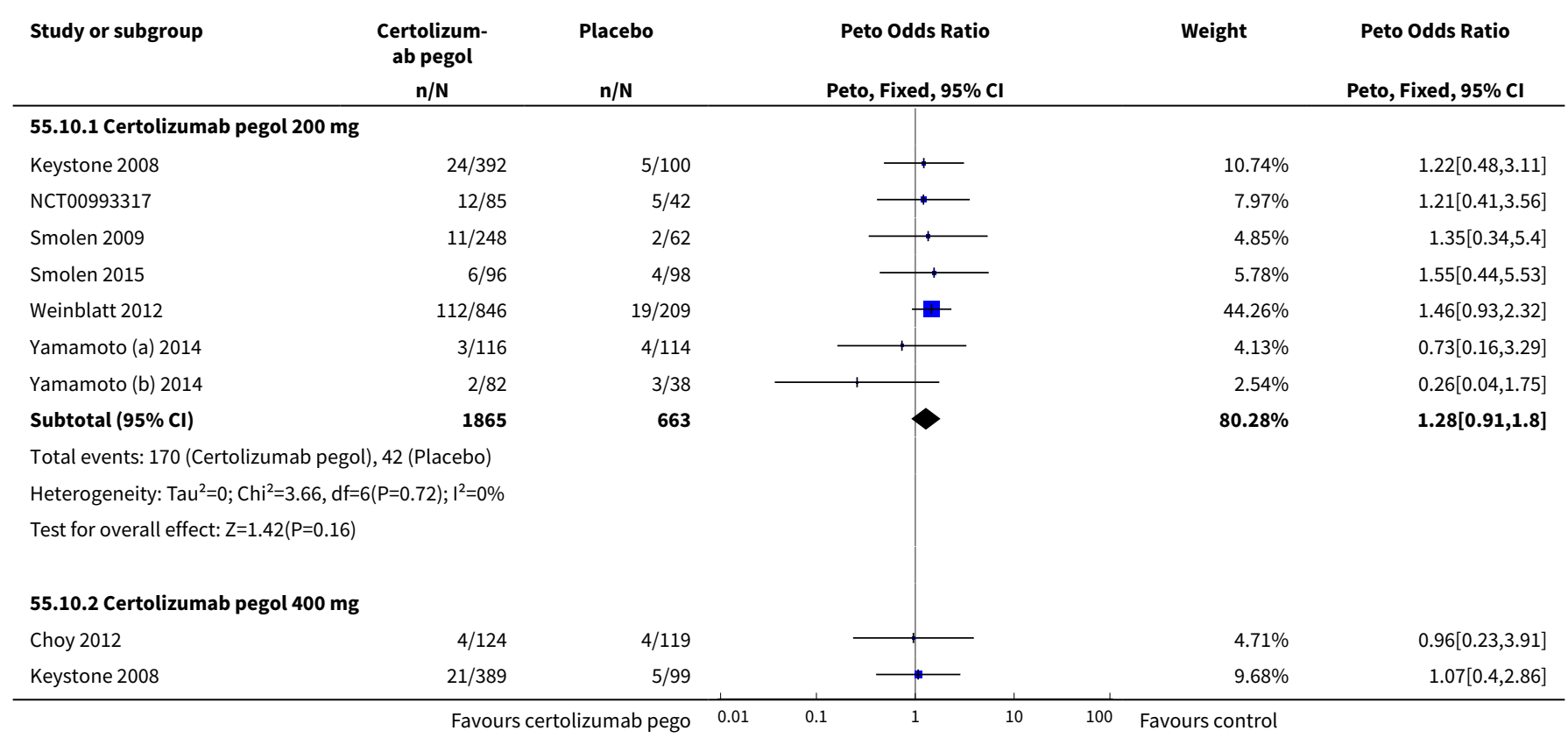




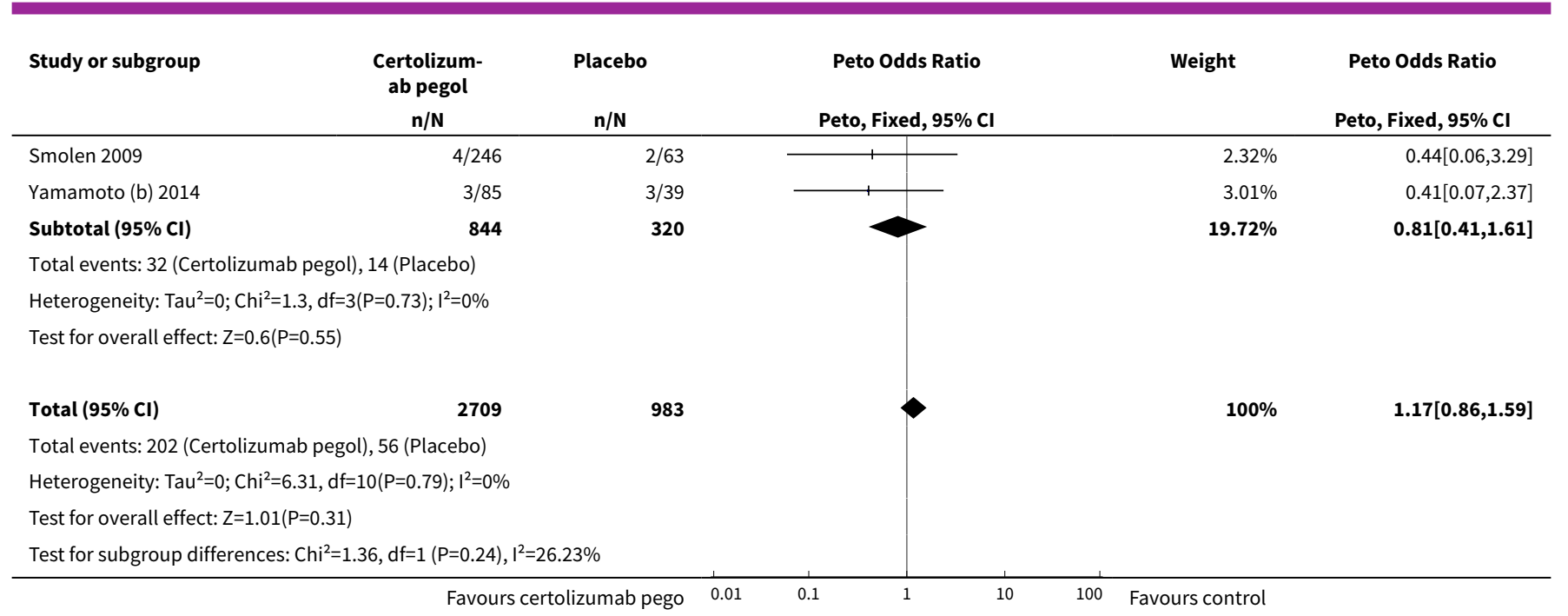

\section{Analysis 55.11. Comparison 55 Summary of findings: certolizumab (with or without MTX) versus placebo (with or without MTX), Outcome 11 Lower respiratory tract infections.}

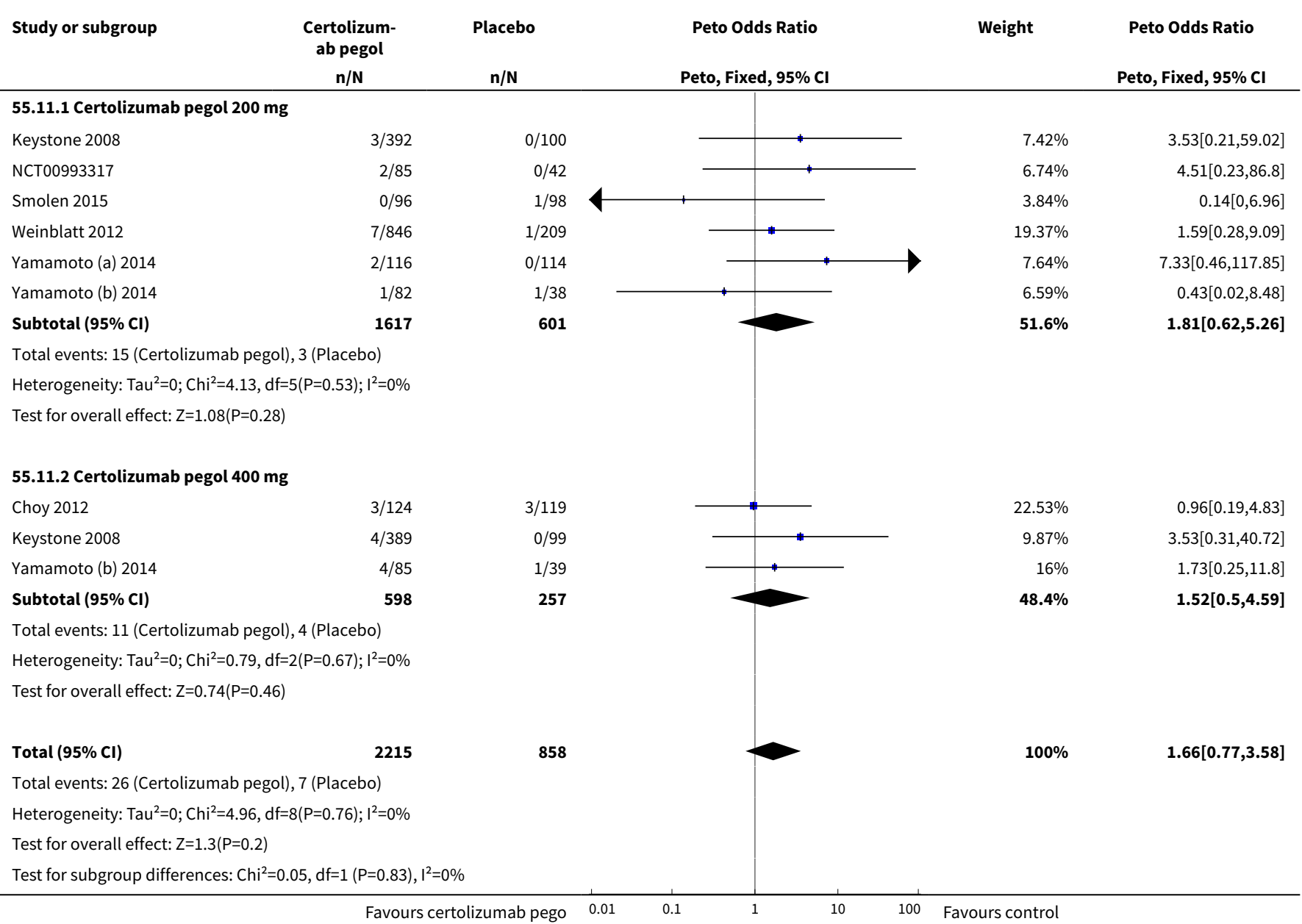




\section{Analysis 55.12. Comparison 55 Summary of findings: certolizumab (with or without MTX) versus placebo (with or without MTX), Outcome 12 Malignancies including lymphoma.}

\begin{tabular}{|c|c|c|c|c|c|}
\hline Study or subgroup & $\begin{array}{c}\text { Certolizum- } \\
\text { ab pegol } \\
n / N\end{array}$ & $\begin{array}{l}\text { Placebo } \\
\text { n/N }\end{array}$ & $\begin{array}{c}\text { Peto Odds Ratio } \\
\text { Peto, Fixed, } 95 \% \mathrm{Cl}\end{array}$ & Weight & $\begin{array}{c}\text { Peto Odds Ratio } \\
\text { Peto, Fixed, 95\% Cl }\end{array}$ \\
\hline \multicolumn{6}{|c|}{ 55.12.1 Certolizumab pegol $200 \mathrm{mg}$} \\
\hline Keystone 2008 & $7 / 392$ & $1 / 199$ & & $32.32 \%$ & $2.61[0.6,11.41]$ \\
\hline NCT00993317 & $0 / 85$ & $0 / 42$ & & & Not estimable \\
\hline Smolen 2009 & $1 / 248$ & $1 / 125$ & & $8.13 \%$ & $0.48[0.03,9.01]$ \\
\hline Weinblatt 2012 & $4 / 846$ & $2 / 209$ & & $17.36 \%$ & $0.43[0.06,3.18]$ \\
\hline Yamamoto (a) 2014 & $0 / 116$ & $1 / 114$ & & $4.58 \%$ & $0.13[0,6.7]$ \\
\hline Subtotal $(95 \% \mathrm{Cl})$ & 1783 & 787 & & $71.49 \%$ & $0.79[0.29,2.12]$ \\
\hline \multicolumn{6}{|c|}{ Total events: 12 (Certolizumab pegol), 7 (Placebo) } \\
\hline \multicolumn{6}{|c|}{ Heterogeneity: $\mathrm{Tau}^{2}=0 ; \mathrm{Chi}^{2}=5.32, \mathrm{df}=4(\mathrm{P}=0.26) ; \mathrm{I}^{2}=24.86 \%$} \\
\hline \multicolumn{6}{|c|}{ Test for overall effect: $Z=0.48(P=0.63)$} \\
\hline \multicolumn{6}{|c|}{ 55.12.2 Certolizumab pegol $400 \mathrm{mg}$} \\
\hline Fleischmann 2009 & $0 / 111$ & $0 / 109$ & & & Not estimable \\
\hline Keystone 2008 & $4 / 389$ & $1 / 199$ & & $20.35 \%$ & $1.86[0.29,11.96]$ \\
\hline Smolen 2009 & $1 / 246$ & $1 / 125$ & & $8.16 \%$ & $0.48[0.03,9.06]$ \\
\hline Subtotal $(95 \% \mathrm{Cl})$ & 746 & 433 & & $28.51 \%$ & $1.26[0.26,6.08]$ \\
\hline \multicolumn{6}{|c|}{ Total events: 5 (Certolizumab pegol), 2 (Placebo) } \\
\hline \multicolumn{6}{|c|}{ Heterogeneity: $\operatorname{Tau}^{2}=0 ; \mathrm{Chi}^{2}=0.58, \mathrm{df}=1(\mathrm{P}=0.44) ; \mathrm{I}^{2}=0 \%$} \\
\hline \multicolumn{6}{|c|}{ Test for overall effect: $Z=0.29(P=0.77)$} \\
\hline Total $(95 \% \mathrm{Cl})$ & 2529 & 1220 & & $100 \%$ & $0.9[0.39,2.08]$ \\
\hline \multicolumn{6}{|c|}{ Total events: 17 (Certolizumab pegol), 9 (Placebo) } \\
\hline \multicolumn{6}{|c|}{ Heterogeneity: $\mathrm{Tau}^{2}=0 ; \mathrm{Chi}^{2}=6.16, \mathrm{df}=6(\mathrm{P}=0.41) ; \mathrm{I}^{2}=2.59 \%$} \\
\hline \multicolumn{6}{|c|}{ Test for overall effect: $\mathrm{Z}=0.25(\mathrm{P}=0.81)$} \\
\hline
\end{tabular}

\section{Comparison 56. Analysis of sensitivity ACR50 24 weeks}

\begin{tabular}{lllll}
\hline Outcome or subgroup title & No. of studies & $\begin{array}{l}\text { No. of partici- } \\
\text { pants }\end{array}$ & Statistical method & Effect size \\
\hline 1 Doses & 8 & 3768 & $\begin{array}{l}\text { Risk Ratio (M-H, Random, 95\% } \\
\text { Cl) }\end{array}$ & $2.89[2.38,3.51]$ \\
\hline 1.1 certolizumab 100 mg sc & 1 & 98 & $\begin{array}{l}\text { Risk Ratio (M-H, Random, 95\% } \\
\text { Cl) }\end{array}$ & $2.89[1.13,7.38]$ \\
\hline 1.2 certolizumab 200 mg sc & 6 & 2295 & $\begin{array}{l}\text { Risk Ratio (M-H, Random, 95\% } \\
\text { Cl) }\end{array}$ & $2.73[2.13,3.51]$ \\
\hline 1.3 certolizumab 400 mg sc & 5 & 1375 & $\begin{array}{l}\text { Risk Ratio (M-H, Random, 95\% } \\
\text { Cl) }\end{array}$ & $3.18[2.29,4.41]$ \\
\hline 2 Size & 8 & 3768 & $\begin{array}{l}\text { Risk Ratio (M-H, Random, 95\% } \\
\text { Cl) }\end{array}$ & $2.89[2.38,3.51]$ \\
\hline
\end{tabular}




\begin{tabular}{|c|c|c|c|c|}
\hline Outcome or subgroup title & No. of studies & $\begin{array}{l}\text { No. of partici- } \\
\text { pants }\end{array}$ & Statistical method & Effect size \\
\hline 2.1 certolizumab $<200$ patients & 2 & 321 & $\begin{array}{l}\text { Risk Ratio (M-H, Random, 95\% } \\
\mathrm{CI})\end{array}$ & $2.44[1.45,4.10]$ \\
\hline 2.2 certolizumab $>200$ patients & 6 & 3447 & $\begin{array}{l}\text { Risk Ratio (M-H, Random, 95\% } \\
\mathrm{CI} \text { ) }\end{array}$ & $2.97[2.41,3.67]$ \\
\hline 3 Use of MTX & 8 & 3768 & $\begin{array}{l}\text { Risk Ratio (M-H, Random, 95\% } \\
\mathrm{Cl})\end{array}$ & $2.89[2.38,3.51]$ \\
\hline 3.1 With MTX & 5 & 3038 & $\begin{array}{l}\text { Risk Ratio (M-H, Random, 95\% } \\
\mathrm{Cl} \text { ) }\end{array}$ & $2.77[2.21,3.46]$ \\
\hline 3.2 Without MTX & 3 & 730 & $\begin{array}{l}\text { Risk Ratio (M-H, Random, 95\% } \\
\mathrm{Cl})\end{array}$ & $3.32[2.23,4.95]$ \\
\hline 4 Population & 8 & 3768 & $\begin{array}{l}\text { Risk Ratio (M-H, Random, 95\% } \\
\mathrm{Cl})\end{array}$ & $2.89[2.38,3.51]$ \\
\hline 4.1 Asian trials & 2 & 443 & $\begin{array}{l}\text { Risk Ratio (M-H, Random, 95\% } \\
\mathrm{Cl} \text { ) }\end{array}$ & $2.66[1.77,4.00]$ \\
\hline 4.2 Other trials & 6 & 3325 & $\begin{array}{l}\text { Risk Ratio (M-H, Random, 95\% } \\
\mathrm{Cl} \text { ) }\end{array}$ & $2.96[2.37,3.70]$ \\
\hline 5 Duration of previous disease & 6 & 3258 & $\begin{array}{l}\text { Risk Ratio (M-H, Random, 95\% } \\
\mathrm{Cl} \text { ) }\end{array}$ & $2.87[2.31,3.57]$ \\
\hline $\begin{array}{l}5.1 \text { Long previous disease duration } \\
\text { (9 years or more) }\end{array}$ & 2 & 467 & $\begin{array}{l}\text { Risk Ratio (M-H, Random, 95\% } \\
\mathrm{Cl} \text { ) }\end{array}$ & $4.02[2.02,7.98]$ \\
\hline $\begin{array}{l}5.2 \text { Short previous disease dura- } \\
\text { tion (less than } 7 \text { years) }\end{array}$ & 4 & 2791 & $\begin{array}{l}\text { Risk Ratio (M-H, Random, 95\% } \\
\mathrm{Cl} \text { ) }\end{array}$ & $2.75[2.18,3.47]$ \\
\hline $\begin{array}{l}6 \text { Published vs unpublished stud- } \\
\text { ies }\end{array}$ & 8 & 3768 & $\begin{array}{l}\text { Risk Ratio (M-H, Random, 95\% } \\
\mathrm{Cl})\end{array}$ & $2.89[2.38,3.51]$ \\
\hline 6.1 Published studies & 5 & 3131 & $\begin{array}{l}\text { Risk Ratio (M-H, Random, 95\% } \\
\mathrm{CI})\end{array}$ & $2.97[2.36,3.73]$ \\
\hline 6.2 Unpublished studies & 3 & 637 & $\begin{array}{l}\text { Risk Ratio (M-H, Random, 95\% } \\
\mathrm{Cl})\end{array}$ & $2.71[1.89,3.90]$ \\
\hline $\begin{array}{l}7 \text { Imputing to ACR50 } 200 \mathrm{mg} \text { from } \\
24 \text { missing values with same pro- } \\
\text { portion as reported outcomes }\end{array}$ & 5 & 1445 & Risk Ratio (M-H, Fixed, 95\% Cl) & $3.34[2.68,4.17]$ \\
\hline $\begin{array}{l}7.1 \text { Imputing missing values with } \\
\text { same proportion as reported out- } \\
\text { comes }\end{array}$ & 5 & 1445 & Risk Ratio (M-H, Fixed, 95\% Cl) & $3.34[2.68,4.17]$ \\
\hline $\begin{array}{l}8 \text { Imputing to ACR50 } 200 \text { mg from } \\
24 \text { weeks } 50 \% \text { of missing out- } \\
\text { comes }\end{array}$ & 5 & 1445 & Risk Ratio (M-H, Fixed, 95\% Cl) & $1.17[1.04,1.32]$ \\
\hline
\end{tabular}




\begin{tabular}{lllll}
\hline Outcome or subgroup title & No. of studies & $\begin{array}{l}\text { No. of partici- } \\
\text { pants }\end{array}$ & Statistical method & Effect size \\
\hline $\begin{array}{l}8.1 \text { Imputing the } 50 \% \text { of missing } \\
\text { outcomes }\end{array}$ & 5 & 1445 & Risk Ratio (M-H, Fixed, 95\% Cl) & 1.17 [1.04, 1.32] \\
\hline $\begin{array}{l}9 \text { Imputing to ACR50 } 200 \mathrm{mg} \text { from } \\
24 \text { weeks: the worst case }\end{array}$ & 5 & 1445 & Risk Ratio (M-H, Fixed, 95\% Cl) & $0.47[0.43,0.52]$ \\
\hline $\begin{array}{l}\text { 9.1 Analysis in the worst case. } \\
\begin{array}{l}\text { All missing values did not reach } \\
\text { ACR50 in certolizumab group and } \\
\text { did in placebo group }\end{array}\end{array}$ & 5 & 1445 & Risk Ratio (M-H, Fixed, 95\% Cl) & $0.47[0.43,0.52]$ \\
\hline
\end{tabular}

Analysis 56.1. Comparison 56 Analysis of sensitivity ACR50 24 weeks, Outcome 1 Doses.

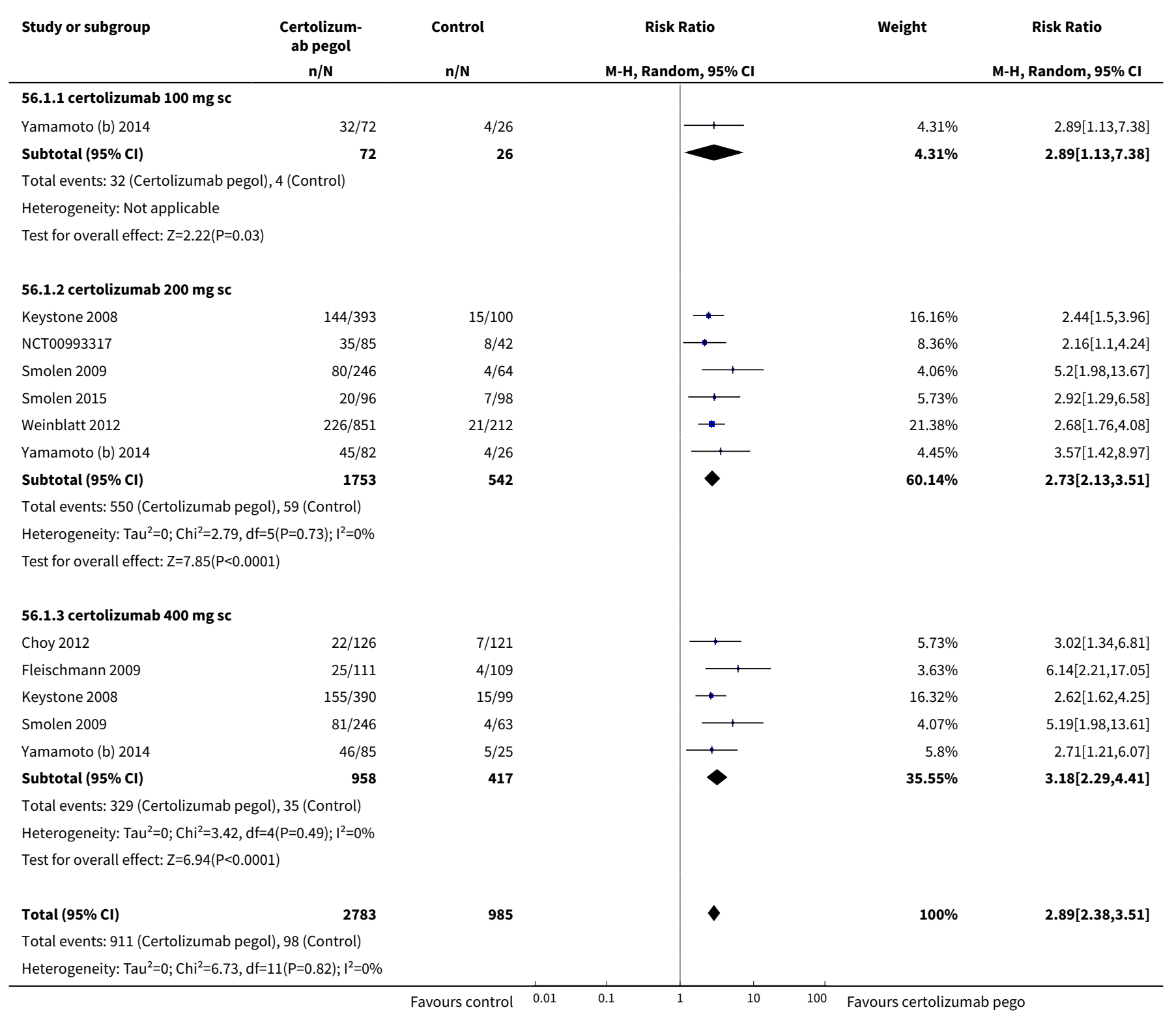




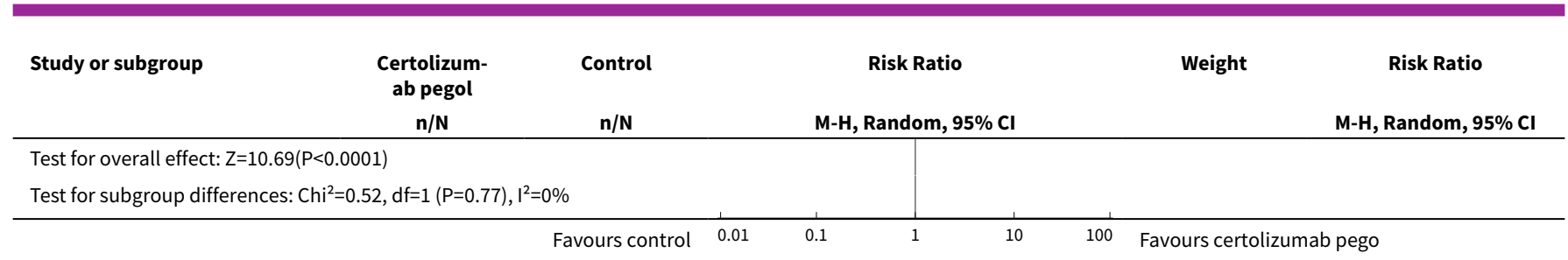

Analysis 56.2. Comparison 56 Analysis of sensitivity ACR50 24 weeks, Outcome 2 Size.

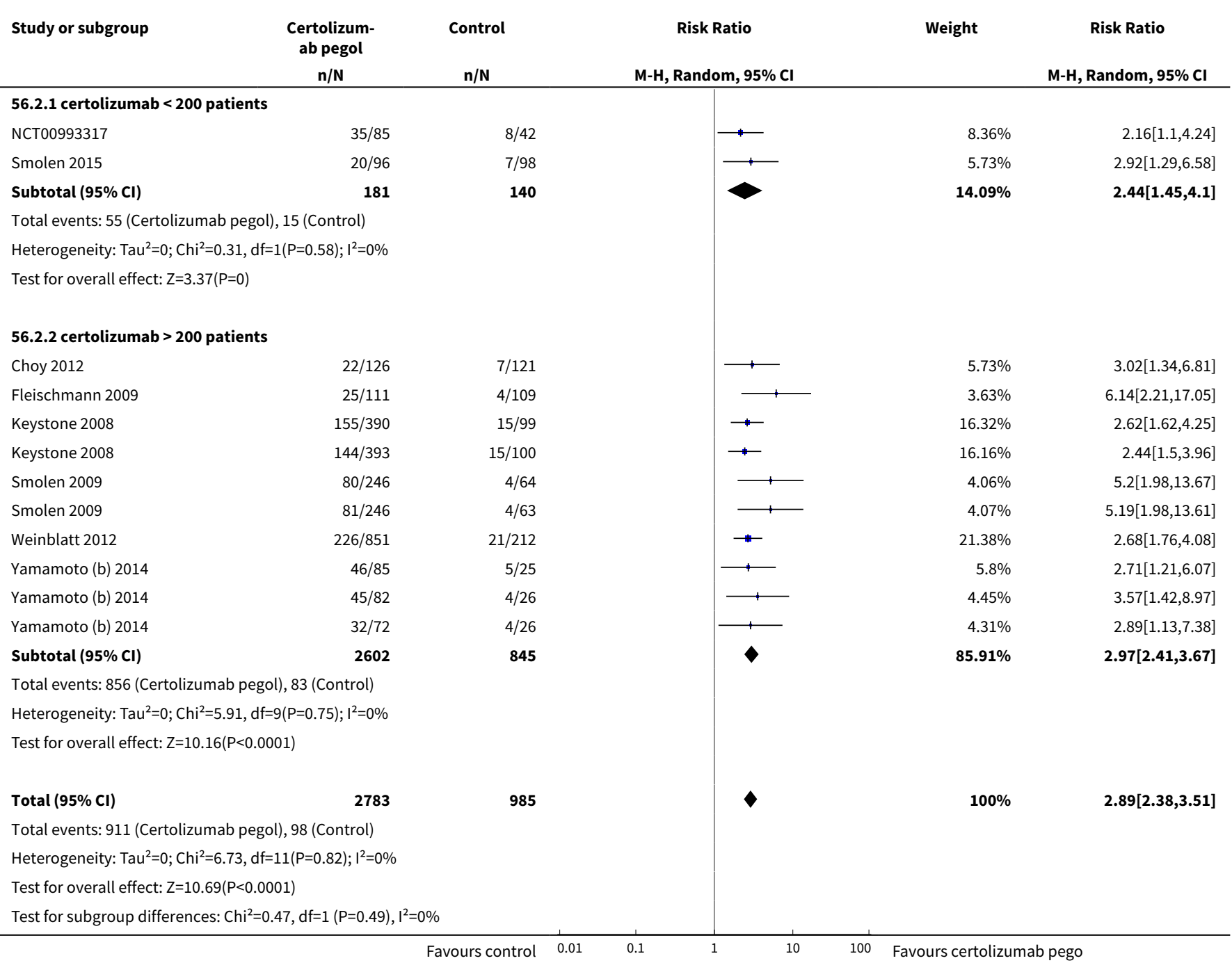

Analysis 56.3. Comparison 56 Analysis of sensitivity ACR50 24 weeks, Outcome 3 Use of MTX.

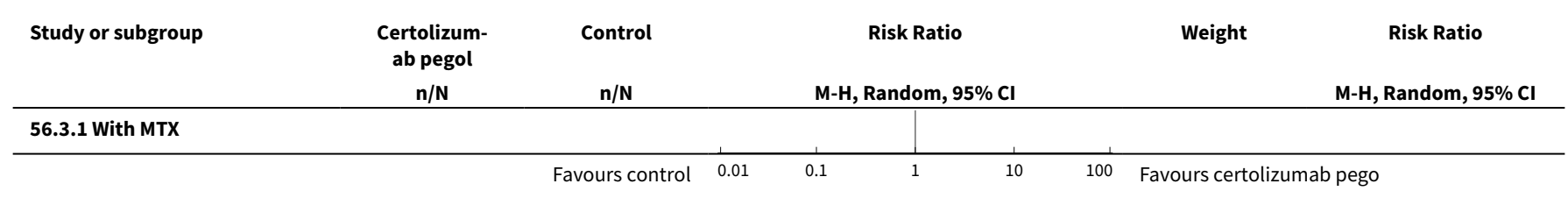




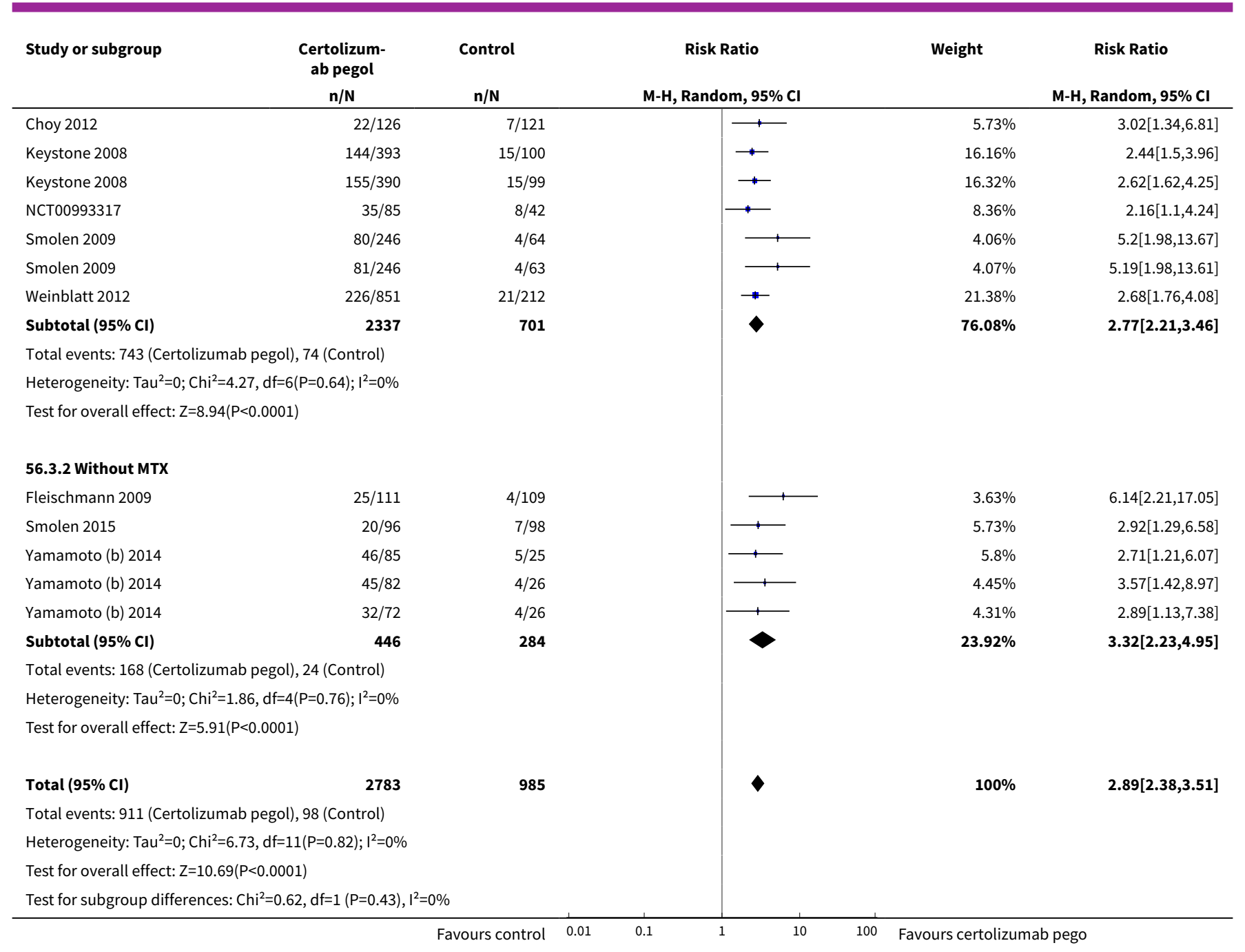

Analysis 56.4. Comparison 56 Analysis of sensitivity ACR50 24 weeks, Outcome 4 Population.

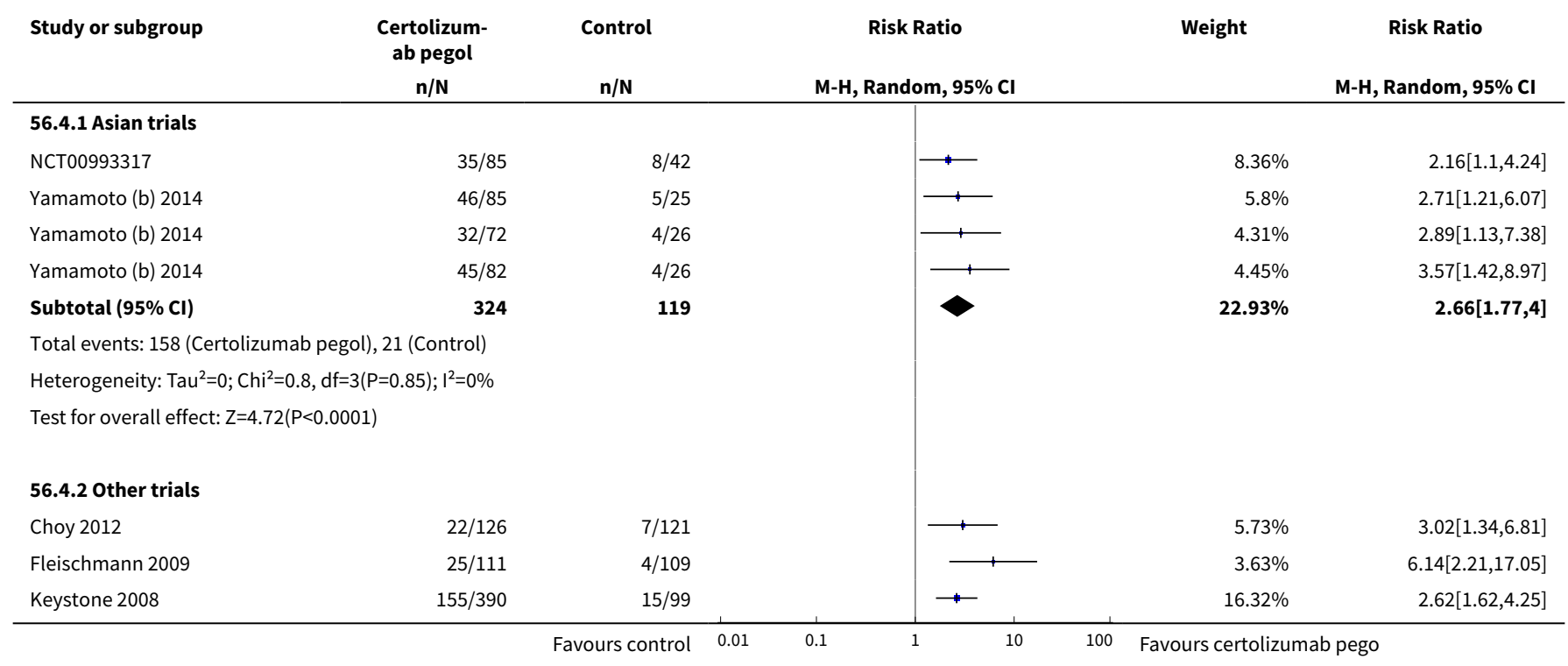




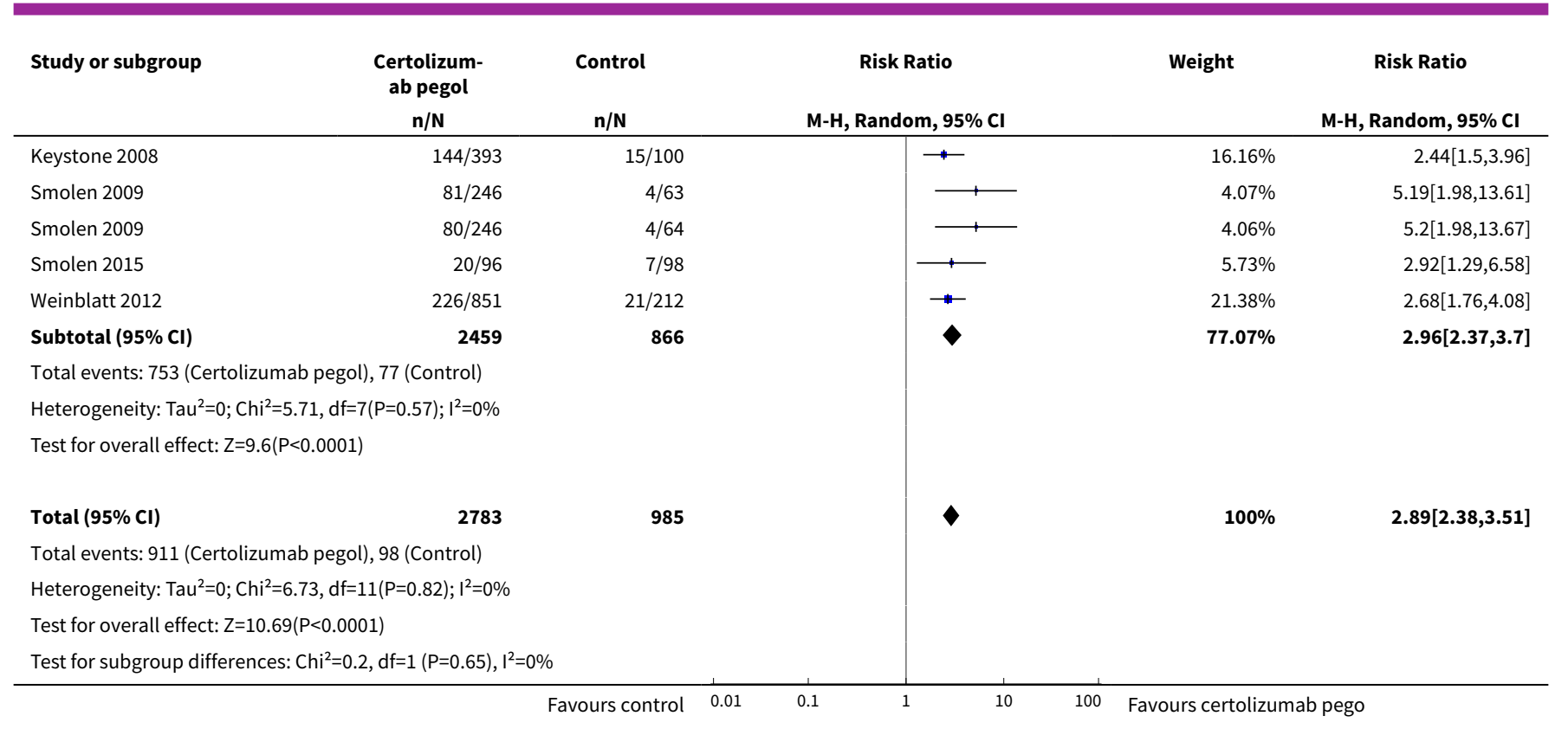

\section{Analysis 56.5. Comparison 56 Analysis of sensitivity ACR50 24 weeks, Outcome 5 Duration of previous disease.}

\begin{tabular}{|c|c|c|c|c|c|}
\hline Study or subgroup & $\begin{array}{c}\text { Certolizum- } \\
\text { ab pegol } \\
n / N\end{array}$ & $\begin{array}{c}\text { Control } \\
n / N\end{array}$ & $\begin{array}{c}\text { Risk Ratio } \\
\text { M-H, Random, } 95 \% \mathrm{CI}\end{array}$ & Weight & $\begin{array}{c}\text { Risk Ratio } \\
\text { M-H, Random, } 95 \% \mathrm{Cl} \\
\end{array}$ \\
\hline \multicolumn{3}{|c|}{ 56.5.1 Long previous disease duration (9 years or more) } & & & \\
\hline Choy 2012 & $22 / 126$ & $7 / 121$ & $\longrightarrow$ & $7.19 \%$ & $3.02[1.34,6.81]$ \\
\hline Fleischmann 2009 & $25 / 111$ & $4 / 109$ & $\longrightarrow$ & $4.55 \%$ & $6.14[2.21,17.05]$ \\
\hline Subtotal $(95 \% \mathrm{CI})$ & 237 & 230 & & $11.75 \%$ & $4.02[2.02,7.98]$ \\
\hline \multicolumn{6}{|c|}{ Heterogeneity: $\mathrm{Tau}^{2}=0.03 ; \mathrm{Chi}^{2}=1.15, \mathrm{df}=1(\mathrm{P}=0.28) ; \mathrm{I}^{2}=13.16 \%$} \\
\hline \multicolumn{6}{|c|}{ Test for overall effect: $Z=3.97(P<0.0001)$} \\
\hline \multicolumn{6}{|c|}{ 56.5.2 Short previous disease duration (less than 7 years) } \\
\hline Keystone 2008 & $144 / 393$ & $15 / 100$ & $\rightarrow-$ & $20.27 \%$ & $2.44[1.5,3.96]$ \\
\hline Keystone 2008 & $155 / 390$ & $15 / 99$ & $\rightarrow$ & $20.47 \%$ & $2.62[1.62,4.25]$ \\
\hline Smolen 2009 & $80 / 246$ & $4 / 64$ & $\longrightarrow$ & $5.1 \%$ & $5.2[1.98,13.67]$ \\
\hline Smolen 2009 & $81 / 246$ & $4 / 63$ & $\longrightarrow$ & $5.11 \%$ & $5.19[1.98,13.61]$ \\
\hline Weinblatt 2012 & $226 / 851$ & $21 / 212$ & $\rightarrow-$ & $26.82 \%$ & $2.68[1.76,4.08]$ \\
\hline Subtotal $(95 \% \mathrm{Cl})$ & 2211 & 580 & $>$ & $88.25 \%$ & $2.75[2.18,3.47]$ \\
\hline \multicolumn{6}{|c|}{ Total events: 721 (Certolizumab pegol), 67 (Control) } \\
\hline \multicolumn{6}{|c|}{ Heterogeneity: $\operatorname{Tau}^{2}=0 ; \mathrm{Chi}^{2}=4.23, \mathrm{df}=5(\mathrm{P}=0.52) ; \mathrm{I}^{2}=0 \%$} \\
\hline \multicolumn{6}{|c|}{ Test for overall effect: $Z=8.53(P<0.0001)$} \\
\hline Total $(95 \% \mathrm{Cl})$ & 2448 & 810 & $\boldsymbol{\gamma}$ & $100 \%$ & $2.87[2.31,3.57]$ \\
\hline \multicolumn{6}{|c|}{ Total events: 768 (Certolizumab pegol), 78 (Control) } \\
\hline \multicolumn{6}{|c|}{ Heterogeneity: $\operatorname{Tau}^{2}=0 ; \mathrm{Chi}^{2}=6.52, \mathrm{df}=7(\mathrm{P}=0.48) ; \mathrm{I}^{2}=0 \%$} \\
\hline \multicolumn{6}{|c|}{ Test for overall effect: $Z=9.47(P<0.0001)$} \\
\hline \multicolumn{6}{|c|}{ Test for subgroup differences: $\mathrm{Chi}^{2}=1.05, \mathrm{df}=1(\mathrm{P}=0.3), \mathrm{I}^{2}=4.99 \%$} \\
\hline
\end{tabular}


Analysis 56.6. Comparison 56 Analysis of sensitivity ACR50 24 weeks, Outcome 6 Published vs unpublished studies.

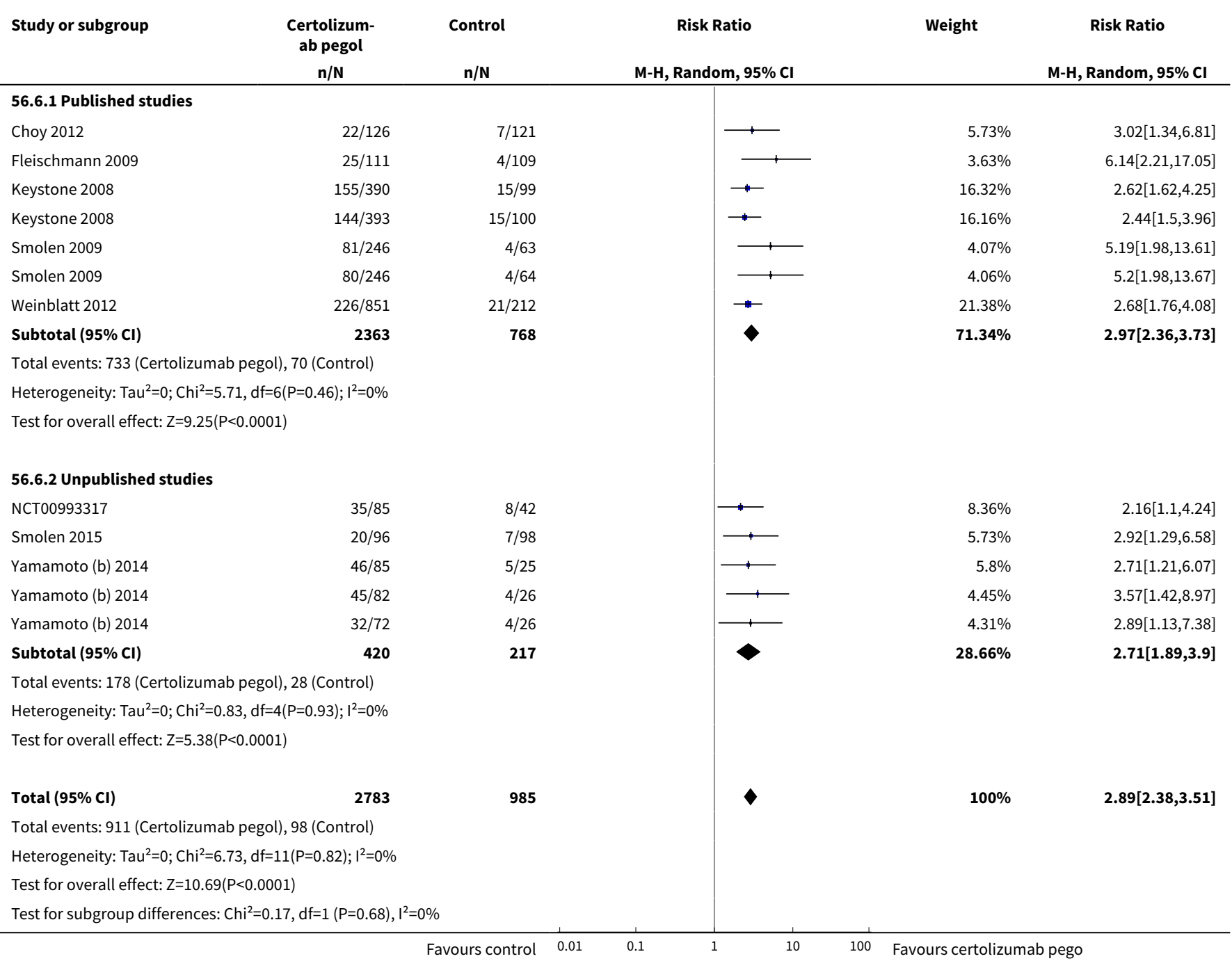

\section{Analysis 56.7. Comparison 56 Analysis of sensitivity ACR50 24 weeks, Outcome 7 Imputing to ACR50 $200 \mathrm{mg}$ from 24 missing values with same proportion as reported outcomes.}

Study or subgroup

Certolizum- Control
ab pegol

Risk Ratio

Weight

Risk Ratio

$n / \mathrm{N}$

$\mathbf{n} / \mathbf{N}$

M-H, Fixed, $95 \% \mathrm{CI}$

M-H, Fixed, $95 \% \mathrm{Cl}$

$\begin{array}{lrr}\begin{array}{l}\text { 56.7.1 Imputing missing values with same proportion as reported out- } \\ \text { comes }\end{array} & \\ \text { Keystone } 2008 & 195 / 393 & 27 / 199 \\ \text { NCT00993317 } & 45 / 85 & 12 / 42 \\ \text { Smolen } 2009 & 103 / 246 & 7 / 127 \\ \text { Smolen } 2015 & 23 / 96 & 8 / 98 \\ \text { Yamamoto (b) 2014 } & 54 / 82 & 22 / 77 \\ \text { Subtotal (95\% Cl) } & \mathbf{9 0 2} & \mathbf{5 4 3}\end{array}$

Subtotal $(95 \% \mathrm{Cl})$

902

543

$39.07 \%$

$17.51 \%$

$10.06 \%$

$8.63 \%$

$24.73 \%$

$100 \%$
$3.66[2.54,5.27]$

$1.85[1.1,3.11]$

$7.6[3.64,15.84]$

$2.93[1.38,6.24]$

$2.3[1.57,3.39]$

$3.34[2.68,4.17]$

Total events: 420 (Certolizumab pegol), 76 (Control)

Heterogeneity: Tau $^{2}=0 ; \mathrm{Chi}^{2}=13.67, \mathrm{df}=4(\mathrm{P}=0.01) ; \mathrm{I}^{2}=70.73 \%$ 


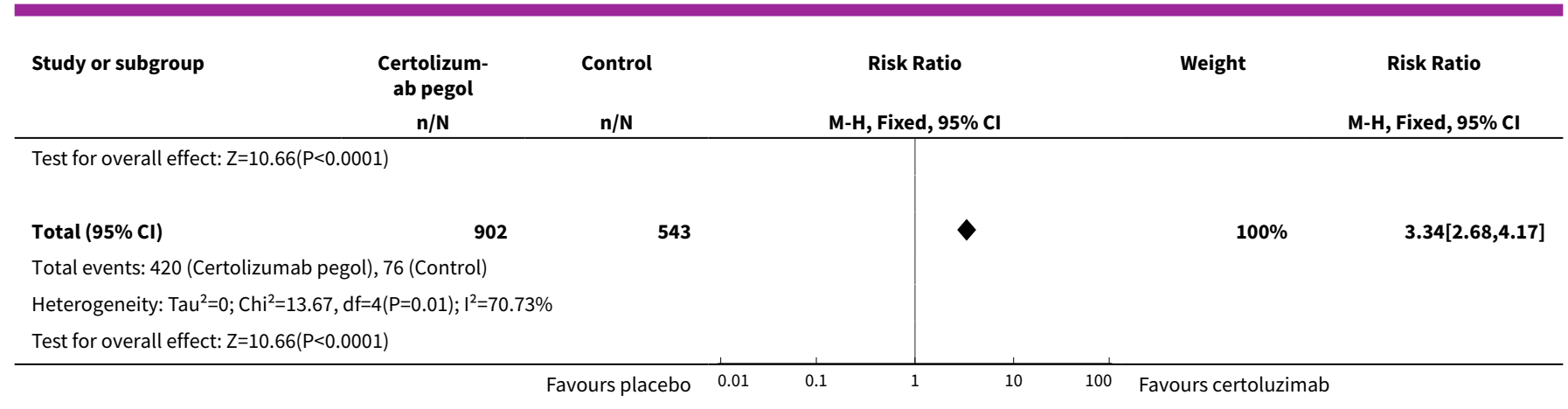

Analysis 56.8. Comparison 56 Analysis of sensitivity ACR50 24 weeks, Outcome 8 Imputing to ACR50 $200 \mathrm{mg}$ from 24 weeks $50 \%$ of missing outcomes.

\begin{tabular}{|c|c|c|c|c|c|}
\hline Study or subgroup & $\begin{array}{c}\text { Certolizum- } \\
\text { ab pegol } \\
n / N\end{array}$ & $\begin{array}{c}\text { Control } \\
n / N\end{array}$ & $\begin{array}{c}\text { Risk Ratio } \\
\text { M-H, Fixed, } 95 \% \mathrm{Cl}\end{array}$ & Weight & $\begin{array}{c}\text { Risk Ratio } \\
\text { M-H, Fixed, 95\% Cl }\end{array}$ \\
\hline \multicolumn{6}{|c|}{ 56.8.1 Imputing the $50 \%$ of missing outcomes } \\
\hline Keystone 2008 & $213 / 393$ & $93 / 199$ & & $43.66 \%$ & $1.16[0.97,1.38]$ \\
\hline NCT00993317 & $48 / 85$ & $19 / 42$ & & $8.99 \%$ & $1.25[0.85,1.83]$ \\
\hline Smolen 2009 & $116 / 246$ & $59 / 127$ & 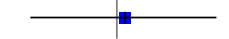 & $27.52 \%$ & $1.02[0.81,1.28]$ \\
\hline Yamamoto (b) 2014 & $53 / 82$ & $39 / 77$ & & $14.22 \%$ & $1.28[0.97,1.68]$ \\
\hline Subtotal $(95 \% \mathrm{Cl})$ & 902 & 543 & & $100 \%$ & $1.17[1.04,1.32]$ \\
\hline \multicolumn{6}{|c|}{ Total events: 456 (Certolizumab pegol), 226 (Control) } \\
\hline \multicolumn{6}{|c|}{ Heterogeneity: $\operatorname{Tau}^{2}=0 ; \mathrm{Chi}^{2}=3.51, \mathrm{df}=4(\mathrm{P}=0.48) ; \mathrm{I}^{2}=0 \%$} \\
\hline \multicolumn{6}{|c|}{ Test for overall effect: $Z=2.69(P=0.01)$} \\
\hline \multicolumn{6}{|c|}{ Total events: 456 (Certolizumab pegol), 226 (Control) } \\
\hline \multicolumn{6}{|c|}{ Heterogeneity: $\operatorname{Tau}^{2}=0 ; \mathrm{Chi}^{2}=3.51, \mathrm{df}=4(\mathrm{P}=0.48) ; \mathrm{I}^{2}=0 \%$} \\
\hline Test for overall effec & & & & & \\
\hline
\end{tabular}

Analysis 56.9. Comparison 56 Analysis of sensitivity ACR50 24 weeks, Outcome 9 Imputing to ACR50 200 mg from 24 weeks: the worst case.

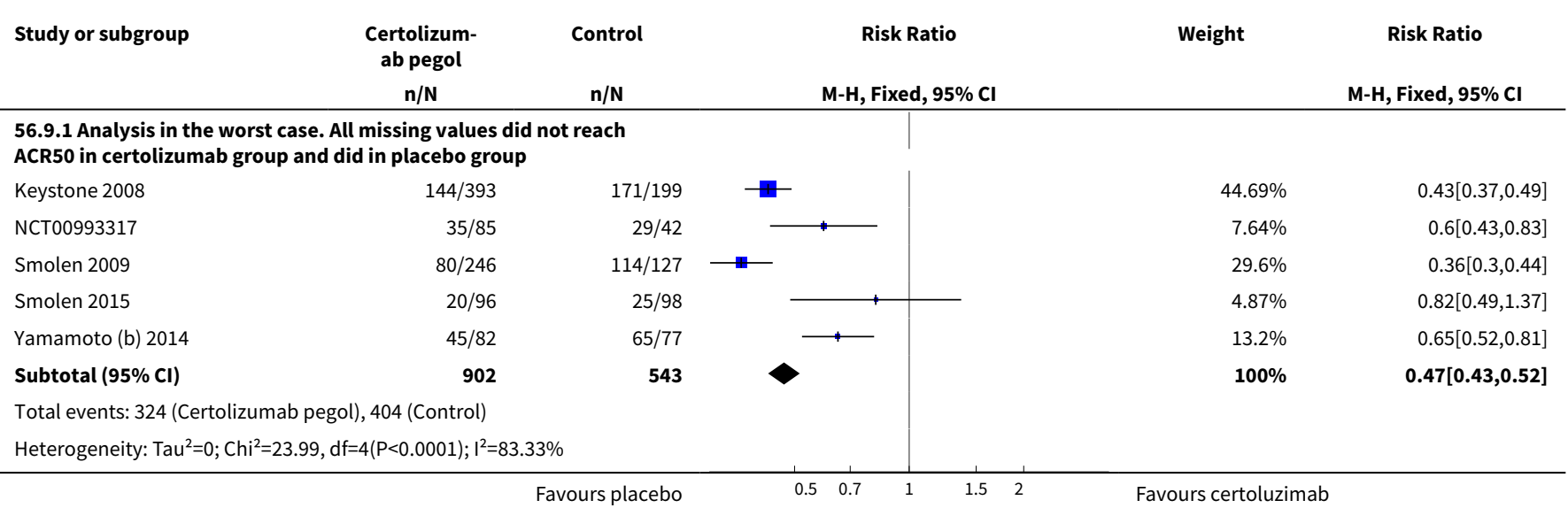




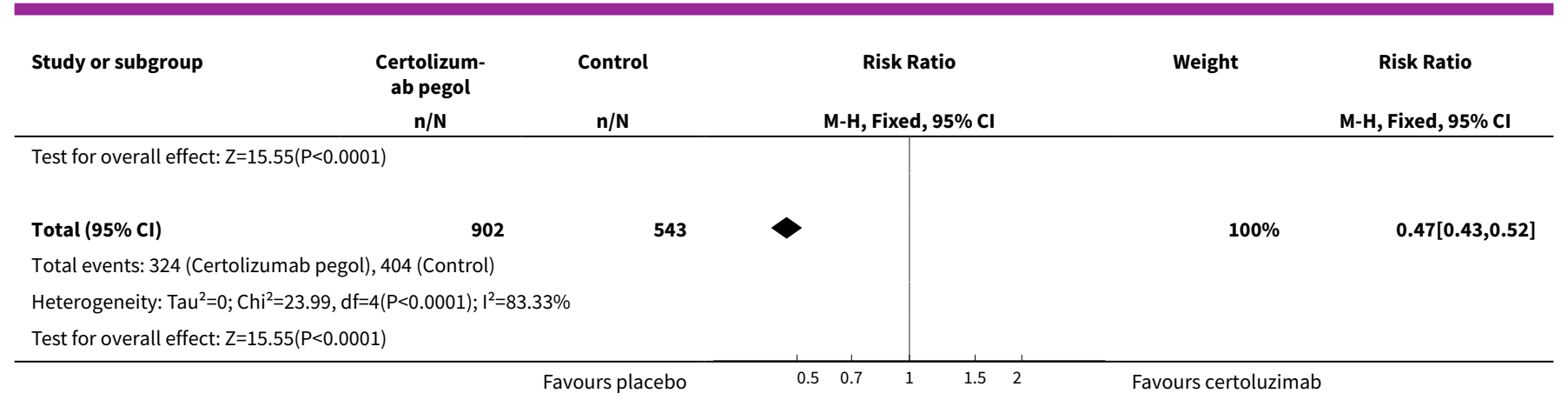

\section{Comparison 57. Analysis of sensitivity ACR50 52 weeks}

\begin{tabular}{|c|c|c|c|c|}
\hline $\begin{array}{l}\text { Outcome or subgroup ti- } \\
\text { tle }\end{array}$ & No. of studies & $\begin{array}{l}\text { No. of partici- } \\
\text { pants }\end{array}$ & Statistical method & Effect size \\
\hline 1 Doses & 3 & 2180 & Risk Ratio (M-H, Random, 95\% Cl) & $1.69[1.22,2.33]$ \\
\hline $\begin{array}{l}1.1 \text { certolizumab } 200 \mathrm{mg} \\
\mathrm{sc}\end{array}$ & 3 & 1691 & Risk Ratio (M-H, Random, 95\% Cl) & $1.48[1.11,1.96]$ \\
\hline $\begin{array}{l}1.2 \text { certolizumab } 400 \mathrm{mg} \\
\mathrm{sc}\end{array}$ & 1 & 489 & Risk Ratio (M-H, Random, 95\% Cl) & $2.62[1.62,4.25]$ \\
\hline 2 Size & 3 & 2180 & Risk Ratio (M-H, Random, 95\% Cl) & $1.69[1.22,2.33]$ \\
\hline $\begin{array}{l}2.1 \text { certolizumab }<200 \text { pa- } \\
\text { tients }\end{array}$ & 0 & 0 & Risk Ratio (M-H, Random, 95\% Cl) & $0.0[0.0,0.0]$ \\
\hline $\begin{array}{l}2.2 \text { certolizumab }>200 \text { pa- } \\
\text { tients }\end{array}$ & 3 & 2180 & Risk Ratio (M-H, Random, 95\% Cl) & $1.69[1.22,2.33]$ \\
\hline 3 Use of MTX & 3 & 2180 & Risk Ratio (M-H, Random, 95\% Cl) & $1.69[1.22,2.33]$ \\
\hline 3.1 Use of MTX & 3 & 2180 & Risk Ratio (M-H, Random, 95\% Cl) & $1.69[1.22,2.33]$ \\
\hline 3.2 Without MTX & 0 & 0 & Risk Ratio (M-H, Random, 95\% Cl) & $0.0[0.0,0.0]$ \\
\hline 4 Population & 3 & 2180 & Risk Ratio (M-H, Random, 95\% Cl) & $1.69[1.22,2.33]$ \\
\hline 4.1 Asian trials & 1 & 319 & Risk Ratio (M-H, Random, 95\% Cl) & $1.41[1.17,1.68]$ \\
\hline 4.2 Other trials & 2 & 1861 & Risk Ratio (M-H, Random, 95\% Cl) & $1.94[1.01,3.72]$ \\
\hline $\begin{array}{l}5 \text { Duration of previous dis- } \\
\text { ease }\end{array}$ & 3 & 2180 & Risk Ratio (M-H, Random, 95\% Cl) & $1.69[1.22,2.33]$ \\
\hline $\begin{array}{l}5.1 \text { Long previous disease } \\
\text { duration ( } 6 \text { years or more) }\end{array}$ & 1 & 982 & Risk Ratio (M-H, Random, 95\% Cl) & $2.58[1.83,3.62]$ \\
\hline $\begin{array}{l}5.2 \text { Short previous disease } \\
\text { duration (less than } 1 \text { year) }\end{array}$ & 2 & 1198 & Risk Ratio (M-H, Random, 95\% Cl) & $1.29[1.10,1.50]$ \\
\hline
\end{tabular}


Analysis 57.1. Comparison 57 Analysis of sensitivity ACR50 52 weeks, Outcome 1 Doses.

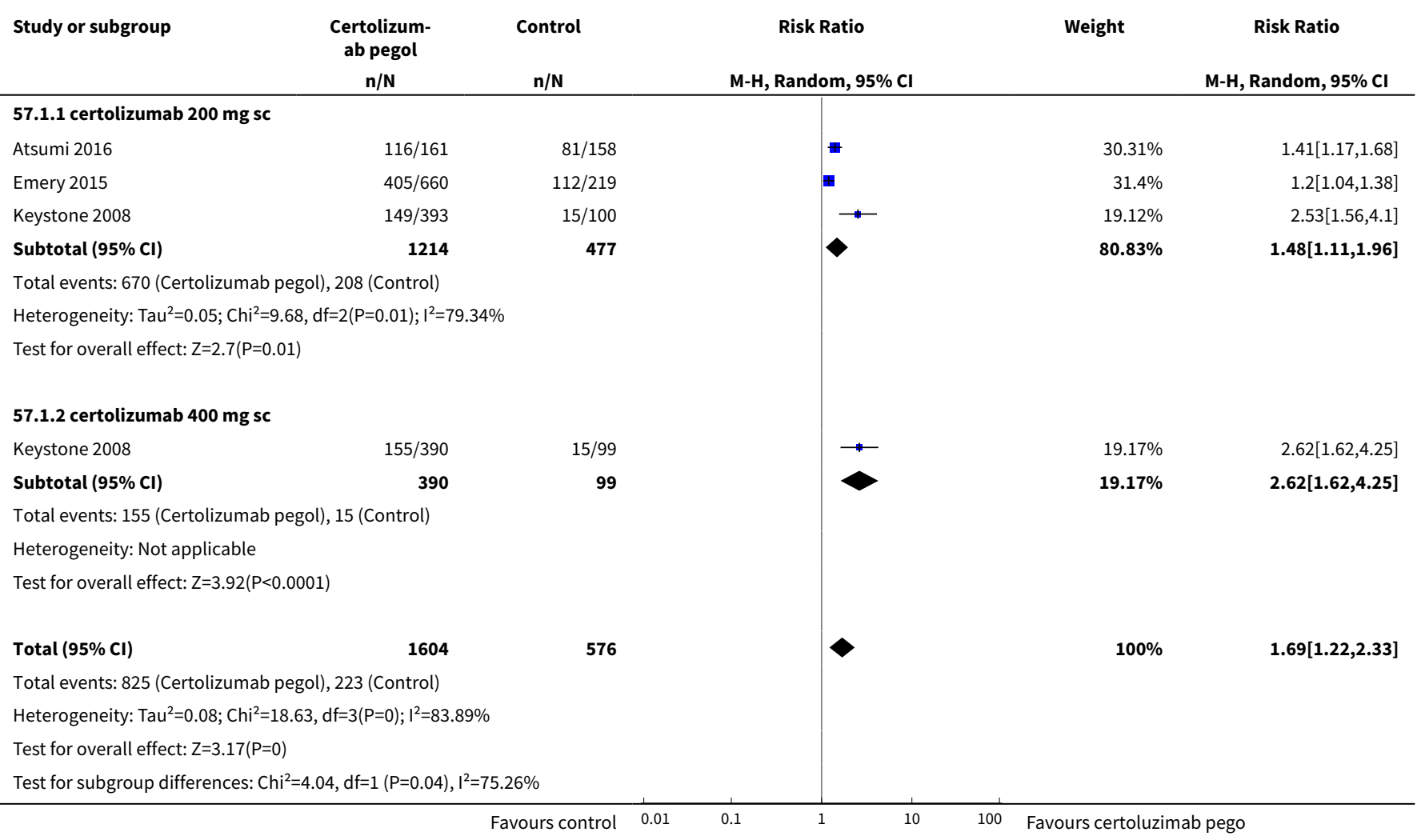

Analysis 57.2. Comparison 57 Analysis of sensitivity ACR50 52 weeks, Outcome 2 Size.

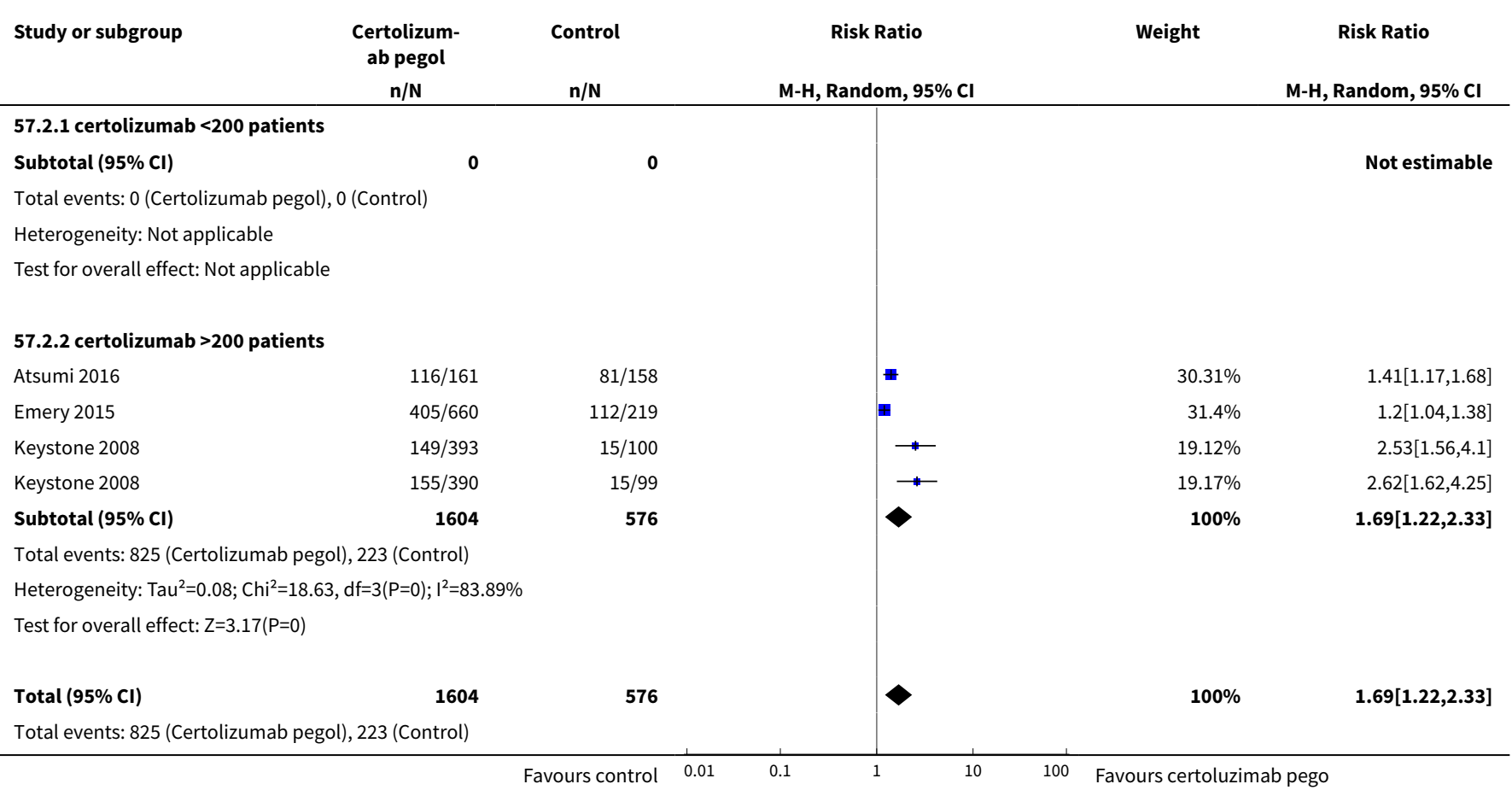




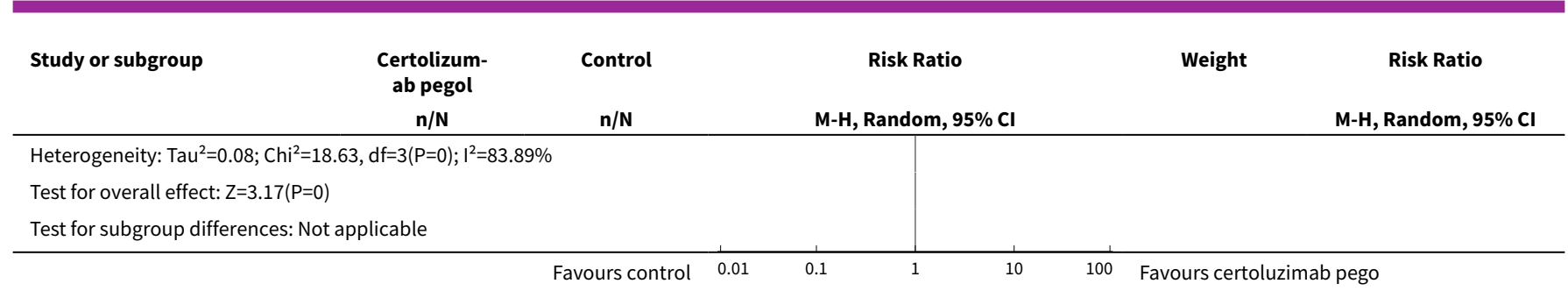

\section{Analysis 57.3. Comparison 57 Analysis of sensitivity ACR50 52 weeks, Outcome 3 Use of MTX.}

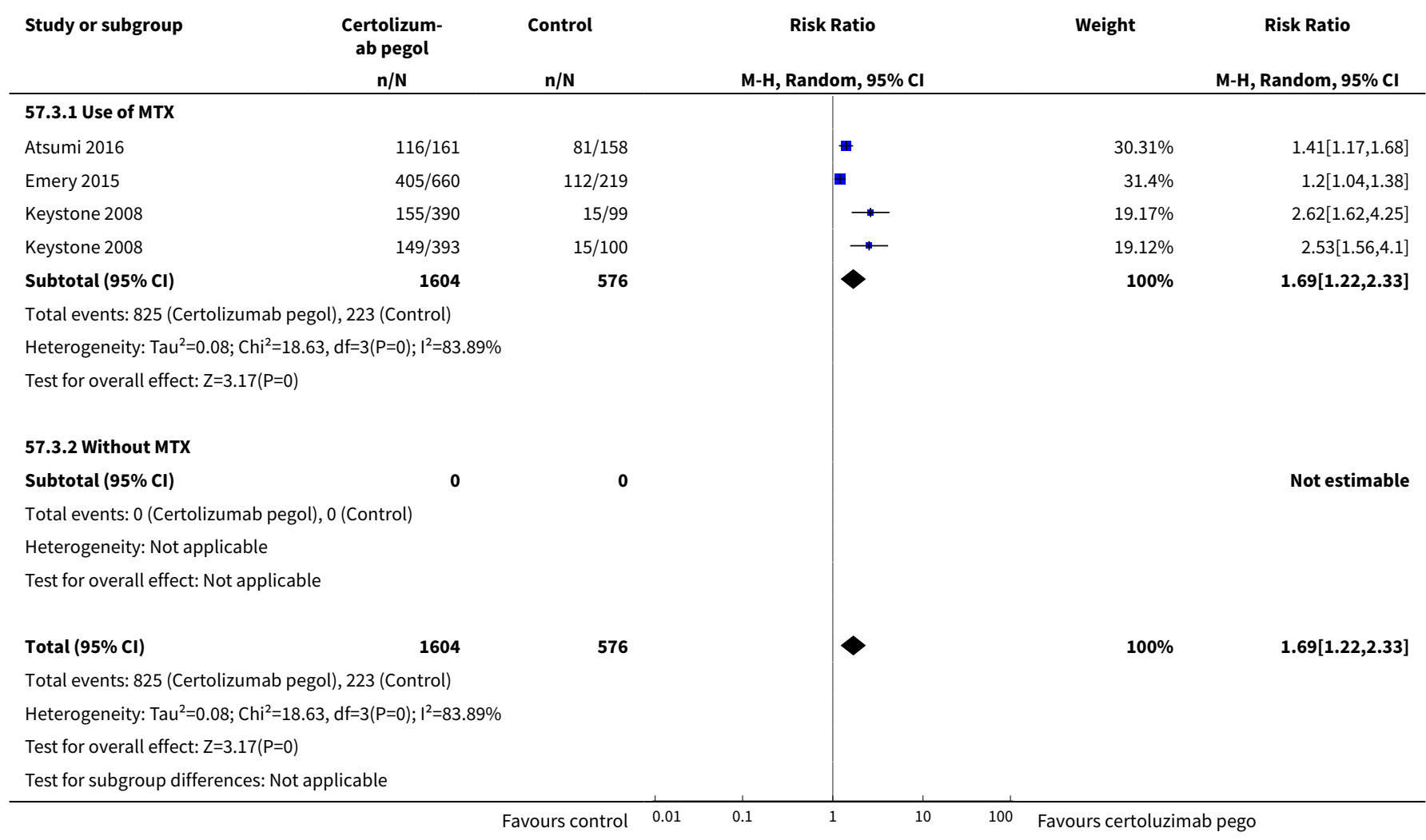

\section{Analysis 57.4. Comparison 57 Analysis of sensitivity ACR50 52 weeks, Outcome 4 Population.}

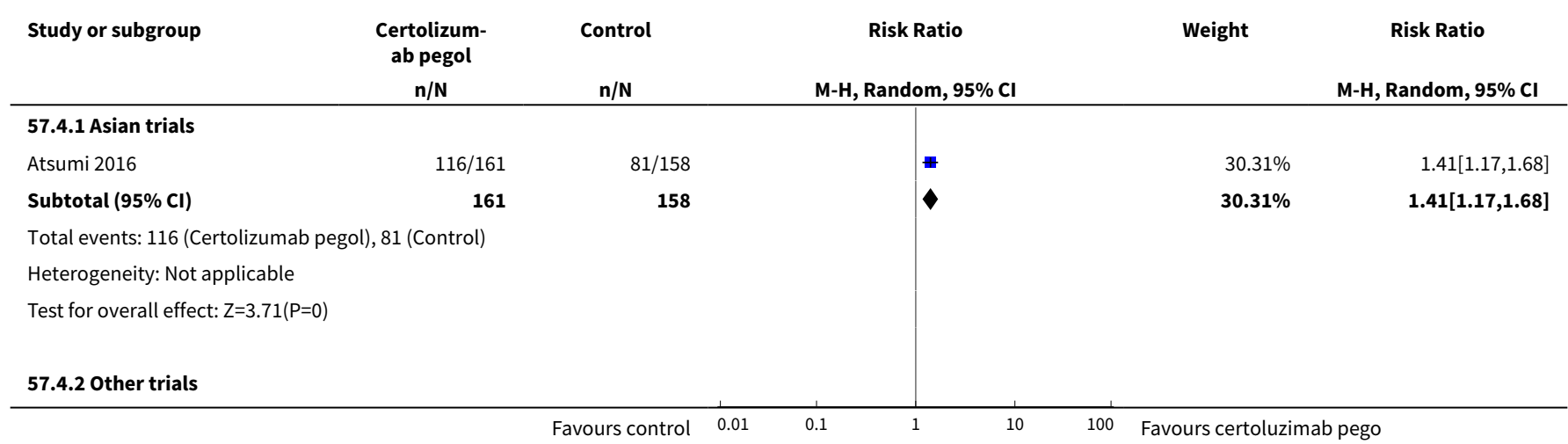




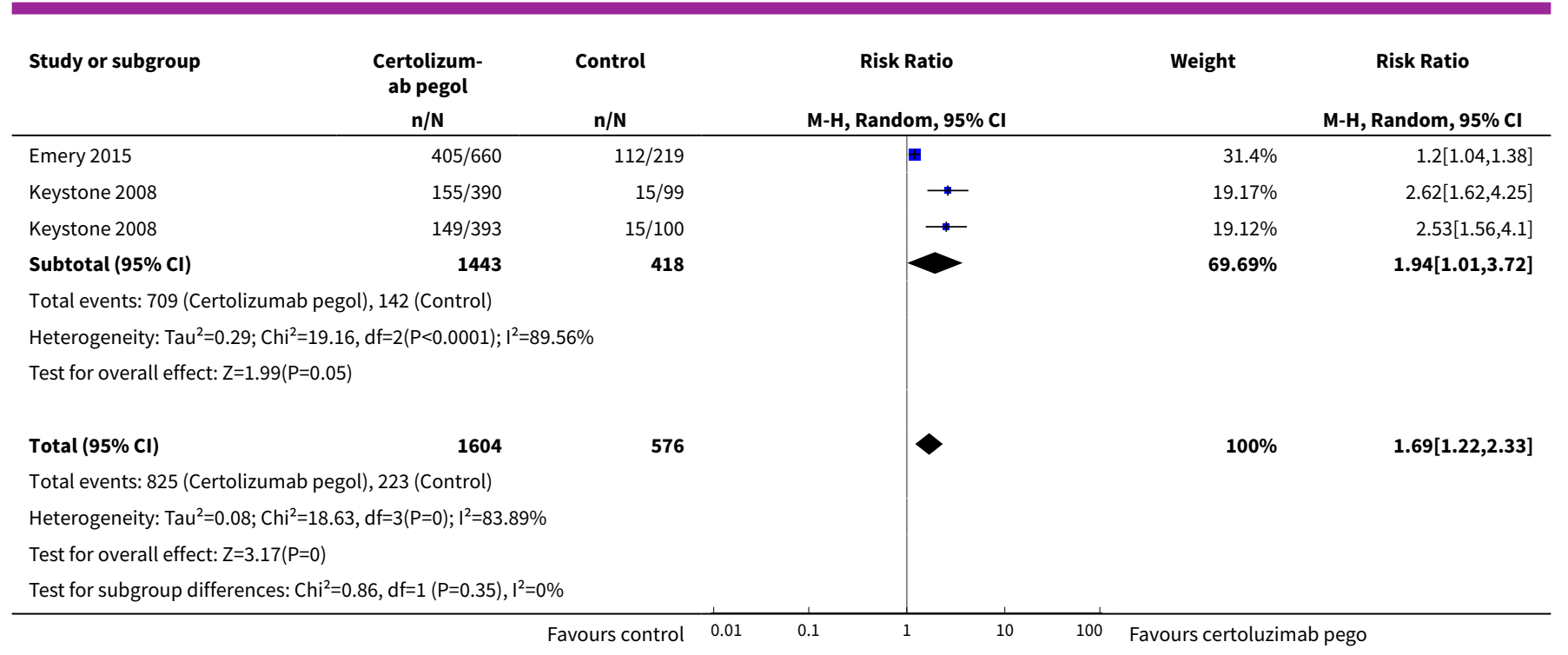

Analysis 57.5. Comparison 57 Analysis of sensitivity ACR50 52 weeks, Outcome 5 Duration of previous disease.

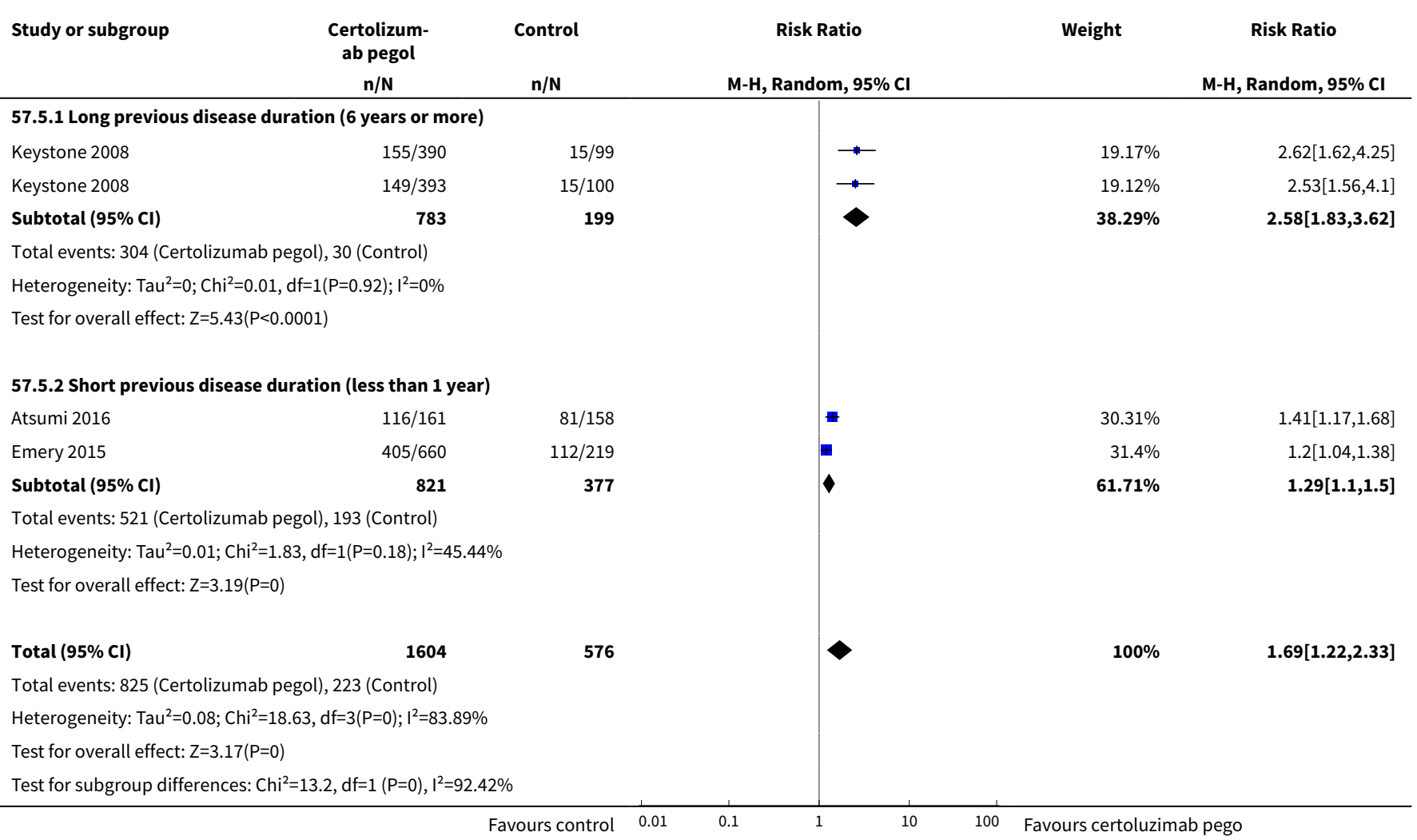

ADDITIONAL TABLES 
Table 1. Contribution of trials

\begin{tabular}{|c|c|c|c|c|}
\hline & \multicolumn{2}{|c|}{ Update 2014} & \multicolumn{2}{|c|}{ Update 2016} \\
\hline & Benefit (B) & Harm (H) & Benefit (B) & Harm (H) \\
\hline Atsumi 2016 & - & - & B & $\mathrm{H}$ \\
\hline CDP870-004 2001 & B & $\mathrm{H}$ & B & - \\
\hline Choy 2002 & - & $\mathrm{H}$ & - & $\mathrm{H}$ \\
\hline Choy 2012 & B & $\mathrm{H}$ & B & $\mathrm{H}$ \\
\hline Emery 2015 & - & - & B & $\mathrm{H}$ \\
\hline Fleischmann 2009 & B & $\mathrm{H}$ & B & $\mathrm{H}$ \\
\hline Keystone 2008 & B & $\mathrm{H}$ & B & $\mathrm{H}$ \\
\hline NCT00993317 & B & $\mathrm{H}$ & B & $\mathrm{H}$ \\
\hline Smolen 2009 & B & $\mathrm{H}$ & B & $\mathrm{H}$ \\
\hline Smolen 2015 & B & $\mathrm{H}$ & B & $\mathrm{H}$ \\
\hline Weinblatt 2012 & B & $\mathrm{H}$ & B & $\mathrm{H}$ \\
\hline Yamamoto (a) 2014 & B & $\mathrm{H}$ & B & $\mathrm{H}$ \\
\hline Yamamoto (b) 2014 & B & $\mathrm{H}$ & B & $\mathrm{H}$ \\
\hline Østergaard 2015 & - & - & - & $\mathrm{H}$ \\
\hline Total trials & 10 & 11 & 12 & 14 \\
\hline Total pooled & 9 & 9 & 11 & 13 \\
\hline
\end{tabular}

The data from the two phase II studies (CDP870-004 2001; Choy 2002) were not pooled with the rest of the studies due to the different follow-ups and doses used. 
Table 2. Demographic and disease characteristics of the included Phase III trials

\begin{tabular}{|c|c|c|c|c|c|c|c|c|c|c|c|c|}
\hline Study & $\begin{array}{l}\text { Atsumi } \\
2016 \\
n=319\end{array}$ & $\begin{array}{l}\text { Choy } \\
2012 n= \\
247\end{array}$ & $\begin{array}{l}\text { Emery } 2015 n= \\
879\end{array}$ & $\begin{array}{l}\text { Fleis- } \\
\text { chmann } \\
2009 n= \\
220\end{array}$ & $\begin{array}{l}\text { Keystone } \\
2008 n= \\
982\end{array}$ & $\begin{array}{l}\text { NCT00993 } \\
n=127\end{array}$ & $\begin{array}{l}\text { STmolen } \\
2009 \mathrm{n}= \\
619\end{array}$ & $\begin{array}{l}\text { Smolen } \\
2015 n= \\
194\end{array}$ & $\begin{array}{l}\text { Wein- } \\
\text { blatt } \\
2012 n= \\
1063\end{array}$ & $\begin{array}{l}\text { Ya- } \\
\text { mamo- } \\
\text { to (a) } \\
2014 n= \\
230\end{array}$ & $\begin{array}{l}\text { Ya- } \\
\text { mamo- } \\
\text { to }(b) \\
2014 n= \\
316\end{array}$ & $\begin{array}{l}\text { Øster- } \\
\text { gaard } \\
2015 n= \\
41\end{array}$ \\
\hline $\begin{array}{l}\text { Age } \\
\text { (years) } \\
\text { Mean } \pm \\
\text { (SD) }\end{array}$ & $\begin{array}{l}\text { CZP 200 } \\
\text { mg plus } \\
\text { MTX 49.4 } \\
(10.6) \\
\text { Placebo } \\
\text { plus MTX } \\
<49.0(10.3)\end{array}$ & $\begin{array}{l}\text { CZP } 400 \\
\text { mg plus } \\
\text { MTX } 53 \\
(12.0) \\
\text { Place- } \\
\text { bo plus } \\
\text { MTX 55.6 } \\
(11.7)\end{array}$ & $\begin{array}{l}\text { CZP 200mg plus } \\
\text { MTX 50.4(13.6) } \\
\text { Placebo plus MTX } \\
51.2(13)\end{array}$ & $\begin{array}{l}53.8 \\
(12.2) \\
\text { CZP 400 } \\
\text { mg } 52.7 \\
(12.7) \\
\text { Place- } \\
\text { bo 54.0 } \\
(11.6)\end{array}$ & $\begin{array}{l}52.0(11.6) \\
\text { CZP 200 } \\
\text { mg plus } \\
\text { MTX 51.4 } \\
\text { (11.6) CZP } \\
400 \mathrm{mg} \\
\text { plus MTX } \\
52.4(11.7) \\
\text { Placebo } \\
\text { plus MTX } \\
52.2(11.2)\end{array}$ & $\begin{array}{l}\text { CZP } 200 \\
\text { mg plus } \\
\text { MTX } 18 \text { - } \\
65 \text { years } \\
=72 ;>65 \\
\text { years = } \\
13 \text { Place- } \\
\text { bo plus } \\
\text { MTX } 18 \text { - } \\
65 \text { years } \\
=38 ;>65 \\
\text { years }=4\end{array}$ & $\begin{array}{l}51.9(11.5) \\
\text { CZP 200 } \\
\text { mg plus } \\
\text { MTX 52.2 } \\
\text { (11.1) CZP } \\
400 \mathrm{mg} \\
\text { plus MTX } \\
51.9(11.8) \\
\text { Placebo } \\
\text { plus MTX } \\
51.5(11.8)\end{array}$ & $\begin{array}{l}\text { CZP } 200 \\
\text { mg } 53.6 \\
(11.9) \\
\text { Place- } \\
\text { bo } 54.0 \\
(12.4)\end{array}$ & $\begin{array}{l}55.1 \\
(12.49) \\
\text { CZP 200 } \\
\text { mg } 55.4 \\
(12.4) \\
\text { Place- } \\
\text { bo } 53.9 \\
(12.7)\end{array}$ & $\begin{array}{l}55.7 \\
(10.0) \\
\text { CZP 200 } \\
\text { mg 56.0 } \\
(10.2) \\
\text { Placebo } \\
55.4(9.8)\end{array}$ & $\begin{array}{l}\text { Total } \\
53.0 \\
(11.0) \\
\text { CZP 100 } \\
\text { mg plus } \\
\text { MTX 54.3 } \\
\text { (10.6) } \\
\text { CZP 200 } \\
\text { mg plus } \\
\text { MTX 50.6 } \\
\text { (11.4) } \\
\text { CZP 400 } \\
\text { mg plus } \\
\text { MTX 55.4 } \\
\text { (10.3) } \\
\text { Place- } \\
\text { bo plus } \\
\text { MTX51.9 } \\
\text { (11.1) }\end{array}$ & $\begin{array}{l}\text { CZP } \\
400 \mathrm{mg} \\
51.3(12.6) \\
\text { Place- } \\
\text { bo } 48.3 \\
(14.4)\end{array}$ \\
\hline $\begin{array}{l}\text { Fol- } \\
\text { low-up }\end{array}$ & $\begin{array}{l}24 \text { and } 52 \\
\text { weeks }\end{array}$ & 24 weeks & 52 weeks & 24 weeks & 52 weeks & 24 weeks & 24 weeks & 24 weeks & 12 weeks & $\begin{array}{l}12 \text { and } \\
24 \text { weeks }\end{array}$ & $\begin{array}{l}12 \text { and } \\
24 \text { weeks }\end{array}$ & 2 weeks \\
\hline $\begin{array}{l}\text { Women } \\
\text { n (\%) }\end{array}$ & $\begin{array}{l}\text { CZP } 200 \\
\text { mg plus } \\
\text { MTX } 129 \\
(81.1 \%) \\
\text { Place- } \\
\text { bo plus } \\
\text { MTX 127 } \\
(80.9 \%)\end{array}$ & $\begin{array}{l}\text { CZP } \\
400 \mathrm{mg} \\
\text { plus MTX } \\
72 \% \\
\text { Placebo } \\
\text { plus MTX } \\
66.1 \%\end{array}$ & $\begin{array}{l}\text { CZP } 200 \text { mg plus } \\
\text { MTX } 497(75.9 \%) \\
\text { Placebo plus MTX } \\
170(79.8 \%)\end{array}$ & $\begin{array}{l}184 \\
(83.6 \%)\end{array}$ & $\begin{array}{l}817 \\
(83.2 \%) \\
\text { CZP } 200 \\
\text { mg 324 } \\
(82.4 \%) \\
\text { CZP 400 } \\
\text { mg } 326 \\
(83.6 \%) \\
\text { Place- } \\
\text { bo } 167 \\
(83.9 \%)\end{array}$ & $\begin{array}{l}112 \\
(88.2 \%) \\
\text { CZP 200 } \\
\text { mg 75 } \\
(59.1 \%) \\
\text { Place- } \\
\text { bo } 37 \\
(29.13 \%)\end{array}$ & $\begin{array}{l}505 \\
(81.6 \%) \\
\text { CZP 200 } \\
\text { mg 206 } \\
(83.7 \%) \\
\text { CZP 400 } \\
\text { mg 192 } \\
(78 \%) \\
\text { Place- } \\
\text { bo 107 } \\
(84.3 \%)\end{array}$ & $\begin{array}{l}156 \\
(80.4 \%) \\
\text { CZP } 200 \\
\text { mg } 81 \\
(41.8 \%) \\
\text { Place- } \\
\text { bo } 75 \\
(38.7 \%)\end{array}$ & $\begin{array}{l}829 \\
(78 \%) \\
\text { CZP 200 } \\
\text { mg 660 } \\
(62.1 \%) \\
\text { Place- } \\
\text { bo } 169 \\
(15.9 \%)\end{array}$ & $\begin{array}{l}171 \\
(74.3 \%) \\
\text { CZP } 200 \\
\text { mg } 83 \\
(36.1 \%) \\
\text { Place- } \\
\text { bo } 88 \\
(38.3 \%)\end{array}$ & $\begin{array}{l}\text { CZP 100 } \\
\text { mg plus } \\
\text { MTX58 } \\
(18.4 \%) \\
\text { CZP 200 } \\
\text { mg plus } \\
\text { MTX69 } \\
\text { (21.8\%) } \\
\text { CZP 400 } \\
\text { mg plus } \\
\text { MTX69 } \\
\text { (21.8\%) }\end{array}$ & $\begin{array}{l}\text { CZP } \\
400 \mathrm{mg} \\
81.5 \% \\
\text { Placebo } \\
76.9 \%\end{array}$ \\
\hline
\end{tabular}




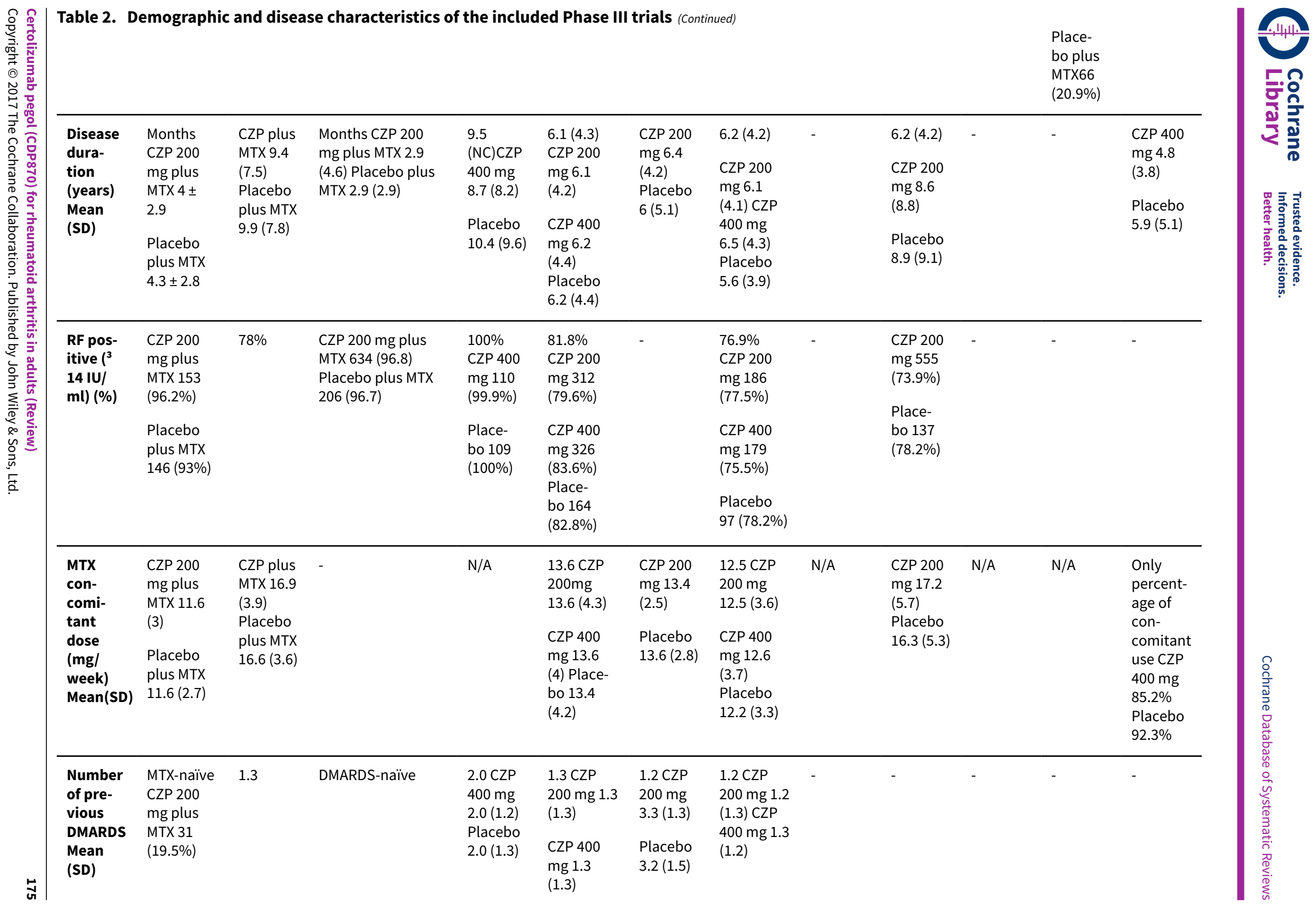




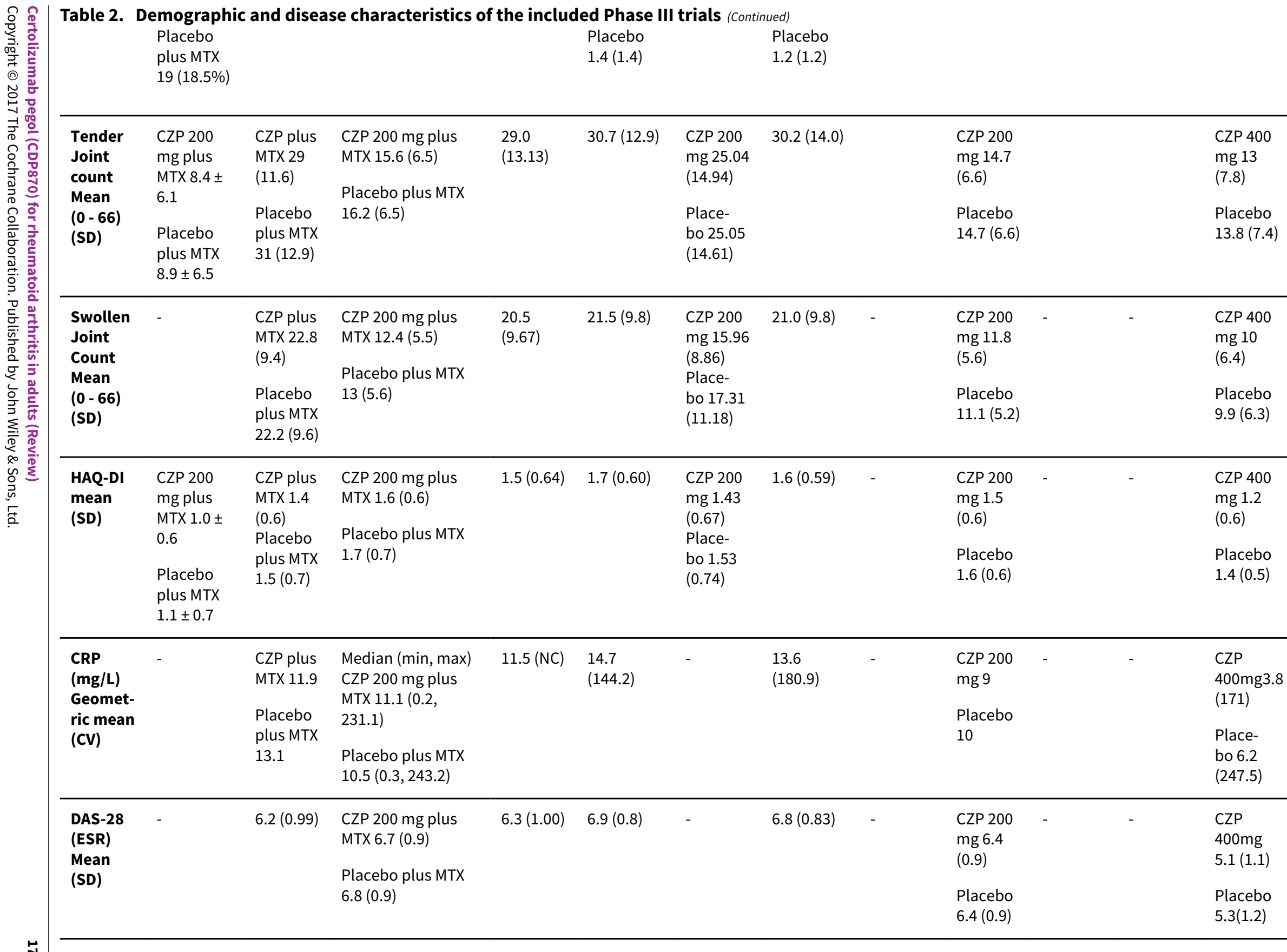




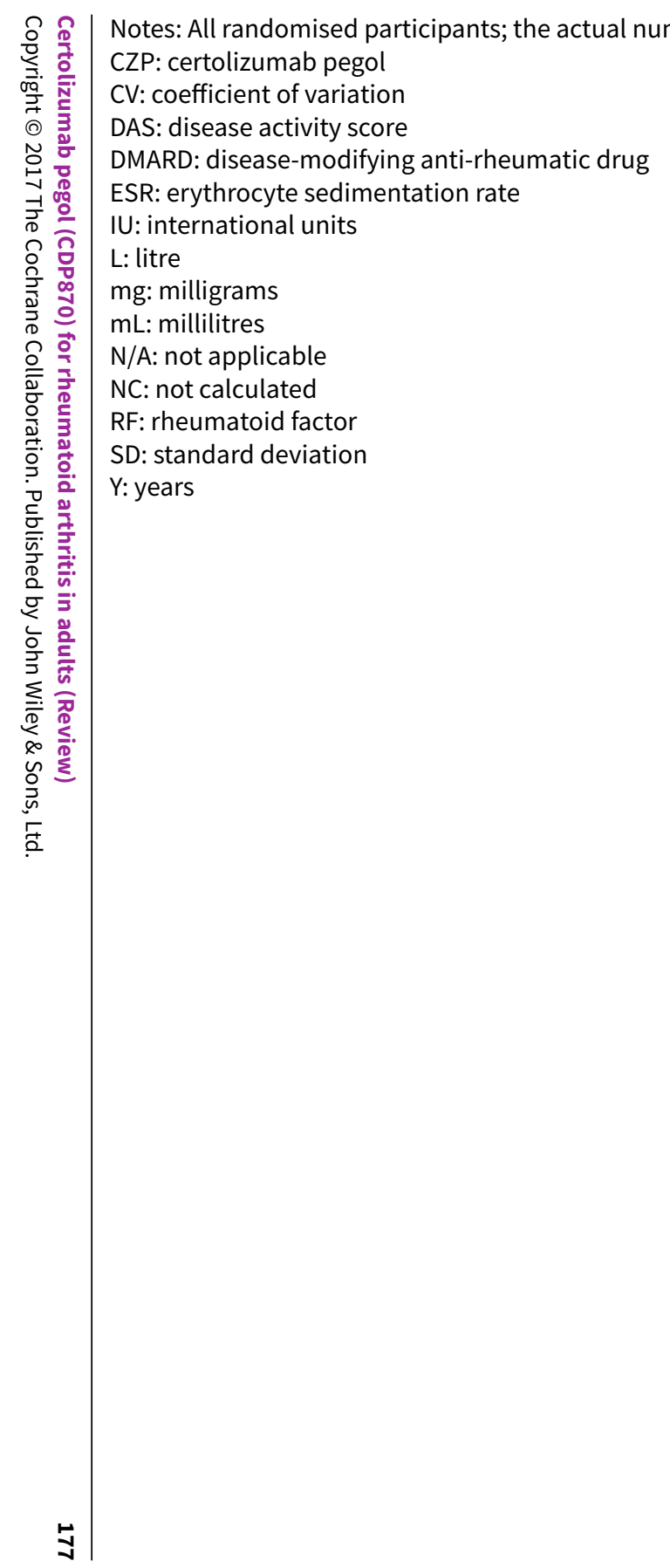


Table 3. Flow of participants in the included Phase III trials

\begin{tabular}{|c|c|c|c|c|}
\hline Study & Placebo & $\begin{array}{l}\text { Certolizumab } \\
\text { pegol } 100 \mathrm{mg}\end{array}$ & Certolizumab pegol $200 \mathrm{mg}$ & $\begin{array}{l}\text { Certolizumab pe- } \\
\text { gol } 400 \mathrm{mg}\end{array}$ \\
\hline \multirow[t]{8}{*}{ Atsumi 2016} & ITT $n=158$ Safety $n=157$ & - & ITT $n=$ 161Safety $n=159$ & - \\
\hline & Discontinued $n=15(\%)$ & - & Discontinued $n=12(7,45 \%)$ & - \\
\hline & Consent withdrawn $=3(2 \%)$ & & Consent withdrawn $=2(1 \%)$ & \\
\hline & Lack of efficacy = $1(0.06 \%)$ & & Lack of efficacy $=0$ & \\
\hline & $\begin{array}{l}\text { Adverse event }=6(4 \%) \text { Other reasons } \\
=5(3 \%)\end{array}$ & & Adverse event = $9(5 \%)$ & \\
\hline & & & Other reasons = $1(0,5 \%)$ & \\
\hline & Moved to rescue $=70(44 \%)$ & & Moved to rescue $=36(22 \%)$ & \\
\hline & Completed $n=73(46.20 \%)$ & - & Completedn $=111(69 \%)$ & - \\
\hline \multirow[t]{11}{*}{ Choy 2012} & ITT $\mathrm{n}=121^{a}$ & - & - & ITT $n=126$ \\
\hline & Safety $n=119$ & & & Safety $n=124$ \\
\hline & All withdrawn & - & - & All withdrawn \\
\hline & $\mathrm{n}=56(46.3 \%)$ & & & $\mathrm{n}=28(22.2 \%)$ \\
\hline & & & & $\begin{array}{l}\text { Lack of efficacy }= \\
16(12.7 \%)\end{array}$ \\
\hline & Adverse event = $6(5 \%)$ & & & \\
\hline & Other reasons $=5(4.1 \%)$ & & & $\begin{array}{l}\text { Adverse event }=7 \\
(5.6 \%)\end{array}$ \\
\hline & & & & $\begin{array}{l}\text { Other reasons }=5 \\
(4 \%)\end{array}$ \\
\hline & Completed & - & - & Completed \\
\hline & $\mathrm{n}=65(53.7 \%)$ & & & $\mathrm{n}=98(77.8 \%)$ \\
\hline & ITT $\mathrm{n}=121^{a}$ Safety $\mathrm{n}=119$ & - & ITT $\mathrm{n}=126^{a}$ Safety $\mathrm{n}=124$ & \\
\hline \multirow[t]{10}{*}{ Emery 2015} & ITT $n=219$ & - & ITT $n=660$ & - \\
\hline & Safety $n=217$ & & Safety $n=659$ & \\
\hline & All withdrawn & - & All withdrawn & - \\
\hline & $n=76(35 \%)$ & & $n=160(24 \%)$ & \\
\hline & Lack of efficacy = $14(6 \%)$ & & Lack of efficacy $=19(3 \%)$ & \\
\hline & Adverse event = $17(8 \%)$ & & Adverse event $=51(8 \%)$ & \\
\hline & Protocol violation $=6(3 \%)$ & & Protocol violation $=18(3 \%)$ & \\
\hline & Lost to follow-up = $6(3 \%)$ & & Lost to follow-up $=14(2 \%)$ & \\
\hline & Consent withdrawn $=15(7 \%)$ & & Consent withdrawn $=35(5 \%)$ & \\
\hline & Other reasons $=18(8 \%)$ & & Other reasons $=23(3 \%)$ & \\
\hline
\end{tabular}


Table 3. Flow of participants in the included Phase III trials (Continued)

\begin{tabular}{|c|c|c|c|c|}
\hline & $\begin{array}{l}\text { Completed } \\
n=143(65 \%)\end{array}$ & - & $\begin{array}{l}\text { Completed } \\
n=500(76 \%)\end{array}$ & - \\
\hline \multirow{11}{*}{$\begin{array}{l}\text { Fleischmann } \\
2009\end{array}$} & ITT $n=109$ & - & - & $\mathrm{ITT} n=111$ \\
\hline & Safety $n=109$ & & & Safety $n=111$ \\
\hline & All withdrawn & - & - & All withdrawn \\
\hline & $\mathrm{n}=81(74 \%)$ & & & $\mathrm{n}=35(31.5 \%)$ \\
\hline & Lack of efficacy = 75 (68.8\%) & & & Lack of efficacy $=$ \\
\hline & Adverse event $=2(1.8 \%)$ & & & $24(21.6 \%)$ \\
\hline & Protocol violation $=1(0.9 \%)$ & & & $\begin{array}{l}\text { Adverse event }=5 \\
(4.5 \%)\end{array}$ \\
\hline & Lost to follow-up = $3(2.8 \%)$ & & & $\begin{array}{l}\text { Protocol violation } \\
=4(3.6 \%)\end{array}$ \\
\hline & & & & $\begin{array}{l}\text { Consent with- } \\
\text { drawn = } 2(1.8 \%)\end{array}$ \\
\hline & Completed & - & - & Completed \\
\hline & $\mathrm{n}=28(25.7 \%)$ & & & $\mathrm{n}=76(68.5 \%)$ \\
\hline \multirow[t]{7}{*}{ Keystone 2008} & ITT $n=199$ & - & ITT $n=393$ & ITT $n=390$ \\
\hline & Safety $n=199$ & & Safety $n=392^{b}$ & Safety $n=389 b$ \\
\hline & $\begin{array}{l}\text { Withdrawn at week } 16 \text { due to lack of } \\
\text { efficacy } \\
n=125(62.8 \%)\end{array}$ & - & $\begin{array}{l}\text { Withdrawn at week } 16 \text { due to } \\
\text { lack of efficacy } \\
n=83(21.1 \%)\end{array}$ & $\begin{array}{l}\text { Withdrawn at } \\
\text { week } 16 \text { due to } \\
\text { lack of efficacy } \\
n=68(17.4 \%)\end{array}$ \\
\hline & All withdrawn & - & All withdrawn & All withdrawn \\
\hline & $\mathrm{n}=156(78.4 \%)$ & & $\mathrm{n}=138(35.1 \%)$ & $\mathrm{n}=116(39.7 \%)$ \\
\hline & Completed & - & Completed & Completed \\
\hline & $\mathrm{n}=43(21.6 \%)$ & & $\mathrm{n}=255(64.9 \%)$ & $\mathrm{n}=274(70.3 \%)$ \\
\hline \multirow[t]{8}{*}{ NCT00993317 } & ITT $n=42$ & - & ITT $n=85$ & - \\
\hline & Safety $n=42$ & & Safety $n=85$ & \\
\hline & All withdrawn & - & All withdrawn & - \\
\hline & $n=21(50 \%)$ & & $\mathrm{n}=25(29.41 \%)$ & \\
\hline & Lack of efficacy = $18(42 \%)$ & & Lack of efficacy = $18(21.8 \%)$ & \\
\hline & Adverse event $=2(4.76 \%)$ & & Adverse event $=4(4.70 \%)$ & \\
\hline & Other reasons $=1(2.38 \%)$ & & Other reasons $=3(3.52 \%)$ & \\
\hline & Completed & - & Completed & - \\
\hline
\end{tabular}


Table 3. Flow of participants in the included Phase III trials (Continued)

$\mathrm{n}=21(50 \%)$

\begin{tabular}{|c|c|c|c|c|}
\hline \multirow[t]{7}{*}{ Smolen 2009} & ITT $n=127$ & - & ITT $n=246$ & ITT n = 246 \\
\hline & Safety $n=125$ & & Safety $n=248^{c}$ & Safety $n=246$ \\
\hline & $\begin{array}{l}\text { Withdrawn at week } 16 \text { due to lack of } \\
\text { efficacy } \\
n=103(81 \%)\end{array}$ & - & $\begin{array}{l}\text { Withdrawn at week } 16 \text { due to } \\
\text { lack of efficacy } \\
n=52(21.1 \%)\end{array}$ & $\begin{array}{l}\text { Withdrawn at } \\
\text { week } 16 \text { due to } \\
\text { lack of efficacy } \\
n=52(21.1 \%)\end{array}$ \\
\hline & All withdrawn & - & All withdrawn & All withdrawn \\
\hline & $n=110(86 \%)$ & & $n=72(29.3 \%)$ & $\mathrm{n}=65(26.4 \%)$ \\
\hline & Completed & - & Completed & Completed \\
\hline & $\mathrm{n}=17(13.4 \%)$ & & $\mathrm{n}=174(70.7 \%)$ & $\mathrm{n}=181(73.6 \%)$ \\
\hline \multirow[t]{6}{*}{ Smolen 2015} & ITT $n=98$ Safety $n=98$ & - & ITT $n=96$ Safety $n=96$ & - \\
\hline & All withdrawnn $=18(18.36 \%)$ & - & All withdrawnn = $12(12.5 \%)$ & - \\
\hline & Lack of efficacy $=7(7.14 \%)$ & & Lack of efficacy $=2(2.08 \%)$ & \\
\hline & Adverse event $=6(6.12 \%)$ & & Adverse event $=6(6.25 \%)$ & \\
\hline & Other reasons $=5(5.10 \%)$ & & Other reasons $=4(4.16 \%)$ & \\
\hline & $\begin{array}{l}\text { Completed } \\
\mathrm{n}=80(81.63 \%)\end{array}$ & - & Completedn $=84(87.5 \%)$ & - \\
\hline
\end{tabular}

\begin{tabular}{|c|c|c|c|c|}
\hline \multirow[t]{8}{*}{ Weinblatt 2012} & $\begin{array}{l}\text { ITT } n=212 \\
\text { Safety } n=209\end{array}$ & - & $\begin{array}{l}\text { ITT } n=851 \\
\text { Safety } n=846\end{array}$ & - \\
\hline & All withdrawn & - & All withdrawn & - \\
\hline & $\mathrm{n}=28(13.20 \%)$ & & $\mathrm{n}=80(9.41 \%)$ & \\
\hline & Lack of efficacy $=6(2.83 \%)$ & & Lack of efficacy $=6(0.70 \%)$ & \\
\hline & Adverse event $=6(2.83 \%)$ & & Adverse event $=33(3.87 \%)$ & \\
\hline & Other reasons $=16(7.54 \%)$ & & Other reasons $=41(4.81 \%)$ & \\
\hline & Completed & - & Completed & - \\
\hline & $\mathrm{n}=184(86.79 \%)$ & & $\mathrm{n}=771(90.59 \%)$ & \\
\hline \multirow{5}{*}{$\begin{array}{l}\text { Yamamoto (a) } \\
2014\end{array}$} & ITT $n=114$ Safety $n=114$ & - & ITT $n=116$ Safety $n=116$ & - \\
\hline & All withdrawnn = $96(84.2 \%)$ & - & All withdrawnn = $34(29.31 \%)$ & - \\
\hline & Lack of efficacy = 2 (1.75\%) & & Lack of efficacy $=0(0 \%)$ & \\
\hline & Adverse event $=2(1.75 \%)$ & & Adverse event $=8(6.9 \%)$ & \\
\hline & $\begin{array}{l}\text { Other reasons (protocol planned } n= \\
88)=94(82 \%)\end{array}$ & & $\begin{array}{l}\text { Other reasons (protocol } \\
\text { planned } n=24)=26(22.4 \%)\end{array}$ & \\
\hline
\end{tabular}


Table 3. Flow of participants in the included Phase III trials (Continued)

\begin{tabular}{|c|c|c|c|c|}
\hline & Completed $n=18(15.8 \%)$ & - & Completedn $=82(70.69 \%)$ & \\
\hline \multirow{6}{*}{$\begin{array}{l}\text { Yamamoto (b) } \\
2014\end{array}$} & ITT n = 77 & ITT $n=72$ & ITT $n=82$ & ITT $n=85$ \\
\hline & All withdrawn & All withdrawn & All withdrawn & All withdrawn \\
\hline & $\begin{array}{l}\text { Lack of efficacy }=2(2.98 \%) \\
\text { Adverse event }=3(3.90 \%)\end{array}$ & $\begin{array}{l}\text { Lack of efficacy }= \\
3(4.17 \%)\end{array}$ & $\begin{array}{l}\text { Lack of efficacy = } 1(1.22 \%) \\
\text { Adverse event }=3(3.66 \%)\end{array}$ & $\begin{array}{l}\text { Lack of efficacy }= \\
0(0 \%)\end{array}$ \\
\hline & $\begin{array}{l}\text { Other reasons (Protocol planned } \\
\text { withdrawal }=45)=47(61.04 \%)\end{array}$ & $\begin{array}{l}\text { Adverse event }= \\
0(0 \%)\end{array}$ & $\begin{array}{l}\text { Other reasons (Protocol } \\
\text { planned withdrawal }=11 \text { ) = }\end{array}$ & $\begin{array}{l}\text { Adverse event }=7 \\
(8.23 \%)\end{array}$ \\
\hline & Completed & Completed & Completed & Completed \\
\hline & $\mathrm{n}=25(32.47 \%)$ & $\mathrm{n}=51(70.83 \%)$ & $n=66(80.49 \%)$ & $\mathrm{n}=65(76.47 \%)$ \\
\hline \multirow{3}{*}{$\begin{array}{l}\text { Østergaard } \\
2015\end{array}$} & ITT $n=13$ & - & ITT $n=27$ & - \\
\hline & Safety at 12 weeks $n=13$ & & Safety at 12 weeks $n=27$ & \\
\hline & $\begin{array}{l}\text { Only the data obtained at week } 2 \\
\text { were usable }\end{array}$ & & $\begin{array}{l}\text { Only the data obtained at } \\
\text { week } 2 \text { were usable }\end{array}$ & \\
\hline
\end{tabular}

$a$ Manufacturers reported efficacy calculations from placebo $n=119$ and certolizumab pegol $n=124$.

$b$ Two participants in each treatment group did not take study medication.

CTwo participants in the placebo group received certolizumab pegol and were included for safety in the $200 \mathrm{mg}$ group. (d) 


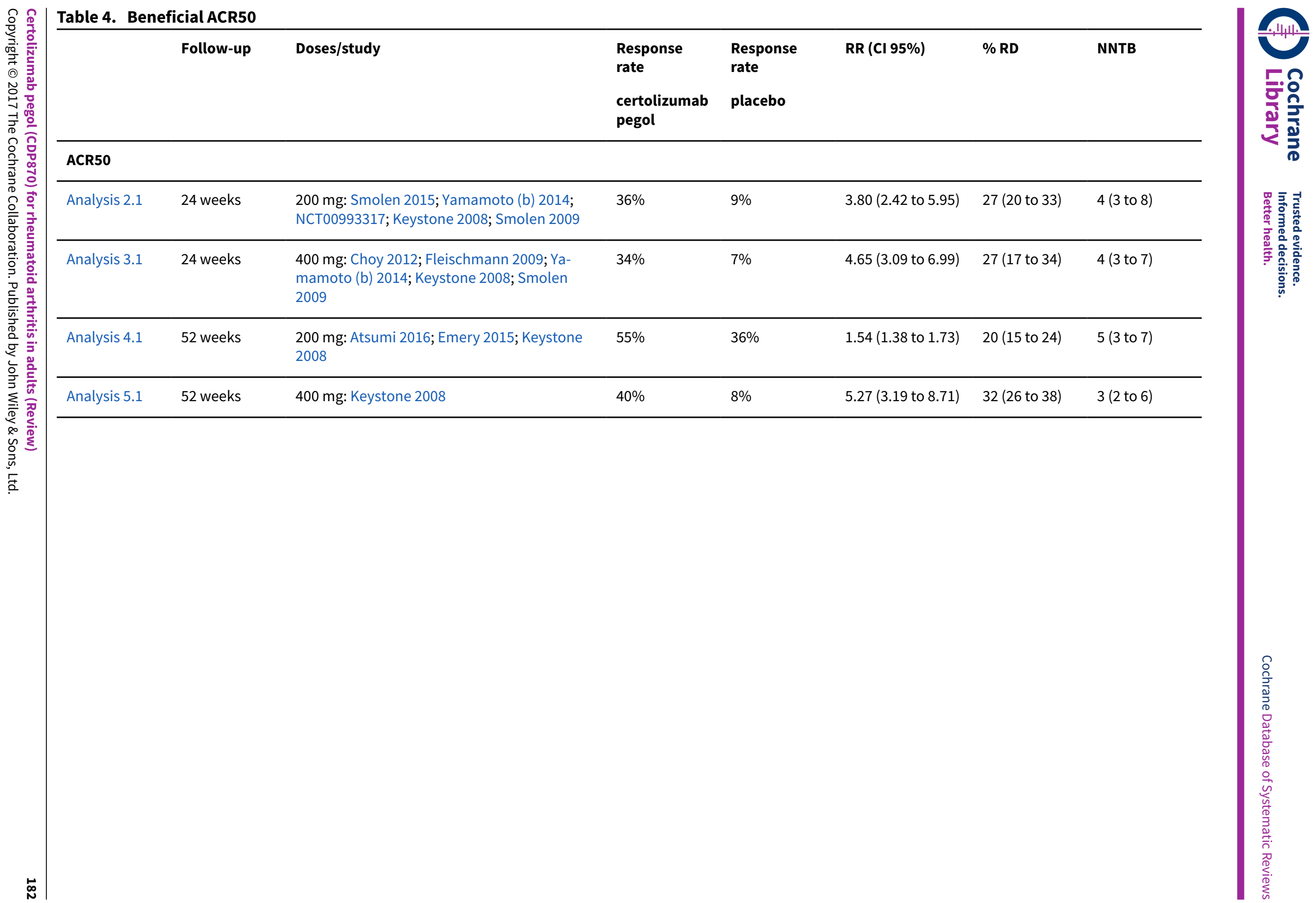


Table 5. Health-related quality of life

\begin{tabular}{|c|c|c|c|}
\hline & Follow-up & Doses/study & Mean differences \\
\hline \multicolumn{4}{|c|}{ HAQ $(0-3)($ Best $=0 ;$ Worst $=3)$} \\
\hline Analysis 7.1 & 24 weeks & $\begin{array}{l}200 \text { mg/ Smolen 2015; NCT00993317; Keystone 2008; } \\
\text { Smolen } 2009\end{array}$ & $-0.35(-0.43$ to -0.26$)$ \\
\hline Analysis 7.2 & 24 weeks & $\begin{array}{l}400 \text { mg/ Choy 2012; Fleischmann 2009; Keystone } \\
\text { 2008; Smolen } 2009\end{array}$ & $-0.38(-0.48$ to -0.28$)$ \\
\hline Analysis 9.1.1 & 52 weeks & 200 mg/ Emery 2015; Keystone 2008 & $-0.27(-0.35$ to -0.20$)$ \\
\hline Analysis 9.1 .2 & 52 weeks & 400 mg/ Keystone 2008 & $-0.45(-0.57$ to -0.33$)$ \\
\hline
\end{tabular}

\section{SF-36 PCS $(0-100)($ Worst $=0$; Best $=100)$}

\begin{tabular}{llll}
\hline Analysis 10.1 & 24 weeks & $200 \mathrm{mg} /$ Smolen 2015; Keystone 2008; Smolen 2009 & 5.03 (3.90 to 6.16) \\
\hline Analysis 10.2 & 24 weeks & $400 \mathrm{mg} /$ Choy 2012; Keystone 2008; Smolen 2009 & 5.54 (4.11 to 6.97) \\
\hline
\end{tabular}

\section{SF-36 MCS $(0-100)($ Worst $=0$; Best $=100)$}

\begin{tabular}{llll}
\hline Analysis 11.1 & 24 weeks & $200 \mathrm{mg} /$ Keystone 2008; Smolen 2009 & 4.18 (2.70 to 5.66) \\
\hline Analysis 11.2 & 24 weeks & $400 \mathrm{mg} /$ Choy 2012; Keystone 2008; Smolen 2009 & 4.05 (2.77 to 5.34) \\
\hline
\end{tabular}

\section{SF-36 PCS}

\begin{tabular}{llll}
\hline Analysis 12.1 & 52 weeks & 200 mg/ Keystone 2008 & 6.06 (4.59 to 7.53) \\
\hline Analysis 12.2 & 52 weeks & $400 \mathrm{mg} /$ Keystone 2008 & 6.88 (5.42 to 8.34) \\
\hline SF-36 MCS (0 - 100) (Worst = 0; Best = 100) & & $4.3(2.4$ to 6.2) \\
\hline 52 weeks & $200 \mathrm{mg} /$ Keystone 2008 & $4.3(2.4$ to 6.2) \\
\hline
\end{tabular}

\section{Participants' VAS score $(0$ - 100)}

\begin{tabular}{llll}
\hline Analysis 52.1 & 24 weeks & $200 \mathrm{mg} /$ Keystone 2008; Smolen 2009 & $-20.48(-24.26$ to -16.69$)$ \\
\cline { 3 - 4 } & & $\begin{array}{l}400 \mathrm{mg} / \text { Fleischmann 2009; Keystone 2008; Smolen } \\
2009\end{array}$ & $-21.35(-25.08$ to -17.61$)$ \\
\hline Analysis 53.1 & 52 weeks & $200 \mathrm{mg} /$ Keystone 2008 & $-22.20(-27.37$ to -17.03$)$ \\
\cline { 2 - 3 } & & $400 \mathrm{mg} /$ Keystone 2008 & $-24.70(-29.73$ to -19.67$)$ \\
\hline
\end{tabular}

DAS-28 remission $(<2.6)$

\begin{tabular}{|c|c|c|c|}
\hline Analysis 21.2 & 24 weeks & $\begin{array}{l}200 \text { mg/ Smolen 2015; Yamamoto (a) 2014; Atsumi } \\
\text { 2016; Emery 2015; Keystone 2008; Smolen } 2009\end{array}$ & 3.79 (1.90 to 7.56$)$ \\
\hline
\end{tabular}

Analysis $21.3 \quad 400$ mg/Choy 2012; Keystone 2008; Smolen $2009 \quad 7.18$ (3.12 to 16.50)


Table 5. Health-related quality of life (Continued)

\begin{tabular}{|c|c|c|c|}
\hline Analysis 21.4 & 52 weeks & 200 mg/ Atsumi 2016; Emery 2015; Keystone 2008 & $1.83(1.53$ to 2.18$)$ \\
\hline Analysis 21.5 & & 400 mg/ Keystone 2008 & 12.49 (3.99 to 39.12 ) \\
\hline
\end{tabular}

Table 6. Radiological changes

\begin{tabular}{|c|c|c|c|}
\hline & Follow-up & Doses/study & Mean differences \\
\hline \multicolumn{4}{|c|}{$\begin{array}{l}\text { Modified Total Sharp Scores (mTTS) is the sum of the erosion score (ES) and the joint space narrowing (JSN) score and has a } \\
\text { range of } 0-398\end{array}$} \\
\hline Analysis 37.1 & 24 weeks & 200 mg/ Keystone 2008; Smolen 2009 & $-1.06(-1.58$ to -0.55$)$ \\
\hline Analysis 37.2 & 24 weeks & 400 mg/ Keystone 2008; Smolen 2009 & $-1.32(-1.85$ to -0.78$)$ \\
\hline Analysis 36.1 .1 & 52 weeks & 200 mg/ Keystone 2008; Emery 2015 & $-2.4(-4.11$ to -0.69$)$ \\
\hline Analysis 36.1 .2 & 52 weeks & 400 mg/ Keystone 2008 & $-2.6(-4.29$ to -0.91$)$ \\
\hline \multicolumn{4}{|c|}{ Erosion Score is the sum of joint scores collected for $\mathbf{4 6}$ joints and has a range of 0 to 230} \\
\hline Analysis 29.1 & 24 weeks & 200 mg/ Keystone 2008; Smolen 2009 & $-0.35(-0.50$ to -0.21$)$ \\
\hline Analysis 29.2 & 24 weeks & 400 mg/ Keystone 2008; Smolen 2009 & $-0.76(-1.14$ to -0.37$)$ \\
\hline Analysis 29.3 & 52 weeks & 200 mg/ Keystone 2008; Emery 2015 & $-1.14(-1.54$ to -0.74$)$ \\
\hline Analysis 29.4 & 52 weeks & 400 mg/ Keystone 2008 & $-1.5(-2.20$ to -0.80$)$ \\
\hline \multicolumn{4}{|c|}{ Joint space narrowing (JSN) is the sum of joint scores collected for 42 joints and has a range of 0 to 168} \\
\hline Analysis 32.1 & 24 weeks & 200 mg/ Keystone 2008; Smolen 2009 & $-0.45(-0.77$ to -0.13$)$ \\
\hline Analysis 32.2 & 24 weeks & 400 mg/ Keystone 2008; Smolen 2009 & $-0.55(-0.86$ to -0.24$)$ \\
\hline Analysis 32.3 & 52 weeks & 200 mg/ Keystone 2008 & $-1(-1.85$ to -0.15$)$ \\
\hline Analysis 32.4 & 52 weeks & 400 mg/ Keystone 2008 & $-1.2(-1.98$ to -0.42$)$ \\
\hline
\end{tabular}

Table 7. Adverse events

\begin{tabular}{|c|c|c|c|c|c|}
\hline Studies & $\begin{array}{l}\text { Response } \\
\text { rate in \% } \\
\text { (number of } \\
\text { events) } \\
\text { cer- } \\
\text { tolizumab } \\
\text { pegol }\end{array}$ & $\begin{array}{l}\text { Response } \\
\text { rate in \% } \\
\text { (number of } \\
\text { events) } \\
\text { placebo }\end{array}$ & $\begin{array}{l}\text { RR }(95 \% \\
\mathrm{CI})\end{array}$ & \% RD & NNTH \\
\hline
\end{tabular}

\section{Serious adverse \\ events (doses)}

Peto OR 
Table 7. Adverse events (Continued)

\begin{tabular}{|c|c|c|c|c|c|c|}
\hline $\begin{array}{l}\text { Analysis } 41.1 \\
200 \text { mg certolizumab } \\
\text { pegol }\end{array}$ & $\begin{array}{l}\text { Smolen 2015; Yamamoto (a) } \\
\text { 2014; Yamamoto (b) 2014; } \\
\text { NCT00993317; Keystone 2008; } \\
\text { Smolen 2009; Weinblatt 2012; At- } \\
\text { sumi 2016; Emery } 2015\end{array}$ & $8.4 \%(228)$ & $5,8 \%(72)$ & $\begin{array}{l}1.47(1.13 \\
\text { to } 1.91)\end{array}$ & 3 (1 to 4$)$ & $\begin{array}{l}33 \\
(250100)\end{array}$ \\
\hline $\begin{array}{l}\text { Analysis } 42.1 \\
400 \text { mg certolizumab } \\
\text { pegol }\end{array}$ & $\begin{array}{l}\text { Choy 2012; Fleischmann 2009; Ya- } \\
\text { mamoto (b) 2014; Keystone 2008; } \\
\text { Smolen 2009; Østergaard } 2015\end{array}$ & $10 \%(95)$ & $4 \%(31)$ & $\begin{array}{l}1.98(1.36 \\
\text { to } 2.9)\end{array}$ & 5 (2 to 7$)$ & $\begin{array}{l}28 \text { (15 to } \\
74)\end{array}$ \\
\hline
\end{tabular}

\section{Adverse events \\ leading to with- \\ drawal}

\section{Peto OR}

\begin{tabular}{|c|c|c|c|c|c|c|}
\hline $\begin{array}{l}\text { Analysis } 50.15 \\
200 \mathrm{mg} \text { certolizumab } \\
\text { pegol }\end{array}$ & $\begin{array}{l}\text { Emery 2015; Keystone 2008; } \\
\text { NCT00993317; Smolen 2009; } \\
\text { Smolen 2015; Weinblatt 2012; Ya- } \\
\text { mamoto (a) 2014; Yamamoto (b) } \\
2014\end{array}$ & $6 \%(147)$ & $4 \%(46)$ & $\begin{array}{l}1.32(0.95 \\
\text { to } 1.84)\end{array}$ & 1 (0 to 3 ) & NS \\
\hline $\begin{array}{l}\text { Analysis } 50.16 \\
400 \mathrm{mg} \text { certolizumab } \\
\text { pegol }\end{array}$ & $\begin{array}{l}\text { Choy 2012; Fleischmann 2009; Ya- } \\
\text { mamoto (b) 2014; Keystone 2008; } \\
\text { Smolen } 2009\end{array}$ & $5 \%(48)$ & $2 \%(16)$ & $\begin{array}{l}2.01(1.20 \\
\text { to } 3.36)\end{array}$ & 3 (1 to 5 ) & $\begin{array}{l}52 \text { (23 to } \\
257)\end{array}$ \\
\hline Death & & & & Peto OR & & \\
\hline $\begin{array}{l}\text { Analysis 50.17; } \\
200 \text { mg certolizumab } \\
\text { pegol }\end{array}$ & $\begin{array}{l}\text { Emery 2015; Keystone 2008; } \\
\text { Smolen 2009; Smolen 2015; } \\
\text { Weinblatt 2012; Yamamoto (a) } \\
2014\end{array}$ & $0.03 \%(8)$ & $0.1 \%(1)$ & $\begin{array}{l}2.66(0.63 \\
\text { to } 11.16)\end{array}$ & $0(-1$ to 1$)$ & NS \\
\hline $\begin{array}{l}\text { Analysis } 50.18 \\
400 \mathrm{mg} \text { certolizumab } \\
\text { pegol }\end{array}$ & $\begin{array}{l}\text { Choy 2012; Fleischmann 2009; } \\
\text { Keystone 2008; Smolen 2009; } \\
\text { Østergaard } 2015\end{array}$ & $0.5 \%(5)$ & $0 \%(1)$ & $\begin{array}{l}1.87(0.31 \\
\text { to } 11.34)\end{array}$ & $0(-1$ to 1$)$ & NS \\
\hline
\end{tabular}

\begin{tabular}{|c|c|c|c|c|c|c|}
\hline Tuberculosis & & & & Peto OR & & \\
\hline $\begin{array}{l}\text { Analysis 50.20; } \\
200 \text { mg certolizumab } \\
\text { pegol }\end{array}$ & $\begin{array}{l}\text { Emery 2015; Keystone 2008; } \\
\text { NCT00993317; Smolen 2009; } \\
\text { Smolen 2015; Weinblatt } 2012\end{array}$ & $0.4 \%(7)$ & $0 \%(0)$ & $\begin{array}{l}1.90(0.55 \\
\text { to } 6.58)\end{array}$ & $\begin{array}{l}\text { Not calcu- } \\
\text { lated }\end{array}$ & NS \\
\hline $\begin{array}{l}\text { Analysis } 50.21 \\
400 \mathrm{mg} \text { certolizumab } \\
\text { pegol }\end{array}$ & $\begin{array}{l}\text { Fleischmann 2009; Keystone } \\
\text { 2008; Smolen } 2009\end{array}$ & $0.6 \%(5)$ & $0 \%(0)$ & $\begin{array}{l}4.55(0.71 \\
\text { to } 29.11)\end{array}$ & $\begin{array}{l}\text { Not calcu- } \\
\text { lated }\end{array}$ & NS \\
\hline
\end{tabular}

\section{Malignancies (neo- \\ plasias including lymphoma)}

\section{Peto OR}

\begin{tabular}{|c|c|c|c|c|c|c|}
\hline $\begin{array}{l}\text { Analysis } 50.23 \\
200 \mathrm{mg} \text { certolizumab } \\
\text { pegol }\end{array}$ & $\begin{array}{l}\text { Atsumi 2016; Emery 2015; Key- } \\
\text { stone 2008; NCT00993317; } \\
\text { Smolen 2009; Smolen 2015; } \\
\text { Weinblatt 2012; Yamamoto (a) } \\
2014\end{array}$ & $0.7 \%(19)$ & $0.7 \%(9)$ & $\begin{array}{l}0.92(0.40 \\
\text { to } 2.11)\end{array}$ & $0(-1$ to 1$)$ & NS \\
\hline
\end{tabular}


Table 7. Adverse events (Continued)

\begin{tabular}{|c|c|c|c|c|c|c|}
\hline $\begin{array}{l}\text { Analysis } 50.24 \\
400 \mathrm{mg} \text { certolizumab } \\
\text { pegol }\end{array}$ & $\begin{array}{l}\text { Fleischmann 2009; Keystone } \\
\text { 2008; Smolen } 2009\end{array}$ & $0.6 \%(5)$ & $0.4 \%(2)$ & $\begin{array}{l}1.26(0.26 \\
\text { to } 6.08)\end{array}$ & $0(-1$ to 1$)$ & NS \\
\hline $\begin{array}{l}\text { Infections and in- } \\
\text { festations }\end{array}$ & & & & $\mathbf{R R}$ & & \\
\hline $\begin{array}{l}\text { Analysis } 50.71 \\
200 \mathrm{mg} \text { certolizumab } \\
\text { pegol }\end{array}$ & $\begin{array}{l}\text { Atsumi 2016; Emery 2015; Key- } \\
\text { stone 2008; NCT00993317; } \\
\text { Smolen 2009; Smolen 2015; } \\
\text { Weinblatt 2012; Yamamoto (a) } \\
\text { 2014; Yamamoto (b) } 2014\end{array}$ & $35 \%(891)$ & $29 \%$ (389) & $\begin{array}{l}1.27(1.10 \\
\text { to } 1.46)\end{array}$ & 7 (1 to 13$)$ & $\begin{array}{l}14 \\
\text { (8 to } 58)\end{array}$ \\
\hline $\begin{array}{l}\text { Analysis } 50.72 \\
400 \mathrm{mg} \text { certolizumab } \\
\text { pegol }\end{array}$ & $\begin{array}{l}\text { Choy 2012; Keystone 2008; } \\
\text { Smolen 2009; Yamamoto (b) } \\
\text { 2014; Østergaard } 2015\end{array}$ & $34 \%$ (298) & $21 \%(183)$ & $\begin{array}{l}1.43(1.03 \\
\text { to } 1.98)\end{array}$ & 10 (1 to 20$)$ & 10 (5 to 44$)$ \\
\hline
\end{tabular}

\section{A P PE N DICES}

\section{Appendix 1. MEDLINE search strategy}

Database: Ovid MEDLINE(R) In-Process \& Other Non-Indexed Citations and Ovid MEDLINE(R) <1946 to Present>

Search Strategy:

1 (CDP870 or CDP 870 or "certolizumab pegol" or certolizumab or CDP-870 or cimzia).mp. (393)

2 ("Rheumatoid Arthritis" or (Caplan\$ and Syndrome?) or (Felty\$ and S?ndrome) or (Rheumatoid and Nodule?) or (Sjogren\$ and S? ndrome?) or (Sicca\$ and S?ndrome?) or (Ankylos\$ and Spondylit\$) or (Spondylarthritis and Ankylopoietica) or (Rheumatoid\$ and Spondylit \$) or (Bechterew\$ and Disease?) or (Marie-Struempell and Disease?) or (Adult and Onset and Still\$ and Disease?)).mp. (98824)

3 exp Arthritis, Rheumatoid/ (94528)

42 or $3(126632)$

51 and $4(131)$

6 Clinical trial.pt. (473242)

7 randomized.ab. (256728)

8 Placebo.ab. (140242)

9 dt.fs. (1573096)

10 randomly.ab. (187872)

11 trial.ab. (264547)

12 groups.ab. (1216413)

13 or/6-12 (3112539)

145 and 13 (114)

15 limit 14 to yr="2009 -Current" (99)

\section{Search date: 2009 - February 12, 2013}




\section{Appendix 2. Embase search strategy}

1. 'rheumatoid arthritis'/exp/

2. 'certolizumab pegol'/exp/

3. (CDP870 OR 'CDP 870' OR CDP-870 OR 'certolizumab pegol' OR certolizumab OR cimzia).mp.

4. 2 OR 3

5. 4 AND 1

6. random:.tw.

7. clinical trial:.mp.

8. exp health care quality

9. or/6-8

10. 5 AND 9

Search date: 2009 - February 12, 2013

\section{Appendix 3. CINAHL search strategy}

1.'rheumatoid arthritis'/exp/

2."rheumatoid arthritis".mp.

3. (CDP870 OR 'CDP 870' OR CDP-870 OR 'certolizumab pegol' OR certolizumab OR cimzia).mp.

4.(1 or 2$)$ and 3

5.exp prognosis

6.exp study design

7.random:.mp.

8.or/ 5-7

9.4 and 8

\section{Search date: 2009 - February 12, 2013}

\section{Appendix 4. Search strategy for CDSR and CENTRAL, HTA, DARE, NHS EED}

\section{Last search in November 2009}

\#1 certolizumab or cimzia

\#2 cdp870

$\# 3$ cdp next 870

\#4 (\#1 OR \#2 OR \#3)

\#5 rheumatoid next arthritis

\#6 MeSH descriptor Arthritis, Rheumatoid explode all trees

\#7 (\#5 OR \#6)

\#8 (\#4 AND \#7)

\section{Search date: 2009 - February 12, 2013}

\section{Appendix 5. SCOPUS search strategy}

Search strategy for benefits:

SCOPUS will be searched up to August of 2007, without limits of years:

KEY((certolizumab OR cimzia OR CDP-870 OR CDP870 OR "CDP 870") AND ("rheumatoid arthritis" ))

Web of Knowledge (WOK), was searched up to August of 2007, without limits of years. The search strategy is as follows:

topic=((certolizumab OR cimzia OR CDP-870 OR CDP870 OR "CDP 870") AND ("rheumatoid arthritis" )

Databases=MEDLINE, Current Contents Connect, Web of Science, Derwent Innovations Index, ISI Proceedings; Timespan=All Years

\section{Search date: 2009 - February 12, 2013}

\section{Appendix 6. TOXLINE (TOXNET) search strategy}

Search strategy for safety:

TOXLINE (TOXNET) will be searched up to October 2007. The search strategy will combine index and text terms for CDP870:

\#1. certolizumab OR "certolizumab pegol" OR CDP870 OR CDP-870 OR "CDP 870" OR cimzia

Search date: 2009 - February 12, 2013

\section{Appendix 7. Web of Knowledge}

Web of Knowledge (Science Citation Index and Social Science Citation Index) 1900 - February 2013 
Search terms: TS= (certolizumab OR cimzia OR or CDP870 OR cdp 870) and ("rheumatoid arthritis")

Search date: 2009-February 12, 2013

Appendix 8. Results of searches 2013

\begin{tabular}{|c|c|c|}
\hline Database name and coverage & Search date & Total Retrieved \\
\hline $\begin{array}{l}\text { Ovid MEDLINE(R) In-Process \& Other Non-Indexed Citations } \\
\text { and Ovid MEDLINE(R) } 1946 \text { to present }\end{array}$ & 2009-February 12, 2013 & 315 \\
\hline $\begin{array}{l}\text { Ovid Embase Classic+Embase } \\
1947 \text { to } 2013 \text { January } 16\end{array}$ & 2009 - February 12, 2013 & 1365 \\
\hline $\begin{array}{l}\text { Wiley Cochrane Library - CENTRAL } \\
\text { Issue } 1 \text { of } 12 \text { - Jan. } 2013\end{array}$ & 2009 - February 12, 2013 & 11 \\
\hline $\begin{array}{l}\text { EbscoHost CINAHL } \\
\text { 1982-January } 2013\end{array}$ & 2009 - February 12, 2013 & 32 \\
\hline Toxline (TOXNET) & $\begin{array}{l}2007 \text { - February 12, } \\
2013\end{array}$ & 34 \\
\hline Web of Knowledge & $\begin{array}{l}2009 \text { - February 12, } \\
2013\end{array}$ & 189 \\
\hline SCOPUS & 2009 - February 12, 2013 & 814 \\
\hline \multirow[t]{2}{*}{1966 to 2013 January } & Total & 2760 \\
\hline & Total without duplicates & 1300 \\
\hline
\end{tabular}

\section{Appendix 9. Searches updated to June 2014}

\begin{tabular}{|c|c|c|c|}
\hline Database name and coverage & Search date & Total Retrieved & $\begin{array}{l}\text { Total without Du- } \\
\text { plicates }\end{array}$ \\
\hline $\begin{array}{l}\text { Ovid MEDLINE(R) In-Process \& Other Non-Indexed Citations } \\
\text { and Ovid MEDLINE(R) }\end{array}$ & June 5, 2014 & 29 & 28 \\
\hline \multicolumn{4}{|l|}{ 2013-2014 } \\
\hline $\begin{array}{l}\text { Ovid Embase Classic+Embase } \\
\text { 2013-2014 }\end{array}$ & June 5, 2014 & 208 & 192 \\
\hline EbscoHost CINAHL & June 5, 2014 & 1 & 1 \\
\hline \multicolumn{4}{|l|}{ 2013-2014 } \\
\hline $\begin{array}{l}\text { Wiley Cochrane Library - CENTRAL } \\
\text { 2013-2014 }\end{array}$ & June 6, 2014 & 4 & 4 \\
\hline
\end{tabular}


(Continued)
SCOPUS
June 10, 2014
233
124

2013-2014

\begin{tabular}{|c|c|c|c|}
\hline Web of Knowledge & June 10, 2014 & 94 & 54 \\
\hline $2013-2014$ & Total & 569 & 403 \\
\hline
\end{tabular}

Appendix 10. Medline search strategy January 25, 2016

\section{MEDLINE Total retrieved $=\mathbf{7 0}$}

1. exp Arthritis, Rheumatoid/

2. ((Arthritis adj2 Rheumatoid) or (caplan* adj2 s?ndrome?) or (Familial and felty* and s?ndrome?) or (felty ${ }^{\star}$ adj2 s?ndrome?) or (Rheumatoid and arthritis and splenomegaly and neutropenia) or (rheumatoid and nodul*) or (rheumatoid and vasculiti*) or (sic$\mathrm{ca}^{\star}$ and s?ndrome?) or (sjogren ${ }^{\star}$ and s?ndrome?) or (adult* and onset and still ${ }^{\star}$ disease?) or (ankylo* and spondylarthriti ${ }^{\star}$ ) or (anky$10^{\star}$ and spondylistis) or (ankylosing and spondylorthriti ${ }^{\star}$ ) or (spondylitis and rheumatoid) or (bechterew* and disease?) or (marie* struempell and disease?) or (rheumatoid and spondylitis) or (spondylarthriti ${ }^{\star}$ and ankylo*)).mp.

3. exp Spondylitis, Ankylosing/

4. exp Certolizumab Pegol/

5. (pegylated tumo?r necrosis factor alpha antibody Fab fragment or pha 738144 or $\left(870^{\star}\right.$ adj1 cdp $)$ or cdp?870? or certolizumab pegol* $^{*}$ or cimzia* or pegol* adj1certolizumab).mp. [mp=title, abstract, original title, name of substance word, subject heading word, keyword heading word, protocol supplementary concept word, rare disease supplementary concept word, unique identifier]

6.4 or 5

7. 1 or 2 or 3

8. 6 and 7

9. limit 8 to $y r=" 2014$-Current"

10. Clinical trial.pt. or randomized.ab. or placebo.ab. or dt.fs. or randomly.ab. or trial.ab. or groups.ab.

11. 9 and 10

Appendix 11. Embase search strategy January 25, 2016

\section{EMBASE Total retrieved $=304$}

1. ((Arthritis adj2 Rheumatoid) or (caplan* adj2 s?ndrome?) or (Familial and felty* and s?ndrome?) or (felty* adj2 s?ndrome?) or (Rheumatoid and arthritis and splenomegaly and neutropenia) or (rheumatoid and nodul*) or (rheumatoid and vasculiti*) or (sic$\mathrm{ca}^{\star}$ and s?ndrome?) or (sjogren ${ }^{\star}$ and s?ndrome?) or (adult* and onset and still ${ }^{\star}$ disease?) or (ankylo* and spondylarthriti*) or (anky$10^{\star}$ and spondylistis) or (ankylosing and spondylorthriti ${ }^{\star}$ ) or (spondylitis and rheumatoid) or (bechterew ${ }^{\star}$ and disease?) or (marie* struempell and disease?) or (rheumatoid and spondylitis) or (spondylarthriti ${ }^{\star}$ and ankylo*)).mp.

2. (arthritis deformans or arthrosis deformans or (beauvais adj2 disease?) or (chronic adj2 poly?arthritis) or (chronic adj2 rheumatoid adj2 arthritis) or inflammatory arthritis or (polyarthritis adj2 primary adj2 chronic) or (progressive adj2 polyarthritis adj2 chronic) or rheumarthritis or rheumatism, chronic articular or (rheumatic adj2 arthritis) or (rheumatic adj1 polyarthritis)).mp.

\section{1 or 2}


4. exp rheumatoid arthritis/

5. exp pneumoconiosis/

6. exp Felty syndrome/

7. exp rheumatoid nodule/

8. exp rheumatoid vasculitis/

9. exp Sjoegren syndrome/

10. exp adult onset Still disease/

11. exp ankylosing spondylitis/

12. or/4-11

13. 3 or 12

14. exp certolizumab pegol/

15. (pegylated tumo?r necrosis factor alpha antibody Fab fragment or pha?738144 or $\left(870^{\star}\right.$ adj1 cdp*) or cdp?870? or certolizumab

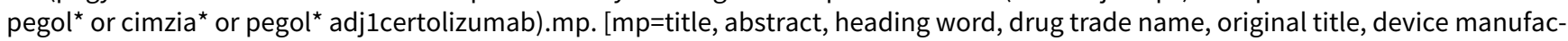
turer, drug manufacturer, device trade name, keyword]

16. 14 or 15

17.13 and 16

18. limit 17 to $y r=" 2014$-Current"

19. random:.tw. or clinical trial:.mp. or exp health care quality/

20. 18 and 19

Appendix 12. Central search strategy January 22, 2016

\section{COCHRANE retrieved $=36$}

\#1 $\left(870^{\star}\right.$ next cdp*) or cdp?870? or certolizumab or cimzia*

\#2 MeSH descriptor: [Arthritis, Rheumatoid] explode all trees

\#3 ((Arthritis next Rheumatoid) or (caplan* next syndrome*) or (Familial and felty* and syndrome*) or (felty* next syndrome*) or (Rheumatoid and arthritis and splenomegaly and neutropenia) or (rheumatoid and nodul*) or (rheumatoid and vasculiti*) or (sic$\mathrm{ca}^{\star}$ and syndrome ${ }^{\star}$ ) or (sjogren ${ }^{\star}$ and $\mathrm{s}^{\star}$ ndrome ${ }^{\star}$ ) or (adult* and onset and still disease $^{\star}$ ) or (ankylo* and spondylarthriti $\left.{ }^{\star}\right)$ or (anky$10^{\star}$ and spondylistis) or (ankylosing and spondylorthriti ${ }^{\star}$ ) or (spondylitis and rheumatoid) or (bechterew* and disease ${ }^{\star}$ ) or (marie* struempell and disease*) or (rheumatoid and spondylitis) or (spondylarthriti* and ankylo*))

\#4 arthritis deformans or arthrosis deformans or (beauvais next disease*) or (chronic next polyarthritis) or (chronic next rheumatoid next arthritis) or inflammatory arthritis or (polyarthritis next primary next chronic) or (progressive next polyarthritis next chronic) or rheumarthritis or rheumatism, chronic articular or (rheumatic next arthritis) or (rheumatic next polyarthritis)

\#5 \#2 or \#3 or \#4

\#6 \#1 and \#5

\#7 ((Arthritis next Rheumatoid) or (caplan ${ }^{\star}$ next syndrome*) or (Familial and felty ${ }^{\star}$ and syndrome*) or (felty ${ }^{\star}$ next syndrome ${ }^{\star}$ ) or (Rheumatoid and arthritis and splenomegaly and neutropenia) or (rheumatoid and nodul*) or (rheumatoid and vasculiti*) or (sic$\mathrm{ca}^{\star}$ and syndrome ${ }^{\star}$ ) or (sjogren ${ }^{\star}$ and s* ${ }^{\star} d r o m e^{\star}$ ) or (adult* and onset and still ${ }^{\star}$ disease ${ }^{\star}$ ) or (ankylo* and spondylarthriti ${ }^{\star}$ ) or (anky- 
lo* and spondylistis) or (ankylosing and spondylorthriti ${ }^{\star}$ ) or (spondylitis and rheumatoid) or (bechterew* and disease ${ }^{\star}$ ) or (marie* struempell and disease* ${ }^{\star}$ or (rheumatoid and spondylitis) or (spondylarthriti* and ankylo*))

\title{
Appendix 13. WOK search strategy January 22, 2016
}

\section{wOK retrieved $=$}

Web of Knowledge (Science Citation Index and Social Science Citation Index) 1900 - January 2016

\begin{abstract}
\#1 Topic: $\left(\left(()\left(\left(()\left(\left(()\left(\left(\left(\left(\left(\right.\right.\right.\right.\right.\right.\right.\right.\right.\right.\right.$ Arthritis NEAR Rheumatoid) OR (caplan* NEAR s?ndrome?)) OR ((Familial AND felty $\left.{ }^{\star}\right)$ AND s?ndrome?)) OR (felty ${ }^{\star}$ NEAR s?ndrome?)) OR (((Rheumatoid AND arthritis) AND splenomegaly) AND neutropenia)) OR (rheumatoid AND nodul $\left.\left.{ }^{\star}\right)\right)$ OR (rheumatoid AND vasculiti $)$ ) OR (sicca* AND s?ndrome?)) OR (sjogren* AND s?ndrome?)) OR ((adult* AND onset) AND still* disease?)) OR (ankylo* AND spondylarthriti*)) OR (ankylo* AND spondylitis)) OR (ankylosing AND spondylorthriti*)) OR (spondylitis AND rheumatoid)) OR (bechterew* AND disease?)) OR (marie\$struempell AND disease?)) OR ((rheumatoid AND spondylitis spondylarthriti^) AND ankylo*)) OR (((()(((((arthritis deformans OR arthrosis deformans) OR (beauvais NEAR disease?)) OR (chronic NEAR poly?arthritis)) OR ((chronic NEAR rheumatoid) NEAR arthritis)) OR inflammatory arthritis) OR ((polyarthritis NEAR primary) NEAR chronic)) OR ((progressive NEAR polyarthritis) NEAR chronic)) OR rheumarthritis) OR rheumatism, chronic articular) OR (rheumatic NEAR arthritis)) OR (rheumatic NEAR polyarthritis)))
\end{abstract}

limit=2016

\#2 Topic: ((pegylated tumo?r necrosis factor alpha antibody Fab fragment or pha?738144 or (870* NEAR cdp*) or cdp?870? or certolizumab pegol* or cimzia* or (pegol ${ }^{\star}$ NEAR certolizumab)))

Time $=2016$

\#3 \#2 AND \#1

\#4 Refined by: Document (CLINICAL TRIAL)

\section{Appendix 14. Search strategy Clinicaltrials.gov}

certolizumab pegol AND Rheumatoid arthritis

\section{Appendix 15. Searches on International Clinical Trials Registry Platform}

certolizumab pegol/Intervention AND Rheumatoid arthritis/Condition | Studies updated from to 12/31/2016

\section{Appendix 16. Results of searches updated to January 2016}

\begin{tabular}{lll}
\hline Database name and coverage & Search date & Total Retrieved \\
\hline Ovid MEDLINE(R) In-Process \& Other Non-Indexed Citations and Ovid & January 25, 2016 & 70 \\
MEDLINE(R) & & 70 \\
\end{tabular}


2014-2016

Wiley Cochrane Library - CENTRAL 2014-2016

Web of Knowledge

2014-2016
January 25, 2016

January 25, 2016

January 25, 2016

28

2014-2016
36

25

\section{Appendix 17. Results of searches updated to September 2016}

\begin{tabular}{|c|c|c|}
\hline Database name and coverage & Search date & Total Retrieved \\
\hline $\begin{array}{l}\text { Ovid MEDLINE(R) In-Process \& Other Non-Indexed Citations and Ovid } \\
\text { MEDLINE(R) }\end{array}$ & September 26, 2016 & 21 \\
\hline \multicolumn{3}{|l|}{ From 1 January 2016 to 26 September 2016} \\
\hline $\begin{array}{l}\text { Ovid Embase Classic+Embase } \\
\text { 2014-2016 }\end{array}$ & September 26, 2016 & 97 \\
\hline \multicolumn{3}{|l|}{ Embase Classic+Embase } \\
\hline \multicolumn{3}{|l|}{1947 to 201626 September 2016} \\
\hline Wiley Cochrane Library - CENTRAL & September 26, 2016 & 4 \\
\hline \multicolumn{3}{|l|}{ From 1 January 2016 to 26 September 2016} \\
\hline Web of Knowledge & September 27, 2016 & 2 \\
\hline \multicolumn{3}{|l|}{ From 1 January 2016 to 27 September 2016} \\
\hline Clinicaltrials.gov & October 1, 2016 & 28 \\
\hline \multicolumn{3}{|l|}{ From 1 January 2016 to 27 September 2016} \\
\hline ICTRP & Decemeber 31, 2016 & 42 \\
\hline \multicolumn{3}{|l|}{ to 31 December 2016} \\
\hline & Total & 194 \\
\hline
\end{tabular}

WHAT'S NEW 


\begin{tabular}{lll}
\hline Date & Event & Description \\
\hline 26 September 2016 & $\begin{array}{l}\text { New citation required but conclusions } \\
\text { have not changed }\end{array}$ & $\begin{array}{l}\text { For this update, we changed the authors in the team: José Anto- } \\
\text { nio Bernal is new. }\end{array}$ \\
\hline 26 September 2016 New search has been performed & $\begin{array}{l}\text { We include } 14 \text { trials, } 3 \text { more than in the previous review. All of } \\
\text { them have information about harm, but we have only pooled } 12 \\
\text { trials. 12 trials gave information on benefits, but we have only } \\
\text { pooled } 11 . \text { We have more information regarding the quality of tri- } \\
\text { als because UCB } \odot \text { gave us further data. We have used this infor- } \\
\text { mation to update our assessment of the quality of trials. }\end{array}$ \\
& $\begin{array}{l}\text { For the new trials we obtained unpublished data about the quali- } \\
\text { ty and results, including withdrawals and serious adverse events } \\
\text { from clinicaltrials.gov. We checked this information with UCB }{ }^{\odot} .\end{array}$ \\
&
\end{tabular}

\section{H IST ORY}

Protocol first published: Issue 1, 2009

Review first published: Issue 2, 2011

\begin{tabular}{lll}
\hline Date & Event & Description \\
\hline 3 April 2008 & New search has been performed & CMSG ID: C001-R \\
\hline
\end{tabular}

\section{CONTRIBUTIONSOF AUTHORS}

Design the protocol: Juan Cabello; Vicente Ruiz; Amanda Burls Write the Background: Paloma Vela and José Antonio Bernal

Develop the search strategy: Tamara Rader

Trial search (two people): Vicente Ruiz; Sylvia Bort

Obtain copies of the trials: Sylvia Bort

Selection of trials for inclusion (two plus one): Vicente Ruiz; Sylvia Bort. If data discrepancies were to be resolved by involvement of a third person: Amanda Burls

Retrieval of trial data on benefits (two plus one): Vicente Ruiz; Sylvia Bort. If data discrepancies were to be resolved by involvement of a third person: Amanda Burls

Data input in Review Manager 5: Sylvia Bort

Carry out analyses: Vicente Ruiz

Interpret analyses: Vicente Ruiz

Write up results: Vicente Ruiz; ; Paloma Vela; Amanda Burls; Juan Cabello; Sylvia Bort; José Antonio Bernal

Update review: Vicente Ruiz; José Antonio Bernal; Paloma Vela

\section{DECLARATIONS OF INTEREST}

UCB paid Dr Vicente Ruiz's registration for the Cochrane meeting in Madrid 2011. In 2011 and 2012 he attended the UCB Advisory Board meetings in Madrid when the sponsor explained details and preliminary results for the new trials of certolizumab pegol. He did not receive any economic or other kind of compensation for these meetings.

Burls A: none known.

Cabello JB: none known.

Vela Casasempere P: "I have participated as a member of advisory boards for Roche and Pfizer. I have also received fees for development of educational presentations for Roche, Abbvie, UCB, BMS and MSD, and travel and accommodations expenses to attend scientific meetings from Pfizer, Abbvie and Roche".

Bort-Marti S: none known. 
Bernal JA: "I have received travel and accommodations expenses to attend scientific meetings from Pfizer and MSD".

\section{SOURCES OF SUPPORT}

\section{Internal sources}

- Grant from, Spain.

Instituto de Salud Carlos III. Ministerio de Sanidad. FIS number PI08_90617 in the first previous systematic review.

\section{External sources}

- No sources of support supplied

\section{DIFFERENCES BETWEEN PROTOCOLANDREVIEW}

\section{Types of participants}

Protocol specified adults with RA who have persistent disease activity, despite current or previous use of conventional DMARDs. We have included two studies (Atsumi 2016; Emery 2015) with MTX-naïve participants. This approach is now considered justified in early RA, as data are available showing differences in outcome when remission is obtained as soon as possible.

\section{Types of outcomes}

In the protocol we stated that we "We will review also this list of adverse events: headache, fever, blood disorders, laboratory disorders, abdominal pain, nasopharyngitis, nausea, respiratory tract infections, urinary tract infections, neck pain, congestive heart failure, pruritus and anaphylaxis". In the previous update and with the approval of the editors, we made serious adverse events, DAS and radiological changes of major outcomes. DAS28 is used as an indicator of RA disease activity and a response to treatment.

\section{Searches}

We did not perform the searches in CINHAL nor in SCOPUS, because although we covered these database in the original protocol they did not yield any additional information in our previous searches. Following MECIR criteria, we conducted searches on the WHO international clinical trials registry platform.

\section{Data synthesis}

We decided to perform a random-effects model analysis, despite low values of the 12 statistic. Although the trials used the same drug, there was clear clinical heterogeneity (different doses, allowing MTX or not, different follow-up, different duration of RA, etc.).

\section{Subgroup analysis}

Subgroup analyses were planned for the duration of the illness (approximately three years evolution), participants' sex, drug dose and administration, and methodological quality; but we performed only a subgroup analysis for dosage of certolizumab pegol. All Phase III trials were conducted in participants with a long mean duration of RA (from 6.1 to 9.5 years) and we could not obtain any data categorised by sex. All Phase III trials allowed previous DMARD treatment (mean 1.2 to 2 years). We rated all the Phase III trials included in the metaanalysis as high quality and so we did not perform subgroup analysis based on methodological quality.

\section{N DEX TERMS}

\section{Medical Subject Headings (MeSH)}

Antirheumatic Agents [adverse effects] [ ${ }^{\star}$ therapeutic use]; Arthritis, Rheumatoid [ ${ }^{\star}$ drug therapy]; Certolizumab Pegol [adverse effects] [*therapeutic use]; Immunoglobulin Fab Fragments [ ${ }^{\star}$ therapeutic use]; Methotrexate [therapeutic use]; Randomized Controlled Trials as Topic; Withholding Treatment [statistics \& numerical data]

\section{MeSH check words}

Adult; Humans 